\title{
Integrated and Comprehensive Pain Management Programs: Effectiveness and Harms
}


Number 251

\section{Integrated and Comprehensive Pain Management Programs: Effectiveness and Harms}

Prepared for:

Agency for Healthcare Research and Quality

U.S. Department of Health and Human Services

5600 Fishers Lane

Rockville, MD 20857

www.ahrq.gov

Contract No. 75Q80120D00006

Prepared by:

Pacific Northwest Evidence-based Practice Center

Portland, OR

Investigators:

Andrea C. Skelly, Ph.D., M.P.H.

Roger Chou, M.D.

Joseph R. Dettori, Ph.D., M.P.H., M.P.T.

Erika D. Brodt, B.S.

Andrea Diulio-Nakamura, Ph.D.

Kim Mauer, M.D.

Rongwei Fu, Ph.D.

Yun $\mathrm{Yu}, \mathrm{M} . \mathrm{S}$.

Ngoc Wasson, M.P.H.

Shelby Kantner, B.A.

Shay Stabler-Morris, B.A.

AHRQ Publication No. 22-EHC002

October 2021 
This report is based on research conducted by the Pacific Northwest Evidence-based Practice Center (EPC) under contract to the Agency for Healthcare Research and Quality (AHRQ), Rockville, MD (Contract No. 75Q80120D00006). The findings and conclusions in this document are those of the authors, who are responsible for its contents; the findings and conclusions do not necessarily represent the views of AHRQ. Therefore, no statement in this report should be construed as an official position of AHRQ or of the U.S. Department of Health and Human Services.

\section{None of the investigators have any affiliations or financial involvement that conflicts with the material presented in this report.}

The information in this report is intended to help healthcare decision makers - patients and clinicians, health system leaders, and policymakers, among others-make well-informed decisions and thereby improve the quality of healthcare services. This report is not intended to be a substitute for the application of clinical judgment. Anyone who makes decisions concerning the provision of clinical care should consider this report in the same way as any medical reference and in conjunction with all other pertinent information, i.e., in the context of available resources and circumstances presented by individual patients.

This report is made available to the public under the terms of a licensing agreement between the author and the Agency for Healthcare Research and Quality. This report may be used and reprinted without permission except those copyrighted materials that are clearly noted in the report. Further reproduction of those copyrighted materials is prohibited without the express permission of copyright holders.

AHRQ or U.S. Department of Health and Human Services endorsement of any derivative products that may be developed from this report, such as clinical practice guidelines, other quality enhancement tools, or reimbursement or coverage policies, may not be stated or implied.

AHRQ appreciates appropriate acknowledgment and citation of its work. Suggested language for acknowledgment: This work was based on an evidence report, Integrated and Comprehensive Pain Management Programs: Effectiveness and Harms, by the Evidence-based Practice Center Program at the Agency for Healthcare Research and Quality (AHRQ).

Suggested citation: Skelly AC, Chou R, Dettori JR, Brodt ED, Diulio-Nakamura A, Mauer K, Fu R, Yu Y, Wasson N, Kantner S, Stabler-Morris S. Integrated and Comprehensive Pain Management Programs: Effectiveness and Harms. Comparative Effectiveness Review No. 251. (Prepared by the Pacific Northwest Evidence-based Practice Center under Contract No. 75Q80120D00006.) AHRQ Publication No. 22-EHC002. Rockville, MD: Agency for Healthcare Research and Quality; October 2021. DOI: https://doi.org/10.23970/AHRQEPCCER251. Posted final reports are located on the Effective Health Care Program search page. 


\section{Preface}

The Agency for Healthcare Research and Quality (AHRQ), through its Evidence-based Practice Centers (EPCs), sponsors the development of evidence reports and technology assessments to assist public- and private-sector organizations in their efforts to improve the quality of healthcare in the United States.

The Centers for Disease Control and Prevention requested this report from the EPC Program at AHRQ. AHRQ assigned this report to the following EPC: Pacific Northwest Evidence-based Practice Center (Contract Number: 75Q80120D00006).

The reports and assessments provide organizations with comprehensive, evidence-based information on common medical conditions and new healthcare technologies and strategies. They also identify research gaps in the selected scientific area, identify methodological and scientific weaknesses, suggest research needs, and move the field forward through an unbiased, evidence-based assessment of the available literature. The EPCs systematically review the relevant scientific literature on topics assigned to them by AHRQ and conduct additional analyses when appropriate prior to developing their reports and assessments.

To bring the broadest range of experts into the development of evidence reports and health technology assessments, AHRQ encourages the EPCs to form partnerships and enter into collaborations with other medical and research organizations. The EPCs work with these partner organizations to ensure that the evidence reports and technology assessments they produce will become building blocks for healthcare quality improvement projects throughout the Nation. The reports undergo peer review and public comment prior to their release as a final report.

AHRQ expects that the EPC evidence reports and technology assessments, when appropriate, will inform individual health plans, providers, and purchasers as well as the healthcare system as a whole by providing important information to help improve healthcare quality.

If you have comments on this systematic review, they may be sent by mail to the Task Order Officer named below at: Agency for Healthcare Research and Quality, 5600 Fishers Lane, Rockville, MD 20857, or by email to epc@ahrq.hhs.gov.

David Meyers, M.D. Acting Director Agency for Healthcare Research and Quality

Craig A. Umscheid, M.D., M.S.

Director

Evidence-based Practice Center Program

Center for Evidence and Practice Improvement Agency for Healthcare Research and Quality
Arlene S. Bierman, M.D., M.S.

Director

Center for Evidence and Practice Improvement

Agency for Healthcare Research and Quality

Suchitra Iyer, Ph.D.

Task Order Officer

Center for Evidence and Practice

Improvement

Agency for Healthcare Research and Quality 


\section{Investigator Affiliations}

Andrea C. Skelly, Ph.D., M.P.H.

Aggregate Analytics, Inc.

Fircrest, WA

Roger Chou, M.D.

Department of Medical Informatics and Clinical Epidemiology

Oregon Health \& Science University

Portland, OR

Joseph R. Dettori, Ph.D., M.P.H, M.P.T.

Spectrum Research, Inc.

Tacoma, WA

Erika D. Brodt, B.S.

Aggregate Analytics, Inc.

Fircrest, WA

Andrea Diulio-Nakamura, Ph.D.

Comprehensive Pain Center

Oregon Health \& Science University

Portland, OR

Kim Mauer, M.D.

Comprehensive Pain Center

Oregon Health \& Science University

Portland, OR

Rongwei Fu, Ph.D.

OHSU-PSU School of Public Health

Oregon Health \& Science University

Portland, OR

Yun Yu, M.S.

OHSU-PSU School of Public Health

Oregon Health \& Science University

Portland, OR

Ngoc Wasson, M.P.H.

Department of Medical Informatics and Clinical Epidemiology

Oregon Health \& Science University

Portland, OR

Shelby Kantner, B.A.

Aggregate Analytics, Inc.

Fircrest, WA 
Shay Stabler-Morris, B.A.

Aggregate Analytics, Inc.

Fircrest, WA

\section{Acknowledgments}

The authors gratefully acknowledge the following individuals for their contributions to this project: Elaine Graham, M.L.S., for program guidance and collaborative support; Tracy Dana, M.L.S., for assistance with the literature search; Leah Williams, B.S., for editorial support; our Task Order Officer, Suchitra Iyer, Ph.D., for her support and guidance in developing this report; and EPC Associate Editor Eric Bass, M.D., M.P.H., for his review of this report.

\section{Technical Expert Panel}

In designing the study questions and methodology at the outset of this report, the EPC consulted several technical and content experts. Broad expertise and perspectives were sought. Divergent and conflicted opinions are common and perceived as healthy scientific discourse that results in a thoughtful, relevant systematic review. Therefore, in the end, study questions, design, methodologic approaches, and/or conclusions do not necessarily represent the views of individual technical and content experts.

Technical Experts must disclose any financial conflicts of interest greater than $\$ 5,000$ and any other relevant business or professional conflicts of interest. Because of their unique clinical or content expertise, individuals with potential conflicts may be retained. The TOO and the EPC work to balance, manage, or mitigate any potential conflicts of interest identified.

The list of Technical Experts who provided input to this report follows:

G. Caleb Alexander, M.D., M.S.*

Professor of Epidemiology and Medicine

Co-Director, Center for Drug Safety and Effectiveness

Johns Hopkins Bloomberg School of Public Health

Baltimore, MD

Kelli Allen, Ph.D.*

Research Professor of Medicine

Division of Rheumatology, Allergy and Immunology

University of North Carolina School of Medicine

Chapel Hill, NC

Center of Innovation to Accelerate Discovery and Practice Transformation

Durham VA Health Care System

Durham, NC

Robert Bonakdar, M.D.*

Director of Pain Management

Scripps Center for Integrative Medicine

La Jolla, CA 
Daniel Clauw, M.D.*

Professor of Anesthesiology, Rheumatology and Psychiatry

Director of the Chronic Pain and Fatigue Research Center

University of Michigan

Ann Arbor, MI

Christine Goertz, D.C., Ph.D.

Professor of Musculoskeletal Research and Chiropractic Care

Director of System Development and Coordination for Spine Health

Department of Orthopedic Surgery

Duke University

Durham, NC

Debra Gordon, R.N., D.N.P.*

Department of Anesthesiology and Pain Medicine

Harborview Integrated Pain Care Program

University of Washington

Seattle, WA

Kurt Kroenke, M.D.

Professor of Medicine

Center for Health Services and Outcomes Research

Regenstrief Institute

Indiana University

Indianapolis, IN

Shari Ling, M.D.

Geriatrics and Rheumatology

Deputy Chief Medical Officer, Center for Clinical Standards and Quality

Centers for Medicare \& Medicaid Services

Baltimore, MD

Douglas Olson, M.D.

Primary Care and Addiction Medicine

Chief Medical Officer, Medicaid and CHIP

Centers for Medicare \& Medicaid Services

Baltimore, MD

Joanna Starrels, M.D., M.S.

Associate Professor, Department of Medicine

Albert Einstein College of Medicine

Bronx, NY

Mark Sullivan, M.D., Ph.D.*

Professor of Psychiatry and Behavioral Sciences 
Adjunct Professor of Anesthesiology and Pain Medicine

Adjunct Professor of Bioethics and Humanities

University of Washington

Seattle, WA

*Provided input on Draft Report.

\section{Peer Reviewers}

Prior to publication of the final evidence report, EPCs sought input from independent Peer Reviewers without financial conflicts of interest. However, the conclusions and synthesis of the scientific literature presented in this report do not necessarily represent the views of individual reviewers.

Peer Reviewers must disclose any financial conflicts of interest greater than $\$ 5,000$ and any other relevant business or professional conflicts of interest. Because of their unique clinical or content expertise, individuals with potential nonfinancial conflicts may be retained. The TOO and the EPC work to balance, manage, or mitigate any potential nonfinancial conflicts of interest identified.

The list of Peer Reviewers follows:

Beth Darnall, Ph.D.

Associate Professor of Anesthesiology

Director of the Stanford Pain Relief Innovations Lab

Stanford Pain Management Center

Stanford University

Redwood City, CA

Steven Dobscha, M.D.

Professor of Psychiatry

Oregon Health and Science University

Director, VA Center to Improve Veteran Involvement in Care

Portland, OR

Emily Hurstak, M.D., M.P.H.

Assistant Professor of Medicine

Boston University School of Medicine

Boston, MA

Katherine Mackey, M.D.

Internal Medicine

Allopathic and Osteopathic Medicine

Veterans Affairs Medical Center

Portland, OR

Terri Pigott, Ph.D. 
Professor

School of Public Health

Georgia State University

Atlanta, GA 


\section{Integrated and Comprehensive Pain Management Programs: Effectiveness and Harms}

\section{Structured Abstract}

Objectives. To evaluate the effectiveness and harms of pain management programs that are based on the biopsychosocial model of care, particularly in the Medicare population.

Data sources. Electronic databases $\left(\right.$ Ovid $^{\circledR} \mathrm{MEDLINE}^{\circledR}$, PsycINFO $^{\circledR}, \mathrm{CINAHL}^{\circledR}, \mathrm{Cochrane}$ Central Register of Controlled Trials, and Cochrane Database of Systematic Reviews) from 1989 to May 24, 2021; reference lists; and a Federal Register notice.

Review methods. Given lack of consensus on terminology and program definition for pain management, we defined programs as integrated (based in and integrated with primary care) and comprehensive (referral based and separate from primary care) pain management programs (IPMPs and CPMPs). Using predefined criteria and dual review, we selected randomized controlled trials (RCTs) comparing IPMPs and CPMPs with usual care or waitlist, physical activity, pharmacologic therapy, and psychological therapy in patients with complex acute/subacute pain or chronic nonactive cancer pain. Patients needed to have access to medication support/review, psychological support, and physical function support in programs. Meta-analyses were conducted to improve estimate precision. We classified the magnitude of effects as small, moderate, or large based on predefined criteria. Strength of evidence (SOE) was assessed for the primary outcomes of pain, function, and change in opioid use.

Results. We included 57 RCTs; 8 evaluated IPMPs and 49 evaluated CPMPs. Compared with usual care or waitlist, IPMPs were associated with small improvements in pain in the short and intermediate term (SOE: low) and in function in the short term (SOE: moderate), but there were no clear differences at other time points. CPMPs were associated with small improvements in pain immediately postintervention (SOE: moderate) but no differences in the short, intermediate, and long term (SOE: low); for function, improvements were moderate immediately postintervention and in the short term; there were no differences in the intermediate or long term (SOE: low at all time points). CPMPs were associated with small to moderate improvements in function and pain versus pharmacologic treatment alone at multiple time frames (SOE: moderate for function intermediate term; low for pain and function at all other times), and with small improvements in function but no improvements in pain in the short term when compared with physical activity alone (SOE: moderate). There were no differences between CPMPs and psychological therapy alone at any time (SOE: low). Serious harms were not reported, although evidence on harms was insufficient. The mean age was 57 years across IPMP RCTs and 45 years across CPMP RCTs. None of the trials specifically enrolled Medicare beneficiaries. Evidence on factors related to program structure, delivery, coordination, and components that may impact outcomes is sparse and there was substantial variability across studies on these factors.

Conclusions. IPMPs and CPMPs may provide small to moderate improvements in function and small improvements in pain in patients with chronic pain compared with usual care. Formal pain management programs have not been widely implemented in the United States for general 
populations or the Medicare population. To the extent that programs are tailored to patients' needs, our findings are potentially applicable to the Medicare population. Programs that address a range of biopsychosocial aspects of pain, tailor components to patient need, and coordinate care may be of particular importance in this population. 


\section{Contents}

Evidence Summary ........................................................................................................................... ES-1

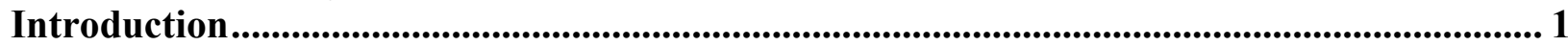

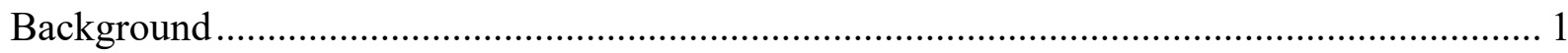

Purpose and Scope of the Systematic Review ………….................................................... 3

Methods............................................................................................................................................. 4

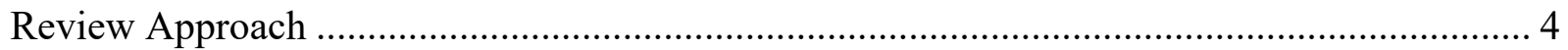

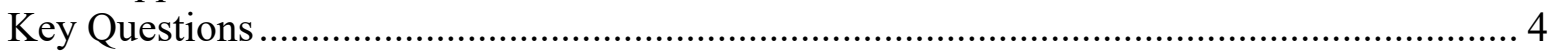

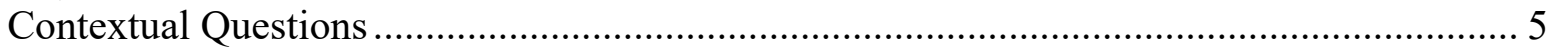

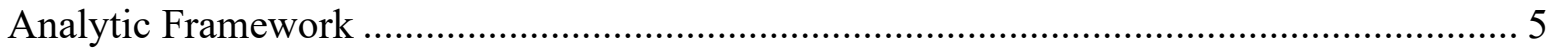

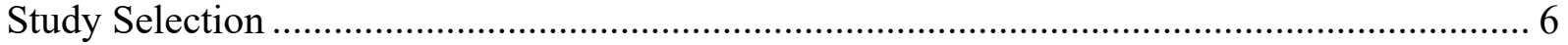

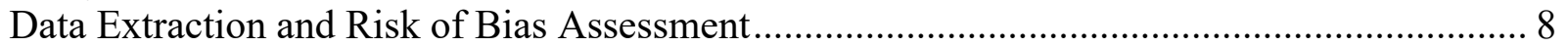

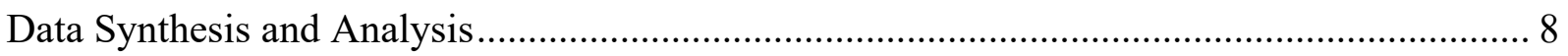

Grading the Strength of the Body of Evidence.................................................................... 9

Results ............................................................................................................................................ 10

Key Question 1. Effectiveness and Harms of Integrated or Comprehensive Pain Management

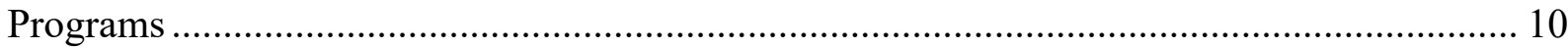

Integrated Pain Management Programs ………………............................................... 10

Comprehensive Pain Management Programs ……………………………………….... 18

Key Question 2. Program Factors ...................................................................................... 38

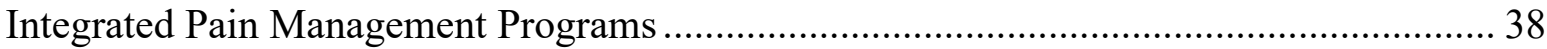

Comprehensive Pain Management Programs …………………………………………... 40

Contextual Question 1. Pain Management Program Types ........................................................ 51

Program Definitions ………………………………………………………………..... 51

Program Components................................................................................................. 51

What Pain Management Models or Mechanisms Are Most Commonly Used in Clinical

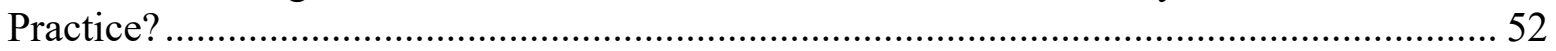

What Types of Programs/Models May Be Most Applicable to Medicare Beneficiaries?.... 52

What Theoretical Advantages and Disadvantages Do Various Programs/Models Have Compared With Current Practice? ................................................................................... 52

Are There Any Potential Safety Issues? ......................................................................... 53

Contextual Question 2. Cost Effectiveness....................................................................... 53

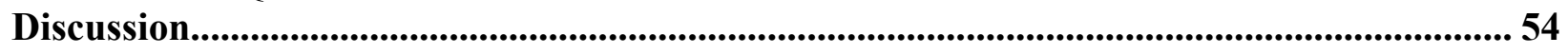

Findings in Relation to the Decisional Dilemmas .............................................................. 54

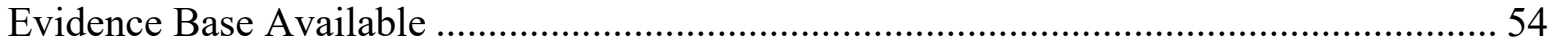

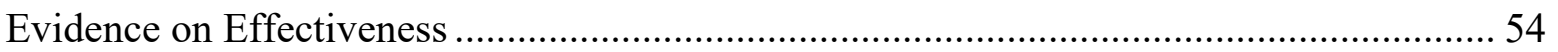

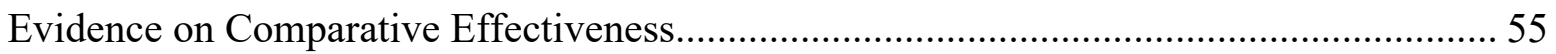

Evidence on Factors Related to Care Delivery or IPMP/CPMP Components ...................... 58

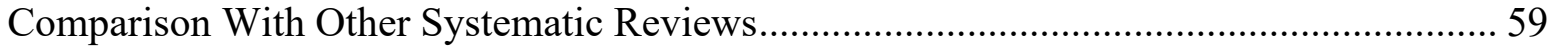

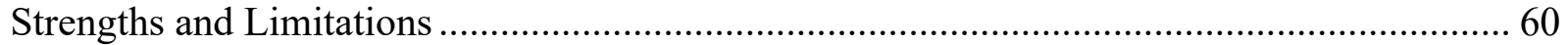

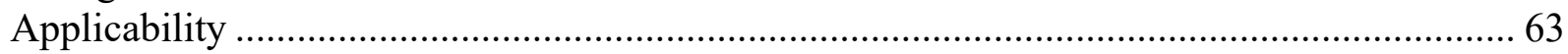

Implications for Clinical Practice, Education, Research, or Health Policy .............................. 64

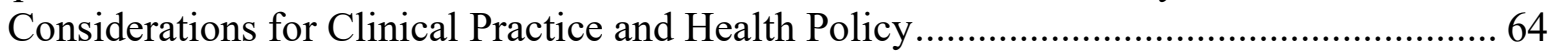

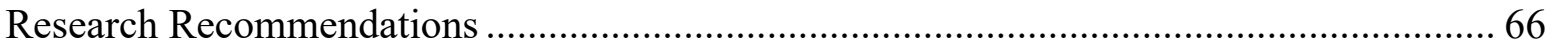

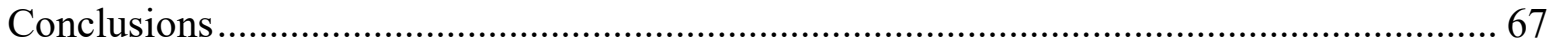

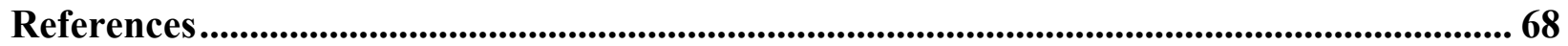




\section{Tables}

Table A. Summary of outcomes with at least low strength of evidence for IPMPs for noncancer pain: Key Question 1 (pain, function, opioid use) ........................................................ ES-2 Table B. Summary of outcomes with at least low strength of evidence for CPMPs for noncancer pain: Key Question 1 (pain, function, opioid use). ES-3

Table 1. Criteria for population, intervention, comparison, and outcomes of eligible studies. 7 Table 2. Summary of evidence of IPMPs for noncancer pain: Key Question 1 (pain, function, opioid use)..... 56 Table 3. Summary of evidence of CPMPs for noncancer pain: Key Question 1 (pain, function, opioid use) 56 Table 4. Overview of reported treatment-related adverse events/harms from included trials...... 57

\section{Figures}

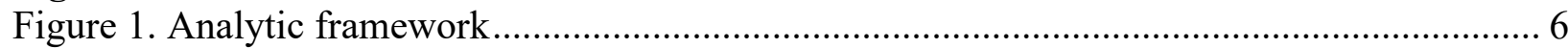

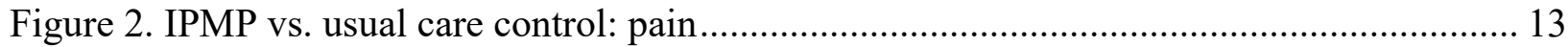

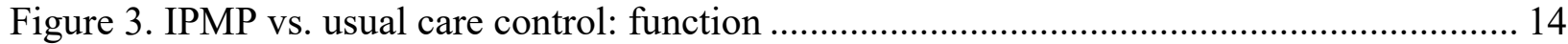

Figure 4. IPMP vs. usual care control: function success ................................................ 15

Figure 5. CPMP versus usual care or waitlist control: pain................................................... 22

Figure 6. CPMP versus usual care or waitlist control: function ............................................. 23

Figure 7. CPMP versus physical activity: pain ................................................................ 27

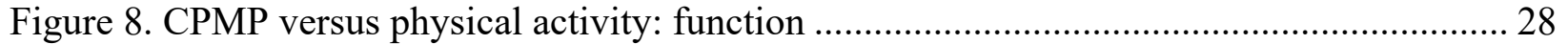

Figure 9. CPMP versus pharmacologic therapy alone: pain................................................. 32

Figure 10. CPMP versus pharmacologic therapy alone: function ...................................... 33

Figure 11. CPMP versus psychological therapy alone: pain ............................................. 36

Figure 12. CPMP versus psychological therapy alone: function .......................................... 37

\section{Appendixes}

Appendix A. Methods

Appendix B. Results Overview

Appendix C. Contextual Questions

Appendix D. Included Studies List

Appendix E. Evidence Tables

Appendix F. Risk of Bias Assessments

Appendix G. Strength of Evidence

Appendix H. Excluded Studies List

Appendix I. Forest Plots

Appendix J. Definitions of Magnitudes of Effect

Appendix K. Appendix References 


\section{Evidence Summary}

\section{Main Points}

- Integrated pain management programs improved both pain and function in patients with chronic pain at some, but not all, time frames compared with usual care or waitlist.

- Comprehensive pain management programs also improved function at multiple time frames and pain immediately after the program compared with usual care.

- Comprehensive programs also improved function and pain compared with medications alone at multiple time frames.

- Comprehensive programs were associated with improvement in function in the short term compared with physical activity alone but not in the intermediate or long term. There was no improvement in pain at any time point.

- There were no differences in pain or function between comprehensive programs and psychological support alone at any time.

- Beneficial effects were usually considered small to moderate for both program types.

- Although evidence was limited, serious harms were not reported for either program.

- Formal pain management programs have not been widely implemented in the United States for either general populations or the Medicare population.

\section{Background and Purpose}

Pain affects millions of adults. It impacts physical and mental function and is influenced by multiple factors (e.g., age, sex, comorbidities, and psychosocial factors). Optimal pain management should address biopsychosocial aspects of pain. The U.S. Department of Health and Human Services has been directed to evaluate ways to improve Medicare coverage and payment for pain treatment, particularly through formal pain management programs. Our review assesses the effectiveness and harms of pain management programs that address multiple aspects of pain. The intended audiences for this review are the Centers for Medicare \& Medicaid Services (CMS) and other stakeholders including clinicians, policymakers, patients, and their caregivers, and researchers. This review is part of the Dr. Todd Graham Pain Management Study and was sponsored by CMS.

\section{Methods}

We employed methods consistent with those outlined in the Agency for Healthcare Research and Quality Evidence-based Practice Center Program methods guidance (https://effectivehealthcare.ahrq.gov/topics/cer-methods-guide/overview). We describe these in the full report. Our searches covered publication dates up to May 2021. We sought studies in patients with complex acute/subacute pain or chronic nonactive cancer pain. Given the lack of consensus in terminology and program definitions for pain management, we defined two program categories $a$ priori, which differ in terms of where care is delivered and how it is coordinated: integrated pain management programs (IPMPs), which are centered in, coordinated by and integrated with primary care and have embedded or easy access to multidisciplinary providers and services, and comprehensive pain management programs (CPMPs) which receive referrals from primary care or other sources and provide multidisciplinary services separate from a primary care environment. Programs needed to have the following components available to patients: medication review and/or management, psychology support, and physical reconditioning. Other multimodal programs that did 
not meet our definitions for IPMPs or CPMPs (i.e., they did not include a psychological and exercise component or were not delivered by different disciplines) were not included. We analyzed effects and assessed strength of evidence (SOE) for the primary outcomes of function, pain, and changes in opioid use immediately after the intervention, at short term ( 1 to $<6$ months following treatment completion), intermediate term ( $\geq 6$ to $<12$ months), and long term ( $\geq 12$ months). Contextual Questions related to program models and components, their cost, safety and applicability to the Medicare population were also addressed.

\section{Results}

We included 57 mostly fair-quality randomized controlled trials (RCTs) (78 publications); 8 RCTs (11 publications) evaluated IPMPs and 49 RCTs (67 publications) evaluated CPMPs. Key findings with at least low strength of evidence (SOE) are summarized in Tables A and $\mathrm{B}$. Three IPMP trials enrolled older Veterans Affairs (VA) patients (mean ages 61 to 63 years); the mean age across IPMP trials was 57 years. One CPMP trial enrolled older VA patients (mean age 69 years); the mean age across CPMP trials was 45 years. Patients in most trials had moderate chronic pain, mostly musculoskeletal pain and fibromyalgia.

Table A. Summary of outcomes with a least low strength of evidence for IPMPs for noncancer pain: Key Question 1 (pain, function, opioid use)

\begin{tabular}{|c|c|c|c|c|}
\hline Outcome & Time Point & $\begin{array}{l}\text { IPMP Versus } \\
\text { UC }\end{array}$ & $\begin{array}{c}\text { IPMP Versus Physical } \\
\text { Activity }\end{array}$ & $\begin{array}{c}\text { IPMP Versus Telephone- } \\
\text { CBT }\end{array}$ \\
\hline \multirow[t]{4}{*}{$\begin{array}{l}\text { Pain } \\
\text { (Effect Size/SOE) }^{a}\end{array}$} & Postintervention & $\begin{array}{c}\text { None } \\
++\end{array}$ & No evidence & No evidence \\
\hline & $\begin{array}{l}\text { Short term } \\
\text { (1 to <6 months) }\end{array}$ & $\begin{array}{c}\text { Small } \\
+ \\
\end{array}$ & No evidence & No evidence \\
\hline & $\begin{array}{l}\text { Intermediate term } \\
\text { ( } \geq 6 \text { to }<12 \text { months) }\end{array}$ & $\begin{array}{c}\text { Small } \\
+ \\
\end{array}$ & No evidence & No evidence \\
\hline & $\begin{array}{l}\text { Long term } \\
\text { ( } \geq 12 \text { months) }\end{array}$ & $\begin{array}{c}\text { None } \\
+ \\
\end{array}$ & No evidence & No evidence \\
\hline \multirow[t]{4}{*}{$\begin{array}{l}\text { Function } \\
\text { (Effect Size/SOE) }^{a}\end{array}$} & Postintervention & $\begin{array}{c}\text { Small } \\
++\end{array}$ & $\begin{array}{c}\text { None } \\
+\end{array}$ & $\begin{array}{c}\text { None } \\
+\end{array}$ \\
\hline & $\begin{array}{l}\text { Short term } \\
\text { (1 to <6 months) }\end{array}$ & $\begin{array}{c}\text { Small } \\
++\end{array}$ & $\begin{array}{c}\text { None } \\
+\end{array}$ & $\begin{array}{c}\text { None } \\
+\end{array}$ \\
\hline & $\begin{array}{l}\text { Intermediate term } \\
\text { ( } \geq 6 \text { to }<12 \text { months) }\end{array}$ & $\begin{array}{c}\text { None } \\
+\end{array}$ & No evidence & No evidence \\
\hline & $\begin{array}{l}\text { Long term } \\
\text { ( } \geq 12 \text { months) }\end{array}$ & $\begin{array}{c}\text { None } \\
+\end{array}$ & $\begin{array}{c}\text { None } \\
+\end{array}$ & $\begin{array}{c}\text { None } \\
+\end{array}$ \\
\hline $\begin{array}{l}\text { Opioid Use } \\
\text { (Effect Size/SOE) }^{a}\end{array}$ & Postintervention & $\begin{array}{c}\text { None } \\
+\end{array}$ & No evidence & No evidence \\
\hline
\end{tabular}

$\mathrm{CBT}=$ cognitive pain management program; IPMP = integrated pain management program; $\mathrm{SOE}=$ strength of evidence; UC $=$ usual care.

${ }^{a}$ Effect size: None, small, moderate, or large difference favoring IPMP; SOE: $+=$ low,$++=$ moderate, $+++=$ high 
Table B. Summary of outcomes with a least low strength of evidence for CPMPs for noncancer pain: Key Question 1 (pain and function)

\begin{tabular}{|c|c|c|c|c|c|c|}
\hline Outcome & Time Point & $\begin{array}{l}\text { CPMPs } \\
\text { Versus } \\
\text { UC/ WL }\end{array}$ & $\begin{array}{l}\text { CPMPs } \\
\text { Versus } \\
\text { Physical } \\
\text { Activity }\end{array}$ & $\begin{array}{c}\text { CPMPs Versus } \\
\text { Pharmacologic } \\
\text { Therapy }\end{array}$ & $\begin{array}{c}\text { CPMPs Versus } \\
\text { Pharmacologic } \\
\text { Therapy and } \\
\text { Passive PT }\end{array}$ & $\begin{array}{c}\text { CPMPs Versus } \\
\text { Psychological } \\
\text { Therapy }\end{array}$ \\
\hline \multirow{4}{*}{$\begin{array}{l}\text { Pain } \\
\text { (Effect } \\
\text { Size/SOE)a }\end{array}$} & Postintervention & $\begin{array}{c}\text { Small } \\
++\end{array}$ & $\begin{array}{c}\text { None } \\
++\end{array}$ & $\begin{array}{c}\text { Moderate } \\
+\end{array}$ & $\begin{array}{c}\text { Moderate } \\
+\end{array}$ & $\begin{array}{c}\text { None } \\
+\end{array}$ \\
\hline & $\begin{array}{l}\text { Short term } \\
\text { ( } 1 \text { to }<6 \text { months) }\end{array}$ & $\begin{array}{c}\text { None } \\
+\end{array}$ & $\begin{array}{c}\text { None } \\
+\end{array}$ & $\begin{array}{c}\text { None } \\
+\end{array}$ & No evidence & No evidence \\
\hline & $\begin{array}{l}\text { Intermediate term } \\
\text { ( } \geq 6 \text { to }<12 \text { months) }\end{array}$ & $\begin{array}{c}\text { None } \\
+\end{array}$ & $\begin{array}{c}\text { None } \\
+\end{array}$ & $\begin{array}{c}\text { Small } \\
+\end{array}$ & $\begin{array}{c}\text { Moderate } \\
+\end{array}$ & $\begin{array}{c}\text { None } \\
+\end{array}$ \\
\hline & $\begin{array}{l}\text { Long term } \\
\text { ( } \geq 12 \text { months) }\end{array}$ & $\begin{array}{c}\text { None } \\
+\end{array}$ & $\begin{array}{c}\text { None } \\
++\end{array}$ & $\begin{array}{c}\text { None } \\
+\end{array}$ & $\begin{array}{c}\text { Moderate } \\
+\end{array}$ & $\begin{array}{c}\text { None } \\
+\end{array}$ \\
\hline \multirow{4}{*}{$\begin{array}{l}\text { Function } \\
\text { (Effect } \\
\text { Size/SOE)a }\end{array}$} & Postintervention & $\begin{array}{c}\text { Moderate } \\
+\end{array}$ & $\begin{array}{c}\text { None } \\
++\end{array}$ & $\begin{array}{c}\text { Moderate }^{\mathrm{b}} \\
+\end{array}$ & $\begin{array}{c}\text { None } \\
+\end{array}$ & $\begin{array}{c}\text { None } \\
+\end{array}$ \\
\hline & $\begin{array}{l}\text { Short term } \\
\text { (1 to <6 months) }\end{array}$ & $\begin{array}{c}\text { Moderate } \\
+ \\
\end{array}$ & $\begin{array}{c}\text { Small } \\
++\end{array}$ & $\begin{array}{c}\text { Small } \\
+\end{array}$ & No evidence & No evidence \\
\hline & $\begin{array}{l}\text { Intermediate term } \\
(\geq 6 \text { to }<12 \text { months) }\end{array}$ & $\begin{array}{c}\text { None } \\
+\end{array}$ & $\begin{array}{c}\text { None } \\
++\end{array}$ & $\begin{array}{c}\text { Small } \\
++\end{array}$ & $\begin{array}{c}\text { None } \\
+\end{array}$ & $\begin{array}{c}\text { None } \\
+\end{array}$ \\
\hline & $\begin{array}{l}\text { Long term } \\
\text { ( } \geq 12 \text { months) }\end{array}$ & $\begin{array}{c}\text { None } \\
+\end{array}$ & $\begin{array}{c}\text { None } \\
++\end{array}$ & $\begin{array}{c}\text { Small } \\
+\end{array}$ & $\begin{array}{c}\text { None } \\
+\end{array}$ & $\begin{array}{c}\text { None } \\
+\end{array}$ \\
\hline
\end{tabular}

$\mathrm{CPMP}=$ comprehensive pain management program; $\mathrm{PT}=$ physical therapy; $\mathrm{SOE}=$ strength of evidence; $\mathrm{UC}=$ usual care; $\mathrm{WL}=$ waitlist.

${ }^{a}$ Effect size: None, small, moderate, or large difference favoring CPMP; SOE: $+=$ low, $++=$ moderate, $+++=$ high

${ }^{\mathrm{b}}$ Based on 1 fair-quality trial in which patients got antidepressants and sedatives in conjunction with basic analgesics.

${ }^{\mathrm{c}}$ Based on 1 fair-quality trial in which patients got antidepressants only.

Contextual Question results reaffirmed that there is substantial variability in program terminology, structure, components employed and how they were delivered. Common components reported in systematic reviews of chronic pain management programs included psychological and mental health support and physical activity and less commonly, medication optimization or monitoring. Coordination and communication across multiple providers were considered key in assuring collaborative, interdisciplinary care. Information on cost-effectiveness was sparse.

Evidence on the impact of program types/components, coordination, and methods of care delivery on patient outcomes as well as potential risks or harms is sparse. These factors were rarely evaluated or were poorly described in included studies.

\section{Strengths and Limitations}

We established internal operational definitions for IPMP and CPMP a priori based on care setting and focused on trials where the primary components of pain management that would most generally address the biopsychosocial needs of patients were available. Our review appears to be the most complete summary of RCTs describing IPMPs. We categorized average effect sizes for function and pain using the system described in our previous reviews to facilitate interpretation of results across trials. The proportions of patients achieving a clinically meaningful improvement for measures of pain and function (i.e., responders) was rarely reported.

There are limitations to the review and evidence. No trial specifically recruited adults eligible for Medicare. Most patients had moderate intensity chronic low back pain, musculoskeletal pain, osteoarthritis, or fibromyalgia. Studies rarely described psychological comorbidities (including suicidal behaviors) or medical comorbidities and many excluded patients with comorbidities. Specifics of pain diagnoses or characteristics and patient factors were not generally reported in studies; we could not evaluate their impact on function, pain, or opioid use. It was not possible to fully capture the diversity of programs potentially available in clinical practice in this review. This 
is in part due to the wide variety of programs available clinically, many of which may not be evaluated in the peer-reviewed literature. There was little evidence to evaluate the impact of specific program structures, components, or their delivery. Details regarding program components were often poorly described. Although multiple investigators reviewed programs against prespecified criteria, some misclassification was possible.

\section{Implications and Conclusions}

Our review suggested that IPMPs and CPMPs may provide small to moderate improvements in function and small improvements in pain for patients with chronic pain compared with usual care and may be more effective than some medications alone. The average improvements in function and pain in our review were consistent with those reported for other therapies for pain, including opioids for chronic pain, nonpharmacologic treatments, and surgery.

Usual care for pain consists of providing selected individual treatments (e.g., medications) or services (e.g., physical therapy, psychological support) prescribed or recommended by a patient's provider (primary care or specialty provider), generally with little or no coordination between multidisciplinary providers or active monitoring of patient progress. Some patients may benefit from a broader range of therapies that address the full range of biopsychosocial concerns that are available through and coordinated in formal programs. Neither IPMPs nor CPMPs have been widely implemented in the United States. Reasons include the costs, logistics, leadership support, staffing, and provider training required to develop and implement them as well as the current feefor-service reimbursement structure. Programs may not be accessible to many populations based on locations, the availability of pain specialists, and socioeconomic factors.

Medicare-eligible patients and beneficiaries are a diverse population. Many older adults may be active, employed, and in good health but require assistance with pain management; others may be disabled or have substantial comorbid conditions that require ongoing support for pain management. Programs that address a range of biopsychosocial aspects of pain, tailor components to patient need, and coordinate care may be of particular importance in this population. Included IPMP programs in particular focused on patient-tailored care and were generally low intensity. To the extent that programs are tailored to patient's needs, our findings may be applicable to the Medicare population. Research in the Medicare population and in patients with a broader range of pain conditions is needed, however. Additional evidence from primary care-based programs is needed. 


\section{Introduction}

\section{Background}

Pain is a monumental public health challenge in the United States, affecting millions of adults, and leading to disability. Conservative estimates suggest costs of \$560-635 billion annually. ${ }^{1}$ Low back and neck pain accounted for the highest healthcare spending in 2016 across 154 conditions. ${ }^{2}$ Low back pain prevalence estimates in elderly adults range from 21 percent to 75 percent. ${ }^{3}$ Estimates of chronic pain and high impact chronic pain (i.e., chronic pain that frequently limits life or work activities) prevalence in adults 65 to 84 years of age were 27.6 percent and 10.7 percent respectively, based on 2016 National Health Interview Survey Data. ${ }^{4}$ Estimates of acute pain in those 65 years and older range from 7 to 52 percent, varying by site with headache, joint, and neuropathic pain most commonly cited. ${ }^{5}$ Opioids are frequently prescribed for acute and chronic pain but there is concern about the safety and efficacy of opioid management of pain. In adults 65 years and older, there were substantial increases in opioidrelated hospitalizations (34\%) and emergency department visits (74\%) between 2010 and 2015, ${ }^{6}$ and a 53 percent increase in the proportion of older adults seeking treatment for opioid use disorder from 2013 to $2015 .^{7}$ Across a sample of 1,776,790 Medicare enrollees under 65 years old who qualified for Medicare secondary to disability, 38.5 percent had a pain diagnosis. In the sample, opioid overdose deaths increased from 57.4 per 100,000 in 2012 to 77.6 per 100,000 in 2016. ${ }^{8}$

Pain is complex. It substantially impacts physical and mental function and is influenced by multiple factors (e.g., genetic, central nervous system, psychological, and environmental) and individual characteristics (e.g., age, sex, presence of comorbidities, and psychosocial factors). Such factors impact a person's pain experience and are collectively considered as part of a variety of biopsychosocial models of pain. ${ }^{9-12}$ Understanding how these factors impact pain is important for informing optimal approaches to management. The National Academy of Sciences workshop on Non-Pharmacological Approaches to Pain Management, ${ }^{13}$ the recent Pain Management Best Practices Inter-Agency Task Force report, ${ }^{14}$ the National Pain Strategy (NPS) report, ${ }^{15}$ and others recommend that optimal pain management be integrated, multi-modal, interdisciplinary, evidence-based, and individualized in keeping with the biopsychosocial model of pain. In keeping with this model, primary components of care may include medication management (e.g., oral pain medications, topical products), physical activity to promote and maintain functional capacity and decrease pain (e.g., movement and body awareness strategies), and pain psychology support (e.g., methods to develop and improve pain management skills such as cognitive behavioral therapy, relaxation, mindfulness-based stress reduction). In addition to these primary components, complementary and integrative health modalities (e.g., acupuncture, massage, spinal manipulation), patient education (e.g., understanding pain, life-style modification, implementation of self-management tools), and other treatments (e.g., physical modalities, injections, surgical procedures) may be part of pain management. Individual patients' need for and success with any given component or set of components may vary and patients likely benefit most from incorporation of multiple methods of pain management combined versus relying on one specific treatment to manage pain. Delivery of these diverse components requires involvement of professionals from multiple disciplines and, ideally, integration, communication, and coordination of care across these disciplines to outline the most appropriate care pathway(s) for a given patient, ${ }^{1,15-18}$ taking into account individual susceptibility and treatment responses. 
There is substantial heterogeneity in the terminology used in the literature and in clinical practice to describe and categorize pain management programs that address a biopsychosocial pain model. There is not a standardized set of terms, program definitions, or categorizations for pain management programs. For purposes of this review we conceptualized pain management programs that potentially address care consistent with a biopsychosocial model into two general categories - integrated pain management programs (IPMPs), centered in, and integrated with primary care which have embedded or easy access to multidisciplinary providers and services; and comprehensive pain management programs (CPMPs), not centered in primary care but based on referral from primary care or other sources (e.g., insurance) to a set of multidisciplinary services separate from the primary care environment. Thus, these programs are different regarding where care is delivered and how it is coordinated. The U.S. Department of Veterans Affairs (VA) Whole Health System is an example of an integrated program for chronic pain management. ${ }^{19-21}$ A stepped care model is used which involves primary care delivered using Patient Aligned Clinical Teams (PACTs) ${ }^{21}$ and provides a basis for patient assessment, medication management and referral to a range of multidisciplinary providers and services (e.g., behavioral pain management) and for advanced diagnostics and interventions as needed. Traditional multidisciplinary or interdisciplinary rehabilitation programs are examples of CPMPs. Both IPMPs and CPMPs usually include access to appropriate medication and/or a medication management component as well as psychological care (pain psychology and mental health support), and physical rehabilitative methods such as physical therapy or occupational therapy and have some mechanism of care coordination or formal communication between multidisciplinary providers. Both IPMPs and CPMPs may incorporate patient education and selfmanagement components as well as various individual complementary and integrative health therapies (e.g., acupuncture). Integrative pain management differs from integrated pain management programs. Integrative management takes a holistic, person-centered approach to patient care as do the individual complementary and integrative health therapies employed. Integrative pain management generally focuses on a broader range of integrative therapies and practices (e.g., manipulation, mindfulness, acupuncture, massage, mind-body therapies, nutritional counseling, etc.) than integrated pain management programs. Such therapies may be part of formal programs or models that are coordinated by integrative health clinicians and may include consultation with allopathic providers. ${ }^{22}$ As with IPMPs and CPMPs, integrative pain management may incorporate providers from multiple disciplines. Unless such formal integrative programs also met our definitions for IPMP, they were excluded from this review. IPMPs and CPMPs that included individual integrative therapies in addition to the primary components of psychological care and physical rehabilitative methods and/or medication management were included in this review.

Given the high prevalence of pain in older adults eligible for Medicare and those under 65 years old who qualify for Medicare due to disability, use of effective, safe, and cost-effective pain management becomes imperative. Unique challenges in assessing and managing pain in older adults ${ }^{5,23}$ include age-related changes in pain perception and thresholds and responses to medication, comorbidities (medical and psychological), polypharmacy, psychosocial concerns, and lack of care coordination. Older adults may also be predisposed to transitioning from acute to chronic, persistent pain. ${ }^{24,25}$ Thus, an integrated, coordinated, and individualized approach may be particularly important in the Medicare population to assure optimal pain management.

The U.S. Department of Health and Human Services has been directed to evaluate ways to improve Medicare coverage and payment for treatment of acute and chronic pain, particularly 
through integrated pain management programs and multidisciplinary, multimodal treatment models that involve care coordination. Requisite to addressing this decisional dilemma is understanding the types/components and methods of care delivery as well as benefits, potential risks and costs related to such programs for Medicare Parts A and B beneficiaries with complex acute/subacute pain or chronic nonactive cancer pain.

\section{Purpose and Scope of the Systematic Review}

This systematic review evaluated the effectiveness and harms of pain management programs and described contextual, process and structural factors that may impact outcomes particularly in the Medicare population. The intended audiences for this review were the Centers for Medicare $\&$ Medicaid Services (CMS) and other stakeholders including clinicians, policymakers, patients, their caregivers, and researchers. This review is part of the Dr. Todd Graham Pain Management Study and was sponsored by CMS. 


\section{Methods}

\section{Review Approach}

The methods for this systematic review followed the Agency for Healthcare Research and Quality (AHRQ) Methods Guide for Effectiveness and Comparative Effectiveness Reviews (https://effectivehealthcare.ahrq.gov/products/cer-methods-guide/overview). This systematic review is in accordance with the Preferred Items for Reporting in Systematic Reviews and MetaAnalyses (PRISMA). ${ }^{26}$

\section{Key Questions}

A Technical Expert Panel provided comments on the scope of the review. The following Key Questions and inclusion criteria reflect suggestions received and are in the final protocol. The final protocol was posted on the Effective Health Care website on November 10, 2020 (https://effectivehealthcare.ahrq.gov/products/integrated-pain-management/protocol).

Key Question 1. What are the effectiveness and harms of integrated or comprehensive pain management programs for Medicare beneficiaries with complex acute/subacute pain or chronic, nonactive cancer pain? Population subgroups of interest include those with disabilities (including ESRD), prior substance use disorder, psychological co-morbidities (including suicidal behaviors), and nociplasticity (i.e., pain resulting from altered nociception without underlying tissue damage resulting in hypersensitivity [e.g., fibromyalgia]).

Key Question 2. Have any of the following factors been evaluated and/or shown to impact outcomes in studies of comprehensive or integrated pain management models?

a. Treatment delivery including session formats (group, one-on-one), duration, intensity and frequency of sessions, number of sessions; general structure and scope of sessions

b. Treatment components (e.g., medication review and/or management, including transition from opioid to nonopioid medications; psychological support or mental health services; physical reconditioning, such as physical therapy and occupational therapy; use of complementary and integrative medicine treatments; patient education; use of medical procedures or devices)

c. Care provision

i. Care coordination methods or decision support

ii. Provider types involved

iii. Personalization, care pathways

d. Program characteristics

i. Program emphasis/goals

ii. Target population

iii. Referral sources 
iv. Staffing characteristics (e.g., turnover)

\section{Contextual Questions}

Following the methods of the U.S. Preventive Services Task Force (USPSTF), ${ }^{27}$ Contextual Questions represent issues in a review for which a valid, but not necessarily systematic, summary of current research is needed in order to provide context on the issue. See the Methods Appendix A for more details.

Contextual Question 1. What different types of comprehensive, integrated approaches to complex acute/subacute pain or chronic, nonactive cancer pain management have been proposed or used in clinical practice?

a. How are comprehensive and integrated pain management programs defined?

b. What are considered the most important components of integrated pain management programs?

c. What pain management models or mechanisms are most commonly used in clinical practice?

d. What types of programs/models may be most applicable to Medicare beneficiaries?

e. What theoretical advantages and disadvantages do various programs/models have compared with current practice?

f. Are there any potential safety issues?

Contextual Question 2. Is there information on the costs or costeffectiveness of integrated pain management programs in the Medicare or general population?

\section{Analytic Framework}

The analytic framework (Figure 1) illustrates the population, interventions, outcomes, and adverse effects that guided the literature search and synthesis. 
Figure 1. Analytic framework

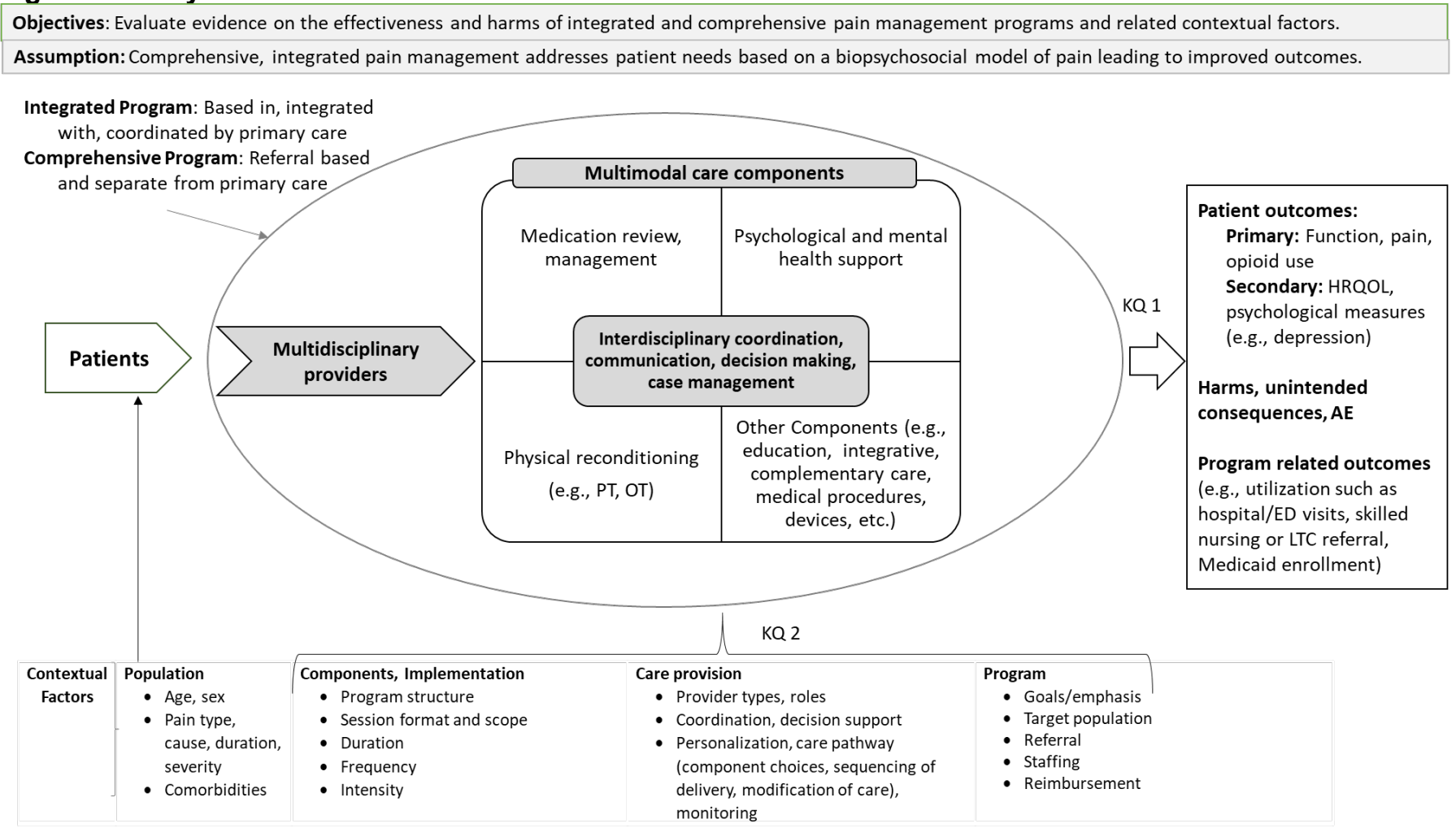

$\mathrm{AE}=$ adverse event; $\mathrm{ED}=$ emergency department; HRQOL $=$ health-related quality of life; KQ $=$ Key Question; LTC $=$ longterm care; OT = occupational therapy; PT = physical therapy

\section{Study Selection}

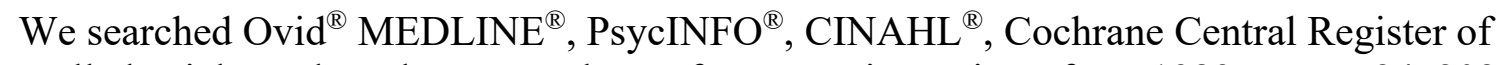
Controlled Trials, and Cochrane Database of Systematic Reviews from 1989 to May 24, 2021. We restricted to English-language articles, given the focus on Medicare eligible patients within the U.S. healthcare system. All searches were conducted by a qualified medical librarian.

In accordance with the Methods Guide for Effectiveness and Comparative Effectiveness Review, ${ }^{28}$ we used the pre-established criteria in Table 1 to identify studies eligible for this review. For all Key Questions, we focused on randomized controlled trials (RCTs), as wellconducted RCTs have the least risk of bias. Nonrandomized studies in pain can be misleading, due to the subjective nature of pain which may exacerbate effects of confounding, selection bias, and attentional and other nonspecific effects. We planned to include comparative nonrandomized studies that controlled for confounding only if RCTs were not available. However, RCTs were identified for each program type and nonrandomized studies were not included. We did not identify single arm studies (e.g., case series, pre-post studies) specifically in the Medicare population that met our inclusion criteria. We excluded very young and nondisabled populations (e.g., military), interventions that were unimodal or confined to a single provider type, or that evaluated the incremental value of adding a single treatment modality to another single treatment modality, and postoperative or post-trauma rehabilitation programs (see Methods Appendix A, Table A-1 for detailed exclusion criteria). We did not identify additional evidence meeting our inclusion criteria from responses to a Federal Register notice requesting Supplemental Evidence and Data for Systematic review (SEADS) or from peer review or public comments. We used 
dual review to select studies. Methods Appendix A contains full details on review methods, including complete search strategies.

Searches were updated for new publications while the draft report was posted for peer review and public comment. Any new literature identified in the update search was assessed using the process described above for the original search and eligible trials were incorporated into the report prior to finalization.

Table 1. Criteria for population, intervention, comparison, and outcomes of eligible studies

\begin{tabular}{|c|c|}
\hline PICOTS & Inclusion \\
\hline Population & 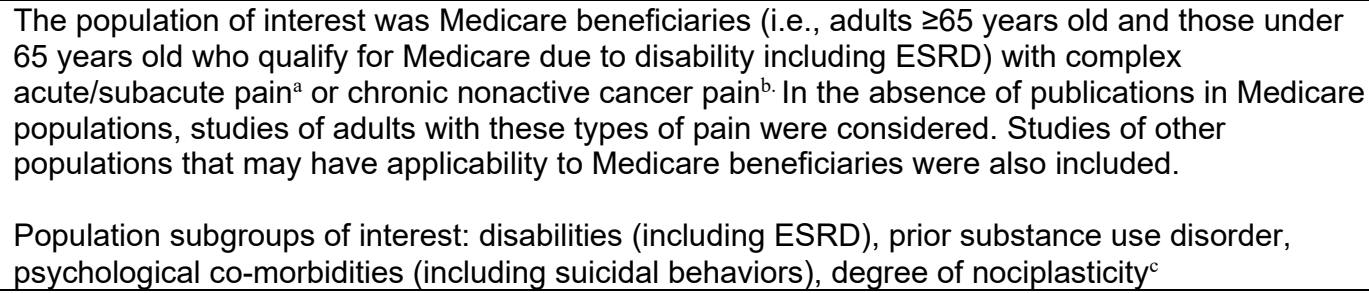 \\
\hline Intervention & $\begin{array}{l}\text { Pain management programs that addressed the biopsychosocial model of pain and included: } \\
\text { - Multidisciplinary (interdisciplinary) teams that at a minimum have the following components } \\
\text { available: pharmacotherapy review and/or management, psychological care (mental health } \\
\text { services), and physical reconditioning (e.g., PT, OT); studies may also include other } \\
\text { components in addition to these; and } \\
\text { - Description of care coordination, case management or mechanisms of multidisciplinary, } \\
\text { interdisciplinary collaboration and communication } \\
\text { IPMPs were defined as those that include the above and are based in primary care. } \\
\text { Comprehensive pain management programs (CPMPs) were defined as those including the above } \\
\text { but are not based in primary care. }\end{array}$ \\
\hline Comparator & Any \\
\hline Outcome & $\begin{array}{l}\text { Primary: Pain, function (focus on "success" if reported), opioid use, harms, adverse events, } \\
\text { unintended consequences } \\
\text { Secondary: HRQOL, emotional function (e.g., depression, anxiety), patient satisfaction, global } \\
\text { improvement, utilization (e.g., pain-related hospital/ED visits or short-term skilled nursing facility } \\
\text { use, long term care facility or institutional care transfer, Medicaid enrollment) }\end{array}$ \\
\hline Timing & $\begin{array}{l}\text { Duration of followup: Focus on persistence of effects evaluated short term ( } 1 \text { to }<6 \text { months), } \\
\text { intermediate term ( } \geq 6 \text { to }<12 \text { months) and long term ( } \geq 12 \text { months) following intervention; } \\
\text { immediate postintervention results are reported as well. }\end{array}$ \\
\hline Setting & Outpatient, inpatient, institutional residence \\
\hline $\begin{array}{l}\text { Study } \\
\text { design, } \\
\text { publication } \\
\text { type }\end{array}$ & $\begin{array}{l}\text { Inclusion focused on RCTs. Prospective cohort studies that controlled for confounding were } \\
\text { considered if RCTs were not available. Comparative cohorts that did not control for confounding } \\
\text { were considered if cohorts controlling for confounding were not available. In the absence of } \\
\text { comparative studies, single arm (e.g., case series, pre-post studies) studies were considered if } \\
\text { they were clearly relevant to the Medicare population. }\end{array}$ \\
\hline
\end{tabular}

$\mathrm{ED}=$ emergency department; ESRD = end stage renal disease; HRQOL = health-related quality of life; IPMPs = integrated pain management programs; OT = occupational therapy; OUD = opioid use disorder; PICOTS = population, intervention, comparator, outcomes, timing, study design; $\mathrm{PT}=$ physical therapy; $\mathrm{RCT}=$ randomized control trial; $\mathrm{SUD}=$ substance use disorder.

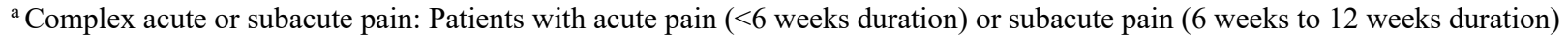
who are at risk of developing chronic pain).

${ }^{\mathrm{b}}$ Chronic, nonactive cancer pain (based on Mersky 1994) ${ }^{29}$ : Pain that persists for at least three months and is not associated with [active] malignant disease"; pain could, however, be resultant from a previous malignancy that is no longer active.

${ }^{c}$ The term nociplasticity has been used to describe pain resulting from altered nociception without underlying tissue damage resulting in hypersensitivity (e.g., fibromyalgia). ${ }^{30}$ Many pain conditions may have a nociplastic component. Some additional terms used in the literature include centralized pain and amplified pain. 


\section{Data Extraction and Risk of Bias Assessment}

Data were abstracted from included studies into evidence tables based on the organizational framework to include study, patient, and model characteristics (e.g., pharmacologic therapy, physical function, care coordination, psychological services) and study results (including harms), with data verified for accuracy and completeness by a second team member. Followup times were defined as immediately postintervention, and short term ( 1 to $<6$ months), intermediate term ( $\geq 6$ to $<12$ months) and long term ( $\geq 12$ months) following intervention. The risk of bias of included studies was assessed according to established methods, ${ }^{28,31}$ with RCTs assessed based on criteria established in the Cochrane Handbook for Systematic Reviews of Interventions. ${ }^{32}$ Based on the risk of bias assessment, individual included studies were rated as being "good," "fair," or "poor" quality. Like many nonpharmacological therapies (e.g., exercise or psychological therapy), it was not possible for studies to effectively blind participants (or providers) with regard to program inclusion. Nonetheless, studies were downgraded to fair for lack of blinding as it may still result in bias from patient expectations of treatment, attentional affects, and performance bias; this is consistent with the approach used in prior AHRQ reviews of nonpharmacological treatments for pain. Full details on data abstraction, data management, and risk of bias assessment can be found in the Methods Appendix A.

\section{Data Synthesis and Analysis}

We analyzed the evidence according to Key Question, using both narrative (qualitative) and quantitative (meta-analysis) methods (where possible). We reviewed and highlighted studies by using a hierarchy-of-evidence approach, focusing our synthesis on the highest quality data for each Key Question. Summary tables were constructed to highlight the main findings.

Meta-analyses, using profile-likelihood random effects models, were conducted to summarize data and obtain more precise estimates where there were at least two studies reporting outcomes that were homogeneous enough to provide a meaningful combined estimate. ${ }^{33,34} \mathrm{We}$ considered clinical and methodological diversity and assessed statistical heterogeneity using Cochran's $\chi^{2}$ test and the $I^{2}$ statistic. ${ }^{35}$ For continuous outcomes (e.g., pain), mean difference was used as the effect measure if the outcomes were reported using the same scale, and standardized mean difference was used when the outcomes were reported in different scales. Pain scales were converted to a common 0 to 10 scale. Risk ratios were used as the effect measure for binary outcomes. Sensitivity and subgroup analyses, including meta-regression, were performed to explore statistical heterogeneity and differences by study quality, intervention differences, patient and model characteristics, longer-term followup, and outcome measurement as data permitted (e.g., at least six to ten studies for continuous variables and four studies for categorical variables). Methods Appendix A contains additional detail of our meta-analysis methods. Consistent with our prior chronic pain report, ${ }^{36,37}$ we considered the impact of higher intensity programs (intensity $\geq 20$ hours/week or $>80$ hours total) versus lower intensity programs ( $<20$ hours/week) by performing meta-regression where data were available. We classified the magnitude of effects for continuous measures of pain and function using the same system as in prior AHRQ reviews on pain. ${ }^{36-40}$ Effects below the threshold for small were categorized as no effect. Where possible, we reported on the proportion of patients meeting thresholds for clinically important differences (e.g., $>30 \%$ pain relief). We did not conduct analyses to evaluate potential markers for publication bias given the substantial heterogeneity in study designs, 
programs, length of followup and patient populations and small number of trials available for most analyses.

Within each Key Question, results were presented separately for programs/models considered to be integrated (based in primary care) and comprehensive (not based in primary care), and any primary outcomes, as prioritized in Table 1, are presented first.

\section{Grading the Strength of the Body of Evidence}

The strength of evidence (SOE) of primary outcome-intervention pairs were evaluated using the AHRQ methods. ${ }^{28}$ Details on the methods used are presented in the Methods Appendix A and primary outcomes are delineated in Table 1, above. Additionally, for bodies of evidence with only a single study, we rated consistency as unknown (rather than not applicable). In these cases, we did not automatically downgrade the evidence to "insufficient" but considered the sample size or number of events available for analysis. The SOE was assigned an overall grade of high, moderate, low, or insufficient by evaluating and weighing the combined results of the following five domains: study limitations, consistency, directness, precision and reporting bias. If only poor-quality trials were available for a given outcome, SOE was considered insufficient. 


\section{Results}

A total of 10,953 abstracts were reviewed, 10,782 from electronic database searches and an additional 171 from handsearching and bibliography review of included studies and systematic reviews and from peer/public review. After dual review of titles and abstracts, 509 articles were selected for full-text review, of which 57 randomized controlled trials (RCTs) (in 78 publications) were included in this review. Eight trials (in 11 publications) evaluated integrated pain management programs (IPMPs) and 49 (in 67 publications) evaluated comprehensive pain management programs (CPMPs). Forty-three trials were rated fair quality (75\%) and 14 were rated poor quality $(25 \%)$. Search results and selection of studies are summarized in the literature flow diagram in Results Appendix B (Figure B-1) and an overview of the number of trials included by Key Question comparison can be found in the same Appendix (Table B-1). In addition, two Contextual Questions are addressed, primarily in the Discussion section with additional information available in Appendix C. Appendix D provides a list of all included studies.

Detailed evidence tables for included studies and quality assessments are available in Appendixes E and F. Appendix G contains details on the strength of evidence (SOE), and Appendix $\mathrm{H}$ lists excluded studies along with reasons for exclusion. Appendix I contains additional forest plots (i.e., pooled analyses) not presented in the report. The definitions of magnitude of effects for continuous measures of pain and function are presented in Appendix J. Appendix K lists all references cited in the Appendixes. Summary results tables for all primary outcomes can be found in Results Appendix B and are organized by Key Question then intervention and comparator.

\section{Key Question 1. Effectiveness and Harms of Integrated or Comprehensive Pain Management Programs}

\section{Integrated Pain Management Programs}

\section{Key Points}

- Integrated pain management was associated with a statistically significant but clinically unimportant effect on pain on a 0 to 10 scale versus usual care postintervention (strength of evidence [SOE]: moderate) but associated with small improvements in pain compared with usual care in the short and intermediate terms. There was no difference between groups long term (SOE: low).

- Integrated pain management was associated with small improvements in function versus usual care postintervention which persisted short term (SOE: moderate), but no clear difference was seen in the intermediate or long term (SOE: low).

- Results regarding proportions of patients experiencing clinically important improvement in function postintervention following integrated pain management versus usual care were conflicting and may be due to differences in outcomes measures used and/or conditions studied. Evidence was very limited at other time points.

- Opioid prescribing during a 12-month intervention in one trial $(\mathrm{N}=397)$ was similar between IPMP and usual care (65\% vs. 61\%) (SOE: low). Evidence was insufficient at other time points.

- No intervention-specific adverse events were seen in two trials (SOE: insufficient). 


\section{Summary of Findings}

Seven RCTs (reported in 10 publications) (1-50 $^{4}$ provided evidence on the effectiveness of IPMPs for Key Question 1 (Results Appendix B, Table B-4; Appendix E, Table E-1). Four cluster RCTs (three system-based ${ }^{41,42,45-47}$ and one practice-based ${ }^{48}$ ) randomized primary care providers (PCP) or practices to a multidisciplinary intervention or to usual care. In two of these trials by the same author group, the provider intervention group received patient-specific osteoarthritis (OA) treatment recommendations from the study team based on their assessment, published treatment guidelines, and an expert-developed algorithm for care at the trial start. ${ }^{41,42}$ Decisions regarding whether to recommend treatments to patients were at the PCP's discretion over the 12-month trial duration. Although the primary care team was not multidisciplinary, patients could be referred for or receive care from providers from multiple disciplines. The third trial randomized PCPs to receive collaborative, multidisciplinary assistance with pain management of patients with musculoskeletal pain diagnoses experiencing moderate or greater pain intensity or disability lasting 12 weeks or longer using a stepped-care model or provision of usual care for 12 months. ${ }^{45-47}$ Patients with subacute low back pain in primary care centers randomized to intervention in the fourth trial received a single 10-hour group session provided by a multidisciplinary team that included information and implementation recommendations for the primary components (medication, physical activity, psychological support) before initiating care with their PCP. ${ }^{48}$ In the remaining three practice-based RCTs, randomization to an IPMP or usual care/waitlist was performed at the patient-level. ${ }^{43,44,49,50} \mathrm{Six}$ trials ${ }^{41-43,45-48,50}$ received government funding and one trial ${ }^{44,49}$ received funding from a nonprofit organization.

Sample sizes ranged from 63 to 501 (total sample=2484). All seven trials compared IPMPs with usual care or waitlist control $(\mathrm{N}=2263)$, and one trial ${ }^{44,49}$ also compared an IPMP with physical activity alone $(\mathrm{N}=218)$ and psychological therapy alone $(\mathrm{N}=221)$. Pain diagnoses included OA (2 trials), ${ }^{41,42}$ subacute low back pain (LBP) (1 trial), ${ }^{48}$ chronic LBP (1 trial), ${ }^{50}$ chronic musculoskeletal pain ( 2 trials), ${ }^{43,45-47}$ and chronic widespread pain ( 1 trial). ${ }^{44,49}$ One trial in Veterans Affairs (VA) patients reported comorbidities including posttraumatic stress disorder (PTSD), anxiety, depression, and prior substance use treatment. ${ }^{45-47}$ All but one of the trials ${ }^{50}$ excluded patients with major psychiatric disorders, and one trial ${ }^{43}$ excluded patients with substance use disorder. None of the trials specifically included Medicare patients, however, three trials enrolled older VA patients (mean ages 61 to 63 years), ${ }^{41,42,45-47}$ with varying proportions of patients on disability $(8 \%, 33 \%$ and $65 \%)$. The pooled mean age across trials was 56.7 years (range, 47 to 63 years), 52 percent of participants were female (range, $8 \%$ to $65 \%$ ), and the pooled percent of non-White individuals across the four trials ${ }^{41,42,45-47,50}$ that provided information on race and/or ethnicity was 31 percent (range, 11\% to 50\%). Across the six trials that provided information on disability status, the pooled proportion of patients reported to be disabled was 33 percent (range, $8 \%$ to $65 \%$ ). Measures of disability included having a status of "disabled" (not otherwise specified) in two trials, ${ }^{41,42}$ being in receipt of disability payment in two trials, ${ }^{43,45-47}$ and being of Grade III or IV on the Chronic Pain Grade questionnaire (moderately or severely limiting high disability) in two ${ }^{44,49,50}$ trials. Trials were conducted in the United States, ${ }^{41,42,45-47,50}$ Canada, ${ }^{43}$ the United Kingdom/Scotland, ${ }^{44,49}$ and Spain. ${ }^{48}$

Programs delivered the treatment components to patients individually in four trials, ${ }^{41,42,44,49,50}$ via group sessions in two trials, ${ }^{43,48}$ and via a combination of group and individual sessions in one trial. ${ }^{45-47}$ All programs were based in primary care and considered to be low intensity $(<20$ hours per week). One program took place primarily at a local gym and/or the patient's home. ${ }^{44,49}$ None of the trials included a vocational rehabilitation or work hardening component. Across all 
trials, intervention durations ranged from one, 10-hour group session ${ }^{48}$ to 52 weeks $^{41,42,45-47}$ of individual sessions. The contents of the program components varied substantially across trials (Appendix E, Table E-1). The psychological support components generally involved using cognitive behavioral therapy (CBT) principles and relaxation techniques in five trials. ${ }^{41-44,48,49}$ Five trials $\mathrm{s}^{41-43,45-48}$ provided details regarding medication review or management, and one trial $^{44,49}$ indicated that there were no drugs approved for use in fibromyalgia (FM) patients in the United Kingdom. The remaining trial did not provide any information about medication management. ${ }^{50}$ Additional components available to patients as needed included a weight management program in two trials, ${ }^{41,42}$ and referral to the specialty pain clinic, orthopedics, or neurosurgery for evaluation for a procedural approach and access to medical social workers in one trial. ${ }^{45-47}$ Six trials provided descriptions of care coordination and/or communication between providers. ${ }^{41-49}$

Six RCTs ${ }^{41,42,44,46-50}$ were rated fair quality and one ${ }^{43}$ poor quality (Appendix F, Table F-1). The major methodological limitation in the fair-quality trials was the inability to effectively blind patients and caregivers to the CPMP. Other methodological shortcomings included unclear randomization and unclear allocation concealment methods. In addition, the poor-quality trial had high attrition. Within each cluster RCT, populations for intervention and comparator arms were comparable with regard to primary risk factors for pain (e.g., symptom duration, pain intensity, baseline scores).

\section{Detailed Synthesis}

\section{IPMPs Versus Usual Care}

\section{Primary Outcomes}

\section{Pain}

Integrated pain management was associated with a statistically significant but clinically unimportant effect on pain compared with usual care postintervention ( 4 trials, $\mathrm{N}=1142$, pooled difference $-0.31,95 \%$ confidence interval $[\mathrm{CI}]-0.51$ to $\left.-0.11, \mathrm{I}^{2}=0 \%\right) .{ }^{41,42,46,47,50}$ IPMPs were associated with a small improvement in pain in the short term ( 2 trials, $\mathrm{N}=721$, pooled difference $-0.59,95 \% \mathrm{CI}-1.17$ to $\left.-0.07, \mathrm{I}^{2}=0 \%\right)^{48,50}$ and the intermediate term ( 1 trial, $\mathrm{N}=197$, difference $-0.70,95 \% \mathrm{CI}-1.13$ to -0.09$)^{50}$ but not in the long term ( 2 trials, $\mathrm{N}=688$, pooled difference $-0.28,95 \%$ CI -0.80 to $0.23, I^{2}=0 \%$ ), ${ }^{48,50}$ (Figure 2). No trials reported the likelihood of experiencing significant improvement in pain. 
Figure 2. IPMP versus usual care control: Pain

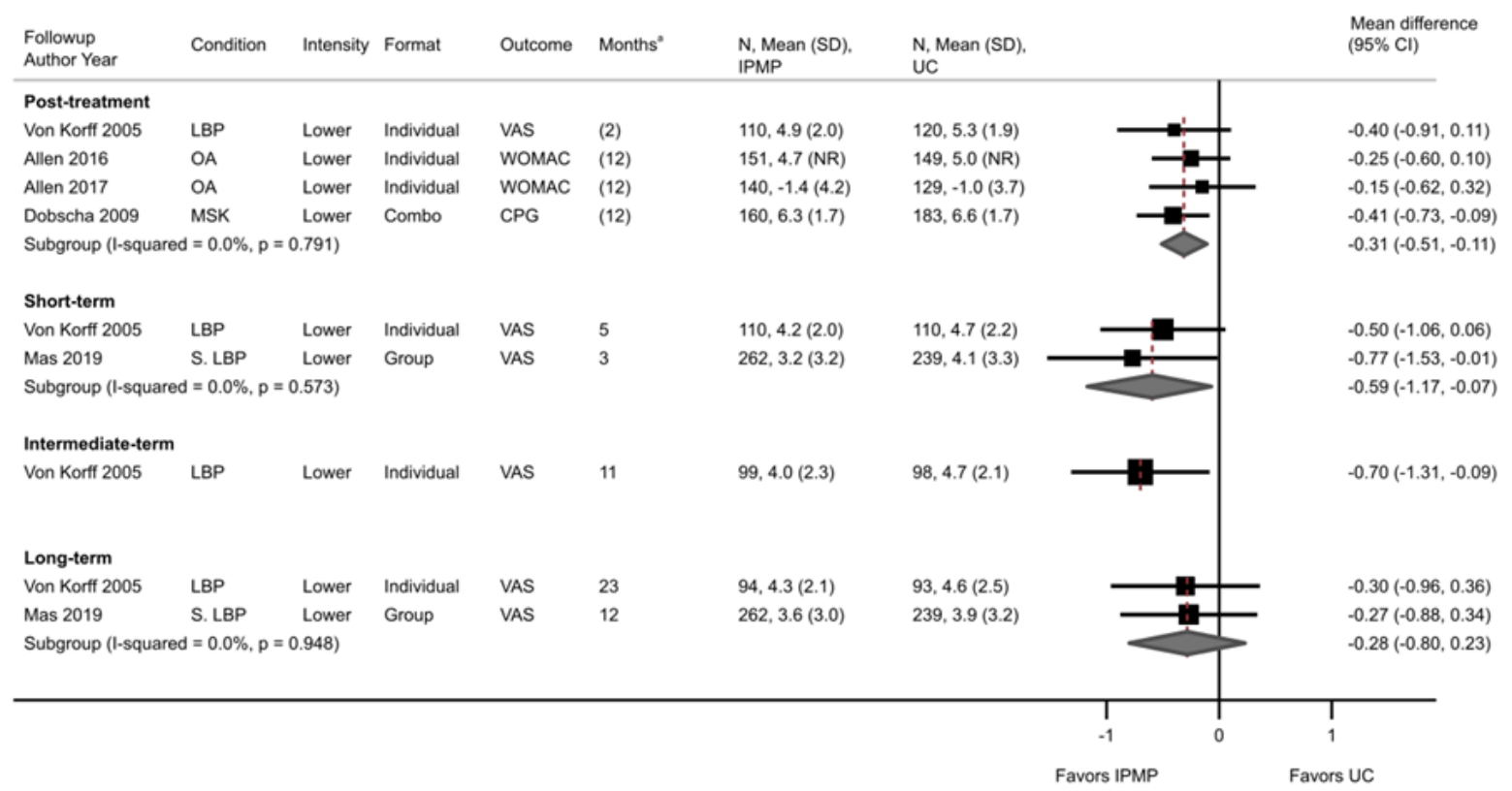

$\mathrm{CI}=$ confidence interval; Combo $=$ combination group and individual sessions; $\mathrm{CPG}=$ Chronic Pain Grade Severity subscale; IPMP = integrated pain management program; LBP = low back pain; MSK = musculoskeletal pain; OA = osteoarthritis; $\mathrm{SD}=$ standard deviation; $\mathrm{S} . \mathrm{LBP}=$ subacute low back pain; $\mathrm{UC}=$ usual care; VAS $=$ visual analog scale; WOMAC $=$ Western Ontario and McMaster Universities Osteoarthritis Index.

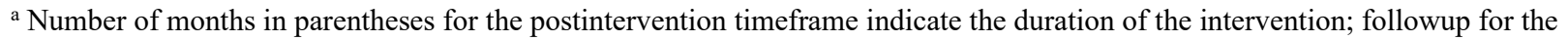
remaining timeframes is in months following the end of the intervention.

\section{Function}

Small improvements in function were associated with IPMP versus usual care for continuous measures of function postintervention ( 4 trials, $\mathrm{N}=1142$, pooled standardized mean difference [SMD] $-0.20,95 \% \mathrm{CI}-0.34$ to $\left.-0.06, \mathrm{I}^{2}=0 \%\right)$ and in the short term ( 2 trials, $\mathrm{N}=721$, pooled SMD $-0.23,95 \% \mathrm{CI}-0.40$ to $\left.-0.02, \mathrm{I}^{2}=0 \%\right),{ }^{48,50}$ but not in the intermediate term ( 1 trial, $\mathrm{N}=220$, pooled SMD $-0.10,95 \% \mathrm{CI}-0.38$ to 0.17$).{ }^{50}$ Long term, a small improvement tended to favor IPMP but was not statistically significant ( 2 trials, $\mathrm{N}=688$, pooled $\mathrm{SMD}-0.19,95 \% \mathrm{CI}-0.36$ to $\left.0.01, I^{2}=0 \%\right)^{48,50}$ (Figure 3). The differences on the original Roland Morris Disability Questionnaire (RMDQ) were -1.0 to -1.3 postintervention, -0.9 to -1.33 in the short term, and -1.0 to -1.11 in the long term. The postintervention difference on the original Western Ontario and McMaster Universities Arthritis Index (WOMAC) Function scale in one trial was -3.3 and the difference in change scores from baseline was -0.2 in another trial. 
Figure 3. IPMP versus usual care control: Function

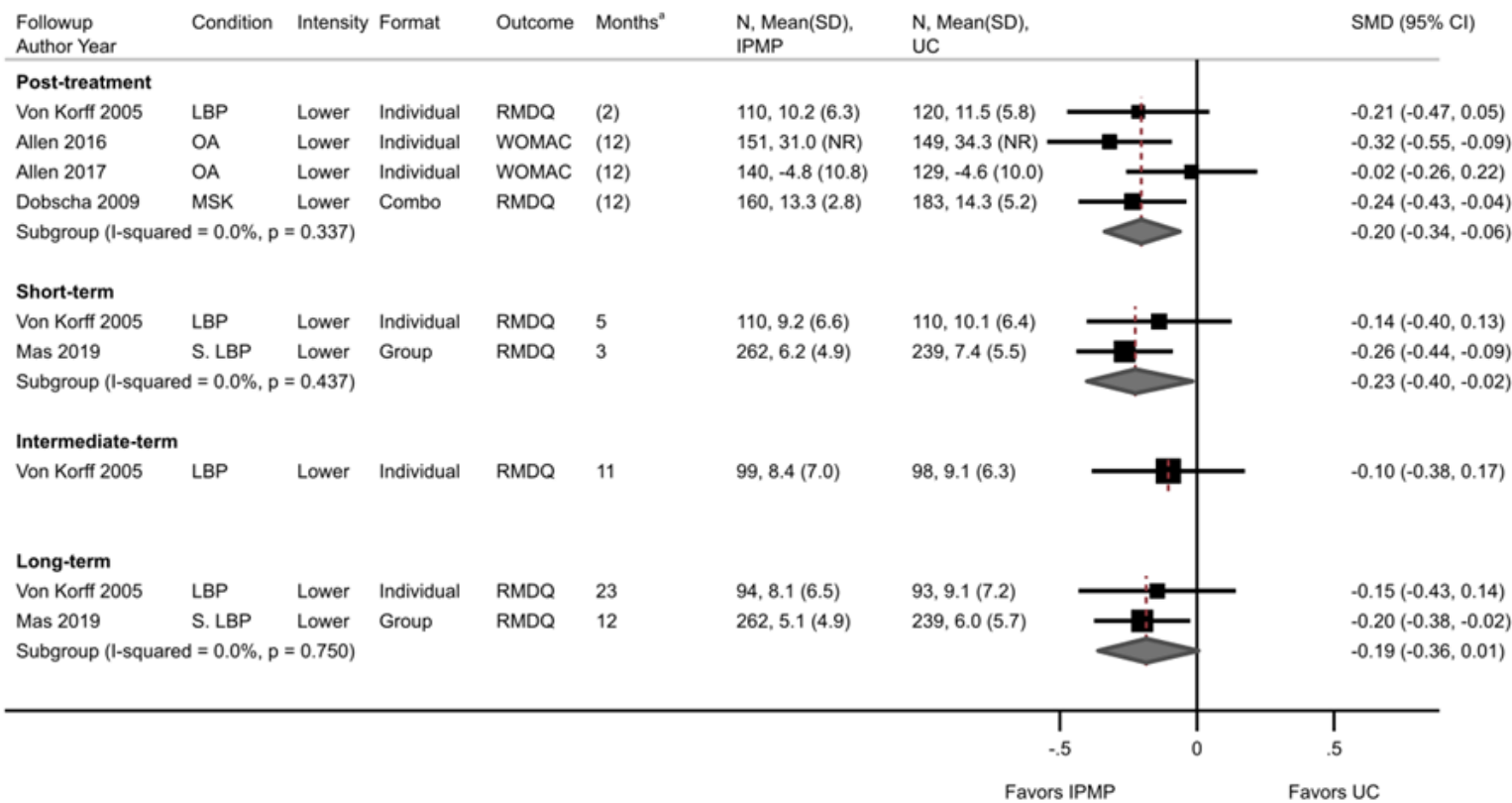

$\mathrm{CI}=$ confidence interval; Combo $=$ combination group and individual sessions; IPMP = integrated pain management program; $\mathrm{LBP}=$ low back pain; MSK = musculoskeletal pain; RMDQ = Roland Morris Disability Questionnaire; $\mathrm{SD}=$ standard deviation; $\mathrm{S}$. LBP = subacute low back pain; $\mathrm{SMD}=$ standardized mean difference; $\mathrm{OA}=$ osteoarthritis; $\mathrm{UC}=$ usual care; WOMAC $=$ Western Ontario and McMaster Universities Osteoarthritis Index.

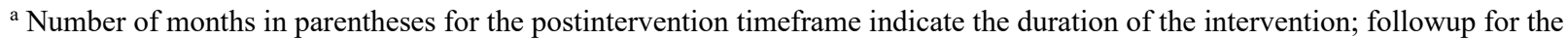
remaining timeframes is in months following the end of the intervention.

More IPMP patients experienced 30 percent or more improvement on the RMDQ ( 0 to 23 or 24 scale) postintervention compared with those receiving usual care across two trials, in patients with chronic LBP (2 month intervention $)^{50}$ or chronic musculoskeletal pain (12 month intervention $)^{46,47}$ ( 2 trials, $\mathrm{N}=608,23 \%$ vs. $13 \%$, pooled risk ratio [RR] $1.73,95 \%$ CI 1.14 to $2.80, \mathrm{I}^{2}=0$ ). In contrast, two trials in patients with OA found no difference between IPMP and usual care based on 18 percent or more improvement on the WOMAC Function (Scale 0 to 68) postintervention ( 2 trials, $\mathrm{N}=399,18 \%$ vs. $21 \%$, pooled RR $1.05,95 \%$ CI 0.69 to $\left.1.65, \mathrm{I}^{2}=0\right) .{ }^{41,42}$ In the RCT of patients with LBP $(\mathrm{N}=207)$, the 30 percent or greater improvement in RMDQ persisted for IPMP participants to the short-term (RR 1.81, 95\% CI 1.20 to 2.72) and intermediate-term (RR 1.97, 95\% CI 1.30 to 2.98), but not to long-term (RR 1.35, 95\% CI 0.98 to 1.85 ) followup after the 2-month intervention (Figure 4). 
Figure 4. IPMP versus usual care control: Function success

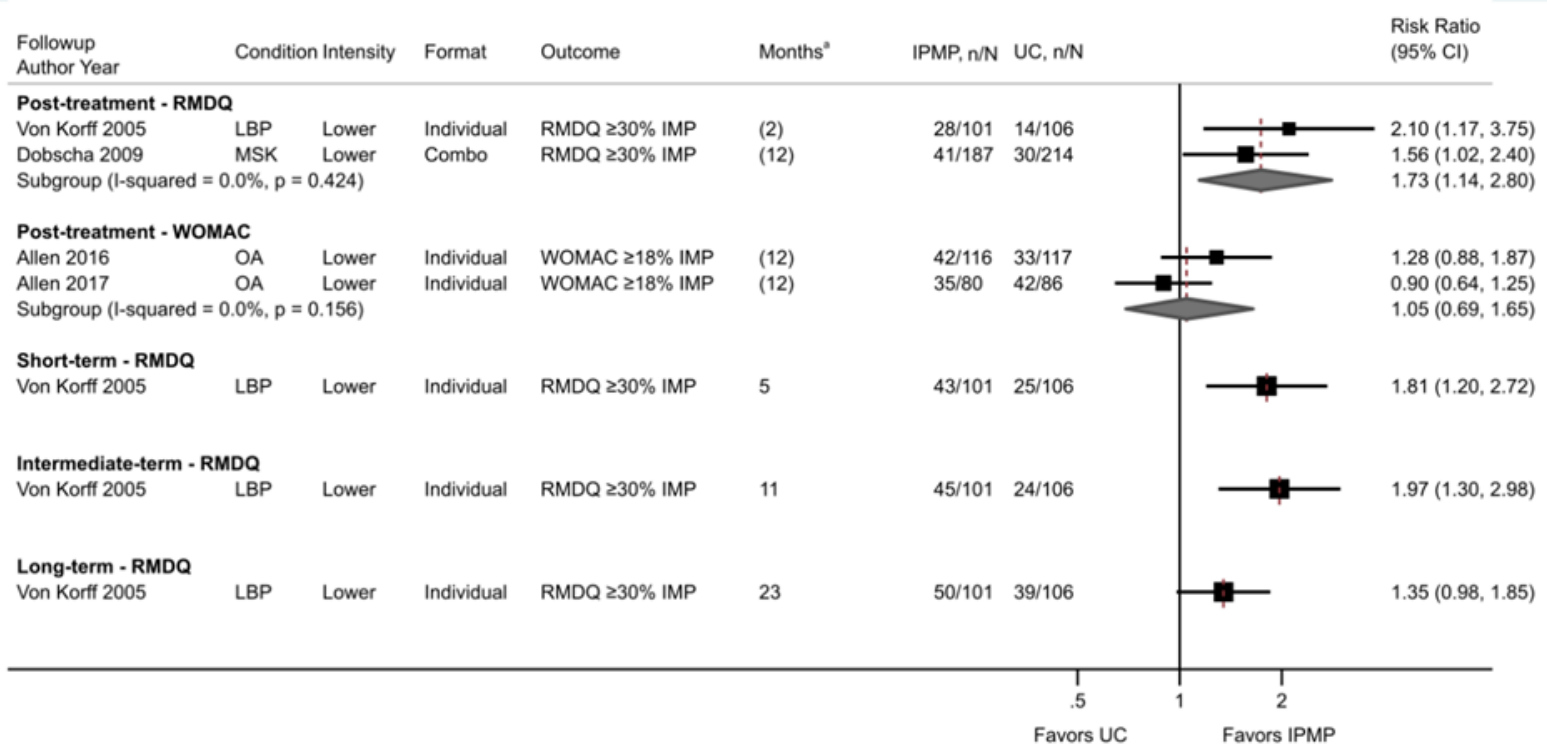

$\mathrm{CI}=$ confidence interval; Combo $=$ combination group and individual sessions; IMP $=$ improvement; IPMP $=$ integrated pain management program; $\mathrm{LBP}=$ low back pain; $\mathrm{MSK}=$ musculoskeletal pain; $\mathrm{RMDQ}=$ Roland Morris Disability Questionnaire; $\mathrm{OA}=$ osteoarthritis; $\mathrm{UC}=$ usual care; WOMAC = Western Ontario and McMaster Universities Osteoarthritis Index.

${ }^{a}$ Number of months in parentheses for the postintervention timeframe indicate the duration of the intervention; followup for the remaining timeframes is in months following the end of the intervention.

One trial in patients with chronic widespread pain $(\mathrm{N}=221)^{44,49}$ reported a measure that combined pain and disability that could not be pooled with other studies. It found no differences between IPMP versus usual care postintervention or long term.

\section{Opioid Use}

One fair quality trial $(\mathrm{N}=397)$ reported no significant differences in opioid prescriptions between IPMP and usual care groups (65\% vs. 61\%) but IPMP participants were more likely to receive long-acting opioids when prescribed (31\% vs. $18 \%$ ) based on adjusted estimates. ${ }^{46,47}$ Prescription of nonsteroidal anti-inflammatory drugs (NSAIDs), antidepressants, and capsaicin were significantly more common in the IPMP group versus usual care. Data are insufficient from one small poor-quality trial, which reported 35 percent attrition. ${ }^{43}$ The trial found no differences between IPMP delivered 2 hours per week for 8 weeks and waitlist regarding early opioid prescription refill $(7.7 \%$ vs. $25 \%)$ at intermediate term. One fair-quality trial reported that similar proportions of patients receiving the IPMP and usual care received new pain medications or that alternative pain medications were discussed during the 12-month intervention, but specific medication types were not described. ${ }^{41}$

\section{Secondary Outcomes}

\section{Health Status}

There was no difference between IPMP and usual care for improved health status based on Short Form (SF)-36 or -12 Physical Component Score (PCS) (0 to 100 scale) postintervention (2 trials, $\mathrm{N}=223$, pooled difference $3.24,95 \% \mathrm{CI}-1.09$ to $\left.6.01, \mathrm{I}^{2}=0 \%\right),{ }^{43,44,49}$ in the short term $(2$ trials, $\mathrm{N}=701$, pooled difference $1.96,95 \% \mathrm{CI}-1.62$ to $\left.5.73, \mathrm{I}^{2}=79.4 \%\right)^{44,48,49}$ or the long term ( 1 trial, $\mathrm{N}=501$, difference $0.53,95 \% \mathrm{CI}-1.20$ to 2.26$)^{48}$ (Appendix I, Figure I-1). Similarly, no 
differences in SF-36 or -12 Mental Component Score (MCS) (0 to 100 scale) were seen postintervention ( 2 trials, $\mathrm{N}=223$, pooled difference $1.92,95 \% \mathrm{CI}-1.92$ to $4.90, \mathrm{I}^{2}=0 \%$ ), $43,44,49$ in the short term ( 2 trials, $\mathrm{N}=701$, pooled difference $1.81,95 \% \mathrm{CI}-0.53$ to $4.25, \mathrm{I}^{2}=0 \%$ ) ${ }^{44,48,49}$ or the long term ( 1 trial, $\mathrm{N}=501$, difference $1.48,95 \% \mathrm{CI}-0.86$ to 3.82$)^{48}$ (Appendix I, Figure I-2). Short term, in one fair-quality trial in patients with chronic widespread pain, IPMP was associated with improvement in PCS (difference $3.60,95 \%$ CI 1.51 to 5.69 ), ${ }^{44,49}$ but in the other fair-quality trial in patients with subacute LBP it was not (difference $0.55,95 \% \mathrm{CI}-1.19$ to $2.29) ;{ }^{48}$ both were compared with usual care. The heterogeneity may be due to conditions studied and/or the differences in intervention. The intervention for subacute LBP consisted of a single 10-hour group session; IPMP in those with chronic widespread pain ranged from 21.25 to 34.75 hours delivered individually. IPMP was associated with improved postintervention PCS (difference 3.50, 95\% CI 1.27 to 5.73) but there was no clear difference in MCS (difference 2.10, $95 \%$ CI 0.28 to 4.48 ) when results were confined to the fair-quality trial. ${ }^{44,49}$ No differences between IPMP and usual care were seen on SF-36 social function subscale or mental health inventory ( 0 to 100 scales, mean differences range -1.4 to 1.4$)$ in the intermediate or long term in one trial, ${ }^{50}$ in EuroQol 5-Dimensions (EQ5D) postintervention in another trial (difference 0.04, $95 \% \mathrm{CI}-0.002$ to 0.08$),{ }^{46,47}$ or on the EQ5D in the long-term in a separate trial. ${ }^{44}$

\section{Depression and Psychological Distress}

There were no differences between IPMP and usual care in postintervention Patient Health Questionnaire (PHQ) depression scores (3 trials across PHQ-8 and -9, N= 912, pooled difference $-0.37,95 \% \mathrm{CI}-1.59$ to $\left.0.37, \mathrm{I}^{2}=0 \%\right)^{41,42,46,47}$ (Appendix I, Figure I-3). One trial reported that IPMP slightly improved psychological distress based on the General Health Questionnaire (scale 0 to 12 ) in the short term (difference $-1.0,95 \% \mathrm{CI}-1.96$ to -0.05 ) versus usual care but this did not persist into the long term (mean 3.0 for each group). ${ }^{44,49}$

\section{Global Improvement and Patient Satisfaction}

Across two studies, IPMPs were associated with improvement in patient-rated global assessment of change versus usual care. Improvement following a 12-month intervention favoring IPMP was seen in one trial (difference $-0.7,95 \% \mathrm{CI}-0.93$ to $-0.47,0$ to 7 scale). ${ }^{46,47}$ Substantially more IPMP recipients reported feeling "very much better" or "much better" (versus little change, no change or feeling worse) compared with those receiving usual care following the 6-month intervention (RR 4.63, 95\% CI 2.17 to 9.87), in the short term (RR 4.37, 95\% CI 2.14 to 8.92 ) and the long term (RR $2.4495 \% \mathrm{CI} 1.33$ to 4.49 ) in the other trial in patients with chronic widespread pain. ${ }^{44,49}$ Patient satisfaction postintervention did not differ between groups in the only trial reporting on this (difference $0.1,95 \% \mathrm{CI}-0.11$ to 0.31 ). ${ }^{46,47}$

\section{Utilization}

No differences in adjusted outcome estimates between IPMP and usual care were seen with respect to hospital admissions ( $12 \%$ vs. $13 \%)$, emergency department visits $(30 \%$ for each group) or total ambulatory visits (14\% each) in one trial. In the IPMP group, 64 percent of patients had phone contact and 21 percent received in-person consultation with a pain specialist. ${ }^{46,47}$ 


\section{Harms and Differential Effectiveness or Safety}

One trial reported that four study-related adverse events occurred, but none were associated with the OA intervention; no further detail was provided. ${ }^{42}$ No intervention-specific events were seen in one other OA trial. ${ }^{41}$

No studies evaluated differential effectiveness or harms of IPMP based on population characteristics of interest.

\section{IPMPs Versus Active Comparators}

One fair-quality RCT conducted in England compared IPMP which incorporated telephonedelivered CBT $($ TCBT $)(\mathrm{N}=112)$ with TCBT $(\mathrm{N}=112)$ alone and exercise alone $(\mathrm{N}=109)$, as well as usual care as described above, in patients with chronic widespread pain (Results Appendix B, Table B-4; Appendix E, Table E-1). ${ }^{44,49}$ Patients were predominately female (71\%), with a mean age of 56 years; approximately one third were retired. TCBT consisted of an initial assessment, seven weekly sessions of 30 to 45 minutes, plus one session at 3 months and another at 6 months post-randomization focused on monitoring progress toward goals, problem solving barriers to improvement and relapse prevention. Exercise was geared toward enhancing cardiorespiratory fitness and consisted of six fitness instructor led monthly assessment appointments to guide and monitor home or leisure facility/gym activity. Exercise types and intensity were tailored to the patient with recommendations for 20 to 60 minutes of exercise at least twice per week on gym facility days plus everyday activities (e.g., brisk walking) on other days. Exercise was logged into patient diaries. The IPMP combined the TCBT and exercise protocols and included provider exchange of information regarding treatment; components were individually tailored and delivered.

\section{Primary Outcomes}

\section{Function}

Evidence for the comparative effectiveness of IPMP is limited to one fair-quality trial. ${ }^{44,49}$ Function was evaluated using the Chronic Pain Grade questionnaire which combines disability and pain intensity using Grades of 0 (no pain), I (low disability/low intensity pain), II (low disability/high intensity pain), III (high disability, low intensity pain), and IV (high disability, high intensity pain). More patients in the IPMP were categorized in Grades 0 to II and fewer in Grades III/VI (high disability) compared with TCBT alone after the 6-month intervention ( $\mathrm{N}=134,92 \%$ vs. $81 \%$, RR $1.14,95 \%$ CI 1.0 to 1.31 ) but differences between groups decreased in the short term $(86 \%$ vs. $79 \%)$ and long term (81\% vs. $82 \%)$. Differences were not statistically significant.

For the comparison of IPMP with exercise alone, again, more IPMP patients had no or low disability (Grades 0 to II) postintervention ( $\mathrm{N}=152,92 \%$ vs. $88 \%$ ) and in the long term ( $81 \%$ vs. $69 \%$ ). In the short term, fewer IPMP patients had no or low disability compared with exercise alone (86\% vs. 92\%). Differences were not statistically significant.

\section{Secondary Outcomes}

\section{Health Status}

No differences in SF-36 PCS or MCS (0 to 100 scales) were seen between IPMP and TCBT groups either postintervention or in the short term. Postintervention and short-term differences for PCS were 1.5 and 2.0 and MCS differences were -0.3 and -1.5. Compared with exercise, 
IPMP was associated with improved PCS postintervention (difference $2.8,95 \%$ CI 0.24 to 5.36) but this did not persist in the long term (difference $0.9,95 \% \mathrm{CI}-1.62$ to 3.42 ). There were no differences between groups on the MCS either postintervention or in the short term (differences were -0.7 and -0.3 , respectively).

\section{Psychological Distress and Global Improvement}

There were no differences in psychological distress based on the General Health Questionnaire (scale 0 to 12) at any time frame for the comparison of IPMP with TCBT or with the comparison to exercise at any time frame. Similarly, there were no differences between IPMP recipients and either TCBT or exercise on global impression of change.

\section{Harms, Utilization, and Differential Effectiveness or Safety}

Two deaths due to cancer were recorded, one in the exercise group and one in the TCBT group. They were not attributed to the interventions.

The trial did not report on healthcare utilization or modification of treatment effects by population characteristics.

\section{Comprehensive Pain Management Programs}

\section{Summary of Findings}

Forty-one RCTs (reported in 58 publications) provided evidence on the effectiveness of CPMPs for Key Question 1 (Appendix E, Table E-2). ${ }^{51-108}$ Twenty-three trials (across 30 publications, $\mathrm{N}=3082$ ) compared CPMPs with usual care, waitlist, or attention control, ${ }^{51,52,54-}$ $57,60,62,65-67,69,70,73,74,82,83,88,89,91-93,100,102-10815$ trials (across 21 publications, $\mathrm{N}=2328$ ) with physical activity alone, $53,56-59,61,68,69,71,72,75,79,84-86,90-92,100,102,103$ five trials (across 6 publications, $\mathrm{N}=531)^{69,74,91,92,100,101}$ with psychological care alone, five trials (across 13 publications, $\mathrm{N}=311)^{63,64,76-78,80,81,87,94-98}$ with pharmacologic therapy alone, and two trials (across 3 publications, $\mathrm{N}=116)^{80,81,99}$ with combined pharmacologic therapy and physical activity. One trial (CPMP vs. physical activity) was a cluster RCT that randomized patients in clusters of four consecutive participants $;{ }^{92}$ the remainder of the trials randomized individual patients. Specific pain diagnoses included chronic low back pain in 19 trials, ${ }^{51,53,55-61,66-68,71,72,80,81,84-86,90-92,94-}$ 98,100,106 fibromyalgia in eight trials, ${ }^{54,63,64,73,76-78,87,88,99,102-105}$ mixed or multiple chronic pain conditions in seven trials, ${ }^{70,75,79,82,83,93,101,108}$ rheumatic disease in three trials, ${ }^{52,65,89}$ chronic nonspecific spinal pain in two trials, ${ }^{69,74}$ and acute low back pain ${ }^{107}$ and traumatic injury ${ }^{62}$ in one trial each.

Sample sizes ranged from 33 to 378 (total sample=5788). None of the studies specifically enrolled Medicare patients, however one trial included older VA patients (mean age 69 years), 20 percent of whom were disabled. ${ }^{106}$ The pooled mean age across trials was 45 years (range, 37 to 69 years). Across all trials, 60 percent of participants were female (range, $4 \%$ to $100 \%$ ). The pooled percent of non-White individuals across the eight trials that provided information on race and/or ethnicity was 6 percent (range, $0 \%$ to 30\%). ${ }^{52,62-64,70,82,83,87,100,106,108}$ Across 22 trials, the mean duration of pain/disease was 69.1 months (range, 9.1 to 195.7 months). ${ }^{51,52,54,55,63,64,66,67,69,70,72-74,76-79,87-89,91,92,94-99,101-103,108}$ One trial ${ }^{80,81}$ excluded patients with a history of substance use disorder, and two trials ${ }^{63,64,87}$ excluded patients with suicidal behaviors. Across 19 trials, the pooled proportion of disabled patients was 57 percent (range, 6\% to $100 \%$ ). Measures of disability included being on sick leave in 10 trials, ${ }^{65,69-71,79,84,85,90-92,100,101}$ 
receiving worker's compensation or disability income in three trials, ${ }^{63,64,87,99,108}$ having a status of "disabled" or "working incapable" in five trials, ${ }^{56-61,76-78,106}$ and holding a self-perception of "disabled" in one trial ${ }^{55}$. Pooled proportion of patients with depression and anxiety was 20 percent (range, $0 \%$ to $59 \%$ ) across eight trials, ${ }^{62,69,71,73,79,89,93,106}$ and 32 percent (range, $4 \%$ to $67 \%$ ) across four trials, ${ }^{69,73,93,106}$ respectively. No trials provided information on the proportion of patients with post-traumatic stress disorder or prior substance use disorder, however, one trial reported that 13 percent of patients engaged in hazardous alcohol consumption. ${ }^{69}$ Only one trial reported on the proportion of patients experiencing suicidal ideation $(15 \%){ }^{62}$ The proportion of smokers across eight trials ranged from 4 to 79 percent (pooled estimate, $32 \%$ ). ${ }^{56-60,71,72,80,81,94-}$ 98,106

Programs delivered the treatment components to patients individually in seven trials, ${ }^{62,80,81,93,97,101,106,107}$ via group sessions in 19 trials, ${ }^{54-61,63,64,66,67,69,70,73,75,82-87,89,94-96,98,102-105,108}$ and via a combination of group and individual sessions in 13 trials. ${ }^{51-53,68,71,72,74,86,88,90-92,99,100}$ Three trials provided no information indicating the delivery format of the CPMP ${ }^{65,76-79}$ Most CPMPs were conducted in an outpatient setting (29 trials), $11,52,54-65,68,69,72-74,76-79,84,85,87-89,91,92,94-$ 98,100-107 six in an inpatient setting, ${ }^{53,71,75,80,81,90,99}$ and four trials evaluated both an inpatient and an outpatient CPMP. ${ }^{66,67,82,83,86,108}$ One trial ${ }^{70}$ delivered the CPMP on either an outpatient or inpatient basis depending on the patient's proximity to the clinic, and another trial ${ }^{93}$ delivered the entire program via an online format. Programs took place at rheumatology clinics in 11 trials, ${ }^{52,54,56-61,65,68,89,94-99}$ rehabilitation clinics in 10 trials, ${ }^{53,66,67,69,70,72,80,81,84-86,90-92}$ pain clinics in six trials, ${ }^{51,55,71,76-78,82,83,101}$ and other settings in eight trials. ${ }^{63,64,73,75,87,88,93,102,103,106,108}$ Six trials provided no information on program location. ${ }^{62,74,79,100,104,105,107}$ Fifteen trials included an occupational, work-hardening, or vocational rehabilitation specific component. ${ }^{53,56-61,65-72,84-}$ 86,107,108 Across all trials, intervention durations ranged from 2 full days to 52 weeks. Twelve trials $^{56-61,68-71,75,79-81,90,102,103}$ evaluated a high-intensity program $(\geq 20$ hours/week or $>80$ hours total) and 25 trials ${ }^{51-55,62-64,72-74,76-78,82-89,91-101,104,105,108}$ evaluated a low-intensity program $(<20$ hours/week or $\leq 80$ hours total). One trial ${ }^{66,67}$ evaluated both a high- and low-intensity program, and three trials ${ }^{65,106,107}$ did not provide enough information to determine program intensity. The contents of the program components varied substantially across trials (Appendix E, Table E-2). Three trials were conducted in the United States, ${ }^{100,106,107}$ one trial in Canada, ${ }^{73}$ three trials in Australia/New Zealand, ${ }^{62,82,83,93}$ two trials in the United Kingdom, ${ }^{101,108} 11$ trials in Northern Europe, ${ }^{52-54,56-61,66,67,69,70,72,74} 14$ trials in Western Europe, ${ }^{55,65,68,71,75,79,84-86,89-92,99,102-105}$ four trials in Eastern/Southern Europe, ${ }^{63,64,76-78,80,81,87,88}$ and three trials in Iran. ${ }^{51,94-98}$ Duration of followup ranged from immediately postintervention to 60 months.

Funding was received from the following sources: nonprofit foundations/associations (15 trials), ${ }^{52,54,56-61,65,70,72,74,79,82,83,93,101,104,105,108}$ government (14 trials), ${ }^{55,62,66,67,75-78,80,81,84-86,89-}$ 92,100,106,107 university (4 trials), ${ }^{51,94-98,102,103}$ and private (1 trial). ${ }^{69}$ Three trials ${ }^{68,71,88}$ reported receiving no funds and four trials ${ }^{53,63,64,73,87,99}$ provided no information on funding.

Twenty-nine trials $\mathrm{s}^{52-61,63-65,68-75,79,84,85,87,88,90-99,102-106}$ were rated fair quality and 12 trials $^{51,62,66,67,76-78,80-83,86,89,100,101,107,108}$ poor quality (Appendix F, Table F-2). The major methodological limitations in the fair-quality trial were unclear allocation concealment methods and the inability to effectively blind patients and caregivers to the CPMP. Other methodological shortcomings in the poor-quality trials included unclear randomization, between-group imbalances in important patient characteristics at baseline, and high attrition. 


\section{CPMPs Versus Usual Care or Waitlist}

\section{Key Points}

- Comprehensive pain management was associated with small improvements in pain on a 0 to 10 scale compared with usual care or waitlist postintervention (SOE: moderate). Differences were below the threshold for small or not statistically significant (or both) at short-term, intermediate-term and long-term followup (SOE: low).

- Comprehensive pain management was associated with moderate improvement in function compared with usual care or waitlist immediately postintervention and in the short term; there was no difference in the intermediate or long term (SOE: low at all time points).

- Data on harms were only reported by one trial (SOE: insufficient).

\section{Detailed Synthesis}

Twenty-three RCTs (reported in 30 publications) $51,52,54-57,60,62,65-67,69,70,73,74,82,83,88,89,91-93,100,102-$ 108 compared CPMPs with usual care or waitlist (Results Appendix B, Table B-5; Appendix E, Table E-2). Randomization occurred at the individual patient-level in all trials. Sample sizes ranged from 39 to 459 (total $\mathrm{N}=2,961$ ). The mean age of participants ranged from 37 to 50 years in all trials except for two, in which the mean age was $59^{52}$ and $69^{106}$ years. The trial in which mean age was 69 years enrolled U.S. veterans 60 years of age or older with LBP. Three trials restricted enrollment to female patients; ${ }^{52,54,88}$ in the other trials, the proportion female ranged from 4 percent ${ }^{106}$ to 96 percent. ${ }^{102,103}$ Race or ethnicity was reported by seven studies. ${ }^{62,82,83,106-}$ ${ }^{108}$ Only five studies reported the proportion of non-White patients (range, $4 \%$ to $30 \%$; two U.S. trials ${ }^{106,107}$ had $30 \%$ African Americans). ${ }^{62,82,83,106-108}$ The pain condition was LBP in nine trials, ${ }^{51,55-57,60,66,67,74,91,92,100,106,107}$ FM in five trials, ${ }^{54,73,88,102-105}$ rheumatoid arthritis in two trials, ${ }^{52,89}$ mixed chronic pain in six trials, ${ }^{65,69,70,82,83,93,108}$ and mixed traumatic injury in one trial. ${ }^{62}$ One trial ${ }^{62}$ enrolled patients with acute $(<4$ weeks) pain following trauma; the other trials enrolled patients with chronic pain (mean duration 3 months ${ }^{74}$ to 168 months ${ }^{66,67}$ in trials that reported this information). Baseline pain intensity ranged from $4.4^{74}$ to $8.2^{88}$ on a 0 to 10 scale. Eleven trials $54,62,65,73,74,88,89,93,100,102,107$ reported baseline depression and patients with psychological comorbidities were excluded in three trials. ${ }^{51,54,70}$ The proportion of patients who used opioids at baseline ranged from 10 percent $^{62}$ to 61 percent $^{108}$ in six trials that reported this information. ${ }^{55,62,82,83,93,106,108}$ Information regarding Medicare-qualifying criteria other than age was not reported. Five trials focused on employed patients or vocational rehabilitation. $56,57,60,65-$ $67,70,107$

Four trials evaluated high intensity ( $\geq 20$ hours per week or $>80$ hours total) CPMPs $^{56,57,60,70,83,102}$ and 17 trials evaluated low intensity $(<20$ hours per

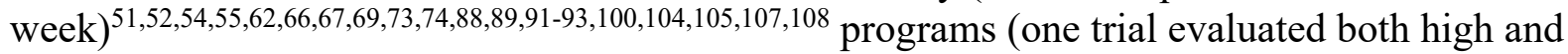
lower intensity programs ${ }^{108}$ ); in two trials the intensity was unclear. ${ }^{65,107}$ In addition to psychological, educational, and exercise components, additional interventions in the CPMP included drug management and medication in 14 trials, $51,52,55-57,60,62,65,70,73,82,83,88,89,93,106-108$ two trials included massage ${ }^{66,67,70}$ and one trial included massage, acupuncture and chiropractic therapy. ${ }^{106}$ The number of sessions varied from one session a week to 5 days of inpatient sessions a week ${ }^{70}$ and duration of treatment ranged from 2 weeks ${ }^{89}$ to 12 months. ${ }^{52}$ Programs were provided individually in four trials, ${ }^{62,93,106,107}$ in group sessions in fourteen trials, ${ }^{51,54,56,57,60,66,67,69,70,73,74,82,83,89,102-105,108}$ and in a combination of group and individual sessions in four trials $;{ }^{52,88,91,92,100}$ one trial did not report delivery format. ${ }^{65}$ Sixteen trials ${ }^{51,52,55-}$ 
57,60,62,65-67,69,73,74,82,83,88,93,102,103,106,107 compared CPMP to usual care and seven trials $\mathrm{s}^{54,70,89,91,92,100,104,105,108}$ to waitlist controls. The majority of CPMPs were conducted in outpatient settings, with the exception of three trials with separate inpatient and outpatient programs, ${ }^{66,67,82,83,108}$ and one that was online. ${ }^{93}$

Trials were conducted in fourteen different countries including three studies in the United States, ${ }^{100,106,107}$ sixteen studies in Europe, ${ }^{52,54-57,60,65-67,69,70,74,82,83,88,89,91,92,102-105,108}$ two in Australia, ${ }^{62,93}$ and one each in Canada, ${ }^{73}$ Iran,${ }^{51}$ New Zealand, ${ }^{82,83}$ and Turkey. ${ }^{88}$ Four trials ${ }^{52,54,56,57,60,89}$ were conducted in rheumatology clinics and the remainder were in pain management or rehabilitation clinic settings with the exception of one trial conducted at the patients home or a local Young Men's Christian Association ${ }^{73}$ and one trial of an online program..$^{93}$ Six trials had followup of 1 year or greater (longest 60 months). ${ }^{56,57,60}$

Fifteen trials were fair quality $52,54-57,60,65,69,70,73,74,88,91-93,102-106$ and eight were poor quality. ${ }^{51,62,82,83,89,100,107,108} 66,67$ The trials were unable to blind care providers or patients; in addition, only two trials blinded outcome assessors. ${ }^{56,57,60,106}$ Other common limitations included unclear randomization and allocation concealment methods and high attrition (Appendix F, Table F-2).

\section{Primary Outcomes}

\section{Pain}

CPMPs were associated with a small improvement in pain compared with usual care or waitlist at postintervention followup ( 11 trials, $\mathrm{N}=764$, pooled difference -0.53 on a 0 to 10 scale, $95 \% \mathrm{CI}-0.80$ to $\left.-0.25, \mathrm{I}^{2}=0 \%\right) .{ }^{51,55,62,70,73,83,92,93,100,103,106}$ At other time points the difference was below the threshold for small effects, was not statistically significant, or both (short term: 6 trials, $\mathrm{N}=943$, pooled difference $-0.39,95 \% \mathrm{CI}-0.83$ to 0.04 , $\mathrm{I}^{2}=36.6 \%,{ }^{54,60,66,70,93,108}$ intermediate term: 4 trials, $\mathrm{N}=690$, pooled difference $-0.85,95 \% \mathrm{CI}$ -2.01 to $0.21, \mathrm{I}^{2}=83.5 \%,{ }^{65,67,88,107}$ long term: 6 trials, $\mathrm{N}=906$, pooled difference $-0.13,95 \% \mathrm{CI}$ -0.71 to $0.22, \mathrm{I}^{2}=19.5 \%{ }^{51,56,65,67,74,103}$ ) (Figure 5). Findings were similar in sensitivity analyses excluding poor-quality trials, ${ }^{51,62,66,67,82,83,100,107,108}$ excluding the trial in patients with acute $(<4$ weeks) trauma, ${ }^{62}$ using the most common duration of followup for the long-term analysis, ${ }^{57,65-67}$ and excluding trials that used the McGill Pain Questionnaire (MPQ) (which is based on pain descriptors, rather than a visual analog scale [VAS] or numerical rating scale [NRS] for pain intensity) ${ }^{100}$ (Appendix I, Figures I-4 to I-7). The effect sizes and associated variability were similar for trials of higher intensity programs ( $>20$ hours/week or $>80$ total hours) and lower intensity programs. Meta-regression confirmed no significant differences in pain effect estimates between higher and lower intensity programs immediately postintervention $(p=0.67)$, in the short-term $(p=0.74)$ or the long-term $(p=0.50)$. Effect sizes and variability were also similar across programs that were delivered individually, as group sessions, or a combination of these.

All trials enrolled patients with chronic pain except for one small $(n=67)$ trial of patients with acute $(<4$ weeks) trauma. In this trial, which only reported results at postintervention, there was no difference between CPMP (lower intensity) versus usual care, but the estimate was imprecise. ${ }^{62}$ One small $(\mathrm{N}=55)$, fair quality trial evaluated effects of CPMP (intensity unclear) in patients 60 and older with chronic LBP. ${ }^{106}$ Postintervention effect on pain (difference $-1.22,95 \%$ CI -2.28 to -0.16 ) was somewhat greater compared to the other trials, which enrolled younger populations. However, evidence was too limited to determine how older age impacts effectiveness. 
Figure 5. CPMP versus usual care or waitlist control: Pain

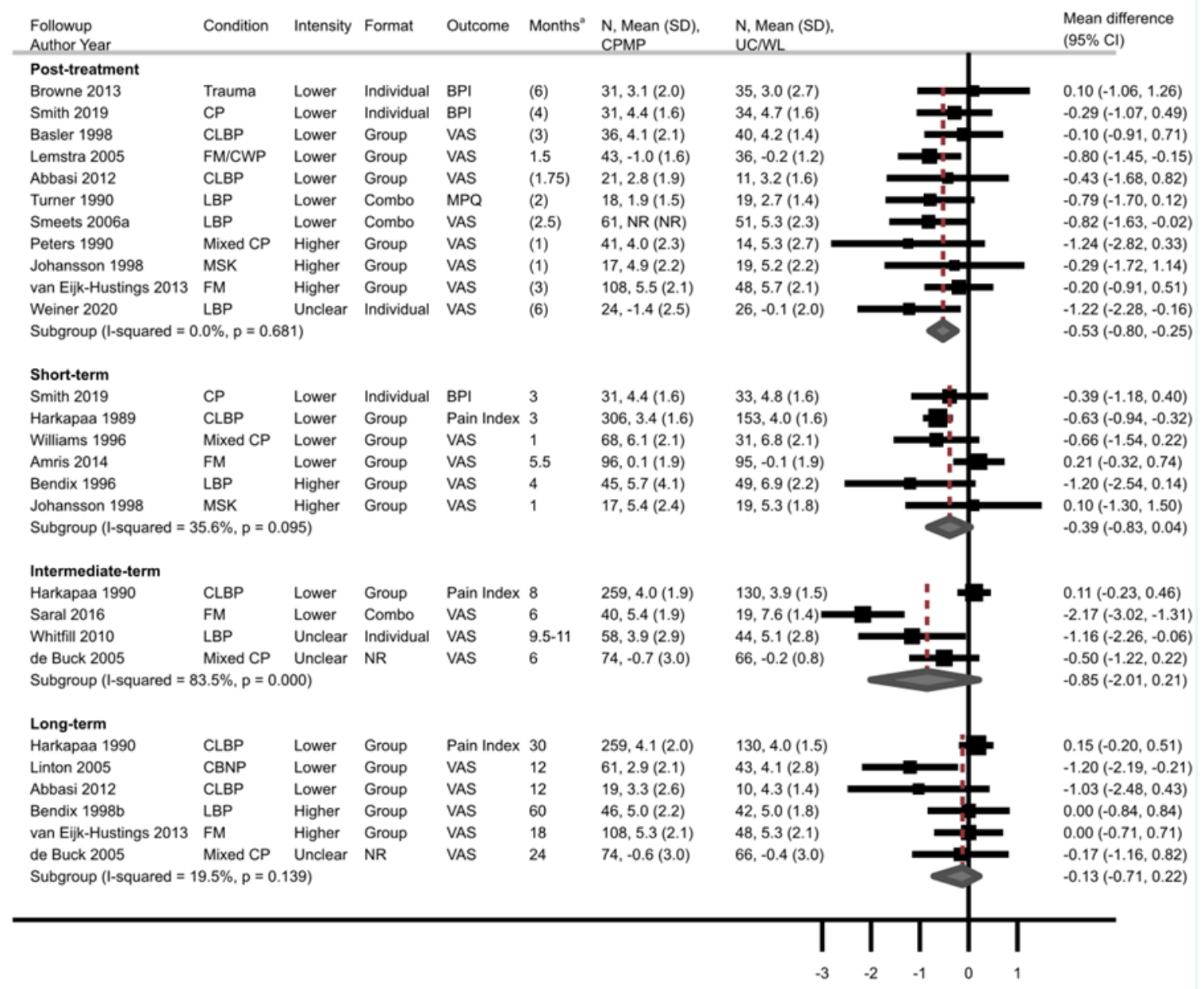

Favors CPMP Favors UC

$\mathrm{BPI}=$ Brief Pain Inventory; $\mathrm{CBNP}=$ chronic back/neck pain; $\mathrm{CI}=$ confidence interval; $\mathrm{CLBP}=$ chronic low back pain; $\mathrm{Combo}=$ combination group and individual sessions; $\mathrm{CP}=$ chronic pain; $\mathrm{CPMP}=$ comprehensive pain management program; $\mathrm{CWP}=$ chronic widespread pain; FM = fibromyalgia; LBP = low back pain; MPQ = The McGill Pain Questionnaire; MSK = musculoskeletal pain; $\mathrm{NR}=$ not reported; $\mathrm{SD}=$ standard deviation; $\mathrm{UC}=$ usual care; $\mathrm{VAS}=$ visual analog scale; $\mathrm{WL}=$ waitlist. ${ }^{a}$ Number of months in parentheses for the postintervention timeframe indicate the duration of the intervention; followup for the remaining timeframes is in months following the end of the intervention.

\section{Function}

CPMPs were associated with moderate improvements in function compared with usual care or waitlist at postintervention ( 13 trials, $\mathrm{N}=981$, pooled $\mathrm{SMD}-0.52,95 \% \mathrm{CI}-0.88$ to -0.16 , $\left.\mathrm{I}^{2}=83.0 \%\right)^{51,52,55,62,73,83,89,92,93,100,103,105,106}$ and short-term followup ( 7 trials, $\mathrm{N}=1,097$, pooled SMD $-0.62,95 \% \mathrm{CI}-1.02$ to $\left.-0.24, \mathrm{I}^{2}=83.7 \%\right) .{ }^{54,60,66,89,93,105,108}$ There was no difference at intermediate-term followup ( 4 trials, $\mathrm{N}=588$, pooled $\mathrm{SMD}-0.33,95 \% \mathrm{CI}-0.81$ to 0.05 , $\left.\mathrm{I}^{2}=66.9 \% \%\right),{ }^{65,67,88,89}$ or at long-term followup ( 6 trials, $\mathrm{N}=974$, pooled SMD $-0.21,95 \% \mathrm{CI}$ -0.47 to $-0.00, \mathrm{I}^{2}=42.1 \%$ ). ${ }^{51,56,65,67,74,89,103}$ The measures used to assess function varied (Figure 6 ). The difference on the original RMDQ scale ranged from -1.40 to 2.80 postintervention and was -1.9 in the short term in one trial. Differences on the original Fibromyalgia Impact Questionnaire (FIQ) ranged from -11.50 to -3.40 postintervention and from -12.1 to 0.10 in the 
short term. For the Sickness Impact Profile (SIP), differences on the original scale ranged from $-68.80^{83}$ to -3.50 postintervention and was -11.50 in the short term in a single trial.

The findings for function were similar in sensitivity analyses excluding an outlier trial, ${ }^{89}$ excluding poor-quality trials, ${ }^{51,62,66,83,89,100,108}$ and analysis excluding the trial in acute trauma $(<4$ weeks). ${ }^{62}$ Using the most common duration of followup (primarily 12 to 18 months) for the longterm analysis ${ }^{65,67}$ the effect size was decreased and again there was no difference between CPMP and usual care or waitlist ( 6 trials pooled SMD $-0.04,95 \% \mathrm{CI}-0.21$ to $0.09, \mathrm{I}^{2}=0 \%$ ) $51,57,65,67,74,103$ (Appendix I, Figures I-8 to I-11). Meta-regression confirmed that there were no significant differences in function estimates between higher and lower intensity programs immediately postintervention $(\mathrm{p}=0.813)$ and in the long-term $(\mathrm{p}=0.154)$. The trial of patients with acute trauma found no difference between CPMP versus usual care at postintervention, but the estimate was imprecise. ${ }^{62}$ The trial of patients 60 years or older reported results for postintervention function that were consistent with the overall pooled results. ${ }^{106}$

Figure 6. CPMP versus usual care or waitlist control: Function

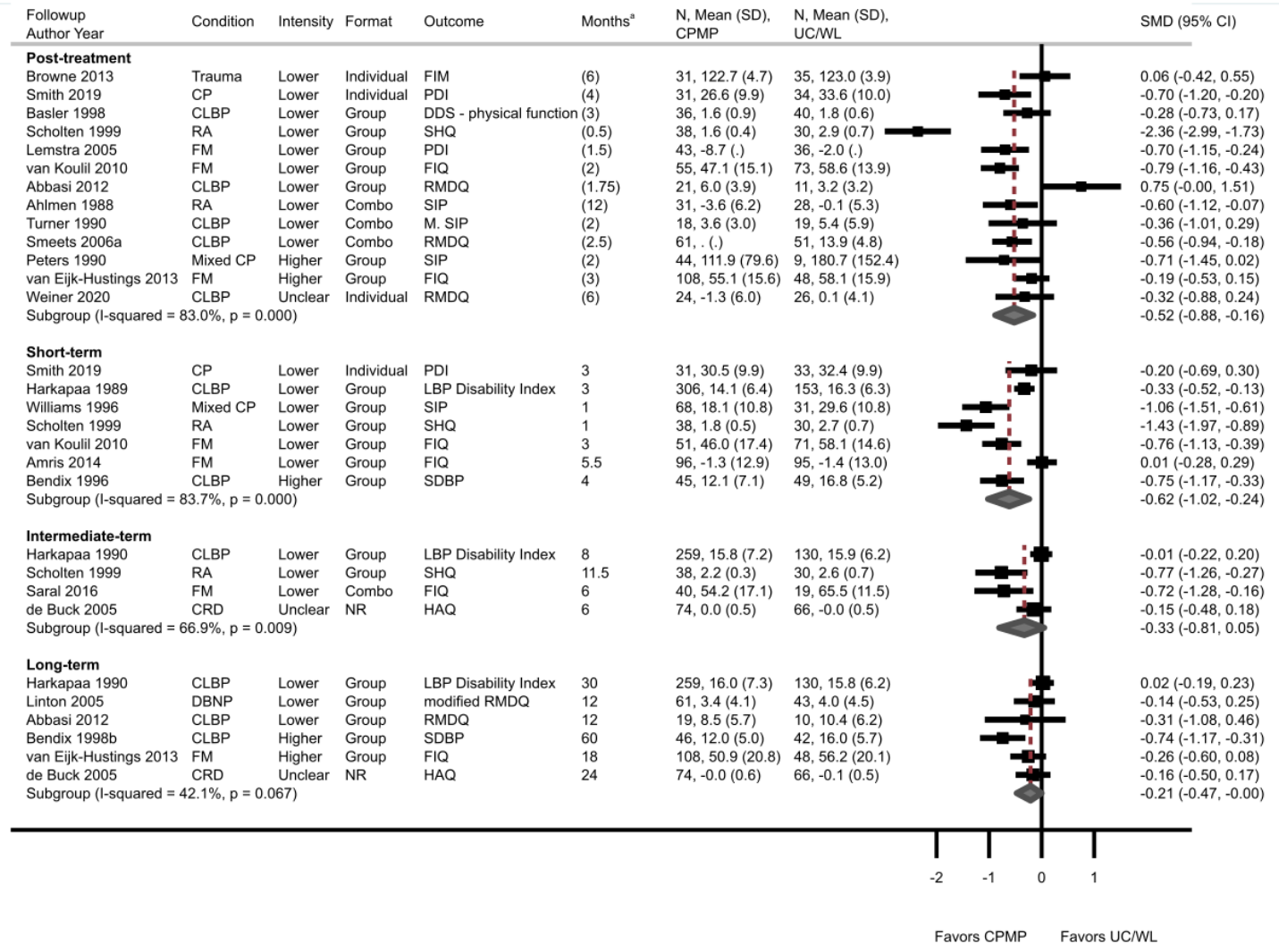

$\mathrm{CBNP}=$ chronic back/neck pain $\mathrm{CI}=$ confidence interval; $\mathrm{CLBP}=$ chronic low back pain ; Combo $=$ combination group and individual sessions; $\mathrm{CP}=$ chronic pain; $\mathrm{CPMP}=$ comprehensive pain management program; $\mathrm{CRD}=$ chronic rheumatoid arthritis; DBNP $=$ back/neck pain; DDS= Dusseldorf Disability Scale; FIM = Functional independence measure; FIQ = Fibromyalgia Impact Questionnaire; FM = Fibromyalgia; HAQ = Health Assessment Questionnaire LPB = low back pain; M. SIP = Modified Sickness Impact Profile; NR = not reported; Sickness Impact Profile; PDI = Pain Disability Index; RA = rheumatoid arthritis; $\mathrm{RMDQ}=$ Roland and Morris Disability Questionnaire; SD = standard deviation; SDBP = self-reported disability of back pain scale; SHQ = Stanford Health Questionnaire; SIP = Sickness Impact Profile; SMD = standardized mean difference; UC = usual care; $\mathrm{WL}=$ waitlist.

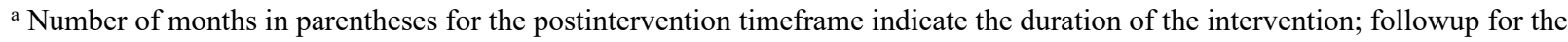
remaining timeframes is in months following the end of the intervention. 


\section{Opioid Use}

Evidence on CPMP and changes in opioid prescribing was very limited. One trial found no difference between CPMP versus usual care in likelihood of opioid use at postintervention (63.3\% vs. $50.0 \%$, RR $1.27,95 \%$ CI 0.82 to 1.95$)$ or 3 months $(60.0 \%$ vs. $51.0 \%$, RR $1.16,95 \%$ CI 0.75 to 1.81$).{ }^{93}$ One other trial reported that among patients randomized to CPMP, the proportion not using opioids at followup increased compared to baseline (at 12 months, the proportion not using opioids increased from $47 \%$ to $80 \%$ for inpatient CPMP and from $33 \%$ to $55 \%$ for outpatient CPMP), but did not report opioid use in the waitlist control arm. ${ }^{108}$

\section{Secondary Outcomes}

\section{Health Status}

Evidence on effects of CPMP on health status (health-related quality of life) was sparse, based on two trials. Estimates were imprecise but indicated no differences in SF-36 PCS or MCS scores (Appendix I, Figures I-13 to I-14).

\section{Depression and Anxiety}

CPMP was associated with small effects on depression and small to moderate effects on anxiety versus waitlist or usual care, with the exception of anxiety at intermediate term, which was only reported in one trial with an imprecise estimate. For depression, based on five to nine trials, the pooled SMD ranged from -0.21 to -0.48 (Appendix I, Figure I-15). For anxiety, based on two or three trials, the pooled SMD was $-0.67\left(95 \% \mathrm{CI}-0.96\right.$ to $\left.-0.38, \mathrm{I}^{2}=0.0 \%\right)$ at postintervention, $-0.45\left(95 \% \mathrm{CI}-1.05\right.$ to $\left.0.12, \mathrm{I}^{2}=75 \%\right)$ at short term and $-0.33(95 \% \mathrm{CI}-0.61$ to $-0.06, \mathrm{I}^{2}=0.0 \%$ ) at long term (Appendix I, Figure I-18). For studies reporting BDI, differences on the original scale ranged from -6.70 to 0.50 postintervention, from -6.60 to -3.70 in the short term and from -2.90 to -1.30 at intermediate term.

\section{Global Improvement and Patient Satisfaction}

One fair-quality trial ${ }^{91,92}$ found CPMP associated with greater global improvement on a 1 to 7 scale (adjusted difference $0.70,95 \%$ CI 0.17 to 1.24 ) and higher patient satisfaction on a 0 to 100 VAS (range of adjusted differences, 19.33 to 27.81, across baseline RMDQ percentiles) versus waitlist at postintervention followup. A second poor-quality trial reported patient satisfaction postintervention in the CPMP group only (mean 5.5 on a 1 to 7 scale). ${ }^{100}$ Both trials were in LBP patients (Appendix B, Table B-5.)

\section{Harms}

One trial reported that three participants $(5.5 \%)$ randomized to CPMP reported increased low back or leg pain leading to withdrawal from the trial. ${ }^{91,92}$ A second trial reported occasional mild increases in pain after some exercise sessions in the CPMP groups ${ }^{88}$ Adverse events related to usual care were not reported by either trial. Harms were not reported in the other trials.

\section{Utilization}

Four studies reported on utilization. ${ }^{56,57,60,62,102,103,108}$ Two fair-quality trials, one for $\mathrm{FM}^{102}$ and one for chronic LBP, ${ }^{56,57,60}$ found no difference between CPMP versus usual care in hospitalization or surgery at 18 for months for $\mathrm{FM}^{102}$ or 60 months for chronic LBP. ${ }^{56}$ One poorquality trial ${ }^{62}$ found CPMP associated with decreased self-reported frequency of outpatient visits for traumatic injury patients versus usual care and another poor-quality trial $^{108}$ that compared 
outpatient and inpatient mixed chronic pain groups to waitlist controls reported no surgery in either group in the long term.

\section{CPMPs Versus Physical Activity}

\section{Key Points}

- There were no differences on a 0 to 10 scale comparing CPMP with physical activity postintervention, at short term, intermediate term or long term (SOE: moderate for postintervention and long term, low for short term and intermediate term).

- CPMP was associated with a small improvement in short-term function compared with physical activity, but not postintervention, intermediate term or long term (SOE: moderate for all timepoints).

- Only two trials reported on harms. One noted no adverse events related to the intervention, and the other reported less pain in new locations in the CPMP group compared to physical activity, RR $0.38,95 \%$ CI 0.08 to 1.7 (SOE: insufficient).

\section{Detailed Synthesis}

Fifteen RCTs (reported in 21 publications) compared 17 CPMPs with physical activity (Results Appendix B, Table B-6; Appendix E, Table E-2). ${ }^{53,56-59,61,68,69,71,72,75,79,84-86,90-92,100,102,103}$ One was a cluster RCT that randomized patients in clusters of four consecutive participants $;{ }^{92}$ the remainder randomized individual patients. Sample sizes ranged from 33 to 409 (total sample=2,344). The diagnosis was FM, ${ }^{103}$ chronic musculoskeletal pain, ${ }^{79}$ and a mix of subacute and chronic LBP pain ${ }^{68}$ in one trial each and chronic LBP with or without leg pain in the remaining 12 trials. Mean symptom duration ranged from 2.2 to 12.9 years across six trials that reported it, ${ }^{69,72,79,92,100,103}$ and in seven trials, pain 3 months or more, ${ }^{71,84-86} 6$ months or more, ${ }^{53,59,90}$ or longer than 6 weeks (for the trial including subacute pain), ${ }^{68}$ was required for inclusion. The remaining two trials ${ }^{61,75}$ only stated that pain was chronic. Baseline pain intensity was moderate in most trials. None of the studies specifically included Medicare patients and mean age in all studies ranged from 40 to 49 years. One trial in FM was comprised of primarily females $(95 \%)^{103}$ and another (chronic LBP) enrolled only females. ${ }^{72}$ The proportion of male participants ranged from 21 to 67 percent across the other trials. Only one trial reported on race (all patients were White). ${ }^{100}$ Comorbidities were poorly reported across the trials and included smoking (range, $31 \%$ to $66 \%$ ) in three trials, ${ }^{59,71,72}$ prior back surgery (15\% to $25 \%$ ) in three trials, ${ }^{59,71,92}$ previous depression diagnosis in one trial $(30 \%),{ }^{71}$ and one trial stated that 39 percent of patients had one or more comorbidity. ${ }^{79}$ In addition, most trials excluded patients with severe psychiatric disorders and contraindications to exercise. Other exclusion criteria related to comorbidities included substance abuse (3 trials), ${ }^{68,69,103}$ severe comorbidities (not further defined) (2 trials) ${ }^{69,90}$ cardiovascular disease ( 1 trial $){ }^{100}$ and current depression ( 1 trial). ${ }^{79}$ Studies inconsistently reported disability. One trial did report that 38 and 24 percent of its population, respectively, was receiving full and partial sick leave/disability pensions. ${ }^{92}$ Nine trials included patients who were either currently sick-listed, at risk for sick leave/work disability, or who had numerous episodes of sick leave prior to study entry. ${ }^{59,61,68,69,71,79,84,86,90}$ One trial ${ }^{100}$ was conducted in the United States and the remainder were conducted in Europe.

Regarding the physical activity components of both groups, eight trials included only active physical activity such as aerobics, strength training, flexibility and stretching exercises, and 
proprioception and coordination exercises ${ }^{59,61,71,84,86,92,100,103}$ and seven included both active and passive modalities (e.g., massage, traction, spinal mobilization, ultrasound, heat therapy, and electrotherapies). ${ }^{53,68,69,72,75,90}$ Ten trials also included an occupational therapy or work therapy component. ${ }^{59,61,68,71,72,75,79,84,86,103}$ The intensity of the programs varied substantially, ranging from 24 to 150 hours. Ten programs met our criteria for high intensity $(\geq 20$ hours/week or $>80$ hours total) ${ }^{56-59,61,68,69,71,75,79,86,90,102,103}$ six for low intensity, ${ }^{53,56-59,72,84-86,100}$ and one unclear. ${ }^{92}$ The duration of the programs ranged from 3 to 12 weeks. Program components were delivered in group sessions in five trials ${ }^{56-59,61,69,84,85,102,103}$ and in a combination of group and individual sessions in ten trials. $53,68,71,72,75,79,86,90-92,100$

Eleven trials were rated fair quality ${ }^{53,56-59,61,68,69,71,72,84,85,90-92,102,103}$ and two poor quality ${ }^{86,100}$ (Appendix F, Table F-2). The major methodological limitation in the fair-quality trials was the inability to effectively blind patients and caregivers to the CPMP. Other methodological shortcomings included unclear randomization, unclear allocation concealment methods, and high attrition.

\section{Primary Outcomes}

\section{Pain}

There were no differences in low back or musculoskeletal pain on a 0 to 10 scale comparing CPMP with physical activity alone postintervention ( 8 trials, $\mathrm{N}=1,312$, pooled difference -0.05 , $95 \% \mathrm{CI}-0.32$ to $\left.0.19, \mathrm{I}^{2}=0 \%\right),{ }^{72,75,79,84,90,91,100,103}$ at short term ( 1 trial, $\mathrm{N}=106$, difference -0.35 , $95 \% \mathrm{CI}-1.49$ to 0.79 ), ${ }^{59}$ intermediate term (4 trials, $\mathrm{N}=341$, pooled difference $-0.15,95 \% \mathrm{CI}$ -0.73 to $\left.0.38, \mathrm{I}^{2}=0 \%\right)^{71,72,91,100}$ or long term ( 9 trials, $\mathrm{N}=2,492$, pooled difference $0.05,95 \% \mathrm{CI}$ -0.30 to $0.42, \mathrm{I}^{2}=0 \%$ ), ${ }^{56,61,72,75,85,86,91,100,103}$ (Figure 7). The findings for pain were similar in sensitivity analyses excluding two poor-quality trials postintervention, and in the intermediate and long term ${ }^{86,100}$ (Appendix I, Figure I-21) and using the most common duration of followup for the long-term analysis ${ }^{56,58,72}$ (Appendix I, Figure I-22). There were three fair-quality trials that assessed pain radiating into the leg on a 0 to 10 scale, which found no difference comparing CPMP with physical activity alone postintervention ( 1 trial, $\mathrm{N}=120$, difference $0.20,95 \% \mathrm{CI}$ -0.75 to 1.15$),{ }^{72}$ short term ( 1 trial, $\mathrm{N}=106$, difference $-0.94,95 \% \mathrm{CI}-2.30$ to 0.42$),{ }^{59}$ intermediate term ( 1 trial, $\mathrm{N}=115$, difference $0.50,95 \% \mathrm{CI}-0.44$ to 1.44$),{ }^{72}$ and long term ( 3 trials, $\mathrm{N}=263$, pooled difference $-0.61,95 \% \mathrm{CI}-1.59$ to $\left.0.37, \mathrm{I}^{2}=0 \%\right)^{56,61,72}$ (Appendix I, Figure I-23). There were no significant differences between higher and lower intensity programs in effects on pain immediately postintervention $(p=0.14)$, at intermediate term $(p=0.30)$ or at long term $(p=0.55)$, based on meta-regression. 
Figure 7. CPMP versus physical activity: Pain

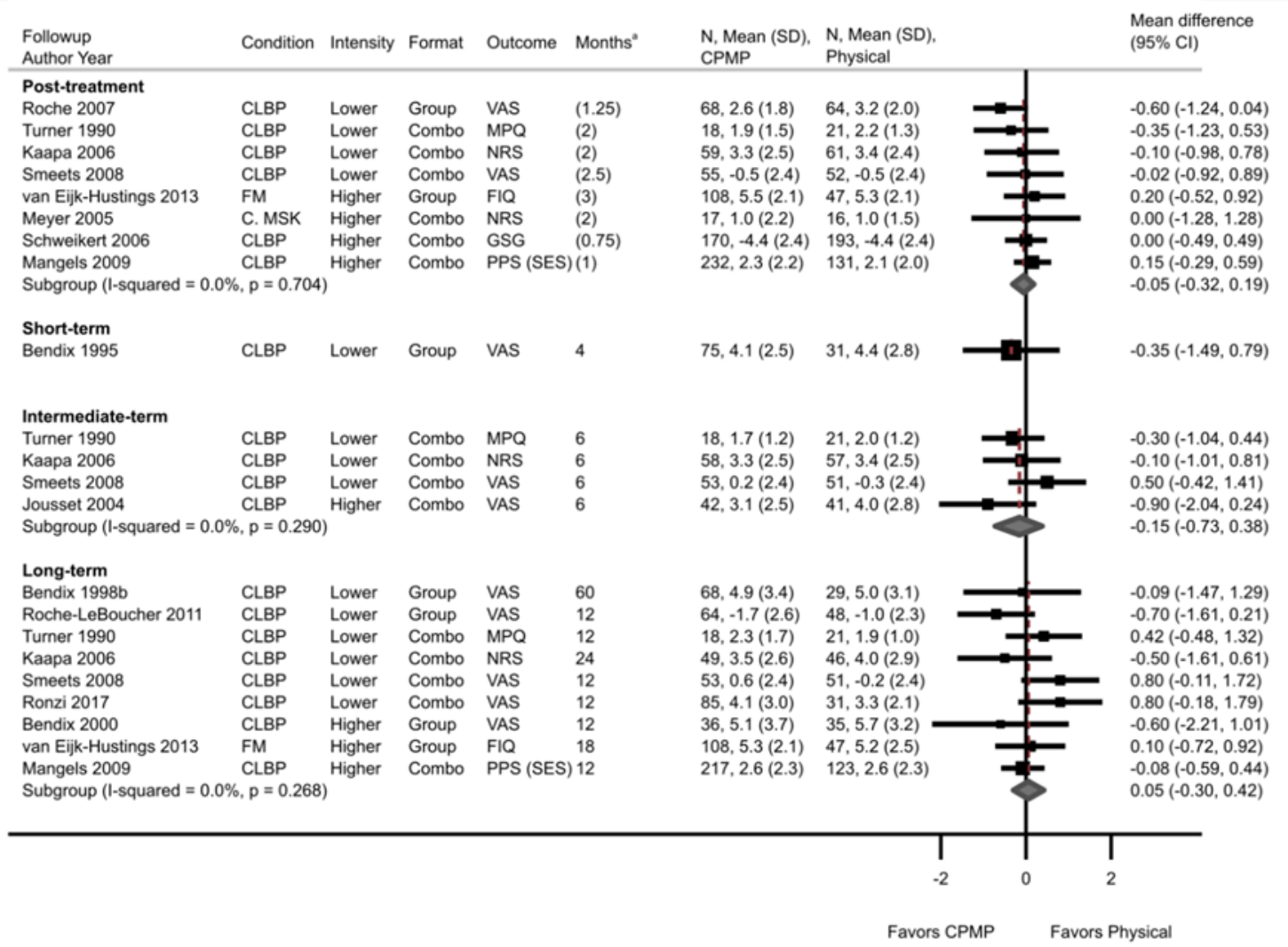

$\mathrm{CI}=$ confidence interval; $\mathrm{CLBP}=$ chronic low back pain; $\mathrm{C} . \mathrm{MSK}=$ chronic musculoskeletal disease; Combo = combination group and individual sessions; CPMP = comprehensive pain management program; FIQ = Fibromyalgia Impact Questionnaire; FM = fibromyalgia; GSG = German school grades pain intensity scale; MPQ = The McGill Pain Questionnaire; NRS = numerical rating scale; PPS $($ SES $)=$ Pain Perception Scale; $S D=$ standard deviation; VAS = visual analog scale.

${ }^{\text {a }}$ Number of months in parentheses for the postintervention timeframe indicate the duration of the intervention; followup for the remaining timeframes is in months following the end of the intervention.

\section{Function}

CPMP was associated with a small improvement in short-term function compared with physical activity alone ( 3 trials, $\mathrm{N}=459$, pooled SMD $-0.37,95 \% \mathrm{CI}-0.61$ to -0.16 , $\mathrm{I}^{2}=0 \%$ ), ${ }^{53,59,68}$ but not postintervention ( 9 trials, $\mathrm{N}=1,379$, pooled $\mathrm{SMD}-0.05,95 \% \mathrm{CI}-0.16$ to $\left.0.05, \mathrm{I}^{2}=0 \%\right),{ }^{68,72,75,79,84,90,91,100,103}$ or in the intermediate ( 6 trials, $\mathrm{N}=695$, pooled SMD -0.11 , $95 \% \mathrm{CI}-0.36$ to $\left.0.13, \mathrm{I}^{2}=38.3 \%\right)^{53,68,71,72,91,100}$ or long term (10 trials, $\mathrm{N}=1,214$, pooled SMD $-0.12,95 \% \mathrm{CI}-0.31$ to $0.06, \mathrm{I}^{2}=43.3 \%$ ), ${ }^{58,61,68,72,75,85,86,91,100,103}$ (Figure 8 ). The findings for function were similar in sensitivity analyses excluding two poor-quality trials ${ }^{86,100}$ (Appendix I, Figure I-24) and using the most common duration of followup for the long-term analysis ${ }^{56,58,72}$ (Appendix I, Figure I-25). There were no significant differences between higher and lower intensity programs in effects on function immediately postintervention $(p=0.50)$ or at long-term $(p=0.91)$, based on meta-regression. 
Figure 8. CPMP versus physical activity: Function

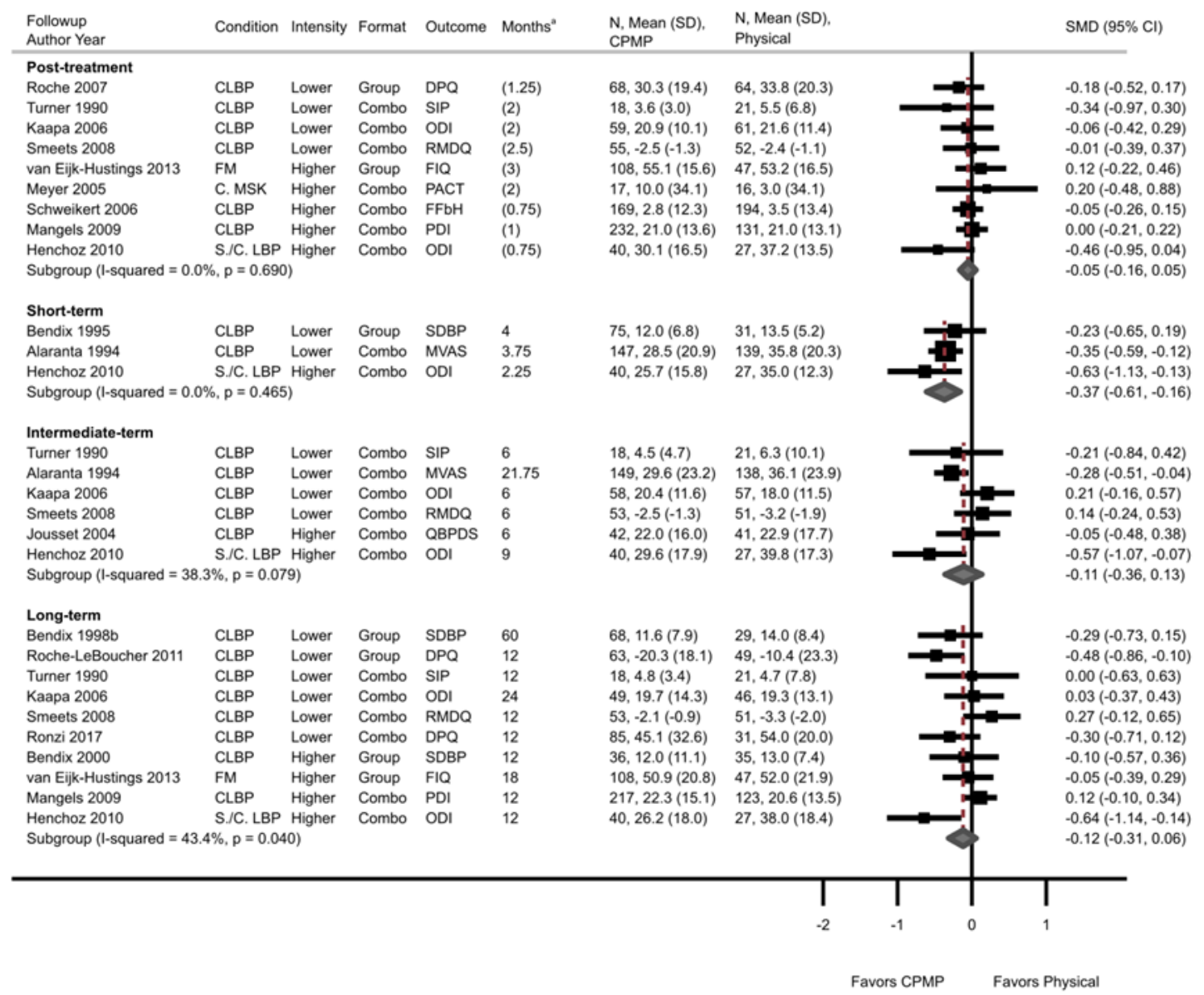

$\mathrm{CI}=$ confidence interval CLBP $=$ chronic low back pain; $\mathrm{C}$. MSK = chronic musculoskeletal disease; Combo = combination group and individual sessions; $\mathrm{CPMP}=$ comprehensive pain management program; $\mathrm{DPQ}=$ Dallas Pain Questionnaire; $\mathrm{FFbH}=$ Hannover functional questionnaire; FIQ = Fibromyalgia Impact Questionnaire; FM = fibromyalgia; S./C. LBP = subacute and chronic low back pain; MVAS = Million visual analog scale; ODI = Oswestry Disability Index; PACT = Performance Assessment of Capacity Testing; PDI = Pain Disability Index; QBPDS = Quebec Back Pain Disability Scale; RMDQ = Roland Morris Disability Index; S/C. LBP = subacute and chronic low back pain; SD = standard deviation; SDBP = self-reported disability of back pain scale; SIP = Sickness Impact Profile; SMD = standardized mean difference.

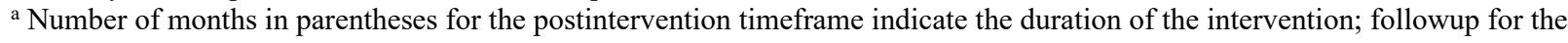
remaining timeframes is in months following the end of the intervention.

\section{Secondary Outcomes}

\section{Health Status}

There were no differences in health status between CPMP and physical activity alone as measured by the SF-12 or SF-36 PCS and MCS postintervention ( 2 trials, $\mathrm{N}=234$, PCS pooled difference $1.17,95 \% \mathrm{CI}-1.61$ to $5.75, \mathrm{I}^{2}=0 \%$; MCS pooled difference $-0.74,95 \% \mathrm{CI}-3.71$ to $\left.4.50, \mathrm{I}^{2}=20.9 \%\right),{ }^{75,79}$ or long term ( 2 trials, $\mathrm{N}=302$, PCS pooled difference $0.58,95 \% \mathrm{CI}-1.97$ to $4.37, \mathrm{I}^{2}=0 \%$; MCS pooled difference $0.19,95 \% \mathrm{CI}-3.20$ to $\left.2.86, \mathrm{I}^{2}=0 \%\right) ;{ }^{.75,86}$ results were similar when the poor-quality trial ${ }^{86}$ was excluded at long term (Appendix I, Figures I-26 to I29). Likewise, there were no differences between CPMP and physical activity in the SF-36 global health in one trial, ${ }^{69}$ or EQ5D in two trials ${ }^{90,103}$ at any time period. 


\section{Depression and Anxiety}

There were no differences in the severity of depression between CPMP and physical activity alone postintervention ( 6 trials, $\mathrm{N}=1,143$, pooled $\mathrm{SMD}-0.07,95 \% \mathrm{CI}-0.20$ to 0.13 , $\left.\mathrm{I}^{2}=0 \%\right),{ }^{72,75,90,91,100,103}$ intermediate term ( 3 trials, $\mathrm{N}=258$, pooled SMD $0.01,95 \% \mathrm{CI}-0.25$ to $\left.0.26, \mathrm{I}^{2}=0 \%\right),{ }^{72,91,100}$ or long term ( 5 trials, $\mathrm{N}=737$, pooled $\mathrm{SMD}-0.04,95 \% \mathrm{CI}-0.24$ to 0.22 , $\left.\mathrm{I}^{2}=14.9 \%\right) ;{ }^{72,75,91,100,103}$ results were similar in sensitivity analyses excluding the poor-quality trial $^{100}$ at all timepoints and using the most common duration of followup for the long-term analysis $^{72}$ (Appendix I, Figures I-30 to I-32). There were no differences at any time between CPMP and physical activity in measures of anxiety ${ }^{71,84-86,90,103}$ (Appendix I, Figure I-33).

\section{Global Improvement}

Patient-reported self-perceived improvement was slightly higher in the CPMP versus physical activity alone in one lower ${ }^{91,92}$ and one higher intensity program ${ }^{61}$ in the long term, while life satisfaction (with health) was not different in another trial. ${ }^{75}$

\section{Harms}

One poor-quality trial reported no adverse effects related to the CPMP, ${ }^{86}$ while one trial reported less pain in new locations in the CPMP group compared to physical activity (RR 0.38 , $95 \%$ CI 0.08 to 1.7$).{ }^{79}$ No other trial reported on program harms.

\section{Utilization}

Five fair-quality trials (4 LBP, 1 FM) reported on utilization. All five trials reported on health system contacts; three found no difference between groups in the number of yearly contacts (two higher intensity ${ }^{61,102,103}$ and one lower intensity ${ }^{72}$ programs) one found fewer contacts in those participating in a higher intensity CPMP program after 12- and 24-month followup, but no difference in those participating in a lower intensity program, ${ }^{56-59}$ and one reported a decrease in the number of visits in both the CPMP and control group but did not compare the groups. ${ }^{53}$ One fair-quality trial found no difference in the proportion of participants hospitalized due to back pain or who underwent back surgery in either a lower or higher intensity program during a 5-year followup period. ${ }^{56-59}$

\section{CPMPs Versus Pharmacologic Therapy and CPMPs Versus Pharmacologic Therapy Plus Physical Activity}

\section{Key Points}

- Comprehensive pain management programs were associated with moderate improvement in average pain (VAS or NRS 0 to 10 scale) versus pharmacologic treatments alone postintervention and small improvement in the intermediate term, but not in the short or long term (SOE: low at all time points).

- More CPMP participants with FM experienced 30 percent or more improvement on 0 to 10 NRS pain scale in one fair-quality trial compared with pharmacologic therapy alone.

- Comprehensive pain management programs were associated with moderate improvement in function based on FIQ Total Score (0 to 100 scale) postintervention and small improvements at short, intermediate, and long term versus pharmacologic therapy alone (SOE: moderate for intermediate term; low for postintervention, short term and long 
term). At all timepoints, more CPMP patients experienced 14 percent or more improvement in function.

- Comprehensive pain management programs were associated with moderate pain improvement postintervention, and in the intermediate and long term compared with the combination of pharmacologic therapy (antidepressants) and physical activity. There were no differences in function between groups at any time point (SOE: low).

- Evidence regarding opioid use from one trial comparing CPMP versus pharmacologic therapy plus physical activity is insufficient.

- No trial reported on harms.

\section{Detailed Synthesis}

Six RCTs (reported in 14 publications) (Appendix E, Table E-2) compared CPMP with pharmacologic therapy alone (5 RCTs, 13 publications) ${ }^{63,64,76-78,80,81,87,94-98}$ (Results Appendix B, Table B-7) or plus physical activity (2 RCTs, 3 publications) ${ }^{80,81,99}$ (Results Appendix B, Table B-8). Randomization occurred at the individual patient-level in all trials. Sample sizes ranged from 63 to 197 (total sample=702). Pain diagnoses included chronic LBP ${ }^{80,81,94-98}$ and FM ${ }^{63,64,76-}$ $78,87,99$ in three trials each. Mean pooled pain duration in the FM trials was 13.4 years (range, 11.7 to 16.3 years) while in the two LBP trials that reported it, pain duration ranged from 9 months ${ }^{97}$ to 6.9 years. ${ }^{94-96,98}$ Mean ages ranged from 44 to 50 years. Across the FM trials, populations were almost entirely female (pooled $97 \%$, range $91 \%$ to $100 \%$ ) while there was more variability across the LBP trials (pooled $75 \%$ female, range $43 \%$ to $100 \%$ ). Comorbidities were reported by three trials, and included hypothyroidism (12\%), high blood pressure (10\%), chronic obstructive pulmonary disease $(12 \%)$, diabetes mellitus $(3 \%)$, rheumatoid arthritis $(3 \%)$, and others $(42 \%)$ in one trial, ${ }^{76-78}$ and smoking (4\% to $6 \%$ ) in two others. ${ }^{94-98}$ In addition, exclusion criteria included major psychiatric disorders in two trials ${ }^{80,81,99}$ and substance abuse disorders in one trial. ${ }^{80,81}$ None of the studies specifically included Medicare patients. Fifteen percent of patients in one trial $^{76-78}$ were on disability and 6 percent ${ }^{63,64,87}$ and 20 percent ${ }^{99}$ were receiving workers' compensation in two other trials, respectively. Four trials ${ }^{63,64,76-78,80,81,87,99}$ were conducted in Europe and two ${ }^{94-98}$ in Iran. Two trials received government funding ${ }^{76-78,80,81}$ and two trials received university funding. ${ }^{94-98}$ Funding was not reported in the remaining two trials. ${ }^{63,64,87,99}$

Intervention durations ranged from 1 to 12 weeks. All but one CPMP ${ }^{80,81}$ were considered low intensity ( $<20$ hours per week) though the total hours varied widely across the trials. Program components were delivered individually (2 RCTs), ${ }^{80,81,97}$ in groups (2 RCTs), ${ }^{63,64,87,94-}$ 96,98 and via a combination of the two (1 RCT) $;{ }^{99}$ the format could not be determined in the sixth trial. $^{76-78}$ Four trials ${ }^{63,64,76-78,87,94-98}$ were conducted in an outpatient setting and two ${ }^{80,81,99}$ in an inpatient setting ( 3 rheumatology, 1 rehabilitation, and 1 pain clinic; unclear location in the last trial).

The content and delivery of program components varied substantially across the trials (Appendix E, Table E-2). Within each trial, patients in both groups received the same medications. Across the five trials that compared CPMP to pharmacologic therapy only, medication regimens included analgesics and/or NSAIDs in all trials as well as combinations of antidepressants in three, ${ }^{63,64,76-78,87,94-96,98}$ sedatives (e.g., benzodiazepine, chlordiazepoxide) in two, ${ }^{63,64,87,97}$ and muscle relaxants, ${ }^{94-96,98}$ omeprazole $^{80,81}$ and tramadol ${ }^{76-78}$ in one trial each. Three trials did not provide information regarding medication dosages. ${ }^{63,64,87,94-98}$ In the two trials evaluating CPMP versus a combination of pharmacologic therapy and physical activity medications included diclofenac, omeprazole, and acetaminophen in one ${ }^{80,81}$ and antidepressants 
only in the other (type and dose not reported).${ }^{99}$ Both trials employed primarily passive physical therapy approaches (e.g., massage, transcutaneous electrical nerve stimulation [TENS]) with more active components not well described ${ }^{80,81,99}$

Four trials were rated fair quality ${ }^{63,64,87,94-99}$ and two poor quality ${ }^{76-78,80,81}$ (Appendix F, Table F-2). The major methodological limitation in the fair-quality trials was the inability to effectively blind patients and caregivers to the CPMP. Other methodological shortcomings included unclear randomization, unclear allocation concealment methods and high attrition.

\section{Primary Outcomes}

\section{Pain}

More CPMP patients achieved 30 percent or more improvement in pain (NRS, 0 to 10 scale) compared with those receiving conventional pharmacologic therapy alone in one fair-quality trial of patients with FM postintervention (22.2\% vs. 6.7\%, RR 3.3, 95\% CI 1.3 to 8.4$)$ and in the intermediate term (16.0\% vs. 5.4\%, RR 3.0, 95\% CI 1.0 to 8.7$).{ }^{64}$ Results at short term (13.6\% vs. $10.8 \%$, RR $1.3,95 \%$ CI 0.53 to 3.0$)$ and long term ( $8.6 \%$ vs. $0 \%$, RR not calculable) were not statistically significant. CPMPs were associated with a moderate improvement in average pain (VAS or NRS 0 to 10 scale) postintervention across one fair-quality trial in FM and one poorquality trial in LBP ( 2 trials, $\mathrm{N}=204$, pooled difference $-1.28,95 \% \mathrm{CI},-2.14$ to -0.63 , $\left.\mathrm{I}^{2}=0 \%\right)^{64,81}$ and a small improvement at intermediate term across one fair- and one-poor quality trial in FM ( 2 trials, $\mathrm{N}=265$, pooled difference $-0.84,95 \% \mathrm{CI}-1.64$ to $\left.-0.15, \mathrm{I}^{2}=0 \%\right)$, ${ }^{64,76}$ (Figure 9). Medications in the fair-quality trial in patients with $\mathrm{FM}^{64}$ included analgesics, antidepressants, benzodiazepines, and nonbenzodiazepine hypnotics and in the poor-quality trial in FM, amitriptyline, paracetamol, and tramadol; ${ }^{76-78}$ the third poor-quality trial of LBP patients ${ }^{81}$ prescribed diclofenac, acetaminophen and omeprazole. The fair-quality trial, in patients with FM $(\mathrm{N}=155)$, reported moderate pain improvement postintervention (difference $-1.20,95 \% \mathrm{CI}-1.78$ to -0.62 ) but no clear difference was found between CPMP and pharmacologic therapy in the short (difference $-0.40,95 \% \mathrm{CI}-0.98$ to 0.18 ), intermediate (difference $-0.60,95 \% \mathrm{CI}-1.20$ to 0.0 ), or long term (difference $-0.40,95 \% \mathrm{CI}-0.94$ to 0.14$)^{64}$ (Figure 9 ).

For the comparison between CPMP and the combination of pharmacologic treatment and physical therapy, CPMP was associated with moderate improvements in Multidimensional Pain Inventory (MPI) pain intensity (differences -1.2 to -2.1 on a 0 to 6 scale) and MPI pain interference (differences -1.9 to -2.5 on 0 to 6 scale) at postintervention, intermediate, and long term in the fair-quality trial in patients with FM; only antidepressants were prescribed in this trial. ${ }^{99}$ In contrast, the poor-quality trial in patients with LBP reported no difference between groups on VAS pain postintervention (difference $0.93,95 \% \mathrm{CI}-0.19$ to 2.1 , on a 0 to 10 scale) ${ }^{81}$ medications in this trial included diclofenac, acetaminophen, and omeprazole. 
Figure 9. CPMP versus pharmacologic therapy alone: Pain

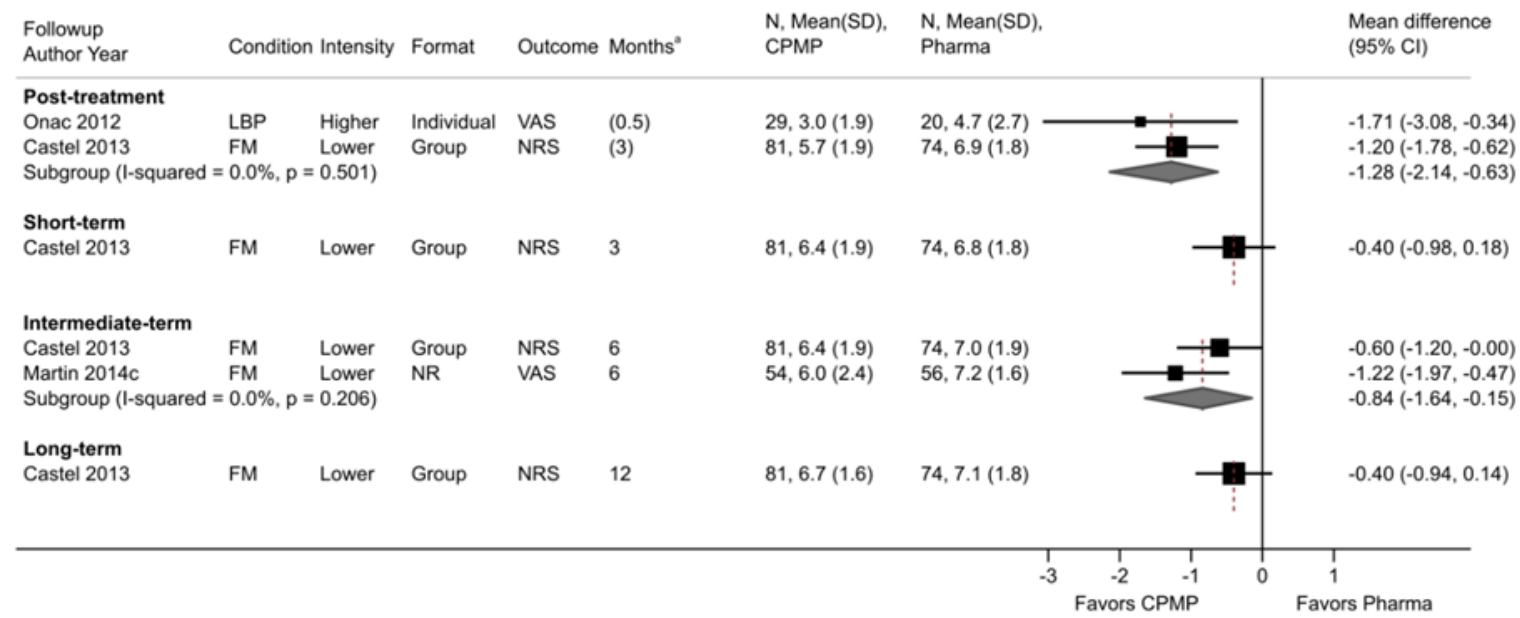

$\mathrm{CI}=$ confidence interval; $\mathrm{CPMP}=$ comprehensive pain management program; $\mathrm{FM}=$ fibromyalgia $\mathrm{LBP}=$ low back pain; $\mathrm{NR}=$ not reported; NRS = numerical rating scale; Pharma = pharmacologic therapy alone; $\mathrm{SD}=$ standard deviation; VAS = visual analog scale.

${ }^{a}$ Number of months in parentheses for the postintervention timeframe indicate the duration of the intervention; followup for the remaining timeframes is in months following the end of the intervention.

\section{Function}

More CPMP patients experienced 14 percent or greater improvement in function (FIQ Total 0 to 100 scale) compared with those receiving conventional pharmacologic therapy alone in one fair-quality trial $(\mathrm{N}=155)$ of patients with FM postintervention $(64.2 \%$ vs. $24.3 \%$, RR 2.6, 95\% CI 1.7 to 4.1$).{ }^{64}$ Improvement persisted to the short (48.1\% vs. $23.0 \%$, RR $2.1,95 \%$ CI 1.3 to $3.4)$, intermediate $(42.0 \%$ vs. $18.9 \%$, RR $2.2,95 \%$ CI 1.3 to 3.8$)$ and long term $(27.2 \%$ vs. $4.0 \%$, RR 6.7, 95\% CI 2.1 to 21.5). No improvement in function was seen with CPMP versus pharmacologic therapy postintervention on continuous outcomes ( 2 trials, $N=204$, pooled SMD $-0.57,95 \% \mathrm{CI}-1.66$ to $\left.0.62, \mathrm{I}^{2}=74.5 \%\right),{ }^{64,81}$ however heterogeneity was substantial across studies with the higher-quality trial in patients with FM having moderate improvement (difference $-18.2,95 \%$ CI -24.0 to -12.4 , on the 0 to 100 FIQ). ${ }^{64}$ The small poor-quality trial in patients with LBP found no difference (difference $-0.24,95 \% \mathrm{CI}-4.2$ to 3.8 , on the 0 to 24 RMDQ). ${ }^{81}$ Heterogeneity may be due to differences in study quality, functional measures used, conditions (FM vs. LBP), program duration and delivery, and/or medications used. Analgesics (acetaminophen), antidepressants, benzodiazepines, and nonbenzodiazepine hypnotics were used in one trial; ${ }^{63,64,87}$ diclofenac, acetaminophen, and omeprazole were used in the other. ${ }^{80,81}$ CPMPs were associated with small improvements in function versus pharmacologic treatment in the short term ( 2 trials, $\mathrm{N}=342$, pooled $\mathrm{SMD}-0.37,95 \% \mathrm{CI}-0.67$ to $\left.-0.08, \mathrm{I}^{2}=0 \%\right),{ }^{64,94}$ intermediate term ( 3 trials, $\mathrm{N}=453$, pooled $\mathrm{SMD}-0.44,95 \% \mathrm{CI}-0.67$ to $\left.-0.22, \mathrm{I}^{2}=0 \%\right),{ }^{64,76,94}$ and long term ( 2 trials, $\mathrm{N}=301$, pooled SMD $-0.46,95 \% \mathrm{CI}-0.76$ to $\left.-0.16, \mathrm{I}^{2}=0 \%\right)^{64,96}$ (Figure 10 ). Sensitivity analyses removing one poor-quality trial ${ }^{76}$ at intermediate term did not impact the effect estimates. Similarly, sensitivity analyses using 12 months versus 30 months followup from one trial $^{96}$ in the long-term estimates lead to similar conclusions (see Appendix I, Figures I-34 to I-35 for sensitivity analyses). Mean differences in RMDQ (0 to 24 scale) were $-1.6,-1.8,-3.1$ in the short, intermediate, and long term (>12 months), respectively. ${ }^{96}$ Mean differences in total FIQ $(0$ to 100 scale) were $-9.1,-12.0$ and -10.8 for these time frames, respectively. ${ }^{64}$ Differences based on these scales suggest small to moderate improvement. Two fair-quality trials also reported on 
additional functional measures. ${ }^{63,64,87,94-96,98}$ CPMPs were consistently associated with at least small functional improvement at all time frames compared with pharmacologic therapy.

For the comparison between CPMP versus the combination of pharmacologic treatment and physical therapy, no differences in function between groups were reported in either study based on MPI total activity at any time frame ( 1 trial, difference ranges -0.27 to -0.22 , on a 0 to 6 scale $)^{99}$ or on postintervention RMDQ ( 1 trial, difference 1.50 on a 0 to 24 scale). ${ }^{81}$

Figure 10. CPMP versus pharmacologic therapy alone: Function

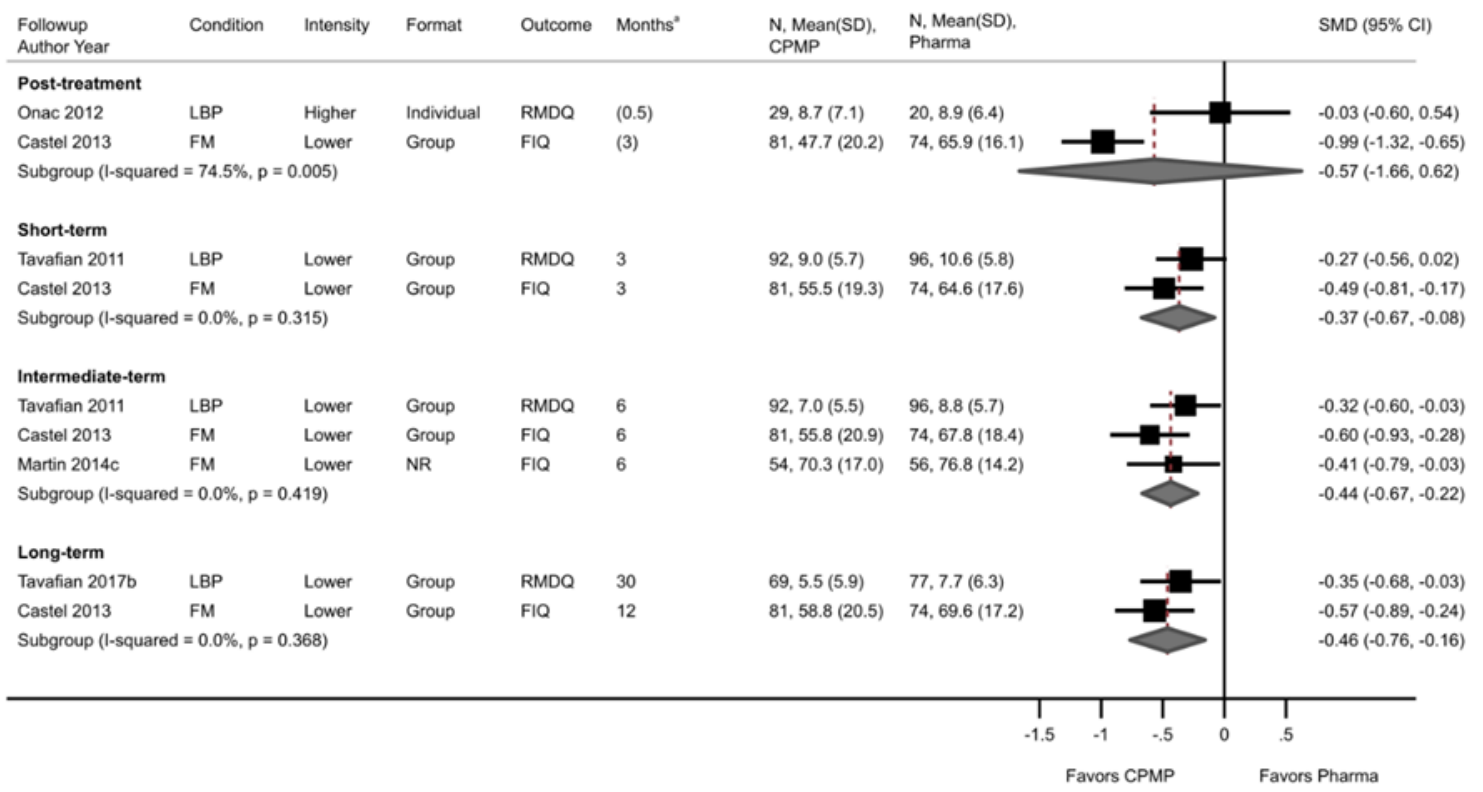

$\mathrm{CI}=$ confidence interval; $\mathrm{CPMP}=$ comprehensive pain management program; FIQ = Fibromyalgia Impact Questionnaire; FM = fibromyalgia; $\mathrm{LBP}=$ low back pain; $\mathrm{NR}=$ not reported; Pharma = pharmacologic therapy alone; $\mathrm{RMDQ}=\mathrm{Roland}$ Morris Disability Questionnaire; $\mathrm{SD}=$ standard deviation; $\mathrm{SMD}=$ standardized mean difference.

${ }^{a}$ Number of months in parentheses for the postintervention timeframe indicate the duration of the intervention; followup for the remaining timeframes is in months following the end of the intervention.

\section{Opioid Use}

Changes in opioid use were not reported in any of the trials comparing CPMP with pharmacologic therapy alone. One trial reported greater overall decrease in medication use for CPMP versus combined medication/physical therapy, indicating that overall opioid use was also reduced with CPMP but did not provide sufficient data for comparisons on use. ${ }^{99}$

\section{Secondary Outcomes}

\section{Health Status}

Evidence on the impact of CPMP on health status and measures of psychologic well-being compared with pharmacologic therapy alone are limited. No differences between CPMP and pharmacologic therapy on the Dartmouth Primary Care Cooperative Information Project/World Organization of National Colleges, Academies, and Academic Associations of General Practice/Family Physicians (COOP/WONCA) measure of health-related quality of life (9 to 45 scale) were seen at any time frame. ${ }^{64} \mathrm{CPMPs}$ were associated with moderate to substantial improvement based on SF-36 PCS in the short and intermediate term and with improvement in SF-36 MCS in the short term (0 to 100 scale for both) versus pharmacologic therapy (range of 
mean differences for significant results, 15.4 to 25.5 ) in one trial. ${ }^{97}$ No differences were seen in the long term. Another trial reported that CPMP was associated with small to moderate improvement in all individual SF-36 domains at one or more time frames (mean difference range 7.2 to 19.3 on a 0 to 100 scale when statistically significant). ${ }^{94-96,98}$

\section{Depression and Anxiety}

CPMPs were associated with improvements in the Hospital Anxiety and Depression Scale (HADS, 0 to 21 scale) versus pharmacologic treatment alone in patients with FM at all time frames (range of mean differences -5.4 to -7.4 ) in a fair-quality trial ${ }^{64}$ but no differences were seen in another poor-quality trial at intermediate term. ${ }^{77} \mathrm{~A}$ third poor-quality trial found no differences in emotional distress based on the Profile of Mood States Short Version (POMS-SV) immediately postintervention. ${ }^{81}$

For the comparison between CPMP versus the combination of pharmacologic treatment and physical therapy, small improvements in MPI affective distress ( 0 to 6 scale) favoring CPMP were seen postintervention, intermediate term, and long term (differences -1.9 to -2.3 ) in one trial $;{ }^{99}$ no difference in emotional distress based on the POMS-SV (scale not reported) was seen in the other trial postintervention. ${ }^{81}$

\section{Utilization}

One trial reported substantially fewer hospital days for CPMP recipients (difference -16.04 , $95 \% \mathrm{CI}-22.3$ to -9.8 ) compared with those in the combined medication/physical therapy group at long-term followup in patients with FM. ${ }^{99}$

\section{Harms and Differential Effectiveness or Safety}

None of the RCTs reported on harms.

No trial evaluated potential modification of treatment effects by population subgroups of interest. One fair-quality trial (CPMP vs. pharmacologic therapy alone) found no modification in treatment response to CPMP based on patient body mass index strata (normal, overweight and obese) ${ }^{63}$ Data from one poor-quality trial (CPMP vs. combined medication/physical therapy) are insufficient to evaluate whether pain catastrophizing may modify treatment response; no tests of interaction were reported. ${ }^{80}$

\section{CPMPs Versus Psychological Therapy}

\section{Key Points}

- There were no differences in pain (on a 0 to 10 scale) or function comparing CPMP versus psychological therapy alone postintervention, intermediate term, and long term (SOE: low for all).

- One trial reported increased low back or radiating leg pain leading to withdrawal in three (5.5\%) CPMP patients compared with no patient who received psychological therapy (SOE: insufficient).

\section{Detailed Synthesis}

Five RCTs compared CPMPs with psychological therapy alone (Results Appendix B, Table B-9; Appendix E, Table E-2). ${ }^{69,74,91,92,100,101}$ Randomization occurred at the individual patientlevel in all trials. Sample sizes ranged from 36 to 138 (total sample=578). Pain diagnoses included chronic LBP ( 2 trials),${ }^{91,92,100}$ chronic nonspecific spinal pain ( 2 trials $),{ }^{69,74}$ and 
nonspecific chronic pain ( 1 trials). ${ }^{101}$ Comorbidities were not reported and none of the studies specifically included Medicare patients. However, four trials reported on patients' disability status with 38 percent and 24 percent receiving a full or partial sick leave/disability pension in one trial $^{91,92}$ and 21 percent currently on sick leave in a second trial; ${ }^{101}$ in the other two trials, inclusion criteria included current and continuous sick leave for at least 1 month (maximum 6 months) before study entry ${ }^{69}$ and "at risk" for long-term disability. ${ }^{74}$ Mean patient age ranged from 42 to 49 years. Intervention durations ranged from 4 to 10 weeks. Program components were delivered via a combination of individual and group formats in two trials ${ }^{91,92,100}$ and in a group format only in the remaining three trials. ${ }^{69,74,101}$ All CPMPs but one ${ }^{69}$ were considered low intensity $(<20$ hours per week). Four trials had psychological components based on CBT and one $^{100}$ on behavioral modification.

Three trials were considered fair quality ${ }^{69,74,91,92}$ and two poor quality ${ }^{100,101}$ (Appendix F, Table F-2). The major methodological limitation in the fair-quality trials was the inability to effectively blind patients and caregivers to the CPMP. Other methodological shortcomings in the poor-quality trials included unclear allocation concealment methods and high attrition.

\section{Primary Outcomes}

\section{Pain}

There were no differences in pain improvement for CPMPs compared with psychological therapy alone postintervention ( 3 trials, $\mathrm{N}=259$, pooled difference 0.03 on a 0 to $10 \mathrm{scale}, 95 \%$ $\mathrm{CI}-0.30$ to $\left.0.31, \mathrm{I}^{2}=0.0 \%\right),{ }^{91,100,101}$ and at intermediate-term ( 3 trials, $\mathrm{N}=228$, pooled difference $-0.09,95 \% \mathrm{CI}-0.50$ to $\left.0.21, \mathrm{I}^{2}=0.0 \%\right)^{91,100,101}$ and long-term ( 3 trials, $\mathrm{N}=256$, pooled difference $0.05,95 \% \mathrm{CI}-0.35$ to $\left.0.47, \mathrm{I}^{2}=26.1 \%\right)^{74,91,100}$ followup (Figure 11 ). Results from sensitivity analyses excluding the two poor-quality trials ${ }^{100,101}$ at postintervention and intermediate term changed the effect estimates somewhat but did not change the conclusions (Appendix I, Figure I36). Likewise, no differences were seen at any timepoint for other measures of pain (MPQ Pain Rating Index, VAS worst pain, number of pain free days in the past week) as reported by two trials $^{74,91}$ (Appendix E, Table E-2). 
Figure 11. CPMP versus psychological therapy alone: Pain

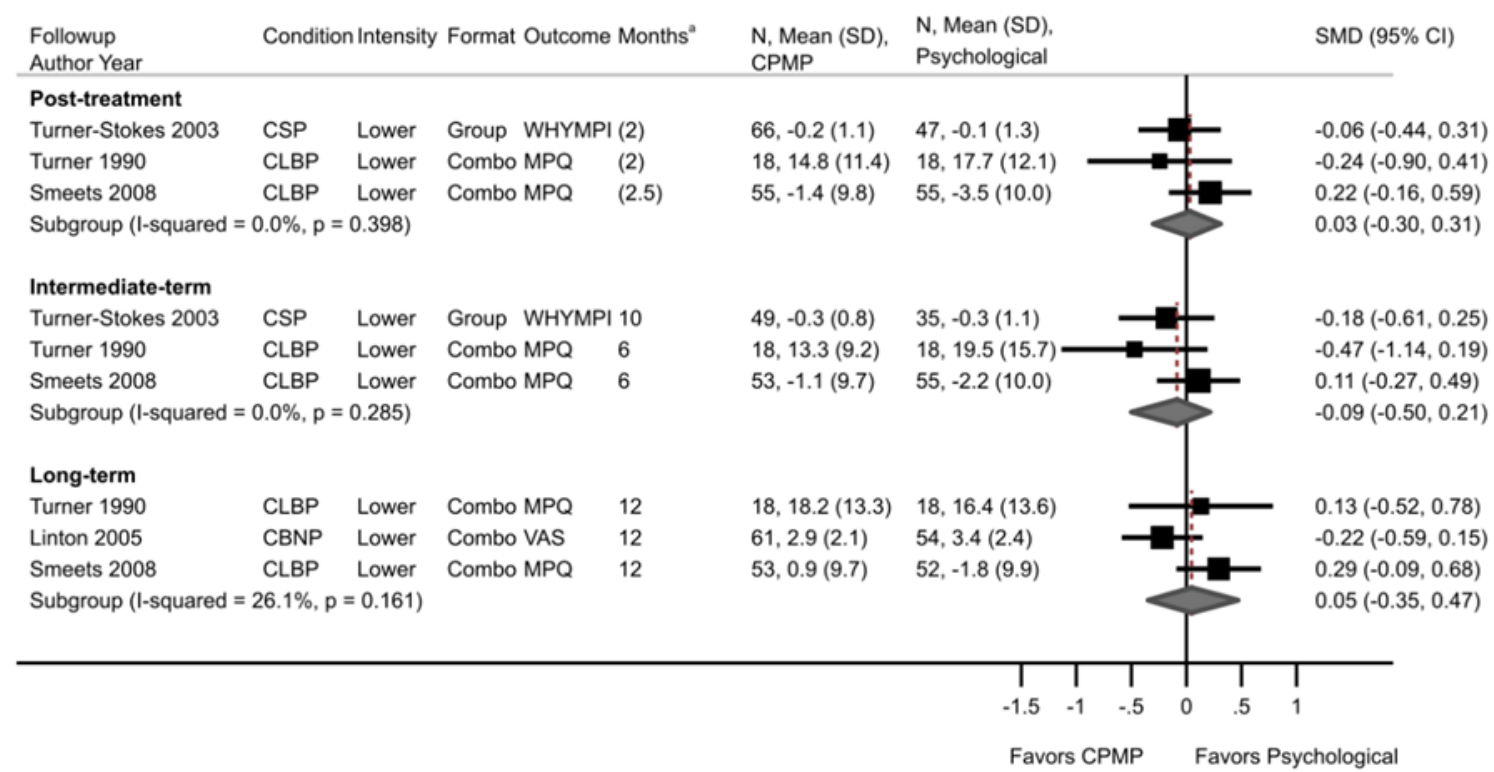

$\mathrm{CBNP}=$ chronic back and neck pain; $\mathrm{CI}=$ confidence interval; $\mathrm{CLBP}=$ chronic low back pain; Combo = combination group and individual sessions; $\mathrm{CPMP}=$ comprehensive pain management program; $\mathrm{CSP}=$ chronic spinal pain; $\mathrm{MPQ}=\mathrm{The} \mathrm{McGill}$ Pain Questionnaire; SD = standard deviation; $\mathrm{SMD}=$ standardized mean difference; VAS = visual analog scale; WHYMPI $=$ West Haven-Yale Multidimensional Pain Inventory.

${ }^{a}$ Number of months in parentheses for the postintervention timeframe indicate the duration of the intervention; followup for the remaining timeframes is in months following the end of the intervention.

\section{Function}

There were no differences in function for CPMPs compared with psychological therapy alone at any time point: postintervention ( 3 trials, $\mathrm{N}=262$, pooled SMD $0.10,95 \% \mathrm{CI}-0.23$ to 0.36 , $\left.\mathrm{I}^{2}=0 \%\right),{ }^{91,100,101}$ intermediate term ( 3 trials, $\mathrm{N}=231$, pooled SMD $0.11,95 \% \mathrm{CI}-0.32$ to 0.41 , $\left.\mathrm{I}^{2}=0 \%\right),{ }^{91,100,101}$ and long term ( 3 trials, $\mathrm{N}=259$, pooled SMD $0.16,95 \% \mathrm{CI}-0.18$ to 0.48 , $\left.\mathrm{I}^{2}=0 \%\right),{ }^{74,91,100}$ (Figure 12 ). Results from sensitivity analyses excluding the two poor-quality trials ${ }^{100,101}$ at postintervention and intermediate term changed the effect estimates somewhat but did not change the conclusions (Appendix I, Figure I-37). There were also no difference between groups on a modified activities of daily living questionnaire in the long term in one fair-quality trial $^{74}$ and pain interference at postintervention and in the intermediate term in one poor-quality trial (Appendix B, Table B-9). ${ }^{101}$ 
Figure 12. CPMP versus psychological therapy alone: Function

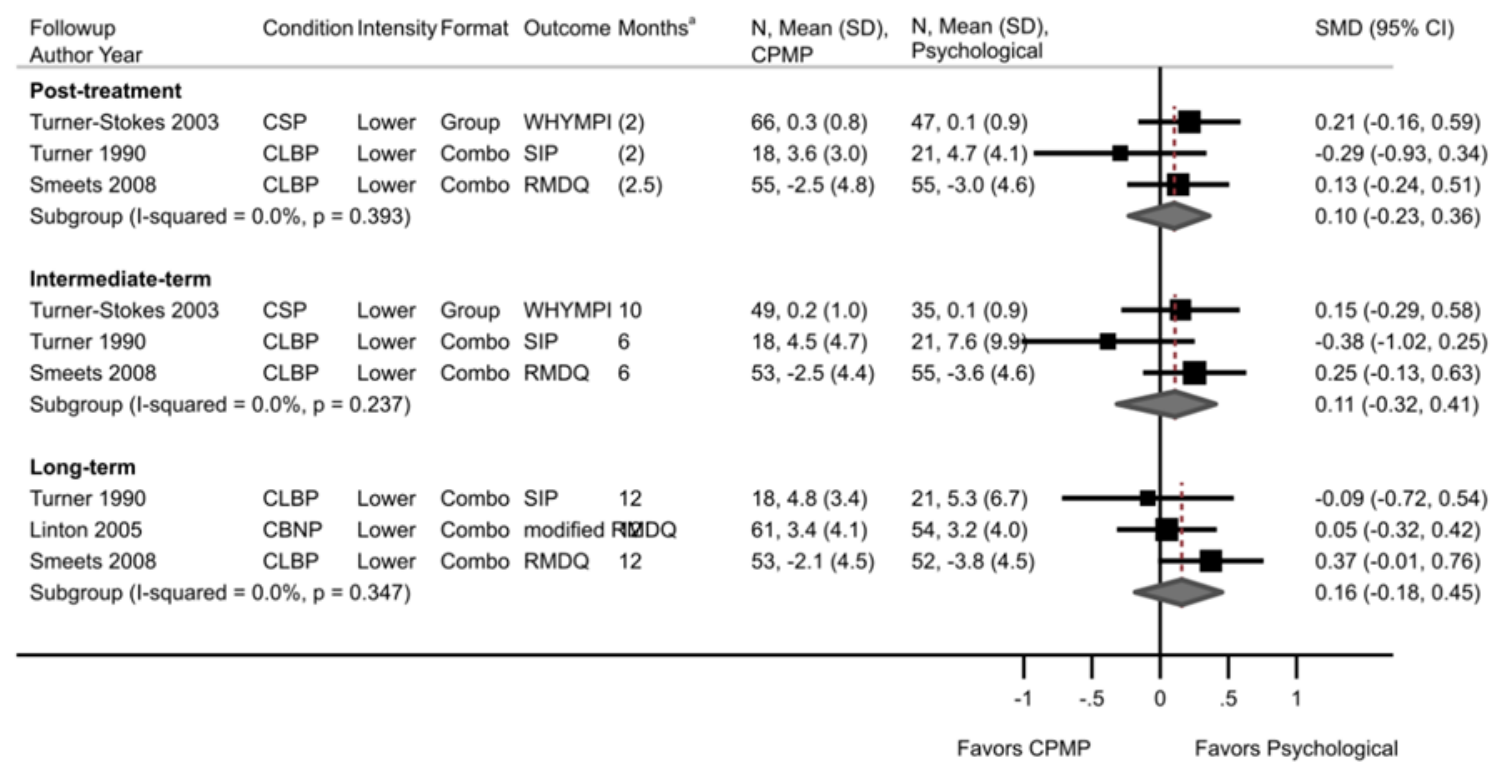

$\mathrm{CBNP}=$ chronic back and neck pain; $\mathrm{CI}=$ confidence interval; $\mathrm{CLBP}=$ chronic low back pain; Combo = combination group and individual sessions; $\mathrm{CPMP}=$ comprehensive pain management program; $\mathrm{CSP}=$ chronic spinal pain; $\mathrm{RMDQ}=\mathrm{Roland} \mathrm{Morris}$ Disability Index; SD = standard deviation; SIP = Sickness Impact Profile; SMD = standardized mean difference; WHYMPI = West Haven-Yale Multidimensional Pain Inventory.

${ }^{a}$ Number of months in parentheses for the postintervention timeframe indicate the duration of the intervention; followup for the remaining timeframes is in months following the end of the intervention.

\section{Secondary Outcomes}

\section{Depression and Anxiety}

There were no differences in depression symptoms for CPMPs versus psychological therapy alone postintervention ( 3 trials, $\mathrm{N}=259$, pooled SMD $0.17,95 \% \mathrm{CI}-0.15$ to $0.43, \mathrm{I}^{2}=0 \%$ ), ${ }^{91,100,101}$ in the intermediate term ( 3 trials, $\mathrm{N}=228$, pooled $\mathrm{SMD}-0.17,95 \% \mathrm{CI}-0.59$ to 0.16 , $\left.\mathrm{I}^{2}=1.5 \%\right),{ }^{91,100,101}$ and the long term ( 3 trials, $\mathrm{N}=256$, pooled SMD $0.00,95 \% \mathrm{CI}-0.25$ to 0.28 , $\left.\mathrm{I}^{2}=0.0 \%\right)^{74,91,100}$ (Appendix I, Figure I-38). Sensitivity analyses excluding the two poor-quality trials $^{100,101}$ did not change the conclusions (Appendix I, Figure I-39). Similarly, there were no differences in anxiety symptoms between groups postintervention (adjusted difference $-2.3,95 \%$ $\mathrm{CI}-6.21$ to 1.59 ) and intermediate term (adjusted difference $-3.43,95 \% \mathrm{CI}-7.81$ to 0.94 ) in one poor-quality trial, ${ }^{101}$ or at long term (difference $0.10,95 \% \mathrm{CI}-1.36$ to 1.56 ) in one fair-quality trial. $^{74}$

\section{Global Improvement and Patient Satisfaction}

There were no differences in SF-36 global score between groups at any timepoint (postintervention, intermediate term or long term) in one fair-quality trial. ${ }^{69}$ There was no difference in global improvement postintervention in a second fair-quality trial, but significantly less improvement was reported by the CPMP group at intermediate- (adjusted difference in change scores $-0.76,95 \% \mathrm{CI}-1.31$ to -0.21 ) and long-term followup (adjusted difference in change scores $-0.65,95 \% \mathrm{CI}-1.21$ to -0.10$)^{91}$ (Appendix B, Table B-9). Of the three trials that reported patient satisfaction, ${ }^{69,92,100}$ only the poor-quality trial found a significant difference favoring CPMP (mean 5.5 vs. 4.0 on a 1 to 7 scale, $p<0.05$ ). ${ }^{100}$ 


\section{Utilization}

One fair-quality trial found no difference between CPMP versus psychological therapy alone regarding mean number of healthcare visits for spinal pain the year following treatment (1.25 vs. $2.5, \mathrm{p}=0.06){ }^{74}$

\section{Harms}

Three patients in the CPMP group (5.5\%, 3/55), compared with none in the psychological therapy group $(0 \%, 0 / 55)$, reported increased pain in the low back or radiating leg pain leading to withdrawal from the program in one fair-quality trial..$^{92}$ No other adverse events occurred in either group.

\section{Differential Effectiveness or Safety}

No trial evaluated differential effectiveness or harms of CPMP in special populations of interest.

\section{Key Question 2. Program Factors}

\section{Integrated Pain Management Programs}

\section{Key Points}

- There were no differences in pain or function for a combined provider-patient intervention compared with a provider only or patient only intervention in one fairquality trial.

- There were no differences in pain or function between IPMP with Web-based support versus the program without such support in one fair-quality trial.

\section{Summary of Findings}

Two RCTs of diverse IPMPs provide limited evidence on the impact of intervention delivery methods on clinical outcomes (Results Appendix B, Table B-10; Appendix E, Table E-1). One trial evaluated the impact of providing multidisciplinary treatment recommendations to primary care providers and patients versus provider only and patient only intervention. ${ }^{41}$ The other evaluated whether Web-based support to reinforce IPMP concepts would improve outcomes versus usual IPMP delivery. ${ }^{109}$ They will be reported separately.

\section{Detailed Synthesis}

One cluster $\mathrm{RCT}^{41,110}$ conducted in the United States randomized 10 community-based primary care clinics to electronically receive patient-specific OA treatment recommendations made by the study team based on patient assessment and treatment guideline care algorithms ( 5 clinics) or to continue usual care (5 clinics). Although the primary care team was not multidisciplinary, patients could be referred for or receive care from providers from multiple disciplines. Patients with OA within each of the 10 clinics' arms were randomized to a 12-month phone-based intervention focused on physical activity, cognitive behavioral strategies for pain management, weight management, or usual care resulting in 4 arms $(\mathrm{N}=537)$. This care was delivered by individuals with training in counseling and/or health education and behavioral change and was overseen by the co-investigators from multiple disciplines. Patient intervention participants also received educational materials, an exercise video, and a compact disc of 
relaxation exercises in addition to the phone-based intervention. Patients in the intervention arms $(\mathrm{N}=408)$ were primarily female $(75 \%)$, White $(59 \%)$, had knee OA $(85 \%)$ with a mean symptom duration of 124.8 months, and mean age of 63 years; 20 percent rated their health as fair or poor. Patients with active psychosis, personality disorder or uncontrolled substance abuse disorder were excluded. The trial was considered fair quality due to lack of blinding and unclear concealment of treatment allocation (Appendix F, Table F-1).

In the cluster RCT, results comparing the provider plus patient intervention group with the provider intervention group alone and provider plus patient intervention versus patient intervention alone showed no difference in pain scores. The difference in change scores from baseline to postintervention (12 months) between the provider plus patient intervention group versus provider intervention only group for pain did not meet the threshold for a small effect (difference $-0.3,95 \% \mathrm{CI}-0.8$ to -0.2 , on a 0 to 10 scale). Further for this comparison, there were no differences in changes scores for WOMAC function ( 0 to 68 scale, difference $-2.5,95 \% \mathrm{CI}$ -5.0 to 0.0 ), or in the proportion of patients achieving 18 percent or more improvement from baseline ( $44 \%$ versus $35 \%$ based on author imputation). PHQ depression scores were also not different. Similarly, there were no differences in change scores for the comparison of provider plus patient intervention versus patient intervention only for pain (difference $-0.05,95 \% \mathrm{CI}$ -0.45 to 0.55 , on a 0 to 10 scale), WOMAC function (difference $0.80,95 \% \mathrm{CI}-1.8$ to 3.4 ) or in the proportion of patients achieving 18 percent or more improvement from baseline (44\% versus $49 \%$ based on author imputation). PHQ depression scores were also not different between groups (Appendix E, Table E-1). Authors reported that no study-related adverse events occurred.

One trial $(\mathrm{N}=109)$ conducted in Sweden compared the impact of adding Web-based behavioral change support to IPMP versus usual IPMP delivery in patients with persistent musculoskeletal pain. ${ }^{109}$ Randomization in this trial occurred at the individual patient-level. For IPMP, a multidisciplinary team conferred with the patient to individualize treatment. In addition to the primary IPMP components (i.e., medication adaptation, psychological care including CBT, and physical activity), treatment could include acupuncture, TENS, manual therapy and others. IPMP in both groups involved a minimum of two or three treatment sessions a week for at least 6 weeks. The 8-module Web-based support program was available for 16 weeks and focused on enhancing patient physical and cognitive activity in their rehabilitation. Only 36 percent of participants opened all modules. Total program intensity was not reported but was considered low based on information provided. Patients were predominantly male (57\%) with a mean age of 43 years. Information on race/ethnicity and comorbidities was not provided. The trial was rated fair due to lack of blinding and unacceptable attrition (27\% at 12 months) (Appendix F, Table F1).

There were no differences between those who did and those who did not have Web-based support in the proportions of patients meeting 30 percent or more improvement on either VAS pain (0 to 10 scale) in the short (22\% vs. $23 \%$; RR $1.00,95 \%$ CI 0.45 to 2.23 ) or long term ( $28 \%$ vs. 22\%; RR 1.23, 95\% CI 0.56 to 2.67) or function based on 30 percent or more improvement in Pain Disability Index (PDI, 0 to 70 scale) in the short (20\% vs. 24\%, RR 0.91, 95\% CI 0.40 to 2.07 ) or long term (31\% vs. 30\%; RR 1.04, 95\% CI 0.54 to 2.01$)$. No differences between groups in average effects for either pain or functional measures were seen. Similarly, there were no differences between groups on mean differences in these measures at either time or on individual SF-36 subscales. Authors did not report on harms (Results Appendix B, Table B-10). 


\section{Comprehensive Pain Management Programs}

For this section (CPMPs addressing Key Question 2), all figures for the meta-analyses can be found in Appendix I, Figures I-38 to I-51.

\section{Greater Versus Fewer Total Program Hours}

\section{Key Points}

- In one fair-quality trial ( $=75), \mathrm{CPMP}$ with greater total hours was associated with moderate improvement in pain and function over the short and long term compared with CPMP with fewer hours. Applicability to the Medicare population might be limited due to high program intensity, inclusion of work-related therapy, and young age.

- Across three lower-intensity trials (total $\mathrm{N}=307$ ), two fair and one poor quality, no differences in pain or function were seen for longer versus shorter CPMPs postintervention and in the intermediate term across different pain diagnoses.

- No trial-related adverse events were reported across two fair-quality trials, although patients in both groups experienced occasional mild increases in pain after exercise sessions.

\section{Detailed Synthesis}

Four RCTs (reported in 7 publications) ${ }^{56-59,88,111,112}$ directly compared CPMPs with greater versus fewer total program hours (Results Appendix B, Table B-11; Appendix E, Table E-2). Sample sizes ranged from 44 to 153 (total sample $=328$ ). The weighted mean age was 42.3 years and 42 percent of the patients were male. Pain diagnoses included chronic LBP (with or without referred leg pain) in two trials, ${ }^{56-59,112} \mathrm{FM}$ in one trial, ${ }^{88}$ and mixed musculoskeletal (mostly neck/shoulder, low back, and lower extremity) pain in the fourth trial. ${ }^{111}$ The duration of symptoms was 6 months or longer in one trial, ${ }^{56-59}$ greater than 1 year in the majority of patients (74\%) in another, ${ }^{111}$ and a mean 7.5 and 8.1 years in two trials. ${ }^{88,112}$ None of the studies specifically included Medicare patients. However, 64 percent of patients were receiving a disability or sickness benefit in one trial, ${ }^{112} 77$ percent were incapable of working due to their condition in a second, ${ }^{56-59}$ and 49 percent were on full or partial sick leave in another trial. ${ }^{111}$ None of the trials reported comorbidities with the exception of smoking (64\%) in one trial. ${ }^{56-59} \mathrm{In}$ one trial, 13 percent had previous spinal surgery. ${ }^{112}$ No trial reported opioid use at baseline. One trial received no funding, ${ }^{88}$ one received hospital funds, ${ }^{111}$ one received nonprofit funding, ${ }^{56-59}$ and one trial ${ }^{12}$ did not report their source of funding. Three trials were conducted in Europe ${ }^{56-}$ $59,111,112$ and one ${ }^{88}$ in Turkey.

Treatment components were delivered to patients in group sessions in two trials, ${ }^{56-59,112}$ individually in one trial, ${ }^{111}$ and via a combination of both in one trial. ${ }^{88}$ All programs were delivered on an outpatient basis, regardless of the group. Only one trial was considered high intensity with 135 total hours (39 hours/week over 3 weeks) in the intervention group (compared with 24 hours in the CPMP of shorter duration). This trial demanded a high level of physical activity, included work-related therapy, and enrolled younger participants (mean 42 years); given the structure and demands of the program this trial will be reported separately below. Across the remaining three lower-intensity programs, the total number of hours ranged from 60 to 70 (over a range of 1 to 10 weeks) and from 10 to 15 (over a range of 2 days to 1 week) across programs with greater versus fewer total hours, respectively, in two trials. The third trial intended to compare two programs of different "dosages" (a conventional program and a "short form") but 
they ultimately ended up with comparable mean durations (11.7 vs. 10.8 weeks) and mean number of patient contact hours with providers (30.7 vs. 29.8). ${ }^{111}$ Two trials included a work rehabilitation component in their program. ${ }^{56-59,111}$

Of the four trials, three were rated fair quality ${ }^{56-59,88,111}$ and one poor quality ${ }^{12}$ (Appendix F, Table F-2). The major methodological limitation in the fair-quality trials was the inability to effectively blind patients and caregivers to the interventions; in addition, two of the trials had unacceptable levels of attrition. Other methodological shortcomings in the poor-quality trial included unclear randomization, unclear allocation concealment methods and lack of an intention-to-treat analysis.

\section{Primary Outcomes}

Across the lower-intensity programs, two trials ${ }^{11,112}$ reported no differences in pain between formats that involved greater versus fewer hours in a CPMP. One fair-quality trial $(\mathrm{N}=153)$ in those with chronic musculoskeletal pain explored tailoring CPMP time based on patient need. ${ }^{111}$ Mean program duration was not substantially different for the longer and shorter forms of the program (mean number of weeks 11.7 vs. 10.8) in this trial and there was no difference in pain following the interventions (difference $-0.01,95 \% \mathrm{CI}-0.70$ to $0.68,0$ to 10 scale). In the other small $(\mathrm{N}=60)$, poor-quality trial VAS pain was similar postintervention for chronic LBP patients attending 60 -, 30 - or 15 -hour programs (medians $4.9,4.3$, and 5.0 on a $0-10$ scale). ${ }^{12}$ There were no differences in pain ( 0 to 10 scale) at intermediate term between longer (60 to 75 hours) and shorter (10 to 15 hours) CPMPs across one fair-quality trial in FM and one poor-quality trial in chronic LBP (2 RCTs, $\mathrm{N}=78$, pooled difference $0.01,95 \% \mathrm{CI}-1.76$ to $\left.1.85, \mathrm{I}^{2}=68.4 \%\right)$, ${ }^{88,112}$ (Appendix I, Figure I-40). Results were similar in a sensitivity analysis including the group who received 30 hours (versus 60 hours) in the poor-quality trial (Appendix I, Figure I-41). ${ }^{112}$

Like the findings for pain, no postintervention improvement in function was seen for the lower-intensity programs with longer versus shorter hours in a fair-quality trial in patients with chronic musculoskeletal pain (11.7 vs. 10.8 weeks, difference $-1.5,95 \% \mathrm{CI}-7.44$ to 4.44 , on the 0 to $70 \mathrm{PDI})^{111}$ or a poor-quality trial in those with chronic LBP (0 to 24 RMDQ, median 8.4 vs. 8.8 for 60 and 15 hours). ${ }^{112}$ There were no differences in function at intermediate term between longer (60 to 75 hours) and shorter (10 to 15 hours) CPMPs across one fair-quality trial in FM and one poor-quality trial in chronic LBP ( 2 RCTs, N=78, pooled SMD $-0.10,95 \% \mathrm{CI}$ -0.62 to $0.42, \mathrm{I}^{2}=0 \%$ ), ${ }^{88,112}$ (Appendix I, Figure I-42). Results of a sensitivity analysis including the group who received 30 hours (versus 60 hours) in the poor-quality trial ${ }^{112}$ did not change the conclusions (Appendix I, Figure I-43).

In the third fair-quality trial $(\mathrm{N}=75)$ evaluating a high-intensity program in patients with chronic LBP ${ }^{59}$ CPMP of 135 hours was associated with moderate improvement in pain in the short term compared with CPMP of 24 hours (medians 2.7, interquartile range [IQR] 1.4 to 4.3 vs. 5.6, IQR 3.8 to 7.6 , on a 0 to 10 scale, $\mathrm{p} \leq 0.001$ ); improvement persisted in the long term (range of medians, 3 to 4 vs. 6 to 6.5 over 12, 24 and 60 months). ${ }^{56-58}$ Similarly, moderate improvements in function based on patient subjective disability due to back pain were seen following the longer CPMP at short-term followup (medians 8.5, IQR 5 to 15 vs. 16.1, IQR 11 to 19 , on a 0 to 30 scale, $\mathrm{p}=0.002$ ) which continued in the long term (range of medians, 8 to $10 \mathrm{vs}$. 16 to 17 over 12, 24, and 60 months). ${ }^{56-58}$ This same trial found no difference in the proportion of patients taking prescription pain medication (not specified) due to LBP in the long term (24 months, $50 \%$ vs. $67 \%$, respectively; RR $0.74,95 \%$ CI 0.50 to 1.09$).{ }^{56}$ 


\section{Secondary Outcomes}

Except for global improvement at long-term followup in one fair-quality trial, which favored a lower-intensity CPMP with greater versus fewer total hours (24 and 60 months: median 2 vs. 3 on a 1 to 5 scale; $p=0.003),{ }^{56,57}$ there were no differences between groups in secondary outcomes at any time point, to include the SF-36 PCS and MCS (1 fair-quality trial), ${ }^{88}$ the EQ5D (1 fairquality trial) $^{111}$ and depression (1 fair- and 1 poor-quality trial) ${ }^{88,112}$ (Results Appendix B, Table B-11; Appendix I, Figures I-44 and I-45).

\section{Harms}

One trial reported that no adverse events occurred although patients in both groups experienced occasional mild increases in pain after exercise sessions. ${ }^{88} \mathrm{~A}$ second trial stated that no trial-related adverse events were reported. ${ }^{111}$

\section{Utilization}

Only one fair-quality trial reported utilization with no differences between CPMP of 135 hours versus 24 hours in prespecified outcomes of interest over the long term (60 months): proportion of patients hospitalized due to LBP (22\% [8/37] vs. 23\% [7/31]; RR 0.96, 95\% CI 0.39 to 2.34$)$ or who underwent surgery (5\% [2/37] vs. $10 \%[3 / 31]$; RR $0.56,95 \%$ CI 0.10 to $3.13) .{ }^{56}$

\section{Differential Effectiveness or Safety}

No trial reported differential effectiveness or safety.

\section{Inpatient Versus Outpatient Setting}

\section{Key Points}

- Evidence comparing CPMPs conducted in an inpatient versus outpatient setting from four poor-quality trials is insufficient to draw conclusions about benefits or safety.

\section{Detailed Synthesis}

Four RCTs (reported in 6 publications) compared CPMPs conducted in an inpatient versus an outpatient setting (Results Appendix B, Table B-11; Appendix E, Table E-2). ${ }^{66,67,82,83,86,108}$ Pain diagnoses included mixed chronic pain (primarily back, neck, head, arms and legs) in two trials $^{82,83,108}$ and chronic LBP in two trials. ${ }^{66,67,86}$ Mean duration of symptoms was 14.4 years in one trial ${ }^{66,67}$ and 8.8 years in another, ${ }^{108}$ in a third trial symptom duration was 3 months or longer ${ }^{86}$ and in the fourth, ranged from 6 months to over 20 years. ${ }^{82,83}$ The weighted mean age of participants was 44.6 years (oldest population mean 50 years) ${ }^{108}$ and 56.9 percent were male; across the two trials that reported race, only 12 percent of participants were non-White. ${ }^{82,83,108}$ While none of the trials specifically included Medicare patients, 60 percent were receiving disability income in one trial. ${ }^{108}$ In two other trials, patients had numerous incidences of sick leave due to their pain condition prior to study enrollment. ${ }^{66,67,86}$ None of the trials reported comorbidities but one reported that 65 percent of patients used opioids, 58 percent reported excess drug use, and 40 percent had undergone at least one prior surgery. ${ }^{108}$ Trials were conducted in New Zealand, ${ }^{82,83}$ France, ${ }^{86}$ Finland, ${ }^{108}$ and the United Kingdom. ${ }^{66,67}$ Two trials ${ }^{82,83,108}$ received government funding and two trials ${ }^{66,67,86}$ received funding from nonprofit organizations. 
Sample sizes ranged from 52 to 306 (total sample size=551). In the inpatient groups, intervention durations ranged from 3 to 5 weeks and were considered higher intensity ( $\geq 20$ hours per week; range, 30 to 40 hours per week). In the outpatient groups, intervention durations ranged from 5 to 9 weeks and were considered lower intensity ( $<20$ hours per week; range, 2 to 11 hours per week) and content differed for the inpatient versus outpatient programs. Two programs ${ }^{66,67,86}$ took place in a rehabilitation clinic, one ${ }^{82,83}$ in a pain clinic, and one ${ }^{108}$ in a general hospital ward. Program components were delivered in groups sessions in three trials ${ }^{66,67,82,83,108}$ and in a combination of individual and group sessions in one. ${ }^{86} \mathrm{~A}$ work rehabilitation component was included in both the inpatient and outpatient programs in one trial, ${ }^{66,67}$ and only in the inpatient group for another trial. ${ }^{82,83}$ The other two trials did not include such a component. In one trial, both groups received multiple reinforcement followup sessions 1.5 years after the initial program. ${ }^{66,67}$

All four trials were rated poor quality (Appendix F, Table F-2). The major methodological limitations included the inability to effectively blind patients and caregivers to the interventions, unclear allocation concealment methods, unacceptable levels of attrition, and lack of an intention-to-treat analysis.

\section{Primary Outcomes}

Evidence regarding the impact of program setting (inpatient vs. outpatient) is insufficient to draw firm conclusions due to substantial heterogeneity across CPMPs and methodological limitations of included trials (all poor quality).

\section{Pain}

There was no difference in pain intensity between inpatient versus outpatient CPMP postintervention in one trial (difference $-0.33,95 \% \mathrm{CI}-1.80$ to 1.14 , on a 0 to 10 scale) ${ }^{83}$ or over the short term in two trials ( 2 RCTs, $\mathrm{N}=374$, pooled difference $-0.44,95 \% \mathrm{CI}-0.88$ to 0.04 , $\mathrm{I}^{2}=0 \%$, on a $0-10$ scale) ${ }^{66,108}$ Individually, the larger trial found an association with inpatient CPMP for pain improvement in the short term but it did not meet our threshold (0.5) for a small improvement $(\mathrm{N}=306$, difference $-0.45,95 \% \mathrm{CI}-0.81$ to -0.09$) .{ }^{66}$ One trial reported no difference in pain between CPMP settings in the intermediate term $(\mathrm{N}=316$, mean 158 vs. 160, on the 0 to 400 Pain Index). ${ }^{67}$ At long-term followup, there was no difference between groups across three trials ( $3 \mathrm{RCTs}, \mathrm{N}=404$; pooled difference $-0.19,95 \% \mathrm{CI}-0.92$ to 0.64 , $\left.\mathrm{I}^{2}=0 \%\right) .{ }^{67,86,108}$ Results of sensitivity analyses excluding an outlier trial ${ }^{86}$ and using the 18 -month followup data (versus 30-month) in another trial ${ }^{67}$ showed somewhat larger effects $(-0.30$ and -0.39) of inpatient CPMP in the long term but did not change conclusions (see Appendix I, Figures I-46 to I-48 for meta-analyses).

\section{Function}

Similarly, for function, there was no difference between inpatient and outpatient groups postintervention in one trial (difference $26.89,95 \% \mathrm{CI}-22.39$ to 76.17 on the SIP, scale not reported) ${ }^{83}$ or at short-term followup across two trials ( 2 RCTs, N=374, pooled SMD $-0.19,95 \%$ $\mathrm{CI}-0.63$ to $\left.0.09, \mathrm{I}^{2}=0 \%\right) .{ }^{66,108}$ One trial reported no difference in function between CPMP settings in the intermediate term $(\mathrm{N}=316$, mean 15.7 vs. 16 , on the 0 to 45 LBP Disability Index). ${ }^{67}$ At long term followup, there was no difference in function between groups across three trials ( 3 RCTs, $\mathrm{N}=404$, pooled SMD $-0.01,95 \% \mathrm{CI}-0.31$ to $0.39, \mathrm{I}^{2}=9.7 \%$ ). ${ }^{67,86,108}$ Results were similar from sensitivity analyses excluding an outlier trial ${ }^{86}$ and using the 18 -month long term 
followup data (versus 30-month) in another trial ${ }^{67}$ (see Appendix I, Figures I-49 to I-51 for metaanalyses).

One small trial reported no difference between the inpatient and outpatient CPMP in the proportion of patients achieving treatment success, defined as appropriate use of medication (i.e., no use of strong opioids, muscle relaxants, or tranquilizers by 6 months), active (e.g., working, exercising, increased recreation), and no increase in pain over the long term (mean 12 months; $68 \%$ [15/22] vs. $61 \%$ [11/18], respectively; RR 1.12 [95\% CI 0.70 to 1.78$]$ ]). ${ }^{82}$

\section{Opioid Use}

Two small trials reported opioid use. ${ }^{82,83,108}$ There were no differences between inpatient versus outpatient CPMP in opioid use in either trial at long-term (12 months) followup. The pooled proportion of patients using any opioid was 28 percent $(17 / 60)$ versus 39 percent $(20 / 51)$, RR 0.72 (95\% CI 0.43 to 1.22 ) and of patients using a "strong" opioid was 7 percent (4/60) versus 14 percent (7/51), RR 0.49 (95\% CI 0.15 to 1.57$).{ }^{82,108}$ Small sample sizes likely played a role in these findings. In one of these trials at short term fewer patients were using opioids in the inpatient versus the outpatient CPMP (18\% [7/38] vs. 42\% [14/33]; RR 0.43, 95\% CI 0.20 to $0.95)$, including when evaluating use of an opioid dose equivalent $>10 \mathrm{mg}$ morphine per day (10.5\% [4/38] vs. 33.3\% [11/33], RR 0.32, 95\% CI 0.11 to 0.90$)$; however the difference did not persist in the long term as reported above. ${ }^{108}$

\section{Secondary Outcomes}

Three small trials reported on secondary outcomes of interest, to include the SF-36 PCS and MCS, Beck Depression Inventory (BDI), State-Trait Anxiety Inventory (STAI), HADS, Dallas Pain Questionnaire (DPQ) depression and anxiety subscale and the General Health Questionnaire (GHQ), with no difference between groups in any trial across followup periods ranging from immediately postintervention to long term (12 months). ${ }^{82,83,86,108}$ See Appendix B, Table B-11 data.

\section{Harms and Utilization}

One trial reported that no intervention-related adverse events occurred. ${ }^{86}$

One trial reported that no patient required surgery for their pain condition (primarily chronic back/neck/leg pain) through long-term followup (12 months) but significantly fewer patients in the inpatient CPMP received subsequent treatments for pain (e.g., nerve blocks, TENS, acupuncture) compared with outpatients (10.3\% [3/29] versus 60.7\% [17/28], RR 0.17 [95\% CI 0.06 to 0.52$]) .{ }^{108}$ Further information related to utilization can be found in Appendix E, Table E2.

\section{Differential Effectiveness or Safety}

No trial reported differential effectiveness or safety.

\section{Additional Psychological or Physical Program Components}

\section{Key Points}

- There were no differences in pain or function at postintervention, intermediate- and longterm followup across two trials $(\mathrm{N}=63)$ comparing $\mathrm{CPMP}$ with and without additional psychological components. 
- There were no differences in function at postintervention, short-, intermediate- and longterm followup in one trial $(\mathrm{N}=78)$ comparing CPMP with and without additional physical components.

- None of the trials provided data on harms.

\section{Detailed Synthesis}

Three RCTs $\mathrm{s}^{51,113,114}$ assessed the effectiveness of adding additional program components to a standard CPMP (Results Appendix B, Table B-11; Appendix E, Table E-2). Sample sizes ranged from 19 to 94 (total sample=158). Two trials assessed the addition of psychological components. One added operant conditioning, relaxation, and biofeedback ${ }^{113}$ and the other ${ }^{51}$ included spouses to assist in the participants' training. The third trial assessed the addition of psychomotor therapy. ${ }^{114}$ Two trials ${ }^{51,113}$ included patients with chronic low back pain and one trial ${ }^{114}$ included patients with any musculoskeletal pain. Trials delivered the program in the outpatient setting in two trials ${ }^{51,114}$ and the inpatient setting in one trial. ${ }^{113}$ Program lengths ranged from 3 to 12 weeks. Program components were delivered in group sessions in two trials $\mathrm{s}^{51,114}$ and via a combination of group and individual session in the third trial. ${ }^{113}$ Two trials ${ }^{113,114}$ administered a high-intensity program ( $\geq 20$ hours/week or $>80$ hours total) and one trial ${ }^{51}$ administered a lowintensity program $(<20$ hours/week or $\leq 80$ hours total). The weighted mean age was 41 years and 18 percent of patients were male (across 2 trials). ${ }^{113,114}$ Symptom duration was reported by two trials; one reported median of 74 months ${ }^{51}$ while in the other, 75 percent of patients had had symptoms for two or more years. ${ }^{114}$ None of the trials specifically included Medicare patients. However, one trial only included patients who were disabled and not working due to pain (for at least 3 but no more than 30 months), ${ }^{113}$ while the other two did not provide information on disability status. No trial reported on comorbidities or opioid use at baseline, but two trials ${ }^{51,113}$ did exclude patients with coexisting psychiatric morbidity and one ${ }^{113}$ stated that no patient entered the program using narcotics. Two trials reported receiving funding from government sources: ${ }^{51,113}$ the third did not report a funding source. Trials were conducted in the United States, ${ }^{113}$ Iran,${ }^{51}$ and the Netherlands. ${ }^{114}$

Two trials were rated fair quality ${ }^{113,114}$ and one poor quality ${ }^{51}$ (Appendix F, Table F-2). The major methodological limitation in the fair-quality trials was the inability to effectively blind patients and caregivers to the interventions. Other methodological shortcomings in the poorquality trial included inadequate randomization methods, unclear allocation concealment methods and lack of an intention-to-treat analysis.

\section{Primary Outcomes}

No differences in various measures of pain or function were found between CPMPs with additional components versus CPMPs with standard content at any timepoint across all three RCTs (Appendix B, Table B-11). ${ }^{51,113,114}$

Across the two small trials (one fair, one poor quality) that compared the addition of psychological components (operant conditioning, relaxation and biofeedback or spouse assistance) to a standard CPMP program in chronic LBP patients, there were no differences between groups in pain intensity postintervention ( $2 \mathrm{RCTs}, \mathrm{N}=63$, pooled difference $0.18,95 \%$ CI -0.81 to $1.25, \mathrm{I}^{2}=0 \%$, on a 0 to 10 scale) ) $^{51,113}$ (Appendix I, Figure I-52), or at intermediate term in the fair-quality trial $(\mathrm{N}=42$, difference $0.33,95 \% \mathrm{CI}-0.22$ to 0.88 , on 1 to $5 \mathrm{MPQ}$ present pain intensity) ${ }^{113}$ or long term in the poor-quality trial $(\mathrm{N}=19$, difference $-0.90,95 \% \mathrm{CI}$ -3.42 to 1.62 , on a 0 to $10 \mathrm{VAS}){ }^{51}$ Like the results for pain, no differences in function were 
found postintervention ( $2 \mathrm{RCTs}, \mathrm{N}=63$, SMD $-0.29,95 \% \mathrm{CI}-0.87$ to $\left.0.35, \mathrm{I}^{2}=0 \%\right)^{51,113}$ (Appendix I, Figure I-53), intermediate term ( 1 trial, $\mathrm{N}=42$, difference $-5.90,95 \% \mathrm{CI}-14.77$ to 2.25 , on the 0 to 130 LBP Rating Scale), ${ }^{113}$ or long term ( 1 trial, $N=19$, difference $-0.60,95 \%$ CI -6.01 to 4.90 , on the 0 to 24 RMDQ). ${ }^{51}$

The third fair-quality RCT, a cluster-randomized trial evaluating the addition of psychomotor therapy to a standard CPMP in patients with chronic musculoskeletal pain, reported no differences between CPMP groups in function (PDI, 0 to 70 scale) postintervention (difference $-3.37,95 \% \mathrm{CI}-7.12$ to 0.38 ) and at short- (difference $-4.13,95 \% \mathrm{CI}-8.84$ to 0.58 ), intermediate- (difference $-1.89,95 \% \mathrm{CI}-7.22$ to 3.44 ) and long-term (difference $-0.30,95 \% \mathrm{CI}$ -5.84 to 5.24 ) followup. ${ }^{114}$

\section{Secondary Outcomes}

CPMP with psychomotor therapy was associated with improvement on the physical component of the RAND-36 at long-term followup (difference 3.09, 95\% CI 0.22 to 5.96, on a 0 to 100 scale) compared with CPMP alone in one fair-quality trial. ${ }^{114}$ There were no other differences between groups for other outcomes in this same trial (RAND-36 mental component, $\mathrm{BDI}$ ) or in a second fair-quality RCT (negative mood) comparing CPMP plus additional psychological components versus the standard program ${ }^{113}$ (Appendix B, Table B-11).

\section{Harms, Utilization, and Differential Effectiveness or Safety}

None of the three studies reported harms, utilization outcomes or differential effectiveness or safety.

\section{Pretreatment Assessment}

\section{Key Points}

- CPMP delivered based on a functional capacity preassessment tool was associated with a small improvement in function in one trial at long term, but there was no difference in pain or function in a second trial that employed a biopsychological preintervention assessment.

- None of the trials provided data on harms.

\section{Detailed Synthesis}

Two RCTs compared CPMPs conducted with versus without a pretreatment assessment to help inform subsequent therapy (Results Appendix B, Table B-10; Appendix E, Table E-2). ${ }^{115,116}$ Sample sizes ranged from 207 to 222 (total sample=429). The mean age in one trial was 46 years and the median age in the other trial was 40 years. Across the two trials, 54 percent of patients were male. Neither trial reported on race/ethnicity, comorbidities, or opioid use at baseline. Both trials included patients with various chronic musculoskeletal pain conditions (primarily back pain in one ${ }^{116}$ ). Only one trial reported duration of symptoms (median of 18 months). ${ }^{115}$ Neither trial specifically included Medicare patients. In one trial, 81 percent of patients were on sick leave at the start of the trial. ${ }^{116}$ One trial ${ }^{116}$ utilized a preintervention assessment in the intervention group designed to evaluate patients' functional capacity in relation to their workplace (or intended workplace) based on a kinesiophysical approach. The CPMPs in this trial were 3-week, lower intensity (50-60 hours total, 3-4 hours/day) inpatient programs and treatment was delivered on an individual basis. The second trial ${ }^{115}$ employed a preintervention assessment 
in the intervention group based on a multidisciplinary, biopsychosocial approach to guide subsequent therapies. The CPMPs in this trial were outpatient programs delivered to groups of patients; program intensity was unclear in this trial but considered to be lower intensity. One trial received nonprofit funds ${ }^{116}$ and the other did not report funding information. Both trials were conducted in Europe.

Both trials were rated fair quality (Appendix F, Table F-2). The major methodological limitation was the inability to effectively blind patients and caregivers to the interventions. In addition, one trial had a high attrition rate. ${ }^{116}$

\section{Primary Outcomes}

Only long-term followup data were reported by both trials. CPMP delivery based on a functional capacity assessment preintervention was associated with a small improvement in function compared with CPMP with no such assessment in one trial (adjusted mean difference $-6.5,95 \% \mathrm{CI}-12.6$ to -0.4 , on the 0 to $70 \mathrm{PDI}$ ). ${ }^{116}$ The second trial found no difference in pain (adjusted odds ratio [OR] of improvement from baseline $1.20,95 \%$ CI 0.63 to $2.30,0$ to 10 scale) or function (adjusted OR of improvement from baseline 1.61, 95\% CI 0.84 to $3.07,0$ to 100 Oswestry Disability Index [ODI]) between CPMPs with or without a biopsychosocial-based preintervention assessment. ${ }^{115}$ Differences in pain conditions, program components, intensity and delivery across trials may have contributed to some of these findings.

\section{Secondary Outcomes}

No differences between groups were seen over the long term for the SF-36 PCS and MCS, the Zung Depression Scale, or the Stress and Crisis Inventory in one trial; patient satisfaction was higher $(\mathrm{p}<0.001)$ in the group that received the biopsychological assessment compared with the group that received the standard pretreatment assessment (Appendix B, Table B-11). ${ }^{115}$

\section{Harms, Utilization, and Differential Effectiveness or Safety}

Neither RCT reported harms, utilization outcomes or differential effectiveness or safety.

\section{Other Comparisons}

\section{Key Points}

- CPMP using a function-centered approach was associated with small improvements in pain and function postintervention, but not pain in the short term, compared with CPMP using a pain-centered approach in one trial.

- There were no differences in pain or function postintervention or at intermediate-term followup comparing CPMP using an "exposure in vivo" versus a graded activity approach in one trial.

- One patient (2\%) in the graded activity group (vs. none in the "exposure in vivo" group) deteriorated during treatment (i.e., treatment counterproductive).

\section{Detailed Synthesis}

Two RCTs (reported in 3 publications) assessed different approaches to CPMPs (Results Appendix B, Table B-11; Appendix E, Table E-2). ${ }^{17-119}$ One trial compared a function-centered versus a pain-centered approach to therapy ${ }^{117,118}$ while the other compared an "exposure in vivo" approach (i.e., systematically reducing pain-related fear using Pavlovian conditioning and CBT) versus a graded activity approach (i.e., increase healthy behavior via operant learning 
principles). ${ }^{119}$ Sample sizes ranged from 85 to 174 (total sample=259). Both trials included patients with chronic LBP, with pain radiating to the legs in 83 percent of patients in one trial ${ }^{117,118}$ and 98 percent in the other trial. ${ }^{119}$ Weighted mean age was 43 years and 70 percent of patients were male. Neither trial specifically included Medicare patients. In one trial, 54 percent of patients were on sick leave or in receipt of a disability pension ${ }^{119}$ and in the other, patients were required to have a minimum of 6 weeks of sick leave in the 6 months prior to enrollment. ${ }^{117,118}$ Neither trial provided information on comorbidities, however, one trial excluded patients with substance abuse, medical disorders or cardiovascular disease preventing physical exercise, and serious psychopathology. ${ }^{119}$ Across the two trials, 73 percent of patients were taking pain medication (not otherwise specified) at baseline and in one trial 31 percent of patients had previous back surgery. ${ }^{119}$ One trial delivered a high-intensity program $(\geq 20$ hours/week or $>80$ hours total) in an inpatient setting for 3 weeks. ${ }^{117,118}$ The program in the other trial was considered low intensity ( $<20$ hours/week or $\leq 80$ hours total) and was delivered over 8 to 12 weeks in an outpatient setting. ${ }^{119}$ In both trials, program components were delivered to patients individually. One trial received government funding ${ }^{119}$ and the other did not report funding information. Both trials were conducted in Europe.

Both trials were rated fair quality (Appendix F, Table F-2). The major methodological limitations were the inability to effectively blind patients and caregivers to the interventions and high attrition rates.

\section{Primary Outcomes}

\section{Pain}

Postintervention, there was a small improvement in pain favoring function-centered versus pain-centered approach to CPMP in one trial $(\mathrm{N}=171$, difference -0.80 on a 0 to 10 scale, $95 \%$ CI -1.40 to -0.20$)^{118}$ while the second trial, which compared CPMP using an "exposure in vivo" versus a graded activity approach, found no difference between programs $(\mathrm{N}=77$, difference -0.04 on a 0 to 10 scale, $95 \% \mathrm{CI}-1.03$ to 0.96$).{ }^{119}$ Neither trial found a significant difference in pain between CPMP groups at later timepoints - short term in one trial (difference in change scores from baseline $-0.54,95 \% \mathrm{CI}-1.35$ to 0.27 , on a 0 to $10 \mathrm{NRS}$; function- versus paincentered) ${ }^{118}$ and intermediate term in the other (difference $0.07,95 \% \mathrm{CI}-0.97$ to 1.11 , on the 0 to 10 MPQ; "exposure in vivo" versus graded activity). ${ }^{119}$ Differences in pain conditions, program components, intensity and delivery across trials may have contributed to some of these findings.

\section{Function}

Like the results for pain, function-centered CPMP was associated with a small improvement in function compared with pain-centered CPMP in one trial $(\mathrm{N}=171$, difference in change scores from baseline $-13.3,95 \%$ CI -20.3 to -6.3 , on the 0 to 200 Performance Assessment and Capacity Testing). ${ }^{118}$ In the second trial that compared "exposure in vivo" CPMP versus graded activity CPMP, there were no differences between groups in function on the 0 to 24 RMDQ postintervention (difference in change scores from baseline $-1.95,95 \% \mathrm{CI}-4.61$ to 0.71 ) or at intermediate-term followup (difference in change scores from baseline $-2.11,95 \% \mathrm{CI}-4.76$ to 0.54 ), or in the proportion of patients with clinically relevant improvement on the RMDQ at either timepoint (54\% [22/41] vs. 42\% [15/36], RR 1.29, 95\% CI 0.80 to 2.08; and 50\% [19/38] vs. $34 \%$ [12/35], RR $1.46,95 \%$ CI 0.83 to 2.55 , respectively). ${ }^{119}$ Sample size likely played a role in this finding. 


\section{Secondary Outcomes}

One trial reported a difference in global improvement favoring a function-centered versus a pain-centered approach postintervention (difference $0.80,95 \%$ CI 0.19 to 1.40 , on a 7-point Likert scale) but not at short-term followup (no data provided). ${ }^{118}$ This same trial reported that patients in both groups were equally satisfied with treatment at long-term followup (median 6 , IQR 4 to 7 , on a 1 to 7 scale). ${ }^{117}$

\section{Harms}

One patient $(2 \% ; 1 / 43)$ in the graded activity group (vs. none in the "exposure in vivo" group) deteriorated during treatment (i.e., treatment counterproductive); no other patient experienced any adverse events or side effects related to the interventions. ${ }^{119}$

\section{Utilization and Differential Effectiveness or Safety}

Prespecified utilization outcomes of interest were not reported and neither RCT reported differential effectiveness or safety.

\section{Group Versus Individual Session Format}

\section{Key Points}

- Evidence comparing CPMPs delivered in a group versus an individual format from one small poor-quality trial did not permit conclusions about effectiveness. This trial did not report data on harms.

\section{Detailed Synthesis}

One RCT $(\mathrm{N}=50)$ conducted in the United Kingdom compared outpatient CPMPs delivered in a group versus an individual format (Results Appendix B, Table B-11; Appendix E, Table E2). ${ }^{112}$ Minimal information on the program characteristics were provided. All patients had chronic LBP (mean duration 8.1 years). Population demographics include data on patients included in another study conducted by the same authors. Including those patients, mean age was 42 years and 41 percent were male. Most patients (64\%) were in receipt of sickness or disability benefit and 13 percent had undergone spinal surgery. Funding information for the trial was not reported.

This trial was rated poor quality due to major methodological limitations: unclear randomization, unclear allocation concealment methods, the inability to effectively blind patients and caregivers to the interventions and an unacceptable attrition rate (Appendix F, Table F-2).

\section{Primary Outcomes}

There were no differences in pain ( 0 to $10 \mathrm{VAS}$ ) postintervention (mean $5.8 \mathrm{vs} .4 .7)$ or at intermediate-term followup (mean 6.5 vs. 6.0) or for function at either timepoint (mean 13.3 versus 11.1 for both, 0 to $24 \mathrm{RMDQ}$ ) between patients who received CPMP delivered in a group versus an individual format. ${ }^{112}$

\section{Secondary Outcomes}

There were no differences between groups (group vs. individual format) postintervention or intermediate term on the modified Zung Depression Inventory (scale not reported) (mean 27.0 versus 27.0 and 28.0 versus 26.1 , respectively). ${ }^{112}$ 


\section{Harms, Utilization, and Differential Effectiveness or Safety}

The trial did not report harms, utilization outcomes and differential effectiveness or safety.

\section{Addition of Booster Sessions}

\section{Key Points}

- There were no differences in pain or function postintervention or at long-term followup comparing a CPMP with and without additional booster sessions in one fair-quality trial. This trial did not report data on harms.

\section{Detailed Synthesis}

One RCT $(\mathrm{N}=232)$ conducted in Germany compared a 4-week CPMP with and without the addition of seven, 20-minute booster sessions over the course of a year ${ }^{75}$ (Results Appendix B, Table B-11; Appendix E, Table E-2). The program took place in an inpatient setting and was delivered via a combination of group and individual sessions. All patients had chronic back pain (duration not reported). The mean patient age was 49 years and 23 percent were male. The trial did not report on race, comorbidities or opioid use at baseline. Funding was provided, in part, by government.

This trial was rated fair quality due to the inability to effectively blind patients and caregivers to the interventions (Appendix F, Table F-2).

\section{Primary Outcomes}

There were no differences in pain according to the Pain Perception Scale immediately postintervention (affective pain, difference $-0.30,95 \% \mathrm{CI}-2.71$ to 2.11 , on a 14 to 56 scale; sensory pain, difference $0.0,95 \% \mathrm{CI}-1.36$ to 1.36 , on a 10 to 40 scale) or at long-term followup (affective pain, difference $-1.40,95 \% \mathrm{CI}-3.95$ to 1.15 , on a 14 to 56 scale; sensory pain, difference $-0.70,95 \% \mathrm{CI}-2.23$ to 0.83 , on a 10 to 40 scale) between patients who received booster sessions following CPMP versus those who did not. ${ }^{75}$ Similarly, there were no differences between groups in function at either timepoint, respectively (difference 1.40 [95\% CI -2.12 to 4.12$]$ and difference 0.60 [95\% CI -3.23 to 4.50$]$ on the 0 to $70 \mathrm{PDI}$ ).

\section{Secondary Outcomes}

There were no differences between groups (CPMP with vs. without booster sessions) immediately postintervention or in the long term according to the SF-12 PCS (difference -0.40 , $95 \% \mathrm{CI}-2.90$ to 2.10 and difference $0.0,95 \% \mathrm{CI}-2.61$ to 2.61 , on a 0 to 100 scale, respectively), MCS (difference $-1.90,95 \% \mathrm{CI}-4.84$ to 1.04 and difference $-0.40,95 \% \mathrm{CI}-3.37$ to 2.57 , on a 0 to 100 scale, respectively), and the BDI (difference $0.50,95 \% \mathrm{CI}-1.31$ to 2.31 and difference $0.30,95 \% \mathrm{CI}-1.86$ to 2.46 , respectively, on a 0 to 63 scale) ${ }^{75}$

\section{Harms, Utilization, and Differential Effectiveness or Safety}

The trial did not report harms, utilization outcomes or differential effectiveness or safety. 


\section{Contextual Question 1. Pain Management Program Types}

Answers to this question were informed by peer-reviewed literature captured by our search and reported in the results above, U.S. government reports, conversations with our Technical Expert Panel (TEP), and comments received on our study protocol via the Supplemental Evidence and Data for Systematic review (SEADS). Additional information is found in Appendix $\mathrm{C}$ and in the Discussion section.

\section{Program Definitions}

There was substantial variability in the terminology used in the literature and in clinical practice to describe programs that incorporated methods that may address the biopsychosocial, multidimensional aspects of pain. Terms such as multimodal, multidisciplinary, interdisciplinary, integrated, comprehensive, and collaborative were used in multitude of ways with no firm consensus on their definition or use. Similarly, various descriptions of what constitutes a biopsychosocial model of factors that contribute to a person's experience of pain have been proposed. ${ }^{9-12}$ A myriad of diverse models and descriptions of management of nonactive cancer pain have been reported in the peer-reviewed literature. Some are described in this report. Many others are in use clinically but may not be represented in peer-reviewed publications. No standard terminology or program definitions were identified. Most of the peer-reviewed literature focuses on programs provided in rehabilitation centers such as comprehensive traditional multidisciplinary rehabilitation programs or specialty clinics versus those that are based in and integrated with primary care. Given the lack of consensus in terminology and program definition, we defined integrated pain management programs as programs centered in primary care, that have embedded or easy access to multidisciplinary providers and comprehensive pain management programs as those that are not based in primary care. Studies included in this review provide insight into the complexity and heterogeneity of care models, their focus, populations, components, delivery, and settings for both comprehensive and integrated program models.

\section{Program Components}

There was substantial variability in the components that may be included in programs as well as how they were delivered. No standard set of components was identified. The components and delivery of them in various pain management programs has evolved since early publications and acceptance of pain management programs in the 1970's. ${ }^{120,121}$ Common general components described from two recent reviews ${ }^{121,122}$ across a total of 112 formal multidisciplinary pain management program studies for chronic pain included psychological and mental health support (94\% of studies, primarily CBT-based strategies, relaxation, coping, mindfulness) and physical activity ( $86 \%$ of studies) and less commonly, medication optimization or monitoring (40\%). Education on a range of topics (pain mechanisms, medication, psychological factors) was done in most studies ( $76 \%$ of 85 studies) in the largest review. ${ }^{121}$ TEP discussions re-affirmed that these were likely the most common and important components of a formal, integrated program. The relative importance of individual components in IPMPs is difficult to assess given the substantial variation across programs. Some programs tailor components to patient needs; not all patients may receive a specific component or set of components. Coordination and communication across multiple providers are considered key in assuring collaborative, interdisciplinary care. ${ }^{13,15,16,18,120,123}$ 


\section{What Pain Management Models or Mechanisms Are Most Commonly Used in Clinical Practice?}

The current paradigm for pain care is the provision of selected individual treatments (e.g., medications) or services (e.g., physical therapy, psychological support) prescribed or recommended by a patient's provider (primary care or specialty provider). No consistent models or mechanisms are evident. Treatment may be unimodal or offer a limited range of management options (e.g., medication and physical therapy only or medication and psychological support only). Formal pain management programs have not been widely implemented in the United States for either general populations or the Medicare population. Reasons include the costs, logistics, leadership support, staffing, and provider training required to develop and implement them as well as the current fee-for-service reimbursement structure. Programs may not be accessible to many populations based on locations, the availability of pain specialists, and socioeconomic factors.

\section{What Types of Programs/Models May Be Most Applicable to Medicare Beneficiaries?}

Medicare eligible patients and beneficiaries are a diverse population. This population may include active working seniors as well as individuals with various disabilities, comorbidities, and psychosocial needs, thus, programs that lend themselves to individualized care may be of most benefit. Programs that are likely most applicable are those that provide comprehensive assessment based on the biopsychosocial model in order to create an individualized care plan which provides access to the primary components of most benefit to that patient and is coordinated across disciplines involved in the care plan.

\section{What Theoretical Advantages and Disadvantages Do Various Programs/Models Have Compared With Current Practice?}

Theoretical advantages of formal programs versus usual care are many. Programs may be best suited to evaluate and manage the range of pain complexity and related comorbidities. Coordination of care based on a patient's particular circumstances may lead to optimal management of pain by optimizing the use of appropriate medications and medical procedures, facilitating physical function and providing psychological support to enhance patient selfmanagement of their pain. This approach ${ }^{16}$ may facilitate identification and best use of diverse resources relevant to patient goals for pain management, including improving quality of life and return to important life activities. This may be particularly important in patients with medical or psychological comorbidities. An integrated, collaborative approach provides support for primary care providers and related care teams which may enhance provision of evidence-based, guideline concordant care that includes appropriate assessment, referral to specialty care as needed, and followup. ${ }^{16,124}$

Theoretical disadvantages to formal programs include the costs, logistics, leadership support, staffing, and need for provider training that are involved in the development and implementation of such programs. ${ }^{14,16,18,124}$ Programs may not be accessible to many populations based on location, insurance coverage, and socioeconomic factors. The availability of professionals trained in pain management may also limit accessibility. 


\section{Are There Any Potential Safety Issues?}

Specific harms related to integrated or comprehensive pain management programs are not well reported. Based on included studies, reported safety issues were not considered serious, i.e. did not require medical attention. They are described in the Discussion section below. For example, minor injuries or temporary increases in pain during physical therapy were reported. Potential safety issues that have not been addressed in the literature reviewed here include suicide and impact on opioid dependence or overuse. Similarly, potential harms related to decreasing opioid use or worsening of pain in formal pain management programs were not described in the literature reviewed here. Additional research and evaluation of these outcomes is warranted.

\section{Contextual Question 2. Cost Effectiveness}

There was sparse information on the cost-effectiveness for either the IPMP or the CPMP conducted in the United States in the peer-reviewed literature. The substantial variations across programs and how components were delivered leads to concerns regarding the applicability of costs or cost-effectiveness across either program type. We restricted studies for this Contextual Question to those which evaluated IPMPs or CPMPs which contained the availability of the primary components of medication review/optimization, physical activity and psychological support, and compared such programs to either usual care or active treatment options. Six programs meeting inclusion criteria for the Key Questions reported associated economic data (Appendix C). The most applicable economic assessment to this review, based on a cluster-RCT of a system-based IPMP, was done from the Veterans Affairs (VA) healthcare perspective. ${ }^{45}$ It is the only U.S.-based study. The trial randomized primary care providers to receive collaborative, multidisciplinary assistance with pain treatment (APT) for patients with musculoskeletal pain diagnoses. The mean APT costs were greater than those for usual care, but confidence intervals were wide (mean [standard deviation] for each, $\$ 11,263$ [ $\$ 14,566]$ versus $\$ 8920$ [\$13,131]). APT participants experienced a mean of 16 additional pain disability-free days (PDFDs) over the 12-month period. Predicted adjusted mean incremental cost per pain disability-free day ranged from $\$ 364$ to $\$ 1117$ and predicted adjusted mean incremental increase of intervention costs ranged from $\$ 6035$ to $\$ 18,554$. Authors state that the average increase of $\$ 2300$ per patient for the APT intervention falls on the low end of costs for commonly used chronic pain interventions. The other five studies, three full economic studies and two costing studies, ${ }^{44,49,90,101,102,125}$ were conducted outside of the United States in working populations. Mean ages of included populations ranged from 42 to 46 years. These economic studies based their analyses on outcomes such as "sick leave" and "return to work" and focused on lost productivity due to pain and related impact on indirect costs from a societal perspective for their determination of cost effectiveness. 


\section{Discussion}

\section{Findings in Relation to the Decisional Dilemmas}

The U.S. Department of Health and Human Services has been directed to evaluate ways to improve Medicare coverage and payment for treatment of acute and chronic pain, particularly through pain management programs and multidisciplinary, multimodal treatment models that involve care coordination as part of the Dr. Todd Graham Pain Management Study. Requisite to addressing this decisional dilemma is understanding the types/components and methods of care delivery as well as benefits, potential risks, and related costs of such programs to Medicare Parts $\mathrm{A}$ and $\mathrm{B}$ beneficiaries with complex acute/subacute pain or chronic nonactive cancer pain.

This review synthesized evidence on the effectiveness, comparative effectiveness, and harms of integrated pain management programs (IPMPs) and comprehensive pain management programs (CPMPs), as defined in our methods, in patients with complex acute/subacute pain or chronic nonactive cancer pain. We also synthesized available evidence on program factors which may impact patient outcomes. The key findings and strength of evidence (SOE) for Key Question 1 are summarized in Tables 2 and 3, focusing on the primary outcomes of pain, function, and changes in opioid prescribing stratified by followup duration. Harms are summarized in Table 4. SOE is further detailed in Appendix G. In addition to the Key Questions, two Contextual Questions are addressed, primarily via the discussions below, with additional information and references found in Appendix C.

\section{Evidence Base Available}

Evidence on effectiveness and comparative effectiveness was available from 8 randomized controlled trials (RCTs) (11 publications) of IPMPs and 49 RCTs (67 publications) of CPMPs, most of which compared programs to usual care or waitlist. CPMPs are the traditional way that multidisciplinary pain care has been delivered and have been reported in the peer-reviewed literature for several decades which may explain the difference in the evidence available. IPMPs may be more efficient; because they are centered in primary care, there may be better opportunity for care coordinated between a range of specialty care providers. While none of the included trials specifically enrolled Medicare beneficiaries, some studies enrolled populations over 60 years of age. The average age of patients was 57 years in IPMP studies and 45 years in CPMP studies. While some RCTs reported including patients with disability they did not provide criteria that would be used to determine Medicare eligibility. None of the included trials evaluated differential impact of IPMPs or CPMPs on outcomes based on patient subgroups of interest. The overall SOE for most outcomes was low for both IPMP and CPMP reflecting low certainty about the findings. Methodological limitations and imprecision were commonly seen in these instances.

\section{Evidence on Effectiveness}

Both IPMPs and CPMPs were associated with improved function at multiple time frames compared with usual care or waitlist. Small average functional improvements immediately following IPMPs (SOE: moderate) persisted to short term but not into intermediate or long term followup (SOE: low) in pooled analyses. In patients with chronic low back pain (LBP) a 30 percent or greater improvement on the Roland-Morris Disability Questionnaire (RMDQ) (0 to 23 or 24 scale) was seen postintervention across two trials which persisted into short and 
intermediate terms in one of the trials. In contrast, across two trials in patients with osteoarthritis (OA), there was no difference in the proportion of patients achieving 18 percent or more improvement on the Western Ontario and McMaster Universities Arthritis Index Function (0 to 68 scale) between IPMP and usual care postintervention. For CPMPs, moderate postintervention functional improvements continued short term but were small in the long term; no differences were seen in the intermediate term (SOE: low for all times). IPMPs were associated with small improvements in pain ( 0 to 10 scale) in the short and intermediate terms compared with usual care or waitlist (SOE: low), however the small pain improvements seen following CPMPs (SOE: moderate) were not evident at later time frames (SOE: low). These findings are consistent with data showing that patients can experience improvement in function without experiencing improvements in pain. This may be important to consider as few interventions for pain effectively improve function and the benefits are generally small. We defined small effects as a mean between-group difference following treatment for pain of 0.5 to 1.0 points on a 0 to 10 scale or for function, a standardized mean difference (SMD) of 0.2 to 0.5 or a mean difference of 5 to 10 points on the 0 to 100-point Oswestry Disability Index, 1 to 2 points on the 0 to 24-point RMDQ, or equivalent. Although this definition may not meet proposed thresholds for clinically important effects, estimates of minimum clinically important effects vary across studies and the relevance of effects classified as small may differ between patients based on baseline symptom severity, harms, costs, patient preferences, and other factors. ${ }^{39,126-128}$ Evidence on the impact of programs on changes in opioid prescribing versus usual care was very limited. One IPMP trial reported no difference in opioid prescriptions postintervention (SOE: low); evidence from small and poor-quality trials for CPMPs was insufficient to draw conclusions. Despite the substantial heterogeneity in programs, their delivery and components, for most outcomes and time frames, little statistical heterogeneity was observed.

\section{Evidence on Comparative Effectiveness}

Evidence for comparative effectiveness of IPMPs was confined to a single trial which compared IPMP with telephone-delivered cognitive behavioral therapy (CBT) alone and with exercise. No differences in function between IPMP and either comparator were seen at any time frame (SOE: low). More comparative effectiveness evidence was available for CPMPs.

Compared with physical activity, small functional improvement favoring CPMP was noted in the short term (SOE: moderate), but no difference in either function or pain were seen at other times. CPMPs did not confer improvement in either function or pain compared with psychological therapy alone at any time frame. CPMPs (patients received pharmacologic therapy in addition to other components) were associated with improved function compared with the pharmacologic therapy alone at all time frames. Pharmacologic therapies varied across trials (and patients). Most all reported nonsteroidal anti-inflammatory drug use and none included full opioid agonists; one poor-quality trial included tramadol. Moderate functional improvement following CPMP was seen in one fair-quality trial in patients with fibromyalgia (FM) compared with pharmacologic therapy alone which included analgesics, antidepressants, and/or sedatives. Functional improvements associated with CPMP were small at other time frames based on pooled estimates compared with pharmacologic treatment alone (analgesics, antidepressants, and/or sedatives). Comparisons to specific medications were not reported. Moderate pain improvement was seen post CPMP and in the intermediate term compared with pharmacologic therapy, but no differences were seen in the short or long term. For the comparison of full CPMPs with the combination of pharmacologic therapies and primarily passive physical therapy 
approaches, there were no differences between groups in function at any time; however, CPMPs were associated with moderate pain improvement based on one fair-quality trial in which only antidepressants were used.

Table 2. Summary of evidence of IPMPs for noncancer pain: Key Question 1 (pain, function, opioid use)

\begin{tabular}{|c|c|c|c|c|}
\hline Outcome & Time Point & IPMP Versus UC & $\begin{array}{c}\text { IPMP Versus } \\
\text { Physical Activity }\end{array}$ & $\begin{array}{c}\text { IPMP Versus } \\
\text { Telephone-CBT }\end{array}$ \\
\hline \multirow[t]{4}{*}{$\begin{array}{l}\text { Pain } \\
\text { (Effect Size/SOE) }^{a}\end{array}$} & Postintervention & $\begin{array}{c}\text { None } \\
++\end{array}$ & No evidence & No evidence \\
\hline & $\begin{array}{l}\text { Short term } \\
\text { ( } 1 \text { to }<6 \text { months) }\end{array}$ & $\begin{array}{c}\text { Small } \\
+\end{array}$ & No evidence & No evidence \\
\hline & $\begin{array}{l}\text { Intermediate term } \\
(\geq 6 \text { to }<12 \text { months) }\end{array}$ & $\begin{array}{c}\text { Small } \\
+\end{array}$ & No evidence & No evidence \\
\hline & $\begin{array}{l}\text { Long term } \\
\text { ( } \geq 12 \text { months) }\end{array}$ & $\begin{array}{c}\text { None } \\
+\end{array}$ & No evidence & No evidence \\
\hline \multirow[t]{4}{*}{$\begin{array}{l}\text { Function } \\
\text { (Effect Size/SOE) }^{a}\end{array}$} & Postintervention & $\begin{array}{c}\text { Small } \\
++\end{array}$ & $\begin{array}{c}\text { None } \\
+\end{array}$ & $\begin{array}{c}\text { None } \\
+\end{array}$ \\
\hline & $\begin{array}{l}\text { Short term } \\
\text { (1 to <6 months) }\end{array}$ & $\begin{array}{c}\text { Small } \\
++\end{array}$ & $\begin{array}{c}\text { None } \\
+\end{array}$ & $\begin{array}{c}\text { None } \\
+\end{array}$ \\
\hline & $\begin{array}{l}\text { Intermediate term } \\
\text { ( } \geq 6 \text { to }<12 \text { months) }\end{array}$ & $\begin{array}{c}\text { None } \\
+\end{array}$ & No evidence & No evidence \\
\hline & $\begin{array}{l}\text { Long term } \\
\text { ( } \geq 12 \text { months) }\end{array}$ & $\begin{array}{c}\text { None } \\
+\end{array}$ & $\begin{array}{c}\text { None } \\
+\end{array}$ & $\begin{array}{c}\text { None } \\
+\end{array}$ \\
\hline \multirow[t]{4}{*}{$\begin{array}{l}\text { Opioid Use } \\
\text { (Effect Size/SOE)a }^{a}\end{array}$} & Postintervention & $\begin{array}{c}\text { None } \\
+\end{array}$ & No evidence & No evidence \\
\hline & $\begin{array}{l}\text { Short term } \\
\text { (1 to <6 months) }\end{array}$ & No evidence & No evidence & No evidence \\
\hline & $\begin{array}{l}\text { Intermediate term } \\
\text { ( } \geq 6 \text { to }<12 \text { months) }\end{array}$ & $\begin{array}{c}\text { Insufficient } \\
\text { evidence }\end{array}$ & No evidence & No evidence \\
\hline & $\begin{array}{l}\text { Long term } \\
\text { ( } \geq 12 \text { months) }\end{array}$ & No evidence & No evidence & No evidence \\
\hline
\end{tabular}

$\mathrm{CBT}=$ cognitive pain management program; IPMP = integrated pain management program; $\mathrm{SOE}=$ strength of evidence; $\mathrm{UC}=$ usual care.

${ }^{\text {a }}$ Effect size: None, small, moderate, or large difference favoring IPMP; SOE: $+=$ low, $++=$ moderate,$+++=$ high

Table 3. Summary of evidence of CPMPs for noncancer pain: Key Question 1 (pain, function, opioid use)

\begin{tabular}{|c|c|c|c|c|c|c|}
\hline Outcome & Time Point & $\begin{array}{l}\text { CPMPs } \\
\text { Versus } \\
\text { UC or WL }\end{array}$ & $\begin{array}{l}\text { CPMPs } \\
\text { Versus } \\
\text { Physical } \\
\text { Activity }\end{array}$ & $\begin{array}{c}\text { CPMPs Versus } \\
\text { Pharmacologic } \\
\text { Therapy }\end{array}$ & $\begin{array}{c}\text { CPMPs Versus } \\
\text { Pharmacologic } \\
\text { Therapy and } \\
\text { Passive PT }\end{array}$ & $\begin{array}{c}\text { CPMPs } \\
\text { Versus } \\
\text { Psychological } \\
\text { Therapy }\end{array}$ \\
\hline \multirow{4}{*}{$\begin{array}{l}\text { Pain } \\
\text { (Effect } \\
\text { Size/SOE) }^{a}\end{array}$} & Postintervention & $\begin{array}{c}\text { Small } \\
++\end{array}$ & $\begin{array}{c}\text { None } \\
++\end{array}$ & $\begin{array}{c}\text { Moderate } \\
+\end{array}$ & $\begin{array}{c}\text { Moderate } \\
+\end{array}$ & $\begin{array}{c}\text { None } \\
+\end{array}$ \\
\hline & $\begin{array}{l}\text { Short term } \\
\text { (1 to <6 months) }\end{array}$ & $\begin{array}{c}\text { None } \\
+\end{array}$ & $\begin{array}{c}\text { None } \\
+\end{array}$ & $\begin{array}{c}\text { None } \\
+\end{array}$ & No evidence & No evidence \\
\hline & $\begin{array}{l}\text { Intermediate term } \\
\text { ( } \geq 6 \text { to }<12 \text { months) }\end{array}$ & $\begin{array}{c}\text { None } \\
+ \\
\end{array}$ & $\begin{array}{c}\text { None } \\
+ \\
\end{array}$ & $\begin{array}{c}\text { Small } \\
+ \\
\end{array}$ & $\begin{array}{c}\text { Moderate }^{\mathrm{C}} \\
+ \\
\end{array}$ & $\begin{array}{c}\text { None } \\
+ \\
\end{array}$ \\
\hline & $\begin{array}{l}\text { Long term } \\
\text { ( } \geq 12 \text { months) }\end{array}$ & $\begin{array}{c}\text { None } \\
+\end{array}$ & $\begin{array}{c}\text { None } \\
++\end{array}$ & $\begin{array}{c}\text { None } \\
+\end{array}$ & $\begin{array}{c}\text { Moderate }^{\mathrm{C}} \\
+\end{array}$ & $\begin{array}{c}\text { None } \\
+\end{array}$ \\
\hline \multirow{4}{*}{$\begin{array}{l}\text { Function } \\
\text { (Effect } \\
\text { Size/SOE) }^{a}\end{array}$} & Postintervention & $\begin{array}{c}\text { Moderate } \\
+\end{array}$ & $\begin{array}{c}\text { None } \\
++\end{array}$ & $\begin{array}{c}\text { Moderate }^{b} \\
+\end{array}$ & $\begin{array}{c}\text { None } \\
+\end{array}$ & $\begin{array}{c}\text { None } \\
+\end{array}$ \\
\hline & $\begin{array}{l}\text { Short term } \\
\text { (1 to <6 months) }\end{array}$ & $\begin{array}{c}\text { Moderate } \\
+\end{array}$ & $\begin{array}{c}\text { Small } \\
++\end{array}$ & $\begin{array}{c}\text { Small } \\
+\end{array}$ & No evidence & No evidence \\
\hline & $\begin{array}{l}\text { Intermediate term } \\
(\geq 6 \text { to }<12 \text { months) }\end{array}$ & $\begin{array}{c}\text { None } \\
+ \\
\end{array}$ & $\begin{array}{c}\text { None } \\
++\end{array}$ & $\begin{array}{c}\text { Small } \\
++\end{array}$ & $\begin{array}{c}\text { None } \\
+ \\
\end{array}$ & $\begin{array}{c}\text { None } \\
+ \\
\end{array}$ \\
\hline & $\begin{array}{l}\text { Long term } \\
\text { ( } \geq 12 \text { months) }\end{array}$ & $\begin{array}{c}\text { None } \\
+\end{array}$ & $\begin{array}{c}\text { None } \\
++\end{array}$ & $\begin{array}{c}\text { Small } \\
+\end{array}$ & $\begin{array}{c}\text { None } \\
+\end{array}$ & $\begin{array}{c}\text { None } \\
+\end{array}$ \\
\hline
\end{tabular}




\begin{tabular}{|c|c|c|c|c|c|c|}
\hline Outcome & Time Point & $\begin{array}{l}\text { CPMPs } \\
\text { Versus } \\
\text { UC or WL } \\
\end{array}$ & $\begin{array}{l}\text { CPMPs } \\
\text { Versus } \\
\text { Physical } \\
\text { Activity }\end{array}$ & $\begin{array}{c}\text { CPMPs Versus } \\
\text { Pharmacologic } \\
\text { Therapy }\end{array}$ & $\begin{array}{c}\text { CPMPs Versus } \\
\text { Pharmacologic } \\
\text { Therapy and } \\
\text { Passive PT }\end{array}$ & $\begin{array}{c}\text { CPMPs } \\
\text { Versus } \\
\text { Psychological } \\
\text { Therapy } \\
\end{array}$ \\
\hline \multirow{4}{*}{$\begin{array}{l}\text { Opioid } \\
\text { Use } \\
\text { (Effect } \\
\text { Size/SOE) }^{a}\end{array}$} & Postintervention & $\begin{array}{l}\text { Insufficient } \\
\text { evidence }\end{array}$ & $\begin{array}{c}\text { No } \\
\text { evidence }\end{array}$ & No evidence & No evidence & No evidence \\
\hline & $\begin{array}{l}\text { Short term } \\
\text { (1 to }<6 \text { months) }\end{array}$ & $\begin{array}{c}\text { No } \\
\text { evidence }\end{array}$ & $\begin{array}{c}\text { No } \\
\text { evidence }\end{array}$ & No evidence & No evidence & No evidence \\
\hline & $\begin{array}{l}\text { Intermediate term } \\
\text { ( } \geq 6 \text { to }<12 \text { months) }\end{array}$ & $\begin{array}{c}\text { No } \\
\text { evidence }\end{array}$ & $\begin{array}{c}\text { No } \\
\text { evidence }\end{array}$ & No evidence & No evidence & No evidence \\
\hline & $\begin{array}{l}\text { Long term } \\
\text { ( } \geq 12 \text { months) }\end{array}$ & $\begin{array}{l}\text { Insufficient } \\
\text { evidence }\end{array}$ & $\begin{array}{c}\text { No } \\
\text { evidence }\end{array}$ & No evidence & $\begin{array}{l}\text { Insufficient } \\
\text { evidence }\end{array}$ & No evidence \\
\hline
\end{tabular}

$\mathrm{CPMP}=$ comprehensive pain management program; $\mathrm{PT}=$ physical therapy; $\mathrm{SOE}=$ strength of evidence; $\mathrm{UC}=$ usual care; $\mathrm{WL}=$ waitlist.

a Effect size: None, small, moderate, or large difference favoring CPMP; SOE: $+=$ low,$++=$ moderate, $+++=$ high

${ }^{b}$ Based on 1 fair-quality trial in which patients got antidepressants and sedatives in conjunction with basic analgesics.

${ }^{c}$ Based on 1 fair-quality trial in which patients got antidepressants only.

Table 4. Overview of reported treatment-related adverse events/harms from included trials

\begin{tabular}{|c|c|}
\hline Intervention & Reported Adverse Events \\
\hline IPMP vs. usual care & $\begin{array}{l}\text { No intervention-specific adverse events were seen in two OA trials. } \\
\text { Harms reported in a third trial in CWP were not attributed to the } \\
\text { intervention. }\end{array}$ \\
\hline $\begin{array}{l}\text { IPMP vs. physical activity and vs. } \\
\text { telephone-CBT }\end{array}$ & $\begin{array}{l}\text { No intervention-related harms were seen in one trial in CWP. One patient in } \\
\text { the exercise group died of cancer. }\end{array}$ \\
\hline CPMP vs. UC or $\mathrm{WL}^{\mathrm{a}}$ & $\begin{array}{l}\text { In one trial, three patients in the CPMP group }(5.5 \% ; 3 / 55) \text { reported } \\
\text { increased low back or leg pain leading to withdrawal from the trial. } \\
\text { A second trial reported occasional mild increases in pain after some } \\
\text { exercise sessions in the CPMP groups. } \\
\text { There was no mention of adverse events in the UC/WL groups }\end{array}$ \\
\hline CPMP vs. physical activitya & $\begin{array}{l}\text { In one trial of chronic LBP (with and without radiating leg pain), three } \\
\text { patients randomized to CPMP (5.5\%; } 3 / 55) \text { and three to exercise only } \\
\text { ( } 5.8 \% ; 3 / 52 \text { ) reported increased low back or leg pain leading to withdrawal } \\
\text { from the trial, one of which had a herniated disc and required surgery } \\
\text { (exercise group). In addition, two patients }(3.8 \%) \text { in the exercise group } \\
\text { stopped activities (aerobic exercise or cycling) due to pain. } \\
\text { A second, small trial in mixed CP reported pain in new localizations in two } \\
\text { vs. five patients randomized to CPMP and exercise alone, respectively, } \\
\text { ( } 11.8 \% \text { [2/17] vs. } 31.2 \% \text { [5/16]; RR } 0.38,95 \% \mathrm{Cl} 0.08 \text { to } 1.7) \text {. } \\
\text { One trial reported no intervention-related adverse events and two trials } \\
\text { reported events in the CPMP groups that were likely unrelated to the } \\
\text { intervention, but limited information was provided (one patient died during } \\
\text { inpatient treatment in one trial, and one patient had a right tibial fracture } \\
\text { that occurred at home in the other). All three trials were in CLBP. }\end{array}$ \\
\hline $\begin{array}{l}\text { CPMP vs. pharmacologic therapy } \\
\text { with or without physical activity }\end{array}$ & No evidence \\
\hline CPMP vs. psychological therapy ${ }^{a}$ & $\begin{array}{l}\text { In one trial, three patients in the CPMP group }(5.5 \% ; 3 / 55) \text { reported } \\
\text { increased low back or leg pain leading to withdrawal from the trial; no } \\
\text { adverse events were reported in the psychological therapy group. }\end{array}$ \\
\hline $\begin{array}{l}\text { IPMP provider and patient } \\
\text { intervention vs. } \\
\text { provider intervention only and vs. } \\
\text { patient intervention only }\end{array}$ & No intervention-specific adverse events were seen in one OA trial. \\
\hline $\begin{array}{l}\text { IPMP delivery with Web support vs. } \\
\text { without Web support }\end{array}$ & No evidence \\
\hline
\end{tabular}




\begin{tabular}{|l|l|}
\hline Intervention & Reported Adverse Events \\
\hline $\begin{array}{l}\text { CPMPs, greater vs. fewer total } \\
\text { hours }\end{array}$ & $\begin{array}{l}\text { No trial-related adverse events reported in in one trial in CMSK pain. } \\
\text { A second trial in FM reported occasional mild increases in pain after } \\
\text { exercise sessions in both groups (data NR). }\end{array}$ \\
\hline $\begin{array}{l}\text { CPMPs, inpatient vs. outpatient } \\
\text { setting }\end{array}$ & $\begin{array}{l}\text { No specific adverse events related to the interventions were reported in } \\
\text { one CLBP trial. }\end{array}$ \\
\hline CPMPs, program components & No evidence \\
\hline CPMPs, pretreatment assessments & No evidence \\
\hline $\begin{array}{l}\text { CPMPs with vs. without booster } \\
\text { sessions }\end{array}$ & No evidence \\
\hline CPMPs, other comparisons & $\begin{array}{l}\text { One small trial in CLBP (with leg pain) reported that one patient (2\%) } \\
\text { randomized to graded activity CPMP deteriorated during treatment (i.e., } \\
\text { treatment counterproductive). No other intervention-related adverse events } \\
\text { were reported. }\end{array}$ \\
\hline
\end{tabular}

$\mathrm{CBT}=$ cognitive behavioral therapy; $\mathrm{CI}=$ confidence interval; $\mathrm{CLBP}=$ chronic low back pain; $\mathrm{CMSK}=$ chronic musculoskeletal pain; $\mathrm{CP}=$ chronic pain; $\mathrm{CPMP}=$ comprehensive pain management program; $\mathrm{CWP}=$ chronic widespread pain; $\mathrm{FM}=$ fibromyalgia; $\mathrm{LBP}=$ low back pain; $\mathrm{NR}=$ not reported; $\mathrm{OA}=$ osteoarthritis; $\mathrm{RCT}=$ randomized controlled trial; $\mathrm{RR}=$ risk ratio; $\mathrm{UC}=$ usual care; $\mathrm{WL}=$ waitlist.

a The trial by Smeets et al. 2006/2008 that reported increased low back or leg pain leading to withdrawal compared CPMP with three different comparator groups: versus usual care, versus physical activity alone and versus psychological therapy alone; thus the data on harms for CPMP for each of the these comparisons is from the same trial and does not constitute unique incidences.

\section{Evidence on Factors Related to Care Delivery or IPMP/CPMP Components}

Evidence on factors related to care delivery or components of IPMPs or CPMPs that may impact outcomes is sparse. For the comparison of CPMP with usual care for Key Question 1, effect sizes for pain and function and their variability were similar whether programs were delivered individually, as group sessions, or a combination of these. Regarding the impact of program intensity on outcomes, for the comparison of CPMP versus usual care for Key Question 1, again effect sizes for pain and function and their variability were generally similar for lower intensity ( $<20$ hours/week or $\leq 80$ hours total) and higher intensity programs suggesting that higher program intensity may not result in better outcomes. There was no evidence that greater or fewer hours improved pain or function at intermediate term across two head-to-head trials for Key Question 2. Our findings suggest that high intensity programs may not be necessary to realize benefits from CPMPs. One small fair-quality trial comparing high intensity CPMP (total 135, 39 hours/week for 3 weeks) versus lower intensity ( 24 hours total) reported moderate improvement in function and pain favoring the high intensity program at short and long term. The high intensity CPMP required a high level of physical activity and included work-related therapy/simulations. This, combined with mean patient age of 42 years, may make these findings less applicable to the Medicare population.

Although there was low SOE from a single head-to-head trial comparing aspects of program delivery or approach, the unique nature of the comparisons, and heterogeneity of the trials likely limits their applicability. For IPMP, the combination of multidisciplinary recommendations to primary care providers and a phone-based multidisciplinary patient intervention did not improve pain or functions compared with either component alone in one trial. IPMP with additional Webbased support did not improve pain or function when compared with IPMP alone in another trial. Trials of CPMP comparing variations in the delivery of treatment components (psychological or physical) reported no differences in pain or function. One trial employing pre-treatment functional assessment to inform treatment decisions found an association between the assessment and small functional improvement in the long term. Small improvements in pain and function 
were seen when a function-centered approach versus a pain-centered approach to treatment was used.

\section{Comparison With Other Systematic Reviews}

Comparison with other reviews is challenging given the substantial heterogeneity in the terminology used in the literature and in clinical practice to describe and categorize multidisciplinary pain management programs that address the biopsychosocial pain model as well as in the components offered, and their delivery. ${ }^{21,121,123} \mathrm{We}$ have encountered this heterogeneity throughout our prior pain reports. ${ }^{36,38}$ Our current review differs from previous systematic reviews of pain management programs by defining and making a distinction between programs integrated with primary care (IPMP) and those that are based in other settings such as dedicated pain or specialty clinics (e.g., rheumatology) or rehabilitation settings which are not centered in primary care but are based on referral from primary care and other sources (CPMP). Also, in contrast to other reviews, studies included in this review needed to have, at a minimum, the availability of appropriate medication and/or a medication management component as well as psychological care (pain psychology or mental health support), and physical rehabilitative methods such as physical therapy or occupational therapy based on patient needs consistent with addressing primary aspects of the biopsychosocial pain model. Programs could contain additional components such as patient self-management education, medical procedures, or complementary and integrative care modalities. We also needed to be able to infer some mechanism of care coordination or communication between multidisciplinary providers and medical management. Finally, our review attempted to focus on the persistence of effects while others may focus on postintervention or final followup. Thus, our findings may differ from other reviews.

A rapid review of chronic musculoskeletal pain management was the only review identified that focused on delivery of multimodal chronic pain management in a primary care setting. ${ }^{21}$ Common model components included multidisciplinary case management, pharmacotherapy review algorithms, mental health services, proactive symptom monitoring, and patient selfmanagement resources which were provided as needed to patients. The review focused on decision support mechanisms and found that models incorporating various decision support methods (e.g., increased provider interaction, pain specialist peer support, case management meetings, others) with proactive treatment monitoring resulted in patients experiencing clinically relevant improvement $(\geq 30 \%)$ in pain and function. Our findings are generally consistent with and complement these findings by including a broader range of RCTs on IPMPs but less stringent emphasis on decision making support; we found small improvements in pain and function for IPMP versus usual care at various time frames.

Findings in this review are consistent with our prior review of nonpharmacologic treatments for specific chronic pain conditions ${ }^{36}$ (which focused on persistence of effects postintervention) and our prior review of LBP. ${ }^{38}$ CPMPs in this current review were associated with small improvements in pain and function in the short and intermediate term compared with usual care in patients with chronic LBP. They were also associated with small improvement in function in the short, intermediate, and long term, and pain in the intermediate term in patients with FM, compared with usual care. The majority of patient populations in this current review had chronic musculoskeletal pain, chronic LBP or FM. Consistent with our review, a 2014 Cochrane review $^{129}$ in patients with chronic LBP reports benefit with CPMPs versus usual care and that evidence was insufficient to assess adverse events. They reported magnitudes of effects across 
time points that were somewhat higher for pain ( 0.5 to 1.4 versus our range for pooled analyses of 0.13 to 0.53 on a 0 to 10 scale). Their pooled SMDs for function in the short, intermediate, and long term $(-0.41,-0.43$, and -0.23$)$ also differed from ours at somewhat similar time frames $(-0.62,-0.11$, and -0.29$)$. Differences may be a function of our inclusion of conditions in addition to chronic LBP (i.e., FM, chronic musculoskeletal pain) and/or different criteria for components CPMP (e.g., we required that programs include a psychological and physical components) leading to differences in included studies. Differences in functional outcome measures used may also partially contribute to differences in SMDs for function between our reviews. Our use of more conservative profile-likelihood methods (versus Der Simonian and Laird methods) for meta-analysis could also contribute to some differences in effect sizes and statistical significance particularly when pooling fewer studies with small sample sizes. A more recent meta-analysis of intensive outpatient interdisciplinary programs in adults with diverse chronic pain conditions reported a pooled SMD of 0.67 for pain corresponding to moderate improvement. ${ }^{130}$ Differences in program definition, components required, and included study designs (one RCT and 12 nonrandomized studies) likely explain differences in our findings. The observation of smaller effect sizes in our review versus others may also partly reflect changes in programs, their components and delivery since early publications, and acceptance of pain management programs ${ }^{120,121}$ as well as continued refinement of methods for primary research and systematic reviews. Many early studies and prior reviews of CPMPs focused on job-related functions, contained specific occupational components and return to work as a primary outcome, and may be less applicable to a Medicare population.

\section{Strengths and Limitations}

Our review has some notable strengths. As noted previously, there is substantial heterogeneity across studies with regard to the terminology used to describe programs, various components that may be used, and their delivery. To minimize heterogeneity, we a priori established internal operational definitions for IPMP and CPMP based on care setting and coordination and focused on the available primary components of pain management that would most generally address the biopsychosocial needs of patients. TEP discussions generally reaffirmed our approach, categorization of programs, and perspective on the important primary components of a formal pain management program. Our review appears to be the most complete summary of RCTs describing IPMPs. Interpretation of clinically important differences in mean change for continuous variables is challenging. Another strength of our review is our categorization of the magnitude of effects for function and pain outcomes using the system described in our previous reviews ${ }^{36,38,39,131}$ to facilitate interpretation of results across trials and interventions by providing a level of consistency and objective benchmarks for comparison. We classified effects below the threshold for small as no effect. Based on this system, beneficial effects identified were usually considered small to moderate (Appendix J). These findings are consistent with what is seen for other therapies for pain, including opioids for chronic pain, nonpharmacologic treatments, surgery, and others. We acknowledge that effects that we classify as small (e.g., 5 to 10 points on a 0 to 100 scale) may be below some proposed thresholds for minimum clinically important differences for some measures, however values for minimum clinically important difference vary based on populations and methods used to determine them. They represent "average" effects, and some patients will experience larger effects and patients will differ in how they value small effects. Evaluating the proportion of patients who 
experienced a clinically important improvement in pain or function may provide better insight into patient treatment response. This was also reported when such data were provided.

Our review has some limitations. We did not conduct analyses to evaluate potential markers for publication bias given the substantial heterogeneity in study designs, programs, length of followup, patient populations, and small number of trials available for most analyses. Our searches of study bibliographies and clinical trial registries and evaluation of comments received from public solicitation, peer review or public comment did not identify unpublished studies meeting our inclusion criteria that would suggest publication bias. While we excluded non-English language publications, it is less likely that such publications would describe programs applicable to the U.S. Medicare population.

While our inclusion/exclusion criteria may be considered narrow from some perspectives based on our requirement for specific components, our operationalization of this was fairly liberal. For example, in recognition that not all patients may need all components, studies were included if the availability of the primary components delivered by a multidisciplinary team was described or it could be reasonably inferred based on evaluation of protocols and supplementary information. In general, we inferred that some level of communication and coordination was likely in such programs. In some studies, if a physician, other primary care provider (e.g., nurse practitioner, pharmacist, or similar provider) was part of the team, we inferred that patients had access to appropriate medications based on that professional's review. Descriptions of program components to which patients had access and the disciplines involved in delivering them was suboptimal in a number of studies, particularly those of IPMPs. ${ }^{41,42}$ A minimum of three individuals evaluated each study/program, including review of published protocols, for inclusion in an effort to be as consistent as possible. We erred on the side of inclusion. In addition, there was no restriction on setting for CPMPs and included studies were conducted in a variety of specialty clinics (e.g., rheumatology). We recognize that others may have considered a different categorization of programs and specification of components. Various pain management programs models may be in use but may not be represented in peer-reviewed publications.

We did not include nonrandomized studies; such studies in pain can be misleading due to the subjective nature of pain and the impact of nonspecific effects related to patient expectations regarding treatment and attention received on patient reported outcomes and the potential for selection bias and uncontrolled confounding. There are numerous examples in the pain literature where nonrandomized studies have shown very large responses or estimates for effectiveness in response to a treatment which were disproven in subsequent RCTs. ${ }^{132,133}$

Our review focused on the overall impact of the included pain management programs based on our specified components. It was not possible within our scope to evaluate a broader range of pain management programs that may be offered with different sets of primary components. Clinically, there are efforts to include a broader array of nonpharmacologic treatments and medications to effectively manage pain. While some included studies briefly described access to additional components (e.g., injections, various medical procedures, chiropractic, massage, acupuncture, and others), details of the impact of such components were not described within the context of the full program. There was insufficient evidence to evaluate such components; however, our recent review on nonpharmacologic, noninvasive treatment did find that for specific chronic pain conditions, many nonpharmacologic treatments did improve pain and/or function. ${ }^{36}$ Similarly, evaluation of specific pharmacologic treatments (e.g., use of topical agents) within such programs was not within our scope. Recent reports provide information on the use of pharmacologic agents for pain. ${ }^{39,131}$ No trials of virtually delivered (e.g., telehealth) programs 
meeting our inclusion criteria were identified. We did not include formal integrative pain management programs unless they met the criteria for IPMPs or CPMPs for this review. There was heterogeneity across studies regarding many aspects of program delivery, however there were insufficient data to explore this. Detail regarding program intensity was often vague and usually described in terms of time spent in the program or on various components. While results from our analyses suggest no difference in pain or functional outcomes for higher versus lower intensity programs based on time spent, findings are based on indirect (across-trial) comparisons and should be considered as hypothesis generating; further research from head-to-head trials would be important.

Limitations in the evidence base are reflected in the limitations to the review. In addition to the heterogeneity mentioned previously, evidence from methodologically rigorous comparative studies on primary care-based pain management programs is currently sparse, particularly evaluating outcomes in the long term. Research and evidence on primary care-based programs is still emerging. Much of the evidence for formal pain management programs is from older trials of CPMPs that focused on specific occupational functions (e.g., manual labor tasks) and return to work outcomes and are from health systems outside of the United States These may be less applicable to the Medicare population in particular. Similarly, studies comparing methods of program delivery and other factors were sparse and were generally of poor quality. Coordination and communication within programs were rarely described precluding evaluation of management models and their impact.

Evidence on outcomes other than pain and function was limited, especially for harms; evidence for the impact of programs on medication use, particularly opioids, was also limited. Adverse events and harms were poorly reported in included RCTs. Some RCTs may not be adequately powered to detect rare outcomes or have sufficient length of followup to characterize long term harms. Intervention-related serious adverse events are likely rare in formal pain management programs and likely depend on patient factors (e.g., comorbid conditions) or are related to delivery of specific program components (e.g., medical procedures). While the Visual Analog Scale for pain was the most commonly reported pain measure, it does not adequately characterize or categorize pain and does not capture individuals' response or achievement of a clinically important difference. The majority of trials compared programs to usual care, which was poorly described in most studies. It is possible that a variety of therapies and medications provided as part of usual care or continued in the intervention may have led to an attenuation of the observed effects. Most studies (75\%) were considered fair, primarily due to the inability to effectively blind care givers and participants. While these studies were well done, lack of blinding leaves open the opportunity for reporting bias and the influence of a placebo effect for subjective measures such as pain and function. Consistent with our previous reports, studies were downgraded for lack of participant blinding. Adherence to programs was poorly reported across trials, making its impact difficult to assess. Time constraints, and the need to travel and attend to other obligations were frequently cited as reasons for drop out or lack of adherence. Lack of adherence may attenuate the effect of programs versus usual care. Studies rarely detailed psychological comorbidities (including suicidal behaviors) or medical comorbidities in enrolled populations and many excluded patients with such comorbidities. Similarly, specifics of pain diagnoses, pain characteristics (e.g., nociplasticity) and other patient characteristics were not generally reported in studies, precluding evaluation of their impact on treatment response. Information on race and ethnicity was rarely reported and under-served populations were not identified in included trials. 


\section{Applicability}

The applicability of our findings may be impacted by a number of factors. First, none of the trials specifically recruited adults eligible for Medicare based on age or those under 65 years old who qualify for Medicare due to disability. Three of the eight IPMP trials enrolled older Veterans Affairs (VA) patients (mean ages 61 to 63 years), ${ }^{41,42,45-47}$ and the mean age across IPMP trials was 57 years. In contrast, one CPMP trial enrolled older VA patients (mean age 69 years $)^{106}$ but the mean age across CPMP trials was 45 years and programs were generally geared to working adults. Based on age and work status, results from the IPMP trials may be most applicable to the Medicare population. Disability status was poorly defined; descriptions varied across trials with some basing the determination on sick leave from work, receiving worker's compensation or disability income while some stated that patients were "disabled" or "working incapable" without further description. Based on the descriptions provided, 8 to 65 percent of patients enrolled in three IPMP trials and 6 to 100 percent of patients enrolled in 19 CPMP trials were classified as disabled. It is unclear to what extent these descriptors may coincide with Medicare-defined disability populations. Data were inadequate to evaluate the impact of programs based on disability status or responses of Medicare beneficiaries to various components, particularly medications. Although direct applicability of included trials to Medicare beneficiaries is unclear, several factors should be considered. Many of the IPMP programs in particular focused on patient-tailored care and were generally low intensity. To the extent that IPMPs or CPMPs are tailored to an individual patient's needs for pain management, maintaining function and psychosocial support, our findings are potentially applicable to the Medicare population. Although many of the work-related CPMPs focused on occupational function and work-related issues, components of these programs may generally address needs of a growing number of older adults that continue to be active in the work force and support maintenance of daily activities in older adults in general or those with disability.

The majority of trial patients had moderate chronic pain ( $\sim 5.5$ on $0-10$ scale). Trials of both IPMP and CPMP primarily enrolled patients with chronic LBP (30\% and 52\% respectively), OA (34\% and 7\%), and FM (18\% and 16\%); patients with mixed or multiple pain conditions comprised about 20 percent of enrolled populations. The applicability of findings from included trials to other pain conditions, complex subacute pain, multiple pain diagnoses, or more severe pain is unclear. One CPMP trial ${ }^{62}$ enrolled patients with acute $(<4$ weeks) pain following trauma; sensitivity analyses excluding this trial did not alter effect size or conclusions. Generally, patients with acute or subacute pain are not referred for formal pain management programs and care approaches and goals differ from those used to manage chronic pain. Important patient subgroups seen in clinical practice, such as those with nociplastic pain, psychological comorbidities (including suicidal behaviors), substance use disorder, or specific disabilities (e.g., end stage renal disease) were poorly described or not reported. Trials were not designed to evaluate how these subgroups or patient demographic factors might impact treatment effects and harms. Most CPMP trials were conducted in Europe; differences with the U.S. healthcare system and social structure may impact applicability.

The substantial heterogeneity in programs, their components, and their delivery observed in included trials may reflect some of the diversity in how programs are delivered clinically. While we abstracted information on types of components, frequency, duration, and types of sessions and how they were delivered (e.g., individually or in groups) and other factors, there was insufficient information to evaluate their contribution. Few head-to-head trials evaluated such 
factors. Many were of poor quality; again, there was substantial heterogeneity across programs and factors compared in trials.

\section{Implications for Clinical Practice, Education, Research, or Health Policy}

\section{Considerations for Clinical Practice and Health Policy}

Our review suggests that IPMPs and CPMPs as defined for this review may provide small, sometimes moderate improvements particularly in function in patients with chronic pain compared with usual care. Further, our findings suggest that CPMPs in particular may also be more effective than medications alone or in combination with physical activity. The magnitude of improvement we see is consistent with other treatments for chronic pain such as surgery (e.g., discectomy, vertebroplasty), steroid injections, and medications such as opioids and there is no evidence of serious or important harms. Medicare eligible patients and beneficiaries are a diverse population. Many older adults ( $>65$ years old) may be active, employed, and in good health but may require assistance with pain management; others may be disabled or have substantial comorbid conditions and require ongoing support for pain management. Programs that address a range of biopsychosocial aspects of pain and coordination of care may be of particular importance in this population.

Across the general models as operationalized for this review, there is substantial variation in how programs and their components are delivered, thus, specification of common models or mechanisms is elusive. The models described in this review likely do not fully capture the diversity of programs potentially available in clinical practice. In recent years, government reports such as The National Academy of Sciences workshop on Non-Pharmacological Approaches to Pain Management, ${ }^{13}$ the recent Pain Management Best Practices Inter-Agency Task Force report, ${ }^{14}$ the National Pain Strategy (NPS) report, ${ }^{15}$ guidelines from the American College of Physicians, ${ }^{134}$ the Centers for Disease Control and Prevention (CDC) ${ }^{135}$ and Canadian Guideline for Opioid Use in Chronic Non-Cancer Pain ${ }^{136}$ have recommended integration of nonpharmacologic pain management approaches to include interventions such as exercise, CBT, multidisciplinary rehabilitation mind-body interventions, and some complementary and integrative medicine therapies, such as acupuncture and spinal manipulation, to address patient behavioral and medical needs based on the biopsychosocial concept of care. Implementation of such recommendations has started in a range of programs, adding to the diversity of models, their components, and methods of delivering them that would be difficult to capture in a single systematic review.

The current paradigm for pain management (usual care) is the provision of selected individual treatments (e.g., medications) or services (e.g., physical therapy, psychological support) prescribed or recommended by a patient's provider (primary care or specialty provider), often with little or no coordination between multidisciplinary providers or active monitoring of patient progress. This is true for both general and Medicare populations. Treatment may be variable, unimodal, or confined to a limited range of options (e.g., medication and physical therapy only). For some patients, this model of care may be sufficient. For patients with acute or subacute pain it may be sufficient to improve their pain, function, and quality of life since these types of pain are generally time-limited and are likely managed with less treatment. However, for some patients, while the selected treatments may be individualized, patients may not be offered a broader range of therapies that address the full range of biopsychosocial concerns. 
Theoretically, coordination of care based on a patient's particular circumstances may lead to optimal management of pain by optimizing the use of appropriate medications and medical procedures, facilitating physical function, and providing psychological support to enhance patient self-management of their pain. This approach may facilitate identification and best use of diverse resources relevant to patient goals for pain management, including improving quality of life and return to important life activities. This may be particularly important in patients with medical or psychological comorbidities and the Medicare population.

Chronic pain management may be complex, particularly for Medicare beneficiaries. Patients are generally not treatment naïve. Formal programs may offer advantages over usual care. Anecdotally, in clinical practice, when patients attend such programs within the United States, care is likely tailored to their pain diagnosis and related physical, medical, and psychosocial needs. Although patients may have access to the primary components we identified and there may be some common features that all patients receive, the components recommended, and care plan for a patient with chronic LBP will likely differ from those recommended for a patient with FM or OA. There is a likely a level of coordination and communication across providers on a care plan, followup on patient progress, and support for understanding treatment options and enhancing treatment adherence. Optimization of medications is an important part of pain management. Compared with usual care, formal pain management programs may offer additional support for this in addition to tailoring management to patient needs. Clinically, IPMPs and CPMPs have more recently engaged in evaluation of medication response, weaning of patients from medications that may no longer be effective, and using alternative medications (e.g., buprenorphine) as patients participate in other supportive program therapies/components (e.g., CBT, physical function restoration) that address multiple biopsychosocial aspects. Unfortunately, there is sparse evidence from included trials regarding mechanisms for optimizing medications or the impact of programs on opioid use.

Neither IPMPs or CPMPs have been widely implemented in the United States for a variety of reasons including the costs, logistics, leadership support, staffing, and provider training that are involved in the development and implementation of such programs ${ }^{14,16,18,124}$ as well as the current fee-for-service reimbursement structure. ${ }^{18}$ In addition, programs may not be accessible to many populations based on locations, the availability of pain specialists, and socio-economic factors. Nonpharmacologic or complimentary and integrative health practices may not commonly be considered or recommended ${ }^{13,14,18,137}$ by providers or adopted by patients for a variety of reasons including lack of awareness about effective options and lack of reimbursement. None of the included trials directly addressed the impact of or optimal approaches for education of providers or patients which is necessary for successful implementation of pain management programs.

In theory, programs that align care with patient needs could improve the quality of care and patient outcomes in patients with complex healthcare needs and help reduce per-capita costs, ${ }^{138}$ but little is known about the cost-effectiveness of pain management programs. Based on our search for Contextual Question 2, information on cost-effectiveness for either IPMPs or the CPMPs conducted in the United States in the peer-reviewed literature is sparse (Appendix C). The substantial variations across programs and how components are delivered leads to concerns regarding the applicability of costs or cost-effectiveness across either program type. The most applicable economic assessment to this review, based on a cluster randomized controlled trial of a system-based IPMP, was done from the VA healthcare perspective. ${ }^{45}$ The trial randomized primary care providers to receive collaborative, multidisciplinary assistance with pain treatment 
(APT) of patients with musculoskeletal pain diagnoses experiencing moderate or greater pain intensity or disability lasting 12 weeks or longer using a stepped-care model or usual care for 12 months. The average increase of $\$ 2300$ per patient for the APT intervention falls on the low end of costs for commonly used chronic pain interventions, however the applicability of these findings to other IPMPs, particularly those that are practiced-based is unclear. A systematic review of cost-effectiveness of complex pain management programs for chronic LBP found that full economic studies appear to be sparse and of questionable overall quality. ${ }^{139}$ Authors cite the variability of settings, interventions, comparators and outcomes as factors contributing to the difficulty of assessing cost-effectiveness.

\section{Research Recommendations}

Gaps in the existing evidence for formal pain management programs, particularly those based in primary care (i.e., IPMPs), are many. With regard to populations, future research is needed to understand how formal programs may impact patients with a broader range of pain conditions (e.g., neuropathic pain, nociplastic pain), individuals with complex subacute pain who may be at risk for development of chronic pain, older adults, and Medicare beneficiaries. Factors such as program accessibility, acceptability, intensity, and participant cost need further examination as does the relationship of such factors to program adherence and outcomes. Research on pain management for under-served populations and equity in program delivery is also needed. In addition, trials with sufficient sample size designed to evaluate differential effectiveness and safety of treatments in subpopulations of interest are needed to understand how to best tailor programs. Given the substantial heterogeneity in the terminology used to describe pain management programs, efforts to standardize terminology are needed. Similarly, additional research into the structure, coordination, and implementation of programs within practices and within systems is needed to understand what may optimize delivery of care as well as components and factors that affect adherence and improve outcomes. Research leading to some level of standardization of programs and their delivery may facilitate general understanding of the best combinations of interventions. Well-designed pragmatic trials may provide valuable information. Trials comparing programs with pharmacologic treatments are needed. With regard to outcomes, standardized protocols for types of outcomes to be assessed (including harms) would facilitate evaluation and comparison across studies. In addition, future studies should be encouraged to incorporate measures that reflect understanding of pathophysiological mechanisms and that address multiple domains of pain. Mean changes in outcomes (e.g. visual analog scale) between groups describe how groups respond on average to treatment and small average effects may be associated with larger effects in some patients. Reporting the proportions of patients achieving a clinically meaningful improvement in pain, function, or quality of life as measures of "success" may provide important additional clinical information and be more clinically intuitive. Reporting of the proportions of patients achieving a clinically meaningful improvement for measures of pain and function (i.e., responders) as well as outcomes related to change in use of opioids, healthcare utilization, and quality of life are needed in future studies. Evaluation of the cost-effectiveness of formal pain management programs presents a number of challenges due to the heterogeneity of them but may facilitate a fuller understanding of the balance of benefit and cost. 


\section{Conclusions}

Both IPMPs and CPMPs may provide small, sometimes moderate improvements in function and small improvements in pain for patients with chronic pain compared with usual care at multiple time frames. Harms were poorly reported but were generally minor. Our findings suggested that higher-intensity programs and lower-intensity programs may confer similar benefit, however verification of these findings is needed. While few trials specifically enrolled Medicare beneficiaries, to the extent that less intense programs are tailored to patient's needs, our findings are potentially applicable to the Medicare population. Although use of selected individual treatments may serve some patients, a broader range of therapies that address the full scope of biopsychosocial concerns available in formal programs may benefit others. Research in the Medicare population and in patients with a broader range of pain conditions is needed as is research on the impact of program structures, coordination methods, and components on patient outcomes. Additional evidence from primary care-based programs is particularly needed. 


\section{References}

1. Institute of Medicine of the National Academies. Relieving Pain in America: A Blueprint for Transforming Prevention, Care, Education, and Research. Washington DC: The National Academies Press; 2011. http://www.ncbi.nlm.nih.gov/pubmed/22553 896doi: 10.17226/13172.

2. Dieleman JL, Cao J, Chapin A, et al. US Health Care Spending by Payer and Health Condition, 1996-2016. Jama. 2020 Mar 3;323(9):863-84. doi: 10.1001/jama.2020.0734. PMID: 32125402.

3. de Souza IMB, Sakaguchi TF, Yuan SLK, et al. Prevalence of low back pain in the elderly population: a systematic review. Clinics (Sao Paulo). 2019;74:e789. doi: 10.6061/clinics/2019/e789. PMID: 31664424.

4. Dahlhamer J, Lucas J, Zelaya C, et al. Prevalence of Chronic Pain and HighImpact Chronic Pain Among Adults - United States, 2016. MMWR Morb Mortal Wkly Rep. 2018 Sep 14;67(36):1001-6. doi: 10.15585/mmwr.mm6736a2. PMID: 30212442 .

5. Domenichiello AF, Ramsden CE. The silent epidemic of chronic pain in older adults. Prog Neuropsychopharmacol Biol Psychiatry. 2019 Jul 13;93:284-90. doi: 10.1016/j.pnpbp.2019.04.006. PMID: 31004724 .

6. Weiss AJ, Heslin KC, Barrett ML, et al. Opioid-Related Inpatient Stays and Emergency Eepartment Visits Among Patients Aged 65 Years and Older, 2010 and 2015: Statistical Brief \# 244. In: Healthcare Cost and Utilization Project (HCUP) Statistical Briefs. Rockville, MD: Agency for Healthcare Research and Quality; 2018. PMID: 30475561.

7. Huhn AS, Strain EC, Tompkins DA, et al. A hidden aspect of the U.S. opioid crisis: Rise in first-time treatment admissions for older adults with opioid use disorder. Drug and alcohol dependence. 2018;193:142-7. doi: 10.1016/j.drugalcdep.2018.10.002. PMID: 30384321 .
8. Kuo Y-F, Raji MA, Goodwin JS.

Association of disability with mortality from opioid overdose among US Medicare adults. JAMA network open. 2019;2(11):e1915638e.

9. Gatchel RJ. Comorbidity of chronic pain and mental health disorders: the biopsychosocial perspective. Am Psychol. 2004 Nov;59(8):795-805. doi: 10.1037/0003-066X.59.8.795. PMID: 15554853.

10. Gatchel RJ, Peng YB, Peters ML, et al. The biopsychosocial approach to chronic pain: scientific advances and future directions. Psychol Bull. 2007 Jul;133(4):581-624. doi: 10.1037/0033-2909.133.4.581. PMID: 17592957.

11. Gatchel RJ, Turk DC. Criticisms of the biopsychosocial model in spine care: creating and then attacking a straw person. Spine (Phila Pa 1976). 2008 Dec 1;33(25):2831-6. doi: 10.1097/BRS.0b013e31817d24ad. PMID: 19050589.

12. Miaskowski C, Blyth F, Nicosia F, et al. A Biopsychosocial Model of Chronic Pain for Older Adults. Pain Med. 2020 Sep 1;21(9):1793-805. doi: 10.1093/pm/pnz329. PMID: 31846035.

13. National Academies of Sciences, Engineering, and Medicine; Health and Medicine Division; Board on Global Health; Board on Health Sciences Policy; Global Forum on Innovation in Health Professional Education; Forum on Neuroscience and Nervous System Disorders. Clare Stroud, Sheena M. Posey Norris, Lisa Bain, editors. The Role of Nonpharmacological Approaches to Pain Management: Proceedings of a Workshop. Washington, DC: Press NA; 2019. doi: 10.17226/25406. PMID: 31145569.

14. U.S. Department of Health and Human Services. Pain Management Best Practices Inter-Agency Task Force Report: Updates, Gaps, Inconsistencies, and Recommendations U. S. Department of Health and Human Services. May 9, 2019 2019.

https://www.hhs.gov/sites/default/files/pmtffinal-report-2019-05-23.pdf. 
15. Interagency Pain Research Coordinating Committee. National Pain Strategy: a comprehensive population health-level strategy for pain. Washington, DC:

Department of Health and Human Services. 2015.

16. Dr. Robert Bree Collaborative.

Collaborative Care for Chronic Pain Report and Recommendations. 2018.

https://www.qualityhealth.org/bree/topicareas/chronic-pain/.

17. Kligler B, Bair MJ, Banerjea R, et al. Clinical Policy Recommendations from the VHA State-of-the-Art Conference on NonPharmacological Approaches to Chronic Musculoskeletal Pain. J Gen Intern Med. 2018 May;33(Suppl 1):16-23. doi: 10.1007/s11606-018-4323-z. PMID: 29633133.

18. Lentz T, Goertz C, Sharma I, et al. Managing Multiple Irons in the Fire: Continuing to Address the Opioid Crisis and Improve Pain Management during a Public Health Emergency. NEJM Catalyst Innovations in Care Delivery. June 22, 2020. doi: 10.1056/CAT.20.0208.

19. Bokhour BG, Haun JN, Hyde J, et al. Transforming the Veterans Affairs to a Whole Health System of Care: Time for Action and Research. Med Care. 2020 Apr;58(4):295-300. doi: 10.1097/MLR.0000000000001316. PMID: 32044866 .

20. Krejci LP, Carter K, Gaudet T. Whole health: the vision and implementation of personalized, proactive, patient-driven health care for veterans. Med Care. 2014 Dec;52(12 Suppl 5):S5-8. doi: 10.1097/MLR.0000000000000226. PMID: 25397823

21. Peterson K, Anderson J, Bourne D, et al. Effectiveness of Models Used to Deliver Multimodal Care for Chronic Musculoskeletal Pain: a Rapid Evidence Review. J Gen Intern Med. 2018 May;33(Suppl 1):71-81. doi: 10.1007/s1 1606-018-4328-7. PMID: 29633140.
22. Hansen KA, McKernan LC, Carter SD, et al. A Replicable and Sustainable Whole Person Care Model for Chronic Pain. J Altern Complement Med. 2019 Mar;25(S1):S86S94. doi: 10.1089/acm.2018.0420. PMID: 30870025

23. Schwan J, Sclafani J, Tawfik VL. Chronic Pain Management in the Elderly. Anesthesiol Clin. 2019 Sep;37(3):547-60. doi: 10.1016/j.anclin.2019.04.012. PMID: 31337484 .

24. Grashorn W, Sprenger C, Forkmann K, et al. Age-dependent decline of endogenous pain control: exploring the effect of expectation and depression. PLoS One.

2013;8(9):e75629. doi: 10.1371/journal.pone.0075629. PMID: 24086595.

25. Quiton RL, Roys SR, Zhuo J, et al. Agerelated changes in nociceptive processing in the human brain. Ann N Y Acad Sci. 2007 Feb;1097:175-8. doi: 10.1196/annals.1379.024. PMID: 17413021.

26. Guise JM, Butler ME, Chang C, et al. AHRQ series on complex intervention systematic reviews-paper 6: PRISMA-CI extension statement and checklist. J Clin Epidemiol. 2017 Oct;90:43-50. doi: 10.1016/j.jclinepi.2017.06.016. PMID: 28720516.

27. U.S. Preventive Services Task Force. U.S. Preventive Services Task Force Procedure Manual. 2015.

https://www.uspreventiveservicestaskforce.o rg/uspstf/procedure-manual. Accessed August 252020

28. Methods Guide for Effectiveness and Comparative Effectiveness Reviews. AHRQ Publication No. 10(14)-EHC063-EF. Rockville, MD: Agency for Healthcare Research and Quality. January 2014. Chapters available at: www.effectivehealthcare.ahrq.gov.

29. Merksey H, Bogduk N. Classification of chronic pain. Descriptions of Chronic Pain Syndromes and Definitions of Pain Term. 1994:59-71. 
30. Kosek E, Cohen M, Baron R, et al. Do we need a third mechanistic descriptor for chronic pain states? Pain. 2016

Jul;157(7):1382-6. doi: 10.1097/j.pain.0000000000000507. PMID: 26835783.

31. Viswanathan M, Patnode CD, Berkman ND, et al. Assessing the risk of bias in systematic reviews of health care interventions. Methods guide for effectiveness and comparative effectiveness reviews [Internet]. Agency for Healthcare Research and Quality (US); 2017.

32. Higgins JP, Green S. Cochrane handbook for systematic reviews of interventions: John Wiley \& Sons; 2011.

33. Fu R, Gartlehner G, Grant M, et al. Conducting quantitative synthesis when comparing medical interventions: AHRQ and the Effective Health Care Program. J Clin Epidemiol. 2011 Nov;64(11):1187-97. doi: 10.1016/j.jclinepi.2010.08.010

S0895-4356(11)00029-1 [pii]. PMID: 21477993.

34. Morton SC, Murad MH, O'Connor E, et al. Quantitative Synthesis-An Update. Methods Guide for Comparative Effectiveness Reviews. (Prepared by the Scientific Resource Center under Contract No. 290-2012-0004-C). AHRQ Publication No. 18-EHC007-EF. Rockville, MD: Quality AfHRa; 2018.

35. Higgins JP, Thompson SG, Deeks JJ, et al. Measuring inconsistency in meta-analyses. BMJ. 2003 Sep 6;327(7414):557-60. doi: 10.1136/bmj.327.7414.557. PMID: 12958120 .

36. Skelly AC, Chou R, Dettori JR, et al. Noninvasive Nonpharmacological Treatment for Chronic Pain: A Systematic Review Update. Comparative Effectiveness Review No. 227. (Prepared by the Pacific Northwest Evidence-based Practice Center under Contract No. 290-2015-00009-I.) AHRQ Publication No. 20-EHC009. Rockville, MD: Agency for Healthcare Research and Quality; April 2020. https://effectivehealthcare.ahrq.gov/products /noninvasive-nonpharm-painupdate/research. PMID: 32338846.
37. Skelly AC, Chou R, Dettori JR, et al. Noninvasive Nonpharmacological Treatment for Chronic Pain: A Systematic Review. Comparative Effectiveness Review No. 209. (Prepared by the Pacific Northwest Evidence-based Practice Center under Contract No. 290-2015-00009-I.) AHRQ Publication No 18-EHC013-EF. Rockville, MD: Agency for Healthcare Research and Quality; June 2018.

https://effectivehealthcare.ahrq.gov/topics/n onpharma-treatment-pain/research-2018. PMID: 30179389.

38. Chou R, Deyo R, Friedly J, et al.

Noninvasive Treatment for Low Back Pain. Comparative Effectiveness Review No. 169. (Prepared by the Pacific Northwest Evidence-based Practice Center under Contract No. HHSA 290-2012-00014-I.) AHRQ Publication No. 16-EHC004-EF. Rockville, MD: Agency for Healthcare Research and Quality; February 2016. https://effectivehealthcare.ahrq.gov/products back-pain-treatment/research. PMID: 26985522.

39. Chou R, Hartung D, Turner J, et al. Opioid Treatments for Chronic Pain. Comparative Effectiveness Review No. 229. (Prepared by the Pacific Northwest Evidence-based Practice Center under Contract No. 2902015-00009-I.) AHRQ Publication No. 20EHC011. Rockville, MD: Agency for Healthcare Research and Quality; 2020. doi: 10.23970/AHRQEPCCER229. PMID: 32338848.

40. Chou R, Wagner J, Ahmed AY, et al. Treatments for Acute Pain: A Systematic Review. Comparative Effectiveness Review No. 240. (Prepared by the Pacific Northwest Evidence-based Practice Center under Contract No. 290-2015-00009-I.) AHRQ Publication No. 20(21)-EHC006. Rockville, MD: Agency for Healthcare Research and Quality; December 2020. doi: 10.23970/AHRQEPCCER240. PMID: 33411426.

41. Allen KD, Oddone EZ, Coffman CJ, et al. Patient, Provider, and Combined Interventions for Managing Osteoarthritis in Primary Care: A Cluster Randomized Trial. Annals of Internal Medicine. 2017 Mar 21;166(6):401-11. doi: https://dx.doi.org/10.7326/M16-1245. PMID: 28114648. 
42. Allen KD, Yancy WS, Jr., Bosworth HB, et al. A Combined Patient and Provider Intervention for Management of Osteoarthritis in Veterans: A Randomized Clinical Trial. Annals of Internal Medicine. 2016 Jan 19;164(2):73-83. doi: https://dx.doi.org/10.7326/M15-0378. PMID: 26720751.

43. Angeles RN, Guenter D, McCarthy L, et al. Group interprofessional chronic pain management in the primary care setting: a pilot study of feasibility and effectiveness in a family health team in Ontario. Pain Research \& Management. 2013 SepOct;18(5):237-42. PMID: 23875181.

44. Beasley M, Prescott GJ, Scotland G, et al. Patient-reported improvements in health are maintained 2 years after completing a short course of cognitive behaviour therapy, exercise or both treatments for chronic widespread pain: long-term results from the MUSICIAN randomised controlled trial. RMD Open. 2015;1(1):e000026. doi: 10.1136/rmdopen-2014-000026. PMID: 26509056 .

45. Dickinson KC, Sharma R, Duckart JP, et al. VA healthcare costs of a collaborative intervention for chronic pain in primary care. Medical Care. 2010 Jan;48(1):38-44. doi:

https://dx.doi.org/10.1097/MLR.0b013e318 1bd49e2. PMID: 19952802.

46. Dobscha SK, Corson K, Leibowitz RQ, et al. Rationale, design, and baseline findings from a randomized trial of collaborative care for chronic musculoskeletal pain in primary care. Pain Medicine. 2008 Nov;9(8):105064. doi: https://dx.doi.org/10.1111/j.15264637.2008.00457.x. PMID: 18565008.

47. Dobscha SK, Corson K, Perrin NA, et al. Collaborative care for chronic pain in primary care: a cluster randomized trial. JAMA. 2009 Mar 25;301(12):1242-52. doi: https://dx.doi.org/10.1001/jama.2009.377. PMID: 19318652.
48. Mas RR, Lopez-Jimenez T, Pujol-Ribera E, et al. Effectiveness of a multidisciplinary BIOPSYCHOSOCIAL intervention for nonspecific SUBACUTE low back pain in a working population: a cluster randomized clinical trial. BMC Health Services Research. 2019 Dec 12;19(1):962. doi: https://dx.doi.org/10.1186/s12913-0194810-x. PMID: 31831074.

49. McBeth J, Prescott G, Scotland G, et al. Cognitive behavior therapy, exercise, or both for treating chronic widespread pain. Archives of Internal Medicine. 2012 Jan 09;172(1):48-57. doi: https://dx.doi.org/10.1001/archinternmed.20 11.555. PMID: 22082706.

50. Von Korff M, Balderson BH, Saunders K, et al. A trial of an activating intervention for chronic back pain in primary care and physical therapy settings. Pain. 2005 Feb;113(3):323-30. doi: 10.1016/j.pain.2004.11.007. PMID: 15661440 .

51. Abbasi M, Dehghani M, Keefe FJ, et al. Spouse-assisted training in pain coping skills and the outcome of multidisciplinary pain management for chronic low back pain treatment: a 1-year randomized controlled trial. Eur J Pain. 2012 Aug;16(7):1033-43. doi: 10.1002/j.1532-2149.2011.00097.x. PMID: 22337646.

52. Ahlmen M, Sullivan M, Bjelle A. Team versus non-team outpatient care in rheumatoid arthritis. A comprehensive outcome evaluation including an overall health measure. Arthritis Rheum. 1988 Apr;31(4):471-9. doi: 10.1002/art.1780310403. PMID: 3358810.

53. Alaranta H, Rytökoski U, Rissanen A, et al. Intensive physical and psychosocial training program for patients with chronic low back pain. A controlled clinical trial. Spine (Phila Pa 1976). 1994 Jun 15;19(12):1339-49. doi: 10.1097/00007632-199406000-00007. PMID: 8066514.

54. Amris K, Wæhrens EE, Christensen R, et al. Interdisciplinary rehabilitation of patients with chronic widespread pain: primary endpoint of the randomized, nonblinded, parallel-group IMPROvE trial. Pain. 2014 Jul;155(7):1356-64. doi: 10.1016/j.pain.2014.04.012. PMID: 24727345 . 
55. Basler HD, Jäkle C, Kröner-Herwig B. Incorporation of cognitive-behavioral treatment into the medical care of chronic low back patients: a controlled randomized study in German pain treatment centers. Patient Educ Couns. 1997 Jun;31(2):113-24. doi: 10.1016/s0738-3991(97)00996-8. PMID: 9216352.

56. Bendix AE, Bendix T, Haestrup C, et al. A prospective, randomized 5-year follow-up study of functional restoration in chronic low back pain patients. Eur Spine J. 1998b;7(2):111-9. doi: 10.1007/s005860050040. PMID: 9629934.

57. Bendix AF, Bendix T, Labriola M, et al. Functional restoration for chronic low back pain. Two-year follow-up of two randomized clinical trials. Spine. 1998a Mar 15;23(6):717-25. PMID: 9549794.

58. Bendix AF, Bendix T, Lund C, et al. Comparison of three intensive programs for chronic low back pain patients: a prospective, randomized, observer-blinded study with one-year follow-up. Scand J Rehabil Med. 1997 Jun;29(2):81-9. PMID: 9198257.

59. Bendix AF, Bendix T, Ostenfeld S, et al. Active treatment programs for patients with chronic low back pain: a prospective, randomized, observer-blinded study. European Spine Journal. 1995;4(3):148-52. PMID: 7552649.

60. Bendix AF, Bendix T, Vaegter K, et al. Multidisciplinary intensive treatment for chronic low back pain: a randomized, prospective study. Cleveland Clinic Journal of Medicine. 1996 Jan-Feb;63(1):62-9. PMID: 8590519.

61. Bendix T, Bendix A, Labriola M, et al. Functional restoration versus outpatient physical training in chronic low back pain: a randomized comparative study. Spine. 2000 Oct 01;25(19):2494-500. PMID: 11013502.

62. Browne AL, Appleton S, Fong K, et al. A pilot randomized controlled trial of an early multidisciplinary model to prevent disability following traumatic injury. Disability \& Rehabilitation. 2013 Jul;35(14):1149-63. doi: https://dx.doi.org/10.3109/09638288.2012.7 21047. PMID: 23083416.
63. Castel A, Castro S, Fontova R, et al. Body mass index and response to a multidisciplinary treatment of fibromyalgia. Rheumatology International. 2015 Feb;35(2):303-14. doi: https://dx.doi.org/10.1007/s00296-0143096-x. PMID: 25080875.

64. Castel A, Fontova R, Montull S, et al. Efficacy of a multidisciplinary fibromyalgia treatment adapted for women with low educational levels: a randomized controlled trial. Arthritis Care Res (Hoboken). 2013 Mar;65(3):421-31. doi: 10.1002/acr.21818. PMID: 22899402.

65. de Buck PD, le Cessie S, van den Hout WB, et al. Randomized Comparison of a Multidisciplinary Job-Retention Vocational Rehabilitation Program With Usual Outpatient Care in Patients With Chronic Arthritis at Risk For Job Loss. Arthritis \& Rheumatism: Arthritis Care \& Research. 2005 Oct;53(5):682-90. doi: http://dx.doi.org/10.1002/art.21452. PMID: 2005-13265-006.

66. Härkäpää K, Järvikoski A, Mellin G, et al. A controlled study on the outcome of inpatient and outpatient treatment of low back pain. Part I. Pain, disability, compliance, and reported treatment benefits three months after treatment. Scand J Rehabil Med. 1989;21(2):81-9. PMID: 2526364.

67. Härkäpää K, Mellin G, Järvikoski A, et al. A controlled study on the outcome of inpatient and outpatient treatment of low back pain. Part III. Long-term follow-up of pain, disability, and compliance. Scand J Rehabil Med. 1990;22(4):181-8. PMID: 2148221.

68. Henchoz Y, de Goumoens P, So AK, et al. Functional multidisciplinary rehabilitation versus outpatient physiotherapy for non specific low back pain: randomized controlled trial. Swiss Medical Weekly. 2010;140:w13133. doi: https://dx.doi.org/10.4414/smw.2010.13133. PMID: 21181567.

69. Jensen IB, Bergström G, Ljungquist T, et al. A randomized controlled component analysis of a behavioral medicine rehabilitation program for chronic spinal pain: are the effects dependent on gender? Pain. 2001 Mar;91(1-2):65-78. doi: 10.1016/s0304-3959(00)00420-6. PMID: 11240079 . 
70. Johansson C, Dahl J, Jannert M, et al. Effects of a cognitive-behavioral painmanagement program. Behaviour Research \& Therapy. 1998 Oct;36(10):915-30. PMID: 9714943.

71. Jousset N, Fanello S, Bontoux L, et al. Effects of functional restoration versus 3 hours per week physical therapy: a randomized controlled study. Spine (Phila Pa 1976). 2004 Mar 1;29(5):487-93; discussion 94. doi: 10.1097/01.brs.0000102320.35490.43. PMID: 15129059.

72. Kaapa EH, Frantsi K, Sarna S, et al. Multidisciplinary group rehabilitation versus individual physiotherapy for chronic nonspecific low back pain: a randomized trial. Spine. 2006 Feb 15;31(4):371-6. PMID: 16481945.

73. Lemstra M, Olszynski W. The Effectiveness of Multidisciplinary Rehabilitation in the Treatment of Fibromyalgia: A Randomized Controlled Trial. The Clinical Journal of Pain. 2005 Mar-Apr;21(2):166-74. doi: http://dx.doi.org/10.1097/00002508200503000-00008. PMID: 2005-02073-008.

74. Linton SJ, Boersma K, Jansson M, et al. The effects of cognitive-behavioral and physical therapy preventive interventions on painrelated sick leave: a randomized controlled trial. Clinical Journal of Pain. 2005 MarApr;21(2):109-19. PMID: 15722803.

75. Mangels M, Schwarz S, Worringen U, et al. Evaluation of a behavioral-medical inpatient rehabilitation treatment including booster sessions: a randomized controlled study.

Clinical Journal of Pain. 2009

Jun;25(5):356-64. doi:

https://dx.doi.org/10.1097/AJP.0b013e3181 925791. PMID: 19454868.

76. Martin J, Torre F, Aguirre U, et al. Evaluation of the interdisciplinary PSYMEPHY treatment on patients with fibromyalgia: a randomized control trial. Pain Medicine. 2014c Apr;15(4):682-91. doi: https://dx.doi.org/10.1111/pme.12375. PMID: 24576148.
77. Martin J, Torre F, Padierna A, et al. Impact of interdisciplinary treatment on physical and psychosocial parameters in patients with fibromyalgia: results of a randomised trial. International journal of clinical practice. 2014a;68(5):618-27. PMID: CN-00988924.

78. Martin J, Torre F, Padierna A, et al. Interdisciplinary treatment of patients with fibromyalgia: improvement of their healthrelated quality of life. Pain Practice. 2014b Nov;14(8):721-31. doi: https://dx.doi.org/10.1111/papr.12134. PMID: 24279638.

79. Meyer K, Fransen J, Huwiler H, et al. Feasibility and results of a randomised pilotstudy of a work rehabilitation programme. Journal of Back and Musculoskeletal Rehabilitation. 2005;18(3-4):67-78.

80. Onac I, Moldovan A, Igna R, et al. Mechanism of change following multidisciplinary treatment of low back pain secondary to lumbar disc prolapse. Journal of Evidence-Based Psychotherapies. 2017 Sep;17(2):159-73. doi: http://dx.doi.org/10.24193/jebp.2017.2.10. PMID: 2017-54116-010.

81. Onac I, Moldovan A, Onac I, et al. Medication, physiotherapy and cognitive behavior therapy for the treatment of chronic back pain: a clinical trial. Journal of Cognitive and Behavioral Psychotherapies. 2012 Mar;12(1):23-37.

82. Peters J, Large RG, Elkind G. Follow-up results from a randomised controlled trial evaluating in- and outpatient pain management programmes. Pain. 1992 Jul;50(1):41-50. PMID: 1381070.

83. Peters JL, Large RG. A randomised control trial evaluating in- and outpatient pain management programmes. Pain. 1990 Jun;41(3):283-93. PMID: 1697057.

84. Roche G, Ponthieux A, Parot-Shinkel E, et al. Comparison of a functional restoration program with active individual physical therapy for patients with chronic low back pain: a randomized controlled trial. Arch Phys Med Rehabil. 2007 Oct;88(10):122935. doi: 10.1016/j.apmr.2007.07.014. PMID: 17908562. 
85. Roche-Leboucher G, Petit-Lemanac'h A, Bontoux L, et al. Multidisciplinary intensive functional restoration versus outpatient active physiotherapy in chronic low back pain: a randomized controlled trial. Spine (Phila Pa 1976). 2011 Dec 15;36(26):223542. doi: 10.1097/BRS.0b013e3182191e13. PMID: 21415807.

86. Ronzi Y, Roche-Leboucher G, Begue C, et al. Efficiency of three treatment strategies on occupational and quality of life impairments for chronic low back pain patients: is the multidisciplinary approach the key feature to success? Clinical Rehabilitation. 2017 Oct;31(10):1364-73. doi:

https://dx.doi.org/10.1177/02692155176910 86. PMID: 28592147.

87. Salvat I, Zaldivar P, Monterde S, et al. Functional status, physical activity level, and exercise regularity in patients with fibromyalgia after Multidisciplinary treatment: retrospective analysis of a randomized controlled trial. Rheumatology International. 2017 Mar;37(3):377-87. doi: https://dx.doi.org/10.1007/s00296-0163597-x. PMID: 27844124.

88. Saral I, Sindel D, Esmaeilzadeh S, et al. The effects of long- and short-term interdisciplinary treatment approaches in women with fibromyalgia: a randomized controlled trial. Rheumatology International. 2016 Oct;36(10):1379-89. doi: https://dx.doi.org/10.1007/s00296-0163473-8. PMID: 27055444.

89. Scholten C, Brodowicz T, Graninger W, et al. Persistent functional and social benefit 5 years after a multidisciplinary arthritis training program. Archives of Physical Medicine \& Rehabilitation. 1999 Oct;80(10):1282-7. PMID: 10527088.

90. Schweikert B, Jacobi E, Seitz R, et al. Effectiveness and cost-effectiveness of adding a cognitive behavioral treatment to the rehabilitation of chronic low back pain. Journal of Rheumatology. 2006 Dec;33(12):2519-26. PMID: 17143986.
91. Smeets RJ, Vlaeyen JW, Hidding A, et al. Chronic low back pain: physical training, graded activity with problem solving training, or both? The one-year posttreatment results of a randomized controlled trial. Pain. 2008 Feb;134(3):263-76. PMID: 17498879.

92. Smeets RJ, Vlaeyen JW, Hidding A, et al. Active rehabilitation for chronic low back pain: cognitive-behavioral, physical, or both? First direct post-treatment results from a randomized controlled trial [ISRCTN22714229]. BMC Musculoskeletal Disorders. 2006a Jan 20;7:5. PMID: 16426449.

93. Smith J, Faux SG, Gardner T, et al. Reboot Online: a Randomized Controlled Trial Comparing an Online Multidisciplinary Pain Management Program with Usual Care for Chronic Pain. Pain medicine. 2019 PMID: CN-01987380 NEW.

94. Tavafian SS, Jamshidi AR, Mohammad K. Treatment of chronic low back pain: a randomized clinical trial comparing multidisciplinary group-based rehabilitation program and oral drug treatment with oral drug treatment alone. Clinical Journal of Pain. 2011 Nov-Dec;27(9):811-8. doi: https://dx.doi.org/10.1097/AJP.0b013e3182 1e7930. PMID: 21642845.

95. Tavafian SS, Jamshidi AR, Mohammad K. Treatment of low back pain: randomized clinical trial comparing a multidisciplinary group-based rehabilitation program with oral drug treatment up to 12 months.

International Journal of Rheumatic Diseases. 2014 Feb;17(2):159-64. doi: https://dx.doi.org/10.1111/1756185X.12116. PMID: 24576271.

96. Tavafian SS, Jamshidi AR, Mohammad K. Treatment of low back pain: Second extended follow up of an original trial (NCT00600197) comparing a multidisciplinary group-based rehabilitation program with oral drug treatment alone up to 30 months. International Journal of Rheumatic Diseases. 2017b Dec;20(12):1910-6. doi: https://dx.doi.org/10.1111/1756185X.12540. PMID: 25546488. 
97. Tavafian SS, Jamshidi AR, Montazeri A. A randomized study of back school in women with chronic low back pain: quality of life at three, six, and twelve months follow-up. Spine (Phila Pa 1976). 2008 Jul 1;33(15):1617-21. doi: 10.1097/BRS.0b013e31817bd31c. PMID: 18580739 .

98. Tavafian SS, Jamshidi AR, Shay B. Treatment of low back pain: first extended follow up of an original trial (NCT00600197) comparing a multidisciplinary group-based rehabilitation program with oral drug treatment alone up to 24 months. International journal of rheumatic diseases. 2017a;20(12):1902-9. PMID: CN-01449489 NEW.

99. Thieme K, Gromnica-Ihle E, Flor H. Operant behavioral treatment of fibromyalgia: a controlled study. Arthritis Rheum. 2003 Jun 15;49(3):314-20. doi: 10.1002/art.11124. PMID: 12794785.

100. Turner JA, Clancy S, McQuade KJ, et al. Effectiveness of behavioral therapy for chronic low back pain: a component analysis. Journal of Consulting \& Clinical Psychology. 1990 Oct;58(5):573-9. PMID: 2147702.

101. Turner-Stokes L, Erkeller-Yuksel F, Miles A, et al. Outpatient cognitive behavioral pain management programs: a randomized comparison of a group-based multidisciplinary versus an individual therapy model. Archives of Physical Medicine \& Rehabilitation. 2003 Jun;84(6):781-8. PMID: 12808527.

102. van Eijk-Hustings Y, Kroese M, Creemers A, et al. Resource utilisation and direct costs in patients with recently diagnosed fibromyalgia who are offered one of three different interventions in a randomised pragmatic trial. Clinical Rheumatology. 2016 May;35(5):1307-15. doi: https://dx.doi.org/10.1007/s10067-0153067-y. PMID: 26409883.
103. van Eijk-Hustings Y, Kroese M, Tan F, et al. Challenges in demonstrating the effectiveness of multidisciplinary treatment on quality of life, participation and health care utilisation in patients with fibromyalgia: a randomised controlled trial. Clinical Rheumatology. 2013

Feb;32(2):199-209. doi: https://dx.doi.org/10.1007/s10067-0122100-7. PMID: 23053692.

104. van Koulil S, van Lankveld W, Kraaimaat FW, et al. Tailored cognitive-behavioural therapy and exercise training improves the physical fitness of patients with fibromyalgia. Annals of the Rheumatic Diseases. 2011 Dec;70(12):2131-3. doi: https://dx.doi.org/10.1136/ard.2010.148577. PMID: 21926189.

105. van Koulil S, van Lankveld W, Kraaimaat FW, et al. Tailored cognitive-behavioral therapy and exercise training for high-risk patients with fibromyalgia. Arthritis Care Res (Hoboken). 2010 Oct;62(10):1377-85. doi: 10.1002/acr.20268. PMID: 20521308.

106. Weiner DK, Gentili A, Rossi M, et al. Aging Back Clinics-a Geriatric Syndrome Approach to Treating Chronic Low Back Pain in Older Adults: Results of a Preliminary Randomized Controlled Trial. Pain Med. 2020 Feb 1;21(2):274-90. doi: 10.1093/pm/pnz179. PMID: 31503275.

107. Whitfill T, Haggard R, Bierner SM, et al. Early intervention options for acute low back pain patients: a randomized clinical trial with one-year follow-up outcomes. Journal of Occupational Rehabilitation. 2010 Jun;20(2):256-63. doi: https://dx.doi.org/10.1007/s10926-0109238-4. PMID: 20369277.

108. Williams AC, Richardson PH, Nicholas MK, et al. Inpatient vs. outpatient pain management: results of a randomised controlled trial. Pain. 1996 Jul;66(1):13-22. PMID: 8857627.

109. Calner T, Nordin C, Eriksson MK, et al. Effects of a self-guided, web-based activity programme for patients with persistent musculoskeletal pain in primary healthcare: a randomized controlled trial. European journal of pain. 2017(pagination) PMID: CN-01374487 NEW. 
110. Allen KD, Bosworth HB, Brock DS, et al. Patient and provider interventions for managing osteoarthritis in primary care: protocols for two randomized controlled trials. BMC Musculoskelet Disord. 2012 Apr 24;13:60. doi: 10.1186/1471-2474-1360. PMID: 22530979.

111. Reneman MF, Waterschoot FPC, Burgerhof JGM, et al. Dosage of pain rehabilitation programmes for patients with chronic musculoskeletal pain: a non-inferiority randomised controlled trial. Disability and rehabilitation. 2020;42(6):814-21. PMID: CN-02100807 NEW.

112. Rose MJ, Reilly JP, Pennie B, et al. Chronic low back pain rehabilitation programs: a study of the optimum duration of treatment and a comparison of group and individual therapy. Spine. 1997 Oct 01;22(19):224651; discussion 52-3. PMID: 9346145.

113. Altmaier EM, Lehmann TR, Russell DW, et al. The effectiveness of psychological interventions for the rehabilitation of low back pain: a randomized controlled trial evaluation. Pain. 1992 Jun;49(3):329-35. doi: 10.1016/0304-3959(92)90240-c. PMID: 1408299.

114. Van der Maas LC, Koke A, Pont M, et al. Improving the Multidisciplinary Treatment of Chronic Pain by Stimulating Body Awareness: A Cluster-randomized Trial. Clinical Journal of Pain. 2015 Jul;31(7):6609. doi:

https://dx.doi.org/10.1097/AJP.0000000000 000138. PMID: 25119509.

115. Rothman MG, Ortendahl M, Rosenblad A, et al. Improved quality of life, working ability, and patient satisfaction after a pretreatment multimodal assessment method in patients with mixed chronic muscular pain: A randomized-controlled study. The Clinical Journal of Pain. 2013 Mar;29(3):195-204. PMID: 2013-10222002.

116. Streibelt M, Bethge M. Effects of intensified work-related multidisciplinary rehabilitation on occupational participation: a randomizedcontrolled trial in patients with chronic musculoskeletal disorders. International Journal of Rehabilitation Research. 2014 Mar;37(1):61-6. doi: https://dx.doi.org/10.1097/MRR.000000000 0000031. PMID: 24056065.
117. Kool J, Bachmann S, Oesch P, et al. Function-centered rehabilitation increases work days in patients with nonacute nonspecific low back pain: 1-year results from a randomized controlled trial. Arch Phys Med Rehabil. 2007 Sep;88(9):1089-94. doi: 10.1016/j.apmr.2007.05.022. PMID: 17826451 .

118. Kool JP, Oesch PR, Bachmann S, et al. Increasing days at work using functioncentered rehabilitation in nonacute nonspecific low back pain: a randomized controlled trial. Arch Phys Med Rehabil. 2005 May;86(5):857-64. doi: 10.1016/j.apmr.2004.10.044. PMID: 15895328.

119. Leeuw M, Goossens ME, van Breukelen GJ, et al. Exposure in vivo versus operant graded activity in chronic low back pain patients: results of a randomized controlled trial. Pain. 2008 Aug 15;138(1):192-207. doi: 10.1016/j.pain.2007.12.009. PMID: 18242858 .

120. Gatchel RJ, McGeary DD, McGeary CA, et al. Interdisciplinary chronic pain management: past, present, and future. Am Psychol. 2014 Feb-Mar;69(2):119-30. doi: 10.1037/a0035514. PMID: 24547798.

121. Lewis GN, Bean D, Mowat R. How Have Chronic Pain Management Programs Progressed? A Mapping Review. Pain Practice. 2019;19(7):767-84.

122. Joypaul S, Kelly F, McMillan SS, et al. Multi-disciplinary interventions for chronic pain involving education: A systematic review. PLoS One. 2019;14(10):e0223306. doi: 10.1371/journal.pone.0223306. PMID: 31577827.

123. International Association for the Study of Pain (IASP) Task Force. Task Force on Multimodal Pain Treatment Defines Terms for Chronic Pain Care. December 14, 2017. Accessed at: https://www.iasppain.org/PublicationsNews/NewsDetail.aspx ?ItemNumber=6981. 
124. Purcell N, Zamora K, Tighe J, et al. The Integrated Pain Team: A Mixed-Methods Evaluation of the Impact of an Embedded Interdisciplinary Pain Care Intervention on Primary Care Team Satisfaction, Confidence, and Perceptions of Care Effectiveness. Pain Med. 2018 Sep 1;19(9):1748-63. doi: 10.1093/pm/pnx254. PMID: 29040715.

125. Smeets RJ, Severens JL, Beelen S, et al. More is not always better: cost-effectiveness analysis of combined, single behavioral and single physical rehabilitation programs for chronic low back pain. Eur J Pain. 2009 Jan;13(1):71-81. doi:

10.1016/j.ejpain.2008.02.008. PMID: 18434221 .

126. Escobar A, Quintana JM, Bilbao A, et al. Responsiveness and clinically important differences for the WOMAC and SF-36 after total knee replacement. Osteoarthritis Cartilage. 2007 Mar;15(3):273-80. doi: 10.1016/j.joca.2006.09.001. PMID: 17052924.

127. Jayadevappa R, Cook R, Chhatre S. Minimal important difference to infer changes in health-related quality of life-a systematic review. J Clin Epidemiol. 2017 Sep;89:188-98. doi: 10.1016/j.jclinepi.2017.06.009. PMID: 28676426.

128. Keurentjes JC, Van Tol FR, Fiocco M, et al. Minimal clinically important differences in health-related quality of life after total hip or knee replacement: A systematic review. Bone Joint Res. 2012 May;1(5):71-7. doi: 10.1302/2046-3758.15.2000065. PMID: 23610674.

129. Kamper SJ, Apeldoorn AT, Chiarotto A, et al. Multidisciplinary biopsychosocial rehabilitation for chronic low back pain. Cochrane Database Syst Rev. 2014 Sep 2(9):CD000963. doi: 10.1002/14651858.CD000963.pub3. PMID: 25180773 .

130. Bujak BK, Regan E, Beattie PF, et al. The effectiveness of interdisciplinary intensive outpatient programs in a population with diverse chronic pain conditions: a systematic review and meta-analysis. Pain Manag. 2019 Jul;9(4):417-29. doi: 10.2217/pmt-20180087. PMID: 31237177.
131. McDonagh MS, Selph SS, Buckley DI, et al. Nonopioid Pharmacologic Treatments for Chronic Pain. Comparative Effectiveness Review No. 228. (Prepared by the Pacific Northwest Evidence-based Practice Center under Contract No. 290-2015-00009-I.) AHRQ Publication No. 20-EHC010. Rockville, MD: 2020. doi: 10.23970/AHRQEPCCER228. PMID: 32338847.

132. Carragee EJ. The vertebroplasty affair: the mysterious case of the disappearing effect size. Spine J. 2010 Mar;10(3):191-2. doi: 10.1016/j.spinee.2010.01.002. PMID: 20207329.

133. Deyo RA. Fads in the treatment of low back pain. N Engl J Med. 1991 Oct 3;325(14):1039-40. doi: 10.1056/NEJM199110033251411. PMID: 1832210.

134. Chou R, Deyo R, Friedly J, et al. Nonpharmacologic therapies for low back pain: A systematic review for an American College of Physicians Clinical Practice Guideline. Ann Intern Med. 2017 Feb 14;166:[Epub ahead of print]. doi: 10.7326/M16-2459. PMID: 28192793.

135. Dowell D, Haegerich TM, Chou R. CDC Guideline for Prescribing Opioids for Chronic Pain--United States, 2016. JAMA. 2016 Apr 19;315(15):1624-45. doi: 10.1001/jama.2016.1464. PMID: 26977696.

136. Busse J. The 2017 Canadian Guideline for Opioids for Chronic Non-Cancer Pain.

Access at: https://nationalpaincentre.mcmaster.ca/docu ments/Opioid\%20GL\%20for\%20CMAJ_01 may2017.pdf. 2017.

137. Heyward J, Jones CM, Compton WM, et al. Coverage of Nonpharmacologic Treatments for Low Back Pain Among US Public and Private Insurers. JAMA Netw Open. 2018 Oct 5;1(6):e183044. doi: 10.1001/jamanetworkopen.2018.3044. PMID: 30646222. 
138. Farrell T, Tomoaia-Cotisel A, Scammon D, et al. Care Management: Implications for Medical Practice, Health Policy, and Health Services Research. (Prepared by

Econometrica, Inc. under Contract No. HHSA2902007 TO No. 5.) AHRQ Publication No. 15-0018-EF. Rockville, MD: Agency for Healthcare Research and Quality. February 2015.
139. AlMazrou SH, Elliott RA, Knaggs RD, et al. Cost-effectiveness of pain management services for chronic low back pain: a systematic review of published studies. BMC Health Serv Res. 2020 Mar 12;20(1):194. doi: 10.1186/s12913-0205013-1. PMID: 32164720. 
AHRQ

BDI

BPI

CES-D

CI

CLBP

CMS

COOP/WONCA

CP

CPMP

CWP

DPQ

EQ5D

FIM

FIQ

FM

HADS

HRQOL

IPMP

IQR

LBP

MCID

MCS

MPI

MPQ

MSK

MVAS

NR

NRS

NSAID

OA

ODI

OT

OTC

PACT

\section{Abbreviations and Acronyms}

Agency for Healthcare Research and Quality

Beck Depression Inventory

Brief Pain Inventory

Center for Epidemiological Studies Depression Scale

confidence interval

chronic low back pain

Centers for Medicare \& Medicaid Services

Dartmouth Primary Care Cooperative Information Project/World

Organization of National Colleges, Academies, and Academic

Associations of General Practice/Family Physicians scale

chronic pain

comprehensive pain management program

chronic widespread pain

Dallas Pain Questionnaire

EuroQol-5 dimensions

functional independence measure

Fibromyalgia Impact Questionnaire

fibromyalgia

Hospital Anxiety and Depression Scale

health-related quality of life

integrated pain management program

interquartile range

low back pain

minimal clinically important difference

Mental Component Score

multidimensional pain inventory

McGill Pain Questionnaire

musculoskeletal

million visual analog scale

not reported

numerical rating scale

nonsteroidal anti-inflammatory drug

osteoarthritis

Oswestry Disability Index

occupational therapist

over the counter

Performance Assessment and Capacity Testing 


$\begin{array}{ll}\text { PCP } & \text { primary care provider } \\ \text { PCS } & \text { Physical Component Score } \\ \text { PDI } & \text { Pain Disability Index } \\ \text { PHQ-8, -9 } & \text { Patient Health Questionnaire-8 or -9 } \\ \text { POMS-SV } & \text { Profile of Mood States Short Version } \\ \text { PT } & \text { physical therapist } \\ \text { RCT } & \text { randomized controlled trial } \\ \text { RMDQ } & \text { Roland Morris Disability Questionnaire } \\ \text { SD } & \text { standard deviation } \\ \text { SF-36 } & \text { Short-Form 36 Questionnaire } \\ \text { SIP } & \text { Sickness Impact Profile } \\ \text { SMD } & \text { standardized mean difference } \\ \text { SPPB } & \text { Short Physical Performance Battery } \\ \text { STAI } & \text { State-Trait Anxiety Inventory } \\ \text { TEP } & \text { Technical Expert Panel } \\ \text { VAS } & \text { visual analog scale } \\ \text { WHYMPI } & \text { West Haven-Yale Multidimensional Pain Inventory } \\ \text { WOMAC } & \text { Western Ontario and McMaster Universities Osteoarthritis Index }\end{array}$




\section{Appendix A. Methods}

\section{Details of Study Selection}

\section{Search Strategy}

Literature Databases: Given that complex, multicomponent interventions encompass numerous dimensions and terminology related to them may be inconsistently used, a broad search strategy across multiple data bases - Ovid $^{\circledR}$ MEDLINE $^{\circledR}$, PsycINFO $^{\circledR}$, CINAHL $^{\circledR}$, Cochrane Central Register of Controlled Trials, and Cochrane Database of Systematic Reviews - was used. ${ }^{1}$ Detailed search strategies are listed below. All searches were conducted by a qualified medical librarian.

Publication Date Range: Searches were conducted across all Key Questions, with study dates reaching back to 1989 up to September 23, 2020. The year 1989 corresponds to publication of the earliest RCTs relevant to this topic. ${ }^{2,3}$ Searches were deduplicated and screened for inclusion. Searches were updated (through May 24, 2021) for new publications while the draft report was posted for peer review and public comment. Literature identified during the update search was assessed using the process described below for the original search. Any new eligible literature identified in the update search was incorporated into the report prior to finalization.

Supplemental Evidence and Data for Systematic review (SEADS): Various stakeholder were informed about submitting information relevant to this review using a Federal Register notification. A portal about the opportunity to submit information was made available on the Effective Health Care (EHC) website. We reviewed all citations included in the submissions we received, and none met the inclusion criteria for this report.

Hand Searching: Reference lists of included articles, as well as relevant systematic reviews, were reviewed for includable studies.

Peer Review and Public Comment: References cited by peer and public reviewers were reviewed for includable studies; none met our inclusion criteria.

\section{Medline Search}

\section{Database: Ovid MEDLINE(R) ALL 1946 to September 23, 2020}

1 Chronic Pain/

2 exp arthralgia/ or exp back pain/ or exp headache/ or exp musculoskeletal pain/ or neck pain/ or exp neuralgia/ or exp nociceptive pain/ or pain, intractable/ or fibromyalgia/ or myalgia/

3 Pain/

4 chronic.ti,ab,kw.

53 and 4

6 ((chronic or persistent or intractable or refractory) adj3 pain).ti,ab,kw.

7 (((back or spine or spinal or cervical or leg or musculoskeletal or neuropathic or nociceptive or nociplastic or centralized or radicular or noncancer or "non-cancer" or "non-malignant" or 
diffuse) adj2 pain) or headache or arthriti* or fibromyalgia or osteoarthriti* or neuropathy or neuropathies).ti,ab,kw.

8 or $/ 1-7$

9 exp Patient Care Team/

10 exp Patient Care Planning/

11 Pain Clinics/

12 interdisciplinary communication/

13 Combined Modality Therapy/

14 Case Management/

15 ((integrated or comprehensive or multidisciplin* or multimod* or interdisciplin*or

multicomponent or collaborat* or coordinat* or interprofessional or "inter-professional") adj3 (intervention* or treatment* or therap* or care or program* or model*)).ti,ab,kf.

16 ("pain clinic*" or "pain program*" or "pain management" or biopsychosocial or "stepped care").ti,ab,kf.

17 or/9-16

$18 \quad 8$ and 17

19 exp Medicare/

20 "Centers for Medicare and Medicaid Services, U.S."/

21 (medicare or disabled or disabilit* or kidney or renal or "lou gehrig*" or "amyotrophic lateral sclerosis" or "als").ti,ab.

22 or $/ 19-20$

$23 \quad 18$ and 22

24 (random* or control* or trial).ti,ab,kf,sh.

25 limit 18 to randomized controlled trial

$26 \quad 18$ and 24

27 limit 26 to "all aged (65 and over)"

2825 or 27

29 limit 28 to english language

30 limit 29 to $\mathrm{yr}=" 1989$-Current"

3123 or 30

Database: EBM Reviews - Cochrane Central Register of Controlled Trials August 2020 1 Chronic Pain/

2 exp arthralgia/ or exp back pain/ or exp headache/ or exp musculoskeletal pain/ or neck pain/ or exp neuralgia/ or exp nociceptive pain/ or pain, intractable/ or fibromyalgia/ or myalgia/

3 Pain/

4 chronic.ti,ab,hw.

53 and 4

6 ((chronic or persistent or intractable or refractory) adj3 pain).ti,ab,hw.

7 (( (back or spine or spinal or cervical or leg or musculoskeletal or neuropathic or nociceptive or nociplastic or centralized or radicular or noncancer or "non-cancer" or "non-malignant" or diffuse) adj2 pain) or headache or arthriti* or fibromyalgia or osteoarthriti* or neuropathy or neuropathies).ti,ab,hw.

8 or $1-7$

9 exp Patient Care Team/

10 exp Patient Care Planning/

11 Pain Clinics/ 
12 interdisciplinary communication/

13 Combined Modality Therapy/

14 Case Management/

15 ((integrated or comprehensive or multidisciplin* or multimod* or interdisciplin*or

multicomponent or collaborat* or coordinat* or interprofessional or "inter-professional") adj3 (intervention* or treatment* or therap* or care or program* or model*)).ti,ab,hw.

16 ("pain clinic*" or "pain program*" or "pain management" or biopsychosocial or "stepped care").ti,ab,hw.

17 or/9-16

$18 \quad 8$ and 17

19 exp Medicare/

20 "Centers for Medicare and Medicaid Services, U.S."/

21 (medicare or disabled or disabilit* or kidney or renal or "lou gehrig*" or "amyotrophic lateral sclerosis" or "als").ti,ab.

22 or $/ 19-20$

$23 \quad 18$ and 22

24 limit 18 to medline records

$25 \quad 18$ not 24

26 conference abstract.pt.

27 "journal: conference abstract".pt.

28 "journal: conference review".pt.

29 "http://.www.who.int/trialsearch*".so.

30 "https://clinicaltrials.gov*".so.

3126 or 27 or 28 or 29 or 30

$32 \quad 25$ not 31

33 limit 32 to english language

34 limit 33 to $\mathrm{yr}=" 1989$-Current"

$35 \quad 23$ or 34

\section{Database: EBM Reviews - Cochrane Database of Systematic Reviews 2005 to September 23, 2020}

1 ((chronic or persistent or intractable or refractory) adj3 pain).ti.

2 (((back or spine or spinal or cervical or leg or musculoskeletal or neuropathic or nociceptive or nociplastic or centralized or radicular or noncancer or "non-cancer" or "non-malignant" or diffuse) adj2 pain) or headache or arthriti* or fibromyalgia or osteoarthriti* or neuropathy or neuropathies).ti.

3 ((integrated or comprehensive or multidisciplin* or multimod* or interdisciplin*or multicomponent or collaborat* or coordinat* or interprofessional or "inter-professional") adj3 (intervention* or treatment* or therap* or care or program* or model*)).ti,ab.

4 ("pain clinic*" or "pain program*" or "pain management" or biopsychosocial or "stepped care").ti,ab.

5 (1 or 2$)$ and ( 3 or 4$)$

6 limit 5 to full systematic reviews

Database: APA PsycInfo 1806 to September Week 22020

1 Chronic Pain/ 
2 exp arthralgia/ or exp back pain/ or exp headache/ or exp musculoskeletal pain/ or neck pain/ or exp neuralgia/ or exp nociceptive pain/ or pain, intractable/ or fibromyalgia/ or myalgia/

3 Pain/

4 chronic.ti,ab.

53 and 4

6 ("chronic pain" or "persistent pain" or "intractable pain" or "refractory pain" or "diffuse pain").ti,ab.

7 (( (back or spine or spinal or cervical or leg or musculoskeletal or neuropathic or nociceptive or nociplastic or centralized or radicular or noncancer or "non-cancer" or "non-malignant" or diffuse) adj2 pain) or headache or arthriti* or fibromyalgia or osteoarthriti* or neuropathy or neuropathies).ti,ab.
8 or $1-7$
9 exp Patient Care Planning/
10 exp interdisciplinary treatment approach/
11 exp multimodal treatment approach/
12 exp integrated services/
13 Case Management/ care").ti,ab.
16 or/9-15
$17 \quad 8$ and 16
18 exp Medicare/
19 medicare.ti,ab.
2018 or 19
$21 \quad 17$ and 20
22 limit 17 to peer reviewed journal
23 exp clinical trials/
$24 \quad 22$ and 23
25 (random* or control* or trial).ti,ab.
$26 \quad 22$ and 25
$27 \quad 24$ or 26
28 limit 27 to english language
29 limit 28 to $\mathrm{yr}=" 1989$-Current"

14 ((integrated or comprehensive or multidisciplin* or multimod* or interdisciplin*or

multicomponent or collaborat* or coordinat* or interprofessional or "inter-professional") adj3 (intervention* or treatment* or therap* or care or program* or model*)).ti,ab.

15 ("pain clinic*" or "pain program*" or "pain management" or biopsychosocial or "stepped

\section{Database: EBSCOHost CINAHL Through September 23, 2020}

S1 (MH "Health Care Delivery, Integrated")

S2 (MH "Combined Modality Therapy+")

S3 (MH "Case Management")

S4 (MH "Patient Care Plans+")

S5 multidisciplinary care

S6 (MH "Multidisciplinary Care Team+")

S7 TI integrated or comprehensive or multidisciplin* or multimod* or interdisciplin*or multicomponent or collaborat* or coordinat* or interprofessional or "inter-professional"

S8 TI intervention* or treatment* or therap* or care or program* or model* 
S10 TI "pain clinic*" or "pain program*" or "pain management" or biopsychosocial or "stepped care"

\section{S11 S1 OR S2 OR S3 OR S4 OR S5 OR S6 OR S9 OR S10}

S12 (MH "Pain+")

S13 TI "chronic pain" or "persistent pain" or "intractable pain" or "refractory pain" or "diffuse pain"

S14 TI ( back or spine or spinal or cervical or leg or musculoskeletal or neuropathic or nociceptive or nociplastic or centralized or radicular or noncancer or "non-cancer" or "nonmalignant" ) AND TI pain

S15 TI headache or arthriti* or fibromyalgia or osteoarthriti* or neuropathy or neuropathies

S 16 S12 OR S13 OR S14 OR S15

S17 S11 AND S16

S18 S11 AND S16 Limiters - Exclude MEDLINE records

S19 (MH "Medicare")

S20 TI medicare OR AB medicare

S21 S19 OR S20

S22 S18 AND S21

S23 S11 AND S16 Limiters - Exclude MEDLINE records; Randomized Controlled Trials

\section{Inclusion and Exclusion Criteria}

\section{Criteria for Inclusion/Exclusion of Studies in the Review}

The criteria for inclusion and exclusion of studies for the systematic were based on the Key Questions and on the specific criteria for population, interventions, comparators, outcomes, timing, and settings (PICOTS), listed in Table A-1.

Table A-1. Inclusion and exclusion criteria: population, interventions, comparators, outcomes, timing, and settings

\begin{tabular}{|c|c|c|}
\hline PICOTS & Inclusion & Exclusion \\
\hline Population & 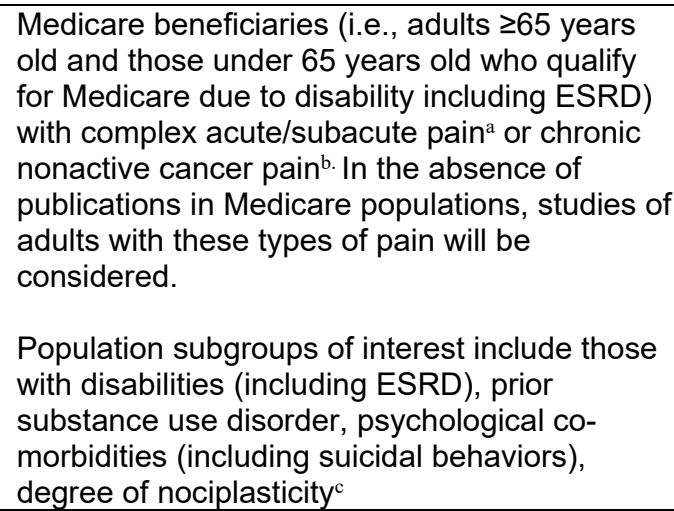 & $\begin{array}{l}\text { - Patients undergoing end-of-life care, } \\
\text { terminally ill (e.g., hospice) patients; } \\
\text { those under supervised palliative care } \\
\text { - Young, nondisabled populations }\end{array}$ \\
\hline
\end{tabular}




\begin{tabular}{|c|c|c|}
\hline PICOTS & Inclusion & Exclusion \\
\hline Intervention & $\begin{array}{l}\text { Pain management programs that address the } \\
\text { biopsychosocial model of pain and include: } \\
\text { - Multidisciplinary (interdisciplinary) teams } \\
\text { that at a minimum have the following } \\
\text { components available: pharmacotherapy } \\
\text { review and/or management, psychological } \\
\text { care (mental health services), and physical } \\
\text { reconditioning (e.g., PT, OT); studies may } \\
\text { also include other components in addition } \\
\text { to these; and } \\
\text { - Description of care coordination, case } \\
\text { management or mechanisms of } \\
\text { multidisciplinary, interdisciplinary } \\
\text { collaboration and communication } \\
\text { Integrated pain management programs (IPMPs) } \\
\text { will be defined as those that include the above } \\
\text { and are based in primary care. Comprehensive } \\
\text { pain management programs (CPMPs) will be } \\
\text { defined as those including the above but are } \\
\text { not based in primary care. }\end{array}$ & $\begin{array}{l}\text { - Unimodal pain management } \\
\text { - Pain management confined to a single } \\
\text { provider type, practice, or isolated } \\
\text { method of management } \\
\text { - Programs focused on functional } \\
\text { restoration and/or occupational health } \\
\text { focused on return to work such as work } \\
\text { hardening programs, unless they are } \\
\text { specifically done in a Medicare eligible } \\
\text { population or are clearly applicable to the } \\
\text { Medicare population } \\
\text { - Programs in very young and nondisabled } \\
\text { populations (e.g., military populations) } \\
\text { - Studies evaluating incremental value of } \\
\text { adding a single treatment modality to } \\
\text { another single treatment modality (e.g., } \\
\text { addition of CBT to PT). } \\
\text { - Postoperative or post-trauma } \\
\text { rehabilitation programs }\end{array}$ \\
\hline Comparator & Any & None \\
\hline Outcome & $\begin{array}{l}\text { Patient oriented outcomes } \\
\text { - Primary: Pain, function (focus on "success" } \\
\text { if reported), opioid use } \\
\text { - Secondary: HRQOL, emotional function } \\
\text { (e.g., depression, anxiety), patient } \\
\text { satisfaction, global improvement } \\
\text { Harms, adverse events, unintended } \\
\text { consequences } \\
\text { Program-related outcomes } \\
\text { - Utilization (e.g., pain-related hospital/ED } \\
\text { visits or short-term skilled nursing facility use, } \\
\text { long term care facility or institutional care } \\
\text { transfer, Medicaid enrollment) }\end{array}$ & $\begin{array}{l}\text { Patient-oriented outcomes } \\
\text { - Nonvalidated instruments for outcomes } \\
\text { (e.g., pain, function, HRQOL, depression, } \\
\text { etc.) } \\
\text { - Intermediate outcomes (e.g., range of } \\
\text { motion, physical strength, etc.) }\end{array}$ \\
\hline Timing & $\begin{array}{l}\text { Duration of followup: Focus on persistence of } \\
\text { effects evaluated short term ( } 1 \text { to }<6 \text { months), } \\
\text { intermediate term ( } \geq 6 \text { to }<12 \text { months) and long } \\
\text { term ( } \geq 12 \text { months) following intervention; } \\
\text { immediate postintervention results are reported } \\
\text { as well. }\end{array}$ & \\
\hline Setting & Outpatient, inpatient, institutional residence & $\begin{array}{l}\text { - Inpatient or outpatient settings } \\
\text { exclusively providing treatment for } \\
\text { SUD/OUD or tertiary care, hospice, or } \\
\text { similar settings }\end{array}$ \\
\hline $\begin{array}{l}\text { Study design, } \\
\text { publication } \\
\text { type }\end{array}$ & $\begin{array}{l}\text { Inclusion will focus on RCTs. Prospective } \\
\text { cohort studies that control for confounding will } \\
\text { be considered if RCTs are not available. } \\
\text { Comparative cohorts that do not control for } \\
\text { confounding will be considered if cohorts } \\
\text { controlling for confounding are not available. In } \\
\text { the absence of comparative studies, single arm } \\
\text { (e.g., case series, pre-post studies) will be } \\
\text { considered if they are clearly relevant to the } \\
\text { Medicare population. }\end{array}$ & $\begin{array}{l}\text { - Case reports } \\
\text { - Case series (unless no comparative } \\
\text { studies) } \\
\text { - Case-control studies, cross-sectional } \\
\text { studies } \\
\text { - Conference proceedings, editorials, } \\
\text { letters, white papers, citations that have } \\
\text { not been peer-reviewed }\end{array}$ \\
\hline
\end{tabular}

$\mathrm{ED}=$ emergency department; $\mathrm{ESRD}=$ end stage renal disease; HRQOL $=$ Health-related quality of life; OT = occupational therapy; OUD = opioid use disorder; PICOTS = population, intervention, comparator, outcomes, timing, study design; PT = physical therapy; RCT = randomized control trial; SUD = substance use disorder. 
${ }^{\text {a }}$ Complex acute or subacute pain: Patients with acute pain ( $<6$ weeks duration) or subacute pain ( 6 weeks to 12 weeks duration) who are at risk of developing chronic pain).

${ }^{\mathrm{b}}$ Chronic, nonactive cancer pain (based on Mersky 1994) ${ }^{4}$ : Pain that persists for at least three months and is not associated with [active] malignant disease"; pain could, however, be resultant from a previous malignancy that is no longer active.

${ }^{c}$ The term nociplasticity has been used to describe pain resulting from altered nociception without underlying tissue damage resulting in hypersensitivity (e.g., fibromyalgia). ${ }^{5}$ Many pain conditions may have a nociplastic component. Some additional terms used in the literature include centralized pain and amplified pain.

Study Design: For all Key Questions, we focused on randomized controlled trials (RCTs) as have the least risk of bias. Nonrandomized studies in pain can be misleading due to the subjective nature of pain which may exacerbate effects of confounding, selection bias, and attentional and other nonspecific effects. We planned to include comparative nonrandomized studies that controlled for confounding only if RCTs were not available. We planned to include comparative nonrandomized studies that controlled for confounding only if RCTs were not available. However, RCTs were identified for each program type and nonrandomized studies were not included.

Single arm studies (i.e., pre-post studies, case series) would have been considered in the absence of comparative studies only if they are clearly relevant to the Medicare population (i.e., those $\geq 65$ or those eligible based on disability as defined for Medicare). No such studies were identified. Systematic reviews recent enough to cover the majority of the available evidence for a given question or subquestion and that evaluated a cohesive group of interventions and outcomes within the scope for this review were considered for inclusion as primary evidence. No such reviews were identified.

Non-English Language Studies: We restricted to English-language articles, given the focus on Medicare eligible patients within the U.S. health care system.

\section{Process for Selecting Studies}

In accordance with the Methods Guide for Effectiveness and Comparative Effectiveness Review, ${ }^{6}$ we used the pre-established criteria above to screen citations (titles and abstracts) identified through our searches or SEADS submissions to determine eligibility for full-text review. No systematic review software (e.g., DistillerSR) was used to assist with abstract and full-text review. To ensure accuracy, any citation deemed not relevant for full-text review was reviewed by a second researcher. All citations deemed potentially eligible for inclusion by at least one of the reviewers were retrieved for full-text screening. Each full-text article was independently reviewed for eligibility by two team members. Any disagreements were resolved by consensus. A flow diagram of study screening and inclusion is below in Appendix B. A record of studies included in the review and those excluded at the full-text level with reasons for exclusion are listed below in Appendix D and $\mathrm{H}$, respectively.

\section{Data Extraction}

After studies were selected for inclusion, given intervention complexity (i.e., pain management models have multiple components, care pathways, participants, organizational levels) and anticipated heterogeneity of model components and delivery, we first constructed a framework to organize key structural features (e.g., setting, locus of coordination, primary care provider involvement) of models and understand relationships between essential components of models (e.g., medications use, monitoring, nonpharmacologic care). For complex interventions, there is no consensus regarding any single best approach for organizing the evidence. ${ }^{1} \mathrm{We}$ 
initially built on the framework from our Medication-Assisted Treatment Models for Opioid Use Disorder technical brief, ${ }^{7}$ which organized models as practice-based (i.e., those implemented in an individual stand-alone clinic) and system-based (involving multiple levels of a healthcare system). We considered whether the program/model is integrated (based in primary care) or comprehensive (not based in primary care).

Organization by key model components (e.g., pharmacologic therapy, physical function, care coordination, psychological services) was considered. Data abstraction reflected these elements, keeping the template for intervention description and replication (TIDieR) checklist in mind. ${ }^{8}$ Elements included, but were not limited to: study design, year, setting, country, sample size, eligibility criteria, attrition, population and clinical characteristics (including age, sex, comorbidities such as medical or psychological disabilities), diagnostic classifications/ information, pain characteristics (e.g., degree of nociplasticity), sociodemographic factors, intervention component characteristics (including the type, number, intensity, duration of and adherence to treatments), processes of care (e.g., provider types, roles, coordination, decision support, sequence of care components, modifications to treatment), comparator characteristics, program/model characteristics (e.g., goals, emphasis, target population, staffing), and results (including harms). Data on outcomes evaluated immediately postintervention and at short term ( 1 to $<6$ months), intermediate term ( $\geq 6$ to $<12$ months) and long term ( $\geq 12$ months) following the intervention were abstracted. Information relevant for assessing applicability was abstracted, including the characteristics of the population, interventions, and the number of patients enrolled relative to the number assessed for eligibility. All study data abstraction was verified for accuracy and completeness by a second team member.

\section{Risk of Bias Assessment of Individual Studies}

Methods from the Methods Guide for Effectiveness and Comparative Effectiveness Review ${ }^{6}$ were used in concordance with the approach recommended in the chapter, Assessing the Risk of Bias of Individual Studies When Comparing Medical Interventions. ${ }^{6,9}$ RCTs were assessed based on criteria established in the Cochrane Handbook for Systematic Reviews of Interventions (Chapter 8.5 Risk of Bias Tool)..$^{10}$ Based on the risk of bias assessment, individual included studies will be rated as being "good," "fair," or "poor" quality as described below in Table A-2. 
Table A-2. Criteria for grading the quality of individual studies

\begin{tabular}{|l|l|}
\hline Rating & Description and Criteria \\
\hline Good & $\begin{array}{l}\text { - Least risk of bias, results generally considered valid } \\
\text { - Employ valid methods for selection, inclusion, and allocation of patients to treatment; report } \\
\text { similar baseline characteristics in different treatment groups; clearly describe attrition and have } \\
\text { low attrition; use appropriate means for preventing bias (e.g., blinding of patients, care providers, } \\
\text { and outcomes assessors); and use appropriate analytic methods (e.g., intention-to-treat analysis) }\end{array}$ \\
\hline Fair & $\begin{array}{l}\text { - Susceptible to some bias but not enough to necessarily invalidate results } \\
\text { - May not meet all criteria for good quality, but no flaw is likely to cause major bias; the study may } \\
\text { be missing information making it difficult to assess limitations and potential problems }\end{array}$ \\
\hline Poor & $\begin{array}{l}\text { Category is broad; studies with this rating will vary in strengths and weaknesses; some fair-quality } \\
\text { studies are likely to be valid, while others may be only possibly valid }\end{array}$ \\
& $\begin{array}{l}\text { - Significant flaws that imply biases of various kinds that may invalidate results; "fatal flaws" in } \\
\text { - Sesious problems with intervention delivery } \\
\text { Studies are at least as likely to reflect flaws in the study design or execution as the true difference } \\
\text { between the compared interventions }\end{array}$ \\
& $\begin{array}{l}\text { Considered to be less reliable than higher quality studies when synthesizing the evidence, } \\
\text { particularly if discrepancies between studies are present }\end{array}$ \\
\hline
\end{tabular}

Like many nonpharmacological therapies (e.g., exercise or psychological therapy), it was not possible for studies to effectively blind participants (or providers) with regard to program inclusion. Nonetheless, studies were downgraded to fair for lack of blinding as it may still result in bias from patient expectations of treatment, attentional affects, and performance bias; this is consistent with the approach used in prior AHRQ reviews of nonpharmacological treatments for pain

\section{Data Synthesis and Analysis}

We constructed evidence tables based on the organizational framework to include study and model characteristics (as discussed above), results of interest, and quality ratings for all included studies, and summary tables to highlight the main findings (Appendix B). We reviewed and highlighted studies by using a hierarchy-of-evidence approach, focusing our synthesis on the highest quality data for each Key Question. Data were qualitatively summarized in tables, using ranges and descriptive analysis and interpretation of the results.

Meta-analyses were conducted to get more precise effect estimates. To determine the appropriateness of meta-analysis, we considered clinical and methodological diversity and assessed statistical heterogeneity. For continuous outcomes (e.g., pain, function, quality of life, depression), mean difference (MD) was used as the effect measure if the outcomes were reported using the same scale, and standardized mean difference (SMD) was used when the outcomes were reported in different scales. Pain scales were converted to a common 0 to 10 scale and pooled using MD when pain intensity was reported on a visual analog scale (VAS) or numerical rating (NRS) scale, or when it was clear that the outcome measured pain intensity. When pain was reported using other instruments, such as the McGill Pain Questionnaire (MPQ) (which is based on pain descriptors, rather than a VAS or NRS for pain intensity), we also conducted sensitivity analyses using SMD. In the meta-analyses, the adjusted or unadjusted mean treatment difference from the analysis of covariance or other appropriate regression models was used if available, followed by the difference in follow-up score and change score between treatment groups. When standard deviation for the followup score was not reported, or could not be calculated from the reported data, it was imputed using the average coefficient of variation from 
the other included studies. For binary outcomes, risk ratio was used as the effect measure. For cluster randomized trials, we used treatment differences accounting for the intracluster correlation if reported; otherwise, we corrected for clustering using the intracluster correlation by calculating the design effect and the effective sample sizes before combining with individually randomized trials. We used reported intracluster correlation or intracluster correlation assumed in the sample size calculation as reported in the original publication. If a study reported results from more than one treatment (intervention) arm that could be combined in the same meta-analysis, results from these treatment arms were combined first so each study was included only once in each meta-analysis.

We used a random effects model based on the profile likelihood method ${ }^{11}$ to combine the included trials. Statistical heterogeneity among the studies was assessed using Cochran's $\chi^{2}$ test and the $I^{2}$ statistic. $^{12}$ Within each Key Question, results were presented separately for programs/models considered to be integrated (based in primary care) and comprehensive (not based in primary care), and any primary outcomes, as prioritized in Table A-1, were presented first. The primary analysis was stratified by the duration of followup (post-treatment, short term when 1 month $<$ followup $\leq 6$ months, intermediate term when 6 month $\leq$ followup $<12$ months, long term when followup $\geq 12$ months). When results were reported at more than one time point that fell in the same time period, we used results from the longest period in the primary analysis, and other time points in the sensitivity analysis as appropriate. One sensitivity analysis included the most frequent time points. Additional sensitivity analyses were conducted by excluding outlying studies and studies rated as poor. Small study effects were not tested when the number of studies was larger than 10 given the heterogeneity related the populations (and sources of pain) as well as program characteristics (e.g., intensity, format) and outcomes reported and small number of trials available for most analyses. Meta-regression was done to formally assess differences between higher and lower intensity programs when sufficient numbers of studies for each intensity were available. All meta-analyses were conducted using Stata/SE 16.1 (StataCorp, College Station, TX). ${ }^{13}$

Consistent with our prior chronic pain report, ${ }^{14,15}$ we considered the impact of higher intensity programs (intensity $\geq 20$ hours/week or $>80$ hours total) versus lower intensity programs $(<20$ hours/week) where data were available. We classified the magnitude of effects for continuous measures of pain and function using the same system as in prior Agency for Healthcare Research and Quality (AHRQ) reviews on pain. ${ }^{14-18}$ Effects below the threshold for small were categorized as no effect. Where possible, we reported on the proportion of patients meeting thresholds for clinically important differences (e.g., $>30 \%$ pain relief). We did not conduct analyses to evaluate potential markers for publication bias given the substantial heterogeneity in study designs, programs, length of followup and patient populations and small number of trials available for most analyses.

\section{Grading the Strength of the Body of Evidence}

Outcomes to be assessed for strength of evidence (SOE) were prioritized based on input from the Technical Expert Panel (TEP). Based on this prioritized list, the strength of evidence for comparison-outcome pairs within each Key Question was initially assessed by one researcher for each clinical outcome (see PICOTS, Table A-1) by using the approach described in the Methods Guide for Effectiveness and Comparative Effectiveness Review. ${ }^{6}$ To ensure consistency and validity of the evaluation, the initial assessment was independently reviewed by at least one other experienced investigator using the following criteria: 
- Study limitations (low, medium, or high level of study limitations)

- Rated as the degree to which studies for a given outcome are likely to reduce bias based on study design and conduct. The aggregate risk of bias across individual studies reporting an outcome is considered.

- Consistency (consistent, inconsistent, or unknown/not applicable)

- Rated by degree to which studies find similar magnitude of effect (i.e., range sizes are similar) or same direction of effect (i.e., effect sizes have the same sign)

- Directness (direct or indirect)

- Rated by degree to which the outcome is directly or indirectly related to health outcomes of interest. Patient centered outcomes are considered direct

- Precision (precise or imprecise)

- Describes the level of certainty of the estimate of effect for a particular outcome with a precise estimate being on that allows a clinically useful conclusion. This may be based on sufficiency of sample size and number of events, and if these are adequate, the interpretation of the confidence interval. When quantitative synthesis is not possible, sample size and assessment of variance within individual studies will be considered.

- Reporting bias (suspected or undetected)

○ Publication bias, selective outcome reporting, and selective analysis reporting are types of reporting bias. Reporting bias is difficult to assess as systematic identification of unpublished evidence is challenging. If sufficient numbers of RCTs ( $>10)$ are available, quantitative funnel plot analysis may be done.

The SOE was assigned an overall grade of high, moderate, low, or insufficient (see Table A3 , below) according to a four-level scale by evaluating and weighing the combined results of the above domains.

Table A-3. Description of strength of evidence grades

\begin{tabular}{|l|l|}
\hline Grade & Definition \\
\hline High & $\begin{array}{l}\text { We are very confident that the estimate of effect lies close to the true effect for this outcome. The } \\
\text { body of evidence has few or no deficiencies. We believe that the findings are stable (i.e., another } \\
\text { study would not change the conclusions). }\end{array}$ \\
\hline Low & $\begin{array}{l}\text { We are moderately confident that the estimate of effect lies close to the true effect for this outcome. } \\
\text { The body of evidence has some deficiencies. We believe that the findings are likely to be stable, but } \\
\text { some doubt remains. }\end{array}$ \\
\hline $\begin{array}{l}\text { We have limited confidence that the estimate of effect lies close to the true effect for this outcome. } \\
\text { The body of evidence has major or numerous deficiencies (or both). We believe that additional } \\
\text { evidence is needed before concluding either that the findings are stable or that the estimate of } \\
\text { effect is close to the true effect. }\end{array}$ \\
$\begin{array}{l}\text { We have no evidence, we are unable to estimate an effect, or we have no confidence in the } \\
\text { estimate of effect for this outcome. No evidence is available, or the body of evidence has } \\
\text { unacceptable deficiencies, precluding reaching a conclusion. }\end{array}$ \\
\hline
\end{tabular}

\section{Assessing Applicability}

Applicability to the Medicare population, i.e., patients eligible for Medicare due to age $\geq 65$ or disability (including end-stage renal disease), was assessed based on the Evidence-based Practice Center (EPC) Methods Guide, ${ }^{6}$ using the PICOTS framework. Applicability refers to the degree to which outcomes associated with the intervention are likely to be similar across patients and settings relevant to the care of the Medicare population based on the populations, 
interventions comparisons and outcomes synthesized across included studies. Factors that may affect applicability which we identified a priori generally reflect the contextual components outlined in the conceptual logic diagram (Figure 1) and include (1) patient factors (e.g., age and disability status, medical and psychiatric comorbidities, symptom severity, duration and underlying pain condition); (2) intervention factors such as program structure and goals (e.g., patient return to work or activities of daily living), delivery of program components (e.g., session or component types, duration, intensity (i.e., hours/week, total hours) and frequency, level of adherence, support and coordination); (3) comparators, including feasibility of comparisons between programs; (4) outcomes (e.g., use of nonstandardized or unvalidated outcomes); and (5) settings (e.g., outpatient versus residential). For example, intensive programs of consisting of multiple daily sessions for 8 weeks geared toward rehabilitation for return to work may have limited applicability to retired populations $>65$ years old with chronic pain. We used information on the factors to assess the extent to which programs and their components are likely most relevant to real-world clinical practice in typical United States settings that include the Medicare population. We provided a qualitative summary of our assessment.

\section{Peer Review and Public Commentary}

Peer reviewers with relevant clinical or methodological expertise were invited to provide external peer review of this systematic review. AHRQ and an associate editor also provided comments. In addition, the draft report was posted on the AHRQ website for 4 weeks for public comment. All comments were reviewed, appraised, and addressed as appropriate. Edits were made for clarity and accuracy; however, no changes were made to the evidence or to our conclusions.

\section{Contextual Questions}

We followed the methods of the U.S. Preventive Services Task Force (USPSTF) to evaluate the Contextual Questions. ${ }^{19} \mathrm{~A}$ targeted search was designed by a medical librarian with experience in searching for contextual question evidence for USPSTF reviews, including searching for systematic and narrative reviews. The team also identified any information relevant to this question opportunistically, while reviewing comprehensive literature searches for Key Questions, and incorporated relevant information from TEP calls. The information on the Contextual Questions were summarized in the introduction of the report and presented in the Results section of the report. Appendix C contains additional information related to the Contextual Questions. 


\section{Appendix B. Results Overview}

\section{Results of Literature Searches}

Figure B-1. Literature flow diagram

Abstracts of potentially relevant articles identified $(\mathrm{N}=10,953)$

Ovid $^{\circledast}$ MEDLINE $^{\circledR}$, Cochrane ${ }^{a}$, PsycINFO $^{\circledR}$, CINAHL $^{\circledast}(n=10,782)$

Handsearching/bibliography review of included studies, systematic reviews $(n=76)$

Peer, public review/comment $(n=95)$

Duplicates $(n=1,158)$

Abstracts of potentially relevant articles reviewed for relevance to Key Questions $(n=9,795)$

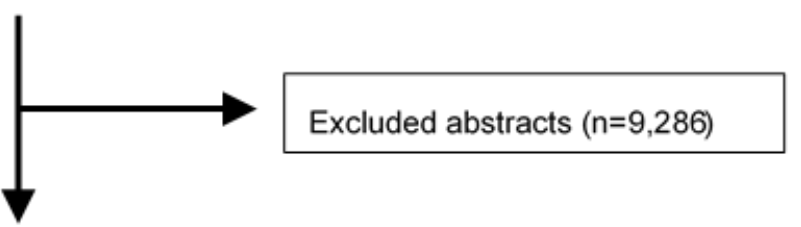

Full text articles reviewed for relevance to Key Questions $(n=509)$

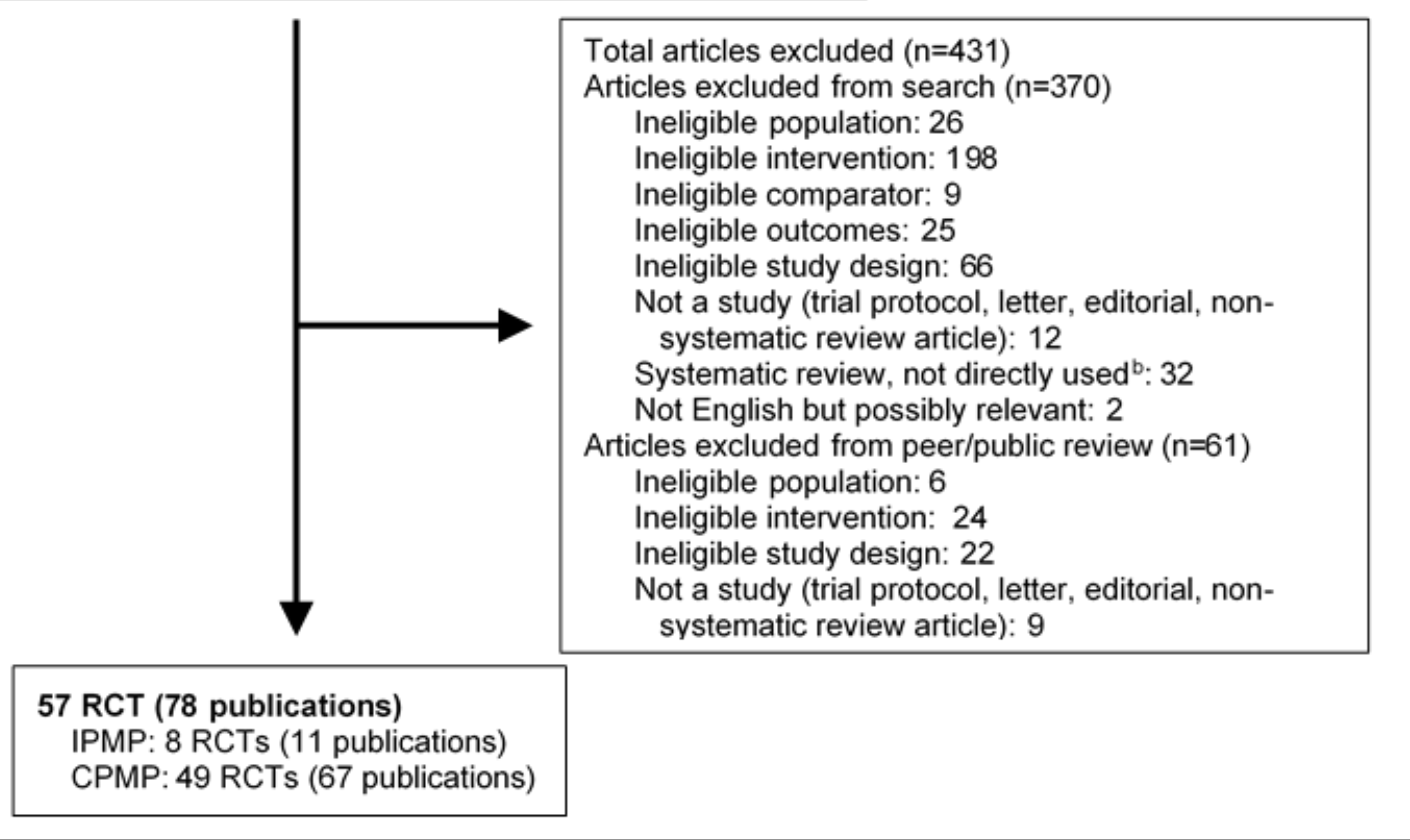

${ }^{a}$ Cochrane databases include the Cochrane Central Register of Controlled Trials and the Cochrane Database of Systematic Reviews

${ }^{\mathrm{b}}$ Studies checked for inclusion. 
A total of 10,953 references were identified, 10,782 from electronic database searches and an additional 76 from handsearching and checking the bibliographies of included studies and systematic reviews, and 95 from peer review/public comment. After dual review of abstracts, 509 articles were evaluated for inclusion at the full-text level. A total of 57 randomized controlled trials (RCTs) (in 78 publications) met inclusion criteria and were included for Key Questions 1 and 2 addressing effectiveness and safety. Forty-three were rated fair quality (75\%) and $14(25 \%)$ were rated poor quality. Search results and selection of studies are summarized in the literature flow diagram above (Figure B-1). A list of included studies appears in Appendix D and excluded studies with reason for exclusion in Appendix $\mathrm{H}$.

Table B-1. Number of studies overall and by Key Question

\begin{tabular}{|c|c|c|c|c|}
\hline Program & Comparator & $\begin{array}{l}\text { KQ1: } \\
\text { n=Number of } \\
\text { RCTs (Number of } \\
\text { Publications) }\end{array}$ & $\begin{array}{l}\text { KQ2: } \\
\text { n=Number of RCTs } \\
\text { (Number of } \\
\text { Publications) }\end{array}$ & $\begin{array}{l}\text { Total: } \\
\text { n=Number } \\
\text { of RCTs } \\
\text { (Number of } \\
\text { Publications) }\end{array}$ \\
\hline \multirow[t]{4}{*}{ IPMP } & Usual care or waitlist & $7(10)^{20-29}$ & NA & NA \\
\hline & Provider vs. patient program & NA & $1^{20}$ & $\mathrm{NA}$ \\
\hline & Program with and without online support & NA & $1^{30}$ & NA \\
\hline & Any & $7(10)^{20-29}$ & $2^{20,30}$ & $8(11)^{20-30}$ \\
\hline \multirow[t]{13}{*}{ CPMP } & Usual care or waitlist & $23(30)^{31-60}$ & NA & $\mathrm{NA}$ \\
\hline & Physical activity & $\begin{array}{l}15(21)^{33,34,38,45,46,48-} \\
50,61-73\end{array}$ & NA & NA \\
\hline & Psychological therapy & $5(6)^{38,45,46,48,60,74}$ & NA & NA \\
\hline & Pharmacologic therapy & $5(13)^{75-87}$ & NA & NA \\
\hline & $\begin{array}{l}\text { Pharmacologic therapy plus physical } \\
\text { activity }\end{array}$ & $2(3)^{80,81,88}$ & NA & NA \\
\hline & Program total hours (greater vs. fewer) & NA & $4(7)^{33,34,43,62,63,89,90}$ & NA \\
\hline & Program setting (inpatient vs. outpatient) & NA & $4(6)^{41,42,55,58,59,70}$ & NA \\
\hline & $\begin{array}{l}\text { Program components (with vs. without } \\
\text { additional psychological or physical } \\
\text { components) }\end{array}$ & NA & $3^{56,91,92}$ & NA \\
\hline & $\begin{array}{l}\text { Preintervention assessment (with vs. } \\
\text { without) }\end{array}$ & NA & $2^{93,94}$ & NA \\
\hline & Session format (group vs. individual) & NA & $1^{90}$ & NA \\
\hline & Booster sessions (with vs. without) & NA & $1^{72}$ & $\mathrm{NA}$ \\
\hline & Other comparisons $^{a}$ & NA & $2(3)^{95,96}$ & NA \\
\hline & Any & $41(58)^{31-55,57-88}$ & $\begin{array}{l}16(22)^{33,34,41-} \\
43,55,56,58,59,62,63,70,72,89-97\end{array}$ & $49(67)^{31-97}$ \\
\hline $\begin{array}{l}\text { IPMP } \\
\text { and } \\
\text { CPMP }\end{array}$ & Any & $48(68)^{20-29,31-55,57-88}$ & $\begin{array}{l}18(24)^{20,30,33,34,41-} \\
43,55,56,58,59,62,63,70,72,89-97\end{array}$ & $57(78)^{20-97}$ \\
\hline
\end{tabular}

$\mathrm{CPMP}=$ comprehensive pain management program; IPMP = integrated pain management program; KQ = Key Question; NA = not applicable; $\mathrm{RCT}=$ randomized controlled trial.

a Comparisons included a function-centered vs. a pain-centered approach to CPMP in one trial and an "exposure in vivo" approach vs. a graded activity approach to CPMP in the other trial.

\section{Description of Included Studies}

Tables B-2 and B-3 below provide an overview of the trial and population characteristics for RCTs that address Key Questions 1 (effectiveness and safety) and 2 (program factors), respectively. Data for trials evaluating integrated pain management programs (IPMPs) and comprehensive pain management programs (CPMPs) are presented separately. Weighted mean 
or proportions are presented for all IPMP or CPMP trials and then for these programs versus each specific comparator. 
Table B-2. Study and population characteristics for trials addressing KQ1 by type of program and comparator

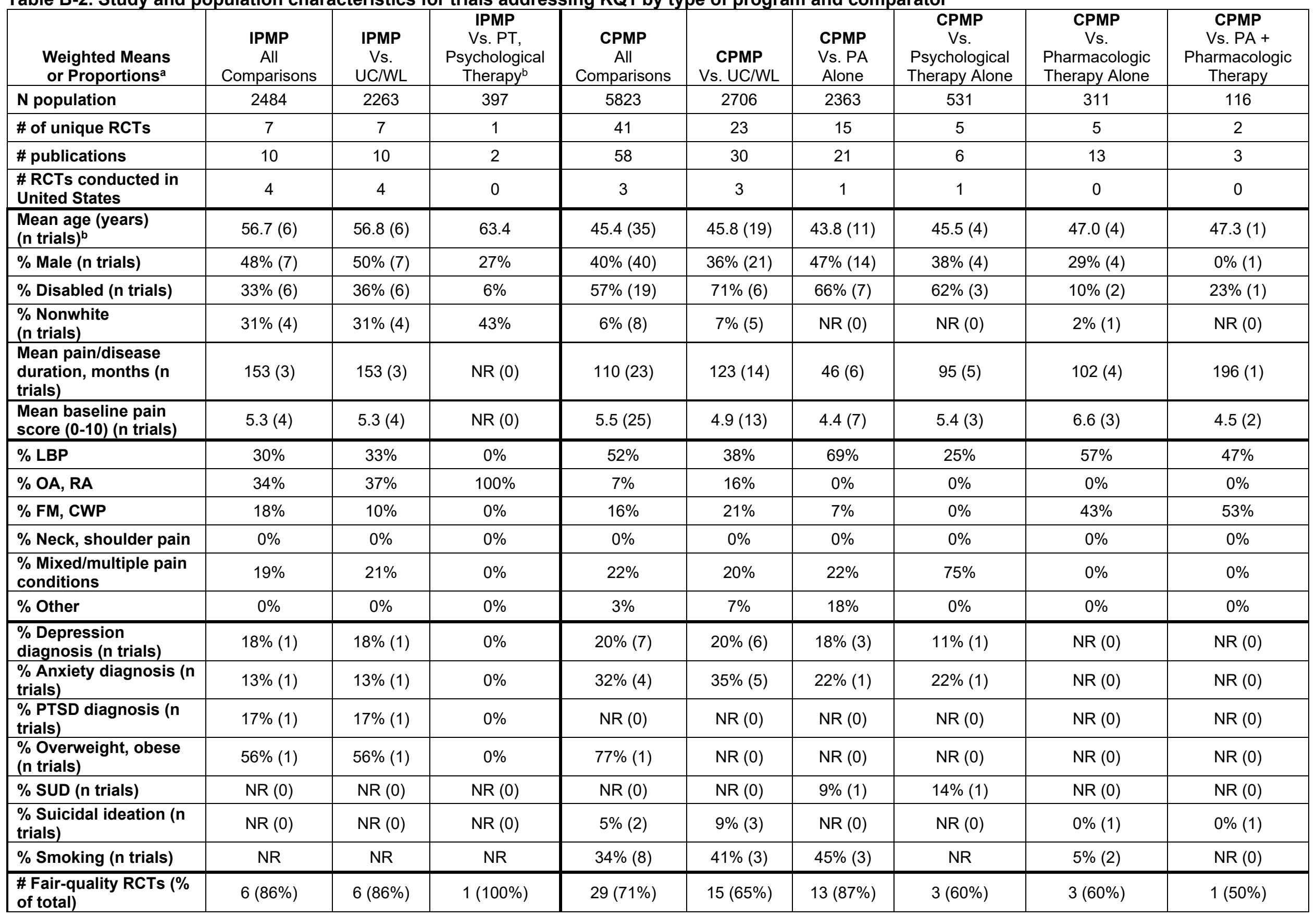




\begin{tabular}{|c|c|c|c|c|c|c|c|c|c|}
\hline $\begin{array}{l}\text { Weighted Means } \\
\text { or Proportions }^{\text {a }}\end{array}$ & $\begin{array}{c}\text { IPMP } \\
\text { All } \\
\text { Comparisons } \\
\end{array}$ & $\begin{array}{l}\text { IPMP } \\
\text { Vs. } \\
\text { UC/WL } \\
\end{array}$ & $\begin{array}{c}\text { IPMP } \\
\text { Vs. PT, } \\
\text { Psychological } \\
\text { Therapy }^{\mathrm{b}} \\
\end{array}$ & $\begin{array}{c}\text { CPMP } \\
\text { All } \\
\text { Comparisons } \\
\end{array}$ & $\begin{array}{c}\text { CPMP } \\
\text { Vs. UC/WL } \\
\end{array}$ & $\begin{array}{l}\text { CPMP } \\
\text { Vs. PA } \\
\text { Alone } \\
\end{array}$ & $\begin{array}{c}\text { CPMP } \\
\text { Vs. } \\
\text { Psychological } \\
\text { Therapy Alone } \\
\end{array}$ & $\begin{array}{c}\text { CPMP } \\
\text { Vs. } \\
\text { Pharmacologic } \\
\text { Therapy Alone } \\
\end{array}$ & $\begin{array}{c}\text { CPMP } \\
\text { Vs. PA + } \\
\text { Pharmacologic } \\
\text { Therapy } \\
\end{array}$ \\
\hline $\begin{array}{l}\text { \# Poor-quality RCTs } \\
\text { (\% of total) }\end{array}$ & $1(14 \%)$ & $1(14 \%)$ & NA & $12(29 \%)$ & $8(35 \%)$ & $2(13 \%)$ & $2(40 \%)$ & $2(40 \%)$ & $1(50 \%)$ \\
\hline
\end{tabular}

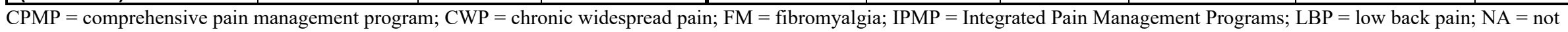
applicable; $\mathrm{NR}=$ Not reported; $\mathrm{OA}=$ osteoarthritis; $\mathrm{PTSD}=$ post-traumatic stress disorder; RA $=$ rheumatoid arthritis; $\mathrm{SUD}=$ substance use disorder

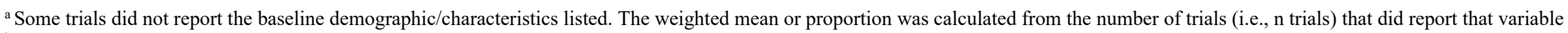

${ }^{\mathrm{b}}$ Data are for one trial with three arms

Table B-3. Study and population characteristics for trials addressing KQ2 by type of program and comparator

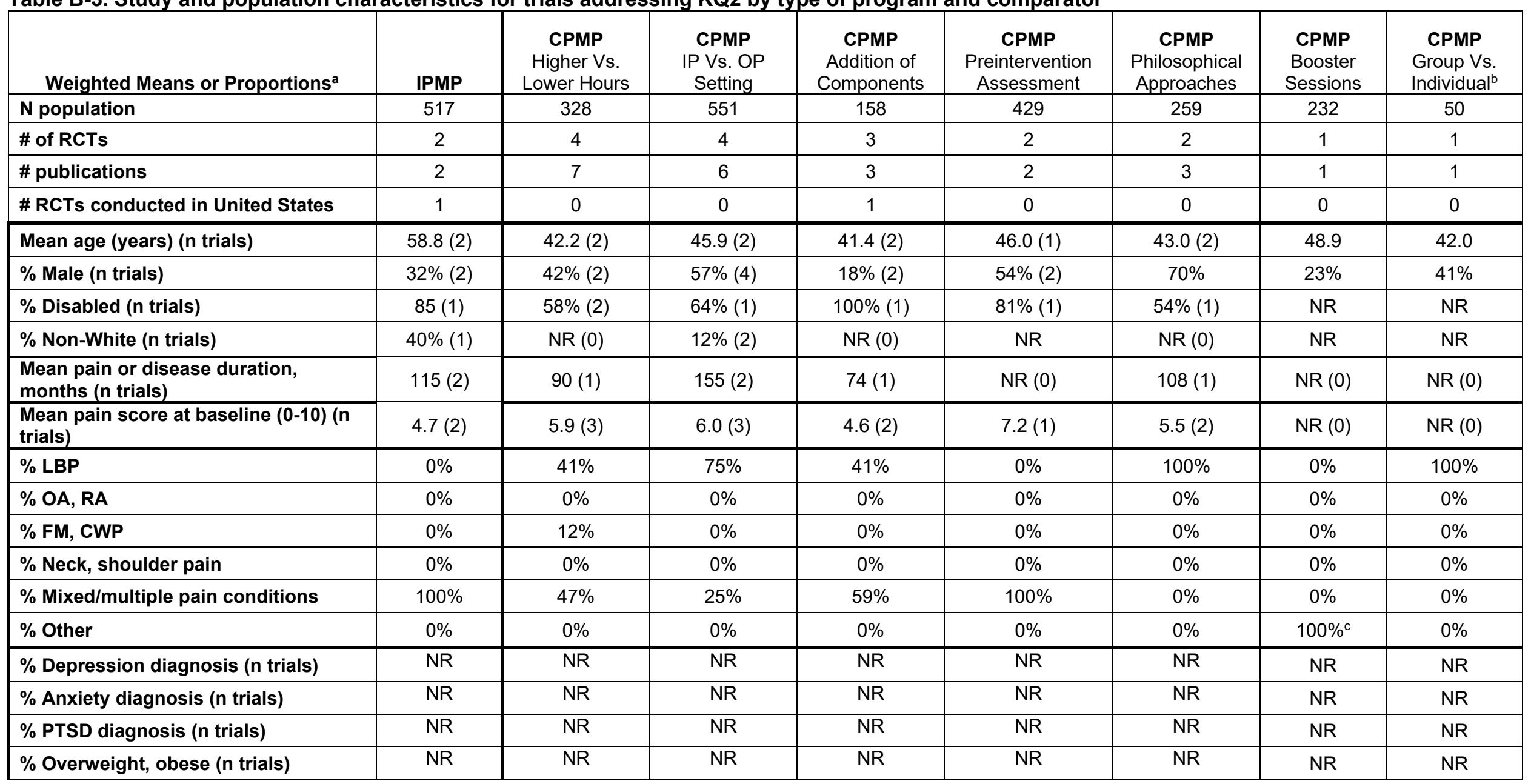




\begin{tabular}{|c|c|c|c|c|c|c|c|c|}
\hline Weighted Means or Proportions ${ }^{a}$ & IPMP & $\begin{array}{c}\text { CPMP } \\
\text { Higher Vs. } \\
\text { Lower Hours }\end{array}$ & $\begin{array}{l}\text { CPMP } \\
\text { IP Vs. OP } \\
\text { Setting }\end{array}$ & $\begin{array}{c}\text { CPMP } \\
\text { Addition of } \\
\text { Components }\end{array}$ & $\begin{array}{c}\text { CPMP } \\
\text { Preintervention } \\
\text { Assessment }\end{array}$ & $\begin{array}{c}\text { CPMP } \\
\text { Philosophical } \\
\text { Approaches }\end{array}$ & $\begin{array}{c}\text { CPMP } \\
\text { Booster } \\
\text { Sessions }\end{array}$ & $\begin{array}{l}\text { CPMP } \\
\text { Group Vs. } \\
\text { Individual }^{\mathrm{b}}\end{array}$ \\
\hline$\%$ SUD (n trials) & NR & NR & $\mathrm{NR}$ & $\mathrm{NR}$ & NR & NR & NR & NR \\
\hline$\%$ Suicidal ideation (n trials) & NR & NR & NR & NR & NR & NR & NR & NR \\
\hline \% Smoking & NR & NR & NR & NR & NR & NR & NR & NR \\
\hline \# Fair-quality RCTs (\% of total) & $2(100 \%)$ & $3(75 \%)$ & 0 & $2(67 \%)$ & $2(100 \%)$ & $2(100 \%)$ & $1(100 \%)$ & 0 \\
\hline \# Poor-quality RCTs (\% of total) & 0 & $1(25 \%)$ & $4(100 \%)$ & $1(33 \%)$ & 0 & 0 & 0 & $1(100 \%)$ \\
\hline
\end{tabular}

$\mathrm{OA}=$ osteoarthritis; PTSD = post-traumatic stress disorder; RA = rheumatoid arthritis; SUD = substance use disorder

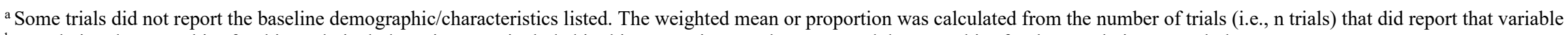

${ }^{b}$ Population demographics for this study include patients not included in this comparison; authors reported demographics for the population as a whole.

${ }^{\mathrm{c}}$ All patients included in this study had chronic back pain (at any location). 


\section{Summary Results Tables}

Table B-4. Summary results for trials addressing KQ1: IPMPs versus usual care, versus physical activity, and versus psychological therapy

\begin{tabular}{|c|c|c|c|c|c|}
\hline $\begin{array}{l}\text { Author, Year } \\
\text { Country } \\
\text { Pain Duration } \\
\text { Study Design } \\
\text { Study Quality } \\
\end{array}$ & $\begin{array}{l}\text { Intervention and } \\
\text { Comparator (n): } \\
\text { Duration/Intensity, } \\
\text { Session Format, } \\
\text { Setting } \\
\end{array}$ & Population & $\begin{array}{l}\text { Primary Outcomes: } \\
\text { Pain, Function, and Opioid Use }\end{array}$ & $\begin{array}{l}\text { Secondary Outcomes: } \\
\text { HRQOL, Psychological } \\
\text { Measures, Global } \\
\text { Improvement, Patient } \\
\text { Satisfaction }\end{array}$ & $\begin{array}{l}\text { Harms } \\
\text { Utilization }\end{array}$ \\
\hline $\begin{array}{l}\text { Allen, } 2016 \\
\text { USA } \\
\text { Mean duration of } \\
\text { pain: } 170 \text { months } \\
\text { Cluster RCT } \\
\text { Fair }\end{array}$ & $\begin{array}{l}\text { A. Patient focused } \\
\text { multidisciplinary } \\
\text { treatment + provider } \\
\text { focused } \\
\text { multidisciplinary } \\
\text { treatment + usual care } \\
\text { (n=151 patients, } 15 \\
\text { providers): } 12 \text { months } \\
\text { (time duration NR), } \\
\text { individual, Community- } \\
\text { based outpatient clinics } \\
\\
\text { B. Usual care ( } n=149 \\
\text { patients, } 15 \text { providers) }\end{array}$ & $\begin{array}{l}\text { Patient Participants } \\
\text { Mean age: } 61 \text { years } \\
\text { Male: } 91 \% \\
\text { Non-White race: } 50 \% \\
\text { Pain etiology/type: Arthritis } \\
\text { Joints with OA: } \\
\text { - Knee only: } 79 \% \\
\text { - Hip only: } 11 \% \\
\text { - Knee and hip: } 10 \% \\
\text { Disabled: } 33 \% \\
\text { Fair or poor health: } 38 \% \\
\text { Mean BMl: } 33.8 \mathrm{~kg} / \mathrm{m} 2 \\
\text { Provider Participants } \\
\text { Mean study patients per } \\
\text { provider: } 10.0 \\
\text { <15\% females in patient } \\
\text { panel: } 83 \% \\
\text { Male: } 40 \% \\
\text { Provider Type: } \\
\text { - Physician: } 63 \% \\
\text { - Physician Assistant: } 23 \% \\
\text { - Nurse Practitioner: } 10 \% \\
\text { - Registered Nurse: } 3 \%\end{array}$ & 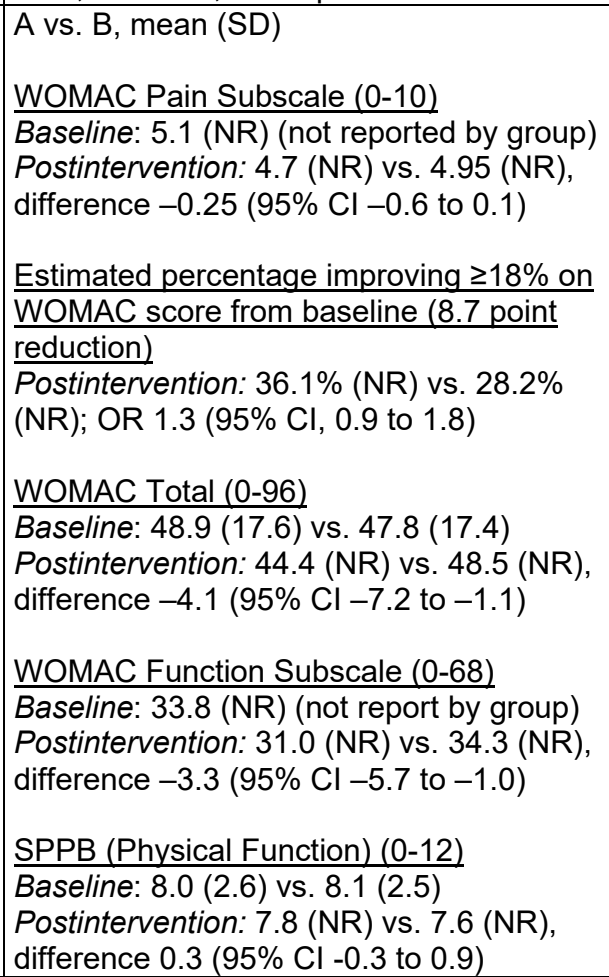 & $\begin{array}{l}\text { A vs. B, mean (SD) } \\
\text { PHQ-8 (0-24) } \\
\text { Baseline: } 7.2(5.6) \text { vs. } 6.4(5.1) \\
\text { Postintervention: } 6.2 \text { (NR) vs. } \\
6.8 \text { (NR), difference }-0.6 \text { (95\% } \\
\mathrm{Cl}-1.5 \text { to } 0.3)\end{array}$ & $\begin{array}{l}\text { A vs. B, \% (n/N) } \\
\text { Adverse Events } \\
4 \text { study-related adverse } \\
\text { events occurred, but } \\
\text { none were associated } \\
\text { with the OA } \\
\text { intervention. } \\
\text { Provider Referrals } \\
\text { Orthopedic visit: } \\
\text { - Referral: } 5.3 \%(8 / 151) \\
\text { vs. } 6.0 \%(9 / 149) \\
\text { - Receipt: } 50.0 \%(4 / 8) \\
\text { vs. } 66.7 \%(6 / 9)\end{array}$ \\
\hline
\end{tabular}




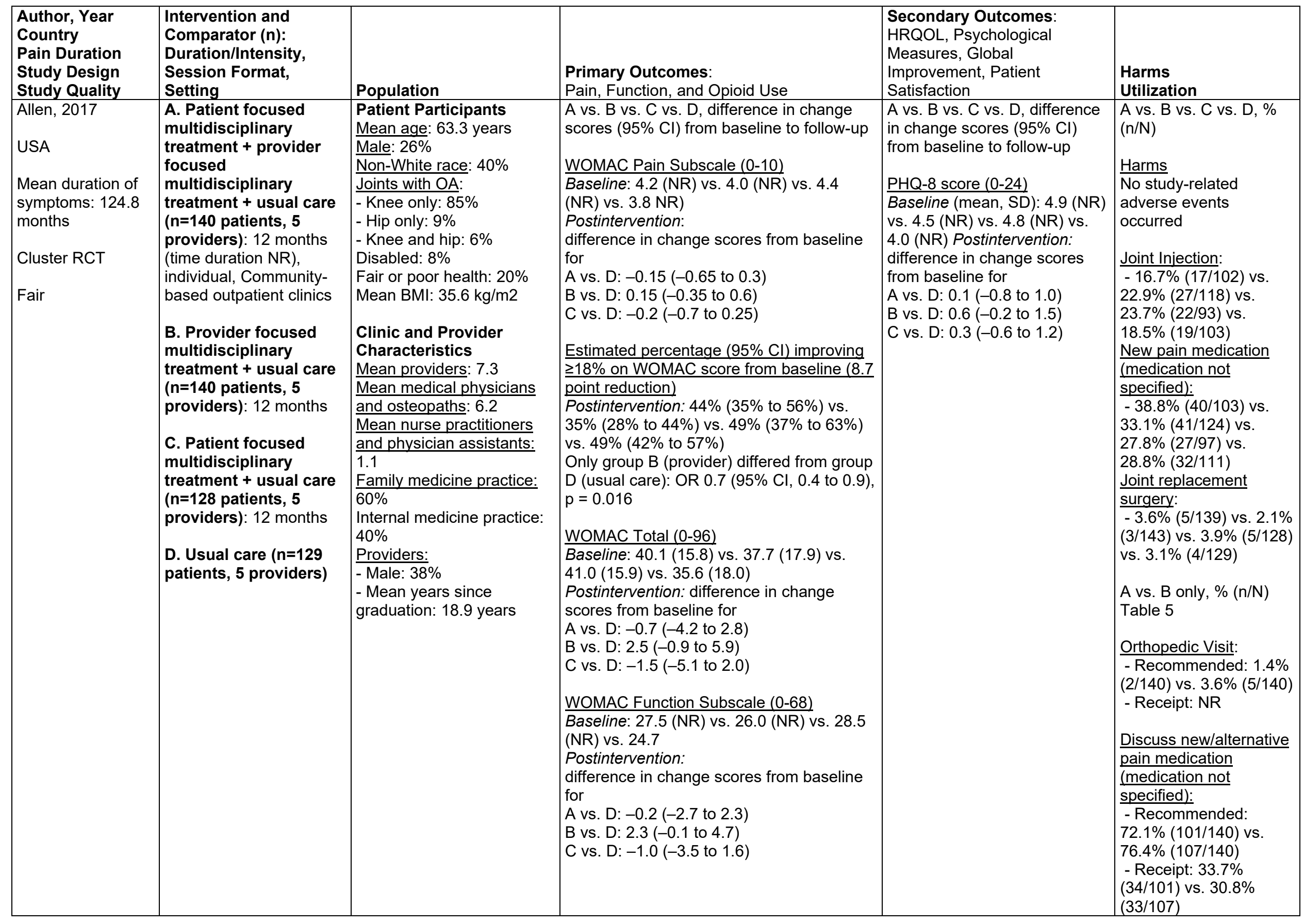




\begin{tabular}{|c|c|c|c|c|c|}
\hline $\begin{array}{l}\text { Author, Year } \\
\text { Country } \\
\text { Pain Duration } \\
\text { Study Design } \\
\text { Study Quality } \\
\end{array}$ & $\begin{array}{l}\text { Intervention and } \\
\text { Comparator (n): } \\
\text { Duration/Intensity, } \\
\text { Session Format, } \\
\text { Setting } \\
\end{array}$ & Population & $\begin{array}{l}\text { Primary Outcomes: } \\
\text { Pain, Function, and Opioid Use }\end{array}$ & $\begin{array}{l}\text { Secondary Outcomes: } \\
\text { HRQOL, Psychological } \\
\text { Measures, Global } \\
\text { Improvement, Patient } \\
\text { Satisfaction } \\
\end{array}$ & $\begin{array}{l}\text { Harms } \\
\text { Utilization }\end{array}$ \\
\hline $\begin{array}{l}\text { Allen, } 2017 \\
\text { (Continued) }\end{array}$ & & & $\begin{array}{l}\text { SPPB (Physical Function) } \\
\text { Baseline: } 8.5 \text { (NR) vs. } 8.8 \text { (NR) vs. } 8.3 \\
\text { (NR) vs. } 8.5 \text { (NR) } \\
\text { Postintervention: } \\
\text { difference in change scores from baseline } \\
\text { for } \\
\text { A vs. D: }-0.3(-0.8 \text { to } 0.3) \\
\text { B vs. D: }-0.4(-1.0 \text { to } 0.1) \\
\text { C vs. D: }-0.3(-0.8 \text { to } 0.3)\end{array}$ & & \\
\hline $\begin{array}{l}\text { Angeles, } 2013 \\
\text { Canada } \\
\text { Mean duration of } \\
\text { pain: NR } \\
\text { RCT } \\
\text { Poor }\end{array}$ & $\begin{array}{l}\text { A. IPMP ( } \mathbf{n}=\mathbf{2 9}) \\
2 \text { months ( } 2 \text { hour group } \\
\text { sessions } 1 \text { time per } \\
\text { week) (16 hours total), } \\
\text { group, Outpatient clinics } \\
\text { B. Waitlist }(\mathbf{n = 3 4 ) :}\end{array}$ & $\begin{array}{l}\text { 40 to } 59 \text { years of age: } 51 \% \\
\text { (mean age NR) } \\
\text { Male: } 27 \% \\
\text { Race/ethnicity: NR } \\
\text { Pain etiology/type: chronic } \\
\text { MSK or neuropathic pain } \\
\text { - work-related accident: } \\
31 \% \\
\text { - non work-related } \\
\text { accident: } 27 \% \\
\text { - disease process: } 60 \% \\
\text { Currently taking } \\
\text { medications for pain: } 91 \% \\
\text { - Taken medications not } \\
\text { prescribed by doctor } \\
\text { (medication not specified): } \\
\text { 37\% } \\
\text { Receiving government } \\
\text { compensation } \\
\text { - before onset of pain: } 8 \% \\
\text { - after onset of pain: } 24 \% \\
\end{array}$ & $\begin{array}{l}\text { A vs. } \mathrm{B}, \%(\mathrm{n} / \mathrm{N}) \\
\text { Early opioid prescription refill: } \\
\text { Intermediate term: } 7.7 \%(1 / 19) \text { vs. } 25 \% \\
(6 / 22), p=0.08 ; \mathrm{RR} 0.19(95 \% \mathrm{Cl} 0.03 \text { to } \\
1.46) \\
\text { Increase in opioid medication dose: } \\
\text { Intermediate term: } 11.5 \%(2 / 19) \text { vs. } 9.4 \% \\
(2 / 22), p=0.56 ; \mathrm{RR} 1.16(95 \% \mathrm{Cl} 0.18 \text { to } \\
7.45)\end{array}$ & $\begin{array}{l}\text { A vs. B, Mean change (SD } \\
\text { NR) } \\
\text { SF-36 PCS } \\
\text { Baseline: NR } \\
\text { Postintervention: change from } \\
\text { baseline }-2.9 \text { vs. }-3.0, p=0.98 \\
\text { SF-36 MCS } \\
\text { Baseline: NR } \\
\text { Postintervention: change from } \\
\text { baseline } 3.6 \text { vs. } 3.6, p=1.00\end{array}$ & $\begin{array}{l}\text { Harms } \\
\text { No study-related } \\
\text { adverse events } \\
\text { occurred. }\end{array}$ \\
\hline
\end{tabular}




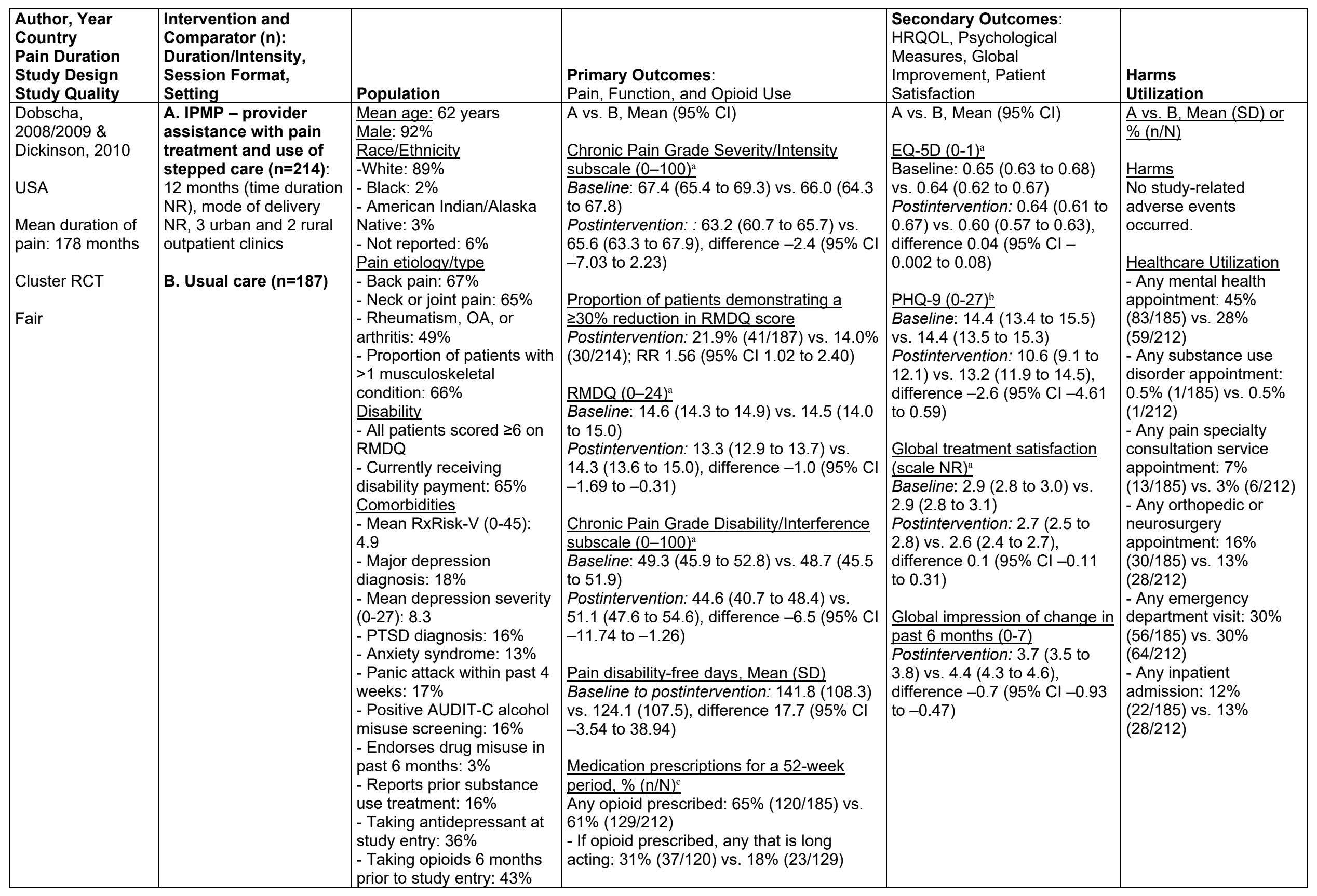




\begin{tabular}{|c|c|c|c|c|c|}
\hline $\begin{array}{l}\text { Author, Year } \\
\text { Country } \\
\text { Pain Duration } \\
\text { Study Design } \\
\text { Study Quality }\end{array}$ & $\begin{array}{l}\text { Intervention and } \\
\text { Comparator (n): } \\
\text { Duration/Intensity, } \\
\text { Session Format, } \\
\text { Setting }\end{array}$ & Population & $\begin{array}{l}\text { Primary Outcomes: } \\
\text { Pain, Function, and Opioid Use }\end{array}$ & $\begin{array}{l}\text { Secondary Outcomes: } \\
\text { HRQOL, Psychological } \\
\text { Measures, Global } \\
\text { Improvement, Patient } \\
\text { Satisfaction }\end{array}$ & $\begin{array}{l}\text { Harms } \\
\text { Utilization }\end{array}$ \\
\hline $\begin{array}{l}\text { Mas, } 2019 \\
\text { Spain } \\
\text { Mean duration of } \\
\text { pain: } 0.5 \text { to } 3 \\
\text { months } \\
\text { Cluster RCT } \\
\text { Fair }\end{array}$ & $\begin{array}{l}\text { A. IPMP ( } \mathbf{n}=\mathbf{2 6 2} \text {; } \mathbf{2 6} \\
\text { primary healthcare } \\
\text { centers): one } 10 \text { hour } \\
\text { session ( } 4 \text { hours physical } \\
\text { therapy, } 4 \text { hours } \\
\text { psychology, } 2 \text { hours for } \\
\text { questions with general } \\
\text { practitioner (10 hours } \\
\text { total), combined group } \\
\text { and individual, outpatient } \\
\text { clinic } \\
\text { B. Usual Care ( } \mathbf{n}=239 ; \\
13 \text { primary healthcare } \\
\text { centers) }\end{array}$ & $\begin{array}{l}\text { Mean age: } 47 \text { years } \\
\text { Male: } 35 \% \\
\text { Race/Ethnicity: NR } \\
\text { Pain etiology/type: } \\
\text { Subacute low back pain } \\
\text { Disability: NR } \\
\text { Comorbidities: } \\
\text { - Obese: } 17 \% \\
\text { - Overweight: } 39 \%\end{array}$ & 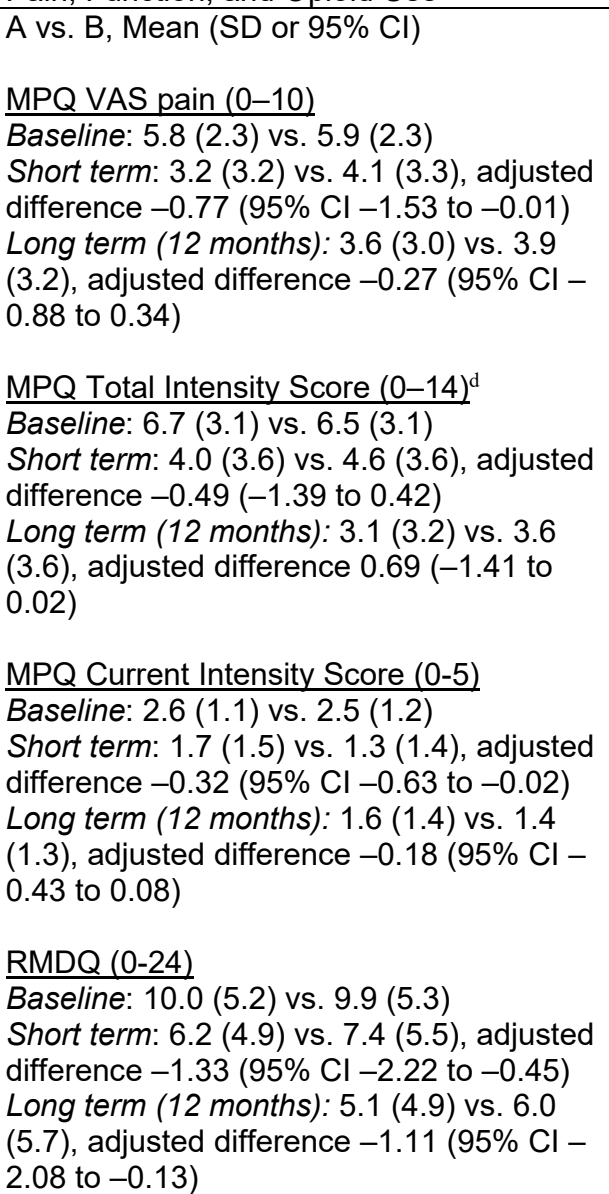 & $\begin{array}{l}\text { A vs. B, Mean (SD) } \\
\text { SF-12 Physical health (0-100) } \\
\text { Baseline: } 41.9 \text { (9.0) vs. } 40.7 \\
\text { (9.3) } \\
\text { Short term: } 46.5 \text { (8.7) vs. } 45.3 \\
\text { (9.8), adjusted difference } 0.55 \\
\text { (95\% Cl -1.19 to 2.29) } \\
\text { Long term (12 months): } 47.0 \\
\text { (8.9) vs. } 46.2(9.5), \text { adjusted } \\
\text { difference } 0.53 \text { (95\% Cl }-1.20 \\
\text { to } 2.27) \\
\text { SF-12 Mental health (0-100) } \\
\text { Baseline: } 43.4 \text { (12.8) vs. } 42.3 \\
\text { (12.4) } \\
\text { Short term: } 48.8(12.0) \text { vs. } \\
45.0 \text { (13.2), adjusted } \\
\text { difference } 2.56 \text { (95\% Cl }-0.33 \\
\text { to } 5.45) \\
\text { Long term (12 months): } 48.9 \\
\text { (11.2) vs. } 47.0(11.9), \text { adjusted } \\
\text { difference } 1.48 \text { (95\% Cl -0.86 } \\
\text { to } 3.83)\end{array}$ & NR \\
\hline
\end{tabular}




\begin{tabular}{|c|c|c|c|c|c|}
\hline $\begin{array}{l}\text { Author, Year } \\
\text { Country } \\
\text { Pain Duration } \\
\text { Study Design } \\
\text { Study Quality }\end{array}$ & $\begin{array}{l}\text { Intervention and } \\
\text { Comparator }(\mathrm{n}) \text { : } \\
\text { Duration/Intensity, } \\
\text { Session Format, } \\
\text { Setting }\end{array}$ & Population & $\begin{array}{l}\text { Primary Outcomes: } \\
\text { Pain, Function, and Opioid Use }\end{array}$ & $\begin{array}{l}\text { Secondary Outcomes: } \\
\text { HRQOL, Psychological } \\
\text { Measures, Global } \\
\text { Improvement, Patient } \\
\text { Satisfaction } \\
\end{array}$ & $\begin{array}{l}\text { Harms } \\
\text { Utilization }\end{array}$ \\
\hline $\begin{array}{l}\text { McBeth } 2012 \text { \& } \\
\text { Beasley, } 2015 \\
\text { England } \\
\text { Mean duration of } \\
\text { pain: NR } \\
\text { RCT } \\
\text { Fair }\end{array}$ & $\begin{array}{l}\text { A. IPMP with telephone } \\
\text { delivered CBT and } \\
\text { exercise ( } \mathrm{n}=112): 6 \\
\text { months, initial } \\
\text { assessment ( } 0.75 \text { to } 1 \\
\text { hour) seven weekly } \\
\text { sessions for } 6 \text { weeks } \\
\text { ( } 0.5 \text { to } 0.75 \text { hours per } \\
\text { session, } 3.5 \text { to } 5.25 \\
\text { hours each week, } 21 \text { to } \\
31.5 \text { hours over } 6 \\
\text { weeks), and a single } 0.5- \\
\text { to } 0.75-h o u r \text { session at } 3 \\
\text { and } 6 \text { months (0.5 to } 1.5 \\
\text { hours) (21.25 to } 34.75 \\
\text { hours total), individual, } \\
\text { patient home and local } \\
\text { gym } \\
\text { B. Usual Care ( } n=109 \text { ) } \\
\text { C. Telephone-delivered } \\
\text { CBT alone ( } n=112 \text { ) } \\
\text { D. Exercise alone } \\
\text { ( } \mathrm{n}=109 \text { ) }\end{array}$ & $\begin{array}{l}\text { Mean age: } 56 \text { years } \\
\text { Male: } 31 \% \\
\text { Race/Ethnicity: NR } \\
\text { Pain etiology/type: Chronic } \\
\text { widespread pain } \\
\text { Disability: NR } \\
\text { Comorbidities: } \\
\text { - Patients with severe } \\
\text { psychiatric disorders were } \\
\text { excluded }\end{array}$ & 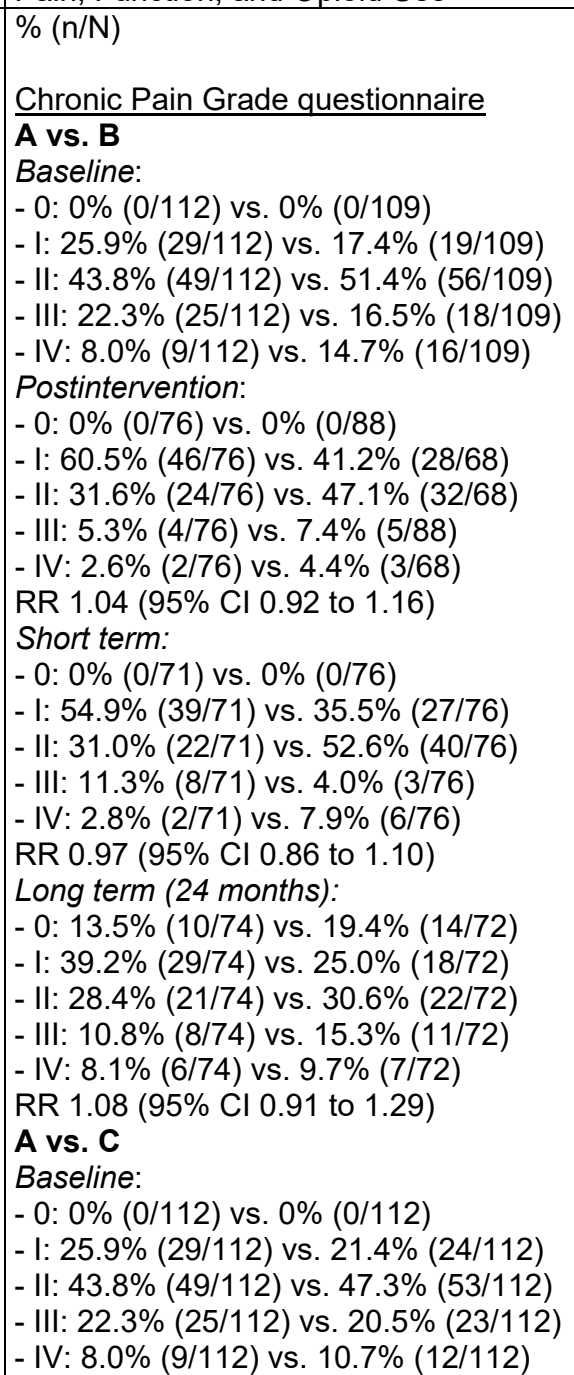 & 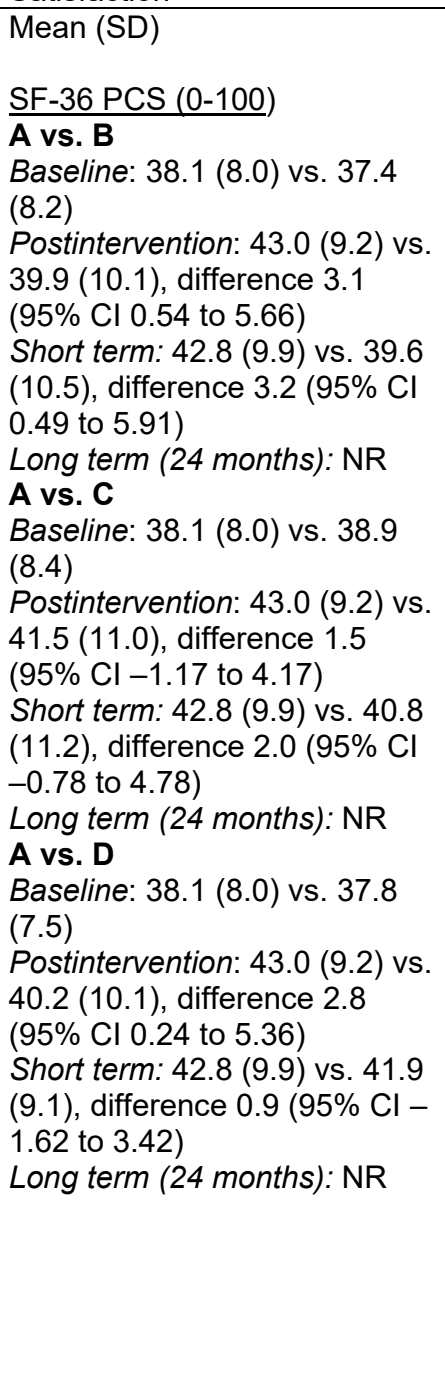 & $\begin{array}{l}\text { Harms } \\
\text { Two deaths due to } \\
\text { cancer were recorded: } \\
\text { ( } 1 \text { in the exercise group } \\
\text { and } 1 \text { in the TCBT } \\
\text { group). None of the } \\
\text { adverse events were } \\
\text { due to the interventions. }\end{array}$ \\
\hline
\end{tabular}




\begin{tabular}{|c|c|c|c|c|c|}
\hline $\begin{array}{l}\text { Author, Year } \\
\text { Country } \\
\text { Pain Duration } \\
\text { Study Design } \\
\text { Study Quality }\end{array}$ & $\begin{array}{l}\text { Intervention and } \\
\text { Comparator }(\mathrm{n}): \\
\text { Duration/Intensity, } \\
\text { Session Format, } \\
\text { Setting }\end{array}$ & Population & $\begin{array}{l}\text { Primary Outcomes: } \\
\text { Pain, Function, and Opioid Use }\end{array}$ & $\begin{array}{l}\text { Secondary Outcomes: } \\
\text { HRQOL, Psychological } \\
\text { Measures, Global } \\
\text { Improvement, Patient } \\
\text { Satisfaction }\end{array}$ & $\begin{array}{l}\text { Harms } \\
\text { Utilization }\end{array}$ \\
\hline $\begin{array}{l}\text { McBeth } 2012 \text { \& } \\
\text { Beasley, } 2015 \\
\text { (Continued) }\end{array}$ & & & 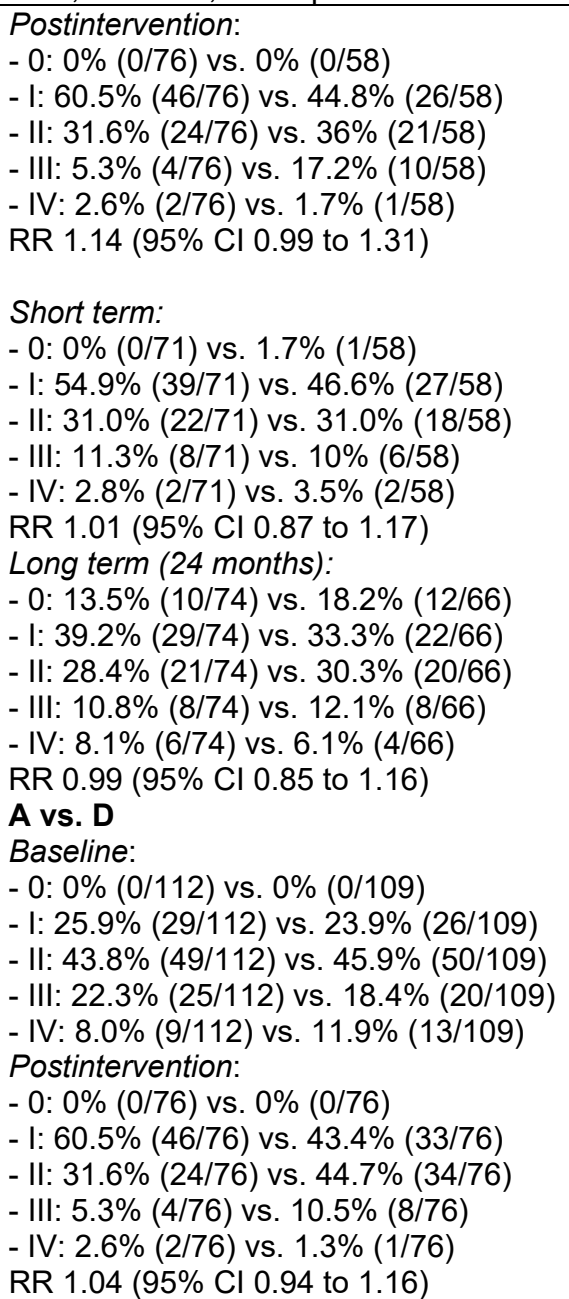 & 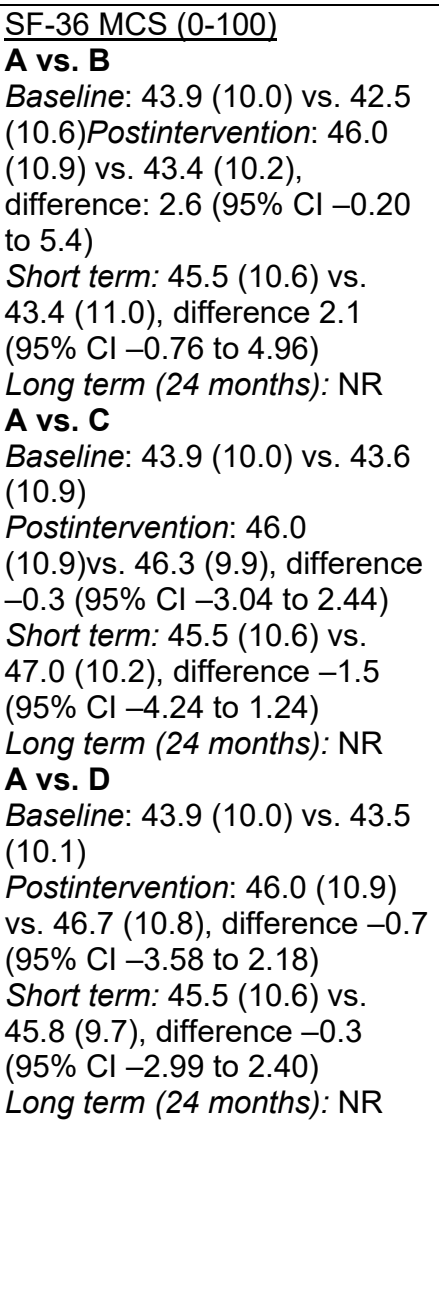 & \\
\hline
\end{tabular}




\begin{tabular}{|c|c|c|c|c|c|}
\hline $\begin{array}{l}\text { Author, Year } \\
\text { Country } \\
\text { Pain Duration } \\
\text { Study Design } \\
\text { Study Quality }\end{array}$ & $\begin{array}{l}\text { Intervention and } \\
\text { Comparator }(\mathrm{n}) \text { : } \\
\text { Duration/Intensity, } \\
\text { Session Format, } \\
\text { Setting }\end{array}$ & Population & $\begin{array}{l}\text { Primary Outcomes: } \\
\text { Pain, Function, and Opioid Use }\end{array}$ & $\begin{array}{l}\text { Secondary Outcomes: } \\
\text { HRQOL, Psychological } \\
\text { Measures, Global } \\
\text { Improvement, Patient } \\
\text { Satisfaction }\end{array}$ & $\begin{array}{l}\text { Harms } \\
\text { Utilization }\end{array}$ \\
\hline $\begin{array}{l}\text { McBeth } 2012 \text { \& } \\
\text { Beasley, } 2015 \\
\text { (Continued) }\end{array}$ & & & $\begin{array}{l}\text { Short term: } \\
\text { - } 0: 0 \%(0 / 71) \text { vs. } 47.3 \%(35 / 74) \\
\text { - I: } 54.9 \%(39 / 71) \text { vs. } 44.6 \%(33 / 74) \\
\text { - II: } 31.0 \%(22 / 71) \text { vs. } 8.1 \%(6 / 74) \\
\text { - III: } 11.3 \%(8 / 71) \text { vs. } 0 \%(0 / 74) \\
\text { - IV: } 2.8 \%(2 / 71) \text { vs. } 3.0 \%(2 / 74) \\
\text { RR } 0.88(95 \% \text { Cl } 0.80 \text { to } 0.97) \\
\text { Long term }(24 \text { months): } \\
\text { - } 0: 13.5 \%(10 / 74) \text { vs. } 14.1 \%(10 / 71) \\
\text { - I: } 39.2 \%(29 / 74) \text { vs. } 32.4 \%(23 / 71) \\
\text { - II: } 28.4 \%(21 / 74) \text { vs. } 22.5 \%(16 / 71) \\
\text { - III: } 10.8 \%(8 / 74) \text { vs. } 21.1 \%(15 / 71) \\
\text { - IV: } 8.1 \%(6 / 74) \text { vs. } 9.9 \%(7 / 71) \\
\text { RR } 1.18(95 \% \text { Cl } 0.97 \text { to } 1.42)\end{array}$ & 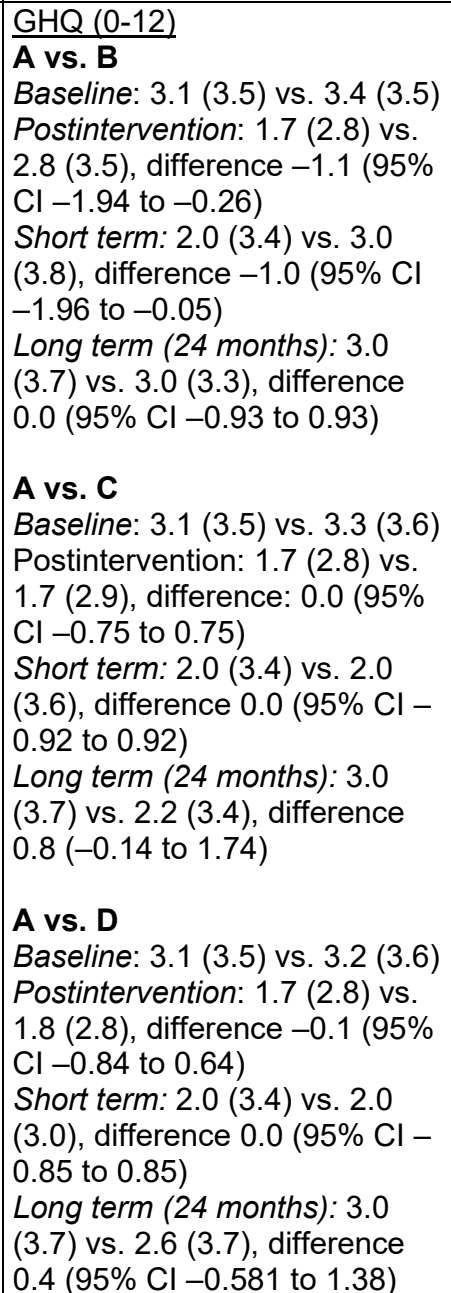 & \\
\hline
\end{tabular}




\begin{tabular}{|c|c|c|c|c|c|}
\hline $\begin{array}{l}\text { Author, Year } \\
\text { Country } \\
\text { Pain Duration } \\
\text { Study Design } \\
\text { Study Quality }\end{array}$ & \begin{tabular}{|l|} 
Intervention and \\
Comparator $(\mathrm{n}):$ \\
Duration/Intensity, \\
Session Format, \\
Setting
\end{tabular} & Population & $\begin{array}{l}\text { Primary Outcomes: } \\
\text { Pain, Function, and Opioid Use }\end{array}$ & $\begin{array}{l}\text { Secondary Outcomes: } \\
\text { HRQOL, Psychological } \\
\text { Measures, Global } \\
\text { Improvement, Patient } \\
\text { Satisfaction }\end{array}$ & $\begin{array}{l}\text { Harms } \\
\text { Utilization }\end{array}$ \\
\hline $\begin{array}{l}\text { McBeth } 2012 \text { \& } \\
\text { Beasley, } 2015 \\
\text { (Continued) }\end{array}$ & & & & 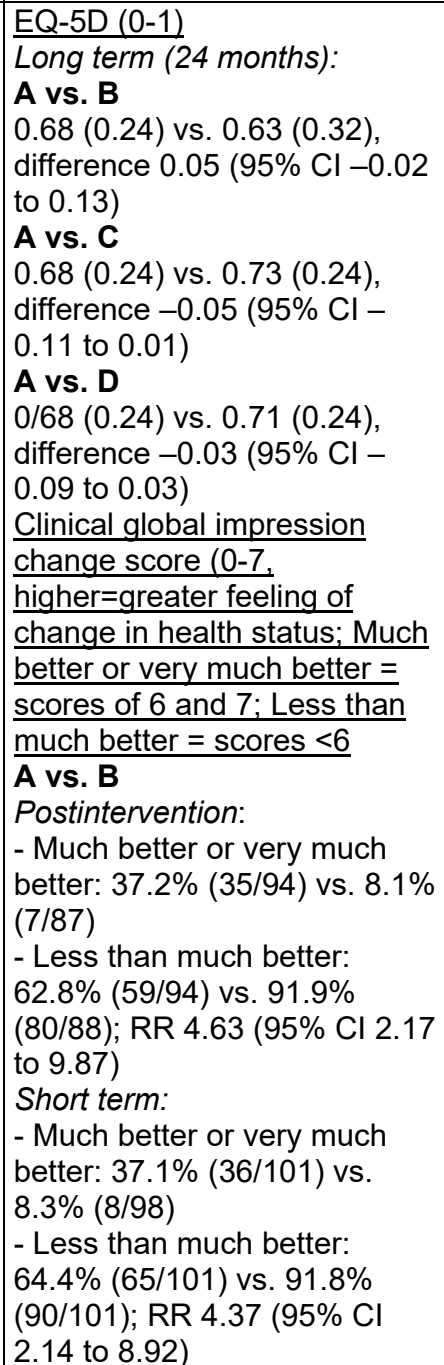 & \\
\hline
\end{tabular}




\begin{tabular}{|c|c|c|c|c|c|}
\hline $\begin{array}{l}\text { Author, Year } \\
\text { Country } \\
\text { Pain Duration } \\
\text { Study Design } \\
\text { Study Quality }\end{array}$ & \begin{tabular}{|l|} 
Intervention and \\
Comparator $(\mathrm{n}):$ \\
Duration/Intensity, \\
Session Format, \\
Setting
\end{tabular} & Population & $\begin{array}{l}\text { Primary Outcomes: } \\
\text { Pain, Function, and Opioid Use }\end{array}$ & $\begin{array}{l}\text { Secondary Outcomes: } \\
\text { HRQOL, Psychological } \\
\text { Measures, Global } \\
\text { Improvement, Patient } \\
\text { Satisfaction }\end{array}$ & $\begin{array}{l}\text { Harms } \\
\text { Utilization }\end{array}$ \\
\hline $\begin{array}{l}\text { McBeth } 2012 \& \\
\text { Beasley, } 2015 \\
\text { (Continued) }\end{array}$ & & & & 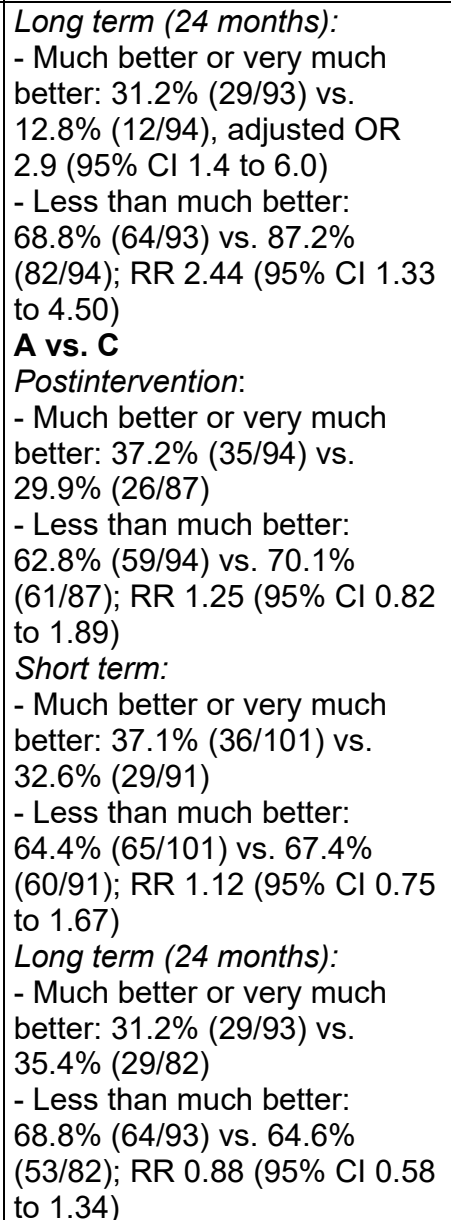 & \\
\hline
\end{tabular}




\begin{tabular}{|c|c|c|c|c|c|}
\hline $\begin{array}{l}\text { Author, Year } \\
\text { Country } \\
\text { Pain Duration } \\
\text { Study Design } \\
\text { Study Quality }\end{array}$ & \begin{tabular}{|l|} 
Intervention and \\
Comparator $(\mathrm{n}):$ \\
Duration/Intensity, \\
Session Format, \\
Setting
\end{tabular} & Population & $\begin{array}{l}\text { Primary Outcomes: } \\
\text { Pain, Function, and Opioid Use }\end{array}$ & $\begin{array}{l}\text { Secondary Outcomes: } \\
\text { HRQOL, Psychological } \\
\text { Measures, Global } \\
\text { Improvement, Patient } \\
\text { Satisfaction }\end{array}$ & $\begin{array}{l}\text { Harms } \\
\text { Utilization }\end{array}$ \\
\hline $\begin{array}{l}\text { McBeth } 2012 \& \\
\text { Beasley, } 2015 \\
\text { (Continued) }\end{array}$ & & & & $\begin{array}{l}\text { A vs. D } \\
\text { Postintervention: } \\
\text { - Much better or very much } \\
\text { better: } 37.2 \% \text { (35/94) vs. } \\
34.8 \% \text { (32/92) } \\
\text { - Less than much better: } \\
62.8 \% \text { (59/94) vs. } 65.2 \% \\
\text { (60/92); RR } 1.07 \text { (95\% Cl } 0.73 \\
\text { to 1.57) } \\
\text { Short term: } \\
\text { - Much better or very much } \\
\text { better: } 37.1 \% \text { (36/101) vs. } \\
24.2 \% \text { (24/99) } \\
\text { - Less than much better: } \\
64.4 \% \text { (65/101) vs. } 75.8 \% \\
\text { (75/99); RR } 1.47 \text { (95\% Cl } 0.95 \\
\text { to 2.27) } \\
\text { Long term (24 months): } \\
\text { - Much better or very much } \\
\text { better: } 31.2 \% \text { (29/93) vs. } \\
29.3 \% \text { (27/92) } \\
\text { - Less than much better: } \\
68.8 \% \text { (64/93) vs. } 70.7 \% \\
\text { (65/92); RR } 1.06 \text { (95\% Cl } 0.69 \\
\text { to } 1.65)\end{array}$ & \\
\hline
\end{tabular}




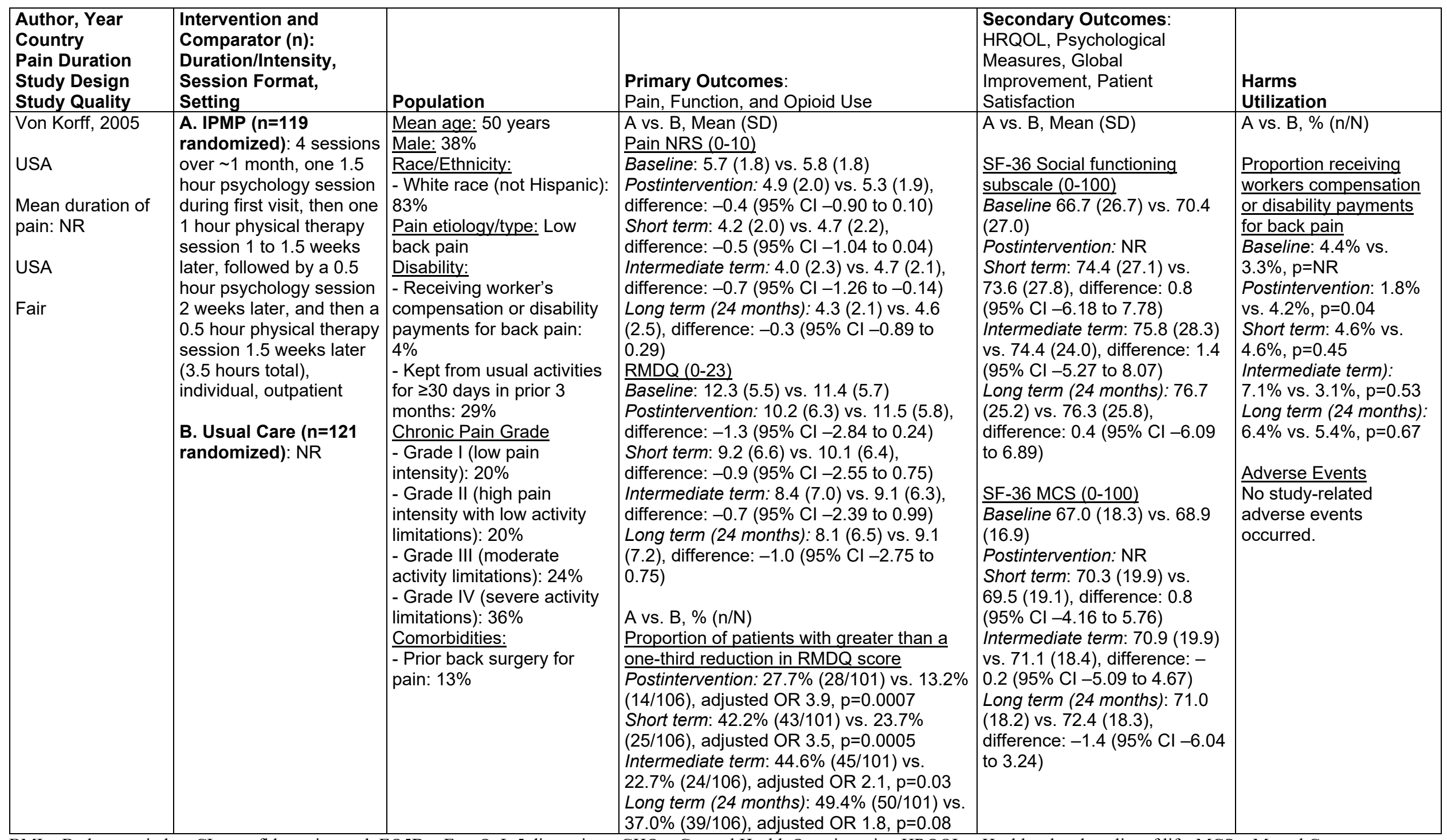

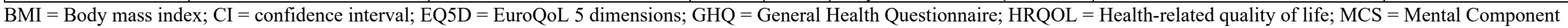

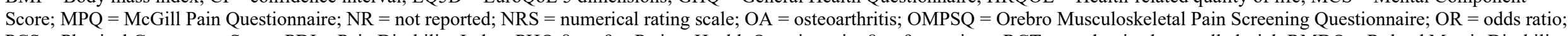

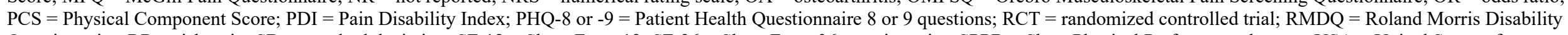

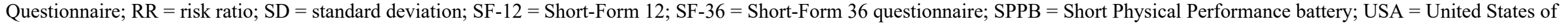
America; VAS = visual analog scale; WOMAC = Western Ontario and McMaster Universities Osteoarthritis Index

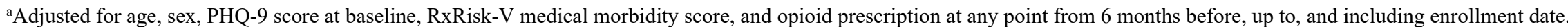

${ }^{\mathrm{b}}$ Adjusted for age, sex RxRisk-V medical morbidity, and opioid prescription withing 6 months prior to the enrollment date.

${ }^{\mathrm{c}}$ Adjusted for age, sex Patient Health Questionnaire 9 score at baseline, RxRisk-V medical morbidity, and baseline opioid prescription status.

${ }^{\mathrm{d} A d j u s t e d ~ f o r ~ a g e, ~ g e n d e r, ~ b a s e l i n e ~ o u t c o m e ~ m e a s u r e m e n t, ~ a n d ~ t h e ~ s i g n i f i c a n t ~ c o n f o u n d e r ~ a n d ~ s i g n i f i c a n t ~ i n t e r a c t i o n ~ v a r i a b l e s . ~}$ 
Table B-5. Summary results for trials addressing KQ1: CPMPs versus usual care or waitlist control

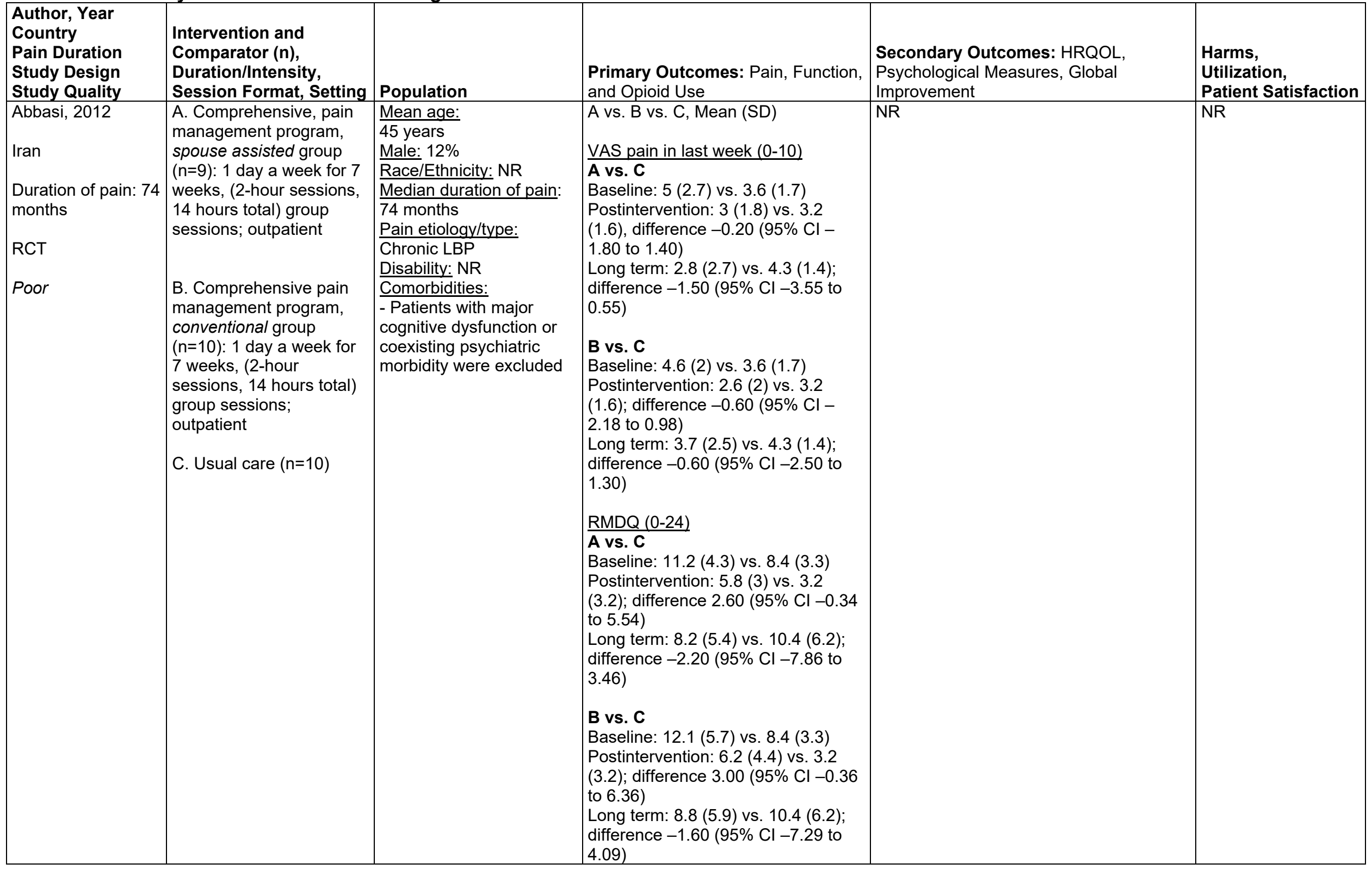




\begin{tabular}{|c|c|c|c|c|c|}
\hline $\begin{array}{l}\text { Author, Year } \\
\text { Country } \\
\text { Pain Duration } \\
\text { Study Design } \\
\text { Study Quality }\end{array}$ & $\begin{array}{l}\text { Intervention and } \\
\text { Comparator (n), } \\
\text { Duration/Intensity, } \\
\text { Session Format, Setting }\end{array}$ & Population & $\begin{array}{l}\text { Primary Outcomes: Pain, Function, } \\
\text { and Opioid Use }\end{array}$ & $\begin{array}{l}\text { Secondary Outcomes: HRQOL, } \\
\text { Psychological Measures, Global } \\
\text { Improvement }\end{array}$ & $\begin{array}{l}\text { Harms, } \\
\text { Utilization, } \\
\text { Patient Satisfaction }\end{array}$ \\
\hline $\begin{array}{l}\text { Ahlmen } 1988 \\
\text { Sweden } \\
\text { Duration of pain: } 11 \\
\text { years ( } \geq 10 \text { years: } \\
38 \% \text { ) } \\
\text { RCT } \\
\text { Fair }\end{array}$ & $\begin{array}{l}\text { A. Comprehensive pain } \\
\text { management program, } \\
\text { Long-term ( } n=31) \text { : } \\
\text { Duration of treatment: } 12 \\
\text { months, } \\
2 \text { hours a week ( } 14 \text { hours } \\
\text { total), individual + group } \\
\text { sessions, outpatient } \\
\text { B. Usual care }(n=28)\end{array}$ & $\begin{array}{l}\text { Mean age: } 59 \text { years } \\
\text { Female: } 100 \% \\
\text { Race/Ethnicity: NR } \\
\text { Pain etiology/type: } \\
\text { Rheumatoid arthritis } \\
\text { Disability: NR } \\
\text { Comorbidities: NR } \\
\end{array}$ & $\begin{array}{l}\text { A vs. B, Mean (SD) } \\
\text { SIP Overall Score (0-100) } \\
\text { Baseline: } 22.1(11.8) \text { vs. } 19.8(11.0) \\
\text { Postintervention change score }-3.6 \\
\text { (6.2) vs. }-0.1 \text { (5.3), p<0.05 } \\
\text { SIP, Physical Index (scale NR) } \\
\text { Baseline: } 22.7(15.8) \text { vs. } 19.8(13.1) \\
\text { Postintervention change score: }-4.6 \\
\text { (7.7) vs. } 0.3(5.5), p<0.01 \\
\text { SIP, Psychosocial Index (scale NR) } \\
\text { Baseline: } 11.1(7.8) \text { vs. } 11.2(11.6) \\
\text { Postintervention change score: }-3.3 \\
\text { (7.1) vs. }-0.7 \text { (6.7), p=NS }\end{array}$ & $\begin{array}{l}\text { A vs. B, Mean (SD) } \\
\text { MACL (scale NR) } \\
\text { Baseline: } 3.28(0.33) \text { vs. } 3.16(0.46) \\
\text { Postintervention: } 3.29(0.31) \text { vs. } 3.15 \\
(0.44), p=N S\end{array}$ & $\begin{array}{l}\text { A vs. B, Mean (SD) } \\
\text { Utilization: } \\
\text { No between group } \\
\text { differences in the } \\
\text { following (data NR): } \\
\text { - Drug treatment } \\
\text { - Intraarticular } \\
\text { corticosteroid } \\
\text { injections } \\
\text { - Orthopedic } \\
\text { specialist } \\
\text { consultations } \\
\text { - Referral for } \\
\text { inpatient } \\
\text { rheumatologic care }\end{array}$ \\
\hline
\end{tabular}




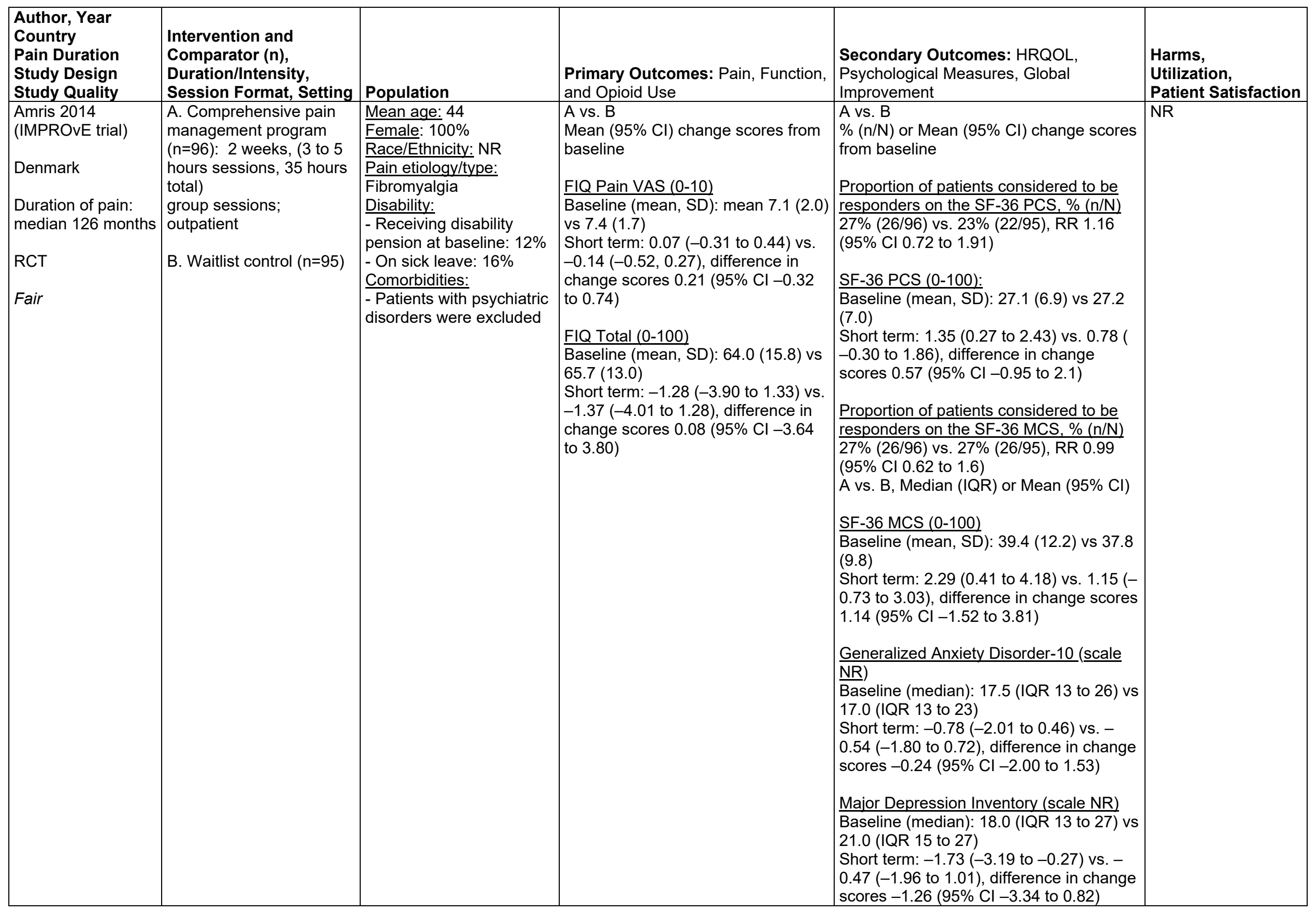




\begin{tabular}{|c|c|c|c|c|c|}
\hline $\begin{array}{l}\text { Author, Year } \\
\text { Country } \\
\text { Pain Duration } \\
\text { Study Design } \\
\text { Study Quality }\end{array}$ & $\begin{array}{l}\text { Intervention and } \\
\text { Comparator (n), } \\
\text { Duration/Intensity, } \\
\text { Session Format, Setting }\end{array}$ & Population & $\begin{array}{l}\text { Primary Outcomes: Pain, Function, } \\
\text { and Opioid Use }\end{array}$ & $\begin{array}{l}\text { Secondary Outcomes: HRQOL, } \\
\text { Psychological Measures, Global } \\
\text { Improvement }\end{array}$ & $\begin{array}{l}\text { Harms, } \\
\text { Utilization, } \\
\text { Patient Satisfaction }\end{array}$ \\
\hline $\begin{array}{l}\text { Basler, } 1997 \\
\text { Germany } \\
\text { Duration of pain: } \\
129.6 \text { months } \\
\text { RCT } \\
\text { Fair }\end{array}$ & $\begin{array}{l}\text { A. Comprehensive pain } \\
\text { management }(n=36) \\
12 \text { weeks (one } 2.5 \text {-hour } \\
\text { sessions per week) } \\
\text { patients attended an } \\
\text { outpatient review } \\
\text { appointment }(2-4 \text { hours) } \\
\text { at } 1 \text { and } 3 \text { months } \\
\text { postdischarge group } \\
\text { sessions, outpatient } \\
\text { B. Usual Care }(n=40)\end{array}$ & $\begin{array}{l}\text { Mean age: } 49 \text { years } \\
\text { \% Male: } 24 \% \\
\text { Race/ethnicity: NR } \\
\text { Pain etiology/type: } \\
\text { Chronic LBP } \\
\text { Quebec Task Force on } \\
\text { Spinal Disorders: } \\
-73 \% \text { chronic pain } \\
\text { syndrome } \\
-26 \% \text { post-surgical spinal } \\
\text { or radicular pain } \\
\text { Disability: NR } \\
\text { - 70\% unemployed } \\
-90.4 \% \text { considered } \\
\text { significantly disabled } \\
\text { - 69\% unemployed } \\
\text { Other characteristics: } \\
\text { - Spinal surgery: } 1.3 \% \\
\text { - Days using an opioid } \\
\text { pain medication per } \\
\text { week, mean (SD): } 3.02 \\
\text { (2.85) vs. } 3.41 \text { (2.76) }\end{array}$ & 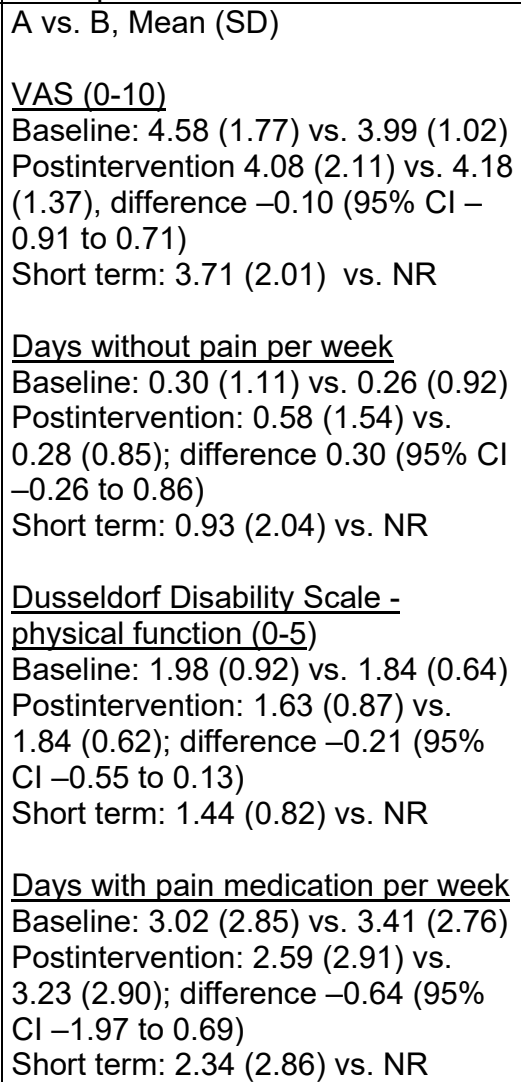 & NR & NR \\
\hline
\end{tabular}




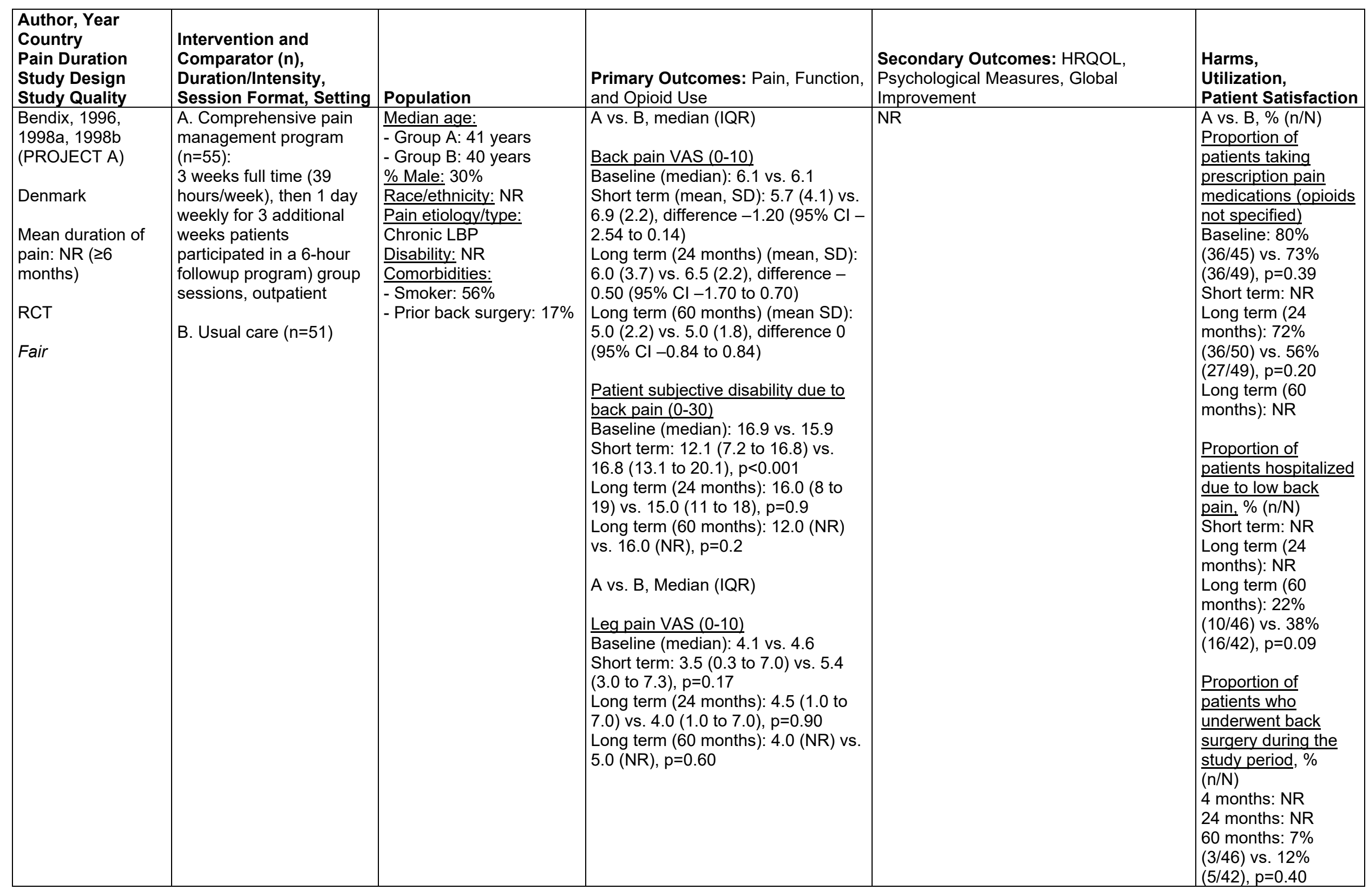




\begin{tabular}{|c|c|c|c|c|c|}
\hline $\begin{array}{l}\text { Author, Year } \\
\text { Country } \\
\text { Pain Duration } \\
\text { Study Design } \\
\text { Study Quality }\end{array}$ & $\begin{array}{l}\text { Intervention and } \\
\text { Comparator (n), } \\
\text { Duration/Intensity, } \\
\text { Session Format, Setting }\end{array}$ & Population & $\begin{array}{l}\text { Primary Outcomes: Pain, Function, } \\
\text { and Opioid Use }\end{array}$ & $\begin{array}{l}\text { Secondary Outcomes: HRQOL, } \\
\text { Psychological Measures, Global } \\
\text { Improvement }\end{array}$ & $\begin{array}{l}\text { Harms, } \\
\text { Utilization, } \\
\text { Patient Satisfaction }\end{array}$ \\
\hline $\begin{array}{l}\text { Browne, } 2013 \\
\text { Australia } \\
\text { Duration of pain: } 1 \\
\text { month } \\
\text { RCT } \\
\text { Poor }\end{array}$ & $\begin{array}{l}\text { A. Comprehensive pain } \\
\text { management program } \\
(\mathrm{n}=69) \text { : } \\
\text { Duration of treatment } \\
\text { unclear ( } 2.5 \text { hours/week; } \\
20 \text { hours total) individual } \\
\text { sessions; outpatient } \\
\text { B. Usual care }(n=73)\end{array}$ & $\begin{array}{l}\text { Mean age: } 37 \text { years } \\
\text { \% Male: } 75 \% \\
\text { Race/Ethnicity: } \\
\text {-Caucasian: } 92 \% \\
\text {-Asian: } 4 \% \\
\text {-Indigenous: } 4 \% \\
\text { Pain etiology/type: } \\
\text { Traumatic injury } \\
\text { Mechanism of injury } \\
\text {-MVA/MBA: } 73 \% \\
\text {-Fall: } 8 \% \\
\text {-Assault: } 7 \% \\
\text {-Sports related: } 6 \% \\
\text {-Work related: } 4 \% \\
\text {-Other } 1 \% \\
\text { Length of hospital stay: } \\
13.87 \text { days } \\
\text { Injury Severity Score: } 9.6 \\
\text { Disability: NR } \\
\text { Comorbidities: NR } \\
\text { Mental Health History: } \\
19 \% \\
\text { Pain Medications at } \\
\text { discharge: } \\
\text {-slow release opioids (i.e., } \\
\text { MS Contin, Kapanol, } \\
\text { Oxycontin, Methadone, } \\
\text { Fentanyl): } 27 \% \\
\text {-antineuropathic } \\
\text { (Gabapentin, Pregabalin, } \\
\text { Clonazepam, } \\
\text { Amitryptaline): } 1 \% \\
\text {-combination of above: } \\
10 \%\end{array}$ & $\begin{array}{l}\text { A vs. B, Mean (SD) } \\
\text { BPI (0-10) } \\
\text { Baseline: } 5.12(2.26) \text { vs. } 5.48(2.11) \\
\text { Postintervention: } 3.13(2.03) \text { vs. } \\
3.03(2.74) \text {, difference } 0.10(95 \% \mathrm{Cl} \\
-1.06 \text { to } 1.26) \\
\\
\text { FIM (scale } 18-126) \\
\text { Baseline: NR } \\
\text { Postintervention: } 122.73(4.74) \text { vs. } \\
123.00 \text { (3.91), difference }-0.27(95 \% \\
\text { Cl }-2.40 \text { to } 1.86)\end{array}$ & 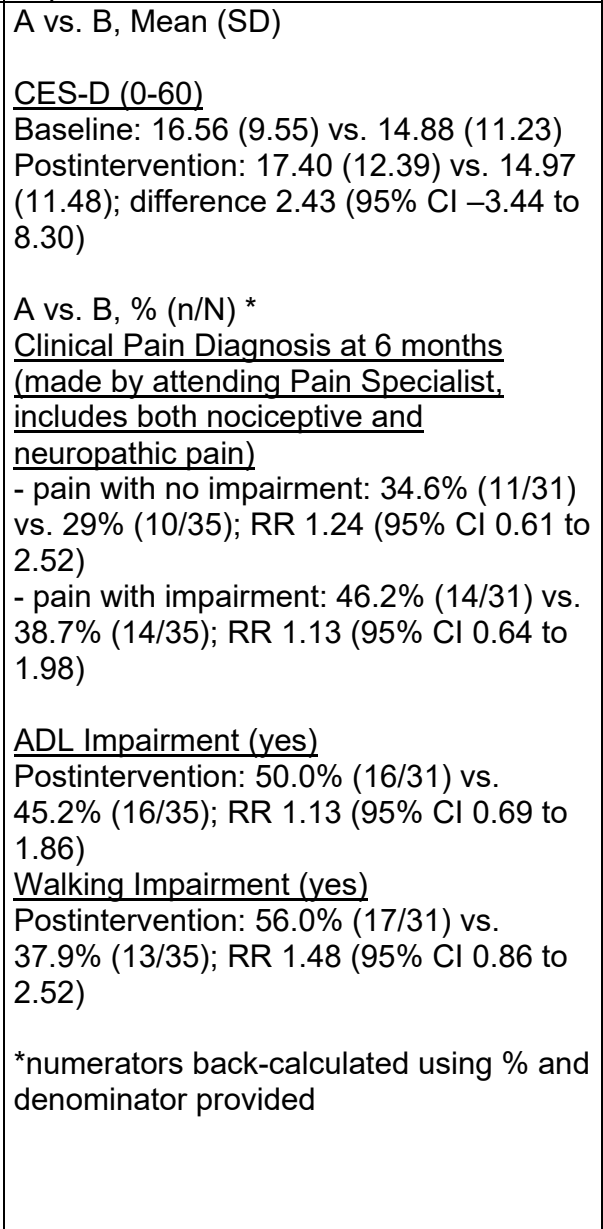 & NR \\
\hline
\end{tabular}




\begin{tabular}{|c|c|c|c|c|c|}
\hline $\begin{array}{l}\text { Author, Year } \\
\text { Country } \\
\text { Pain Duration } \\
\text { Study Design } \\
\text { Study Quality }\end{array}$ & $\begin{array}{l}\text { Intervention and } \\
\text { Comparator }(\mathrm{n}), \\
\text { Duration/Intensity, } \\
\text { Session Format, Setting }\end{array}$ & Population & $\begin{array}{l}\text { Primary Outcomes: Pain, Function, } \\
\text { and Opioid Use }\end{array}$ & $\begin{array}{l}\text { Secondary Outcomes: HRQOL, } \\
\text { Psychological Measures, Global } \\
\text { Improvement }\end{array}$ & $\begin{array}{l}\text { Harms, } \\
\text { Utilization, } \\
\text { Patient Satisfaction }\end{array}$ \\
\hline $\begin{array}{l}\text { de Buck, } 2005 \\
\text { The Netherlands } \\
\text { Duration of pain: } \\
\text { Group A. } 11.0 \\
\text { months } \\
\text { Group B. } 19.5 \\
\text { months } \\
\text { RCT } \\
\text { Fair }\end{array}$ & $\begin{array}{l}\text { A. Comprehensive pain } \\
\text { management program } \\
(n=74) \text { : } \\
\text { Duration NR (average } \\
\text { between } 4 \text { and } 12 \text { weeks) } \\
\text { Individual or group } \\
\text { sessions NR; outpatient } \\
\text { B. Usual care }(n=66)\end{array}$ & $\begin{array}{l}\text { Median age: } \\
\text { - Group A: } 43 \text { years } \\
\text { - Group B: } 44 \text { years } \\
\text { \% Male: } 44 \% \\
\text { Race/Ethnicity: NR } \\
\text { Median duration of } \\
\text { disease: } \\
\text { - Group A: } 11.0 \text { months } \\
\text { - Group B: } 19.5 \text { months } \\
\text { Pain etiology/type: Mixed } \\
\text { chronic pain } \\
\text { - Rheumatoid arthritis: } \\
\text { 50\% } \\
\text { - Ankylosing spondylitis, } \\
\text { psoriatic arthritis, or } \\
\text { reactive arthritis: } 21 \% \\
\text { - Systemic lupus } \\
\text { erythematosus, } \\
\text { scleroderma: } 29 \% \\
\text { Disability: } \\
\text { - Partial work disability } \\
\text { benefit: } 16.4 \% \\
\text { - Sick leave: } 55 \% \\
\text { - Complete sick leave: } \\
29 \% \\
\text { Comorbidities: } \\
\text { - Charlson index } \geq 0: 43 \%\end{array}$ & 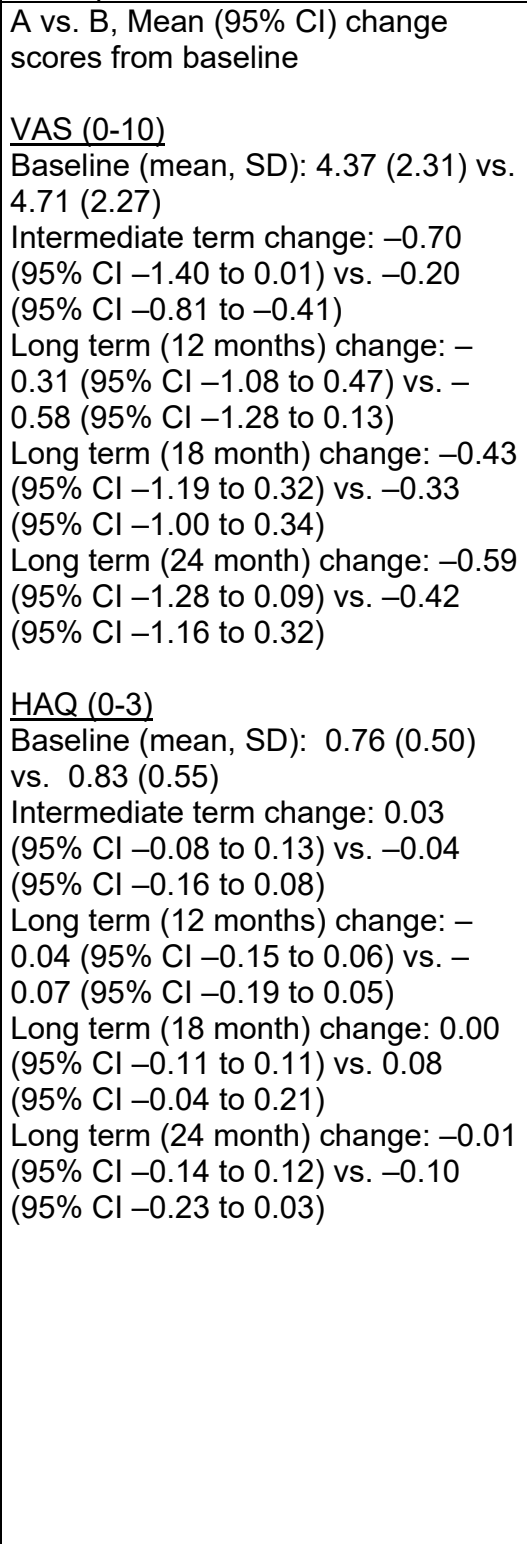 & 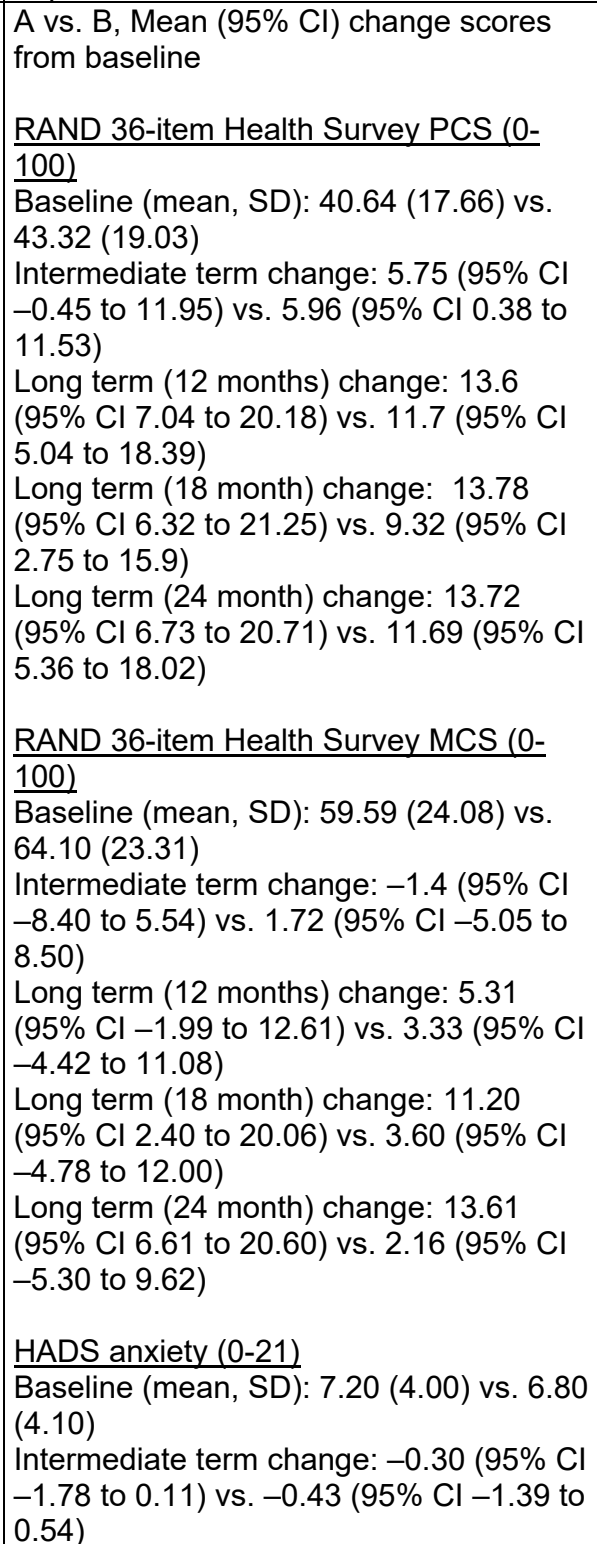 & NR \\
\hline
\end{tabular}




\begin{tabular}{|c|c|c|c|c|c|}
\hline $\begin{array}{l}\text { Author, Year } \\
\text { Country } \\
\text { Pain Duration } \\
\text { Study Design } \\
\text { Study Quality }\end{array}$ & $\begin{array}{l}\text { Intervention and } \\
\text { Comparator }(\mathrm{n}) \text {, } \\
\text { Duration/Intensity, } \\
\text { Session Format, Setting }\end{array}$ & Population & $\begin{array}{l}\text { Primary Outcomes: Pain, Function, } \\
\text { and Opioid Use }\end{array}$ & $\begin{array}{l}\text { Secondary Outcomes: HRQOL, } \\
\text { Psychological Measures, Global } \\
\text { Improvement }\end{array}$ & $\begin{array}{l}\text { Harms, } \\
\text { Utilization, } \\
\text { Patient Satisfaction }\end{array}$ \\
\hline $\begin{array}{l}\text { de Buck, } 2005 \\
\text { (Continued) }\end{array}$ & & & & $\begin{array}{l}\text { HADS anxiety (0-21) (continued) } \\
\text { Long term (12 months) change: }-0.83 \\
(95 \% \mathrm{Cl}-1.78 \text { to } 0.11) \text { vs. }-0.25(95 \% \mathrm{Cl} \\
-1.37 \text { to } 0.89) \\
\text { Long term }(18 \text { month) change: }-0.94 \\
(95 \% \mathrm{Cl}-1.87 \text { to }-0.02) \text { vs. }-0.34(95 \% \\
\mathrm{Cl}-1.53 \text { to } 0.89) \\
\text { Long term }(24 \text { month) change: }-1.83 \\
(95 \% \mathrm{Cl}-2.86 \text { to }-0.80) \text { vs. }-0.03(95 \% \\
\mathrm{Cl}-1.26 \text { to } 1.34) \\
\text { HADS depression }(0-21) \\
\text { Baseline (mean, SD): } 6.10(3.30) \text { vs. } 5.70 \\
(3.50) \\
\text { Intermediate term change: }-0.02(95 \% \mathrm{Cl} \\
-1.05 \text { to } 1.01) \text { vs. } 0.28 \text { (95\% } \mathrm{Cl}-0.54 \text { to } \\
1.10) \\
\text { Long term }(12 \text { months) change: }-0.46 \\
(95 \% \mathrm{Cl}-1.50 \text { to } 0.57) \text { vs. } 0.02(-0.89 \text { to } \\
0.92) \\
\text { Long term }(18 \text { month) change: }-0.64 \\
(95 \% \mathrm{Cl}-1.71 \text { to } 0.44) \text { vs. }-0.21(95 \% \mathrm{Cl} \\
-0.36 \text { to } 0.93) \\
\text { Long term }(24 \text { month) change: }-1.66 \\
(95 \% \mathrm{Cl}-2.72 \text { to }-0.60) \text { vs. } 0.15(95 \% \mathrm{Cl} \\
-1.12 \text { to } 1.42)\end{array}$ & \\
\hline
\end{tabular}




\begin{tabular}{|c|c|c|c|c|c|}
\hline $\begin{array}{l}\text { Author, Year } \\
\text { Country } \\
\text { Pain Duration } \\
\text { Study Design } \\
\text { Study Quality }\end{array}$ & $\begin{array}{l}\text { Intervention and } \\
\text { Comparator (n), } \\
\text { Duration/Intensity, } \\
\text { Session Format, Setting }\end{array}$ & Population & $\begin{array}{l}\text { Primary Outcomes: Pain, Function, } \\
\text { and Opioid Use }\end{array}$ & $\begin{array}{l}\text { Secondary Outcomes: HRQOL, } \\
\text { Psychological Measures, Global } \\
\text { Improvement }\end{array}$ & $\begin{array}{l}\text { Harms, } \\
\text { Utilization, } \\
\text { Patient Satisfaction }\end{array}$ \\
\hline $\begin{array}{l}\text { Härkäpää, 1989, } \\
1990 \\
\text { Finland } \\
\text { Duration of } \\
\text { pain:168 months } \\
\text { RCT } \\
\text { Poor }\end{array}$ & $\begin{array}{l}\text { A. Comprehensive pain } \\
\text { management program } \\
\text { Inpatient group ( } \mathrm{n}=156) \\
2 \text { times a week for } 2 \\
\text { months (15 sessions) }++ \\
\text { 2-week refresher } \\
\text { sessions after } 1.5 \text { years) } \\
\text { group sessions; inpatient } \\
\\
\text { B. Comprehensive pain } \\
\text { management program } \\
\text { Outpatient group ( } \mathrm{n}=150) \\
2 \text { times a week for } 2 \\
\text { months (15 sessions) }(+8 \\
\text { refresher sessions after } \\
1.5 \text { years) group } \\
\text { sessions; outpatient } \\
\text { C. Usual Care ( } \mathrm{n}=153)\end{array}$ & $\begin{array}{l}\text { Mean age: } 45 \text { years } \\
\text { \% Male: } 63 \% \\
\text { Race/Ethnicity: NR } \\
\text { Pain etiology/type: } \\
\text { Chronic LBP } \\
\text { - Continuous LBP during } \\
\text { past year: } 41 \% \\
\text { - Severe LPB during past } \\
\text { year: } 81 \% \\
\text { Other characteristics: } \\
\text { - \% Disability } \\
\text { compensation, pensions: } \\
\text { 10\% } \\
\text { - Use of opioid } \\
\text { medication: NR } \\
\text { - Use of analgesics: } 65 \% \\
\text { - Work absenteeism due } \\
\text { to LBP in past two years: } \\
34 \% "\end{array}$ & 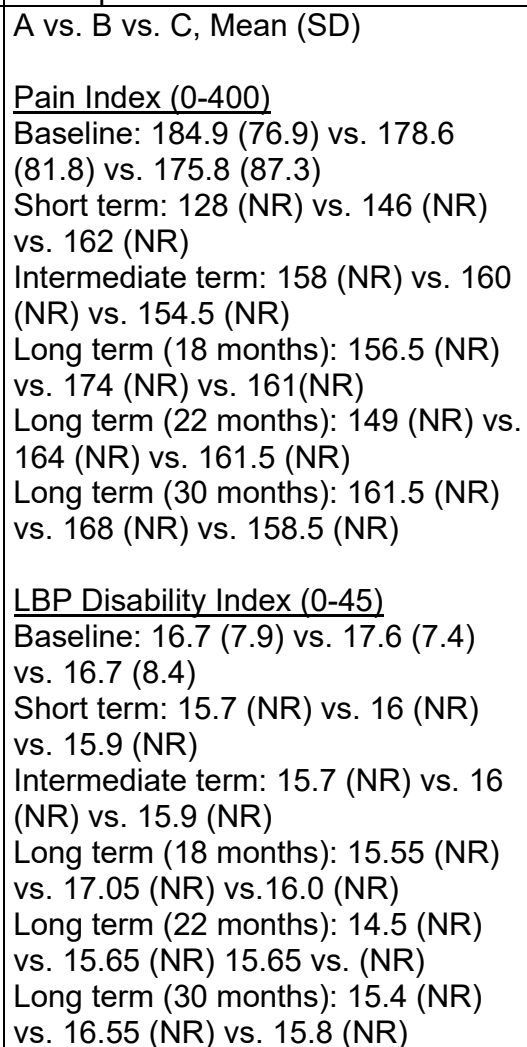 & $\begin{array}{l}\text { A vs. B \%, p-value } \\
\text { Benefits of treatment } \\
\text { Short term: } \\
\text {-Increased knowledge concerning low } \\
\text { back pains } 88 \text { vs. } 84, p=N S \\
\text {-Increased knowledge of factors affecting } \\
\text { low back pain: } 81 \text { vs. } 77, p=N S \\
\text {-Increased motivation for self-care: } 60 \text { vs. } \\
40, p=0.001 \\
\text { - Decrease in low back pain: } 57 \text { vs. } 38 \\
p=0.05 \\
- \text { Mental recreation: } 56 \text { vs. } 25, p=0.001 \\
- \text { Improved physical condition: } 55 \text { vs. } 23, \\
p=0.001 \\
- \text { Improved working capacity: } 45 \text { vs. } 22, \\
p=0.01 \\
- \text {-Decrease in other illness symptoms: } 24 \\
\text { vs. } 10, p=0.02\end{array}$ & NR \\
\hline
\end{tabular}




\begin{tabular}{|c|c|c|c|c|c|}
\hline \begin{tabular}{|l} 
Author, Year \\
Country \\
Pain Duration \\
Study Design \\
Study Quality
\end{tabular} & $\begin{array}{l}\text { Intervention and } \\
\text { Comparator (n), } \\
\text { Duration/Intensity, } \\
\text { Session Format, Setting }\end{array}$ & Population & $\begin{array}{l}\text { Primary Outcomes: Pain, Function, } \\
\text { and Opioid Use }\end{array}$ & $\begin{array}{l}\text { Secondary Outcomes: HRQOL, } \\
\text { Psychological Measures, Global } \\
\text { Improvement }\end{array}$ & $\begin{array}{l}\text { Harms, } \\
\text { Utilization, } \\
\text { Patient Satisfaction }\end{array}$ \\
\hline $\begin{array}{l}\text { Jensen, } 2001 \\
\text { Sweden } \\
\text { Duration of pain: } 31 \\
\text { months } \\
\text { RCT } \\
\text { Fair }\end{array}$ & $\begin{array}{l}\text { A. Comprehensive pain } \\
\text { management program } \\
(\mathrm{n}=63): \\
4 \text { weeks }{ }^{*},(20 \text { hours a } \\
\text { week, } 80 \text { hours total) } \\
\text { group sessions; } \\
\text { outpatient } \\
{ }^{*} \text { (plus six } 90-\text { minute } \\
\text { booster sessions over a } \\
\text { period of } 1 \text { year after } \\
\text { treatment) } \\
\text { B. Usual care }(n=48)\end{array}$ & $\begin{array}{l}\text { Mean age: } 43 \text { years } \\
\text { \% Male: } 45 \% \\
\text { Race/ethnicity: } \\
\text { - Swedish origin: } 67 \% \\
\text { Pain etiology/type: Mixed } \\
\text { chronic pain } \\
\text { (long-term, nonspecific } \\
\text { spinal pain) } \\
\text { - Cervical/thoracic pain: } \\
42 \% \\
\text { - Lumbar pain: } 46 \% \\
\text { - Mixed pain areas: } 12 \% \\
\text { Disability: NR } \\
\text { - Mean total sick leave in } \\
6 \text { months prior to } \\
\text { inclusion in study: } 292 \\
\text { (63) } \\
\text { Comorbidities: NR }\end{array}$ & NR & $\begin{array}{l}\text { A vs. B, Mean (SD) } \\
\text { SF-36 Global health (scale 0-100) } \\
\text { Females only } \\
\text { A vs. B ( } n=30 \text { vs. } 28) \\
\text { Baseline: } 38.1(14.5) \text { vs. } 45.6(16.5) \\
\text { Postintervention: } 47.6 \text { (18.0) vs. } 47.0 \\
\text { (15.2); difference } 0.60(95 \% \mathrm{Cl}-8.12 \text { to } \\
9.40) \\
\text { Intermediate term: } 52.4(21.6) \text { vs. } 46.3 \\
\text { (19.3); difference } 6.10(95 \% \mathrm{Cl}-4.70 \text { to } \\
16.90) \\
\text { Long term (18 months): } 53.1(24.5) \text { vs. } \\
43.4(20.1) ; \text { difference } 9.70(95 \% \mathrm{Cl}- \\
2.14 \text { to } 21.5) \\
\text { Males only } \\
\text { A vs. B (n=33 vs. } 20) \\
\text { Baseline: } 41.6(14.6) \text { vs. } 45.0(14.7) \\
\text { Postintervention: } 48.5(17.2) \text { vs. } 45.1 \\
\text { (13.2); difference } 3.40(95 \% \mathrm{Cl}-5.61 \text { to } \\
12.41) \\
\text { Intermediate term: } 54.3(18.3) \text { vs. } 51.5 \\
\text { (24.2); difference } 2.80(95 \% \mathrm{Cl}-8.97 \text { to } \\
14.57) \\
\text { Long term (18 months): } 57.2(21.8) 45.9 \\
\text { (21.2); difference } 11.30(95 \% \mathrm{Cl}-0.98 \text { to } \\
\text { 23.58) }\end{array}$ & $\begin{array}{l}\text { A vs. B, Mean (SD) } \\
\text { Harms: NR } \\
\text { Perceived } \\
\text { appropriateness of } \\
\text { the treatment } \\
\text { program to treating } \\
\text { patient's pain } \\
\text { Females only } \\
\text { Postintervention: } 6.4 \\
\text { (3.1) vs. NR } \\
\text { Males only } \\
\text { Postintervention: } 6.0 \\
\text { (3.6) vs. NR }\end{array}$ \\
\hline
\end{tabular}




\begin{tabular}{|c|c|c|c|c|c|}
\hline $\begin{array}{l}\text { Author, Year } \\
\text { Country } \\
\text { Pain Duration } \\
\text { Study Design } \\
\text { Study Quality }\end{array}$ & $\begin{array}{l}\text { Intervention and } \\
\text { Comparator }(\mathbf{n}), \\
\text { Duration/Intensity, } \\
\text { Session Format, Setting }\end{array}$ & Population & $\begin{array}{l}\text { Primary Outcomes: Pain, Function, } \\
\text { and Opioid Use }\end{array}$ & $\begin{array}{l}\text { Secondary Outcomes: HRQOL, } \\
\text { Psychological Measures, Global } \\
\text { Improvement }\end{array}$ & $\begin{array}{l}\text { Harms, } \\
\text { Utilization, } \\
\text { Patient Satisfaction }\end{array}$ \\
\hline $\begin{array}{l}\text { Johansson, } 1998 \\
\text { Norway } \\
\text { Duration of pain: } \\
132 \text { months } \\
\text { RCT } \\
\text { Fair }\end{array}$ & $\begin{array}{l}\text { A. Comprehensive pain } \\
\text { management program } \\
(\mathrm{n}=21) \text { : } \\
5 \text { days a week for } 5 \\
\text { weeks, (hours total NR) * } \\
\text { group sessions; inpatient } \\
\text { and outpatient } \\
\text { *(+ booster sessions after } \\
2 \text { months) } \\
\text { B. Waitlist control }(n=21) \text { : }\end{array}$ & $\begin{array}{l}\text { Mean age: } 44 \text { years } \\
\text { \% Male: } 22 \% \\
\text { Race/ethnicity: NR } \\
\text { Pain etiology/type: } \\
\text { Chronic musculoskeletal } \\
\text { pain } \\
\text { Disability: NR } \\
\text { - On sick leave: } 75 \% \\
\text { - Unemployed: } 32 \% \\
\text { Comorbidities: NR } \\
\text { - Patients with psychotic } \\
\text { illness were excluded }\end{array}$ & 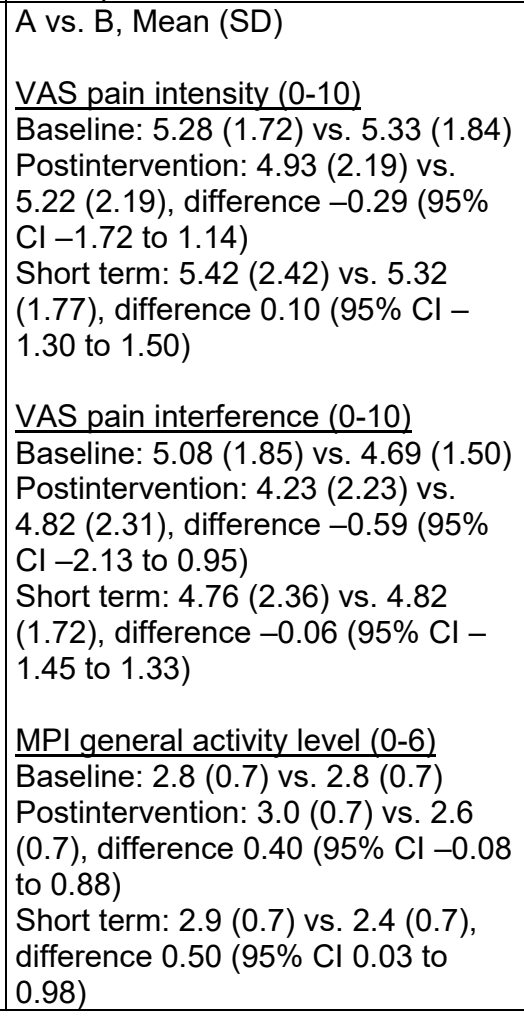 & NR & NR \\
\hline
\end{tabular}




\begin{tabular}{|c|c|c|c|c|c|}
\hline $\begin{array}{l}\text { Author, Year } \\
\text { Country } \\
\text { Pain Duration } \\
\text { Study Design } \\
\text { Study Quality }\end{array}$ & $\begin{array}{l}\text { Intervention and } \\
\text { Comparator (n), } \\
\text { Duration/Intensity, } \\
\text { Session Format, Setting }\end{array}$ & Population & $\begin{array}{l}\text { Primary Outcomes: Pain, Function, } \\
\text { and Opioid Use }\end{array}$ & $\begin{array}{l}\text { Secondary Outcomes: HRQOL, } \\
\text { Psychological Measures, Global } \\
\text { Improvement }\end{array}$ & $\begin{array}{l}\text { Harms, } \\
\text { Utilization, } \\
\text { Patient Satisfaction }\end{array}$ \\
\hline $\begin{array}{l}\text { Lemstra, } 2005 \\
\text { Canada } \\
\text { Duration of pain: } \\
121 \text { months } \\
\text { RCT } \\
\text { Fair }\end{array}$ & $\begin{array}{l}\text { A. Comprehensive pain } \\
\text { management program } \\
(n=43) \text { : } \\
6 \text { weeks, (18 one-hour } \\
\text { sessions } 18 \text { hours total) } \\
\text { group sessions; } \\
\text { outpatient setting } \\
\text { B. Usual care }(n=36) \text { : }\end{array}$ & $\begin{array}{l}\text { Mean age: } 50 \\
\text { \% Male: } 15 \% \\
\text { Race/ethnicity: NR } \\
\text { Pain etiology/type: } \\
\text { Fibromyalgia } \\
\text { Disability: NR } \\
\text { Comorbidities (all self- } \\
\text { reported): } \\
\text { - Fatigue: } 94 \% \\
\text { - Sleep deprivation: } 96 \% \\
\text { - Emotional problems: } \\
64 \% \\
\text { - Headaches: } 77 \% \\
\text { - Morning stiffness: } 94 \% \\
\text { - Depression: } 89 \% \\
\text { - Anxiety: } 67 \% \\
\text { - Frustration: } 82 \%\end{array}$ & 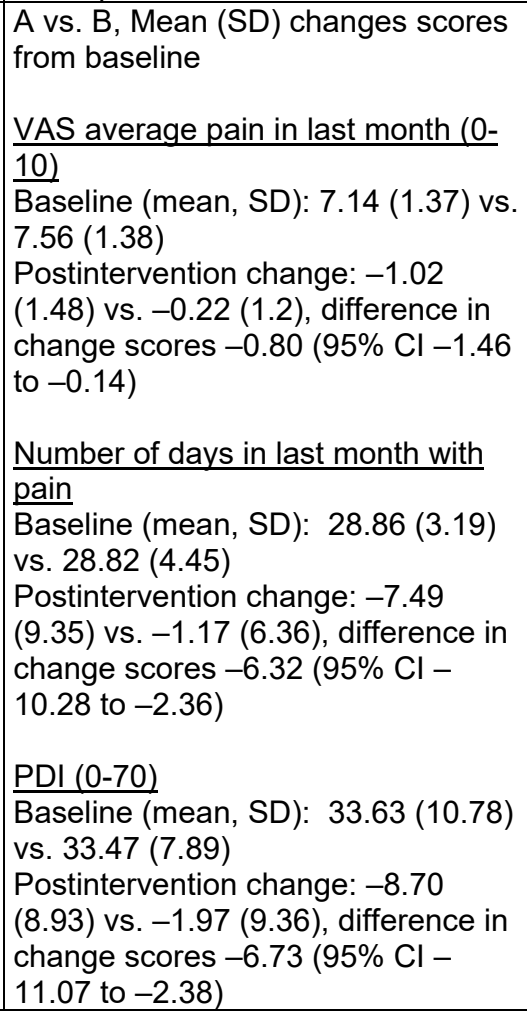 & $\begin{array}{l}\text { A vs. B, Mean (SD) changes scores from } \\
\text { baseline } \\
\text { BDI (0-63) } \\
\text { Baseline (mean, SD): } 18.23 \text { (10.72) vs. } \\
17.89(10.03) \\
\text { Postintervention change: }-7.74(6.92) \text { vs. } \\
-0.97(4.5) \text {, difference in change scores - } \\
6.77(95 \% \mathrm{Cl}-9.67 \text { to }-3.87) \\
\text { Self-reported health status }(0-5) \\
\text { Baseline (mean, SD): } 3.60(1.03) \text { vs. } 3.67 \\
(0.89) \\
\text { Postintervention change: }-0.60(0.12) \text { vs. } \\
0.03(0.11) \text {, difference in change scores - } \\
0.63(95 \% \mathrm{Cl}-0.95 \text { to }-0.31)\end{array}$ & NR \\
\hline
\end{tabular}




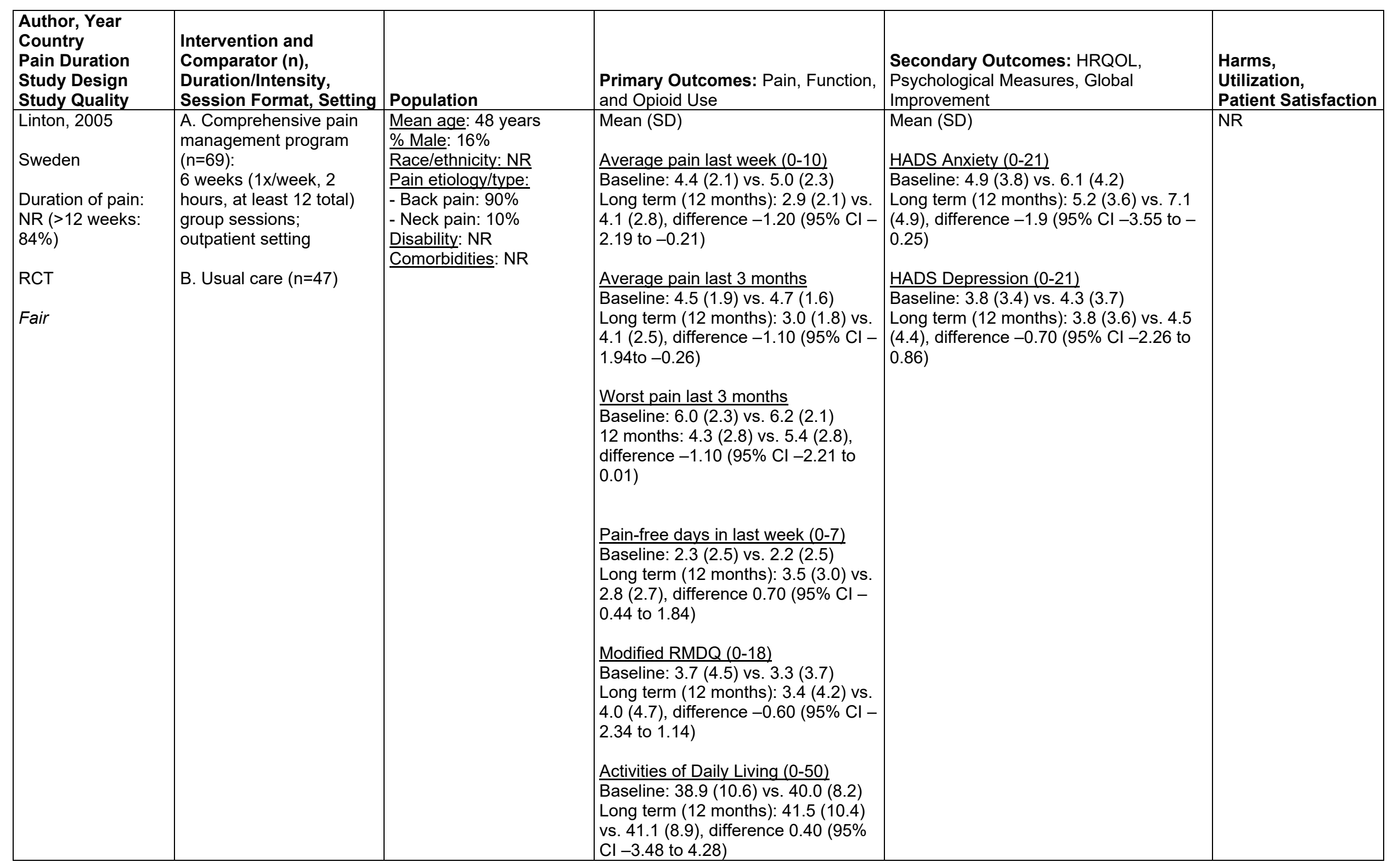




\begin{tabular}{|c|c|c|c|c|c|}
\hline $\begin{array}{l}\text { Author, Year } \\
\text { Country } \\
\text { Pain Duration } \\
\text { Study Design } \\
\text { Study Quality }\end{array}$ & $\begin{array}{l}\text { Intervention and } \\
\text { Comparator (n), } \\
\text { Duration/Intensity, } \\
\text { Session Format, Setting }\end{array}$ & Population & $\begin{array}{l}\text { Primary Outcomes: Pain, Function, } \\
\text { and Opioid Use }\end{array}$ & $\begin{array}{l}\text { Secondary Outcomes: HRQOL, } \\
\text { Psychological Measures, Global } \\
\text { Improvement }\end{array}$ & $\begin{array}{l}\text { Harms, } \\
\text { Utilization, } \\
\text { Patient Satisfaction }\end{array}$ \\
\hline $\begin{array}{l}\text { Peters } 1990,1992 \\
\text { New Zealand } \\
\text { Duration of pain: } 6 \\
\text { to } 48 \text { months, } 49 \% \text {; } \\
48 \text { months to } 240+ \\
\text { months, } 51 \% \\
\text { RCT } \\
\text { Poor }\end{array}$ & $\begin{array}{l}\text { A. Comprehensive pain } \\
\text { management program, } \\
\text { Inpatient group }(\mathrm{n}=23) \text { : } \\
5 \text { days a week for } 4 \\
\text { weeks, (hours total } \\
\text { unclear) } \\
\text { group sessions; inpatient } \\
\text { (Monday - Friday) } \\
\text { B. Comprehensive pain } \\
\text { management program, } \\
\text { Outpatient group ( } \mathrm{n}=29 \text { ) } \\
9 \text { weeks, (one 2-hour } \\
\text { session per week, } 18 \\
\text { hours total) } \\
\text { group sessions; } \\
\text { outpatient } \\
\text { C. Usual care }(n=16)\end{array}$ & $\begin{array}{l}\text { Mean age: } 44 \text { years } \\
\text { \% Male: } 38 \% \\
\text { Race/Ethnicity: } \\
\text { - European: } 93 \% \\
\text { - Maori: } 4 \% \\
\text { - Polynesian: } 3 \% \\
\text { Pain etiology/type Mixed } \\
\text { chronic pain } \\
\text { (patient could have more } \\
\text { than one pain type): } \\
\text { - Back pain: } 43 \% \\
\text { - Head pain: } 35 \% \\
\text { - Arm pain: } 26 \% \\
\text { - Leg pain: } 18 \% \\
\text { - Chest pain: } 10 \% \\
\text { - Abdomen pain: } 6 \% \\
\text { Disability: NR } \\
\text { Comorbidities: NR }\end{array}$ & 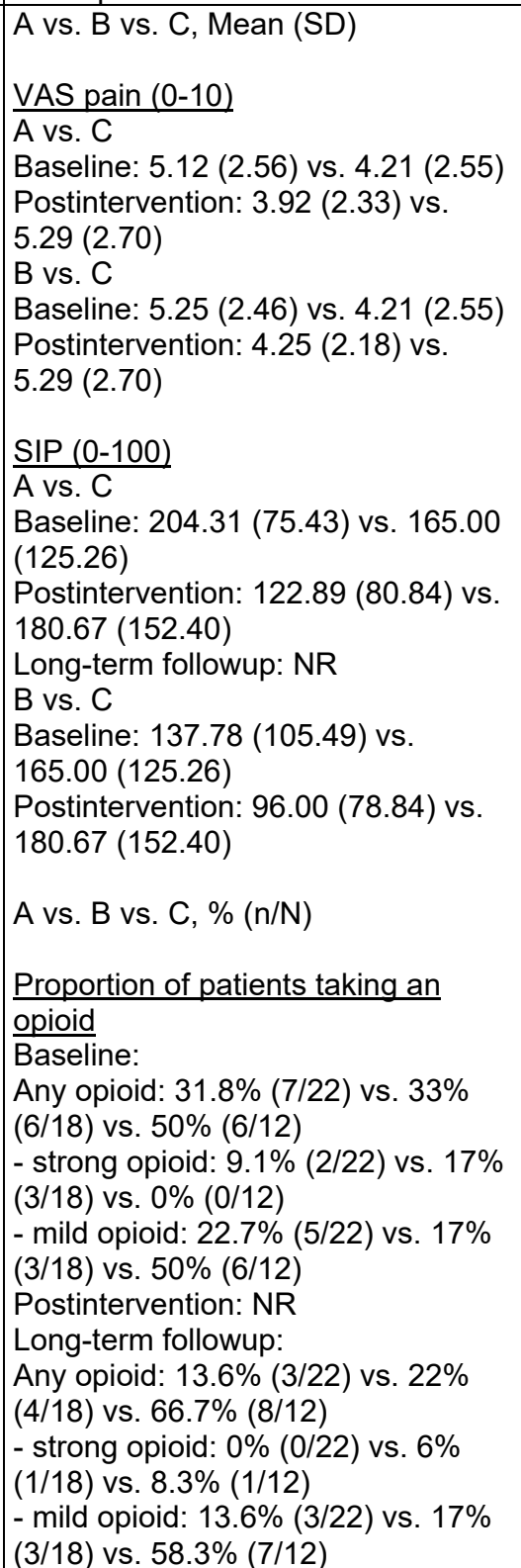 & 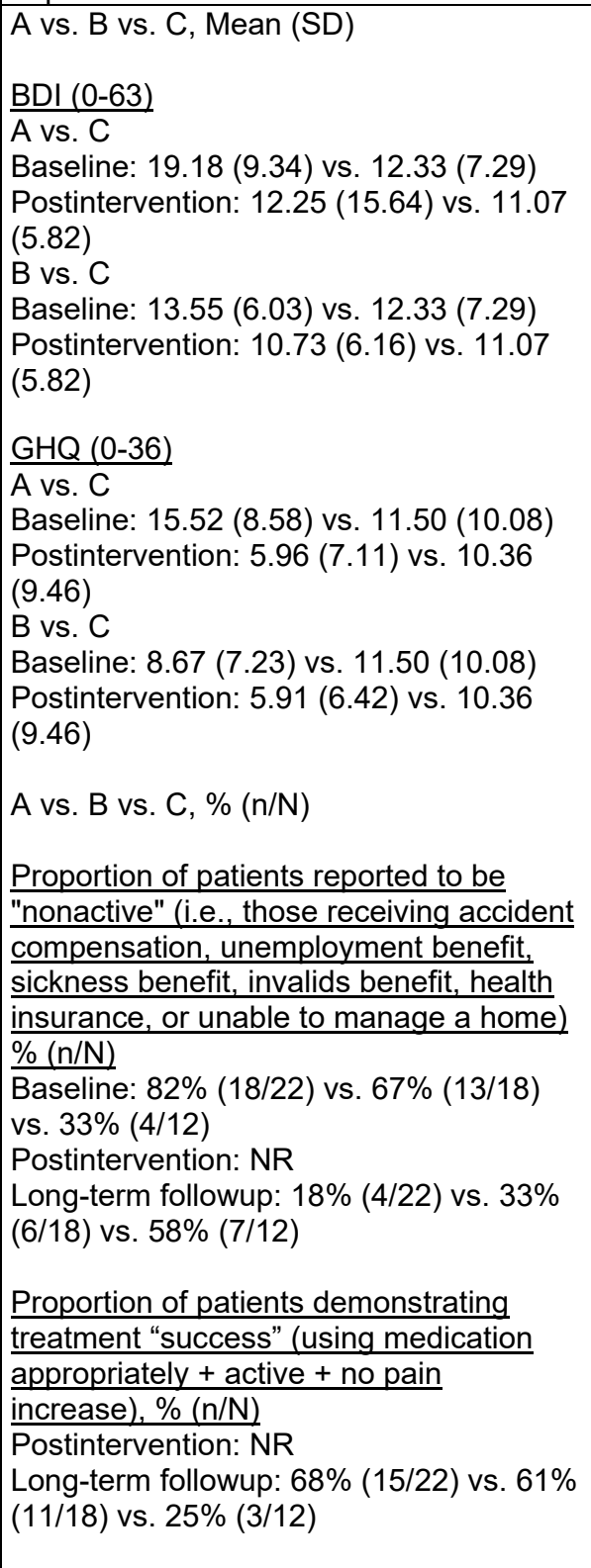 & NR \\
\hline
\end{tabular}




\begin{tabular}{|c|c|c|c|c|c|}
\hline $\begin{array}{l}\text { Author, Year } \\
\text { Country } \\
\text { Pain Duration } \\
\text { Study Design } \\
\text { Study Quality }\end{array}$ & $\begin{array}{l}\text { Intervention and } \\
\text { Comparator (n), } \\
\text { Duration/Intensity, } \\
\text { Session Format, Setting }\end{array}$ & Population & $\begin{array}{l}\text { Primary Outcomes: Pain, Function, } \\
\text { and Opioid Use }\end{array}$ & $\begin{array}{l}\text { Secondary Outcomes: HRQOL, } \\
\text { Psychological Measures, Global } \\
\text { Improvement }\end{array}$ & $\begin{array}{l}\text { Harms, } \\
\text { Utilization, } \\
\text { Patient Satisfaction }\end{array}$ \\
\hline $\begin{array}{l}\text { Saral, } 2016 \\
\text { Turkey } \\
\text { Duration of pain: } 90 \\
\text { months } \\
\text { RCT } \\
\text { Fair }\end{array}$ & $\begin{array}{l}\text { A. Comprehensive pain } \\
\text { management program, } \\
\text { long-term group ( } \mathrm{n}=22) \text { : } \\
10 \text { weeks }(\sim 7.5 \\
\text { hours/week; } 75 \text { hours } \\
\text { total); group + individual } \\
\text { sessions; outpatient } \\
\text { B. Comprehensive pain } \\
\text { management program, } \\
\text { short-term group }(n=22) \text { : } \\
2 \text { days ( 10 hours total); } \\
\text { group + individual } \\
\text { sessions; outpatient } \\
\text { C. Usual care }(n=22)\end{array}$ & $\begin{array}{l}\text { Mean age: } 42 \text { years } \\
\text { \% Male: } 0 \% \text { (female only } \\
\text { for inclusion) } \\
\text { Race/ethnicity: NR } \\
\text { Pain etiology/type: } \\
\text { Fibromyalgia } \\
\text { Disability: NR } \\
\text { Comorbidities: NR Other } \\
\text { characteristics: } \\
\text { - Excluded: history of } \\
\text { severe trauma, advanced } \\
\text { psychiatric diseases, } \\
\text { serious physical } \\
\text { comorbidities }\end{array}$ & $\begin{array}{l}\text { A vs. B vs. C, Mean (SD) } \\
\frac{\text { VAS pain }(0-10)}{\text { Baseline: } 8.2(0.9)} \text { vs. } 7.6(0.8) \text { vs. } \\
7.5(0.9) \\
\text { Intermediate term: } 5.1(2.4) \text { vs. } 5.8 \\
(1.0) \text { vs. } 7.6(1.4) \\
\text { FIQ (0-100) } \\
\text { Baseline: } 71.6(14.2) \text { vs. } 67.7(12.0) \\
\text { vs. } 65.5(13.2) \\
\text { Intermediate term: } 53.9(19.3) \text { vs. } \\
54.5(14.2) \text { vs. } 65.5(11.5)\end{array}$ & $\begin{array}{l}\text { A vs. B vs. C, Mean (SD) } \\
\text { BDI (0-63) } \\
\text { Baseline: } 23.4(11.0) \text { vs. } 20.7 \text { (6.6) vs. } \\
21.4(10.4) \\
\text { Intermediate term:16.6 (9.6) vs. } 15.0 \\
\text { (10.2) vs. } 18.7(9.5) \\
\text { SF-36 PCS (0-100) } \\
\text { Baseline: } 32.8(7.9) \text { vs. } 36.5(8.7) \text { vs. } \\
36.0(7.2) \\
\text { Intermediate term: } 39.9(7.5) \text { vs. } 39.6 \\
(8.1) \text { vs. } 34.3(8.1) \\
\text { SF-36 MCS (0-100) } \\
\text { Baseline: } 30.4(11.7) \text { vs. } 33.2 \text { (8.9) vs. } \\
36.1(9.8) \\
\text { Intermediate term: } 40.7 \text { (12.3) vs. } 40.2 \\
(10.0) \text { vs. } 37.6(10.0)\end{array}$ & NR \\
\hline $\begin{array}{l}\text { Scholten, } 1999 \\
\text { Austria } \\
\text { Duration of pain: } \\
106.8 \text { months } \\
\text { RCT } \\
\text { Poor }\end{array}$ & $\begin{array}{l}\text { A. Comprehensive pain } \\
\text { management program } \\
(n=38) \text { : } \\
9 \text { days over } 2 \text { weeks, } \\
\text { (unclear hours total) } \\
\text { group; outpatient setting } \\
\text { B. Waitlist control }(n=30) \text { : }\end{array}$ & $\begin{array}{l}\text { Mean age: } 48 \text { years } \\
\text { \% Male: } 21 \% \\
\text { Race/ethnicity: NR } \\
\text { Pain etiology/type: } \\
\text { Rheumatoid arthritis } \\
\text { Joint status } \\
\text { (Steinbrocker's criteria): } \\
\text { - functional class I: } 21 \% \\
\text { - functional class II: } 54 \% \\
\text { - functional class III: } 25 \% \\
\text { Disability: NR } \\
\text { Comorbidities: NR }\end{array}$ & $\begin{array}{l}\text { A vs. B, mean (SD) } \\
\text { Stanford Health Assessment } \\
\text { Questionnaire (1-5) } \\
\text { Baseline: } 2.6(0.78) \text { vs. } 2.9(0.62) \\
\text { Postintervention: } 1.6(0.41) \text { vs. } 2.9 \\
(0.68) \\
\text { Short term: } 1.8(0.54) \text { vs. } 2.7(0.71) \\
\text { Intermediate term: } 2.2(0.32) \text { vs. } 2.6 \\
(0.69)\end{array}$ & $\begin{array}{l}\text { A vs. B, mean (SD) } \\
\text { BDI (0-63) } \\
\text { Baseline: } 12.1(6.2) \text { vs. } 12.0(6.4) \\
\text { Postintervention: } 6.9(3.6) \text { vs. } 12.2(6.5) \\
\text { Short term: } 8.2(3.0) \text { vs. } 11.9(7.0) \\
\text { Intermediate term: } 9.6(2.3) \text { vs. } 12.1(6.5) \\
\\
\text { FQCl, Depression (5-25) } \\
\text { Baseline: } 12.7(6.6) \text { vs. } 11.9(5.3) \\
\text { Postintervention: } 10.7(4.2) \text { vs. } 12.4(5.9) \\
\text { Short term; } 10.0(3.7) \text { vs. } 12.3(5.9) \\
\text { Intermediate term: } 10.8(2.0) \text { vs. } 12.7 \\
\text { (6.2) }\end{array}$ & NR \\
\hline
\end{tabular}




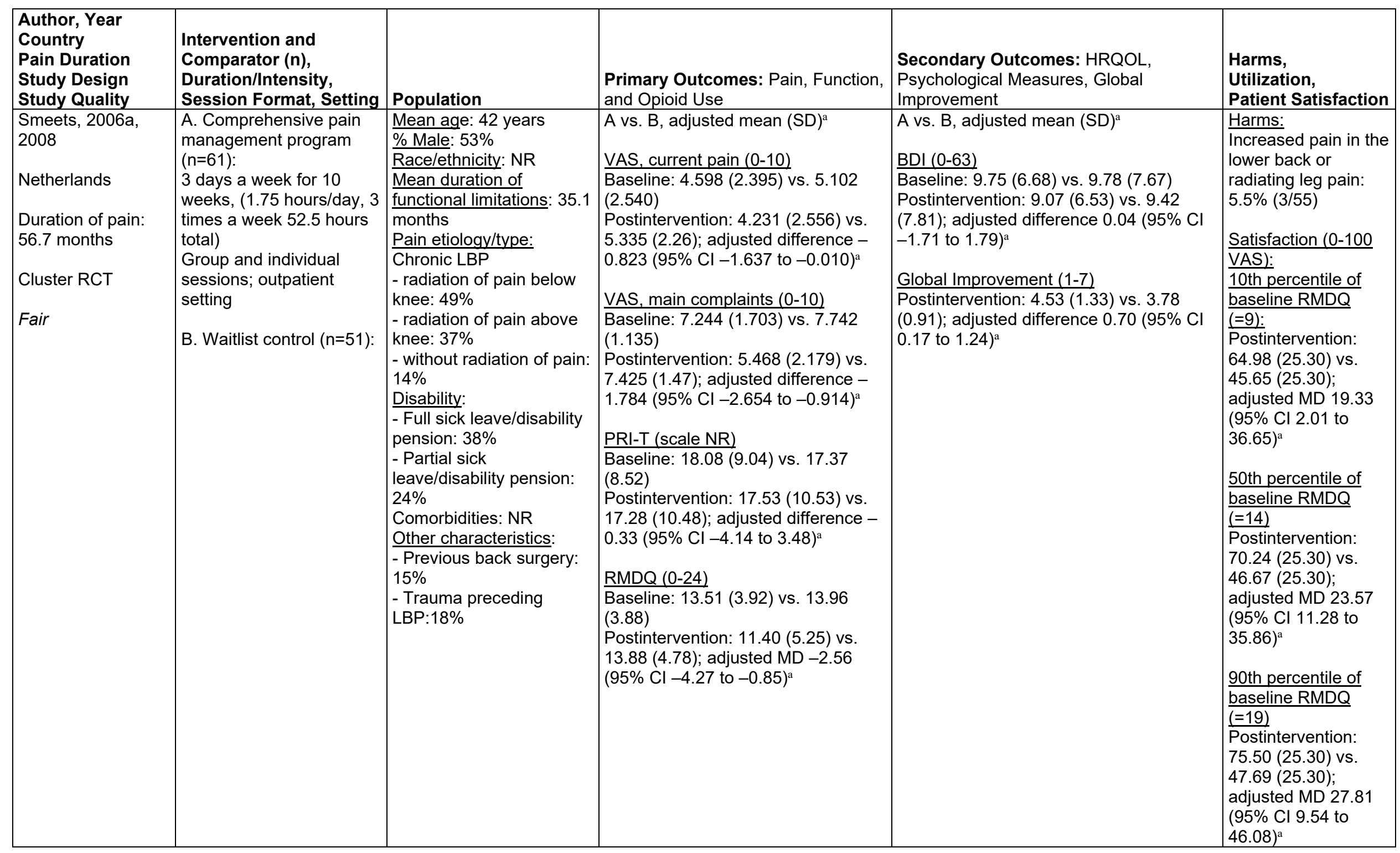




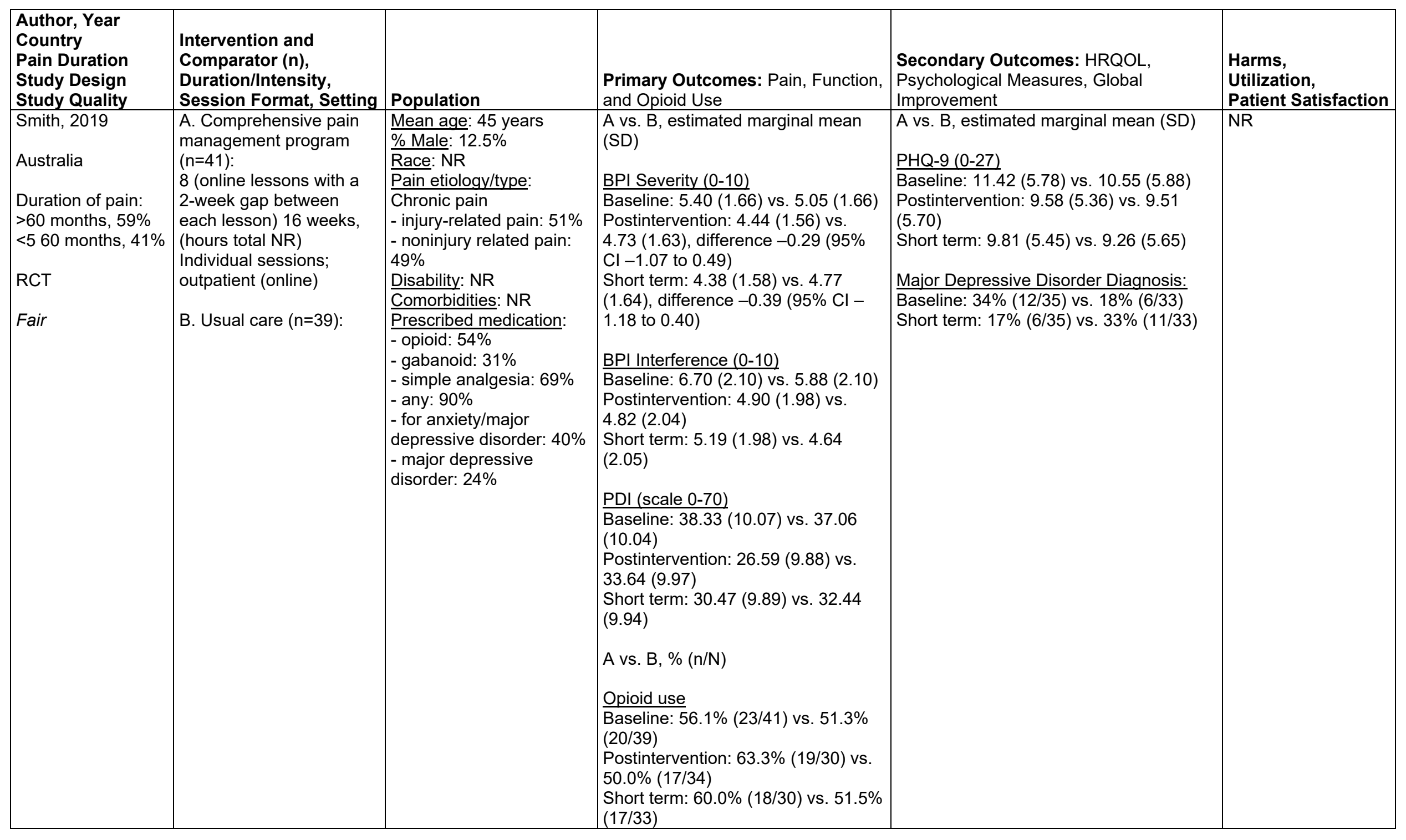




\begin{tabular}{|c|c|c|c|c|c|}
\hline $\begin{array}{l}\text { Author, Year } \\
\text { Country } \\
\text { Pain Duration } \\
\text { Study Design } \\
\text { Study Quality }\end{array}$ & $\begin{array}{l}\text { Intervention and } \\
\text { Comparator (n), } \\
\text { Duration/Intensity, } \\
\text { Session Format, Setting }\end{array}$ & Population & $\begin{array}{l}\text { Primary Outcomes: Pain, Function, } \\
\text { and Opioid Use }\end{array}$ & $\begin{array}{l}\text { Secondary Outcomes: HRQOL, } \\
\text { Psychological Measures, Global } \\
\text { Improvement }\end{array}$ & $\begin{array}{l}\text { Harms, } \\
\text { Utilization, } \\
\text { Patient Satisfaction }\end{array}$ \\
\hline $\begin{array}{l}\text { Turner, } 1990 \\
\text { USA } \\
\text { Duration of pain: } \\
155 \text { months } \\
\text { RCT } \\
\text { Poor }\end{array}$ & $\begin{array}{l}\text { A. Comprehensive pain } \\
\text { management program } \\
(n=18): 8 \text { weeks ( } 4 \\
\text { hours/week, } 32 \text { hours } \\
\text { total); group + individual } \\
\text { sessions; outpatient } \\
\text { B. Waitlist control }(n=39)\end{array}$ & $\begin{array}{l}\text { Mean age: } 44 \text { years } \\
\text { \% Male: } 52.1 \% \\
\text { Race/ethnicity: } \\
\text { White: } 100 \% \\
\text { Pain etiology/type: } \\
\text { Chronic LBP } \\
\text { Disability: NR } \\
\text { Comorbidities: NR }\end{array}$ & $\begin{array}{l}\text { A vs B, Mean (SD) } \\
\text { MPQ (0-78) } \\
\text { Baseline: } 25.54 \text { (12.41) vs. } 21.17 \\
(8.84) \\
\text { Postintervention: } 14.78 \text { (11.44) vs. } \\
20.95 \text { (10.62) [on a } 0-10 \text { scale, } 1.9 \\
(1.5) \text { vs. } 2.7 \text { (1.4), difference }-0.79 \\
(95 \% \text { Cl -1.70 to 0.12)] } \\
\text { SIP (scale NR) } \\
\text { Baseline: } 8.50 \text { (4.59) vs. } 6.24 \text { (4.99) } \\
\text { Postintervention: } 3.63 \text { (2.98) vs. } \\
5.37 \text { (5.93) }\end{array}$ & $\begin{array}{l}\text { A vs. B, Mean (SD) } \\
\text { CES-D (0-60) } \\
\text { Baseline: } 12.38(7.31) \text { vs.10.48 (4.19) } \\
\text { Postintervention: } 7.36 \text { (5.89) vs. } 7.03 \\
(5.02)\end{array}$ & $\begin{array}{l}\text { A vs. B, Mean (SD) } \\
\frac{\text { Patient satisfaction }}{\frac{(1-7)}{5.50}(\mathrm{NR}) \text { vs. NR }}\end{array}$ \\
\hline
\end{tabular}




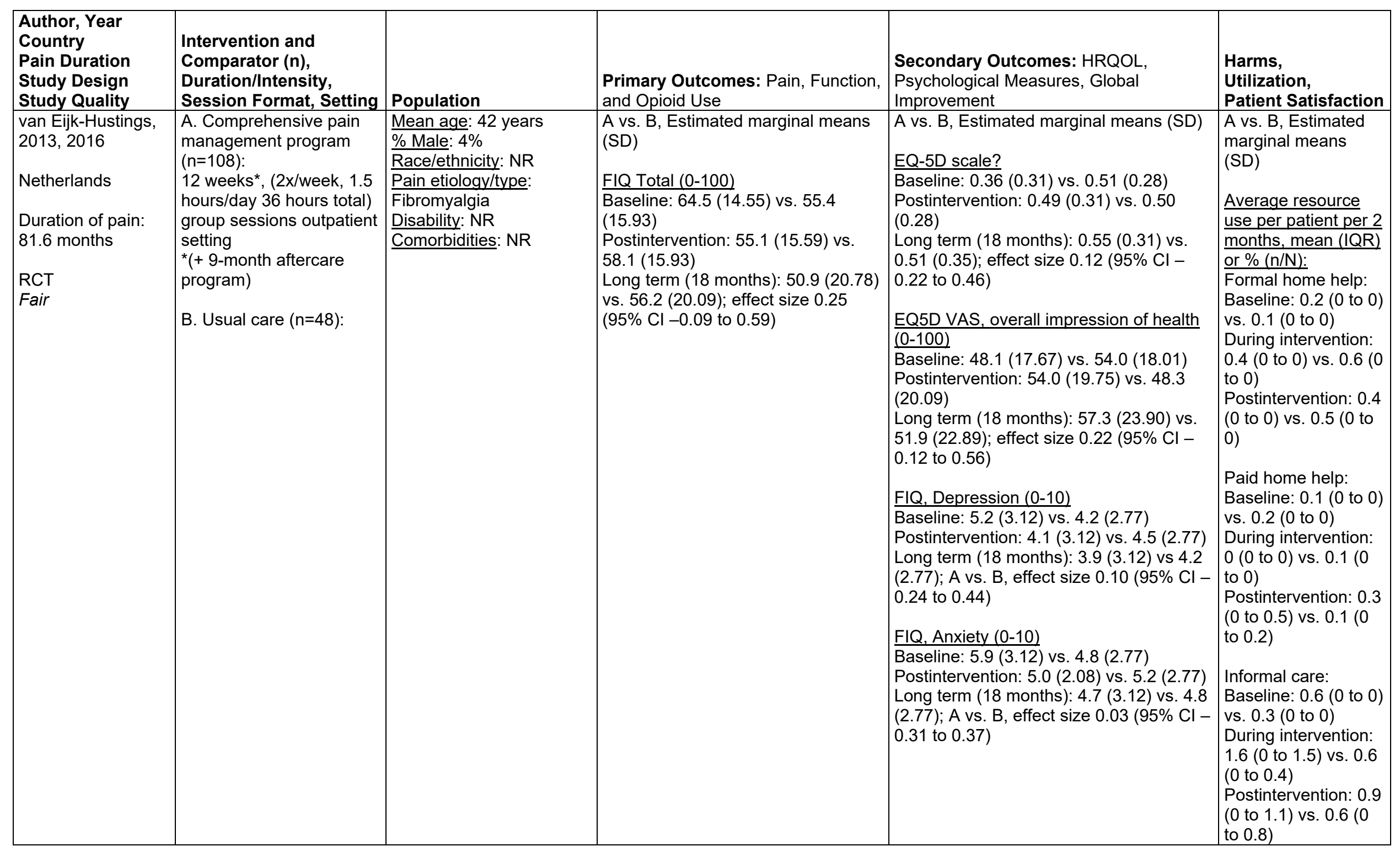




\begin{tabular}{|c|c|c|c|c|c|}
\hline $\begin{array}{l}\text { Author, Year } \\
\text { Country } \\
\text { Pain Duration } \\
\text { Study Design } \\
\text { Study Quality }\end{array}$ & $\begin{array}{l}\text { Intervention and } \\
\text { Comparator (n), } \\
\text { Duration/Intensity, } \\
\text { Session Format, Setting }\end{array}$ & Population & $\begin{array}{l}\text { Primary Outcomes: Pain, Function, } \\
\text { and Opioid Use }\end{array}$ & $\begin{array}{l}\text { Secondary Outcomes: HRQOL, } \\
\text { Psychological Measures, Global } \\
\text { Improvement }\end{array}$ & $\begin{array}{l}\text { Harms, } \\
\text { Utilization, } \\
\text { Patient Satisfaction }\end{array}$ \\
\hline $\begin{array}{l}\text { van Koulil, 2010, } \\
2011 \\
\text { Netherlands } \\
\text { Duration of pain: } \\
\text { NR } \\
\text { Cluster RCT } \\
\text { Fair }\end{array}$ & 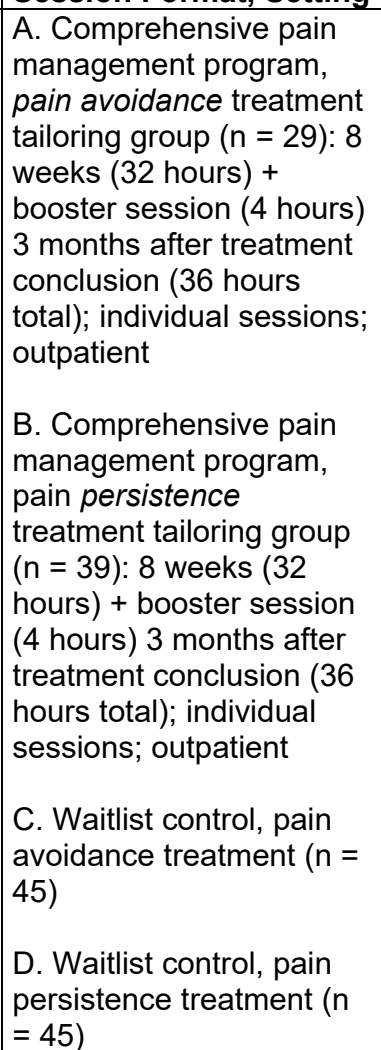 & $\begin{array}{l}\frac{\text { Mean age: }}{\text { Male: } 6 \%} \text { years } \\
\text { Race/ethnicity: NR } \\
\text { Pain etiology/type: } \\
\text { Fibromyalgia } \\
\text { Disability: NR } \\
\text { Comorbidities: } \\
\text {-self-reported heightened } \\
\text { psychological distress } \\
\text { (part of inclusion criteria) }\end{array}$ & $\begin{array}{l}\text { A vs. B vs. C vs. D, Mean (SD) } \\
\text { IRGL pain (6-25) } \\
\text { Baseline: } 20.3(2.4) \text { vs. } 19.1(3.7) \\
\text { vs. } 19.8(3.1) \text { vs. } 17.6(3.4) \\
\text { Postintervention: } 16.0(3.2) \text { vs. } 15.9 \\
\text { (3.8) vs. } 20.0(4.3) \text { vs. } 17.4(3.5) \\
\text { Short term: } 17.2(3.3) \text { vs. } 16.4(5.1) \\
\text { vs. } 20.4(3.4) \text { vs. } 16.4(3.6) \\
\\
\text { FIQ (0 to } 100) \\
\text { Baseline: } 66.3(11.6) \text { vs. } 57.2(11.0) \\
\text { vs. } 67.0(11.8) \text { vs. } 54.1(14.7) \\
\text { Postintervention: } 47.6(14.7) \text { vs. } \\
46.8(15.3) \text { vs. } 63.6(14.9) \text { vs. } 53.9 \\
(12.8) \\
\text { Short term: } 50.0(15.6) \text { vs. } 43.2 \\
\text { (18.5) vs. } 66.0(13.9) \text { vs. } 50.8(15.2) \\
\\
\text { IRGL mobility (7-28) } \\
\text { Baseline: } 13.6(3.0) \text { vs. } 18.6(4.5) \\
\text { vs. } 13.6(3.0) \text { vs. } 18.7(4.3) \\
\text { Postintervention: } 18.4(3.5) \text { vs. } 21.4 \\
(4.0) \text { vs. } 14.5(4.3) \text { vs. } 19.4(4.1) \\
\text { Short term: } 19.3(3.8) \text { vs. } 22.2(4.8) \\
\text { vs. } 14.5(4.2) \text { vs. } 19.8(4.4)\end{array}$ & $\begin{array}{l}\text { A vs. B vs. C vs. D, Mean (SD) } \\
\text { IRGL negative mood scale (0-24) } \\
\text { Baseline: } 8.9(3.8) \text { vs. } 5.9(3.3) \text { vs. } 10.5 \\
\text { (5.7) vs. } 5.6(3.6) \\
\text { Postintervention: } 4.7(3.7) \text { vs. } 4.0(3.5) \\
\text { vs. } 8.8(6.2) \text { vs. } 6.3(3.7) \\
\text { Short term: } 5.0(3.5) \text { vs. } 3.5(2.6) \text { vs. } 8.4 \\
\text { (5.2) vs. } 6.1(4.5) \\
\text { IRGL anxiety scale (10-40) } \\
\text { Baseline: } 26.3(5.9) \text { vs. } 23.2(4.3) \text { vs. } \\
27.0(6.4) \text { vs. } 23.9(5.1) \\
\text { Postintervention: } 21.6(5.9) \text { vs. } 20.6 \text { (4.3) } \\
\text { vs. } 25.6(6.7) \text { vs. } 23.6(5.2) \\
\text { Short term: } 20.3(5.6) \text { vs. } 19.0(4.4) \text { vs. } \\
26.0(5.4) \text { vs. } 22.7(5.4)\end{array}$ & NR \\
\hline
\end{tabular}




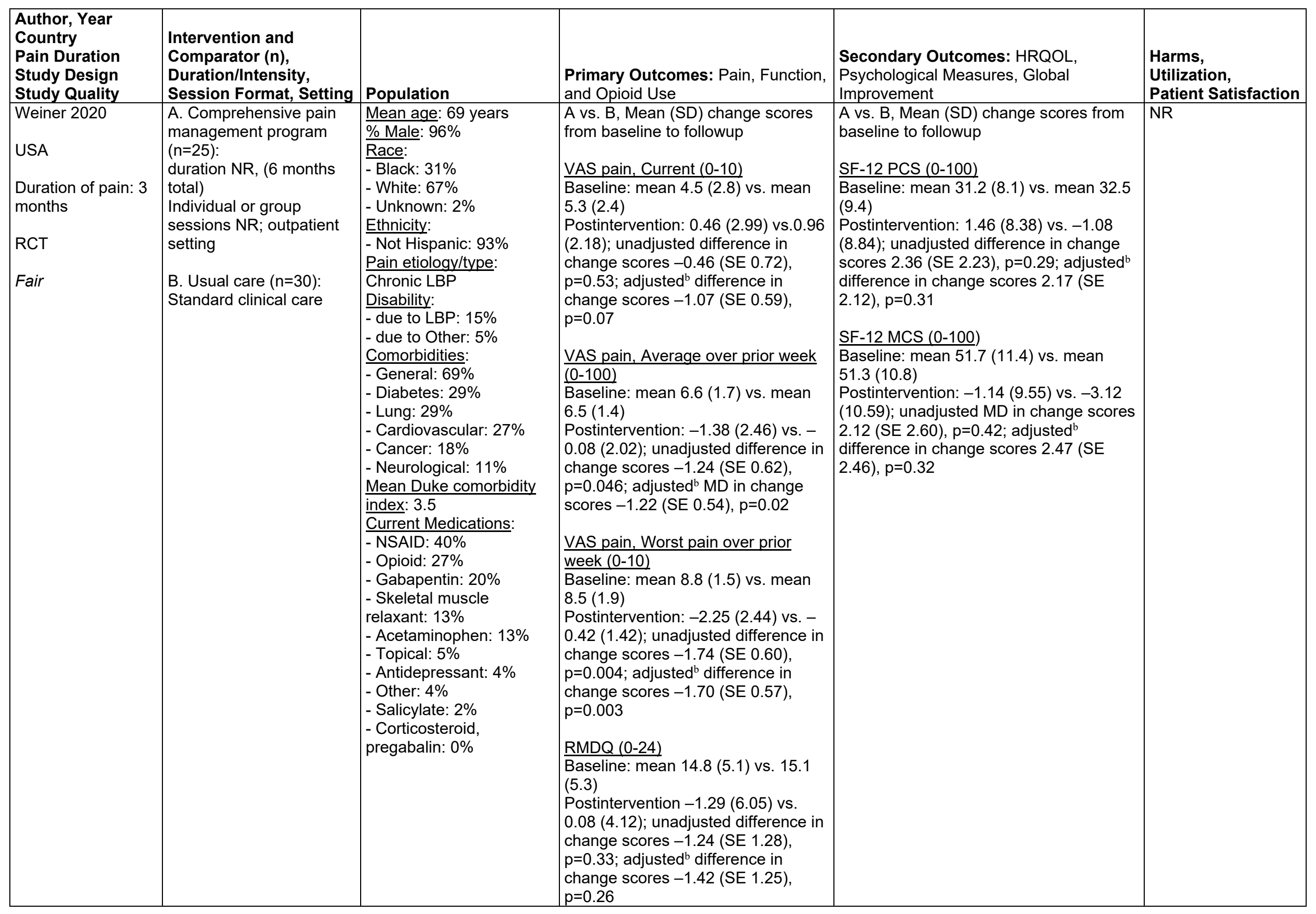




\begin{tabular}{|c|c|c|c|c|c|}
\hline $\begin{array}{l}\text { Author, Year } \\
\text { Country } \\
\text { Pain Duration } \\
\text { Study Design } \\
\text { Study Quality }\end{array}$ & $\begin{array}{l}\text { Intervention and } \\
\text { Comparator (n), } \\
\text { Duration/Intensity, } \\
\text { Session Format, Setting }\end{array}$ & Population & $\begin{array}{l}\text { Primary Outcomes: Pain, Function, } \\
\text { and Opioid Use }\end{array}$ & $\begin{array}{l}\text { Secondary Outcomes: HRQOL, } \\
\text { Psychological Measures, Global } \\
\text { Improvement }\end{array}$ & $\begin{array}{l}\text { Harms, } \\
\text { Utilization, } \\
\text { Patient Satisfaction }\end{array}$ \\
\hline $\begin{array}{l}\text { Whitfill, } 2010 \\
\text { USA } \\
\text { Duration of pain: } \\
\text { NR } \\
\text { RCT } \\
\text { Poor }\end{array}$ & $\begin{array}{l}\text { A. Comprehensive pain } \\
\text { management program } \\
(n=90): 4 \text { to } 10 \text { weeks; } \\
\text { intensity unclear; } \\
\text { individual sessions; } \\
\text { outpatient } \\
\text { B. Usual care }(n=52)\end{array}$ & $\begin{array}{l}\text { Mean age: } 40 \text { years } \\
\text { \% Male: } 50 \% \\
\text { Race/ethnicity: } \\
\text { - Caucasian: } 51.7 \% \text { vs. } \\
40.3 \% \\
\text { - Latino: } 19.0 \% \text { vs. } 20.7 \% \\
\text { - African American: } \\
27.6 \% \text { vs. } 32.0 \% \\
\text { - Asian: } 1.7 \% \text { vs. } 7.0 \% \\
\text { - Other: } 0.0 \% \text { vs. } 0.0 \% \\
\text { Pain etiology/type: Low } \\
\text { back pain } \\
\text { Disability: NR } \\
\text { Other characteristics: NR }\end{array}$ & $\begin{array}{l}\text { A vs. B, Mean (SD) } \\
\text { VAS pain }(0-10) \\
\text { Baseline: } 6.00(2.07) \text { vs. } 5.95(1.95) \\
\text { Intermediate term: } 3.91(2.86) \text { vs. } \\
5.07(2.78) \text {, difference }-1.16(95 \% \\
\mathrm{Cl}-2.26 \text { to }-0.06) \\
\text { CPI (0-10) } \\
\text { Baseline: } 5.23(2.51) \text { vs. } 2.50(2.45) \\
\text { Intermediate term: } 2.96(2.82) \text { vs. } \\
4.27(3.01)\end{array}$ & $\begin{array}{l}\text { A vs. B, Mean (SD) } \\
\text { SF-36 (0 to } 100) \\
\text { Baseline: } 33.00(8.09) \text { vs. } 35.99(10.13) \\
\text { Intermediate term: } 40.47(11.47) \text { vs. } \\
39.45(10.59) \\
\text { BDI (0 to } 63) \\
\text { Baseline: } 11.63(9.30) \text { vs. } 9.43(9.58) \\
\text { Intermediate term: } 8.81(9.49) \text { vs. } 10.11 \\
(10.23)\end{array}$ & NR \\
\hline
\end{tabular}




\begin{tabular}{|c|c|c|c|c|c|}
\hline $\begin{array}{l}\text { Author, Year } \\
\text { Country } \\
\text { Pain Duration } \\
\text { Study Design } \\
\text { Study Quality }\end{array}$ & $\begin{array}{l}\text { Intervention and } \\
\text { Comparator }(\mathrm{n}), \\
\text { Duration/Intensity, } \\
\text { Session Format, Setting }\end{array}$ & Population & $\begin{array}{l}\text { Primary Outcomes: Pain, Function, } \\
\text { and Opioid Use }\end{array}$ & $\begin{array}{l}\text { Secondary Outcomes: HRQOL, } \\
\text { Psychological Measures, Global } \\
\text { Improvement }\end{array}$ & $\begin{array}{l}\text { Harms, } \\
\text { Utilization, } \\
\text { Patient Satisfaction }\end{array}$ \\
\hline $\begin{array}{l}\text { Williams, } 1996 \\
\text { UK } \\
\text { Duration of pain: } \\
93.7 \text { months } \\
\text { RCT } \\
\text { Poor }\end{array}$ & $\begin{array}{l}\text { A. Comprehensive pain } \\
\text { management program, } \\
\text { inpatient group }(n=43) \text { : } \\
4.5 \text { days a week for } 4 \\
\text { weeks, }(28 \text { hours total) } \\
\text { group sessions; inpatient } \\
\\
\text { B. Comprehensive pain } \\
\text { management program, } \\
\text { outpatient group ( } n=45) \text { : } 8 \\
\text { weeks, }(3.5 \text { hours a } \\
\text { week, } 28 \text { hours total) } \\
\text { group sessions outpatient } \\
\\
\text { C. Waitlist control }(n=33)\end{array}$ & $\begin{array}{l}\text { Mean age: } 50 \text { years } \\
\text { \% Male: } 47 \% \\
\text { Race/ethnicity: } \\
\text { Afro-Caribbean or Asian: } \\
12 \%-16 \% \\
\text { White: } 84 \%-88 \% \\
\text { Pain etiology/type: Mixed } \\
\text { chronic pain } \\
\text { - back/neck/legs: } 74 \% \\
\text { - } \geq 1 \text { surgery: } 39 \% \\
\text { - central/peripheral nerve } \\
\text { system damage: } 26 \% \\
\text { - other tissue damage: } \\
16 \% \\
\text { - unknown mechanism: } \\
58 \% \\
\text { Receiving disability } \\
\text { income: } 62 \% \\
\text { Litigation related to pain: } \\
21 \% \\
\text { Opioid use: } 61 \% \\
\text { Excess drug use: } 59 \%\end{array}$ & 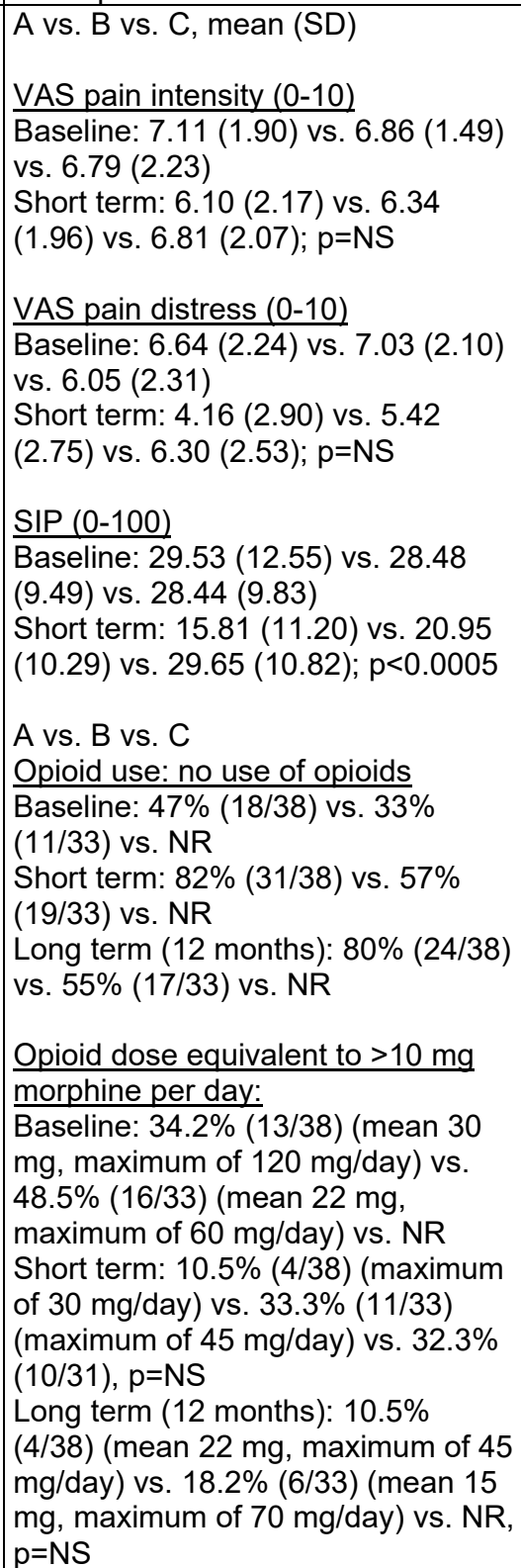 & $\begin{array}{l}\text { A vs. B vs. C, mean (SD) } \\
\text { BDI (0-63) } \\
\text { Baseline: } 17.8(8.0) \text { vs. } 16.8(5.6) \text { vs. } \\
16.6(6.5) \\
\text { Short term: } 9.5(7.8) \text { vs. } 12.2(6.3) \text { vs. } \\
17.3(7.0) ; p<0.0005 \\
\text { STAl (20-80) } \\
\text { Baseline: } 45.1(10.7) \text { vs. } 45.7(8.2) \text { vs. } \\
44.8(11.6) \\
\text { Short term: } 36.8(13.6) \text { vs. } 42.3(10.6) \text { vs. } \\
45.0(11.7), p<0.05 \text { for all }\end{array}$ & $\begin{array}{l}\text { A vs. B vs. C } \\
\text { Utilization: } \\
\text { Subsequent } \\
\text { treatments: } \\
\text { - Surgery: } 0 \% \text { for all } \\
\text { groups } \\
\text { - Any, to include the } \\
\text { above as well as } \\
\text { prescribed and } \\
\text { nonprescribed } \\
\text { analgesics: } 44.8 \% \\
(13 / 29) \text { vs. } 85.7 \% \\
\text { vs. NR (24/28); RR } \\
0.52(95 \% \mathrm{Cl} 0.34 \text { to } \\
0.80)\end{array}$ \\
\hline
\end{tabular}




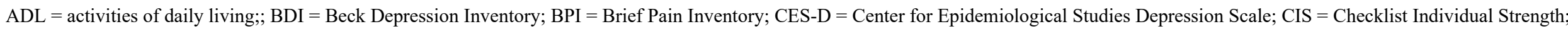

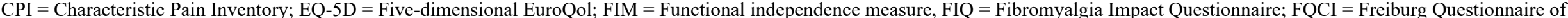

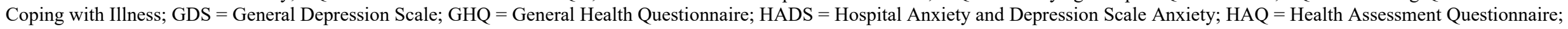

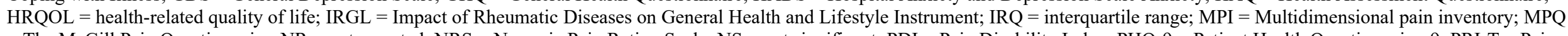

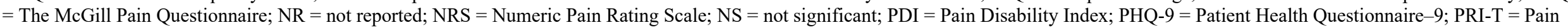

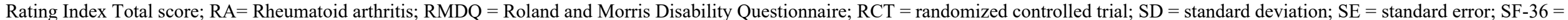
Short-Form 36 questionnaire; SIP = Sickness Impact Profile; STAI = Spielberger State-Trait Anxiety Inventory; VAS = visual analog scale.

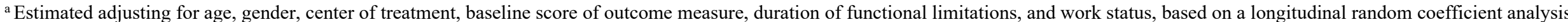
with an extra random intercept for clusters of four patients being randomized together.

${ }^{\mathrm{b}}$ Adjusted for baseline scores. 
Table B-6. Summary results for trials addressing KQ1: CPMPs versus physical activity

\begin{tabular}{|c|c|c|c|c|c|}
\hline $\begin{array}{l}\text { Author, Year } \\
\text { Country } \\
\text { Pain Duration } \\
\text { Study Design } \\
\text { Study Quality } \\
\end{array}$ & $\begin{array}{l}\text { Intervention }(\mathrm{n}) \\
\text { Comparator (n) } \\
\text { Duration/Intensity } \\
\text { Session Format } \\
\text { Setting (IP/OP) } \\
\end{array}$ & Population & $\begin{array}{l}\text { Primary Outcomes: } \\
\text { Pain, Function, and Opioid Use }\end{array}$ & $\begin{array}{l}\text { Secondary Outcomes: HRQOL, } \\
\text { Psychological Measures, Global } \\
\text { Improvement }\end{array}$ & $\begin{array}{l}\text { Harms, } \\
\text { Utilization, } \\
\text { Patient Satisfaction }\end{array}$ \\
\hline $\begin{array}{l}\text { Alaranta, } 1994 \\
\text { Finland } \\
\text { Mean duration of } \\
\text { pain: NR ( } \geq 6 \\
\text { months) } \\
\text { RCT } \\
\text { Fair } \\
\end{array}$ & $\begin{array}{l}\text { A. CPMP }(n=152) \\
6 \text { weeks, } 52 \text { hours } \\
\text { total; group }+ \\
\text { individual; } 3 \text { weeks at } \\
\text { home }+3 \text { weeks } \\
\text { inpatient } \\
\\
\text { B. PA ( } n=141) \\
3 \text { weeks } 45-60 \text { hours } \\
\text { total, session format } \\
\text { NR, inpatient }\end{array}$ & $\begin{array}{l}\text { Mean age: } 40 \text { years } \\
\text { Male: } 45 \% \\
\text { Race/Ethnicity: NR } \\
\text { Pain etiology/type: } \\
\text { Chronic LBP } \\
\text { Disability: } \\
\text { - Million index (0-100): } \\
58.1 \\
\text { Comorbidities: } \\
- \text { NR }\end{array}$ & $\begin{array}{l}\text { A vs. B, Mean (SD) } \\
\text { MVAS disability (0-100) } \\
\text { (f/u data estimated by EPC from Fig } 4 \text { of } \\
\text { study publication) } \\
\text { Baseline: } 45.5(18.8) \text { vs. } 45.1(20.8) \\
\text { Short term: } 28.5(20.9) \text { vs. } 35.8(20.3), \\
\text { difference }-7.3(95 \% \mathrm{Cl}-12.1 \text { to } 2.5) \\
\text { Intermediate term: } 29.6(23.2) \text { vs. } 36.1 \\
(23.9) \text {, difference }-6.5(95 \% \mathrm{Cl}-12.0 \text { to - } \\
1.0)\end{array}$ & $\begin{array}{l}\frac{\text { Symptom Check List Pain and }}{\text { Anxiety Subscales: Data not }} \\
\text { reported }(p>0.05) \\
\text { BDI: Data not reported. }\end{array}$ & $\begin{array}{l}\text { A vs. B } \\
\text { Harms: NR } \\
\text { Decrease (\%) in the } \\
\text { number of yearly visits } \\
\text { Physician: } \\
74 \% \text { vs. } 67 \% \\
\text { Outpatient PT: } \\
69 \% \text { vs. } 77 \%\end{array}$ \\
\hline
\end{tabular}




\begin{tabular}{|c|c|c|c|c|c|}
\hline $\begin{array}{l}\text { Author, Year } \\
\text { Country } \\
\text { Pain Duration } \\
\text { Study Design } \\
\text { Study Quality }\end{array}$ & \begin{tabular}{|l|} 
Intervention (n) \\
Comparator (n) \\
Duration/Intensity \\
Session Format \\
Setting (IP/OP) \\
\end{tabular} & Population & $\begin{array}{l}\text { Primary Outcomes: } \\
\text { Pain, Function, and Opioid Use }\end{array}$ & $\begin{array}{l}\text { Secondary Outcomes: HRQOL, } \\
\text { Psychological Measures, Global } \\
\text { Improvement }\end{array}$ & $\begin{array}{l}\text { Harms, } \\
\text { Utilization, } \\
\text { Patient Satisfaction }\end{array}$ \\
\hline $\begin{array}{l}\text { Bendix, 1995, } \\
\text { 1997, 1998a, } \\
\text { 1998b } \\
\text { (PROJECT B) } \\
\text { Denmark } \\
\text { Mean duration of } \\
\text { pain: NR ( } \geq 6 \\
\text { months) } \\
\text { RCT } \\
\text { Fair }\end{array}$ & $\begin{array}{l}\text { A. CPMP, high } \\
\text { intensity ( } n=46) \\
3 \text { weeks (39 } \\
\text { hours/week + one 6- } \\
\text { hour session /week for } \\
3 \text { weeks, group, } \\
\text { outpatient } \\
\text { B. CPMP, lower } \\
\text { intensity ( } n=43 \text { ) } \\
\text { Twice weekly for } 6 \\
\text { weeks (total } 24 \text { hours), } \\
\text { group, outpatient } \\
\\
\text { C. PA (n=43) } \\
\text { Twice weekly for } 6 \\
\text { weeks (total } 24 \text { hours), } \\
\text { group, outpatient }\end{array}$ & $\begin{array}{l}\text { Mean age: } 42 \text { years } \\
\text { Male: } 25 \% \\
\text { Race/Ethnicity: NR } \\
\text { Pain etiology/type: } \\
\text { Chronic LBP } \\
\text { Disability: } \\
\text { Patient's perception of } \\
\text { disability due to back } \\
\text { pain (0-30): } 15 \\
\text { Comorbidities: } \\
\text { Smoker: } 66 \% \\
\text { Prior back surgery: } 21 \%\end{array}$ & 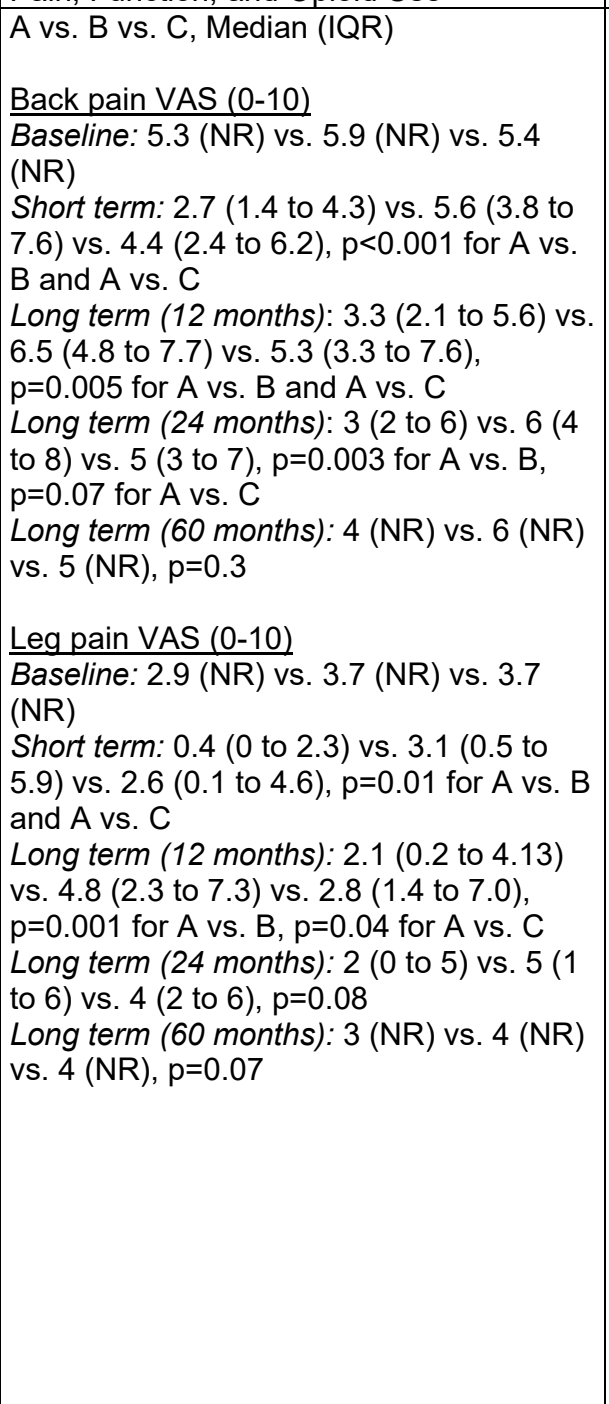 & NR & 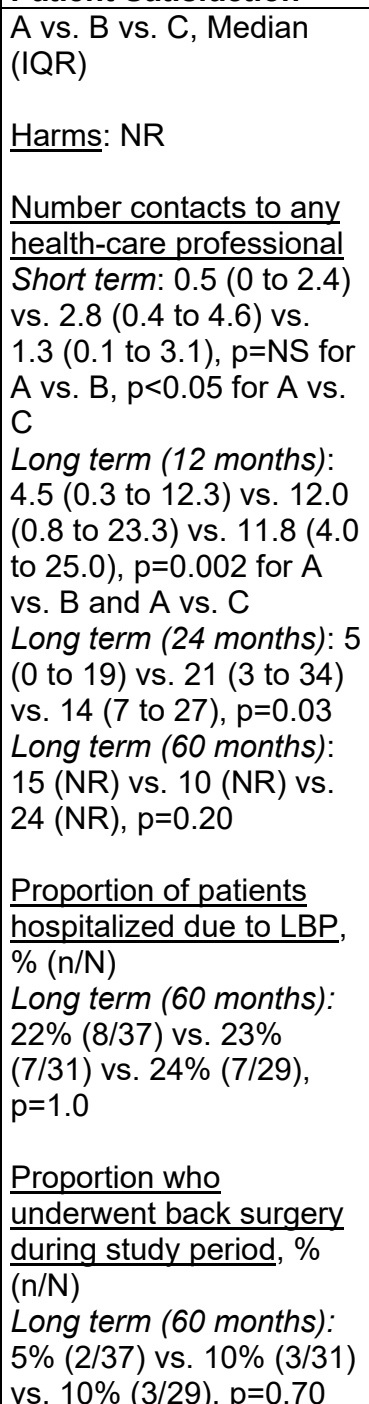 \\
\hline
\end{tabular}




\begin{tabular}{|c|c|c|c|c|c|}
\hline $\begin{array}{l}\text { Author, Year } \\
\text { Country } \\
\text { Pain Duration } \\
\text { Study Design } \\
\text { Study Quality }\end{array}$ & $\begin{array}{l}\text { Intervention (n) } \\
\text { Comparator (n) } \\
\text { Duration/Intensity } \\
\text { Session Format } \\
\text { Setting (IP/OP) }\end{array}$ & Population & $\begin{array}{l}\text { Primary Outcomes: } \\
\text { Pain, Function, and Opioid Use }\end{array}$ & $\begin{array}{l}\text { Secondary Outcomes: HRQOL, } \\
\text { Psychological Measures, Global } \\
\text { Improvement }\end{array}$ & $\begin{array}{l}\text { Harms, } \\
\text { Utilization, } \\
\text { Patient Satisfaction }\end{array}$ \\
\hline $\begin{array}{l}\text { Bendix, 1995, } \\
\text { 1997, 1998a, } \\
\text { 1998b } \\
\text { (PROJECT B) } \\
\text { (Continued) }\end{array}$ & & & 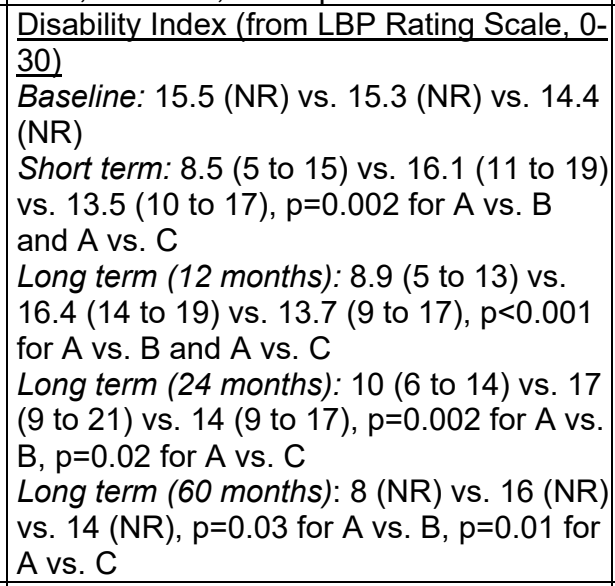 & & \\
\hline $\begin{array}{l}\text { Bendix, } 2000 \\
\text { Denmark } \\
\text { Mean duration of } \\
\text { pain: NR ( } \geq 6 \\
\text { months) } \\
\text { RCT } \\
\text { Fair }\end{array}$ & $\begin{array}{l}\text { A. CPMP }(\mathbf{n}=59) \\
3 \text { weeks (40 } \\
\text { hours/week), then } 1 \\
\text { day weekly for } 3 \text { weeks } \\
\text { + a } 6 \text {-hour follow-up, } \\
\text { group, outpatient } \\
\\
\text { B. PA ( } \mathbf{n}=68) \\
8 \text { weeks }(1.5 \text { hour per } \\
\text { day, } 3 \times / \text { week, } 36 \text { hours } \\
\text { total), group, outpatient }\end{array}$ & $\begin{array}{l}\text { Median age: } 41 \text { years } \\
\text { Male: } 35 \% \\
\text { Race/Ethnicity: NR } \\
\text { Pain etiology/type: } \\
\text { Chronic LBP } \\
\text { Disability: } \\
\text { - Working capable: } 46 \% \\
\text { Comorbidities: NR }\end{array}$ & 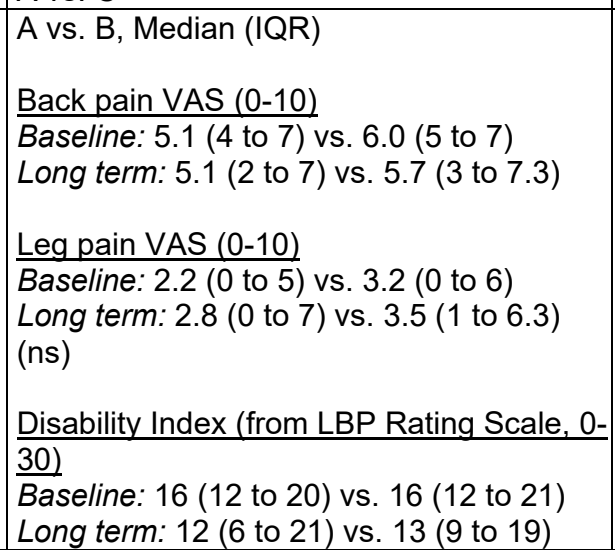 & 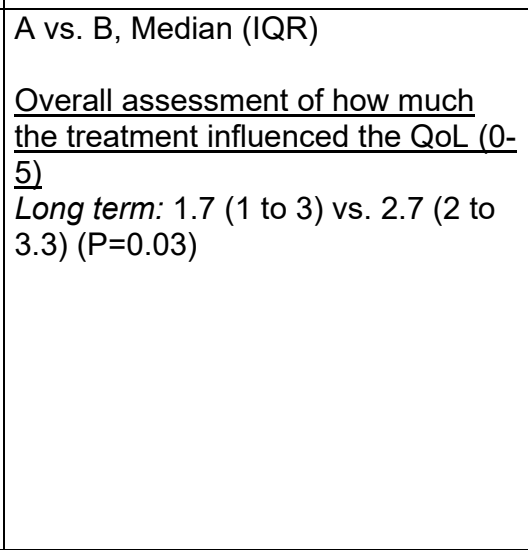 & $\begin{array}{l}\text { A vs. B, Median (IQR) } \\
\text { Harms: NR } \\
\frac{\text { Number contacts to any }}{\text { health-care professional }} \\
\begin{array}{l}\text { Long term: } 2.5(0 \text { to } 10) \\
\text { vs. } 4 \text { (0 to } 12.3)(\mathrm{ns})\end{array}\end{array}$ \\
\hline
\end{tabular}




\begin{tabular}{|c|c|c|c|c|c|}
\hline $\begin{array}{l}\text { Author, Year } \\
\text { Country } \\
\text { Pain Duration } \\
\text { Study Design } \\
\text { Study Quality }\end{array}$ & $\begin{array}{l}\text { Intervention (n) } \\
\text { Comparator (n) } \\
\text { Duration/Intensity } \\
\text { Session Format } \\
\text { Setting (IP/OP) } \\
\end{array}$ & Population & $\begin{array}{l}\text { Primary Outcomes: } \\
\text { Pain, Function, and Opioid Use }\end{array}$ & $\begin{array}{l}\text { Secondary Outcomes: HRQOL, } \\
\text { Psychological Measures, Global } \\
\text { Improvement }\end{array}$ & $\begin{array}{l}\text { Harms, } \\
\text { Utilization, } \\
\text { Patient Satisfaction }\end{array}$ \\
\hline $\begin{array}{l}\text { Henchoz, } 2010 \\
\text { Switzerland } \\
\text { Mean duration of } \\
\text { pain: NR (>6 } \\
\text { weeks) } \\
\text { RCT } \\
\text { Fair }\end{array}$ & $\begin{array}{l}\text { A. CPMP }(\mathbf{n}=\mathbf{4 0}) \\
3 \text { weeks }(\sim 31 \\
\text { hours/week, } 93 \text { hours } \\
\text { total), group }+ \\
\text { individual, outpatient } \\
\\
\text { B. PA ( } \mathbf{n}=27) \\
9 \text { weeks (1.5 } \\
\text { hours/week, } 13.5 \text { hours } \\
\text { total), individual, } \\
\text { outpatient }\end{array}$ & $\begin{array}{l}\text { Mean age: } 40 \text { years } \\
\text { Male: } 67 \% \\
\text { Race/Ethnicity: NR } \\
\text { Pain etiology/type: LBP } \\
\text { Disability: NR } \\
\text { Comorbidities: NR }\end{array}$ & $\begin{array}{l}\text { A vs. B, Mean (SD) } \\
\text { ODI (0-100) } \\
\text { Baseline: } 37.6(15.8) \text { vs. } 39.1(14.7) \\
\text { Postintervention: } 30.1 \text { (16.5) vs. } 37.2 \\
\text { (13.5), difference }-7.1 \text { (95\% Cl }-14.75 \text { to } \\
0.55) \\
\text { Short term: } 25.7(15.8) \text { vs. } 35.0(12.3), \\
\text { difference }-9.3(95 \% \mathrm{Cl}-16.51 \text { to }-2.09) \\
\text { Intermediate term (6 months): } 28.6(18.4) \\
\text { vs. } 35.4(15.0) \text {, difference }-6.8,(95 \% \mathrm{Cl}- \\
15.32 \text { to } 1.72) \\
\text { Intermediate term (9 months): } 29.6(17.9) \\
\text { vs. } 39.8(17.3), \text { difference }-10.2,(95 \% \mathrm{Cl} \\
-18.99 \text { to }-1.41) \\
\text { Long term (12 months): } 26.2(18.0) \text { vs. } \\
38.0(18.4), \text { difference }-11.8(95 \% \mathrm{Cl}- \\
20.83 \text { to }-2.77)\end{array}$ & $\mathrm{NR}$ & NR \\
\hline
\end{tabular}




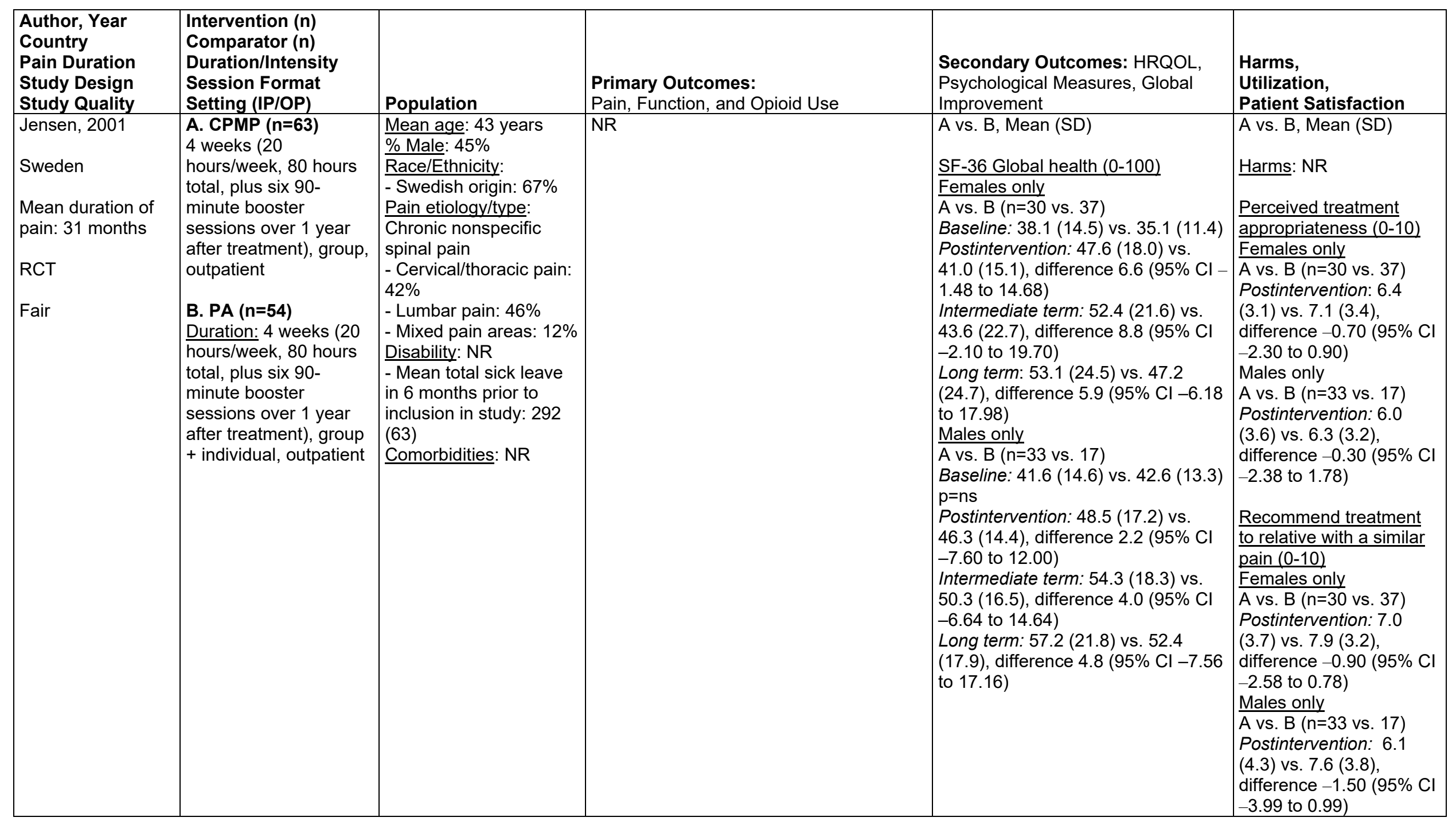




\begin{tabular}{|c|c|c|c|c|c|}
\hline $\begin{array}{l}\text { Author, Year } \\
\text { Country } \\
\text { Pain Duration } \\
\text { Study Design } \\
\text { Study Quality }\end{array}$ & $\begin{array}{l}\text { Intervention (n) } \\
\text { Comparator (n) } \\
\text { Duration/Intensity } \\
\text { Session Format } \\
\text { Setting (IP/OP) }\end{array}$ & Population & $\begin{array}{l}\text { Primary Outcomes: } \\
\text { Pain, Function, and Opioid Use }\end{array}$ & $\begin{array}{l}\text { Secondary Outcomes: HRQOL, } \\
\text { Psychological Measures, Global } \\
\text { Improvement }\end{array}$ & $\begin{array}{l}\text { Harms, } \\
\text { Utilization, } \\
\text { Patient Satisfaction }\end{array}$ \\
\hline $\begin{array}{l}\text { Jousett, } 2004 \\
\text { France } \\
\text { Mean duration of } \\
\text { pain: NR } \\
\text { RCT } \\
\text { Fair }\end{array}$ & $\begin{array}{l}\text { A. CPMP ( } \mathbf{n}=43) \\
5 \text { weeks ( } 6 \text { hours per } \\
\text { day, } 30 \text { hours total), } \\
\text { group + individual, } \\
\text { outpatient } \\
\text { B. PA ( } n=41) \\
5 \text { weeks ( } 3 \text { hours per } \\
\text { week in clinic, } 2 \text { hours } \\
\text { per week at home, } 25 \\
\text { hours total), individual, } \\
\text { outpatient }\end{array}$ & $\begin{array}{l}\text { Mean age: } 41 \text { years } \\
\text { \% Male: } 67 \% \\
\text { Race/Ethnicity: NR } \\
\text { Pain etiology/type: } \\
\text { Chronic LBP } \\
\text { Disability: } \\
\text { - On sick leave } \\
\text { - Mean sick leave days } \\
\text { in the } 2 \text { prior years: } 198 \\
\text { days } \\
\text { Comorbidities: } \\
\text { - Prior surgery: } 25 \% \\
\text { - Prior depression: } 30 \% \\
\text { - Smoking: } 39 \%\end{array}$ & 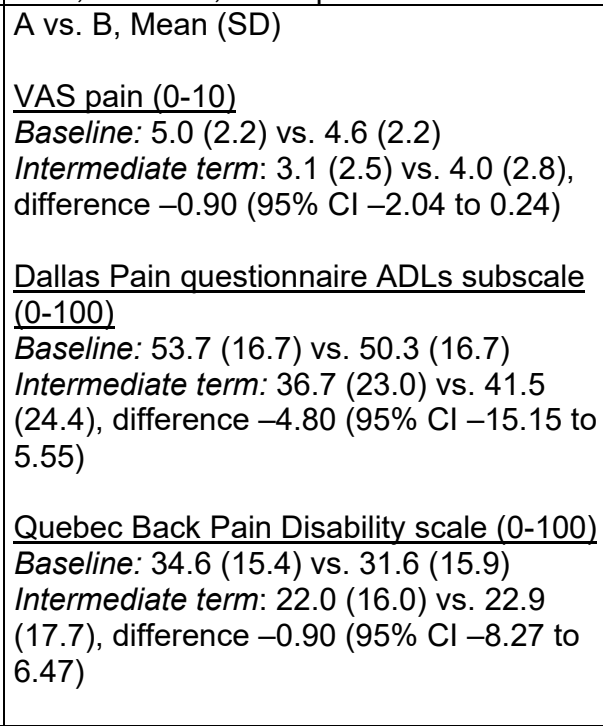 & 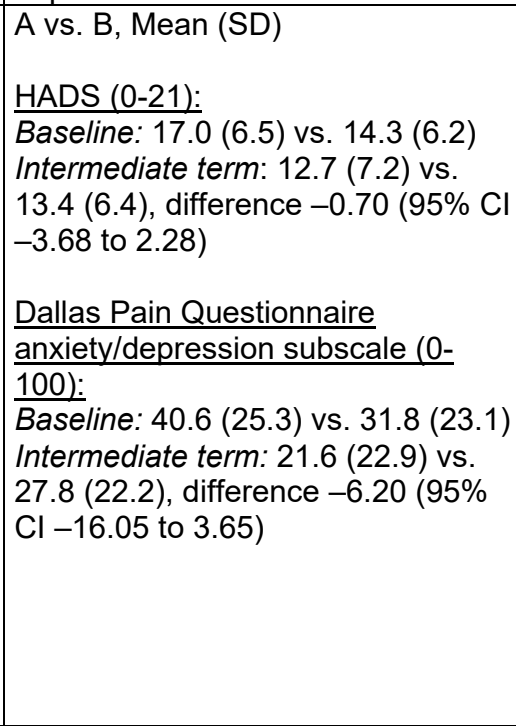 & $\begin{array}{l}\text { A vs. B, \% (n/N) or Mean } \\
\text { (SD) } \\
\text { Harms: NR } \\
\text { Proportion seeking pain } \\
\text { treatments } \\
\text { Baseline: } 85.7 \%(63 / 42) \\
\text { vs. } 78.0 \%(32 / 41) \\
\text { Intermediate term: } 64.3 \% \\
(27 / 42) \text { vs. } 61.0 \% \\
(25 / 41), \text { RR } 1.05(95 \% \\
\text { Cl } 0.76 \text { to } 1.47) \\
\\
\frac{\text { Treatment appreciation }}{(1-5)} \\
\text { Intermediate term: } 1.9 \\
(0.8) \text { vs. } 2.3(0.9), \\
\text { difference }-0.40(95 \% \mathrm{Cl} \\
-0.77 \text { to }-0.03)\end{array}$ \\
\hline
\end{tabular}




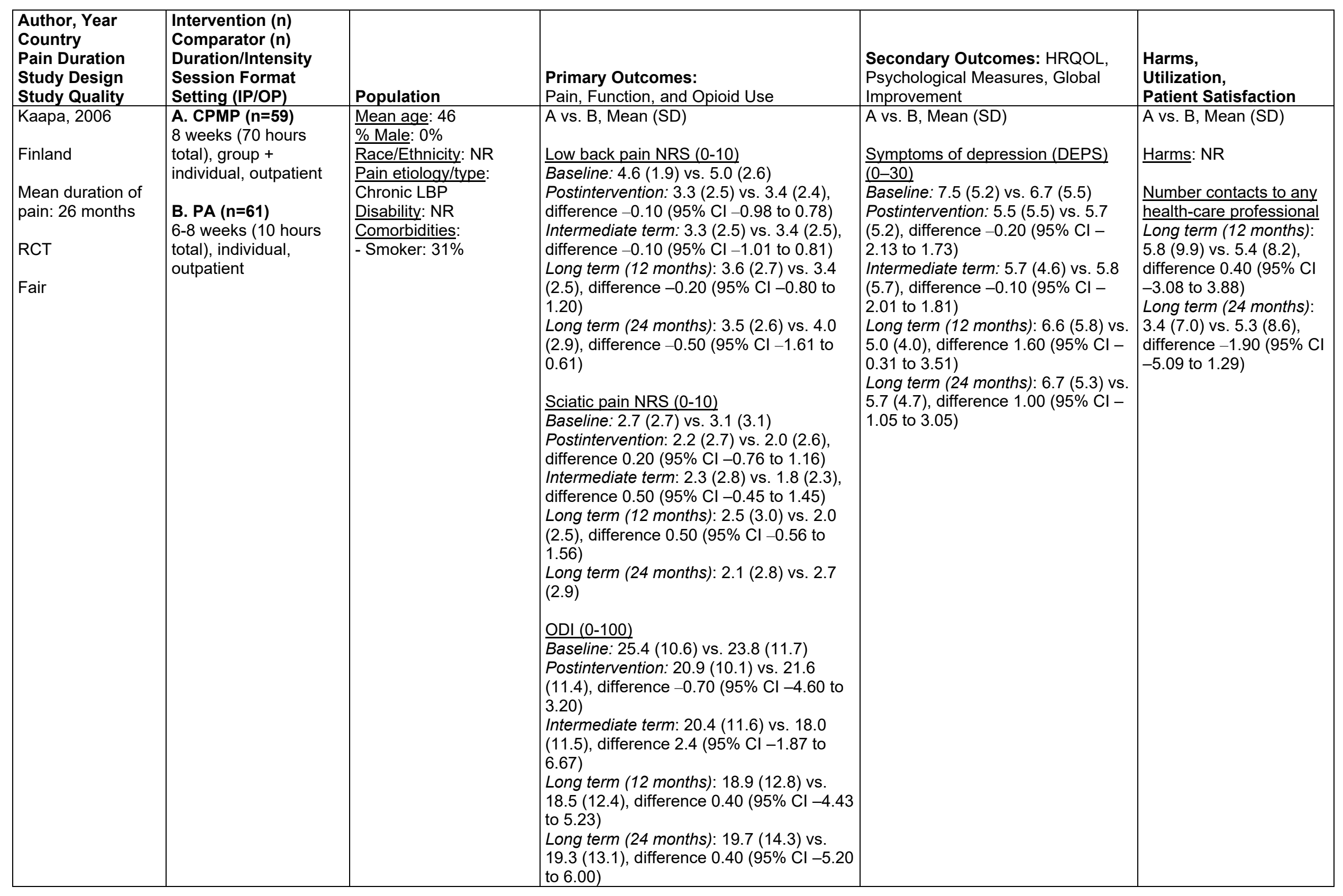




\begin{tabular}{|c|c|c|c|c|c|}
\hline $\begin{array}{l}\text { Author, Year } \\
\text { Country } \\
\text { Pain Duration } \\
\text { Study Design } \\
\text { Study Quality }\end{array}$ & $\begin{array}{l}\text { Intervention (n) } \\
\text { Comparator (n) } \\
\text { Duration/Intensity } \\
\text { Session Format } \\
\text { Setting (IP/OP) }\end{array}$ & Population & $\begin{array}{l}\text { Primary Outcomes: } \\
\text { Pain, Function, and Opioid Use }\end{array}$ & $\begin{array}{l}\text { Secondary Outcomes: HRQOL, } \\
\text { Psychological Measures, Global } \\
\text { Improvement }\end{array}$ & $\begin{array}{l}\text { Harms, } \\
\text { Utilization, } \\
\text { Patient Satisfaction }\end{array}$ \\
\hline $\begin{array}{l}\text { Mangels, } 2009 \\
\text { Germany } \\
\text { Mean duration of } \\
\text { pain: NR } \\
\text { RCT } \\
\text { Fair }\end{array}$ & $\begin{array}{l}\text { A. CPMP + booster } \\
\text { sessions ( } n=119) \\
\sim 4 \text { weeks }+7,20- \\
\text { minute booster } \\
\text { sessions over } 12 \\
\text { months, group + } \\
\text { individual, inpatient } \\
\text { B. CPMP ( } n=113 \text { ) } \\
\sim 4 \text { weeks, group + } \\
\text { individual, inpatient } \\
\text { C. PA ( } n=131 \text { ) } \\
\sim 3.5 \text { weeks, group + } \\
\text { individual, inpatient }\end{array}$ & $\begin{array}{l}\frac{\text { Mean age: } 49 \text { years }}{\% \text { Male: } 22 \%} \\
\text { Race/Ethnicity: NR } \\
\text { Pain etiology/type } \\
\text { Dorsalgia: } 84 \% \\
\text { Other dorsopathies, } \\
71 \% \\
\text { Arthrosis: } 34 \% \\
\text { Disability: NR } \\
\text { Comorbidities: NR }\end{array}$ & $\begin{array}{l}\text { A vs. B vs. C, Mean (SD) } \\
\text { PPS - Affective pain perception (14-56) } \\
\text { Baseline: } 30.9 \text { (9.4) vs. } 30.9 \text { (10.4) vs. } \\
29.6 \text { (9.0) } \\
\text { Postintervention: } 23.4(9.4) \text { vs. } 23.7(9.2) \\
\text { vs. } 22.9 \text { (8.2) } \\
\text { A vs. C: difference } 0.50(95 \% \mathrm{Cl}-1.69 \text { to } \\
2.69) \\
\text { B vs. C: difference } 0.80(95 \% \mathrm{Cl}-1.39 \text { to } \\
2.99) \\
12 \text { months: } 24.1 \text { (9.8) vs. } 25.5(9.9) \text { vs. } \\
25.1 \text { (9.6) } \\
\text { A vs. C: difference }-0.10(95 \% \mathrm{Cl}-3.50 \text { to } \\
1.50) \\
\text { B vs. C: difference } 0.40(95 \% \mathrm{Cl}-2.14 \text { to } \\
2.94) \\
\text { PPS - Sensory pain perception (10-40) } \\
\text { Baseline: } 18.9 \text { (5.9) vs. } 18.5(5.6) \text { vs. } 18.8 \\
\text { (5.9) } \\
\text { Postintervention: } 15.9(5.3) \text { vs. } 15.9(5.2) \\
\text { vs. } 16.4 \text { (5.8) } \\
\text { A vs. C: difference }-0.50(95 \% \mathrm{Cl}-1.89 \text { to } \\
0.89) \\
\text { B vs. C: difference }-0.50(95 \% \mathrm{Cl}-1.90 \text { to } \\
0.90) \\
\text { Long term: } 16.3(5.7) \text { vs. } 17.0(6.1) \text { vs. } \\
17.3 \text { (6.1) } \\
\text { A vs. C: difference }-1.0(95 \% \mathrm{Cl}-2.53 \text { to } \\
0.53) \\
\text { B vs. C: difference }-0.30(95 \% \mathrm{Cl}-1.89 \text { to } \\
1.29)\end{array}$ & $\begin{array}{l}\text { A vs. B vs. C, Mean (SD) } \\
\text { SF-12 PCS (0-100) } \\
\text { Baseline: } 33.5(9.1) \text { vs. } 33.6(7.4) \\
\text { vs. } 33.9 \text { (8.7) } \\
\text { Postintervention: } 38.9(9.4) \text { vs. } 39.3 \\
\text { (9.9) vs. } 38.6(8.6) \\
\text { A vs. C: difference } 0.30(95 \% \mathrm{Cl}- \\
1.94 \text { to } 2.54) \\
\text { B vs. C: difference } 0.70(95 \% \mathrm{Cl}- \\
\text { 1.63 to } 3.03) \\
\text { Long term: } 38.4(10.4) \text { vs. } 38.4(9.7) \\
\text { vs. } 38.4(10.1) \\
\text { A vs. C: difference } 0(95 \% \mathrm{Cl}-2.64 \\
\text { to } 2.64) \\
\text { B vs. C: difference } 0(95 \% \mathrm{Cl}-2.59 \\
\text { to } 2.59) \\
\text { SF-12 MCS (0-100) } \\
\text { Baseline: } 43.9(12.1) \text { vs. } 44.0(11.1) \\
\text { vs. } 44.5(11.5) \\
\text { Postintervention: } 48.9(12.1) \text { vs. } \\
50.8 \text { (10.5) vs. } 50.9(10.5) \\
\text { A vs. C: difference }-2.0(95 \% \mathrm{Cl}- \\
4.82 \text { to } 0.82) \\
\text { B vs. C: difference }-0.10(95 \% \mathrm{Cl}- \\
2.76 \text { to } 2.56) \\
\text { Long term: } 45.6(11.7) \text { vs. } 46.0 \\
(11.2) \text { vs. } 45.0(11.7) \\
\text { A vs. C: difference } 0.60(95 \% \mathrm{Cl}- \\
2.42 \text { to } 3.62) \\
\text { B vs. C: difference } 1.0(95 \% \mathrm{Cl}- \\
2.00 \text { to } 4.00)\end{array}$ & $\begin{array}{l}\text { A vs. B vs. C, Mean (SD) } \\
\text { Harms: One death during } \\
\text { inpatient treatment } \\
\text { (group A); no other } \\
\text { details provided } \\
\text { German Life Satisfaction } \\
\text { (with health) } \\
\text { Questionnaire (scale NR) } \\
\text { Baseline: } 28.4 \text { (9.5) vs. } \\
28.1 \text { (9.0) vs. } 28.9 \text { (8.5) } \\
\text { Postintervention: } 33.1 \\
\text { (8.8) vs. } 33.1 \text { (9.2) vs. } \\
32.5 \text { (8.8) } \\
\text { A vs. C: } 0.60 \text { (95\% Cl - } \\
1.60 \text { to } 2.80) \\
\text { B vs. C: } 0.60 \text { (95\% Cl - } \\
1.67 \text { to } 2.87 \text { ) } \\
\text { Long term: } 33.3(9.5) \text { vs. } \\
31.7 \text { (9.8) vs. } 31.2 \text { (8.4) } \\
\text { A vs. C: difference } 2.1 \\
\text { (95\% Cl }-0.21 \text { to } 4.41) \\
\text { B vs. C: difference } 0.50 \\
\text { (95\% Cl -1.87 to } 2.87 \text { ) }\end{array}$ \\
\hline
\end{tabular}




\begin{tabular}{|c|c|c|c|c|c|}
\hline $\begin{array}{l}\text { Author, Year } \\
\text { Country } \\
\text { Pain Duration } \\
\text { Study Design } \\
\text { Study Quality }\end{array}$ & \begin{tabular}{|l|} 
Intervention (n) \\
Comparator (n) \\
Duration/Intensity \\
Session Format \\
Setting (IP/OP) \\
\end{tabular} & Population & $\begin{array}{l}\text { Primary Outcomes: } \\
\text { Pain, Function, and Opioid Use }\end{array}$ & $\begin{array}{l}\text { Secondary Outcomes: HRQOL, } \\
\text { Psychological Measures, Global } \\
\text { Improvement }\end{array}$ & $\begin{array}{l}\text { Harms, } \\
\text { Utilization, } \\
\text { Patient Satisfaction }\end{array}$ \\
\hline $\begin{array}{l}\text { Mangels, } 2009 \\
\text { (Continued) }\end{array}$ & & & $\begin{array}{l}\text { PDI (0-70) } \\
\text { Baseline: } 26.9 \text { (13.9) vs. } 26.1 \text { (11.8) vs. } \\
24.8(12.5) \\
\text { Postintervention: } 21.7(13.3) \text { vs. } 20.3 \\
\text { (13.9) vs. } 21.0(13.1) \\
\text { A vs. C: difference } 0.70(95 \% \mathrm{Cl}-2.59 \text { to } \\
\text { 3.99) } \\
\text { B vs. C: difference }-0.70(95 \% \mathrm{Cl}-4.12 \text { to } \\
2.71) \\
\text { Long term: } 22.6(16.0) \text { vs. } 22.0(14.0) \text { vs. } \\
20.6(13.5) \\
\text { A vs. C: difference } 2.0(95 \% \mathrm{Cl}-1.80 \text { to } \\
5.80) \\
\text { B vs. C: difference } 1.4(95 \% \mathrm{Cl}-2.19 \text { to } \\
\text { 4.99) }\end{array}$ & $\begin{array}{l}\text { BDI (0-63) } \\
\text { Baseline: } 11.4(9.8) \text { vs. } 11.1(10.1) \\
\text { vs. } 10.1(8.1) \\
\text { Postintervention: } 7.2(7.8) \text { vs. } 6.7 \\
\text { (6.0) vs. } 7.8(7.8) \\
\text { A vs. C: difference }-0.60(95 \% \mathrm{Cl}- \\
4.08 \text { to } 2.88) \\
\text { B vs. C: difference }-1.10(95 \% \mathrm{Cl}- \\
4.56 \text { to } 2.36) \\
\text { Long term: } 10.7(8.8) \text { vs. } 10.4(7.8) \\
\text { vs. } 11.4(8.2) \\
\text { A vs. C: difference }-0.70(95 \% \mathrm{Cl}- \\
2.89 \text { to } 1.49) \\
\text { B vs. C: difference }-1.0(95 \% \mathrm{Cl}- \\
3.09 \text { to } 1.09)\end{array}$ & \\
\hline
\end{tabular}




\begin{tabular}{|c|c|c|c|c|c|}
\hline $\begin{array}{l}\text { Author, Year } \\
\text { Country } \\
\text { Pain Duration } \\
\text { Study Design } \\
\text { Study Quality }\end{array}$ & \begin{tabular}{|l|} 
Intervention (n) \\
Comparator (n) \\
Duration/Intensity \\
Session Format \\
Setting (IP/OP) \\
\end{tabular} & Population & $\begin{array}{l}\text { Primary Outcomes: } \\
\text { Pain, Function, and Opioid Use }\end{array}$ & $\begin{array}{l}\text { Secondary Outcomes: HRQOL, } \\
\text { Psychological Measures, Global } \\
\text { Improvement }\end{array}$ & $\begin{array}{l}\text { Harms, } \\
\text { Utilization, } \\
\text { Patient Satisfaction }\end{array}$ \\
\hline $\begin{array}{l}\text { Roche, 2007, } \\
\text { Roche-LeBoucher } \\
2011 \\
\text { France } \\
\text { RCT } \\
\text { Mean duration of } \\
\text { pain: NR ( } \geq 3 \\
\text { months) } \\
\text { Fair }\end{array}$ & $\begin{array}{l}\text { A. CPMP ( } \mathbf{n}=68) \\
5 \text { weeks ( } 6 \text { hours/day, } \\
150 \text { hours total), group, } \\
\text { outpatient } \\
\text { B. PA ( } \mathbf{n = 6 4 )} \\
5 \text { weeks (5 } \\
\text { hours/week, } 25 \text { hours } \\
\text { total), individual, } \\
\text { outpatient + home } \\
\text { setting }\end{array}$ & $\begin{array}{l}\text { Mean age: } 40 \text { years } \\
\% \text { Male: } 65 \% \\
\text { Race/ethnicity: NR } \\
\text { Pain etiology/type: } \\
\text { Chronic LBP } \\
\text { Disability: } \\
\text { - Excluded if receiving } \\
\text { disability pension. } \\
\text { Other characteristics: } \\
\text { - Excluded if acute LBP } \\
\text { or sciatica, } \\
\text { spondylolisthesis, or } \\
\text { cardiac or respiratory } \\
\text { insufficiency, or } \\
\text { psychiatric disorders } \\
\text { preventing participation }\end{array}$ & $\begin{array}{l}\text { A vs. B, Mean (SD) } \\
\text { VAS pain (0-10) } \\
\text { Baseline: } 4.7(2.1) \text { vs. } 4.5(2.1) \\
\text { Postintervention change score: }-1.9 \text { (NR) } \\
\text { vs. }-1.5 \text { (NR) } \\
\text { Long term change score: }-1.7 \text { (2.6) vs. - } \\
1.0(2.3) \text {, difference in change scores - } \\
0.70(95 \% \mathrm{Cl}-1.64 \text { to } 0.24) \\
\text { DPQ, daily activities }(0 \%-100 \%) \\
\text { Baseline: } 51.8 \% \text { (NR) vs. } 51.0 \%(\mathrm{NR}) \\
\text { Postintervention change score: }-21.5 \% \\
\text { (NR) vs. }-17.2 \% \text { (NR) } \\
\text { Long term change score: }-20.3 \%(18.1 \%) \\
\text { vs. }-10.4 \%(23.3 \%) \text {, difference in change } \\
\text { scores }-9.9(95 \% \text { Cl - } 17.62 \text { to }-2.19)\end{array}$ & $\begin{array}{l}\text { A vs. B } \\
\text { DPQ, anxiety and depression } 10 \%- \\
100 \%) \\
\text { Baseline: } 36.9 \%(\mathrm{NR}) \text { vs. } 30.9 \% \\
\text { (NR) } \\
\text { Postintervention change score: - } \\
17.6 \%(\mathrm{NR}) \text { vs. }-7.4 \%(\mathrm{NR}) \\
\text { Long term change score: }-15.6 \% \\
(21.4 \%) \text { vs. }-4.8 \%(23.5 \%), \\
\text { difference in change scores }-10.8 \\
(95 \% \mathrm{Cl}-19.20 \text { to }-2.40)\end{array}$ & $\begin{array}{l}\text { Harms: one right tibial } \\
\text { fracture in Group A } \\
\text { which occurred at home; } \\
\text { no other details provided }\end{array}$ \\
\hline
\end{tabular}




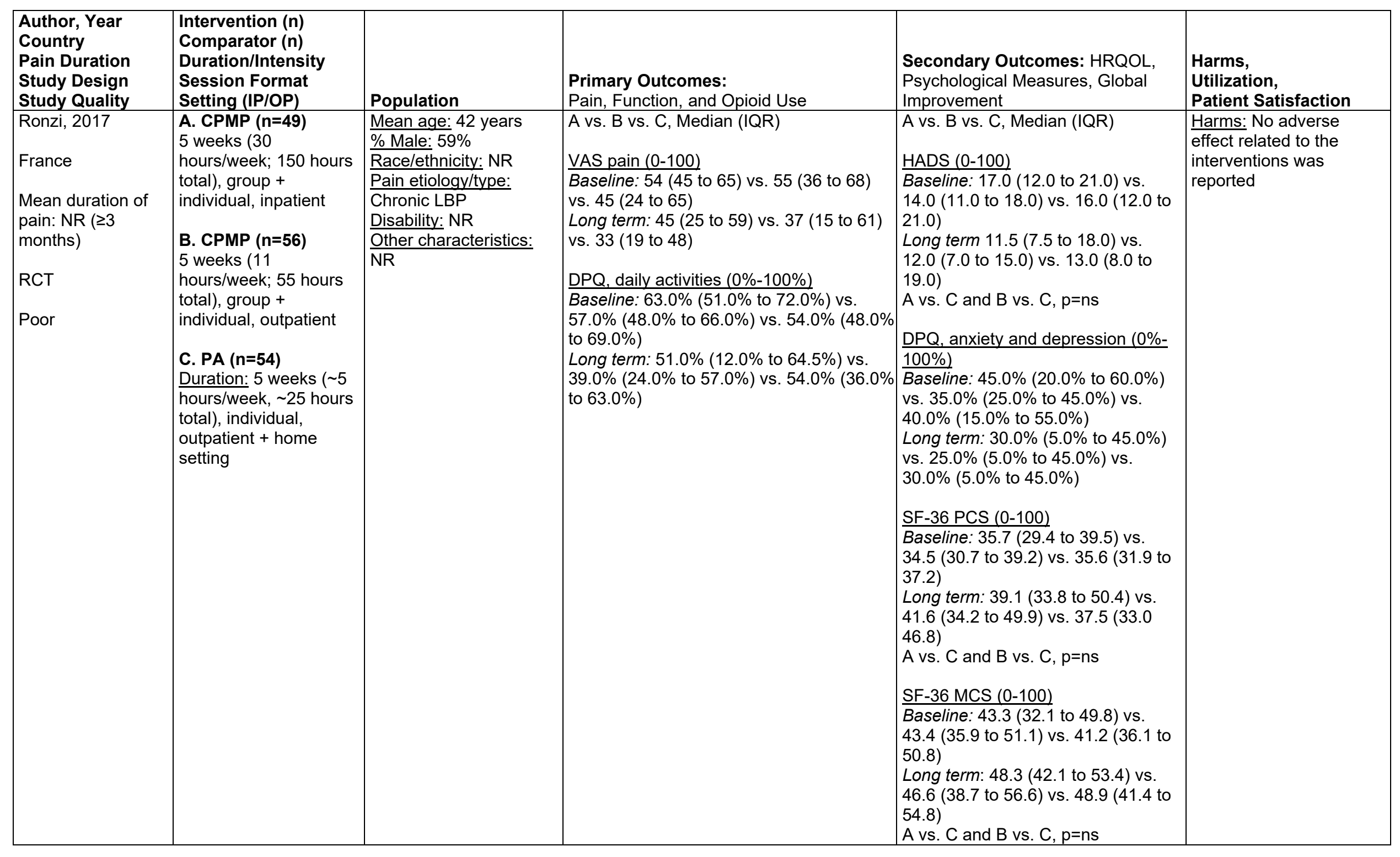




\begin{tabular}{|c|c|c|c|c|c|}
\hline $\begin{array}{l}\text { Author, Year } \\
\text { Country } \\
\text { Pain Duration } \\
\text { Study Design } \\
\text { Study Quality }\end{array}$ & \begin{tabular}{|l|} 
Intervention (n) \\
Comparator (n) \\
Duration/Intensity \\
Session Format \\
Setting (IP/OP)
\end{tabular} & Population & $\begin{array}{l}\text { Primary Outcomes: } \\
\text { Pain, Function, and Opioid Use }\end{array}$ & $\begin{array}{l}\text { Secondary Outcomes: HRQOL, } \\
\text { Psychological Measures, Global } \\
\text { Improvement }\end{array}$ & $\begin{array}{l}\text { Harms, } \\
\text { Utilization, } \\
\text { Patient Satisfaction }\end{array}$ \\
\hline $\begin{array}{l}\text { Schweikert, } 2006 \\
\text { Germany } \\
\text { RCT } \\
\text { Mean duration of } \\
\text { pain: NR ( } \geq 6 \\
\text { months) } \\
\text { Fair }\end{array}$ & $\begin{array}{l}\text { A. CPMP }(\mathbf{n = 2 0 0 )} \\
3 \text { weeks } \\
\text { hours total NR), group, } \\
\text { inpatient } \\
\text { B. PA ( } \mathbf{n}=\mathbf{2 0 9}) \\
3 \text { weeks } \\
\text { hours total NR), group } \\
+ \text { individual, inpatient }\end{array}$ & $\begin{array}{l}\text { Mean age: } 47 \text { years } \\
\text { Male: } 83 \% \\
\text { Race/Ethnicity: NR } \\
\text { Pain etiology/type: } \\
\text { Chronic LBP } \\
\text { Disability: } \\
\text { - Sick listed }<6 \text { months } \\
\text { in last year: } 69 \% \\
\text { - Sick listed }>6 \text { months } \\
\text { in last year: } 6 \% \\
\text { Comorbidities: NR }\end{array}$ & 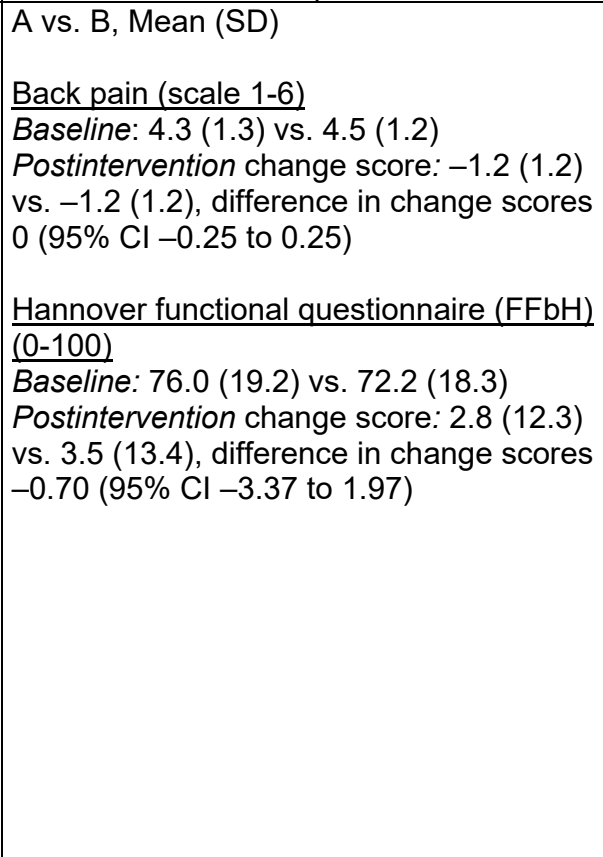 & 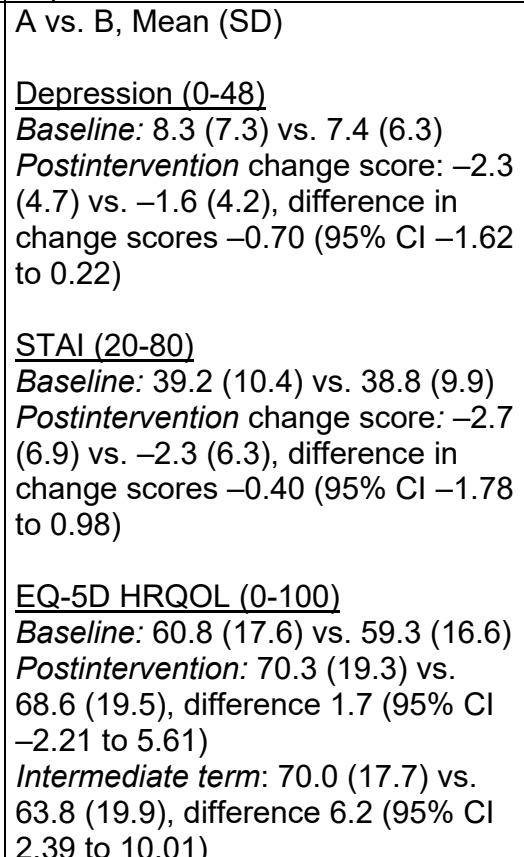 & NR \\
\hline
\end{tabular}




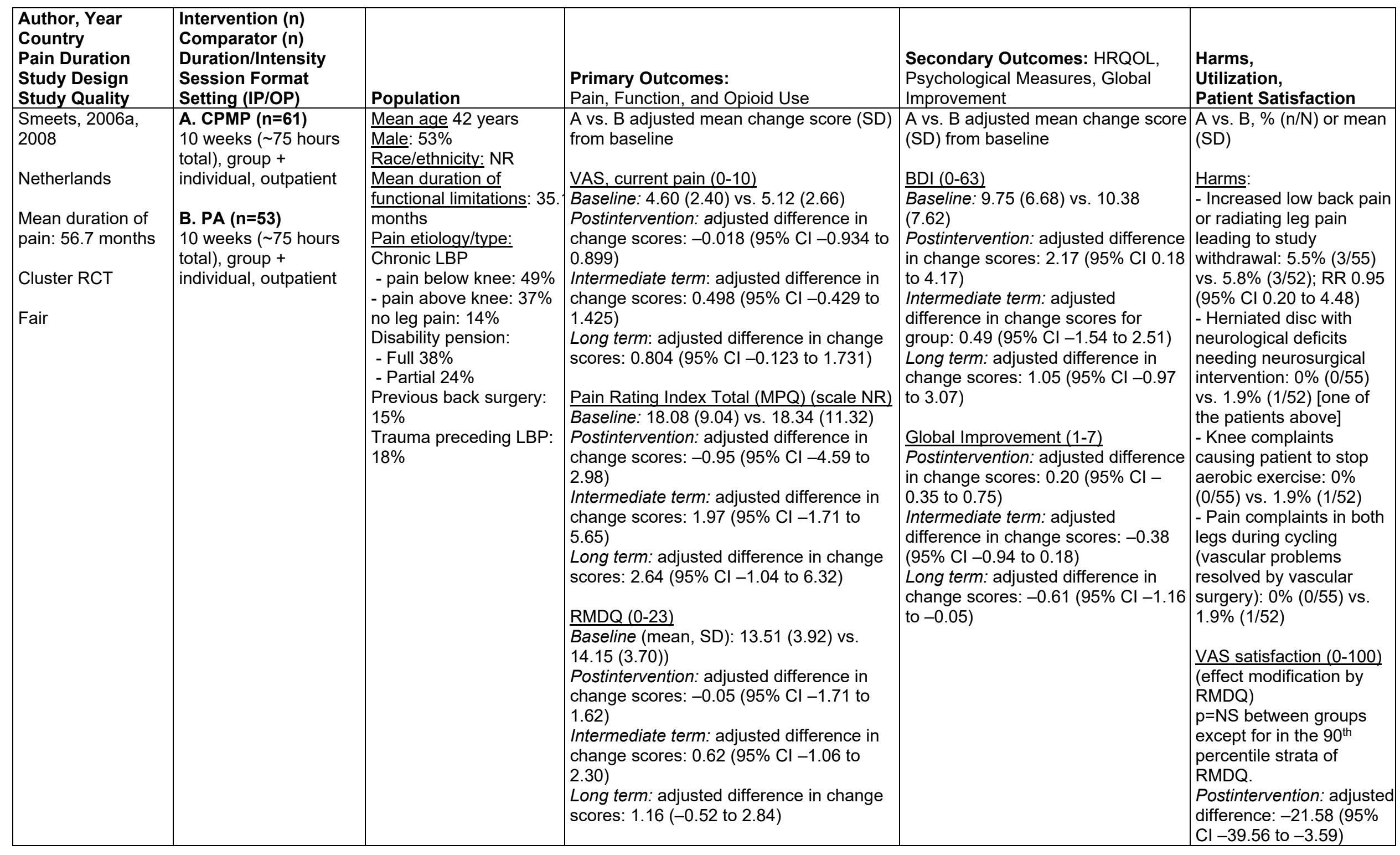




\begin{tabular}{|c|c|c|c|c|c|}
\hline $\begin{array}{l}\text { Author, Year } \\
\text { Country } \\
\text { Pain Duration } \\
\text { Study Design } \\
\text { Study Quality }\end{array}$ & $\begin{array}{l}\text { Intervention (n) } \\
\text { Comparator (n) } \\
\text { Duration/Intensity } \\
\text { Session Format } \\
\text { Setting (IP/OP) } \\
\end{array}$ & Population & $\begin{array}{l}\text { Primary Outcomes: } \\
\text { Pain, Function, and Opioid Use }\end{array}$ & $\begin{array}{l}\text { Secondary Outcomes: HRQOL, } \\
\text { Psychological Measures, Global } \\
\text { Improvement }\end{array}$ & $\begin{array}{l}\text { Harms, } \\
\text { Utilization, } \\
\text { Patient Satisfaction }\end{array}$ \\
\hline $\begin{array}{l}\text { Smeets, 2006a, } \\
2008 \\
\text { (Continued) }\end{array}$ & & & $\begin{array}{l}\text { VAS, main (activity) complaints (0-10) } \\
\text { Baseline: } 7.24(1.70) \text { vs. } 7.45 \\
\text { Postintervention: adjusted difference in } \\
\text { change scores: }-0.64(95 \% \mathrm{Cl}-1.60 \text { to } \\
0.33) \\
\text { Intermediate term: adjusted difference in } \\
\text { change scores: }-0.23(95 \% \mathrm{Cl}-1.19 \text { to } \\
0.74) \\
\text { Long term: adjusted difference in change } \\
\text { scores: } 0.03(95 \% \mathrm{Cl}-0.94 \text { to } 1.00)\end{array}$ & & \\
\hline $\begin{array}{l}\text { Turner, } 1990 \\
\text { USA } \\
\text { Mean duration of } \\
\text { pain: } 12.9 \text { years } \\
\text { RCT } \\
\text { Poor }\end{array}$ & $\begin{array}{l}\text { A. CPMP }(\mathbf{n = 1 8}) \\
8 \text { weeks }(4 \\
\text { hours/week, } 32 \text { hours } \\
\text { total), group and } \\
\text { individual, outpatient } \\
\\
\text { B. PA ( } \mathbf{n = 2 1 )} \\
8 \text { weeks }(2 \\
\text { hours/week, } 16 \text { hours } \\
\text { total). group + } \\
\text { individual, outpatient }\end{array}$ & $\begin{array}{l}\text { Mean age: } 44 \text { years } \\
\text { Male: } 52.1 \% \\
\text { Race/ethnicity: } \\
\text { White: } 100 \% \\
\text { Pain etiology/type: } \\
\text { Chronic LBP } \\
\text { Disability: N/A }\end{array}$ & 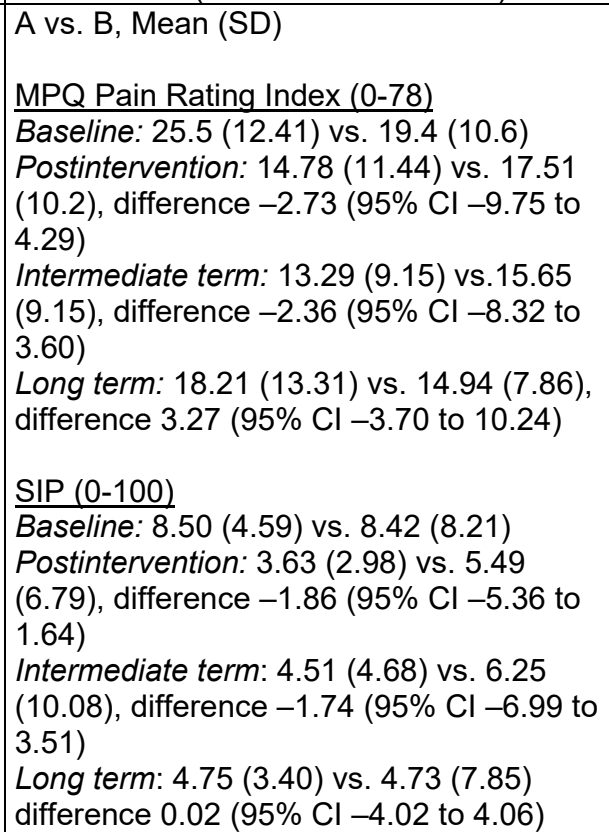 & $\begin{array}{l}\text { A vs. B, Mean (SD) } \\
\text { CES-D (0-60) } \\
\text { Baseline: } 12.38 \text { (7.31) vs. } 11.95 \\
\text { (7.68) } \\
\text { Postintervention: } 7.36(5.89) \text { vs. } \\
7.38(4.57) \text {, difference }-0.02(95 \% \\
\text { Cl }-3.42 \text { to } 3.38) \\
\text { Intermediate term: } 8.29(7.94) \mathrm{vs.} \\
9.29(8.30), \text { difference }-1.0(95 \% \mathrm{Cl} \\
-6.30 \text { to } 4.30) \\
\text { Long term: } 10.00(7.57) \text { vs. } 9.31 \\
(7.73), \text { difference } 0.69(95 \% \mathrm{Cl}- \\
4.29 \text { to } 5.67)\end{array}$ & $\begin{array}{l}\text { A vs. B, Mean (SD) } \\
\text { Harms: NR } \\
\text { Patient satisfaction (1-7) } \\
\begin{array}{l}\text { Postintervention: } 5.50 \\
\text { (NR) vs. } 4.48 \text { (NR), } \\
p=N S\end{array}\end{array}$ \\
\hline
\end{tabular}




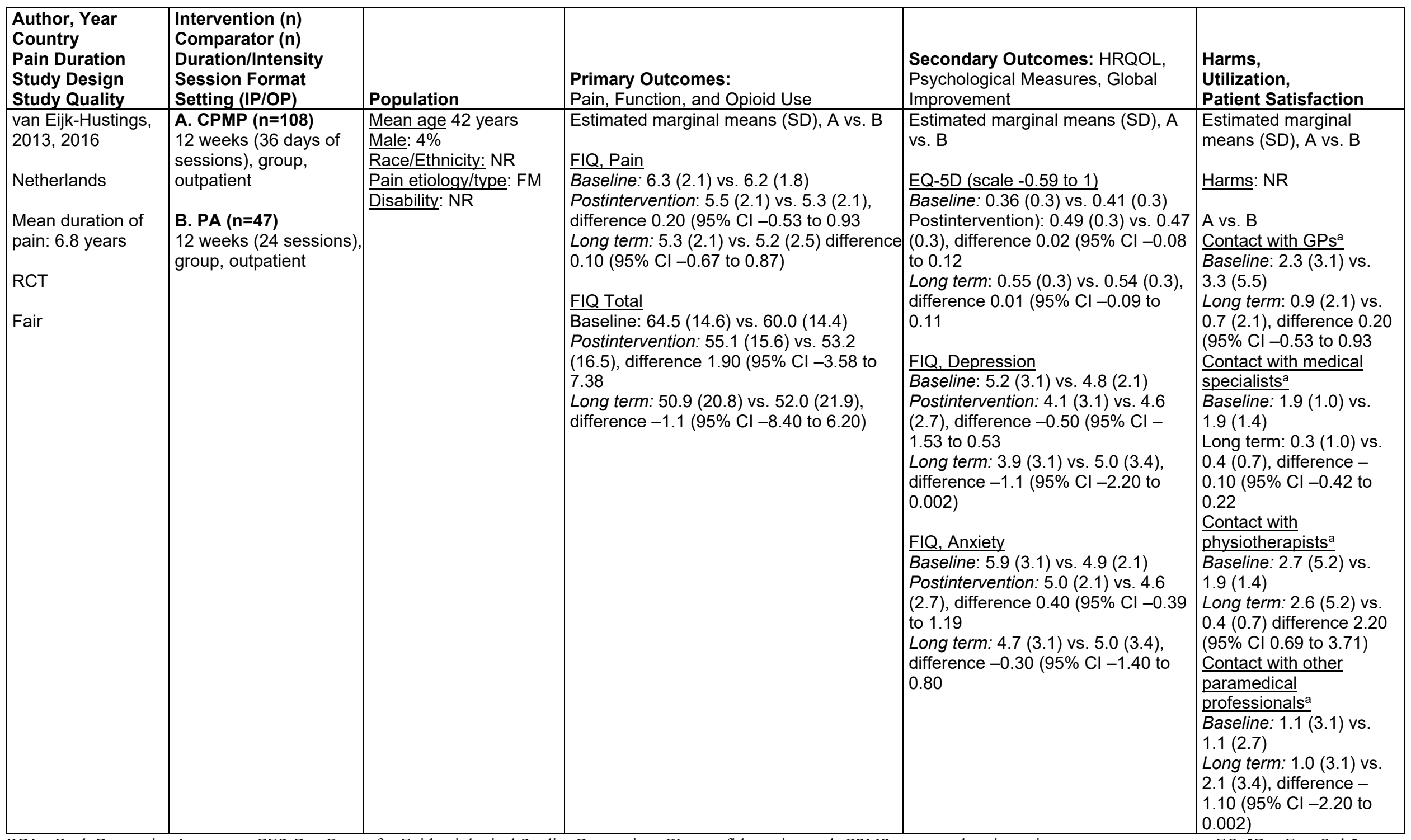

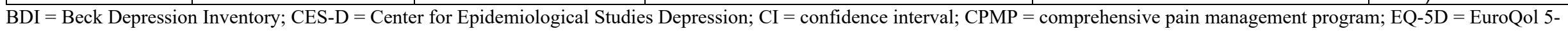

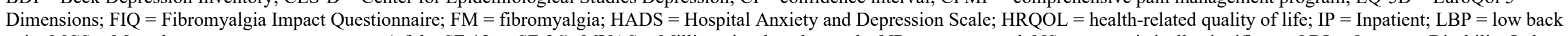

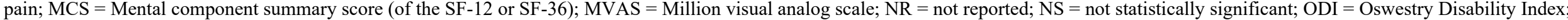

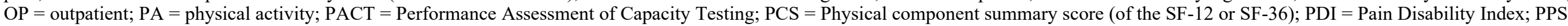

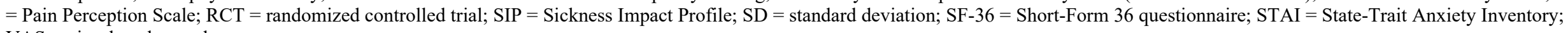
$\mathrm{VAS}=$ visual analog scale

${ }^{\text {a }}$ Total number of consultations over a period of 2 months prior to measurement 
Table B-7. Summary results for trials addressing KQ1: CPMPs versus pharmacologic therapy alone

\begin{tabular}{|c|c|c|c|c|c|}
\hline $\begin{array}{l}\text { Author, Year } \\
\text { Country } \\
\text { Pain Duration } \\
\text { Study Design } \\
\text { Study Quality } \\
\end{array}$ & $\begin{array}{l}\text { Intervention (n), } \\
\text { Comparator (n), } \\
\text { Duration/Intensity, } \\
\text { Session Format, } \\
\text { Setting }\end{array}$ & Population & $\begin{array}{l}\text { Primary Outcomes: } \\
\text { Pain, Function, and Opioid Use }\end{array}$ & $\begin{array}{l}\text { Secondary Outcomes: } \\
\text { HRQOL, Psychological Measures, Global Improvement }\end{array}$ & $\begin{array}{l}\text { Harms } \\
\text { Utilization } \\
\text { Patient } \\
\text { Satisfaction }\end{array}$ \\
\hline $\begin{array}{l}\text { Castel, 2013, } \\
2015 \text {, Salvat, } \\
2017 \\
\text { Spain } \\
\text { Mean pain } \\
\text { duration: } 140 \\
\text { months } \\
\text { RCT } \\
\text { Fair }\end{array}$ & $\begin{array}{l}\text { A. Comprehensive } \\
\text { pain management } \\
\text { program ( } \mathbf{n}=74) \\
12 \text { weeks, } 4 \\
\text { hours/week, } 48 \text { hours } \\
\text { total, group, outpatient } \\
\\
\text { B. Pharmacological } \\
\text { therapy only (n=81) } \\
12 \text { weeks, } \\
\text { Analgesics, } \\
\text { antidepressants, } \\
\text { benzodiazepine and } \\
\text { nonbenzodiazepine } \\
\text { hypnotics (dosages } \\
\text { NR); adjusted as } \\
\text { recommend by } \\
\text { guidelines }\end{array}$ & $\begin{array}{l}\text { Mean age: } 49 \text { years } \\
\text { \% Female: } 100 \% \\
\text { Race/Ethnicity: NR } \\
\text { Pain etiology/type: } \\
\text { Fibromyalgia } \\
\text { Disability: NR } \\
\text { - Receiving workers } \\
\text { compensation: } 6 \% \\
\text { Comorbidities: NR }\end{array}$ & 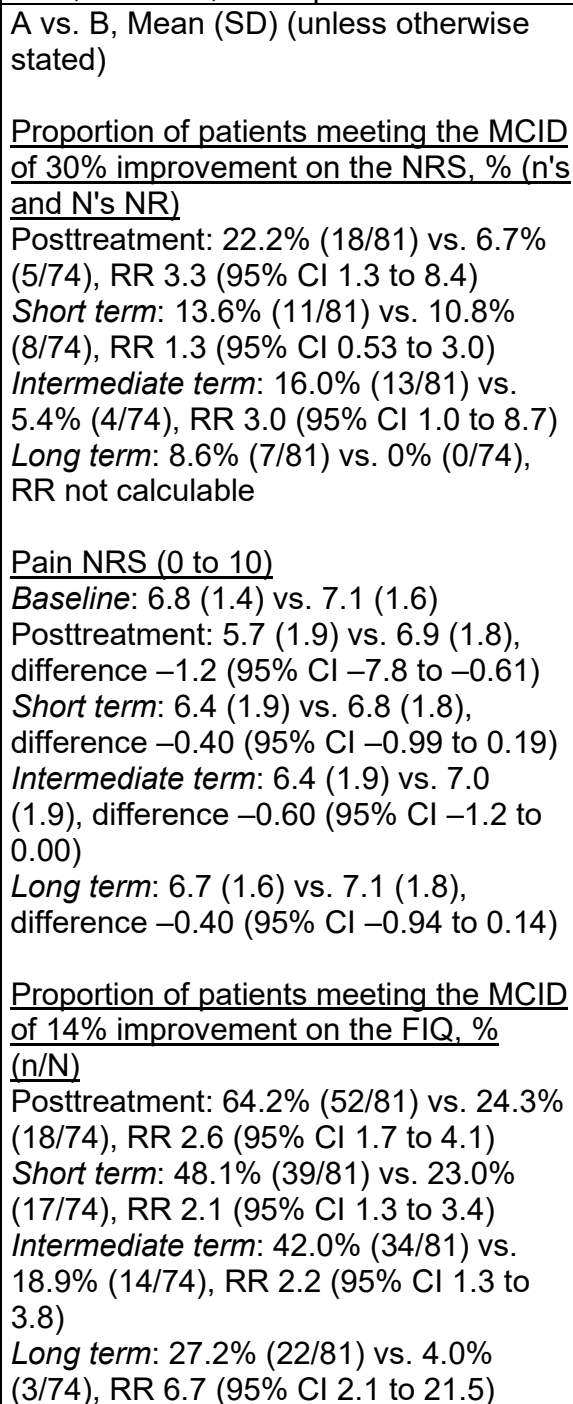 & 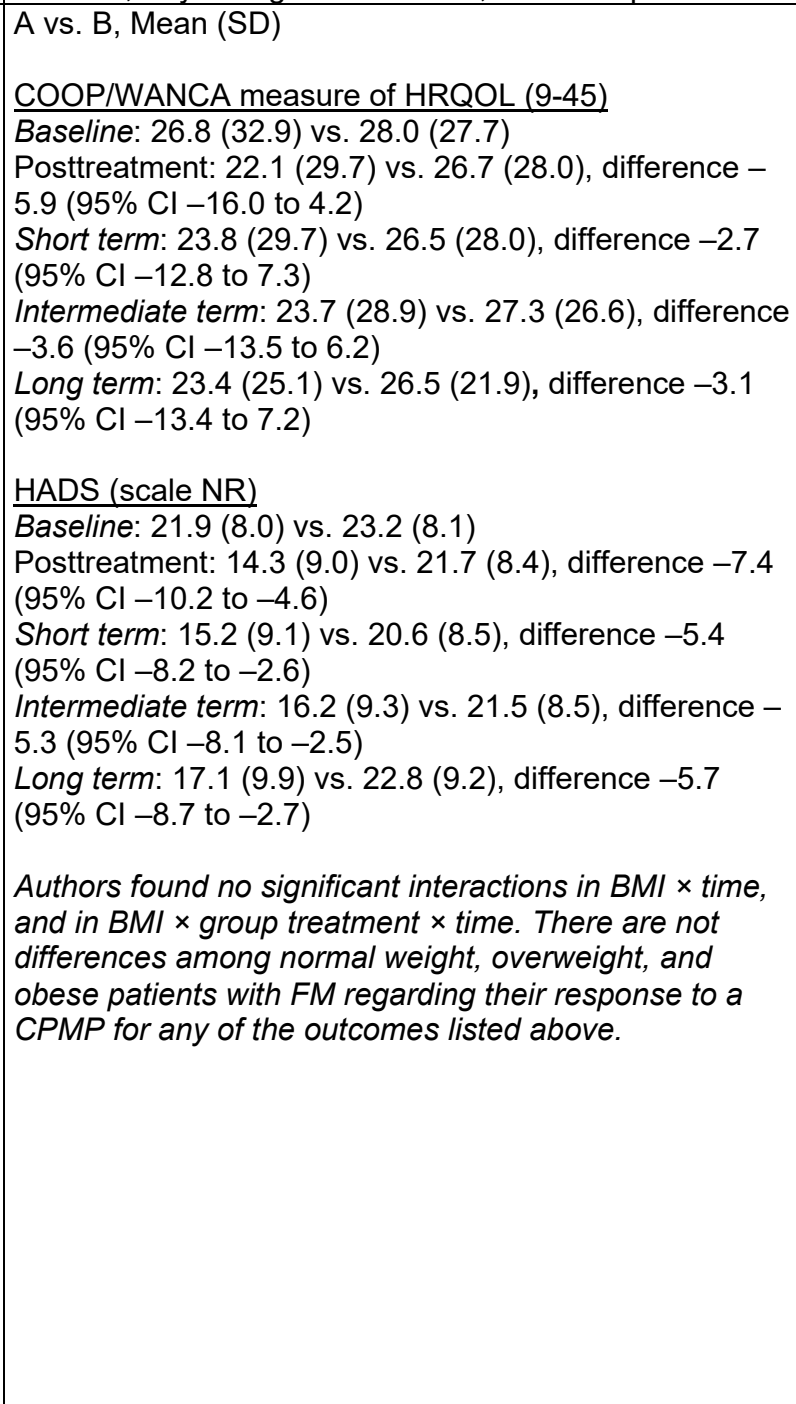 & NR \\
\hline
\end{tabular}




\begin{tabular}{|c|c|c|c|c|c|}
\hline $\begin{array}{l}\text { Author, Year } \\
\text { Country } \\
\text { Pain Duration } \\
\text { Study Design } \\
\text { Study Quality }\end{array}$ & $\begin{array}{l}\text { Intervention (n), } \\
\text { Comparator (n), } \\
\text { Duration/Intensity, } \\
\text { Session Format, } \\
\text { Setting }\end{array}$ & Population & $\begin{array}{l}\text { Primary Outcomes: } \\
\text { Pain, Function, and Opioid Use }\end{array}$ & $\begin{array}{l}\text { Secondary Outcomes: } \\
\text { HRQOL, Psychological Measures, Global Improvement }\end{array}$ & \begin{tabular}{|l} 
Harms \\
Utilization \\
Patient \\
Satisfaction
\end{tabular} \\
\hline $\begin{array}{l}\text { Castel, 2013, } \\
2015, \text { Salvat, } \\
2017 \\
\\
\text { (Continued) }\end{array}$ & & & 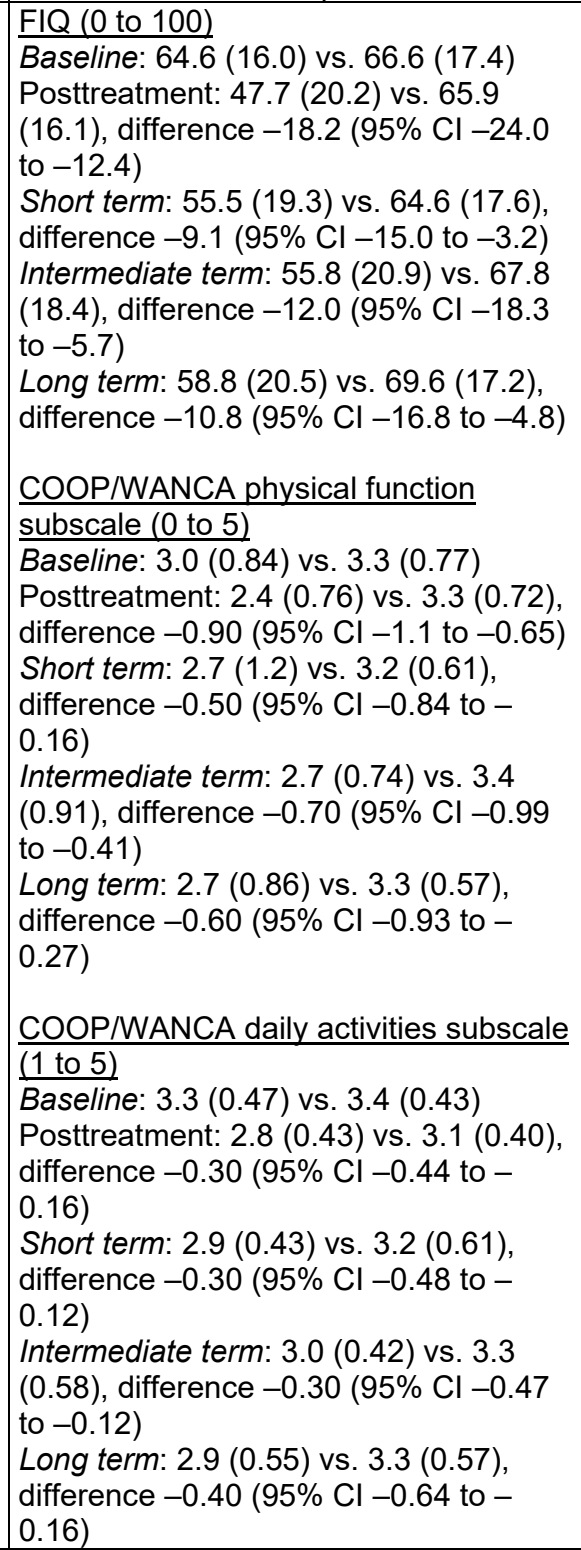 & & \\
\hline
\end{tabular}




\begin{tabular}{|c|c|c|c|c|c|}
\hline $\begin{array}{l}\text { Author, Year } \\
\text { Country } \\
\text { Pain Duration } \\
\text { Study Design } \\
\text { Study Quality } \\
\end{array}$ & $\begin{array}{l}\text { Intervention (n), } \\
\text { Comparator (n), } \\
\text { Duration/Intensity, } \\
\text { Session Format, } \\
\text { Setting } \\
\end{array}$ & Population & $\begin{array}{l}\text { Primary Outcomes: } \\
\text { Pain, Function, and Opioid Use }\end{array}$ & $\begin{array}{l}\text { Secondary Outcomes: } \\
\text { HRQOL, Psychological Measures, Global Improvement }\end{array}$ & $\begin{array}{l}\text { Harms } \\
\text { Utilization } \\
\text { Patient } \\
\text { Satisfaction }\end{array}$ \\
\hline $\begin{array}{l}\text { Castel, 2013, } \\
2015, \text { Salvat, } \\
2017 \\
\text { (Continued) }\end{array}$ & & & $\begin{array}{l}\text { Authors found no significant interactions } \\
\text { in BMI } \times \text { time, and in BMI } \times \text { group } \\
\text { treatment } \times \text { time. There are not } \\
\text { differences among normal weight, } \\
\text { overweight, and obese patients with FM } \\
\text { regarding their response to a CPMP for } \\
\text { any of the outcomes listed above. }\end{array}$ & & \\
\hline $\begin{array}{l}\text { Martin, 2014a, } \\
2014 b, 2014 c \\
\text { Spain } \\
\text { Mean duration } \\
\text { of pain: } 170 \\
\text { months } \\
\text { RCT } \\
\text { Poor }\end{array}$ & $\begin{array}{l}\text { A. Comprehensive } \\
\text { pain management } \\
\text { program }(\mathrm{n}=54) \\
6 \text { weeks, } 2.75 \\
\text { hours/week, } 16.5 \\
\text { hours total, session } \\
\text { format NR, outpatient } \\
\\
\text { B. Pharmacological } \\
\text { therapy only ( } \mathrm{n}=56 \text { ) } \\
6 \text { weeks, } \\
\text { Amitriptyline } \\
\text { (maximum dose } 75 \\
\text { mg/day), Paracetamol, } \\
\text { (maximum dose } 4 \\
\text { g/day), Tramadol, } \\
\text { (maximum dose } 400 \\
\text { mg/day) }\end{array}$ & $\begin{array}{l}\text { Mean age: } 50 \text { years } \\
\text { \% Male: } 9 \% \\
\text { Race/Ethnicity: NR } \\
\text { Pain etiology/type: } \\
\text { Fibromyalgia } \\
\text { Disability: } 15 \% \\
\text { Comorbidities: } \\
\text { - Hypothyroidism: } 12 \% \\
\text { - High blood pressure: } \\
\text { 10\% } \\
\text { - COPD: } 12 \% \\
\text { - Diabetes mellitus: } 3 \% \\
\text { - Rheumatoid arthritis: } \\
\text { 3\% } \\
\text { - Other: } 42 \%\end{array}$ & $\begin{array}{l}\text { A vs. B, Mean (SD) } \\
\text { VAS current pain (0 to 10) } \\
\text { Baseline: } 6.76(1.98) \text { vs. } 7.06(2.04) \\
\text { Intermediate term: } 5.99(2.37) \text { vs. } 7.21 \\
(1.56) \text {, difference }-1.2(95 \% \mathrm{Cl}-2.0 \text { to - } \\
0.46) \\
\text { FIQ Total }(0 \text { to } 100) \\
\text { Baseline: } 76.28(13.17) \text { vs. } 76.23 \\
(14.80) \\
\text { Intermediate term: } 70.33(16.98) \text { vs. } \\
76.81(14.18), \text { difference }-6.5(95 \% \mathrm{Cl}- \\
12.4 \text { to }-0.58)\end{array}$ & 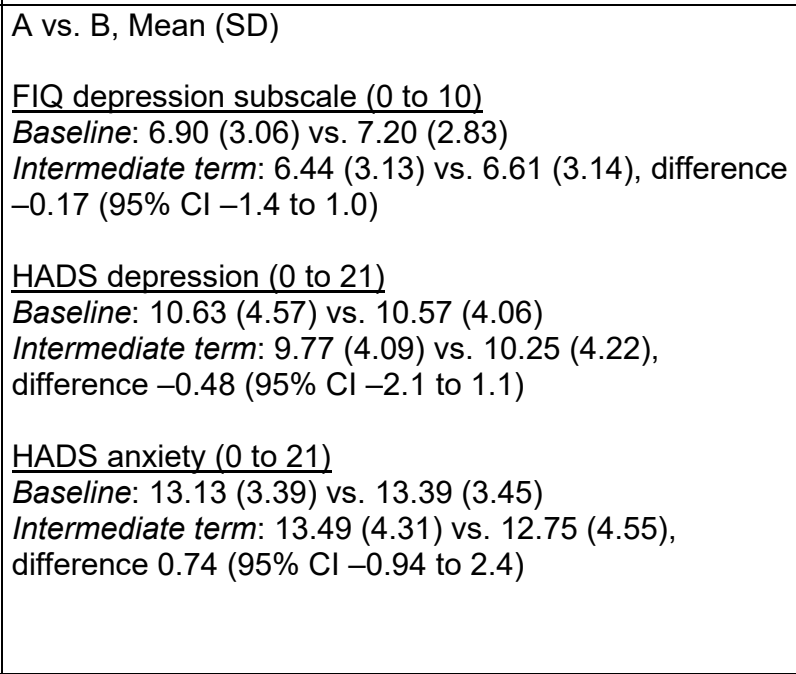 & NR \\
\hline
\end{tabular}




\begin{tabular}{|c|c|c|c|c|c|}
\hline $\begin{array}{l}\text { Author, Year } \\
\text { Country } \\
\text { Pain Duration } \\
\text { Study Design } \\
\text { Study Quality } \\
\end{array}$ & $\begin{array}{l}\text { Intervention (n), } \\
\text { Comparator (n), } \\
\text { Duration/Intensity, } \\
\text { Session Format, } \\
\text { Setting }\end{array}$ & Population & $\begin{array}{l}\text { Primary Outcomes: } \\
\text { Pain, Function, and Opioid Use }\end{array}$ & $\begin{array}{l}\text { Secondary Outcomes: } \\
\text { HRQOL, Psychological Measures, Global Improvement }\end{array}$ & $\begin{array}{l}\text { Harms } \\
\text { Utilization } \\
\text { Patient } \\
\text { Satisfaction }\end{array}$ \\
\hline $\begin{array}{l}\text { Onac, 2012, } \\
2017 \\
\text { Romania } \\
\text { Mean duration } \\
\text { of pain: NR } \\
\text { RCT } \\
\text { Poor }\end{array}$ & $\begin{array}{l}\text { A. Comprehensive } \\
\text { pain management } \\
\text { program ( } \mathbf{n = 2 9 )} \\
2 \text { weeks, } 5 \text { days/week } \\
\text { (total hours NR), } \\
\text { individual, inpatient } \\
\\
\text { B. Pharmacological } \\
\text { therapy ( } \mathbf{n}=20) \\
2 \text { weeks, } \\
\text { Diclofenac ( } 50 \mathrm{mg}, 3 \\
\text { times/day), } \\
\text { Omeprazole ( } 20 \mathrm{mg}, 1 \\
\text { time/day), and } \\
\text { Acetaminophen (1000 } \\
\text { mg, } 4 \text { times/day) }\end{array}$ & $\begin{array}{l}\text { Population data } \\
\text { include patients from } \\
\text { the third arm of this } \\
\text { trial } \\
\text { Mean age: } 47 \text { years } \\
\% \text { Male: } 57 \% \\
\text { Race/ethnicity: NR } \\
\text { Pain etiology/type: } \\
\text { Lumbar disk hernia } \\
\text { Disability: NR } \\
\text { Other characteristics: } \\
\\
\text { - Excluded: history of } \\
\text { psychotic disorders, } \\
\text { substance abuse } \\
\text { disorders, certain } \\
\text { personality disorders. }\end{array}$ & 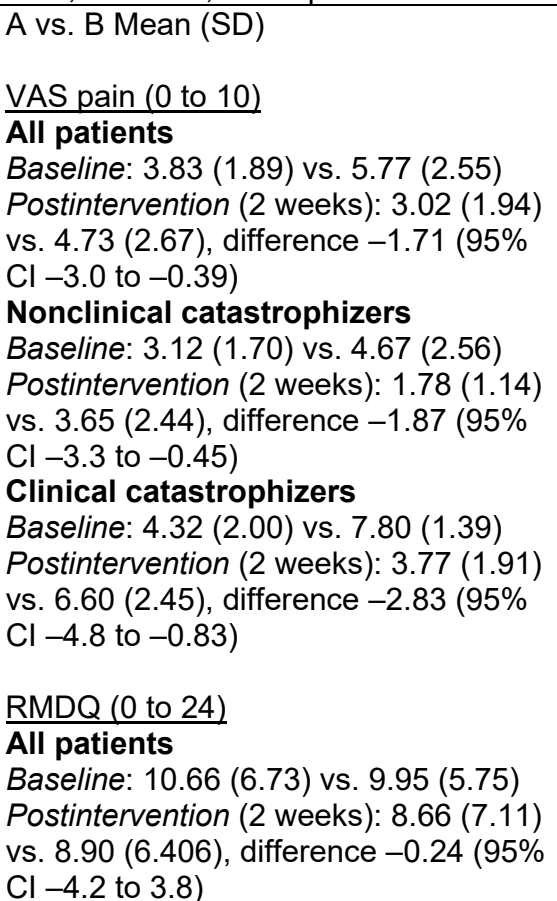 & $\begin{array}{l}\text { A vs. B Mean (SD) } \\
\text { POMS-SV Emotional Distress (scale NR) } \\
\text { All patients } \\
\text { Baseline: } 46.03 \text { (26.52) vs. } 39.85(21.52) \\
\text { Postintervention (2 weeks): } 43.15(31.16) \text { vs. } 37.94 \\
\text { (21.01), difference } 5.2(95 \% \mathrm{Cl}-10.9 \text { to } 21.3)\end{array}$ & NR \\
\hline
\end{tabular}




\begin{tabular}{|c|c|c|c|c|c|}
\hline $\begin{array}{l}\text { Author, Year } \\
\text { Country } \\
\text { Pain Duration } \\
\text { Study Design } \\
\text { Study Quality } \\
\end{array}$ & \begin{tabular}{|l} 
Intervention (n), \\
Comparator (n), \\
Duration/Intensity, \\
Session Format, \\
Setting \\
\end{tabular} & Population & $\begin{array}{l}\text { Primary Outcomes: } \\
\text { Pain, Function, and Opioid Use }\end{array}$ & $\begin{array}{l}\text { Secondary Outcomes: } \\
\text { HRQOL, Psychological Measures, Global Improvement }\end{array}$ & $\begin{array}{l}\text { Harms } \\
\text { Utilization } \\
\text { Patient } \\
\text { Satisfaction } \\
\end{array}$ \\
\hline $\begin{array}{l}\text { Tavafian, } \\
2011,2014, \\
2017 a, 2017 b \\
\text { Iran } \\
\text { Mean duration } \\
\text { of pain: } 82.2 \\
\text { months } \\
\text { RCT } \\
\text { Fair }\end{array}$ & $\begin{array}{l}\text { A. Comprehensive } \\
\text { pain management } \\
\text { program ( } n=97) \\
1 \text { week + monthly } \\
\text { booster sessions, } 69 \\
\text { hours total, session } \\
\text { format NR, outpatient } \\
\text { B. Pharmacologic } \\
\text { therapy alone } \\
\text { ( } n=100) \\
\text { Analgesics, NSAIDS, } \\
\text { muscle relaxants, and } \\
\text { antidepressant drugs }\end{array}$ & $\begin{array}{l}\text { Mean age: } 45 \text { years } \\
\text { \% Male: } 26.08 \% \\
\text { Race/ethnicity: NR } \\
\text { Pain etiology/type: } \\
\text { Chronic LBP } \\
\text { Disability: NR } \\
\text { Comorbidities: } \\
\text { - \% Smokers: } 5.6 \% \\
\text { Other characteristics: } \\
-\% \text { with sciatica: } \\
85.8 \% \\
\text { - Excluded: back } \\
\text { surgery within last } 2 \\
\text { years }\end{array}$ & 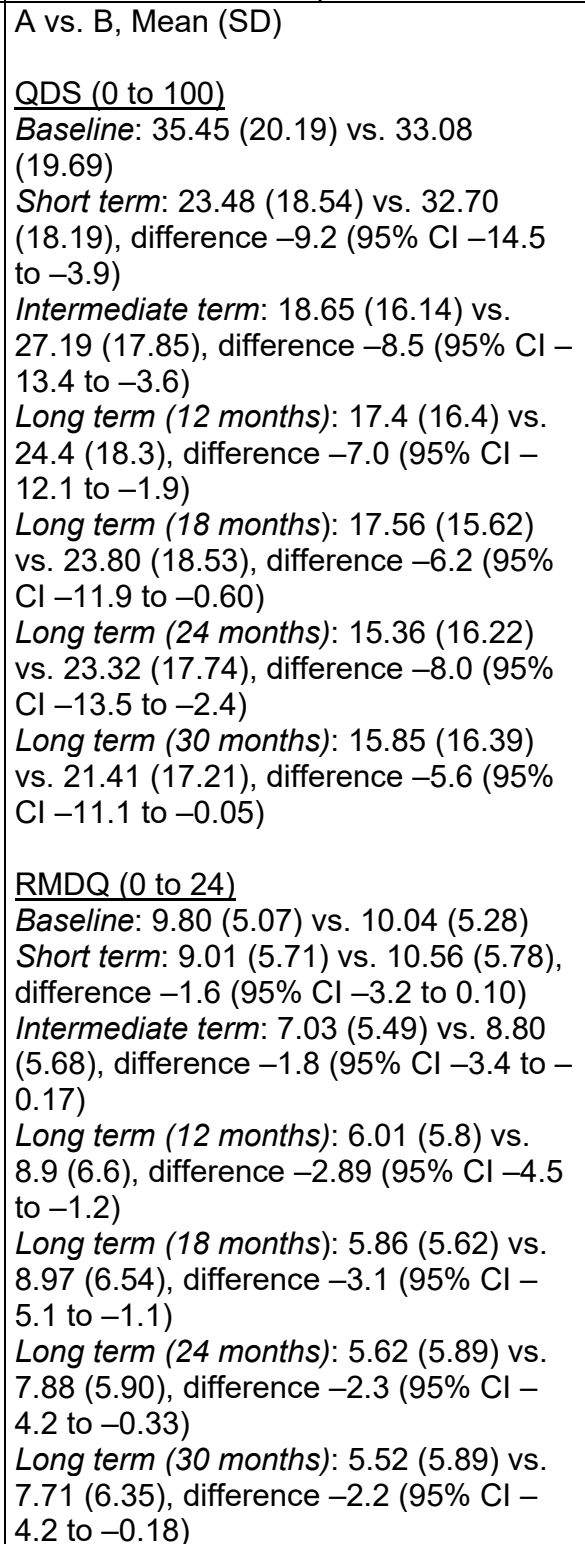 & 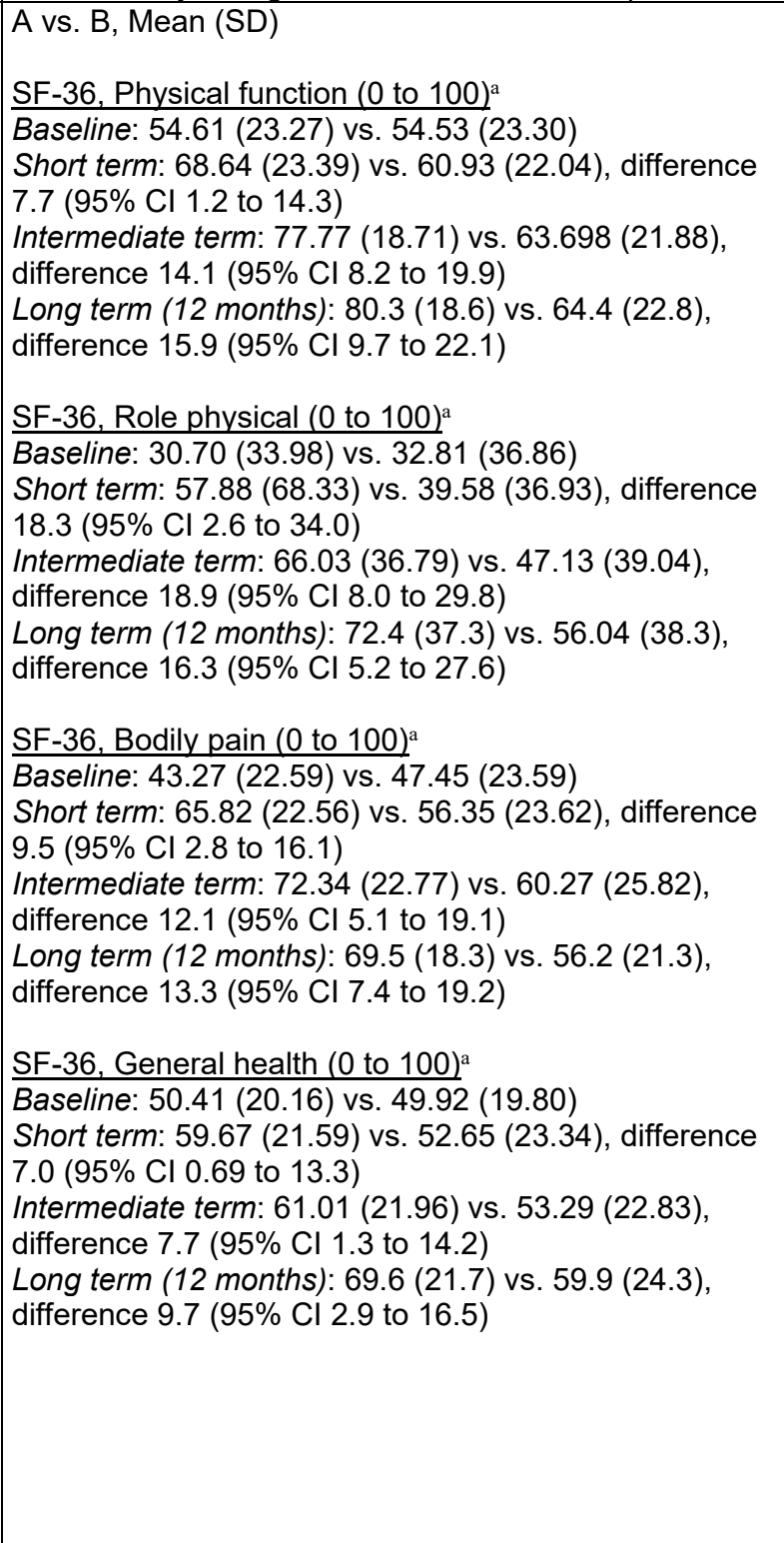 & NR \\
\hline
\end{tabular}




\begin{tabular}{|c|c|c|c|c|c|}
\hline \begin{tabular}{|l} 
Author, Year \\
Country \\
Pain Duration \\
Study Design \\
Study Quality
\end{tabular} & $\begin{array}{l}\text { Intervention (n), } \\
\text { Comparator (n), } \\
\text { Duration/Intensity, } \\
\text { Session Format, } \\
\text { Setting }\end{array}$ & Population & $\begin{array}{l}\text { Primary Outcomes: } \\
\text { Pain, Function, and Opioid Use }\end{array}$ & $\begin{array}{l}\text { Secondary Outcomes: } \\
\text { HRQOL, Psychological Measures, Global Improvement }\end{array}$ & $\begin{array}{l}\text { Harms } \\
\text { Utilization } \\
\text { Patient } \\
\text { Satisfaction }\end{array}$ \\
\hline $\begin{array}{l}\text { Tavafian, } \\
\text { 2011, 2014, } \\
\text { 2017a, 2017b } \\
\text { (Continued) }\end{array}$ & & & & 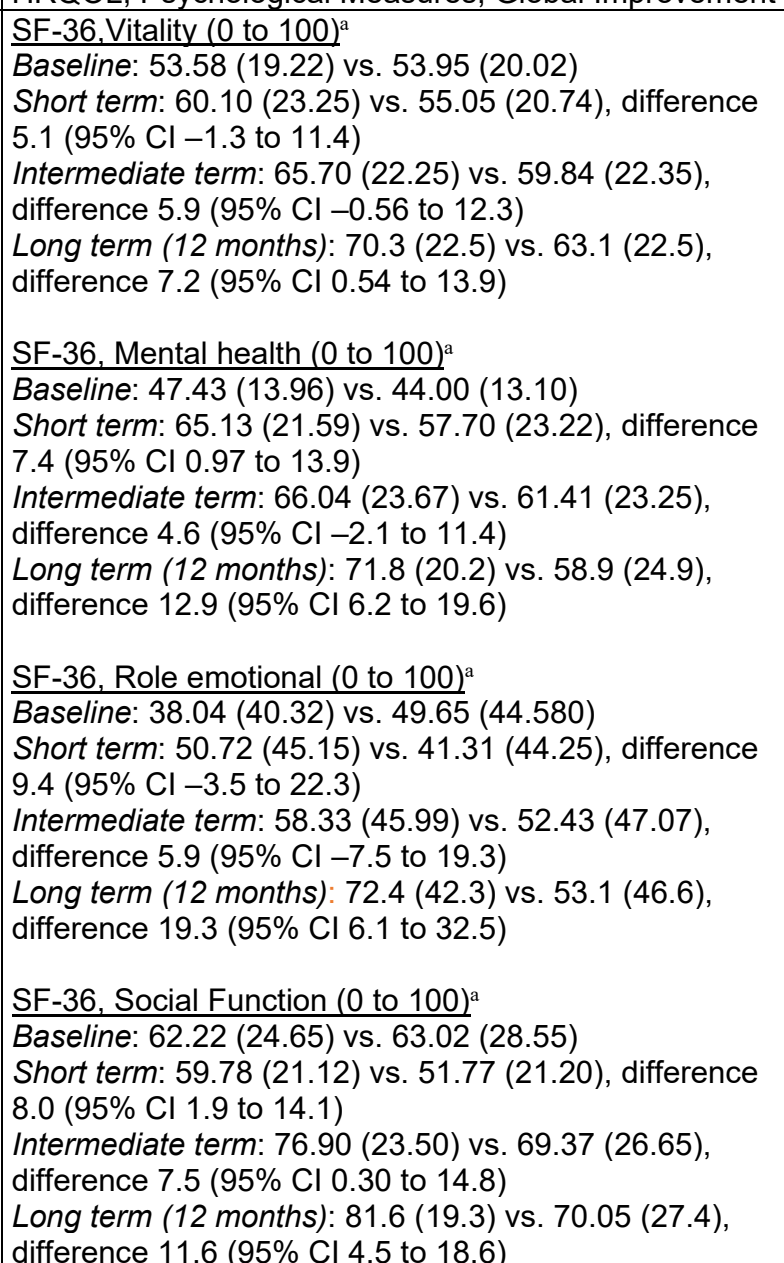 & \\
\hline
\end{tabular}




\begin{tabular}{|c|c|c|c|c|c|}
\hline $\begin{array}{l}\text { Author, Year } \\
\text { Country } \\
\text { Pain Duration } \\
\text { Study Design } \\
\text { Study Quality }\end{array}$ & $\begin{array}{l}\text { Intervention }(n), \\
\text { Comparator }(n), \\
\text { Duration/Intensity, } \\
\text { Session Format, } \\
\text { Setting } \\
\end{array}$ & Population & $\begin{array}{l}\text { Primary Outcomes: } \\
\text { Pain, Function, and Opioid Use }\end{array}$ & $\begin{array}{l}\text { Secondary Outcomes: } \\
\text { HRQOL, Psychological Measures, Global Improvement }\end{array}$ & $\begin{array}{l}\text { Harms } \\
\text { Utilization } \\
\text { Patient } \\
\text { Satisfaction } \\
\end{array}$ \\
\hline $\begin{array}{l}\text { Tavaflan, } 2008 \\
\text { Iran } \\
\text { Mean duration } \\
\text { of pain: } 9.07 \\
\text { months } \\
\text { RCT } \\
\text { Fair }\end{array}$ & $\begin{array}{l}\text { A. Comprehensive } \\
\text { pain management } \\
\text { program }(n=50) \\
4 \text { days, intensity NR, } \\
\text { individual, } \\
\text { outpatient } \\
\\
\text { B. Pharmacological } \\
\text { therapy alone ( } n=52) \\
\text { Acetaminophen, } \\
\text { NSAID and } \\
\text { chlordiazepoxide }\end{array}$ & $\begin{array}{l}\text { Mean age: } 44 \text { years } \\
\text { \% Female: } 100 \% \\
\text { Race/ethnicity: NR } \\
\text { Pain etiology/type: } \\
\text { Chronic LBP } \\
\text { Disability: NR } \\
\text { Comorbidities: } \\
\text { - \% Smokers: } 3.9 \%\end{array}$ & NR & $\begin{array}{l}\text { A vs. B, Mean (SD) } \\
\text { SF-36 PCS }(0 \text { to } 100) \\
\text { Baseline: } 44.3(16.8) \text { vs. } 42.6(24.0) \\
\text { Short term: } 76.7(17.3) \text { vs. } 51.2(28.1) \text {, difference } 25.5 \\
(95 \% \mathrm{Cl} 14.9 \text { to } 36.3) \\
\text { Intermediate term: } 66.6(27.5) \text { vs. } 51.2 \text { (28.8), difference } \\
15.4(95 \% \mathrm{Cl} 2.4 \text { to } 28.5) \\
\text { Long term: } 64.7(36.3) \text { vs. } 51.1 \text { (28.3), difference } 13.6 \\
(95 \% \mathrm{Cl}-1.5 \text { to } 28.7) \\
\\
\text { SF-36 MCS } 0 \text { to } 100) \\
\text { Baseline: } 47.7(28) \text { vs. } 49.5(23.1) \\
\text { Short term: } 80.4(22.8) \text { vs. } 57.4(29.5) \text {, difference } 23.0 \\
(95 \% \mathrm{Cl} 10.8 \text { to } 35.2) \\
\text { Intermediate term: } 66.9(29.9) \text { vs. } 57.9 \text { (25.5), difference } \\
9.0(95 \% \mathrm{Cl}-3.9 \text { to } 21.9) \\
\text { Long term: } 65.1(27.2) \text { vs. } 60.2(26.6) \text {, difference } 4.9 \\
\text { (95\% Cl }-7.6 \text { to } 17.4)\end{array}$ & NR \\
\hline
\end{tabular}

BMI = body mass index; $\mathrm{CI}$ = confidence interval; COOP/WANCA = Dartmouth Primary Care Cooperative Information Project/World Organization of National Colleges, Academies, and Academic Associations of General Practice/Family Physicians; FIQ = Fibromyalgia Impact Questionnaire; HADS = Hospital Anxiety and Depression Scale; HRQOL = health-related quality of life; LBP = low back pain; MCID = Minimally clinically important difference; $\mathrm{MD}$ = mean difference; $\mathrm{NR}=$ not reported; NRS = Numeric Rating Scale; NRS = numeric rating scale; NSAIDS = nonsteroidal antiinflammatory; POMS-SV = Profile of Mood States Short Version; QDS = Quebec Back Pain Disability Scale; QOL = quality of life; RCT = randomized control trial; RMDQ = Roland Morris Disability Questionnaire; RR = risk ratio; SD = standard deviation; SF-36 = short form 36 item questionnaire; VAS = Visual Analog Scale.

${ }^{a}$ Only 12-month data for long term followup are reported here. For long term followup at 18, 24, and 30 months, see the full data abstraction appendix. 
Table B-8. Summary results for trials addressing KQ1: CPMPs versus pharmacologic therapy plus physical activity

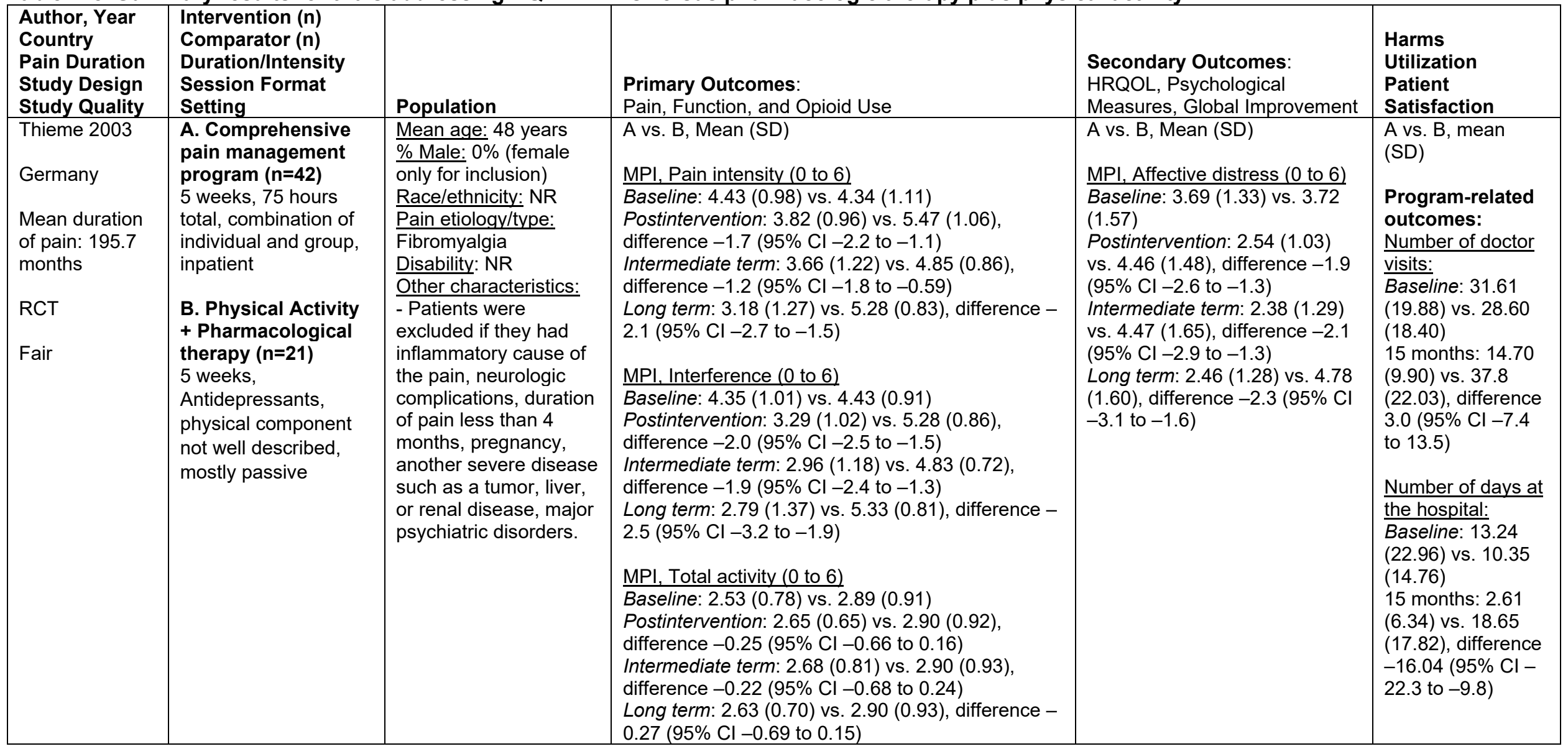




\begin{tabular}{|c|c|c|c|c|c|}
\hline $\begin{array}{l}\text { Author, Year } \\
\text { Country } \\
\text { Pain Duration } \\
\text { Study Design } \\
\text { Study Quality } \\
\end{array}$ & $\begin{array}{l}\text { Intervention (n) } \\
\text { Comparator (n) } \\
\text { Duration/Intensity } \\
\text { Session Format } \\
\text { Setting }\end{array}$ & Population & $\begin{array}{l}\text { Primary Outcomes: } \\
\text { Pain, Function, and Opioid Use }\end{array}$ & $\begin{array}{l}\text { Secondary Outcomes: } \\
\text { HRQOL, Psychological } \\
\text { Measures, Global Improvement }\end{array}$ & $\begin{array}{l}\text { Harms } \\
\text { Utilization } \\
\text { Patient } \\
\text { Satisfaction }\end{array}$ \\
\hline $\begin{array}{l}\text { Onac, } 2012 \text { \& } \\
2017 \\
\text { Romania } \\
\text { Mean duration } \\
\text { of pain: NR } \\
\text { RCT } \\
\text { Poor }\end{array}$ & $\begin{array}{l}\text { A. Comprehensive } \\
\text { pain management } \\
\text { program ( } \mathbf{n}=29 \text { ) } \\
2 \text { weeks, } 5 \text { days/week } \\
\text { (total hours NR), } \\
\text { individual, inpatient } \\
\\
\text { B. Physical Activity } \\
\text { + Pharmacological } \\
\text { therapy ( } \mathbf{n}=26 \text { ) } \\
2 \text { weeks (total hours } \\
\text { NR), } \\
\text { Diclofenac (50mg, } 3 \\
\text { times/day), } \\
\text { Omeprazole (20 mg, } \\
1 \text { time/day), and } \\
\text { Acetaminophen (1000 } \\
\text { mg, } 4 \text { times/day) } \\
\text { physical component } \\
\text { not well described, } \\
\text { mostly passive }\end{array}$ & $\begin{array}{l}\text { Population data include } \\
\text { patients from the third } \\
\text { arm of this trial } \\
\text { Mean age: } 47 \text { years } \\
\text { \% Male: } 57 \% \\
\text { Race/ethnicity: NR } \\
\text { Pain etiology/type: } \\
\text { Lumbar disk hernia } \\
\text { Disability: NR } \\
\text { Other characteristics: } \\
\text { Patients were excluded } \\
\text { if they had history of } \\
\text { psychotic disorders, } \\
\text { substance abuse } \\
\text { disorders in past Short } \\
\text { term, or certain } \\
\text { personality disorders. }\end{array}$ & 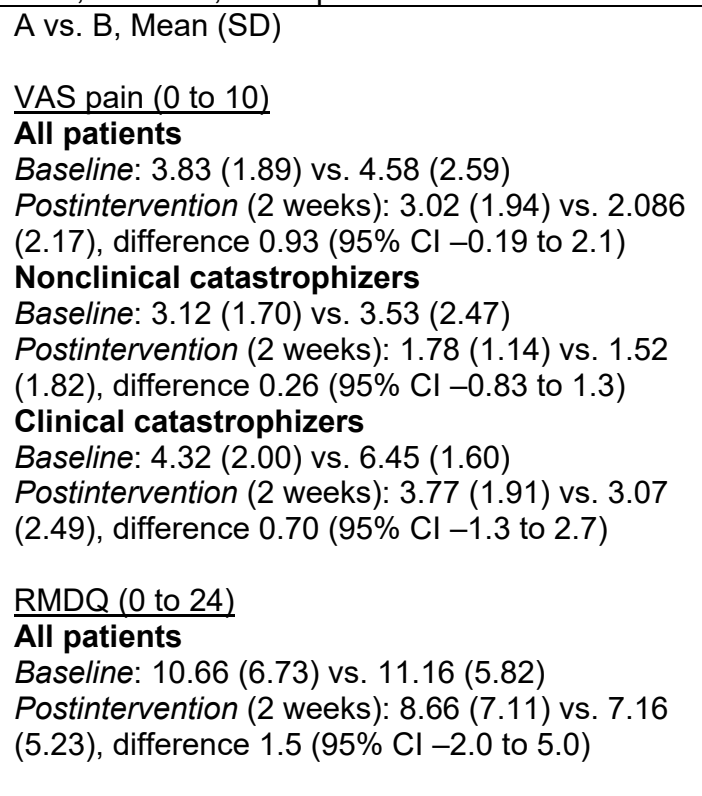 & $\begin{array}{l}\text { A vs. B, Mean (SD) } \\
\text { POMS-SV Emotional Distress } \\
\text { (scale NR) } \\
\text { All patients } \\
\text { Baseline: } 46.03 \text { (26.52) vs. } \\
45.16(28.09) \\
\text { Postintervention (2 weeks): } \\
43.15(31.16) \text { vs. } 41.90(32.18) \text {, } \\
\text { difference } 1.25(95 \% \mathrm{Cl}-16.1 \\
\text { to } 18.6)\end{array}$ & NR \\
\hline
\end{tabular}

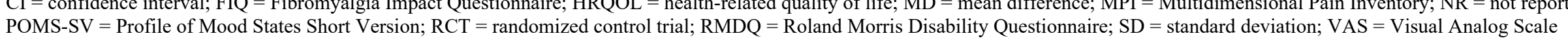


Table B-9. Summary results for trials addressing KQ1: CPMPs versus psychological therapy

\begin{tabular}{|c|c|c|c|c|c|}
\hline $\begin{array}{l}\text { Author, Year } \\
\text { Country } \\
\text { Pain Duration } \\
\text { Study Design } \\
\text { Study Quality }\end{array}$ & $\begin{array}{l}\text { Intervention }(\mathrm{n}) \\
\text { Comparator }(\mathrm{n}) \\
\text { Duration/Intensity } \\
\text { Session Format } \\
\text { Setting }\end{array}$ & Population & $\begin{array}{l}\text { Primary Outcomes: } \\
\text { Pain, Function, and Opioid Use }\end{array}$ & $\begin{array}{l}\text { Secondary Outcomes: } \\
\text { HRQOL, Psychological Measures, } \\
\text { Global Improvement }\end{array}$ & $\begin{array}{l}\text { Harms } \\
\text { Utilization } \\
\text { Patient Satisfaction }\end{array}$ \\
\hline $\begin{array}{l}\text { Jensen, } \\
2001 \\
\text { Sweden } \\
\text { Duration of pain: } 31 \\
\text { months } \\
\text { RCT } \\
\text { Fair }\end{array}$ & $\begin{array}{l}\text { A. Comprehensive pain } \\
\text { management program, } \\
\text { ( } \mathrm{n}=63 \text { ): } 4 \text { weeks (33-34 } \\
\text { hours/week, plus six 90- } \\
\text { minute booster sessions } \\
\text { over a period of } 1 \text { year } \\
\text { after treatment); high } \\
\text { intensity, group; } \\
\text { Rehabilitation clinic } \\
\text { B. Psychological } \\
\text { Therapy (n=49): } 4 \\
\text { weeks, (13-14 } \\
\text { hours/week, plus six } 90- \\
\text { minute booster sessions } \\
\text { over a period of } 1 \text { year } \\
\text { after treatment); low } \\
\text { intensity, group in } \\
\text { person; Rehabilitation } \\
\text { clinic }\end{array}$ & $\begin{array}{l}\text { Mean age: } 43 \text { years } \\
\text { Male: } 45 \% \\
\text { Race/ethnicity: Swedish } \\
\text { origin } 67 \% \\
\text { Pain etiology/type: } \\
\text { Chronic nonspecific } \\
\text { spinal pain } \\
\text {-Cervical/ } \\
\text { thoracic pain: } 42 \% \\
\text { - Lumbar pain: } 46 \% \\
\text { - Mixed pain areas: } 12 \% \\
\text { Disability: NR } \\
\text { - Mean total sick leave in } \\
6 \text { months prior to } \\
\text { inclusion in study: } 292 \\
\text { (63) } \\
\text { Comorbidities: } \\
\text { - NR }\end{array}$ & NR & $\begin{array}{l}\text { A vs. B, Mean (SD) } \\
\text { SF-36 Global health (0-100) } \\
\text { Females only ( } n=30 \text { vs. 22) } \\
\text { Baseline: } 38.1(14.5) \text { vs. } 38.9(13.7) \\
\text { Postintervention: } 47.6(18.0) \text { vs. } 48.8 \\
(16.8) \\
\text { Intermediate term: } 52.4(21.6) \text { vs. } \\
54.2(19.3) \\
\text { Long term: } 53.1 \text { (24.5) vs. } 58.2(18.4) \\
\text { - improvement approached } \\
\text { significance for group B at Long } \\
\text { term(p=0.057) } \\
\text { Males only ( } n=33 \text { vs. } 27) \\
\text { Baseline: } 41.6(14.6) \text { vs. } 43.8(16.0) \\
\text { Postintervention: } 48.5 \text { (17.2) vs. } 44.8 \\
\text { (18.0) } \\
\text { Intermediate term: } 54.3(18.3) \text { vs. } \\
43.5 \text { (19.1)) } \\
\text { Long term: } 57.2 \text { (21.8) vs. } 50.8(27.9) \\
\text { - Nonsignificant group effect via } \\
\text { MANCOVA with a repeated- } \\
\text { measures design } \\
\text { - p=ns between interventions at any } \\
\text { timepoint }\end{array}$ & 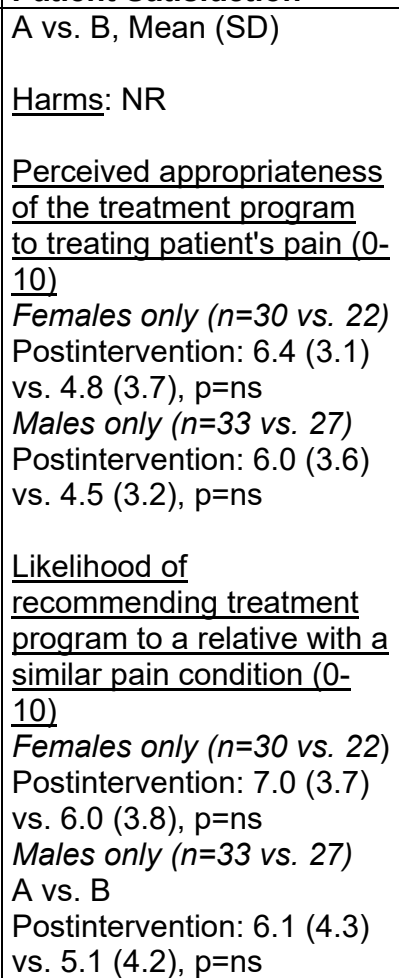 \\
\hline
\end{tabular}




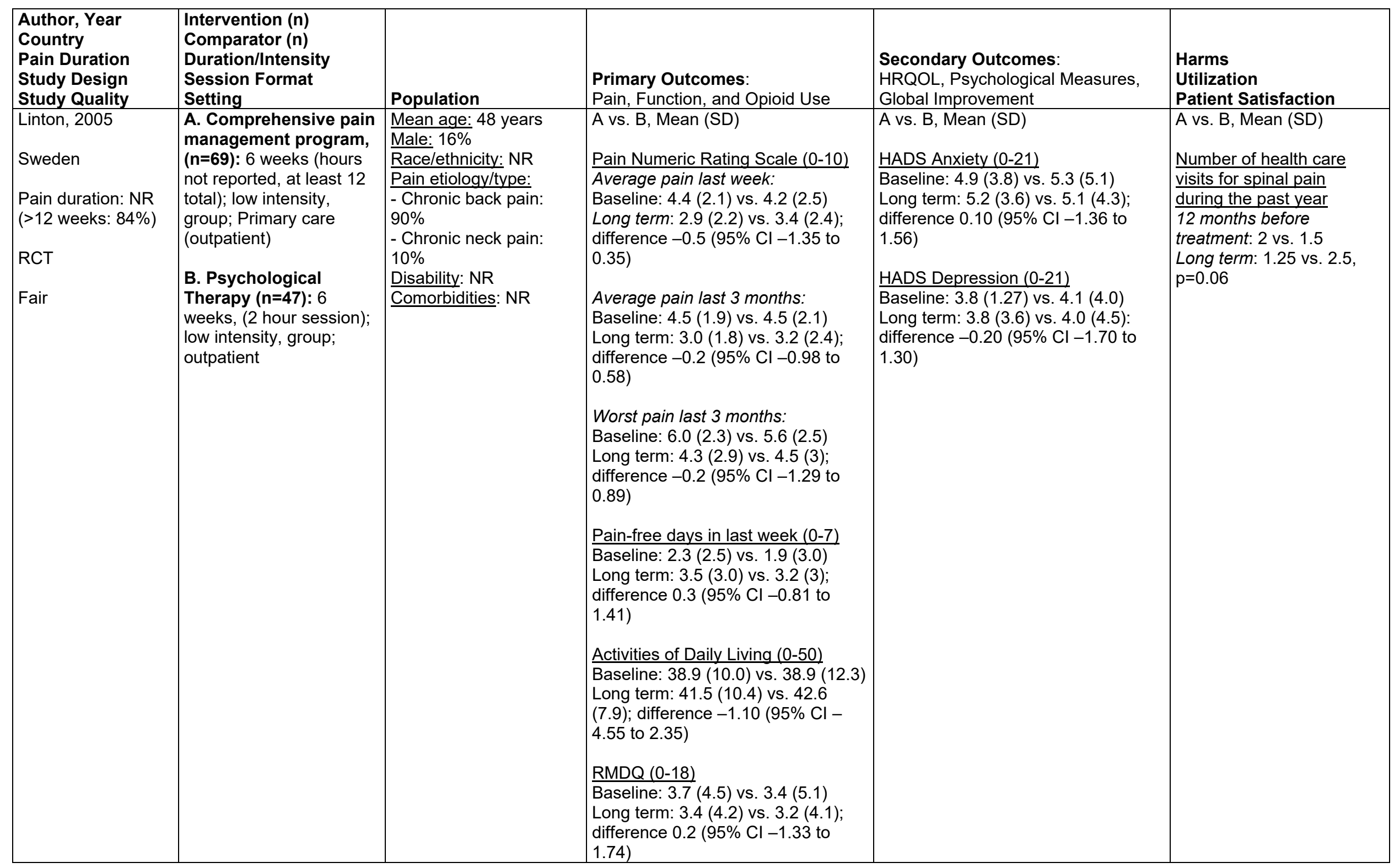




\begin{tabular}{|c|c|c|c|c|c|}
\hline $\begin{array}{l}\text { Author, Year } \\
\text { Country } \\
\text { Pain Duration } \\
\text { Study Design } \\
\text { Study Quality }\end{array}$ & $\begin{array}{l}\text { Intervention (n) } \\
\text { Comparator }(\mathrm{n}) \\
\text { Duration/Intensity } \\
\text { Session Format } \\
\text { Setting } \\
\end{array}$ & Population & $\begin{array}{l}\text { Primary Outcomes: } \\
\text { Pain, Function, and Opioid Use }\end{array}$ & $\begin{array}{l}\text { Secondary Outcomes: } \\
\text { HRQOL, Psychological Measures, } \\
\text { Global Improvement }\end{array}$ & $\begin{array}{l}\text { Harms } \\
\text { Utilization } \\
\text { Patient Satisfaction }\end{array}$ \\
\hline $\begin{array}{l}\text { Smeets, 2006a, } \\
2008 \\
\text { Netherlands } \\
\text { Pain duration: } 56.7 \\
\text { months } \\
\text { RCT } \\
\text { Fair }\end{array}$ & $\begin{array}{l}\text { A. Comprehensive pain } \\
\text { management program } \\
\text { ( } \mathbf{n = 6 1 ) :} 10 \text { weeks ( } 79 \\
\text { hours total), low intensity, } \\
\text { group and individual, } \\
\text { outdoor rehabilitation } \\
\text { centers } \\
\text { B. Psychological } \\
\text { Therapy only (CBT) } \\
\text { only ( } \mathbf{n}=58 \text { ): } \\
10 \text { weeks (26.5 hours } \\
\text { total), low intensity, group } \\
\text { and individual, } \\
\text { rehabilitation center }\end{array}$ & $\begin{array}{l}\text { Mean age: } 42 \text { years } \\
\text { Male: } 53 \% \\
\text { Race/ethnicity: NR } \\
\text { Pain etiology/type: Pain } \\
\text { etiology/type: LBP } \\
\text { - radiation of pain below } \\
\text { knee: } 49 \% \\
\text { - radiation of pain above } \\
\text { knee: } 37 \% \\
\text { - without radiation of } \\
\text { pain: } 14 \% \\
\text { Disability: } \\
\text { Full sick leave/disability } \\
\text { pension: } 38 \% \\
\text { Partial sick } \\
\text { leave/disability pension: } \\
24 \% \\
\text { Comorbidities: NR }\end{array}$ & 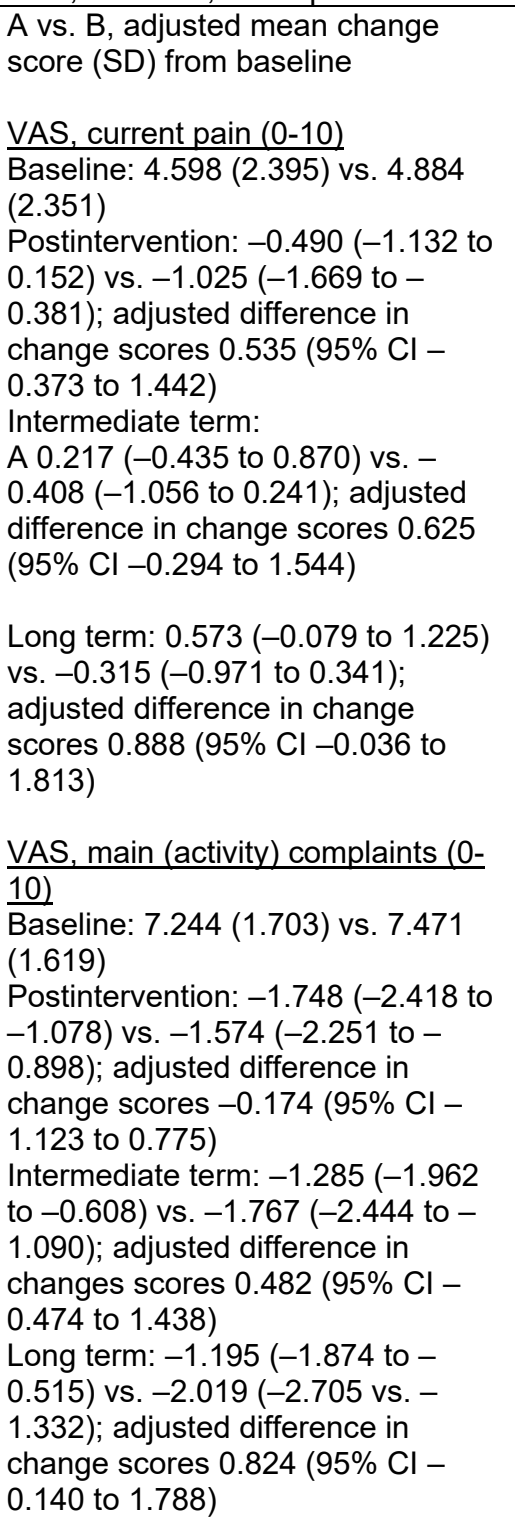 & 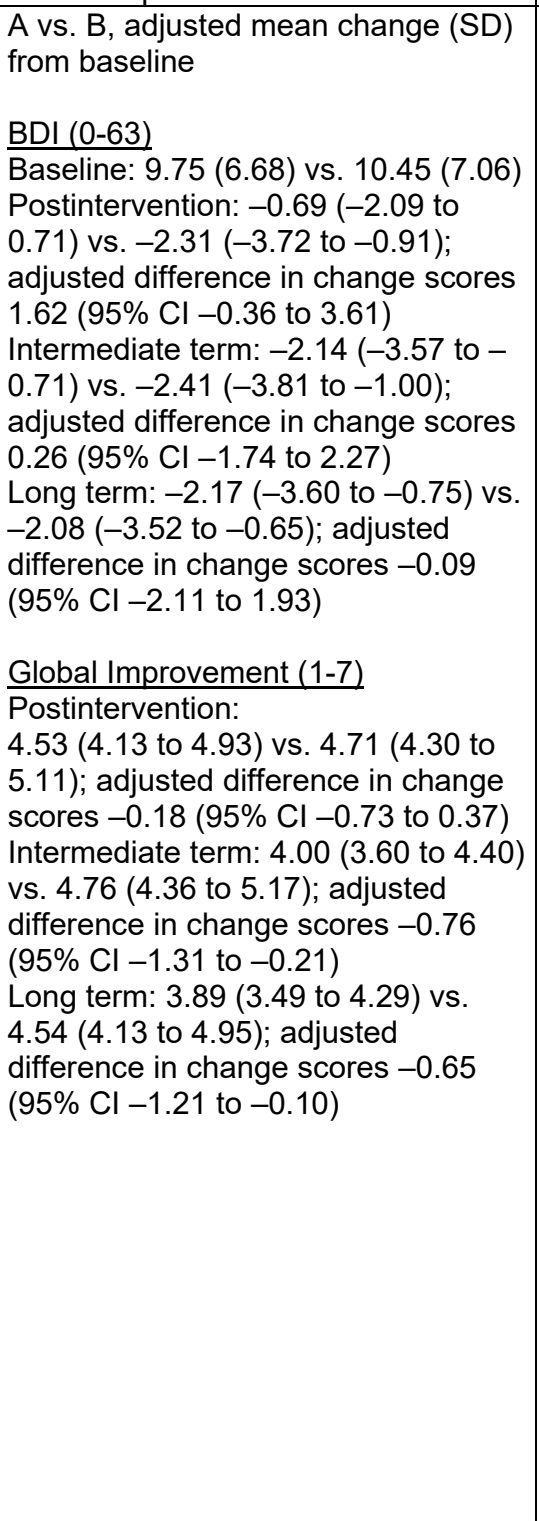 & $\begin{array}{l}\text { A vs. B, \% (n/N) or mean } \\
\text { (SD) } \\
\text { Harms } \\
\text { - Increased pain in the } \\
\text { lower back or radiating leg } \\
\text { pain leading to withdrawal } \\
\text { from the trial: } 5.5 \% \text { (3/55) } \\
\text { vs. } 0 \% \text { (0/55) } \\
\text { - No other adverse events } \\
\text { were reported in either } \\
\text { group } \\
\text { VAS Satisfaction (0-100) } \\
\text { (effect modification by } \\
\text { RDQ) } \\
\text { - 10th percentile of } \\
\text { baseline RMDQ (=9) } \\
\text { Postintervention: } 64.98 \\
\text { (25.30) vs. NR; adjusted } \\
\text { difference for group B } \\
\text { versus group A: }-0.99 \\
\text { (95\% Cl -18.55 to 16.56) } \\
\text { - 50th percentile of } \\
\text { baseline RMDQ (=14) } \\
\text { Postintervention: } 70.24 \\
\text { (25.30) vs. NR; adjusted } \\
\text { difference for group B } \\
\text { versus group A: -0.89 } \\
\text { (95\% Cl -13.16 to } 11.28) \\
\text { - 90th percentile of } \\
\text { baseline RMDQ (=19) } \\
\text { Postintervention: } 70.24 \\
\text { (25.30) vs. NR; adjusted } \\
\text { difference for group B } \\
\text { versus group A: -0.78 } \\
\text { (95\% Cl -19.11 to } 17.55 \text { ) }\end{array}$ \\
\hline
\end{tabular}




\begin{tabular}{|c|c|c|c|c|c|}
\hline $\begin{array}{l}\text { Author, Year } \\
\text { Country } \\
\text { Pain Duration } \\
\text { Study Design } \\
\text { Study Quality } \\
\end{array}$ & \begin{tabular}{|l|} 
Intervention (n) \\
Comparator (n) \\
Duration/Intensity \\
Session Format \\
Setting \\
\end{tabular} & Population & $\begin{array}{l}\text { Primary Outcomes: } \\
\text { Pain, Function, and Opioid Use }\end{array}$ & $\begin{array}{l}\text { Secondary Outcomes: } \\
\text { HRQOL, Psychological Measures, } \\
\text { Global Improvement }\end{array}$ & $\begin{array}{l}\text { Harms } \\
\text { Utilization } \\
\text { Patient Satisfaction }\end{array}$ \\
\hline $\begin{array}{l}\text { Smeets, 2006a, } \\
2008 \\
\text { (Continued) }\end{array}$ & & & 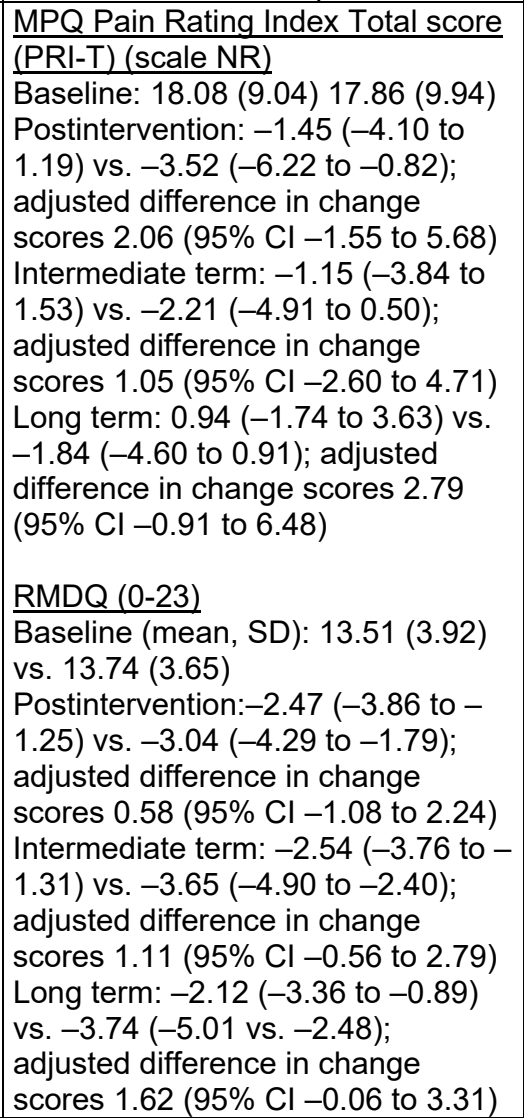 & & \\
\hline
\end{tabular}




\begin{tabular}{|c|c|c|c|c|c|}
\hline $\begin{array}{l}\text { Author, Year } \\
\text { Country } \\
\text { Pain Duration } \\
\text { Study Design } \\
\text { Study Quality }\end{array}$ & $\begin{array}{l}\text { Intervention (n) } \\
\text { Comparator (n) } \\
\text { Duration/Intensity } \\
\text { Session Format } \\
\text { Setting }\end{array}$ & Population & $\begin{array}{l}\text { Primary Outcomes: } \\
\text { Pain, Function, and Opioid Use }\end{array}$ & $\begin{array}{l}\text { Secondary Outcomes: } \\
\text { HRQOL, Psychological Measures, } \\
\text { Global Improvement }\end{array}$ & $\begin{array}{l}\text { Harms } \\
\text { Utilization } \\
\text { Patient Satisfaction }\end{array}$ \\
\hline $\begin{array}{l}\text { Turner, } 1990 \\
\text { USA } \\
\text { Duration of pain: } \\
155 \text { months } \\
\text { RCT } \\
\text { Poor }\end{array}$ & $\begin{array}{l}\text { A. Comprehensive pain } \\
\text { management program } \\
(\mathrm{n}=18): 8 \text { weeks (4 } \\
\text { hours/week, } 32 \text { hours } \\
\text { total), low intensity, } \\
\text { Individual and group, } \\
\text { Outpatient } \\
\\
\text { B. Psychological } \\
\text { Therapy alone ( } \mathrm{n}=18): 8 \\
\text { weeks (2 hours/week, } 16 \\
\text { hours total), low intensity, } \\
\text { Individual and group, } \\
\text { outpatient }\end{array}$ & $\begin{array}{l}\text { Mean age: } 44 \text { years } \\
\text { Male: } 52.1 \% \\
\text { Race/ethnicity: White } \\
100 \% \\
\text { Pain etiology/type: } \\
\text { Chronic LBP ( } \geq 6 \\
\text { months) } \\
\text { Disability: N/A } \\
\text { Comorbidities: NR }\end{array}$ & $\begin{array}{l}\text { A vs. B, Mean (SD) } \\
\text { MPQ Pain Rating Index (0-78) } \\
\text { Baseline: } 25.54 \text { (12.41) vs. } 20.96 \\
(9.95) \\
\text { Postintervention: } 14.78 \text { (11.44) vs. } \\
17.71 \text { (12.08) } \\
\text { Intermediate term: } 13.29 \text { (9.15) vs. } \\
19.50(15.72) \\
\text { Long term: } 18.21 \text { (13.31) vs. } 16.41 \\
(13.63) \\
\text { SIP (0-100) } \\
\text { Baseline: } 8.50 \text { (4.59) vs. } 7.90 \text { (6.43) } \\
\text { Postintervention: } 3.63 \text { (2.98) vs. } \\
4.72(4.12) \\
\text { Intermediate term: } 4.51 \text { (4.68) vs. } \\
7.60(9.86) \\
\text { Long term: } 4.75 \text { (3.40) vs. } 5.25 \\
(6.72)\end{array}$ & $\begin{array}{l}\text { A vs. B, Mean (SD) } \\
\text { CES-D (0-60) } \\
\text { Baseline: } 12.38 \text { (7.31) vs. } 10.40 \\
(7.51) \\
\text { Postintervention: } 7.36(5.89) \text { v vs. } \\
8.08 \text { (4.95) } \\
\text { Intermediate term: } 8.29(7.94) \text { vs. } \\
11.36(8.30) \\
\text { Long term: } 10.00 \text { (7.57) vs. } 8.29 \\
(7.74) \\
\text { Patient satisfaction (1-7) } \\
\text { Postintervention: } 5.50(\mathrm{NR}) \text { vs. } 4.00 \\
\text { (NR), p<0.05 }\end{array}$ & $\begin{array}{l}\text { A vs. B, Mean (SD) } \\
\text { Harms: NR } \\
\text { Patient satisfaction (1-7) } \\
\text { Postintervention: } 5.50(N R) \\
\text { vs. } 4.00(N R), p<0.05\end{array}$ \\
\hline
\end{tabular}




\begin{tabular}{|c|c|c|c|c|c|}
\hline $\begin{array}{l}\text { Author, Year } \\
\text { Country } \\
\text { Pain Duration } \\
\text { Study Design } \\
\text { Study Quality }\end{array}$ & $\begin{array}{l}\text { Intervention (n) } \\
\text { Comparator }(\mathrm{n}) \\
\text { Duration/Intensity } \\
\text { Session Format } \\
\text { Setting }\end{array}$ & Population & $\begin{array}{l}\text { Primary Outcomes: } \\
\text { Pain, Function, and Opioid Use }\end{array}$ & $\begin{array}{l}\text { Secondary Outcomes: } \\
\text { HRQOL, Psychological Measures, } \\
\text { Global Improvement }\end{array}$ & $\begin{array}{l}\text { Harms } \\
\text { Utilization } \\
\text { Patient Satisfaction }\end{array}$ \\
\hline \begin{tabular}{|l|} 
Turner-Stokes, \\
2003 \\
UK \\
Pain duration: 105.6 \\
months \\
RCT \\
Poor
\end{tabular} & $\begin{array}{l}\text { A. Comprehensive pain } \\
\text { management program } \\
\text { (73): } 8 \text { weeks ( } 2 \text { full } \\
\text { afternoons per week, } ~ 72 \\
\text { hours total), low intensity, } \\
\text { group, Outpatient pain } \\
\text { management clinics } \\
\\
\text { B. Psychological } \\
\text { Therapy alone ( } \mathbf{n}=53): 8 \\
\text { weeks (1 hour every } \\
\text { other week, } 4 \text { hours } \\
\text { total), low intensity, } \\
\text { group, outpatient }\end{array}$ & $\begin{array}{l}\frac{\text { Mean age: } 47 \text { years }}{\text { Male: } 31 \%} \\
\text { Race/ethnicity: NR } \\
\text { Pain etiology/type: } \\
\text { Chronic pain } \\
\text { Disability: NR } \\
\text { Comorbidities: NR }\end{array}$ & 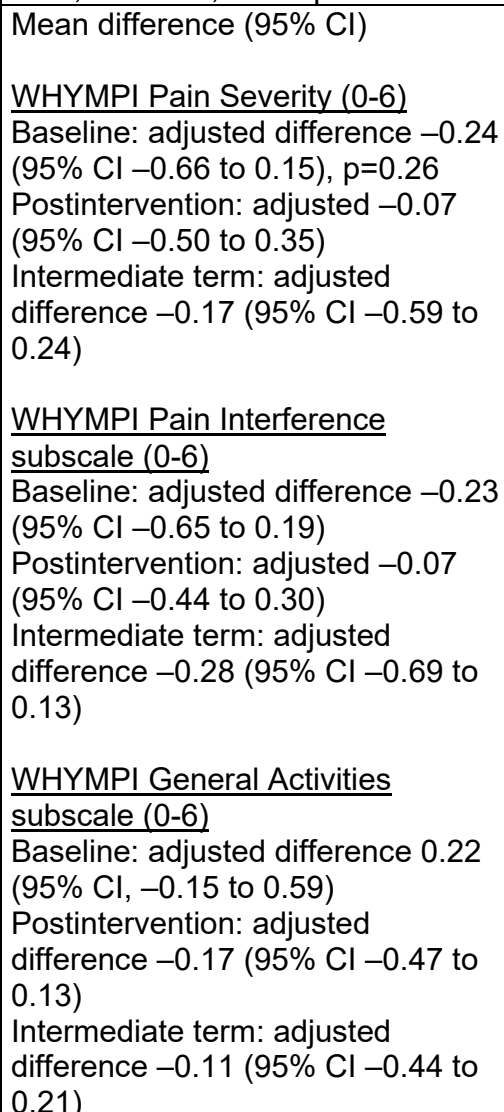 & 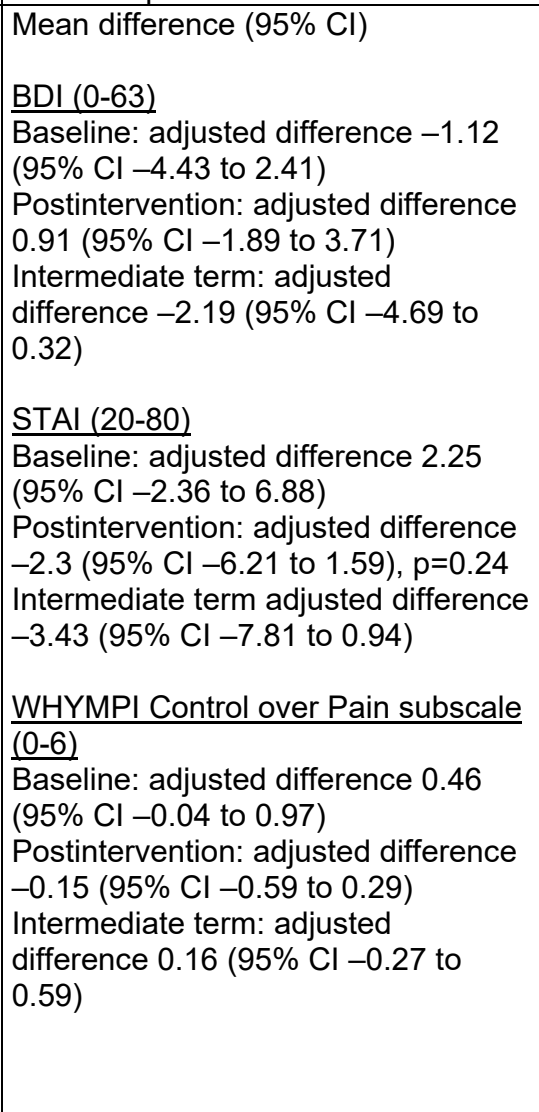 & NR \\
\hline
\end{tabular}

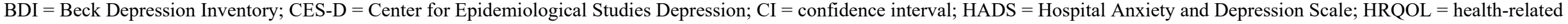

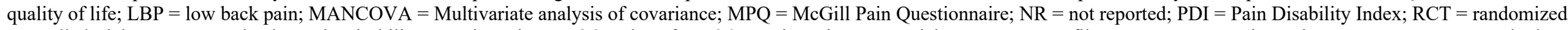

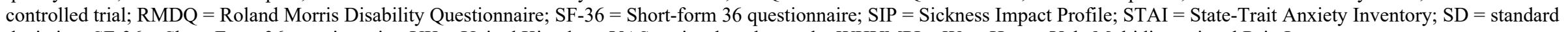
deviation; SF-36 = Short-Form 36 questionnaire; UK = United Kingdom; VAS = visual analog scale; WHYMPI = West Haven-Yale Multidimensional Pain Inventory. 
Table B-10. Summary results for trials addressing KQ2: IPMPs

\begin{tabular}{|c|c|c|c|c|c|}
\hline $\begin{array}{l}\text { Author, Year } \\
\text { Country } \\
\text { Pain Duration } \\
\text { Study Design } \\
\text { Study Quality }\end{array}$ & $\begin{array}{l}\text { Intervention (n) } \\
\text { Comparator ( } \mathrm{n}) \\
\text { Duration/Intensity } \\
\text { Session Format } \\
\text { Setting } \\
\end{array}$ & Population & $\begin{array}{l}\text { Primary Outcomes: } \\
\text { Pain, Function, and Opioid Use }\end{array}$ & $\begin{array}{l}\text { Secondary Outcomes: } \\
\text { HRQOL, Psychological Measures, Global } \\
\text { Improvement }\end{array}$ & $\begin{array}{l}\text { Harms } \\
\text { Utilization } \\
\text { Patient Satisfaction }\end{array}$ \\
\hline $\begin{array}{l}\text { Allen, } 2017 \\
\text { USA } \\
\text { Mean duration } \\
\text { of symptoms: } \\
124.8 \text { months } \\
\text { Cluster RCT } \\
\text { Fair }\end{array}$ & $\begin{array}{l}\text { A. Patient } \\
\text { focused } \\
\text { multidisciplinary } \\
\text { treatment + } \\
\text { provider focused } \\
\text { multidisciplinary } \\
\text { treatment + usual } \\
\text { care ( } \mathrm{n}=140 \\
\text { patients, } 5 \\
\text { providers): } 12 \\
\text { months (time } \\
\text { duration NR), } \\
\text { individual, } \\
\text { community-based } \\
\text { outpatient clinics } \\
\\
\text { B. Provider } \\
\text { focused } \\
\text { multidisciplinary } \\
\text { treatment + usual } \\
\text { care ( } n=140 \\
\text { patients, } 5 \\
\text { providers): } 12 \\
\text { months } \\
\text { C. Patient } \\
\text { focused } \\
\text { multidisciplinary } \\
\text { treatment + usual } \\
\text { care ( }=128 \\
\text { patients, } 5 \\
\text { providers): } 12 \\
\text { months }\end{array}$ & $\begin{array}{l}\text { Patient Participants } \\
\text { Mean age: } 63.1 \text { years } \\
\text { Male: } 25 \% \\
\text { Non-White race: } 41 \% \\
\text { Joints with OA: } \\
\text { - Knee only: } 85 \% \\
\text { - Hip only: } 9 \% \\
\text { - Knee and hip: } 6 \% \\
\text { Disabled: } 7 \% \\
\text { Fair or poor health: } \\
20 \% \\
\text { Mean BMI: } 35.6 \\
\text { kg/m2 } \\
\text { Clinic and Provider } \\
\text { Characteristics } \\
\text { Mean providers: } 7.3 \\
\text { Mean medical } \\
\text { physicians and } \\
\text { osteopaths: } 6.2 \\
\text { Mean nurse } \\
\text { practitioners and } \\
\text { physician assistants: } \\
\text { 1.1 } \\
\text { Family medicine } \\
\text { practice: } 60 \% \\
\text { Internal medicine } \\
\text { practice: } 40 \% \\
\text { Providers: } \\
\text { - Male: } 38 \% \\
\text { - Mean years since } \\
\text { graduation: } 18.9 \\
\text { years }\end{array}$ & 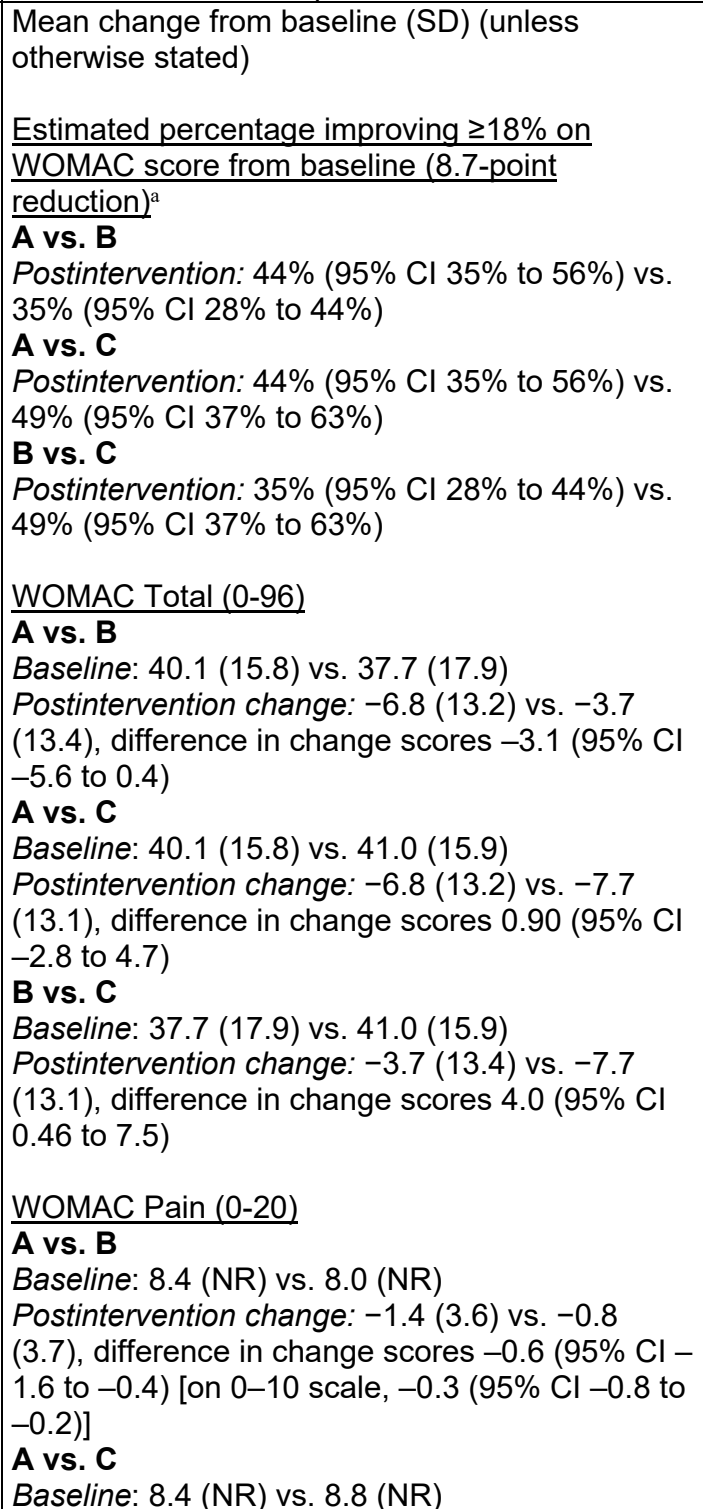 & $\begin{array}{l}\text { Mean change from baseline (SD) } \\
\text { PHQ-8 (0-24) } \\
\text { A vs. B } \\
\text { Baseline: } 4.9 \text { (NR) vs. } 4.5 \text { (NR) } \\
\text { Postintervention change: }-0.6(3.1) \text { vs. } 0 \\
(3.1) \text {, difference in change scores -0.6 } \\
(95 \% \mathrm{Cl}-1.4 \text { to } 0.2) \\
\text { A vs. C } \\
\text { Baseline: } 4.9 \text { (NR) vs. } 4.8 \text { (NR) } \\
\text { Postintervention change: }-0.6(3.1) \text { vs. } \\
-0.4(3.0), \text { difference in change scores - } \\
0.2(95 \% \text { Cl }-1.1 \text { to } 0.7) \\
\text { B vs. C } \\
\text { Baseline: } 4.5 \text { (NR) vs. } 4.8 \text { (NR) } \\
\text { Postintervention change: } 0 \text { (3.1) vs. }-0.4 \\
(3.0), \text { difference in change scores } 0.4 \\
(95 \% \mathrm{Cl}-0.4 \text { to } 1.2)\end{array}$ & 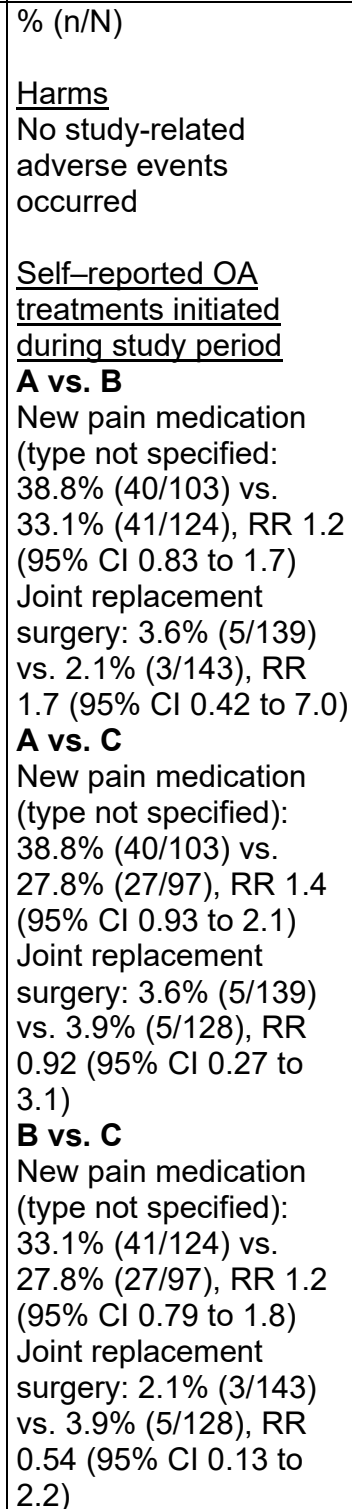 \\
\hline
\end{tabular}




\begin{tabular}{|c|c|c|c|c|c|}
\hline $\begin{array}{l}\text { Author, Year } \\
\text { Country } \\
\text { Pain Duration } \\
\text { Study Design } \\
\text { Study Quality }\end{array}$ & $\begin{array}{l}\text { Intervention (n) } \\
\text { Comparator (n) } \\
\text { Duration/Intensity } \\
\text { Session Format } \\
\text { Setting }\end{array}$ & Population & $\begin{array}{l}\text { Primary Outcomes: } \\
\text { Pain, Function, and Opioid Use }\end{array}$ & $\begin{array}{l}\text { Secondary Outcomes: } \\
\text { HRQOL, Psychological Measures, Global } \\
\text { Improvement }\end{array}$ & $\begin{array}{l}\text { Harms } \\
\text { Utilization } \\
\text { Patient Satisfaction }\end{array}$ \\
\hline $\begin{array}{l}\text { Allen, } 2017 \\
\text { (Continued) }\end{array}$ & & & 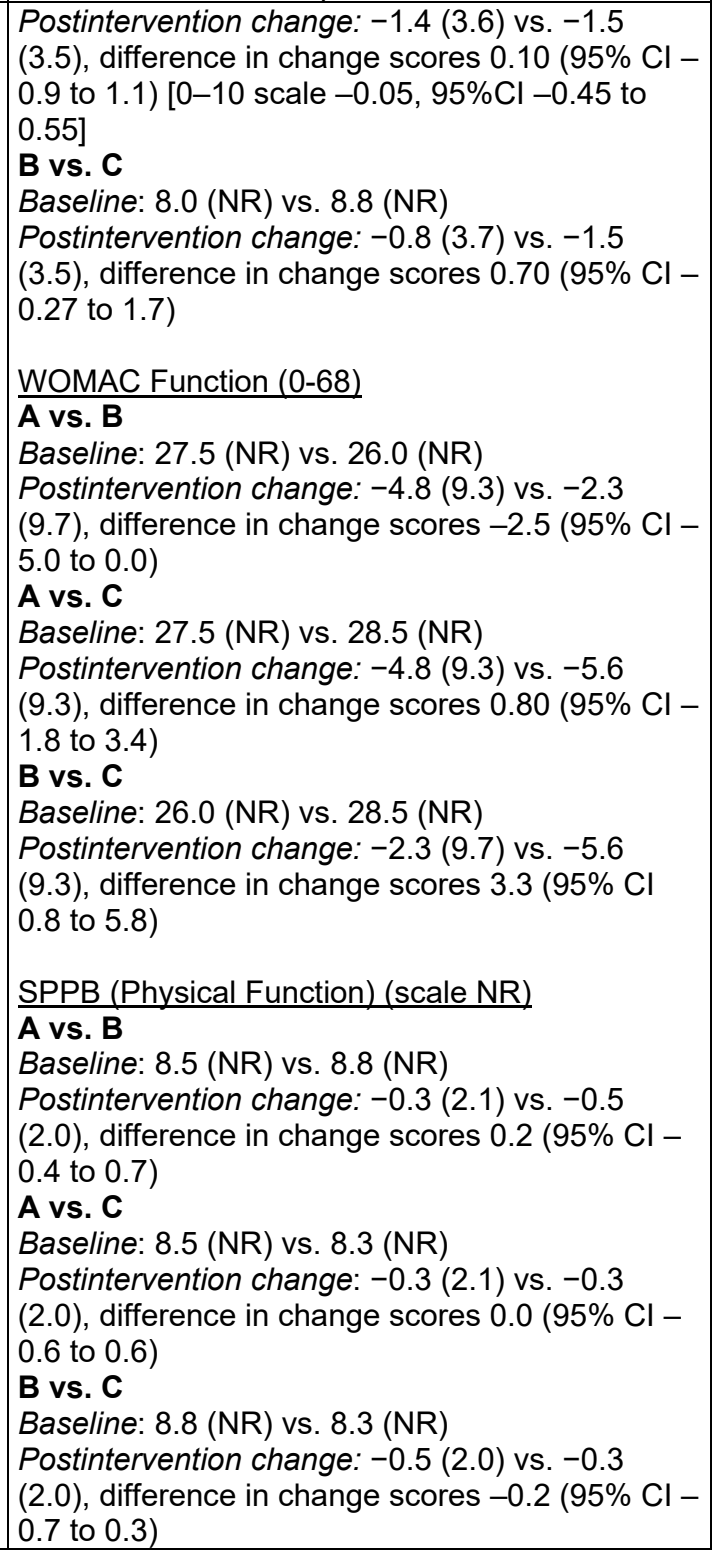 & & \\
\hline
\end{tabular}




\begin{tabular}{|c|c|c|c|c|c|}
\hline $\begin{array}{l}\text { Author, Year } \\
\text { Country } \\
\text { Pain Duration } \\
\text { Study Design } \\
\text { Study Quality }\end{array}$ & $\begin{array}{l}\text { Intervention (n) } \\
\text { Comparator (n) } \\
\text { Duration/Intensity } \\
\text { Session Format } \\
\text { Setting }\end{array}$ & Population & $\begin{array}{l}\text { Primary Outcomes: } \\
\text { Pain, Function, and Opioid Use }\end{array}$ & $\begin{array}{l}\text { Secondary Outcomes: } \\
\text { HRQOL, Psychological Measures, Global } \\
\text { Improvement }\end{array}$ & $\begin{array}{l}\text { Harms } \\
\text { Utilization } \\
\text { Patient Satisfaction }\end{array}$ \\
\hline $\begin{array}{l}\text { Calner, } 2017 \\
\text { Sweden } \\
\text { Mean duration } \\
\text { of pain: } 79 \\
\text { months } \\
\text { RCT } \\
\text { Fair }\end{array}$ & $\begin{array}{l}\text { A. } \\
\text { Multidisciplinary } \\
\text { rehab + web- } \\
\text { based } \\
\text { intervention } \\
\text { (Web-BCPA) } \\
\text { (n=60): minimum } \\
1.5 \text { months of 2-3 } \\
\text { sessions/week; } \\
\text { total duration } 12 \\
\text { months (time } \\
\text { duration NR), } \\
\text { mode of delivery } \\
\text { NR, Outpatient } \\
\text { healthcare centers } \\
\text { B. } \\
\text { Multidisciplinary } \\
\text { rehab alone } \\
\text { ( } \mathrm{n}=49 \text { ) }\end{array}$ & $\begin{array}{l}\frac{\text { Mean age: } 43 \text { years }}{\text { Male: } 57 \%} \\
\text { Race/Ethnicity: NR } \\
\text { Disability: NR } \\
\text { - Working: } 28 \% \\
\text { - Unemployed: } 7 \% \\
\text { - Temporarily } \\
\text { - Unemployed: } 4 \%\end{array}$ & 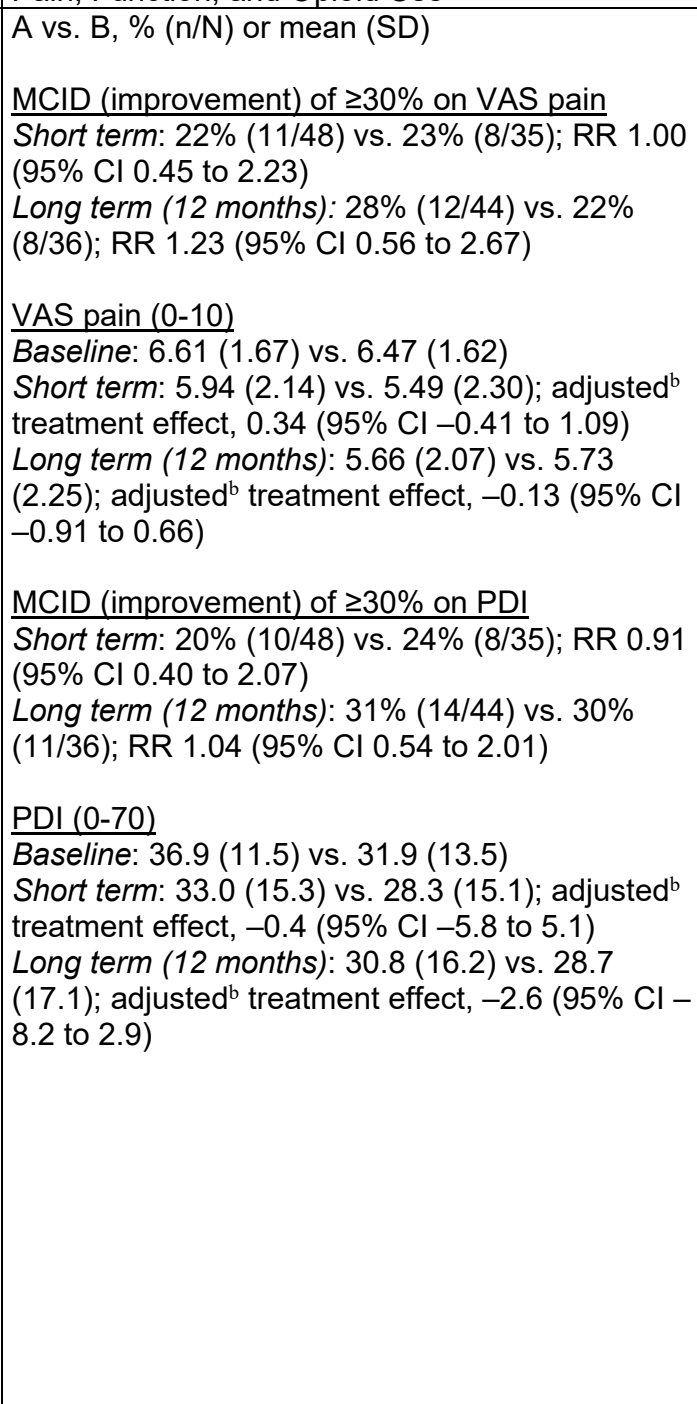 & 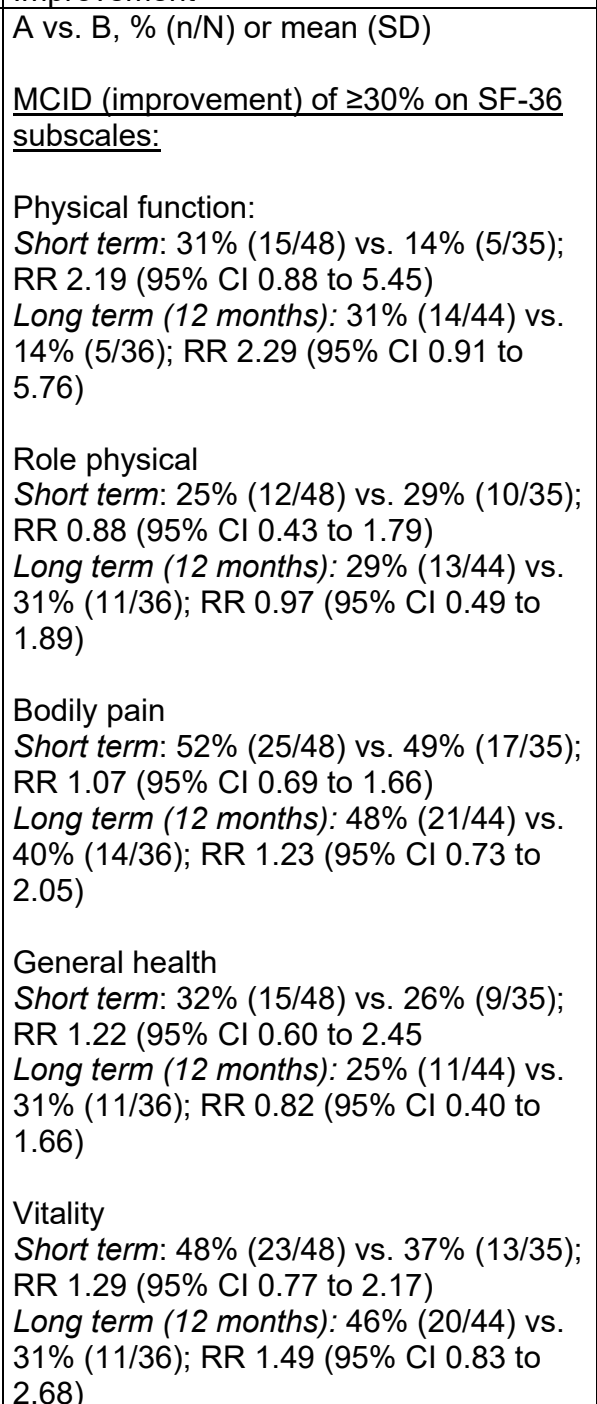 & NR \\
\hline
\end{tabular}




\begin{tabular}{|c|c|c|c|c|c|}
\hline $\begin{array}{l}\text { Author, Year } \\
\text { Country } \\
\text { Pain Duration } \\
\text { Study Design } \\
\text { Study Quality }\end{array}$ & $\begin{array}{l}\text { Intervention (n) } \\
\text { Comparator (n) } \\
\text { Duration/Intensity } \\
\text { Session Format } \\
\text { Setting }\end{array}$ & Population & $\begin{array}{l}\text { Primary Outcomes: } \\
\text { Pain, Function, and Opioid Use }\end{array}$ & $\begin{array}{l}\text { Secondary Outcomes: } \\
\text { HRQOL, Psychological Measures, Global } \\
\text { Improvement }\end{array}$ & $\begin{array}{l}\text { Harms } \\
\text { Utilization } \\
\text { Patient Satisfaction }\end{array}$ \\
\hline $\begin{array}{l}\text { Calner, } 2017 \\
\text { (Continued) }\end{array}$ & & & & 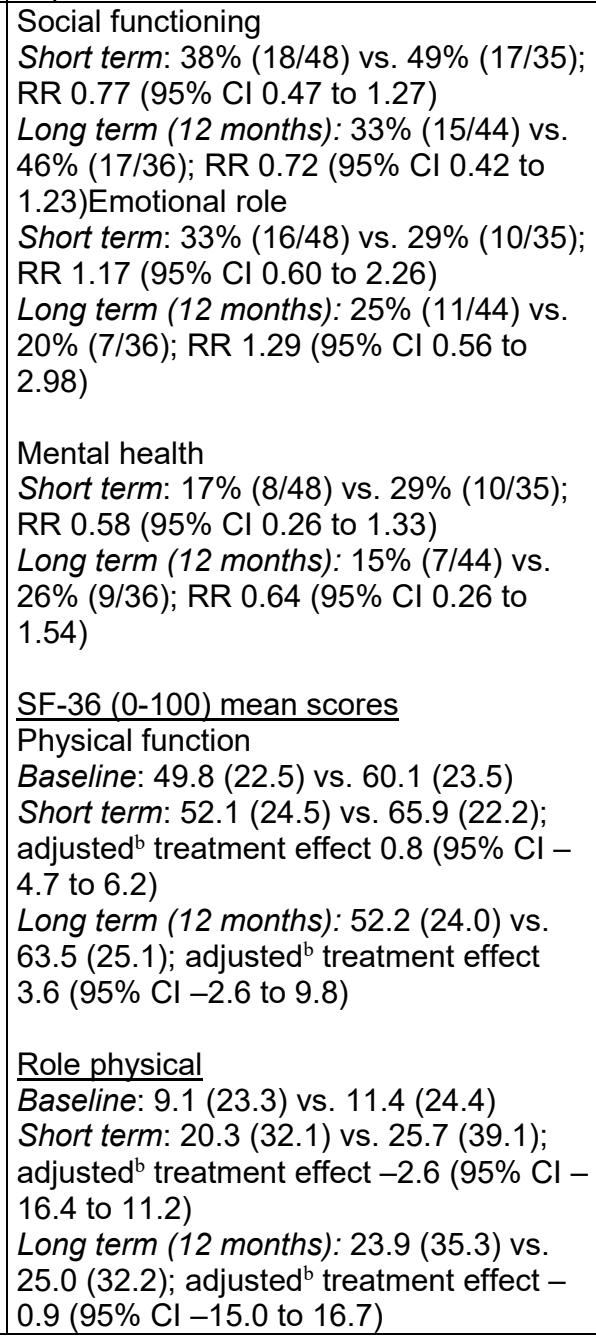 & \\
\hline
\end{tabular}




\begin{tabular}{|c|c|c|c|c|c|}
\hline $\begin{array}{l}\text { Author, Year } \\
\text { Country } \\
\text { Pain Duration } \\
\text { Study Design } \\
\text { Study Quality }\end{array}$ & \begin{tabular}{|l|} 
Intervention $(\mathrm{n})$ \\
Comparator $(\mathrm{n})$ \\
Duration/Intensity \\
Session Format \\
Setting
\end{tabular} & Population & $\begin{array}{l}\text { Primary Outcomes: } \\
\text { Pain, Function, and Opioid Use }\end{array}$ & $\begin{array}{l}\text { Secondary Outcomes: } \\
\text { HRQOL, Psychological Measures, Global } \\
\text { Improvement }\end{array}$ & $\begin{array}{l}\text { Harms } \\
\text { Utilization } \\
\text { Patient Satisfaction }\end{array}$ \\
\hline $\begin{array}{l}\text { Calner, } 2017 \\
\text { (Continued) }\end{array}$ & & & & 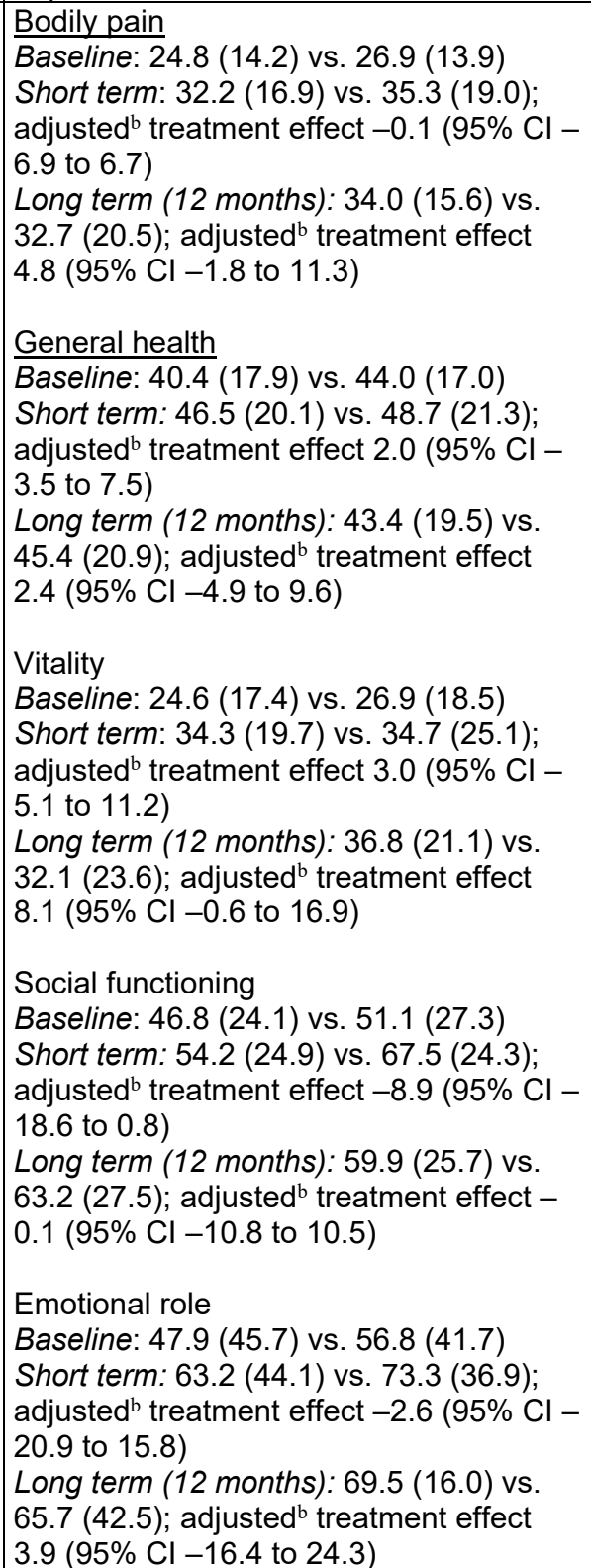 & \\
\hline
\end{tabular}




\begin{tabular}{|c|c|c|c|c|c|}
\hline $\begin{array}{l}\text { Author, Year } \\
\text { Country } \\
\text { Pain Duration } \\
\text { Study Design } \\
\text { Study Quality } \\
\end{array}$ & \begin{tabular}{|l|} 
Intervention (n) \\
Comparator (n) \\
Duration/Intensity \\
Session Format \\
Setting \\
\end{tabular} & Population & $\begin{array}{l}\text { Primary Outcomes: } \\
\text { Pain, Function, and Opioid Use }\end{array}$ & $\begin{array}{l}\text { Secondary Outcomes: } \\
\text { HRQOL, Psychological Measures, Global } \\
\text { Improvement }\end{array}$ & $\begin{array}{l}\text { Harms } \\
\text { Utilization } \\
\text { Patient Satisfaction }\end{array}$ \\
\hline $\begin{array}{l}\text { Calner, } 2017 \\
\text { (Continued) }\end{array}$ & & & & 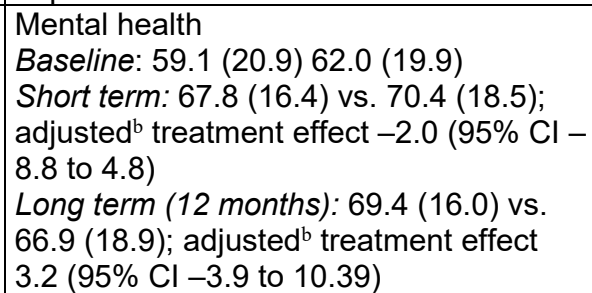 & \\
\hline
\end{tabular}

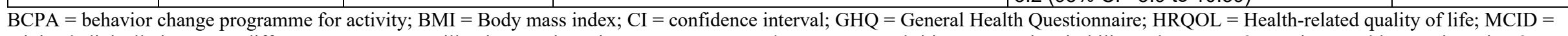

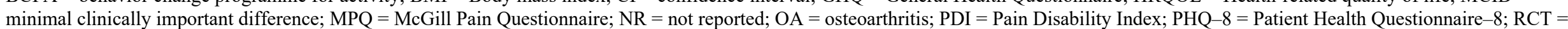

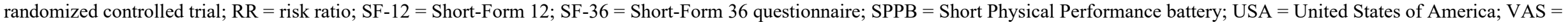
visual analog scale; WOMAC $=$ Western Ontario and McMaster Universities Osteoarthritis Index

${ }^{a}$ Data calculated by hierarchical linear mixed models and based on author imputation

${ }^{\mathrm{b}}$ Linear mixed model was adjusted for sex and interaction between time and group 
Table B-11. Summary results for trials addressing KQ2: CPMPs

\begin{tabular}{|c|c|c|c|c|c|}
\hline $\begin{array}{l}\text { Author, Year } \\
\text { Country } \\
\text { Pain Duration } \\
\text { Study Design } \\
\text { Study Quality } \\
\text { Comparison } \\
\end{array}$ & $\begin{array}{l}\text { Intervention ( } \mathrm{n}) \\
\text { Comparator (n) } \\
\text { Duration/Intensity } \\
\text { Session Format } \\
\text { Setting } \\
\end{array}$ & Population & $\begin{array}{l}\text { Primary Outcomes: } \\
\text { Pain, Function, and Opioid Use }\end{array}$ & $\begin{array}{l}\text { Secondary Outcomes: } \\
\text { HRQOL, Psychological Measures, } \\
\text { Global Improvement }\end{array}$ & $\begin{array}{l}\text { Harms } \\
\text { Utilization } \\
\text { Patient Satisfaction }\end{array}$ \\
\hline $\begin{array}{l}\text { Bendix 1995, 1997, } \\
\text { 1998a, 1998b } \\
\text { (PROJECT B) } \\
\text { Denmark } \\
\text { Duration of pain: } \geq 6 \\
\text { months (NOS) } \\
\text { RCT } \\
\text { Fair } \\
\text { Higher vs. Lower } \\
\text { Total Program } \\
\text { Hours }\end{array}$ & $\begin{array}{l}\text { A. CPMP - } 135 \text { hours } \\
\text { (n=46) } \\
3 \text { weeks full time, } 39 \\
\text { hours/week + } 1 \text { day }(6 \\
\text { hours) weekly for } 3 \text { (135 } \\
\text { hours total), group, } \\
\text { outpatient rheumatology } \\
\text { clinic } \\
\text { B. CPMP - } 24 \text { hours } \\
\text { (n=43) } \\
6 \text { weeks, } 4 \text { hours/week (24 } \\
\text { hours total), group, } \\
\text { outpatient rheumatology } \\
\text { clinic }\end{array}$ & $\begin{array}{l}\text { Mean age: } 42 \text { years } \\
\text { \% Male: } 25 \% \\
\text { Race/Ethnicity: NR } \\
\text { Pain etiology/type: } \\
\text { Chronic LBP } \\
\text { Disability: } 68 \% \text { (working } \\
\text { incapable) } \\
\text { Comorbidities: } \\
\text { Smoker: } 64 \% \\
\text { Prior back surgery: } 16 \%\end{array}$ & 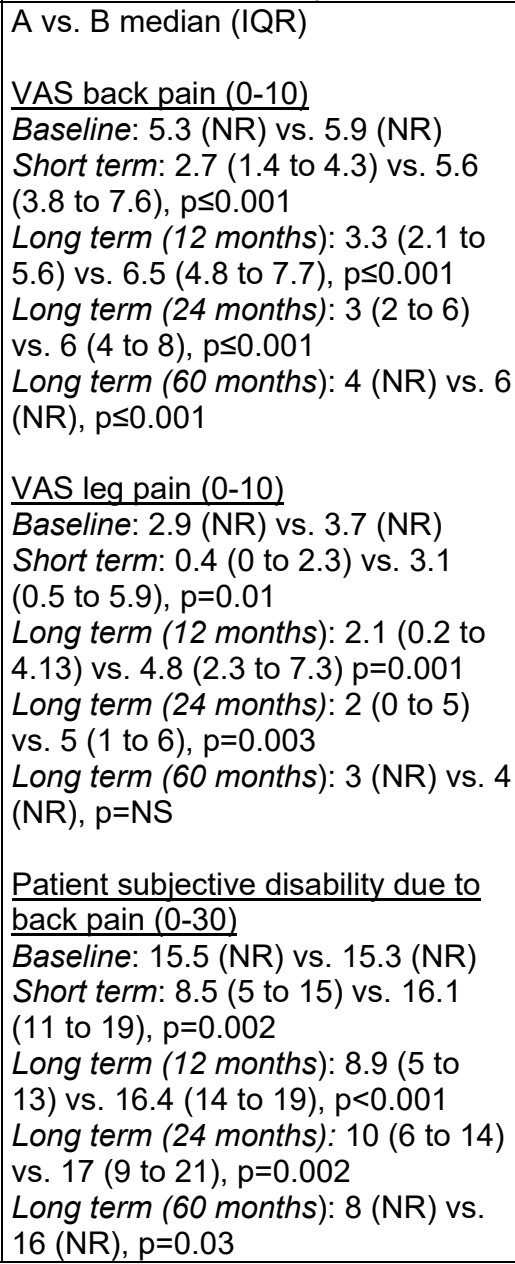 & $\begin{array}{l}\text { A vs. B, median (IQR) } \\
\text { Global improvement (1-5) } \\
\text { Long term (24 months): } 2 \text { (1 to } 3) \\
\text { vs. } 3 \text { ( } 2 \text { to } 3), p=0.003 \\
\text { Long term }(60 \text { months): } 2 \text { (NR) vs. } 3 \\
\text { (NR), } p=0.003\end{array}$ & 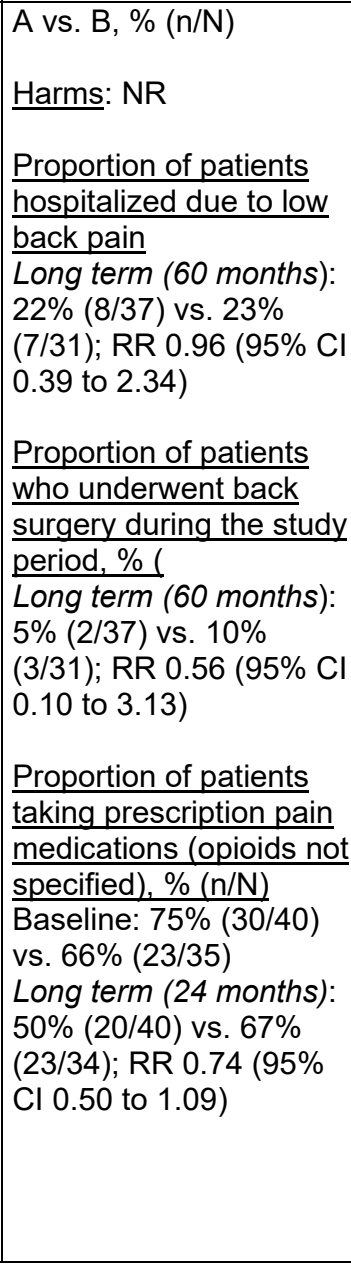 \\
\hline
\end{tabular}




\begin{tabular}{|c|c|c|c|c|c|}
\hline $\begin{array}{l}\text { Author, Year } \\
\text { Country } \\
\text { Pain Duration } \\
\text { Study Design } \\
\text { Study Quality } \\
\text { Comparison } \\
\end{array}$ & $\begin{array}{l}\text { Intervention (n) } \\
\text { Comparator (n) } \\
\text { Duration/Intensity } \\
\text { Session Format } \\
\text { Setting } \\
\end{array}$ & Population & $\begin{array}{l}\text { Primary Outcomes: } \\
\text { Pain, Function, and Opioid Use }\end{array}$ & $\begin{array}{l}\text { Secondary Outcomes: } \\
\text { HRQOL, Psychological Measures, } \\
\text { Global Improvement }\end{array}$ & \begin{tabular}{|l} 
Harms \\
Utilization \\
Patient Satisfaction \\
\end{tabular} \\
\hline $\begin{array}{l}\text { Rose } 1997 \text { (Part 2) }^{\mathrm{a}} \\
\text { UK } \\
\text { Mean duration of } \\
\text { pain: } 97 \text { months } \\
\text { RCT } \\
\text { Poor } \\
\text { Higher vs. Lower } \\
\text { Total Program } \\
\text { Hours }\end{array}$ & $\begin{array}{l}\text { A. CPMP - } 60 \text { hours: } 10 \\
\text { consecutive workdays, } 60 \\
\text { hours total, group, } \\
\text { outpatient } \\
\text { B. CPMP - } 30 \text { hours: } 5 \\
\text { consecutive full days, } 30 \\
\text { hours total, group, } \\
\text { outpatient } \\
\text { C. CPMP - } 15 \text { hours: } 5 \\
\text { consecutive half days, } 15 \\
\text { hours total, group, } \\
\text { outpatient }\end{array}$ & $\begin{array}{l}\text { Mean age: } 42 \text { years } \\
\text { \% Male: } 41 \% \\
\text { Race/Ethnicity: NR } \\
\text { Pain etiology/type: } \\
\text { Chronic LBP and/or } \\
\text { referred leg pain } \\
\text { Disability or sickness } \\
\text { benefit: } 64 \% \text { (completers } \\
\text { only) } \\
\text { Comorbidities: NR } \\
\text { Prior spinal surgery: } 13 \%\end{array}$ & $\begin{array}{l}\text { Mean (SDs NR) } \\
\text { VAS pain (0-10) } \\
\text { A vs. B } \\
\text { Baseline: } 5.0 \text { vs. } 6.0 \\
\text { Postintervention: } 5.0 \text { vs. } 4.3 \\
\text { Intermediate term: } 5.1 \text { vs. } 5.2 \\
\text { p=ns for all comparisons } \\
\text { A vs. C } \\
\text { Baseline: } 5.0 \text { vs. } 5.3 \\
\text { Postintervention: } 5.0 \text { vs. } 4.9 \\
\text { Intermediate term: } 5.1 \text { vs. } 4.3 \\
\text { p=ns for all comparisons } \\
\\
\text { RMDQ (0-24) } \\
\text { A vs. B } \\
\text { Baseline: } 10.7 \text { vs. } 14.0 \\
\text { Postintervention: } 8.4 \text { vs. } 9.9 \\
\text { Intermediate term: } 9.5 \text { vs. } 11.5 \\
\text { p=ns for all comparisons } \\
\text { A vs. C } \\
\text { Baseline: } 10.7 \text { vs. } 10.8 \\
\text { Postintervention: } 8.4 \text { vs. } 8.8 \\
\text { Intermediate term: } 9.5 \text { vs. } 10.0 \\
\text { p=ns for all comparisons }\end{array}$ & $\begin{array}{l}\text { Mean (SDs NR) } \\
\text { The Modified Zung Depression } \\
\text { Inventory (Scale NR) } \\
\text { A vs. B } \\
\text { Baseline: } 25.0 \text { vs. } 26.0 \\
\text { Postintervention: } 22.0 \text { vs. } 21.0 \\
\text { Intermediate term: } 21.1 \text { vs. } 22.0 \\
\text { p=ns for all comparisons } \\
\text { A vs. C } \\
\text { Baseline: } 25.0 \text { vs. } 24.0 \\
\text { Postintervention: } 22.0 \text { vs. } 21.1 \\
\text { Intermediate term: } 21.1 \text { vs. } 21.1 \\
\text { p=ns for all comparisons }\end{array}$ & NR \\
\hline $\begin{array}{l}\text { Saral } 2016 \\
\text { Turkey } \\
\text { Mean duration of } \\
\text { pain } 90 \text { months } \\
\text { RCT } \\
\text { Fair } \\
\text { Higher vs. Lower } \\
\text { Total Program } \\
\text { Hours }\end{array}$ & $\begin{array}{l}\text { A. CPMP - } 75 \text { hours } \\
\text { ( } \mathbf{n = 2 2 )} \\
10 \text { weeks, } 75 \text { hours total, } \\
\text { group, outpatient } \\
\text { B. CPMP - } 10 \text { hours } \\
\text { ( } \mathbf{n = 2 2 )} \\
2 \text { days, } \sim 10 \text { hours total, } \\
\text { group, outpatient }\end{array}$ & $\begin{array}{l}\text { Mean age: } 40 \text { years } \\
\text { \% Female: } 100 \% \\
\text { Race/ethnicity: NR } \\
\text { Pain etiology/type: } \\
\text { Fibromyalgia } \\
\text { Disability: NR } \\
\text { Comorbidities: NR } \\
\text { - Excluded: advanced } \\
\text { psychiatric diseases and } \\
\text { serious physical } \\
\text { comorbidities }\end{array}$ & $\begin{array}{l}\text { A vs. B, Mean (SD) } \\
\text { VAS pain (0 to } 10) \\
\text { Baseline: } 8.2(0.9) \text { vs. } 7.6(0.8) \\
\text { Intermediate term: } 5.1(2.4) \text { vs. } 5.8 \\
\text { (1.0), difference }-0.70(95 \% \mathrm{Cl}- \\
1.82 \text { to } 0.42) \\
\text { FIQ (0-100) } \\
\text { Baseline: } 71.6(14.2) \text { vs. } 67.7(12.0) \\
\text { Intermediate term: } 53.9(19.3) \text { vs. } \\
54.5(14.2), \text { difference }-0.03(95 \% \\
\mathrm{Cl}-0.66 \text { to } 0.59)\end{array}$ & $\begin{array}{l}\text { A vs. B, Mean (SD) } \\
\text { SF-36 PCS (0-100) } \\
\text { Baseline: } 32.8(7.9) \text { vs. } 36.5(8.7) \\
\text { Intermediate term: } 39.9(7.5) \text { vs. } \\
39.6(8.1), \text { difference } 0.30(95 \% \mathrm{Cl} \\
-4.69 \text { to } 5.29) \\
\\
\text { SF-36 MCS (0-100) } \\
\text { Baseline: } 30.4(11.7) \text { vs. } 33.2(8.9) \\
\text { Intermediate term: } 40.7(12.3) \text { vs. } \\
40.2(10.0), \text { difference } 0.50(95 \% \mathrm{Cl} \\
-6.72 \text { to } 7.72) \\
\text { BDI (0-63) } \\
\text { Baseline: } 23.4(11.0) \text { vs. } 20.7(6.6) \\
\text { Intermediate term: } 16.6(9.6) \text { vs. } \\
15.0(10.2), \text { difference } 0.16(95 \% \\
\mathrm{Cl}-0.46 \text { to } 0.78)\end{array}$ & $\begin{array}{l}\text { Harms: None reported; } \\
\text { Occasional mild } \\
\text { increases in pain after } \\
\text { some exercise sessions } \\
\text { in both groups A and B. }\end{array}$ \\
\hline
\end{tabular}




\begin{tabular}{|c|c|c|c|c|c|}
\hline $\begin{array}{l}\text { Author, Year } \\
\text { Country } \\
\text { Pain Duration } \\
\text { Study Design } \\
\text { Study Quality } \\
\text { Comparison }\end{array}$ & $\begin{array}{l}\text { Intervention (n) } \\
\text { Comparator (n) } \\
\text { Duration/Intensity } \\
\text { Session Format } \\
\text { Setting } \\
\end{array}$ & Population & $\begin{array}{l}\text { Primary Outcomes: } \\
\text { Pain, Function, and Opioid Use }\end{array}$ & $\begin{array}{l}\text { Secondary Outcomes: } \\
\text { HRQOL, Psychological Measures, } \\
\text { Global Improvement }\end{array}$ & $\begin{array}{l}\text { Harms } \\
\text { Utilization } \\
\text { Patient Satisfaction }\end{array}$ \\
\hline $\begin{array}{l}\text { Reneman, } 2020 \\
\text { The Netherlands } \\
\text { Mean duration of } \\
\text { pain: NR (>1 year = } \\
74 \% \text { ) } \\
\text { RCT } \\
\text { Fair } \\
\text { Higher vs. Lower } \\
\text { Total Program } \\
\text { Hours }\end{array}$ & $\begin{array}{l}\text { A. CPMP - Higher hours } \\
\text { (n=81) } \\
\text { Intended duration: } 12,16 \\
\text { or } 20 \text { weeks (4 weeks } 10 \\
\text { contact hours more than } \\
\text { Group B) } \\
\text { Actual duration: } \\
12 \text { or } 16 \text { weeks: } 96.3 \% \\
\text { (78/81) } \\
\text { Mean (SD) number of } \\
\text { weeks of treatment: } 11.7 \\
\text { (4.5) } \\
\text { Mean (SD) number of } \\
\text { contact hours with } \\
\text { providers: } 30.7(11.3) \\
\text { Outpatient, Individual } \\
\text { B. CPMP - Lower hours } \\
\text { (n=72) } \\
\text { Intended duration: } 8,12 \text { or } \\
16 \text { weeks (4 weeks } / 10 \\
\text { contact hours less than } \\
\text { Group A) } \\
8 \text { or } 12 \text { weeks: } 98.6 \% \\
(71 / 72) \\
\text { Mean (SD) number of } \\
\text { weeks of treatment: } 10.8 \\
\text { (3.9) } \\
\text { Mean (SD) number of } \\
\text { contact hours with } \\
\text { providers: } 29.8 \text { (10.4) } \\
\text { Outpatient, Individual } \\
\end{array}$ & $\begin{array}{l}\text { Mean age: } 44 \text { years } \\
\% \text { Male: } 53 \% \\
\text { Race/ethnicity: NR } \\
\text { Pain etiology/type: } \\
\text { Chronic MSK pain } \\
\text { Disability: } \\
\text { - Partial sick } \\
\text { leave/disability pension: } \\
37 \% \\
\text { - Full sick leave/disability } \\
\text { pension: } 13 \% \\
\text { Other characteristics: } \\
\text { - Excluded: comorbidities } \\
\text { such as heart failure, } \\
\text { rheumatoid arthritis, or } \\
\text { psychiatric disorders } \\
\text { preventing participation. }\end{array}$ & $\begin{array}{l}\text { A vs. B, Mean (SD) } \\
\text { EQ5D VAS pain (0-10) } \\
\text { Baseline: } 5.45(1.78) \text { vs. } 5.44(1.77) \\
\text { Post-treatment: } 6.75(1.61) \text { vs. } 6.76 \\
\text { (1.87), difference }-0.01(95 \% \mathrm{Cl} \text { - } \\
0.697 \text { to } 0.677) \\
\\
\text { PDI (0-70) } \\
\text { Baseline: } 36.1(12.5) \text { vs. } 37.9(14.2) \\
\text { Post-treatment: } 25.1(15.0) \text { vs. } 26.6 \\
(17.7) \text {, difference }-1.5(95 \% \mathrm{Cl}- \\
7.44 \text { to } 4.44)\end{array}$ & $\begin{array}{l}\text { EQ5D Index }(0-1) \\
\text { Baseline: } 0.56(0.19) \text { vs. } 0.54(0.21) \\
\text { Post-treatment: } 0.70(0.17) \text { vs. } 0.70 \\
(0.20) \text {, difference } 0.00(95 \% \mathrm{Cl}- \\
0.073 \text { to } 0.073)\end{array}$ & $\begin{array}{l}\text { Harms: No trial-related } \\
\text { adverse events were } \\
\text { reported. }\end{array}$ \\
\hline
\end{tabular}




\begin{tabular}{|c|c|c|c|c|c|}
\hline $\begin{array}{l}\text { Author, Year } \\
\text { Country } \\
\text { Pain Duration } \\
\text { Study Design } \\
\text { Study Quality } \\
\text { Comparison }\end{array}$ & $\begin{array}{l}\text { Intervention (n) } \\
\text { Comparator (n) } \\
\text { Duration/Intensity } \\
\text { Session Format } \\
\text { Setting }\end{array}$ & Population & $\begin{array}{l}\text { Primary Outcomes: } \\
\text { Pain, Function, and Opioid Use }\end{array}$ & $\begin{array}{l}\text { Secondary Outcomes: } \\
\text { HRQOL, Psychological Measures, } \\
\text { Global Improvement }\end{array}$ & $\begin{array}{l}\text { Harms } \\
\text { Utilization } \\
\text { Patient Satisfaction }\end{array}$ \\
\hline $\begin{array}{l}\text { Harkapaa, 1989, } \\
1990 \\
\text { Finland } \\
\text { Mean duration of } \\
\text { pain: } 173 \text { months } \\
\text { RCT } \\
\text { Poor } \\
\text { Inpatient vs. } \\
\text { Outpatient Setting }\end{array}$ & $\begin{array}{l}\text { A. CPMP - Inpatient } \\
\text { (n=156) } \\
\text { 2x/week for } 2 \text { months (15 } \\
\text { sessions) + 2-week } \\
\text { refresher sessions after } 1.5 \\
\text { years, group, inpatient } \\
\text { B. CPMP - Outpatient } \\
\text { (n=150) } \\
\text { 2x/week for } 2 \text { months (15 } \\
\text { sessions) + } 8 \text { refresher } \\
\text { sessions after } 1.5 \text { years, } \\
\text { group, outpatient }\end{array}$ & \begin{tabular}{|l} 
Mean age: 45 years \\
\% Male: $62 \%$ \\
Race/Ethnicity: NR \\
Pain etiology/type: \\
Chronic LBP \\
- Continuous LBP past \\
year: $41 \%$ \\
- Severe LBP past year: \\
$84 \%$ \\
Disability: NR \\
Comorbidities: NR \\
Other characteristics: \\
- Mean number of days of \\
absenteeism due to LBP \\
in past 2 years: 36 \\
- Use of analgesics \\
(opioids NR): $63 \%$
\end{tabular} & $\begin{array}{l}\text { A vs. B, Mean (SD) } \\
\text { Pain Index (0-400) } \\
\text { Baseline (mean, SD): } 184.9(76.9) \\
\text { vs. } 178.6(81.8)[4.6(1.9) \text { vs. } 4.5 \\
(2.0) \text { on a } 0-10 \text { scale] } \\
\text { Short term: } 128 \text { (NR) vs. } 146 \text { (NR) } \\
{[3.2(1.3) \text { vs. } 3.6 \text { (1.8) on a 0-10 }} \\
\text { scale] } \\
\text { Intermediate term: } 158 \text { (NR vs. } 160 \\
\text { (NR) [3.9 (NR) vs. } 4.0 \text { (NR) on a 0- } \\
10 \text { scale] } \\
\text { Long term (18 months): } 156.5(\mathrm{NR}) \\
\text { vs. } 174 \text { (NR) [3.9 (1.6) vs. } 4.3(2.2) \\
\text { on a 0-10 scale] } \\
\text { Long term (22 months): } 149 \text { (NR) } \\
\text { vs. } 164 \text { (NR) [3.7 (NR) vs. } 4.1 \text { (NR) } \\
\text { on a 0-10 scale] } \\
\text { Long term (30 months): } 161.5 \text { (NR) } \\
\text { vs. } 168 \text { (NR) [4.0 (1.7) vs. } 4.2(2.1) \\
\text { on a 0-10 scale] }\end{array}$ & NR & NR \\
\hline & & & $\begin{array}{l}\text { LBP Disability Index (0-45) } \\
\text { Baseline (mean SD): } 16.7 \text { (7.9) vs. } \\
17.6(7.4) \\
\text { Short term: } 13.6 \text { (9.7) vs. } 14.7(7.7) \\
\text { Intermediate term: } 15.7 \text { (NR) vs. } 16 \\
\text { (NR) } \\
\text { Long term (18 months): } 15.6(11.1) \\
\text { vs. } 17.1 \text { (9.0) } \\
\text { Long term (22 months): } 14.5 \text { (NR) } \\
\text { vs. } 15.65 \text { (NR) } \\
\text { Long term (30 months): } 15.4(11.0) \\
\text { vs. } 16.55 \text { (8.7) }\end{array}$ & & \\
\hline
\end{tabular}




\begin{tabular}{|c|c|c|c|c|c|}
\hline $\begin{array}{l}\text { Author, Year } \\
\text { Country } \\
\text { Pain Duration } \\
\text { Study Design } \\
\text { Study Quality } \\
\text { Comparison }\end{array}$ & $\begin{array}{l}\text { Intervention (n) } \\
\text { Comparator (n) } \\
\text { Duration/Intensity } \\
\text { Session Format } \\
\text { Setting }\end{array}$ & Population & $\begin{array}{l}\text { Primary Outcomes: } \\
\text { Pain, Function, and Opioid Use }\end{array}$ & $\begin{array}{l}\text { Secondary Outcomes: } \\
\text { HRQOL, Psychological Measures, } \\
\text { Global Improvement }\end{array}$ & $\begin{array}{l}\text { Harms } \\
\text { Utilization } \\
\text { Patient Satisfaction }\end{array}$ \\
\hline $\begin{array}{l}\text { Peters, 1990, } 1992 \\
\text { New Zealand } \\
\text { Mean duration of } \\
\text { pain: NR (6 to } 48 \\
\text { months, } 49 \% \text {; } \\
48 \text { to } \geq 240 \text { months, } \\
51 \% \text { ) } \\
\text { RCT } \\
\text { Poor } \\
\text { Inpatient vs. } \\
\text { Outpatient Setting }\end{array}$ & $\begin{array}{l}\text { A. CPMP - Inpatient } \\
\text { Setting ( } \mathrm{n}=23 \text { ) } \\
4 \text { weeks, intensity NR, } \\
\text { group, inpatient } \\
\\
\text { B. CPMP - Outpatient } \\
\text { Setting (n=29) } \\
9 \text { weeks, } 2 \text { hours/week (18 } \\
\text { hours total), outpatient }\end{array}$ & $\begin{array}{l}\frac{\text { Mean age: } 44 \text { years }}{\% \text { Male: } 38 \%} \\
\text { Race/Ethnicity: } \\
\text { - European: } 92 \% \\
\text { - Maori: } 6 \% \\
\text { - Polynesian: } 2 \% \\
\text { Pain etiology/type (patient } \\
\text { could have } \geq 1 \text { pain type): } \\
\text { - Back pain: } 44 \% \\
\text { - Head pain: } 31 \% \\
\text { - Arm pain: } 27 \% \\
\text { - Leg pain: } 19 \% \\
\text { - Chest pain: } 13 \% \\
\text { - Abdomen pain: } 8 \% \\
\text { Disability: NR } \\
\text { Comorbidities: NR } \\
\text { - Excluded: Psychotic } \\
\text { illness }\end{array}$ & 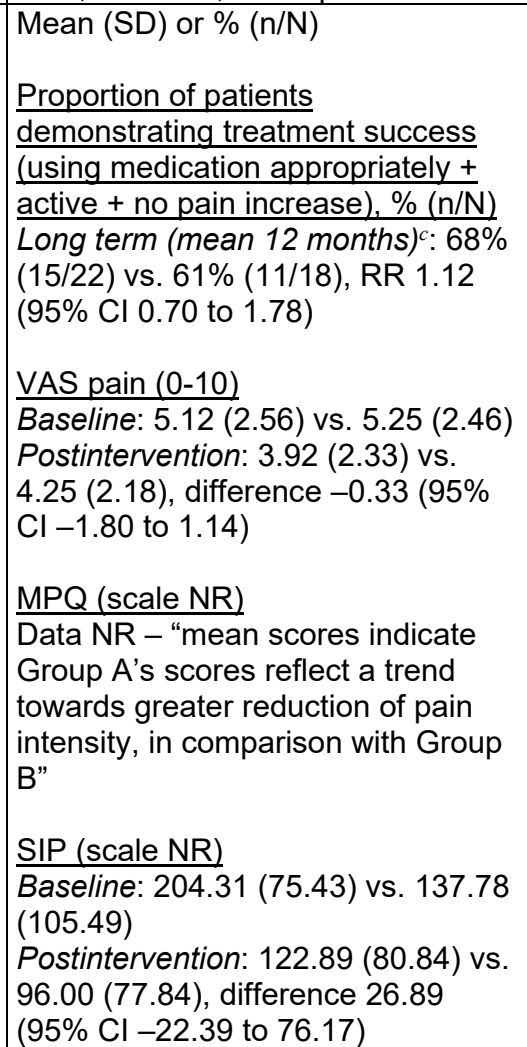 & $\begin{array}{l}\text { Mean (SD) } \\
\text { BDI } \\
\text { Baseline: } 19.18(9.34) \text { vs. } 13.55 \\
(6.03) \\
\text { Postintervention: } 12.25(15.64) \text { vs. } \\
10.73(6.16) \text {, difference } 1.52(95 \% \\
\mathrm{Cl}-5.59 \text { to } 8.63) \\
\\
\text { General Health Questionnaire } \\
\text { Baseline: } 15.52(8.58) \text { vs. } 8.67 \\
(7.23) \\
\text { Postintervention: } 5.96(7.11) \text { vs. } \\
5.91(6.42), \text { difference } 0.05(95 \% \mathrm{Cl} \\
-4.09 \text { to } 4.19)\end{array}$ & NR \\
\hline
\end{tabular}




\begin{tabular}{|c|c|c|c|c|c|}
\hline $\begin{array}{l}\text { Author, Year } \\
\text { Country } \\
\text { Pain Duration } \\
\text { Study Design } \\
\text { Study Quality } \\
\text { Comparison }\end{array}$ & $\begin{array}{l}\text { Intervention }(n) \\
\text { Comparator }(n) \\
\text { Duration/Intensity } \\
\text { Session Format } \\
\text { Setting }\end{array}$ & Population & $\begin{array}{l}\text { Primary Outcomes: } \\
\text { Pain, Function, and Opioid Use }\end{array}$ & $\begin{array}{l}\text { Secondary Outcomes: } \\
\text { HRQOL, Psychological Measures, } \\
\text { Global Improvement }\end{array}$ & $\begin{array}{l}\text { Harms } \\
\text { Utilization } \\
\text { Patient Satisfaction }\end{array}$ \\
\hline $\begin{array}{l}\text { Peters, 1990, } 1992 \\
\text { (Continued) }\end{array}$ & & & $\begin{array}{l}\text { Proportion of patients taking an } \\
\text { opioid, \% (n/N) } \\
\text { Baseline: } \\
\text { Any opioid: } 31.8 \%(7 / 22) \text { vs. } 33 \% \\
\text { (6/18), RR } 0.95(95 \% \mathrm{Cl} 0.39 \text { to } \\
\text { 2.34) } \\
\text { - strong opioid: } 9.1 \%(2 / 22) \text { vs. } 17 \% \\
\text { (3/18), RR } 0.55(95 \% \mathrm{Cl} 0.10 \text { to } \\
\text { 2.92) } \\
\text { - mild opioid: } 22.7 \%(5 / 22) \text { vs. } 17 \% \\
\text { (3/18), RR } 1.36(95 \% \mathrm{Cl} 0.38 \text { to } \\
4.95) \\
\text { Long term (mean } 12 \text { months): } \\
\text { Any opioid: } 13.6 \%(3 / 22) \text { vs. } 22 \% \\
\text { (4/18), RR } 0.61(95 \% \mathrm{Cl} 0.16 \text { to } \\
\text { 2.39) } \\
\text { - strong opioid: } 0 \%(0 / 22) \text { vs. } 6 \% \\
\text { (1/18), RR NC } \\
\text { - mild opioid: } 13.6 \%(3 / 22) \text { vs. } 17 \% \\
\text { (3/18), } 0.82(95 \% \mathrm{Cl} 0.19 \text { to } 3.57)\end{array}$ & & \\
\hline
\end{tabular}




\begin{tabular}{|c|c|c|c|c|c|}
\hline $\begin{array}{l}\text { Author, Year } \\
\text { Country } \\
\text { Pain Duration } \\
\text { Study Design } \\
\text { Study Quality } \\
\text { Comparison }\end{array}$ & $\begin{array}{l}\text { Intervention (n) } \\
\text { Comparator (n) } \\
\text { Duration/Intensity } \\
\text { Session Format } \\
\text { Setting }\end{array}$ & Population & $\begin{array}{l}\text { Primary Outcomes: } \\
\text { Pain, Function, and Opioid Use }\end{array}$ & $\begin{array}{l}\text { Secondary Outcomes: } \\
\text { HRQOL, Psychological Measures, } \\
\text { Global Improvement }\end{array}$ & $\begin{array}{l}\text { Harms } \\
\text { Utilization } \\
\text { Patient Satisfaction }\end{array}$ \\
\hline $\begin{array}{l}\text { Ronzi, } 2017 \\
\text { France } \\
\text { Mean duration of } \\
\text { pain: NR (>5 years: } \\
60 \% \text { ) } \\
\text { RCT } \\
\text { Poor } \\
\text { Inpatient vs. } \\
\text { Outpatient Setting }\end{array}$ & $\begin{array}{l}\text { A. CPMP - Inpatient } \\
\text { setting ( } \mathrm{n}=49) \\
5 \text { weeks, } 30 \text { hours/week } \\
\text { (150 hours total), combo, } \\
\text { inpatient } \\
\text { B. CPMP - Outpatient } \\
\text { setting ( } \mathrm{n}=56) \\
5 \text { weeks, } 11 \text { hours/week } \\
\text { ( } 55 \text { hours total), combo, } \\
\text { outpatient }\end{array}$ & \begin{tabular}{|l|} 
Median age: 40 years \\
\% Male: $59 \%$ \\
Race/ethnicity: NR \\
Pain etiology/type: \\
Chronic LBP ( $\geq 3$ months) \\
Disability: NR \\
Sick leave: "almost all \\
patients on sick leave"; \\
median days in past year: \\
233 \\
Comorbidities: \\
- history of depression: \\
$<33 \%$ \\
Prior spine surgery: $<33 \%$
\end{tabular} & $\begin{array}{l}\text { A vs. B } \\
\text { VAS pain (0-10) } \\
\text { Baseline (median, IQR): } 5.4(4.5 \text { to } \\
6.5) \text { vs. } 5.5(3.6 \text { to } 6.8) \\
\text { Long term (mean, } S D): 4.5(2.5) \text { vs. } \\
3.7(3.4) \text {, difference } 0.80(95 \% \mathrm{Cl}- \\
0.48 \text { to } 2.08) \\
\\
\text { DPQ daily activity (\%; lower = lower } \\
\text { impact of pain on QOL) } \\
\text { Baseline (median, IQR): } 63.0 \% \\
(51.0 \% \text { to } 72.0 \%) \text { vs. } 57.0 \%(48.0 \% \\
\text { to } 66.0 \%) \\
\text { Long term (mean, SD): } 51.0 \% \\
(38.9 \%) \text { vs. } 39.0 \%(24.5 \%) ; \\
\text { difference } 0.36 \%(95 \% \mathrm{Cl}-0.06 \% \\
\text { to } 0.79 \%)\end{array}$ & $\begin{array}{l}\text { A vs. B, Median (IQR) } \\
\text { SF-36 PCS }(0-100) \\
\text { Baseline: } 35.7(29.4 \text { to } 39.5) \text { vs. } \\
34.5(30.7 \text { to } 39.2) \\
\text { Long term: } 39.1(33.8 \text { to } 50.4) \text { vs. } \\
41.6(34.2 \text { to } 49.9) \\
p=N S \text { for all } \\
\text { SF-36 MCS }(0-100) \\
\text { Baseline: } 43.3(32.1 \text { to } 49.8) \text { vs. } \\
43.4(35.9 \text { to } 51.1) \\
\text { Long term: } 48.3(42.1 \text { to } 53.4) \text { vs. } \\
46.6(38.7 \text { to } 56.6) \\
p=N S \text { for all } \\
\text { HADS (0-100) } \\
\text { Baseline: } 17.0(12.0 \text { to } 21.0) \text { vs. } \\
14.0(11.0 \text { to } 18.0) \\
\text { Long term: } 11.5(7.5 \text { to } 18.0) \text { vs. } \\
12.0(7.0 \text { to } 15.0) \\
p=N S \text { for all } \\
\text { DPQ anxiety and depression (\%; } \\
\text { lower = lower impact of pain on } \\
\text { QOL) } \\
\text { Baseline: } 45.0 \%(20.0 \% \text { to } 60.0 \%) \\
\text { vs. } 35.0 \%(25.0 \% \text { to } 45.0 \%) \\
\text { Long term: } 30.0 \%(5.0 \% \text { to } 45.0 \%) \\
\text { vs. } 25.0 \%(5.0 \% \text { to } 45.0 \%) \\
p=N S \text { for all }\end{array}$ & Harms: None reported \\
\hline
\end{tabular}




\begin{tabular}{|c|c|c|c|c|c|}
\hline $\begin{array}{l}\text { Author, Year } \\
\text { Country } \\
\text { Pain Duration } \\
\text { Study Design } \\
\text { Study Quality } \\
\text { Comparison }\end{array}$ & $\begin{array}{l}\text { Intervention }(\mathrm{n}) \\
\text { Comparator }(\mathrm{n}) \\
\text { Duration/Intensity } \\
\text { Session Format } \\
\text { Setting }\end{array}$ & Population & $\begin{array}{l}\text { Primary Outcomes: } \\
\text { Pain, Function, and Opioid Use }\end{array}$ & $\begin{array}{l}\text { Secondary Outcomes: } \\
\text { HRQOL, Psychological Measures, } \\
\text { Global Improvement }\end{array}$ & $\begin{array}{l}\text { Harms } \\
\text { Utilization } \\
\text { Patient Satisfaction }\end{array}$ \\
\hline $\begin{array}{l}\text { Williams, } 1996 \\
\text { UK } \\
\text { Mean duration of } \\
\text { pain: } 94 \text { months } \\
\text { RCT } \\
\text { Poor } \\
\text { Inpatient vs. } \\
\text { Outpatient Setting }\end{array}$ & 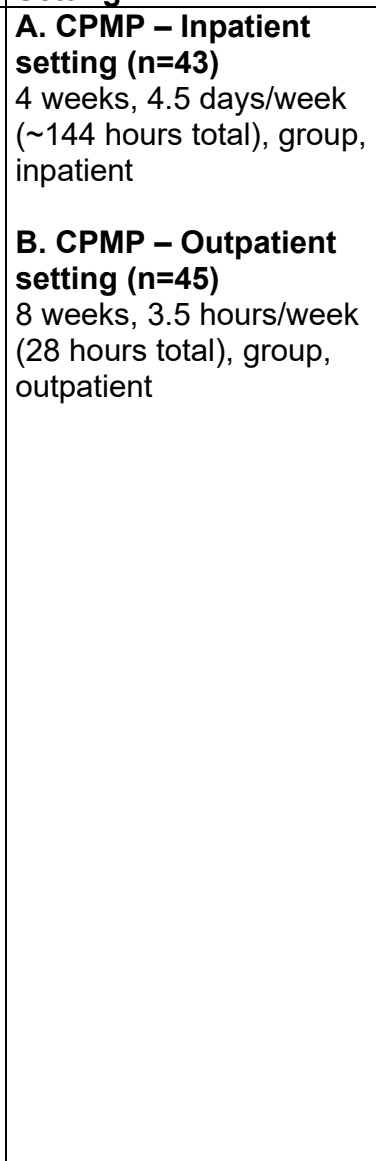 & $\begin{array}{l}\text { Mean age: } 50 \text { years } \\
\text { \% Male: } 49 \% \\
\text { Race/ethnicity: white, } \\
\text { 85\% } \\
\text { Pain etiology/type: } \\
\text { - back/neck/legs: } 76 \% \\
\text { - central/peripheral nerve } \\
\text { system damage: } 25 \% \\
\text { - other tissue damage: } \\
13 \% \\
\text { - unknown mechanism: } \\
61 \% \\
\text { Disability: } 60 \% \\
\text { Opioid use: } 65 \% \\
\text { Excess drug use: } 58 \% \\
\geq 1 \text { prior surgery: } 40 \%\end{array}$ & 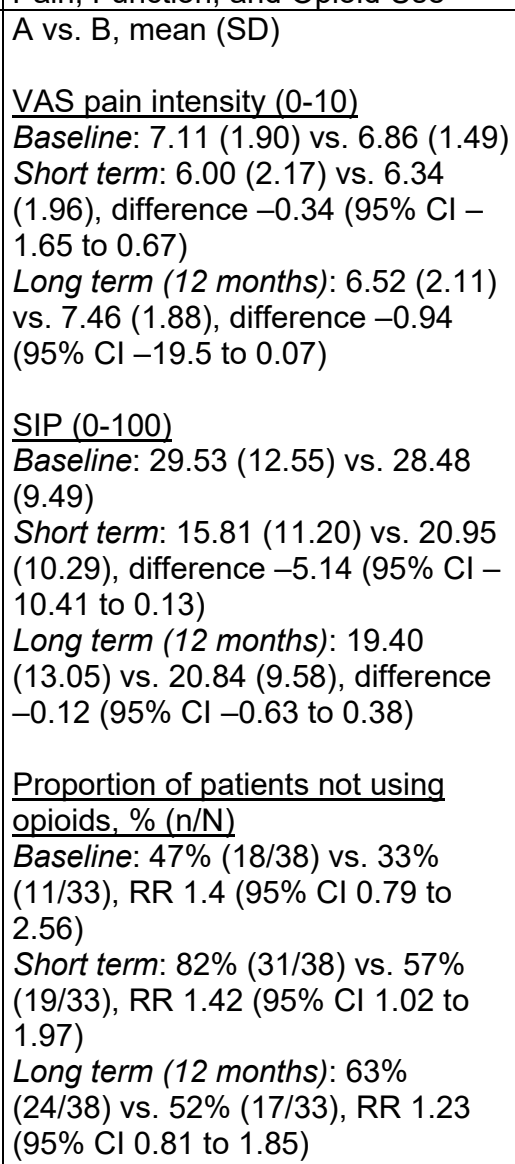 & 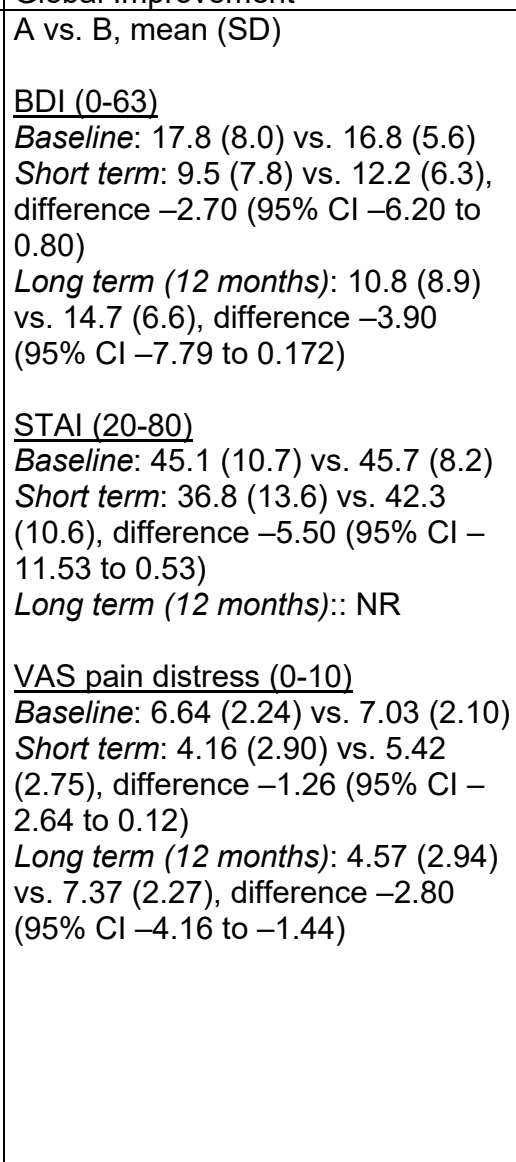 & $\begin{array}{l}\text { A vs. B } \\
\text { Harms: NR } \\
\text { Subsequent treatments } \\
\text { - Surgery: } 0 \% \text { vs. } 0 \% \\
\text { - Pain-relieving } \\
\text { procedures } \\
\text { (acupuncture, TENS, } \\
\text { nerve blocks): } 10.3 \% \\
\text { (3/29) vs. } 60.7 \% \\
(17 / 28), \text { RR } 0.17(95 \% \\
\text { Cl } 0.06 \text { to } 0.52)\end{array}$ \\
\hline
\end{tabular}




\begin{tabular}{|c|c|c|c|c|c|}
\hline $\begin{array}{l}\text { Author, Year } \\
\text { Country } \\
\text { Pain Duration } \\
\text { Study Design } \\
\text { Study Quality } \\
\text { Comparison }\end{array}$ & $\begin{array}{l}\text { Intervention ( } \mathrm{n}) \\
\text { Comparator (n) } \\
\text { Duration/Intensity } \\
\text { Session Format } \\
\text { Setting } \\
\end{array}$ & Population & $\begin{array}{l}\text { Primary Outcomes: } \\
\text { Pain, Function, and Opioid Use }\end{array}$ & $\begin{array}{l}\text { Secondary Outcomes: } \\
\text { HRQOL, Psychological Measures, } \\
\text { Global Improvement }\end{array}$ & $\begin{array}{l}\text { Harms } \\
\text { Utilization } \\
\text { Patient Satisfaction } \\
\end{array}$ \\
\hline $\begin{array}{l}\text { Williams, } 1996 \\
\text { (Continued) }\end{array}$ & & & $\begin{array}{l}\text { Proportion of patients taking an } \\
\text { opioid dose equivalent to }>10 \mathrm{mg} \\
\text { morphine per day } \\
\text { Baseline: } 34.2 \%(13 / 38) \text { vs. } 48.5 \% \\
(16 / 33), \text { RR } 0.71(95 \% \text { Cl } 0.40 \text { to } \\
1.24) \\
\text { Short term: } 10.5 \%(4 / 38) \text { vs. } 33.3 \% \\
(11 / 33), \text { RR } 0.32(95 \% \text { Cl } 0.11 \text { to } \\
0.90) \\
\text { Long term (12 months): } 10.5 \% \\
(4 / 38) \text { vs. } 18.2 \%(6 / 33), \text { RR } 0.58 \\
(95 \% \text { Cl } 0.18 \text { to } 1.88) \\
\text { Mean opioid dose per day (mg } \\
\text { morphine equivalents) } \\
\text { Baseline: } 30 \text { mg vs. } 22 \text { mg } \\
\text { Short term: NR } \\
\text { Long term (12 months): } 22 \mathrm{mg} \\
\text { vs.15 mg, p=NS }\end{array}$ & & \\
\hline $\begin{array}{l}\text { Rose } 1997(\text { Part 1) } \\
\text { UK } \\
\text { Mean duration of } \\
\text { pain } 97 \text { months } \\
\text { RCT } \\
\text { Poor } \\
\text { Group vs. } \\
\text { Individual Session } \\
\text { Format }\end{array}$ & $\begin{array}{l}\text { A. CPMP - Group format } \\
\text { (n=26) } \\
\text { Duration and intensity NR, } \\
\text { group, outpatient } \\
\\
\text { B. CPMP - Individual } \\
\text { format }(\mathbf{n}=\mathbf{2 4 )} \\
\text { Duration and intensity NR, } \\
\text { individual, outpatient }\end{array}$ & $\begin{array}{l}\text { Mean age: } 42 \text { years } \\
\text { \% Male: } 41 \% \\
\text { Race/Ethnicity: NR } \\
\text { Pain etiology/type: } \\
\text { Chronic LBP (and/or } \\
\text { referred leg pain) } \\
\text { Disability or sickness } \\
\text { benefit: } 64 \% \text { (completers } \\
\text { only) } \\
\text { Comorbidities: NR } \\
\text { Prior spinal surgery: } 13 \% \\
\text { Unsuccessful PT: } 91 \%\end{array}$ & $\begin{array}{l}\text { A vs. B, Mean (SDs NR) } \\
\text { VAS-pain } \\
\text { Baseline: } 6.6 \text { vs. } 6.0 \\
\text { Postintervention: } 5.8 \text { vs. } 4.7 \\
\text { Intermediate term: } 6.5 \text { vs. } 6.0 \\
\text { p=NS for all } \\
\text { RDQ (0-24) } \\
\text { Baseline: } 15.8 \text { vs. } 17.0 \\
\text { Postintervention: } 13.3 \text { vs. } 11.1 \\
\text { Intermediate term: } 13.3 \text { vs. } 11.1 \\
\text { p=NS for all }\end{array}$ & $\begin{array}{l}\text { A vs. B, Mean (SDs NR) } \\
\text { The Modified Zung Depression } \\
\text { Inventory (scale NR) } \\
\text { Baseline: } 33.1 \text { vs. } 32.0 \\
\text { Postintervention: } 27.0 \text { vs. } 27.0 \\
\text { Intermediate term: } 28.0 \text { vs. } 26.1 \\
\text { p=NS for all }\end{array}$ & NR \\
\hline
\end{tabular}




\begin{tabular}{|c|c|c|c|c|c|}
\hline $\begin{array}{l}\text { Author, Year } \\
\text { Country } \\
\text { Pain Duration } \\
\text { Study Design } \\
\text { Study Quality } \\
\text { Comparison } \\
\end{array}$ & $\begin{array}{l}\text { Intervention (n) } \\
\text { Comparator ( }(\mathbf{n}) \\
\text { Duration/Intensity } \\
\text { Session Format } \\
\text { Setting } \\
\end{array}$ & Population & $\begin{array}{l}\text { Primary Outcomes: } \\
\text { Pain, Function, and Opioid Use }\end{array}$ & $\begin{array}{l}\text { Secondary Outcomes: } \\
\text { HRQOL, Psychological Measures, } \\
\text { Global Improvement }\end{array}$ & $\begin{array}{l}\text { Harms } \\
\text { Utilization } \\
\text { Patient Satisfaction }\end{array}$ \\
\hline $\begin{array}{l}\text { Abbasi } 2012 \\
\text { Iran } \\
\text { Mean duration of } \\
\text { pain: } 74 \text { months } \\
\text { RCT } \\
\text { Poor } \\
\text { CPMP + additional } \\
\text { components vs. } \\
\text { standard CPMP }\end{array}$ & $\begin{array}{l}\text { A. CPMP - Spouse- } \\
\text { assisted ( } n=9 \text { ) } \\
7 \text { weeks, } 2 \text { hours/week (14 } \\
\text { hours total), combo } \\
\text { (primarily group, individual } \\
\text { if needed), outpatient } \\
\\
\text { B. CPMP - Conventional, } \\
\text { patient-oriented ( } n=10 \text { ) } \\
7 \text { weeks, } 2 \text { hours/week (14 } \\
\text { hours total), combo } \\
\text { (primarily group, individual } \\
\text { if needed), outpatient }\end{array}$ & $\begin{array}{l}\text { Mean age: } 45 \text { years } \\
\text { \% Male: } 12 \% \\
\text { Race/Ethnicity: NR } \\
\text { Pain etiology/type: } \\
\text { Chronic LBP } \\
\text { Disability: NR } \\
\text { Comorbidities: } \\
\text { - Excluded: major } \\
\text { cognitive dysfunction or } \\
\text { coexisting psychiatric } \\
\text { morbidity }\end{array}$ & $\begin{array}{l}\text { A vs. B, Mean (SD) } \\
\text { VAS pain in last week (0-10) } \\
\text { Baseline: } 5(2.7) \text { vs. } 4.6(2) \\
\text { Postintervention: } 3(1.8) \text { vs. } 2.6(2) \text {, } \\
\text { difference } 0.40 \text { (95\% Cl -1.45 to } \\
2.25) \\
\text { Long term: } 2.8(2.7) \text { vs. } 3.7(2.5), \\
\text { difference }-0.90(95 \% \mathrm{Cl}-3.42 \text { to } \\
1.62) \\
\text { RDQ (0-24) } \\
\text { Baseline: } 11.2(4.3) \text { vs. } 12.1(5.7) \\
\text { Postintervention: } 5.8(3) \text { vs. } 6.2 \\
(4.4), \text { difference }-0.40(95 \% \mathrm{Cl}- \\
4.10 \text { to } 3.30) \\
\text { Long term: } 8.2(5.4) \text { vs. } 8.8(5.9), \\
\text { difference }-0.60(95 \% \mathrm{Cl}-6.01 \text { to } \\
4.90)\end{array}$ & NR & NR \\
\hline
\end{tabular}




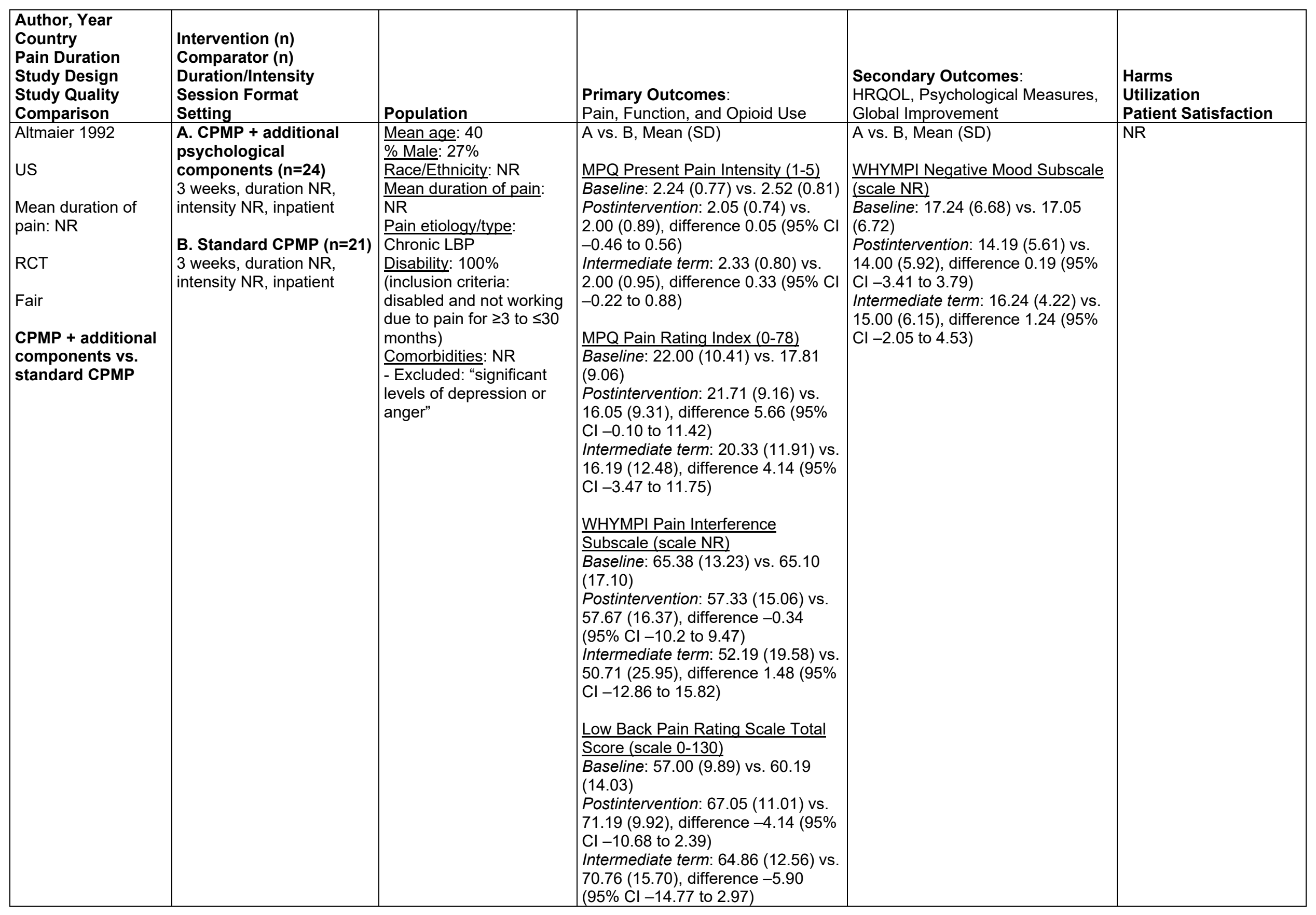




\begin{tabular}{|c|c|c|c|c|c|}
\hline $\begin{array}{l}\text { Author, Year } \\
\text { Country } \\
\text { Pain Duration } \\
\text { Study Design } \\
\text { Study Quality } \\
\text { Comparison }\end{array}$ & $\begin{array}{l}\text { Intervention (n) } \\
\text { Comparator (n) } \\
\text { Duration/Intensity } \\
\text { Session Format } \\
\text { Setting }\end{array}$ & Population & $\begin{array}{l}\text { Primary Outcomes: } \\
\text { Pain, Function, and Opioid Use }\end{array}$ & $\begin{array}{l}\text { Secondary Outcomes: } \\
\text { HRQOL, Psychological Measures, } \\
\text { Global Improvement }\end{array}$ & $\begin{array}{l}\text { Harms } \\
\text { Utilization } \\
\text { Patient Satisfaction }\end{array}$ \\
\hline $\begin{array}{l}\text { Van der Maas } 2015 \\
\text { The Netherlands } \\
\text { Mean duration of } \\
\text { pain: NR ( } \geq 2 \text { years: } \\
75 \% \text { ) } \\
\text { RCT } \\
\text { Fair } \\
\text { CPMP + additional } \\
\text { components vs. } \\
\text { standard CPMP }\end{array}$ & $\begin{array}{l}\text { A. CPMP + Psychomotor } \\
\text { Therapy ( } \mathbf{n = 4 9 )} \\
3 \text { days a week for } 12 \\
\text { weeks, } 104 \text { hours total, } \\
\text { group, outpatient } \\
\\
\text { B. Standard CPMP }(\mathbf{n}=\mathbf{4 5}) \\
3 \text { days a week for } 12 \\
\text { weeks, } 94 \text { hours total, } \\
\text { group, outpatient }\end{array}$ & $\begin{array}{l}\text { Mean age: } 42 \text { years } \\
\% \text { Male: } 14 \% \\
\text { Race/ethnicity: NR } \\
\text { Pain etiology/type: } \\
\text { Chronic MSK pain ( } \geq 3 \\
\text { months) } \\
\text { Disability: NR } \\
\text { Comorbidities: NR } \\
\text { - Excluded: psychiatric } \\
\text { diagnosis which could } \\
\text { interfere with treatment }\end{array}$ & $\begin{array}{l}\text { A vs. B, Mean (SD) } \\
\text { PDI disability (0-70) } \\
\text { Baseline: } 40.00(8) \text { vs. } 40.34(11) \\
\text { Postintervention: } 33.26(7) \text { vs. } \\
36.63(11) \text {, difference }-3.37 \text { ( } 95 \% \\
\mathrm{Cl}-7.12 \text { to } 0.38) \\
\text { Short term: } 31.33(11) \text { vs. } 35.46 \\
\text { (12), difference }-4.13(95 \% \mathrm{Cl}- \\
8.84 \text { to } 0.58) \\
\text { Intermediate term: } 31.82(12) \text { vs. } \\
33.71(14) \text {, difference }-1.89(95 \% \\
\text { Cl }-7.22 \text { to } 3.44) \\
\text { Long term: } 32.10(12) \text { vs. } 32.40 \\
\text { (15), difference }-0.30(95 \% \mathrm{Cl}- \\
5.84 \text { to } 5.24)\end{array}$ & 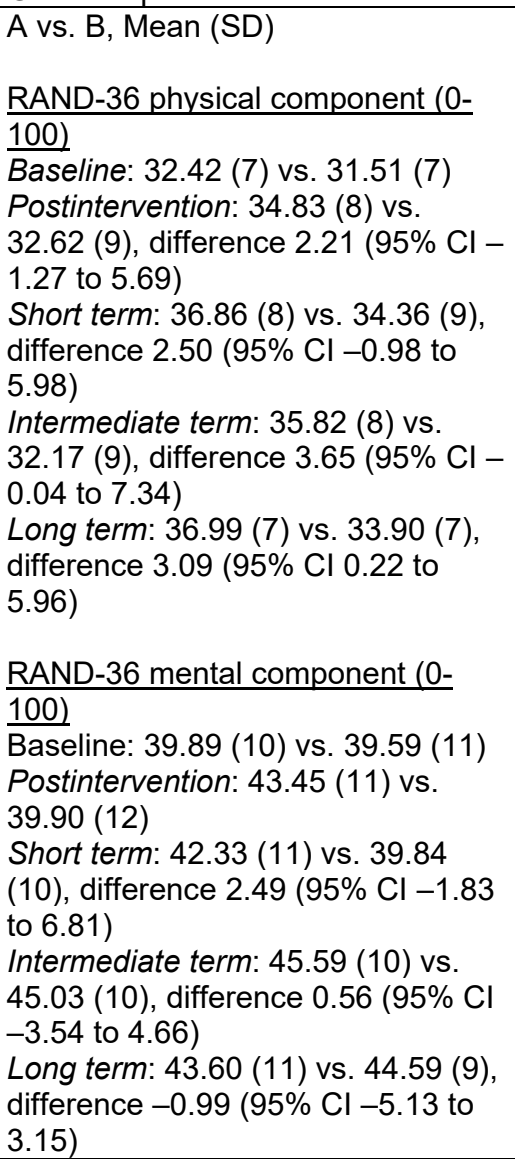 & NR \\
\hline
\end{tabular}




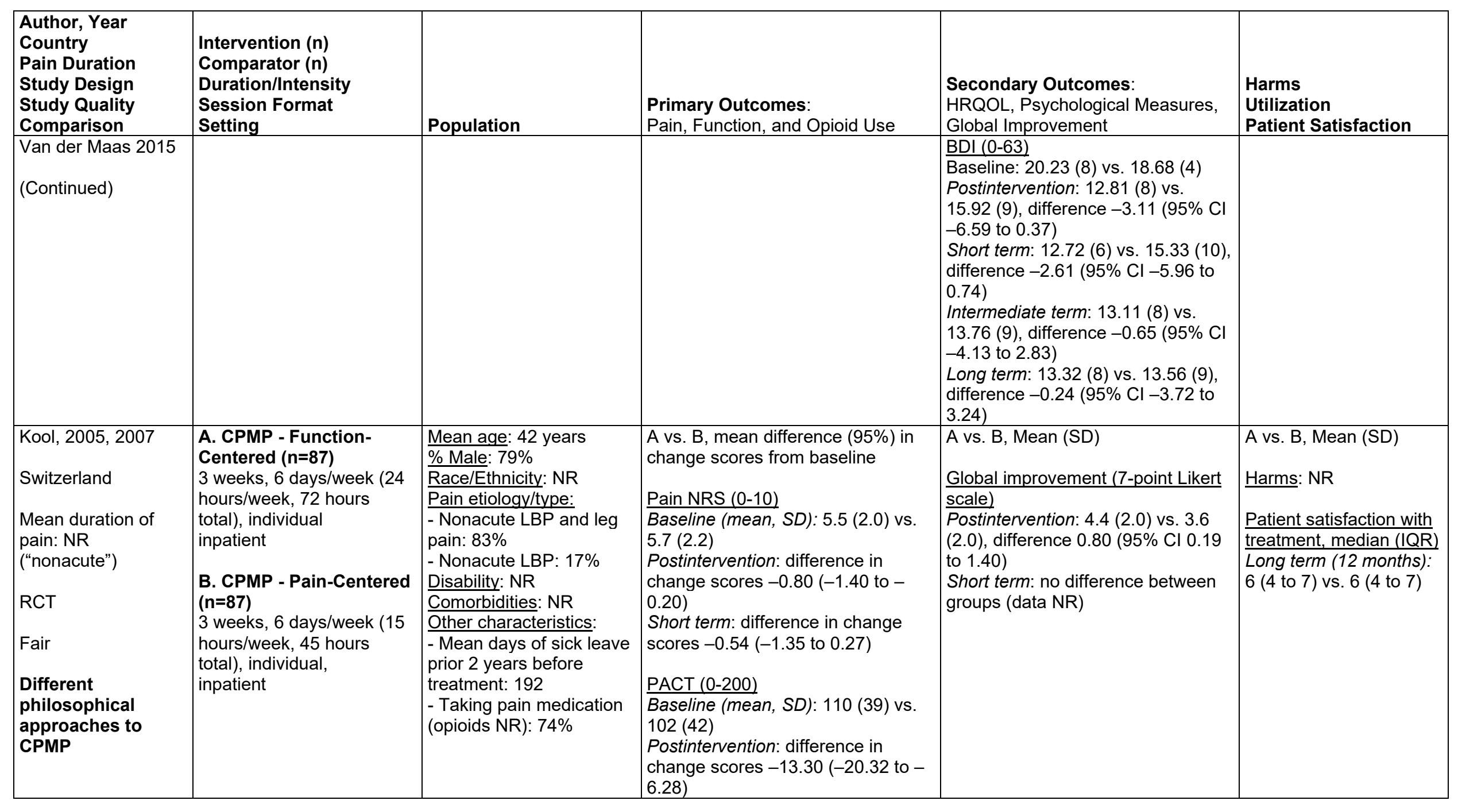




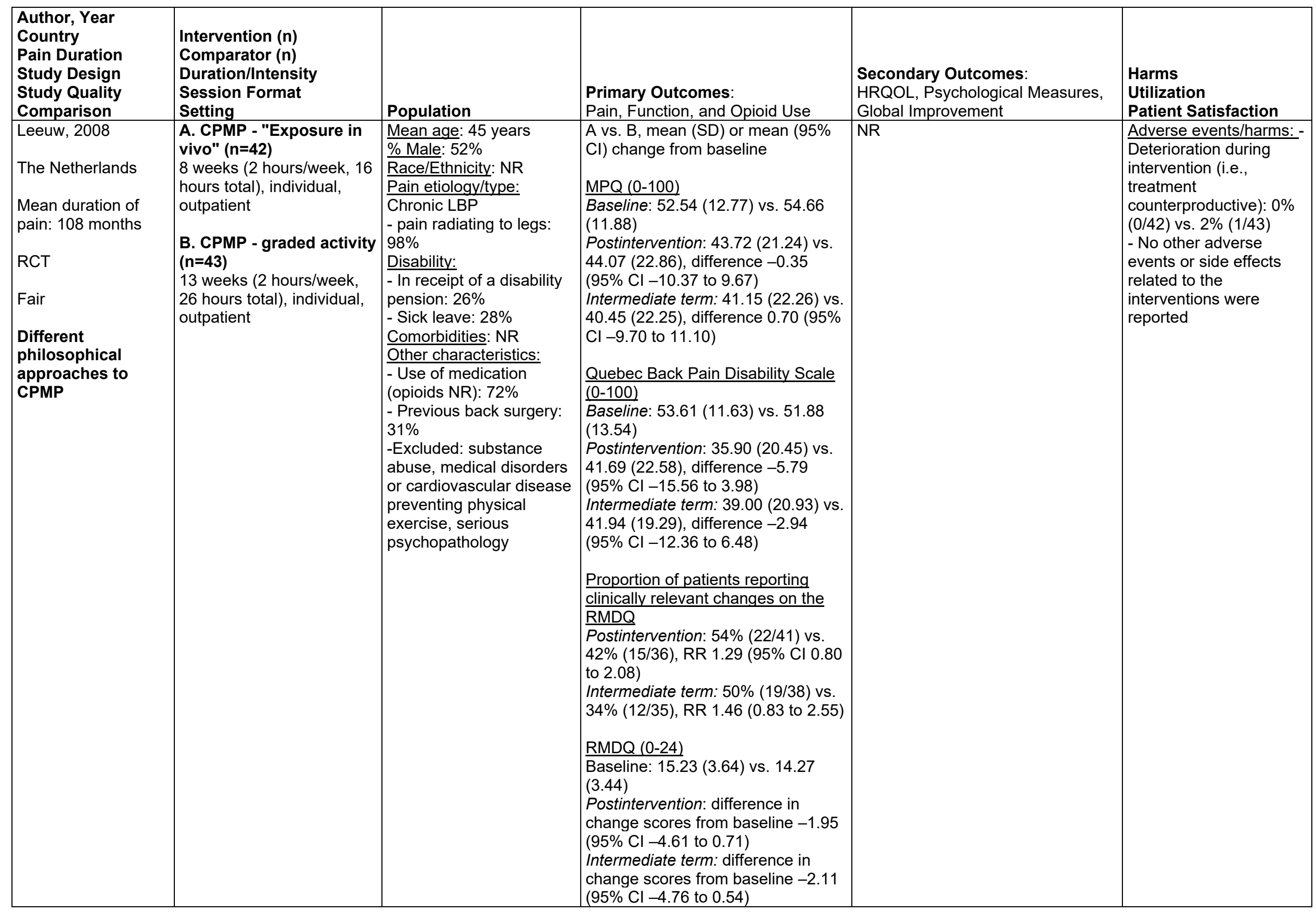




\begin{tabular}{|c|c|c|c|c|c|}
\hline $\begin{array}{l}\text { Author, Year } \\
\text { Country } \\
\text { Pain Duration } \\
\text { Study Design } \\
\text { Study Quality } \\
\text { Comparison }\end{array}$ & $\begin{array}{l}\text { Intervention (n) } \\
\text { Comparator ( }(\mathrm{n}) \\
\text { Duration/Intensity } \\
\text { Session Format } \\
\text { Setting }\end{array}$ & Population & $\begin{array}{l}\text { Primary Outcomes: } \\
\text { Pain, Function, and Opioid Use }\end{array}$ & $\begin{array}{l}\text { Secondary Outcomes: } \\
\text { HRQOL, Psychological Measures, } \\
\text { Global Improvement }\end{array}$ & $\begin{array}{l}\text { Harms } \\
\text { Utilization } \\
\text { Patient Satisfaction }\end{array}$ \\
\hline $\begin{array}{l}\text { Rothman } 2013 \\
\text { Sweden } \\
\text { Median duration of } \\
\text { pain: } 18 \text { months } \\
\text { RCT } \\
\text { Fair } \\
\text { CPMP with vs. } \\
\text { without } \\
\text { pretreatment } \\
\text { assessment }\end{array}$ & $\begin{array}{l}\text { A. CPMP with } \\
\text { "multimodal" } \\
\text { pretreatment assessment } \\
\text { (n=99) } \\
\text { Duration NR, Intensity NR, } \\
\text { combo, outpatient } \\
\\
\text { B. CPMP using standard } \\
\text { process ( }=108 \text { ) } \\
\text { Duration NR, Intensity NR, } \\
\text { combo, outpatient }\end{array}$ & $\begin{array}{l}\text { Median age (IQR): } \\
\text { Treatment: } 40 \text { (32 to 47) } \\
\text { years, Control: } 40 \text { (33-48) } \\
\text { years } \\
\% \text { Male: } 23.6 \% \\
\text { Race/ethnicity: NR } \\
\text { Pain etiology/type: } \\
\text { Chronic muscular pain } \\
\text { Disability: NR } \\
\text { Comorbidities: NR }\end{array}$ & $\begin{array}{l}\text { A vs. B, Median (IQR) } \\
\text { VAS pain }(0-10) \\
\text { Baseline: } 6.95 \text { (5.90 to } 8.00) \text { vs. } \\
7.45(6.00 \text { to } 8.10) \\
\text { Long term: } 6.0 \text { ( }(3.0 \text { to } 8.1) \text { vs. } 6.55 \\
\text { (3.80 to } 8.00) \text {, adjusted OR of } \\
\text { improvement from baseline } 1.20 \\
(0.63 \text { to } 2.30)^{\mathrm{f}} \\
\text { ODI }(0-100) \\
\text { Baseline: } 40 \text { ( } 28 \text { to } 50) \text { vs. } 38 \text { ( } 28 \text { to } \\
50) \\
\text { Long term: } 36 \text { (22 to } 49) \text { vs. } 38(28 \\
\text { to } 50) \text {, adjusted OR of improvement } \\
\text { from baseline } 1.61(0.84 \text { to } 3.07)^{\mathrm{f}}\end{array}$ & 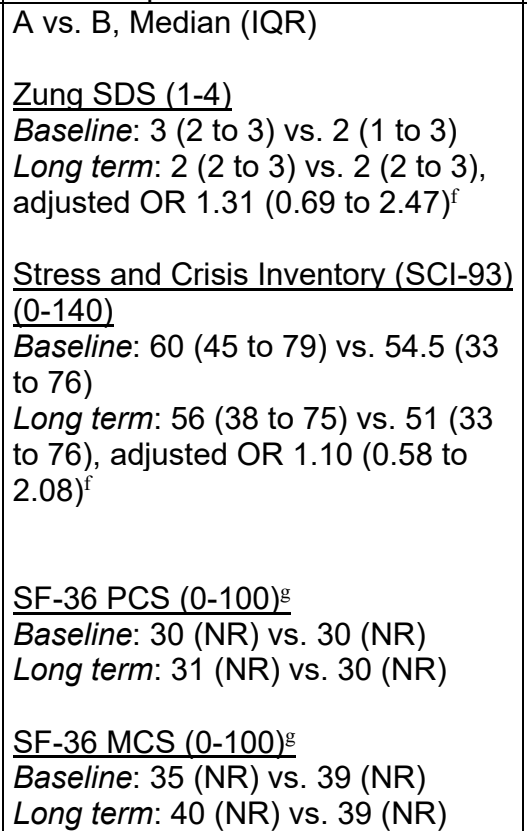 & $\begin{array}{l}\text { Harms: NR } \\
\text { Patient Satisfaction (1- } \\
\text { 7), median (IQR) } \\
\text { - Felt fully medically } \\
\text { assessed: } 5 \text { (3 to 7) vs. } \\
3 \text { (1 to 5), p<0.001 } \\
\text { - Received an } \\
\text { understandable } \\
\text { explanation of the } \\
\text { condition: } 6 \text { (5 to } 7) \text { vs. } 3 \\
\text { (1 to 5), p<0.001 } \\
\text { - Would recommend } \\
\text { assessment to a friend: } \\
7 \text { (5 to } 7) \text { vs. } 5 \text { (2 to } 7), \\
\text { p<0.001 } \\
\text { - Rehabilitation plan } \\
\text { carried out after } \\
\text { assessment: } 5 \text { (3- to 5) } \\
\text { vs. } 4 \text { (2 to 5), p=0.004 } \\
- \text { Assessment was } \\
\text { helpful: } 6 \text { (4 to 7) vs. } 4 \\
\text { (2 to 5), p<0.001 }\end{array}$ \\
\hline $\begin{array}{l}\text { Streibelt } 2014 \\
\text { Germany } \\
\text { Mean duration of } \\
\text { pain: NR } \\
\text { RCT } \\
\text { Fair } \\
\text { CPMP with vs. } \\
\text { without } \\
\text { pretreatment } \\
\text { assessment }\end{array}$ & $\begin{array}{l}\text { A. CPMP plus } \\
\text { pretreatment functional } \\
\text { capacity evaluation } \\
\text { (FCE) ( } \mathrm{n}=109 \text { ) } \\
3 \text { weeks, } 3 \text { to } 4 \text { hours/day } \\
\text { (total } 50 \text { to } 60 \text { hours), } \\
\text { individual, inpatient } \\
\\
\text { B. CPMP alone ( } \mathrm{n}=113 \text { ) } \\
3 \text { weeks, } 3 \text { to } 4 \text { hours/day } \\
\text { (total } 50 \text { to } 60 \text { hours), } \\
\text { individual, inpatient }\end{array}$ & $\begin{array}{l}\frac{\text { Mean age: } 46 \text { years }}{\text { \% Male: } 83 \%} \\
\frac{\text { Race/ethnicity: NR }}{\text { Pain etiology/type: }} \\
\text { Chronic MSK disorders } \\
\text { - M40-M54: 82\% } \\
\text { Disability: NR } \\
\text { Currently sick-listed: } 81 \% \\
\text { Duration of sick leave last } \\
\text { year: 15 weeks } \\
\text { Comorbidities: NR } \\
\text { - Excluded: "physicians' } \\
\text { diagnosis of red flags" }\end{array}$ & $\begin{array}{l}\text { A vs. B, mean } \\
\text { PDI (0-70) } \\
\text { Baseline: } 37.4(14.4) \text { vs. } 33.2(13.6) \\
\text { Long term: } 27.0 \text { vs. 33.5, adjusted } \\
\text { difference }-6.5(95 \% \mathrm{Cl}-12.6 \text { to - } \\
0.4)^{\mathrm{h}}\end{array}$ & NR & NR \\
\hline
\end{tabular}

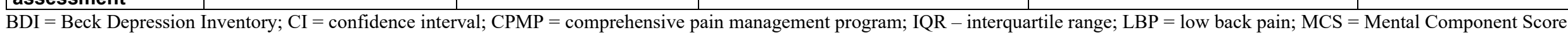

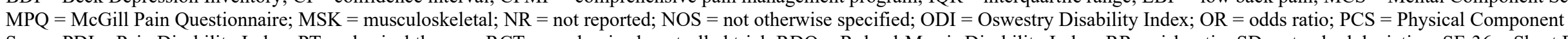

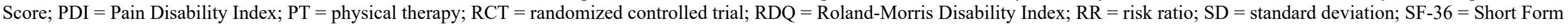
36 Questionnaire; SIP = Sickness Impact Profile; VAS = visual analog scale; WHYMPI = West Haven-Yale Multidimensional Pain Inventory.

${ }^{\text {a }}$ Demographics data include information for patients in both Part 1 and 2 of this study (demographics were not reported separately by the authors). 


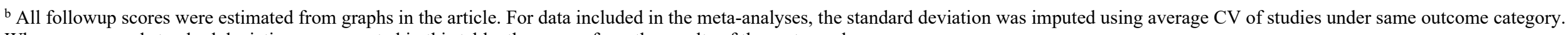
Where means and standard deviations are reported in this table, they came from the results of the meta-analyses.

${ }^{\mathrm{c}}$ Range of 9 to 18 months with the majority at 12 months.

${ }^{\mathrm{d}}$ Data were estimated by the EPC from figures in the article.

e Standard deviations estimated from Figure 2 of article.

${ }^{\mathrm{f}}$ Multivariate regression adjusted for sex, age, smoking status, nationality, education level, and relationship status.

g SF-36 scores estimated from Figure 2 of article.

h Adjusted for baseline score of the outcome, baseline employment status, baseline work ability, baseline PDI and diagnosis. 


\section{Appendix C. Contextual Questions}

\section{Contextual Question 1}

What different types of comprehensive, integrated approaches to complex acute/subacute pain or chronic, nonactive cancer pain management have been proposed or used in clinical practice?

a. How are comprehensive and integrated pain management defined?

b. What are considered the most important components of integrated pain management programs?

c. What pain management models or mechanisms are most commonly used in clinical practice?

d. What types of programs/models may be most applicable to Medicare beneficiaries?

e. What theoretical advantages and disadvantages do various programs/models have compared with current practice?

f. Are there any potential safety issues?

Answers to these questions are informed by peer-reviewed literature captured by our search and reported in the results above, U.S. government reports, conversations with our Technical Expert Panel and comments received on our study protocol via the Supplemental Evidence and Data for Systematic review (SEADS).

A myriad of diverse approaches to management of nonactive cancer pain have been reported in the peer-reviewed literature and are currently used clinically. Most of the peer-reviewed literature on formal pain management programs focuses on those provided in rehabilitation centers such as comprehensive traditional multidisciplinary rehabilitation programs or specialty clinics versus those that are based in and integrated with primary care. There are also a few reports of integrative pain care models, which focus on a broader range of integrative therapies and practices (e.g., mind-body therapies, acupuncture, nutritional counseling, mindfulness training and others). There is an overall lack of standardization with regard to pain management.

\section{Definitions}

There is substantial variability in the terminology used in the literature and in clinical practice to describe programs that incorporate methods that may address the biopsychosocial, multidimensional aspects of pain. Terms such as multimodal, multidisciplinary, interdisciplinary, integrated, comprehensive and collaborative are used in multitude of ways with no firm consensus and variable consistency. The National Pain Strategy (NPS) defines integrated care as the "systematic coordination of medical, psychological and social aspects of health care and includes primary care, mental health care, and, when needed, specialist services." 98 No discrete definition of "comprehensive" pain care was identified, but its use in government reports and peer-reviewed literature implies that assessment as well as patient-tailored treatment which targets the multiple aspects of pain management is based on the biopsychosocial model and is delivered by providers from different disciplines and promotes patient self-management. ${ }^{99,100}$ While across the literature, the terms multidisciplinary and interdisciplinary have frequently been used interchangeably, various publications cite definitions suggested by the International Association for the Study of Pain (IASP) ${ }^{101}$ which distinguishes them based primarily on the level 
of interaction between practitioners from multiple disciplines goals for patient care. IASP defines multidisciplinary treatment as multimodal treatment provided by practitioners from different disciplines, each following their own therapeutic aims for the patient which may or may not include communication between disciplines. In defining interdisciplinary treatment, they make the distinction that the multimodal care delivered by the multidisciplinary team is based on collaboration in assessment and treatment based on a shared biopsychosocial model and goals. Consistent with this, the NPS defines interdisciplinary care as being provided by health professionals from diverse fields who coordinate their skills and resources to meet patient goals. ${ }^{98}$ The 2011 IOM report states that ideally an interdisciplinary model of care includes comprehensive evaluation by providers from multiple disciplines and that is integrated and coordinated. ${ }^{99}$ The term multimodal therapy has been variably defined. For example, the IASP defines it as concurrent use of separate therapeutic interventions with different mechanisms of action within one discipline aimed at different pain mechanisms (e.g., use of medications with different mechanisms of actions) while a recent rapid review defines it more broadly to include use of more than one type of therapy which can in turn include treatments delivered by more than one discipline. ${ }^{102}$

Given the lack of consensus on program definitions, we defined integrated pain management programs (IPMPs) as programs centered in primary care, that have embedded or easy access to multidisciplinary providers and comprehensive pain management programs (CPMPs) as those that are not based in primary care. We further assumed that care in either type of program be provided by professionals from different disciplines and that programs include some level of interdisciplinary collaboration, communication, or coordination across providers and that the programs contain components that correspond to delivery of multimodal care (broadly defined as above) based on the biopsychosocial model. This review does not address integrative pain management programs unless they were explicitly included as part of IPMPs or CPMPs as described above. Integrative models of pain management generally focus on a broader range of integrative therapies and practices (e.g., mind-body therapies, acupuncture, nutritional counseling, mindfulness training and others). To the extent that such therapies and practices were included as a part of IPMPs or CPMPs, they were included.

\section{What Are Considered the Most Important Components?}

There is substantial variability in the types of components that may be included in programs as well as how they are delivered. There is no standard approach or consensus on specific components that should be included. Care components of pain management programs in general, based on a biopsychosocial model, center around the medical/biological, psychological, and social aspects of a patient's pain experience to promote pain relief, maintain or enhance physical function and body awareness, address psychosocial contributors to pain as needed, facilitate selfmanagement, and improve quality of life. The components and delivery of them in various pain management programs has evolved since early publications and acceptance of pain management programs $^{3,103}$ in the 1970 's. Common general treatment components described from two recent reviews ${ }^{3,104}$ across a total of 112 formal multidisciplinary pain management program studies for chronic pain included psychological and mental health support (94\% of studies, primarily CBTbased strategies, relaxation, coping, mindfulness) and physical activity ( $86 \%$ of studies) and less commonly, medication optimization or monitoring (40\%). Education on a range of topics (pain mechanisms, medication, psychological factors) was done in most studies (76\% of 85 studies) in the largest review. ${ }^{3}$ TEP discussions re-affirmed that these were likely the most common and 
important components of a formal, integrated program. Additional components described across review of formal programs include the use of passive treatments (e.g., electrotherapy) and complementary, integrative treatments (e.g., acupuncture, manipulation, massage). ${ }^{3,104}$ The relative importance of individual components in IPMPs is difficult to assess given the substantial variation across programs regarding specific component content and breadth, intensity, frequency, and delivery formats. Individual patient needs also impact which components may be most important and how to best incorporate them optimize their pain management.

In recent years, many programs have attempted to include a broader range of components, particularly related to more integrative and complementary care (e.g., acupuncture, manual therapies), those related to mindfulness and mind-body practices such as Yoga, Tai Chi, and others. Our report on nonpharmacologic, noninvasive treatment of chronic pain found evidence that many of these interventions improved function and/or pain that persisted after the end of treatment, some into long term. ${ }^{14,15}$ Our findings also suggested that evidence was somewhat more robust for "active" interventions that engage patients in movement and address psychological contributors to pain, particularly at longer-term followup, versus more "passive" treatments focused on symptom relief such as massage. Active interventions include exercise, multidisciplinary rehabilitation, psychological interventions (particularly CBT), and mind-body interventions. Our findings also suggested that, because of the heterogeneity of chronic pain, patients with one type of pain may respond differently to a given component than another patient with a different pain diagnosis. In addition, the level of supporting evidence varied from condition to condition. Thus, policy makers may need to consider the degree to which evidence may be reasonably extrapolated across conditions (e.g., effectiveness of psychological therapies for chronic back pain may not necessarily be extrapolated to osteoarthritis pain). This also speaks to the importance of programs considering which components may be best suited to which patients.

Integrative approaches to pain management have been increasingly reported in the literature, mostly via studies of individual modalities such as acupuncture, massage or manipulation or practices such as Yoga, Tai Chi and mindfulness. Integrative pain management differs from the integrated pain management as defined for this review. Integrative management takes a holistic, person-centered approach to patient care as do the individual complimentary and integrative health therapies employed. In contrast, IPMPs may focus more on outcomes such as pain and function. In addition to more recent inclusion of a broader range of individual integrative therapies as part of IPMPs or CPMPs, there are formal integrative pain management programs. ${ }^{105} \mathrm{Such}$ programs focus on a broader range of integrative therapies (e.g., acupuncture, massage, and mindbody practices) and whole-person approaches to well-being (e.g., spiritual and lifestyle counseling, consideration of sleep, diet, gut health and metabolism, and others). Such programs may coordinate treatment components across diverse provider disciplines (e.g., acupuncturists, nutritionists, health coaches, naturopathic physicians, medical doctors, and others). While previous ARHQ reports and other peer-reviewed literature have evaluated individual integrative therapies, there is sparse literature comparing formal integrative pain management programs that focus on coordination of integrative therapies specifically to usual care or active comparators. Further research is needed.

Coordination and communication across multiple providers are considered key in assuring collaborative, interdisciplinary care. ${ }^{98,103,106,107}$ A rapid review ${ }^{102}$ of system components for improving guideline-concordant integrated pain care in primary care settings related to 1) enhanced decision support containing provider education and assistance with treatment planning, including use of care algorithms 2) enhanced care coordination resources, including use of care manager 3) methods of improving patient education and activation and 4) increasing patient 
access to a broader range of treatments including specialty care as needed. They concluded that decision support, coupled with on-going treatment monitoring led to improvement in pain intensity and pain-related function compared with usual care based on five studies in different models of care but that additional research was needed.

\section{What Pain Management Models or Mechanisms Are Most Used in Clinical Practice?}

The current paradigm for pain care is the provision of selected individual treatments (e.g., medications) or services (e.g., physical therapy, psychological support) prescribed or recommended by a patient's provider (primary care or specialty provider). Treatment maybe unimodal or offer a limited range of management options (e.g., medication and physical therapy [PT] only or medication and psychological support only). Provision of individual treatments may be centered more around individual clinical or specific provider skills and/or reimbursement versus collaborative, coordinated management of the problem across disciplines that is focused on patient outcomes. ${ }^{107}$ Formal pain management programs have not been widely implemented in the United States and may not be assessable to many populations based on location, insurance coverage and socio-economic factors.

Models reported in the peer-reviewed literature may include methods of assessment or risk stratification to facilitate individualized treatment and appropriate referral recommendations, formal case management, incorporation of algorithms for pharmacologic $20,21,53,108$ and other care and engagements of patients, monitoring of patient progress with formal, regular communication between primary care providers and other providers is key component. Provider education and support has been cited as important to collaborative, integrated care. ${ }^{20,21,108,109}$ Some models have sought to actively incorporate integrative and complementary health approaches with primary care $^{110}$ and others have explored use of telecare. Programs within Veterans Affairs (VA) have continued to develop, based on the peer-reviewed literature. One such program incorporates most of the features outlined (patient assessment, engagement and symptom monitoring, provider education feedback and recommendations and facilitation of specialty care. ${ }^{24-26}$ Another model ${ }^{111}$ using a stepped-care approach which involves primary care and is delivered via Patient Aligned Clinical Teams (PACTs) ${ }^{102,112,113}$ and provides a basis for patient assessment, medication management and referral to a range of multidisciplinary providers and services (e.g., behavioral pain management) and for advanced diagnostics and interventions as needed. Patient care is individualized. Not all will get the same components.

\section{Contextual Question 2}

\section{Is there information on the costs or cost-effectiveness of integrated pain management programs in the Medicare or general population?}

There is sparse information on the costs and cost-effectiveness for either the IPMP or the CPMP conducted in the United States in the peer-reviewed literature. The substantial variations across programs and how components are delivered leads to concerns regarding the applicability of costs or cost-effectiveness across either program type. The literature search for this report yielded 298 potentially relevant economic studies. We restricted studies for this contextual question to those which evaluated IPMPs or CPMPs which contained the availability of the primary components of medication review/optimization, physical activity and psychological support and compared such programs to either usual care or active treatment options. Six 
programs meeting inclusion criteria for the Key Questions reported associated economic data. Two full economic studies in IPMPs were identified, one conducted in the United States ${ }^{24}$ and one in the United Kingdom..$^{23,28}$ In addition, two full economic studies on CPMPs, one conducted in Germany ${ }^{71}$ and the other in the Netherlands ${ }^{114}$ were identified. The other two were costing studies for CPMPs conducted in the United Kingdom ${ }^{74}$ and the Netherlands ${ }^{49}$ and will not be discussed here as they are not comparative. Three additional studies of CPMP programs, two conducted in Sweden ${ }^{115,116}$ and one in the Netherlands ${ }^{117}$ that are not included in the Key Question portion were also identified. All but one CPMP study ${ }^{116}$ used randomized controlled trial (RCT) clinical data and reported on patients with low back pain. Studies were of varying quality.

The most applicable economic assessment to this review, based on a cluster-randomized controlled trial $(\mathrm{N}=401)^{24}$ of a system-based IPMP, was done from the VA healthcare perspective (Appendix E, Table E1). It is the only U.S.-based study. The trial randomized primary care providers to receive collaborative, multidisciplinary assistance with pain treatment (APT) of patients with musculoskeletal pain diagnoses experiencing moderate or greater pain intensity or disability lasting 12 weeks or longer using a stepped-care model or usual care for 12 months. Patients' mean age was 62 years, 92 percent were male, and 65 percent were receiving disability payments. The most common patient comorbidities reported were major depression $(18 \%)$, panic attack $(17 \%)$, post-traumatic stress disorder $(16 \%)$, anxiety syndrome $(13 \%)$ and prior substance use treatment (16\%); 43 percent of patients reported taking opioids in the 6 months prior to enrollment. APT included a full-time clinical psychologist care manager, an internist who spent up to 1 day/week on APT team activities and a physical therapist. APT practice patients received assessments and care management with APT internist or mental health consultation provided as needed based on a stepped-care model. Participants were encouraged to attend a 4-session workshop co-led by the team. Total VA costs included treatment in the year prior to enrollment, treatment while and intervention team activity costs. The mean APT costs were greater than those for usual care, but confidence intervals were wide: mean (standard deviation) for each, $\$ 11,263(\$ 14,566)$ versus $\$ 8920(\$ 13,131)$. Year for dollar costs was not provided. The primary outcome for the economic analysis was number of pain disability-free days (PDFDs) computed based on RDMQ scores. Models for PDFDs and natural log of total VA costs were adjusted for age, sex, opioid prescription in six months prior and baseline chronic disease burden; the PDFD model also adjusted for baseline Roland Morris Disability Questionnaire scores and costs were also adjusted by prior year treatment costs. APT participants experienced a mean of 16 additional PDFDs over the 12-month period. Predicted adjusted mean incremental cost per pain-disability free day ranged from $\$ 364$ to $\$ 1117$ and predicted adjusted mean incremental increase of intervention costs ranged from $\$ 6035$ to $\$ 18,554$. Baseline medical comorbidities, depression severity and prior year's treatment costs were important drivers of cost. Authors state that the average increase of $\$ 2300$ per patient for the APT intervention falls on the low end of costs for commonly used chronic pain interventions and that identification of subgroups for which APT is most cost-effective is important. The applicability of these findings to other IPMPs, particularly those that are practiced-based is unclear.

A cost-utility analysis of IPMP based on an RCT conducted in the United Kingdom ${ }^{23,28}$ was performed from a provider/health services perspective. The trial randomized patients with chronic widespread pain to IPMP, telephone cognitive behavioral therapy (TCBT) or exercise. Mean patient age was 56 years old, more than $80 \%$ were female and approximately one third were retired. National Health Service cost data were used with utilities-based EuroQol-5 
Dimensions data postintervention, short term ( 3 months postintervention) and long term ( 24 months). Authors' focus was on the TCBT versus usual care. IPMP compared with usual care was not considered cost-effective post-intervention or short term compared with usual care. At long term, TCBT was reported as dominating other interventions, including IPMP; it had the lowest cost and greatest increase in quality adjusted life years (QALYs) compared with usual care. Authors report that cost-effectiveness was sensitive to missing data and used imputation to account for missing data. The applicability of these findings to the U.S. healthcare system, the Medicare population and patients with other pain conditions is unclear.

Two full economic studies based on included CPMP studies ${ }^{71,114}$ adopted a societal perspective as did three other full economic studies for CPMP programs ${ }^{115-117}$ that are not included in the Key Question portion of the report (Table C-1). Mean patient ages ranged from 42 to 46 years and 17 to 64 percent were female. There was substantial heterogeneity in programs, how they were delivered, and how costing was done. All focused-on costeffectiveness based primarily on lost productivity due to pain and related impact on indirect costs. Results across these studies were mixed with most finding no differences in disability or QALYs gained between CPMPs and individual components. Inpatient CPMP was cost-effective compared with physical therapy-based rehabilitation ${ }^{71}$ due to lower indirect costs related to fewer days absent from work in one trial, however, two others suggest that full CPMP programs were not cost effective versus single treatment modalities ${ }^{114}$ for CLPB (PT or CBT alone) or versus clinical assessment and advice. ${ }^{115}$ The observational study found CPMP to be cost effective versus orthopedic manual therapy ${ }^{116}$ for neck or back pain. Given the focus on sick-leave and return to work as well as differences in healthcare costs and delivery compared with the United States, the applicability of these findings, particularly to Medicare beneficiaries, is unclear. 
Table C-1. Overview of formal economic studies for integrated pain management programs (IPMPs)

\begin{tabular}{|c|c|c|}
\hline Components & Dickinson 2010 & McBeth 2012, Beasley 2015 \\
\hline Population & $\begin{array}{l}\text { SEACAP cluster RCT }(\mathrm{N}=401) \\
\text { Chronic MSK pain, mean (SD) duration } 14.8(12.7) \text { years } \\
\text { Mean (SD) age: } 62(12) \text { years } \\
\text { Male: } 92 \% \\
\text { Disability: } 65 \% \\
\text { Opioid prescription: } 43 \% \\
\text { Comorbidities: major depression }(18 \%) \text {, panic attack }(17 \%) \text {, } \\
\text { PTSD }(16 \%) \text {, anxiety syndrome }(13 \%) \text { and prior substance use } \\
\text { treatment }(16 \%)\end{array}$ & $\begin{array}{l}\text { MUSICIAN RCT (N=442) } \\
\text { Chronic widespread pain (duration NR) } \\
\text { Mean (SD) age: } 56(13) \text { years } \\
\text { Female: } 70 \% \\
\text { Disability: NR } \\
\text { Patient with severe psychiatric disorders were excluded }\end{array}$ \\
\hline Intervention(s) & Integrated Pain Management Program (stepped care model) & $\begin{array}{l}\text { Integrated Pain Management Program, TCBT alone (author's focus), } \\
\text { physical therapy }\end{array}$ \\
\hline Comparator(s) & Usual Care & Usual Care \\
\hline Country & United States & United Kingdom \\
\hline Funding & Department of Veteran Affairs & Grant (Arthritis Research UK) \\
\hline Study design & CEA & CUA \\
\hline Perspective & Veteran Affairs healthcare, payer & Health service, payer \\
\hline Time horizon & 12 months & Post 6-month intervention, short term (3 months post), 24 months \\
\hline Analytic model & $\begin{array}{l}\text { Models for PDFDs and natural log of VA costs adjusted for } \\
\text { intervention status, sex, age, depression severity, opioid } \\
\text { prescription in the } 6 \text { months prior to enrollment, and baseline } \\
\text { chronic disease burden (RxRisk-V score); } \\
\text { PDFDs model further adjusted for RMDQ score; } \\
\text { VA treatment costs model further adjusted by prior years' } \\
\text { treatment costs }\end{array}$ & $\begin{array}{l}\text { Generalized linear model, with Poisson family distribution and a } \\
\text { power link function }\end{array}$ \\
\hline $\begin{array}{l}\text { Effectiveness } \\
\text { outcome }\end{array}$ & Number of pain disability-free days (PDFDs) & QALYs \\
\hline $\begin{array}{l}\text { Effectiveness } \\
\text { outcome } \\
\text { components }\end{array}$ & $\begin{array}{l}\text { RMDQ scores at the beginning and end of 3-, 6-, 12-month } \\
\text { intervals used to estimate pain disability for each day in that } \\
\text { interval. The } 12-\text { month evaluation was postintervention } \\
\text { Score } \leq 5=\text { fully pain disability free } \\
\text { Score } 6 \text { to } 18=\text { proportion of days spent in pain disability } \\
\text { assumed to increase linearly with the score. } \\
\text { Score } \geq 19=\text { fully pain disabled } \\
\text { Total number of PDFDs = sum of PDFDs across time intervals. }\end{array}$ & EQ-5D scores at baseline, postintervention, short and long term \\
\hline $\begin{array}{l}\text { Source for } \\
\text { effectiveness data }\end{array}$ & Authors' own trial (SEACAP) & Authors' own trial (MUSICIAN) \\
\hline Costing year & Not reported & 2009-2010 \\
\hline Currency & USD & Pounds sterling \\
\hline Discounting & Not reported; 1 year time frame & Costs and QALYs beyond 12 months were discounted at $3.5 \%$ \\
\hline
\end{tabular}




\begin{tabular}{|c|c|c|}
\hline Components & Dickinson 2010 & McBeth 2012, Beasley 2015 \\
\hline $\begin{array}{l}\text { Components of cost } \\
\text { data }\end{array}$ & $\begin{array}{l}\text { Cumulative cost of individual components of the intervention } \\
\text { (microcosting via DSS), telephone and in-person contacts } \\
\text { (duration and clinician profession taken into account), weekly } \\
\text { case conferences, group educational meetings, training time for } \\
\text { team care manager, internist and PT, video/DVD, travel } \\
\text { expenses }\end{array}$ & $\begin{array}{l}\text { Provider contact time, therapist training and supervision, production } \\
\text { of printed materials, health service resource use (community and } \\
\text { hospital), hospital admission }\end{array}$ \\
\hline Cost sources & $\begin{array}{l}\text { VA's DSS and Microsoft Access database for activities not } \\
\text { recorded in DSS }\end{array}$ & United Kingdom National Health Service \\
\hline Sensitivity analysis & $\begin{array}{l}\text { Nonlinear regression modeling to evaluate key predictors for } \\
\text { selected patient profiles }\end{array}$ & $\begin{array}{l}\text { Nonparametric bootstrapping, multivariate regression; Chained } \\
\text { equations to assess sensitivity to missing data }\end{array}$ \\
\hline ICER & $\begin{array}{l}\text { Predicted adjusted mean incremental cost per pain-disability } \\
\text { free day: range, } \$ 364 \text { to } \$ 1117 \\
\text { Predicted adjusted mean incremental increase of intervention } \\
\text { costs: range, } \$ 6,035 \text { to } \$ 18,554\end{array}$ & $\begin{array}{l}\text { Additional cost per QALY versus usual care based on complete } \\
\text { cases } \\
\text { Post } 6 \text {-month intervention: IPMP } £ 63,858 \text {, exercise } £ 114,303 \text {, TCBT } \\
£ 76,695 \\
\text { Short term: IPMP } £ 34,731 \text {, exercise } £ 72,270 \text {, TCBT } £ 16,542 \\
\text { Long term ( } 24 \text { months): IPMP- dominated, exercise dominated, TCBT } \\
£ 5917\end{array}$ \\
\hline $\begin{array}{l}\text { Sensitivity analysis } \\
\text { results }\end{array}$ & $\begin{array}{l}\text { Incremental effects (across selected patient profiles), range for } \\
\text { intervention vs. usual care: } \\
\text { Female: } \$ 3,998-\$ 12,291 \text { vs. } \$ 2,332-\$ 7,168 \\
\text { 10-year increase in age: } \$ 626-\$ 4,305 \text { vs. } \$ 366-\$ 2,510 \\
\text { 6-point increase in depression: } \$ 878-\$ 6,036 \text { vs. } \$ 512-\$ 3,521 \\
\text { Opioid prescription } 6 \text { months prior to enrollment: } \$ 307-\$ 2,103 \\
\text { vs. } \$ 178-\$ 1,226 \\
\text { 3-point increase in chronic disease score: } \$ 1,727-\$ 11,867 \text { vs. } \\
\$ 1,008-\$ 11,867 \\
\$ 10,000 \text { increase in treatment costs in year prior to } \\
\text { enrollment: } \$ 4,863-\$ 6,605 \text { vs. } \$ 4,179-\$ 6,268\end{array}$ & $\begin{array}{l}\text { Sensitivity to missing data } \\
\text { Additional cost per QALY versus usual care based on imputed data } \\
\text { Short-term: IPMP £49,220, exercise } £ 61,165 \text {, TCBT } £ 39,868 \\
\text { Long term ( } 24 \text { months): IPMP- dominated, exercise dominated, TCBT } \\
£ 3957 \\
\text { IPMP was not considered cost-effective at the end of treatment } \\
\text { based on a willingness to pay } £ 30000 \text { ( } \$ 46770) / \text { extra QALY or short } \\
\text { term; TCBT had an estimated } 70 \% \text { chance of being cost effective vs. } \\
\text { usual care short term and } 75 \% \text { chance of being cost-effective at } \\
\text { ceiling of } £ 20000 / \text { extra QALY versus usual care. }\end{array}$ \\
\hline Author's Conclusion & $\begin{array}{l}\text { More PDFDs and higher costs with the integrated program } \\
\text { versus usual care over a 12-month followup. } \\
\text { The wide range in cost to obtain an additional PDFD suggests } \\
\text { that the intervention may be quite costly for older people with } \\
\text { many comorbidities and long-standing pain. }\end{array}$ & $\begin{array}{l}\text { McBeth (postintervention and short-term results: There were } \\
\text { nonsignificant increases in QALYs versus usual care. Conclusions } \\
\text { regarding cost-effectiveness were sensitive to missing data } \\
\text { Beasley (24-month results): TCBT was associated with the lowest } \\
\text { cost and highest QALY gain compared with usual care and was } \\
\text { considered to be highly cost-effective, and improvement could partly } \\
\text { be predicted by patient characteristics. Authors report that TCBT was } \\
\text { cost-effective in the long term with cost/QALY ranging from } \sim £ 4 \mathrm{~K} \text { to } \\
£ 6 \mathrm{~K} \text { depending on the method of analysis }\end{array}$ \\
\hline
\end{tabular}




\begin{tabular}{|c|c|c|}
\hline Components & Dickinson 2010 & McBeth 2012, Beasley 2015 \\
\hline Limitations & $\begin{array}{l}\text { - Generalizability of the VA health system and VA population } \\
\text { to other healthcare systems/populations. } \\
\text { - Estimated the costs of the intervention, which were not } \\
\text { captured in the VA costing system, leading to possible } \\
\text { overestimates } \\
\text { - Did not take into account effects of the intervention on } \\
\text { healthcare received outside of the VA } \\
\text { - Short time horizon } \\
\text { - Calculations/model not clearly specified } \\
\text { - Use of calculated, nonstandard effect measures }\end{array}$ & $\begin{array}{l}\text { - Generalizability of U.K. health system costs and procedures to } \\
\text { U.S.-based programs is unclear } \\
\text { - Limited sensitivity analyses and documentation of those done } \\
\text { - Additional QALYs accrued between short term ( } 3 \text { months) and } 24 \\
\text { months were calculated assuming a linear change. No sensitivity } \\
\text { analysis around this was reported } \\
\text { - CUA models were sensitive to missing data; other factors were not } \\
\text { well evaluated }\end{array}$ \\
\hline
\end{tabular}

CEA = cost-effectiveness analysis; CUA = cost-utility analysis; DVD = digital video disc; GDP = gross domestic product; EQ-5D = EuroQol-5 Dimensions; ICER = incremental cost-effectiveness ratio; IPMP = Integrated Pain Management Program; NR = not reported; PDFDs = pain disability-free days; PSA = probabilistic sensitivity analysis; QALY = quality-adjusted life years; RCT = randomized controlled trial; RMDQ = Roland Morris Disabilty Questionnaire; $\mathrm{SD}=\mathrm{standard}$ deviation; TCBT = telephone cognitive behavioral therapy; U.K. = United Kingdom; U.S. = United States; USD = United States dollar; VA = Veteran's Affairs. 


\section{Appendix D. Included Studies List}

1. Abbasi M, Dehghani M, Keefe FJ, et al. Spouse-assisted training in pain coping skills and the outcome of multidisciplinary pain management for chronic low back pain treatment: a 1-year randomized controlled trial. Eur J Pain. 2012 Aug;16(7):1033-43. doi: $10.1002 / \mathrm{j} .1532-2149.2011 .00097 . x$. PMID: 22337646.

2. Ahlmen M, Sullivan M, Bjelle A. Team versus non-team outpatient care in rheumatoid arthritis. A comprehensive outcome evaluation including an overall health measure. Arthritis Rheum. 1988 Apr;31(4):471-9. doi: 10.1002/art.1780310403. PMID: 3358810.

3. Alaranta H, Rytökoski U, Rissanen A, et al. Intensive physical and psychosocial training program for patients with chronic low back pain. A controlled clinical trial. Spine (Phila Pa 1976). 1994 Jun 15;19(12):1339-49. doi: 10.1097/00007632-199406000-00007. PMID: 8066514.

4. Allen KD, Oddone EZ, Coffman CJ, et al. Patient, Provider, and Combined Interventions for Managing Osteoarthritis in Primary Care: A Cluster Randomized Trial. Annals of Internal Medicine. 2017 Mar 21;166(6):401-11. doi: https://dx.doi.org/10.7326/M16-1245. PMID: 28114648.

5. Allen KD, Yancy WS, Jr., Bosworth HB, et al. A Combined Patient and Provider Intervention for Management of Osteoarthritis in Veterans: A Randomized Clinical Trial. Annals of Internal Medicine. 2016 Jan 19;164(2):73-83. doi: https://dx.doi.org/10.7326/M15-0378. PMID: 26720751.

6. Altmaier EM, Lehmann TR, Russell DW, et al. The effectiveness of psychological interventions for the rehabilitation of low back pain: a randomized controlled trial evaluation. Pain. 1992 Jun;49(3):329-35. doi: 10.1016/0304-3959(92)90240-c. PMID: 1408299 .
7. Amris K, Wæhrens EE, Christensen R, et al. Interdisciplinary rehabilitation of patients with chronic widespread pain: primary endpoint of the randomized, nonblinded, parallel-group IMPROvE trial. Pain. 2014 Jul;155(7):1356-64. doi: 10.1016/j.pain.2014.04.012. PMID: 24727345.

8. Angeles RN, Guenter D, McCarthy L, et al. Group interprofessional chronic pain management in the primary care setting: a pilot study of feasibility and effectiveness in a family health team in Ontario. Pain Research \& Management. 2013 SepOct;18(5):237-42. PMID: 23875181.

9. Basler HD, Jäkle C, Kröner-Herwig B. Incorporation of cognitive-behavioral treatment into the medical care of chronic low back patients: a controlled randomized study in German pain treatment centers. Patient Educ Couns. 1997 Jun;31(2):113-24. doi: 10.1016/s0738-3991(97)00996-8. PMID: 9216352.

10. Beasley M, Prescott GJ, Scotland G, et al. Patient-reported improvements in health are maintained 2 years after completing a short course of cognitive behaviour therapy, exercise or both treatments for chronic widespread pain: long-term results from the MUSICIAN randomised controlled trial. RMD Open. 2015;1(1):e000026. doi: 10.1136/rmdopen-2014-000026. PMID: 26509056.

11. Bendix AE, Bendix T, Haestrup C, et al. A prospective, randomized 5-year follow-up study of functional restoration in chronic low back pain patients. Eur Spine J. 1998b;7(2):111-9. doi: 10.1007/s005860050040. PMID: 9629934.

12. Bendix AF, Bendix T, Labriola M, et al. Functional restoration for chronic low back pain. Two-year follow-up of two randomized clinical trials. Spine. 1998a Mar 15;23(6):717-25. PMID: 9549794. 
13. Bendix AF, Bendix T, Lund C, et al. Comparison of three intensive programs for chronic low back pain patients: a prospective, randomized, observer-blinded study with one-year follow-up. Scand J Rehabil Med. 1997 Jun;29(2):81-9. PMID: 9198257.

14. Bendix AF, Bendix T, Ostenfeld S, et al. Active treatment programs for patients with chronic low back pain: a prospective, randomized, observer-blinded study. European Spine Journal. 1995;4(3):148-52. PMID: 7552649.

15. Bendix AF, Bendix T, Vaegter K, et al. Multidisciplinary intensive treatment for chronic low back pain: a randomized, prospective study. Cleveland Clinic Journal of Medicine. 1996 Jan-Feb;63(1):62-9. PMID: 8590519.

16. Bendix T, Bendix A, Labriola M, et al. Functional restoration versus outpatient physical training in chronic low back pain: a randomized comparative study. Spine. 2000 Oct 01;25(19):2494-500. PMID: 11013502.

17. Browne AL, Appleton S, Fong K, et al. A pilot randomized controlled trial of an early multidisciplinary model to prevent disability following traumatic injury. Disability \& Rehabilitation. 2013 Jul;35(14):1149-63. doi:

https://dx.doi.org/10.3109/09638288.2012.7 21047. PMID: 23083416.

18. Calner T, Nordin C, Eriksson MK, et al. Effects of a self-guided, web-based activity programme for patients with persistent musculoskeletal pain in primary healthcare: a randomized controlled trial. European journal of pain. 2017(pagination) PMID: CN-01374487 NEW.

19. Castel A, Castro S, Fontova R, et al. Body mass index and response to a multidisciplinary treatment of fibromyalgia. Rheumatology International. 2015 Feb;35(2):303-14. doi: https://dx.doi.org/10.1007/s00296-0143096-x. PMID: 25080875.
20. Castel A, Fontova R, Montull S, et al. Efficacy of a multidisciplinary fibromyalgia treatment adapted for women with low educational levels: a randomized controlled trial. Arthritis Care Res (Hoboken). 2013 Mar;65(3):421-31. doi: 10.1002/acr.21818. PMID: 22899402.

21. de Buck PD, le Cessie S, van den Hout WB, et al. Randomized Comparison of a Multidisciplinary Job-Retention Vocational Rehabilitation Program With Usual Outpatient Care in Patients With Chronic Arthritis at Risk For Job Loss. Arthritis \& Rheumatism: Arthritis Care \& Research. 2005 Oct;53(5):682-90. doi: http://dx.doi.org/10.1002/art.21452. PMID: 2005-13265-006.

22. Dickinson KC, Sharma R, Duckart JP, et al. VA healthcare costs of a collaborative intervention for chronic pain in primary care. Medical Care. 2010 Jan;48(1):38-44. doi:

https://dx.doi.org/10.1097/MLR.0b013e318 1bd49e2. PMID: 19952802.

23. Dobscha SK, Corson K, Leibowitz RQ, et al. Rationale, design, and baseline findings from a randomized trial of collaborative care for chronic musculoskeletal pain in primary care. Pain Medicine. 2008 Nov;9(8):105064. doi: https://dx.doi.org/10.1111/j.15264637.2008.00457.x. PMID: 18565008.

24. Dobscha SK, Corson K, Perrin NA, et al. Collaborative care for chronic pain in primary care: a cluster randomized trial. JAMA. 2009 Mar 25;301(12):1242-52. doi: https://dx.doi.org/10.1001/jama.2009.377. PMID: 19318652.

25. Härkäpää K, Järvikoski A, Mellin G, et al. A controlled study on the outcome of inpatient and outpatient treatment of low back pain. Part I. Pain, disability, compliance, and reported treatment benefits three months after treatment. Scand J Rehabil Med. 1989;21(2):81-9. PMID: 2526364.

26. Härkäpää K, Mellin G, Järvikoski A, et al. A controlled study on the outcome of inpatient and outpatient treatment of low back pain. Part III. Long-term follow-up of pain, disability, and compliance. Scand J Rehabil Med. 1990;22(4):181-8. PMID: 2148221. 
27. Henchoz Y, de Goumoens P, So AK, et al. Functional multidisciplinary rehabilitation versus outpatient physiotherapy for non specific low back pain: randomized controlled trial. Swiss Medical Weekly. 2010;140:w13133. doi: https://dx.doi.org/10.4414/smw.2010.13133. PMID: 21181567.

28. Jensen IB, Bergström G, Ljungquist $\mathrm{T}$, et al. A randomized controlled component analysis of a behavioral medicine rehabilitation program for chronic spinal pain: are the effects dependent on gender? Pain. 2001 Mar;91(1-2):65-78. doi: 10.1016/s0304-3959(00)00420-6. PMID: 11240079 .

29. Johansson C, Dahl J, Jannert M, et al. Effects of a cognitive-behavioral painmanagement program. Behaviour Research \& Therapy. 1998 Oct;36(10):915-30. PMID: 9714943.

30. Jousset N, Fanello S, Bontoux L, et al. Effects of functional restoration versus 3 hours per week physical therapy: a randomized controlled study. Spine (Phila Pa 1976). 2004 Mar 1;29(5):487-93; discussion 94. doi: 10.1097/01.brs.0000102320.35490.43. PMID: 15129059.

31. Kaapa EH, Frantsi K, Sarna S, et al. Multidisciplinary group rehabilitation versus individual physiotherapy for chronic nonspecific low back pain: a randomized trial. Spine. 2006 Feb 15;31(4):371-6. PMID: 16481945.

32. Kool J, Bachmann S, Oesch P, et al. Function-centered rehabilitation increases work days in patients with nonacute nonspecific low back pain: 1-year results from a randomized controlled trial. Arch Phys Med Rehabil. 2007 Sep;88(9):1089-94. doi: 10.1016/j.apmr.2007.05.022. PMID: 17826451.

33. Kool JP, Oesch PR, Bachmann S, et al. Increasing days at work using functioncentered rehabilitation in nonacute nonspecific low back pain: a randomized controlled trial. Arch Phys Med Rehabil. 2005 May;86(5):857-64. doi: 10.1016/j.apmr.2004.10.044. PMID: 15895328 .
34. Leeuw M, Goossens ME, van Breukelen GJ, et al. Exposure in vivo versus operant graded activity in chronic low back pain patients: results of a randomized controlled trial. Pain. 2008 Aug 15;138(1):192-207. doi: 10.1016/j.pain.2007.12.009. PMID: 18242858 .

35. Lemstra M, Olszynski W. The Effectiveness of Multidisciplinary Rehabilitation in the Treatment of Fibromyalgia: A Randomized Controlled Trial. The Clinical Journal of Pain. 2005 Mar-Apr;21(2):166-74. doi: http://dx.doi.org/10.1097/00002508200503000-00008. PMID: 2005-02073-008.

36. Linton SJ, Boersma K, Jansson M, et al. The effects of cognitive-behavioral and physical therapy preventive interventions on painrelated sick leave: a randomized controlled trial. Clinical Journal of Pain. 2005 MarApr;21(2):109-19. PMID: 15722803.

37. Mangels $\mathrm{M}$, Schwarz $\mathrm{S}$, Worringen $\mathrm{U}$, et al. Evaluation of a behavioral-medical inpatient rehabilitation treatment including booster sessions: a randomized controlled study. Clinical Journal of Pain. 2009

Jun;25(5):356-64. doi: https://dx.doi.org/10.1097/AJP.0b013e3181 925791. PMID: 19454868.

38. Martin J, Torre F, Aguirre U, et al. Evaluation of the interdisciplinary PSYMEPHY treatment on patients with fibromyalgia: a randomized control trial. Pain Medicine. 2014c Apr;15(4):682-91. doi: https://dx.doi.org/10.1111/pme.12375. PMID: 24576148.

39. Martin J, Torre F, Padierna A, et al. Impact of interdisciplinary treatment on physical and psychosocial parameters in patients with fibromyalgia: results of a randomised trial. International journal of clinical practice. 2014a;68(5):618-27. PMID: CN-00988924.

40. Martin J, Torre F, Padierna A, et al. Interdisciplinary treatment of patients with fibromyalgia: improvement of their healthrelated quality of life. Pain Practice. 2014b Nov;14(8):721-31. doi: https://dx.doi.org/10.1111/papr.12134. PMID: 24279638. 
41. Mas RR, Lopez-Jimenez T, Pujol-Ribera E, et al. Effectiveness of a multidisciplinary BIOPSYCHOSOCIAL intervention for nonspecific SUBACUTE low back pain in a working population: a cluster randomized clinical trial. BMC Health Services Research. 2019 Dec 12;19(1):962. doi: https://dx.doi.org/10.1186/s12913-0194810-x. PMID: 31831074.

42. McBeth J, Prescott G, Scotland G, et al. Cognitive behavior therapy, exercise, or both for treating chronic widespread pain. Archives of Internal Medicine. 2012 Jan 09;172(1):48-57. doi: https://dx.doi.org/10.1001/archinternmed.20 11.555. PMID: 22082706.

43. Meyer K, Fransen J, Huwiler H, et al. Feasibility and results of a randomised pilotstudy of a work rehabilitation programme. Journal of Back and Musculoskeletal Rehabilitation. 2005;18(3-4):67-78.

44. Onac I, Moldovan A, Igna R, et al. Mechanism of change following multidisciplinary treatment of low back pain secondary to lumbar disc prolapse. Journal of Evidence-Based Psychotherapies. 2017 Sep;17(2):159-73. doi: http://dx.doi.org/10.24193/jebp.2017.2.10. PMID: 2017-54116-010.

45. Onac I, Moldovan A, Onac I, et al. Medication, physiotherapy and cognitive behavior therapy for the treatment of chronic back pain: a clinical trial. Journal of Cognitive and Behavioral Psychotherapies. 2012 Mar;12(1):23-37.

46. Peters J, Large RG, Elkind G. Follow-up results from a randomised controlled trial evaluating in- and outpatient pain management programmes. Pain. 1992 Jul;50(1):41-50. PMID: 1381070.

47. Peters JL, Large RG. A randomised control trial evaluating in- and outpatient pain management programmes. Pain. 1990 Jun;41(3):283-93. PMID: 1697057.

48. Reneman MF, Waterschoot FPC, Burgerhof JGM, et al. Dosage of pain rehabilitation programmes for patients with chronic musculoskeletal pain: a non-inferiority randomised controlled trial. Disability and rehabilitation. 2020;42(6):814-21. PMID: CN-02100807 NEW.
49. Roche G, Ponthieux A, Parot-Shinkel E, et al. Comparison of a functional restoration program with active individual physical therapy for patients with chronic low back pain: a randomized controlled trial. Arch Phys Med Rehabil. 2007 Oct;88(10):122935. doi: 10.1016/j.apmr.2007.07.014. PMID: 17908562 .

50. Roche-Leboucher G, Petit-Lemanac'h A, Bontoux L, et al. Multidisciplinary intensive functional restoration versus outpatient active physiotherapy in chronic low back pain: a randomized controlled trial. Spine (Phila Pa 1976). 2011 Dec 15;36(26):223542. doi: 10.1097/BRS.0b013e3182191e13. PMID: 21415807.

51. Ronzi Y, Roche-Leboucher G, Begue C, et al. Efficiency of three treatment strategies on occupational and quality of life impairments for chronic low back pain patients: is the multidisciplinary approach the key feature to success? Clinical Rehabilitation. 2017 Oct;31(10):1364-73. doi:

https://dx.doi.org/10.1177/02692155176910 86. PMID: 28592147.

52. Rose MJ, Reilly JP, Pennie B, et al. Chronic low back pain rehabilitation programs: a study of the optimum duration of treatment and a comparison of group and individual therapy. Spine. 1997 Oct 01;22(19):224651; discussion 52-3. PMID: 9346145.

53. Rothman MG, Ortendahl M, Rosenblad A, et al. Improved quality of life, working ability, and patient satisfaction after a pretreatment multimodal assessment method in patients with mixed chronic muscular pain: A randomized-controlled study. The Clinical Journal of Pain. 2013

Mar;29(3):195-204. PMID: 2013-10222002.

54. Salvat I, Zaldivar P, Monterde S, et al. Functional status, physical activity level, and exercise regularity in patients with fibromyalgia after Multidisciplinary treatment: retrospective analysis of a randomized controlled trial. Rheumatology International. 2017 Mar;37(3):377-87. doi: https://dx.doi.org/10.1007/s00296-0163597-x. PMID: 27844124. 
55. Saral I, Sindel D, Esmaeilzadeh S, et al. The effects of long- and short-term interdisciplinary treatment approaches in women with fibromyalgia: a randomized controlled trial. Rheumatology International. 2016 Oct;36(10):1379-89. doi: https://dx.doi.org/10.1007/s00296-0163473-8. PMID: 27055444.

56. Scholten C, Brodowicz T, Graninger W, et al. Persistent functional and social benefit 5 years after a multidisciplinary arthritis training program. Archives of Physical Medicine \& Rehabilitation. 1999 Oct;80(10):1282-7. PMID: 10527088.

57. Schweikert B, Jacobi E, Seitz R, et al. Effectiveness and cost-effectiveness of adding a cognitive behavioral treatment to the rehabilitation of chronic low back pain. Journal of Rheumatology. 2006 Dec;33(12):2519-26. PMID: 17143986.

58. Smeets RJ, Vlaeyen JW, Hidding A, et al. Chronic low back pain: physical training, graded activity with problem solving training, or both? The one-year posttreatment results of a randomized controlled trial. Pain. 2008 Feb;134(3):263-76. PMID: 17498879.

59. Smeets RJ, Vlaeyen JW, Hidding A, et al. Active rehabilitation for chronic low back pain: cognitive-behavioral, physical, or both? First direct post-treatment results from a randomized controlled trial

[ISRCTN22714229]. BMC Musculoskeletal Disorders. 2006a Jan 20;7:5. PMID: 16426449 .

60. Smith J, Faux SG, Gardner T, et al. Reboot Online: a Randomized Controlled Trial Comparing an Online Multidisciplinary Pain Management Program with Usual Care for Chronic Pain. Pain medicine. 2019 PMID: CN-01987380 NEW.

61. Streibelt M, Bethge M. Effects of intensified work-related multidisciplinary rehabilitation on occupational participation: a randomizedcontrolled trial in patients with chronic musculoskeletal disorders. International Journal of Rehabilitation Research. 2014 Mar;37(1):61-6. doi: https://dx.doi.org/10.1097/MRR.000000000 0000031. PMID: 24056065.
62. Tavafian SS, Jamshidi AR, Mohammad K. Treatment of chronic low back pain: a randomized clinical trial comparing multidisciplinary group-based rehabilitation program and oral drug treatment with oral drug treatment alone. Clinical Journal of Pain. 2011 Nov-Dec;27(9):811-8. doi: https://dx.doi.org/10.1097/AJP.0b013e3182 1e7930. PMID: 21642845.

63. Tavafian SS, Jamshidi AR, Mohammad K. Treatment of low back pain: randomized clinical trial comparing a multidisciplinary group-based rehabilitation program with oral drug treatment up to 12 months.

International Journal of Rheumatic Diseases. 2014 Feb;17(2):159-64. doi: https://dx.doi.org/10.1111/1756185X.12116. PMID: 24576271.

64. Tavafian SS, Jamshidi AR, Mohammad K. Treatment of low back pain: Second extended follow up of an original trial (NCT00600197) comparing a multidisciplinary group-based rehabilitation program with oral drug treatment alone up to 30 months. International Journal of Rheumatic Diseases. 2017b

Dec;20(12):1910-6. doi: https://dx.doi.org/10.1111/1756185X.12540. PMID: 25546488.

65. Tavafian SS, Jamshidi AR, Montazeri A. A randomized study of back school in women with chronic low back pain: quality of life at three, six, and twelve months follow-up. Spine (Phila Pa 1976). 2008 Jul 1;33(15):1617-21. doi: 10.1097/BRS.0b013e31817bd31c. PMID: 18580739.

66. Tavafian SS, Jamshidi AR, Shay B. Treatment of low back pain: first extended follow up of an original trial (NCT00600197) comparing a multidisciplinary group-based rehabilitation program with oral drug treatment alone up to 24 months. International journal of rheumatic diseases. 2017a;20(12):1902-9. PMID: CN-01449489 NEW.

67. Thieme K, Gromnica-Ihle E, Flor H. Operant behavioral treatment of fibromyalgia: a controlled study. Arthritis Rheum. 2003 Jun 15;49(3):314-20. doi: 10.1002/art.11124. PMID: 12794785. 
68. Turner JA, Clancy S, McQuade KJ, et al. Effectiveness of behavioral therapy for chronic low back pain: a component analysis. Journal of Consulting \& Clinical Psychology. 1990 Oct;58(5):573-9. PMID: 2147702.

69. Turner-Stokes L, Erkeller-Yuksel F, Miles A, et al. Outpatient cognitive behavioral pain management programs: a randomized comparison of a group-based multidisciplinary versus an individual therapy model. Archives of Physical Medicine \& Rehabilitation. 2003 Jun;84(6):781-8. PMID: 12808527.

70. Van der Maas LC, Koke A, Pont M, et al. Improving the Multidisciplinary Treatment of Chronic Pain by Stimulating Body Awareness: A Cluster-randomized Trial. Clinical Journal of Pain. 2015 Jul;31(7):6609. doi:

https://dx.doi.org/10.1097/AJP.0000000000 000138. PMID: 25119509.

71. van Eijk-Hustings Y, Kroese M, Creemers $\mathrm{A}$, et al. Resource utilisation and direct costs in patients with recently diagnosed fibromyalgia who are offered one of three different interventions in a randomised pragmatic trial. Clinical Rheumatology. 2016 May;35(5):1307-15. doi: https://dx.doi.org/10.1007/s10067-0153067-y. PMID: 26409883.

72. van Eijk-Hustings Y, Kroese M, Tan F, et al. Challenges in demonstrating the effectiveness of multidisciplinary treatment on quality of life, participation and health care utilisation in patients with fibromyalgia: a randomised controlled trial. Clinical Rheumatology. 2013 Feb;32(2):199-209. doi: https://dx.doi.org/10.1007/s10067-0122100-7. PMID: 23053692.

73. van Koulil S, van Lankveld W, Kraaimaat FW, et al. Tailored cognitive-behavioural therapy and exercise training improves the physical fitness of patients with fibromyalgia. Annals of the Rheumatic Diseases. 2011 Dec;70(12):2131-3. doi: https://dx.doi.org/10.1136/ard.2010.148577. PMID: 21926189.
74. van Koulil S, van Lankveld W, Kraaimaat FW, et al. Tailored cognitive-behavioral therapy and exercise training for high-risk patients with fibromyalgia. Arthritis Care Res (Hoboken). 2010 Oct;62(10):1377-85. doi: 10.1002/acr.20268. PMID: 20521308.

75. Von Korff M, Balderson BH, Saunders K, et al. A trial of an activating intervention for chronic back pain in primary care and physical therapy settings. Pain. 2005 Feb;113(3):323-30. doi: 10.1016/j.pain.2004.11.007. PMID: 15661440.

76. Weiner DK, Gentili A, Rossi M, et al. Aging Back Clinics-a Geriatric Syndrome Approach to Treating Chronic Low Back Pain in Older Adults: Results of a Preliminary Randomized Controlled Trial. Pain Med. 2020 Feb 1;21(2):274-90. doi: 10.1093/pm/pnz179. PMID: 31503275.

77. Whitfill T, Haggard R, Bierner SM, et al. Early intervention options for acute low back pain patients: a randomized clinical trial with one-year follow-up outcomes. Journal of Occupational Rehabilitation. 2010 Jun;20(2):256-63. doi: https://dx.doi.org/10.1007/s10926-0109238-4. PMID: 20369277.

78. Williams AC, Richardson PH, Nicholas MK, et al. Inpatient vs. outpatient pain management: results of a randomised controlled trial. Pain. 1996 Jul;66(1):13-22. PMID: 8857627. 


\section{Appendix E. Evidence Tables}

Shown in associated Excel file. 


\section{Appendix F. Risk of Bias Assessments}

Shown in associated Excel file. 


\section{Appendix G. Strength of Evidence}

All outcomes were considered direct; therefore, the Directness domain is not shown on the strength of evidence tables. See Appendix D. Included Studies for references.

\begin{tabular}{|c|c|c|c|c|c|c|c|c|}
\hline Comparison & Outcome & $\begin{array}{c}\text { Number of RCTs } \\
\text { (Patients) } \\
\text { Author Year }\end{array}$ & $\begin{array}{c}\text { Study } \\
\text { Limitations }\end{array}$ & Consistency & Precision & $\begin{array}{c}\text { Reporting } \\
\text { Bias }\end{array}$ & $\begin{array}{l}\text { Strength } \\
\text { of } \\
\text { Evidence }\end{array}$ & $\begin{array}{c}\text { Findings, Direction, and Magnitude } \\
\text { of Effect }\end{array}$ \\
\hline \multirow[t]{5}{*}{$\begin{array}{l}\text { IPMPs vs. usual } \\
\text { care }\end{array}$} & $\begin{array}{l}\text { Pain } \\
\text { Postintervention }^{a}\end{array}$ & $\begin{array}{l}4(\mathrm{~N}=1142) \\
\\
\text { Allen, } 2016 \\
\text { Allen, } 2017 \\
\text { Dobscha, } 2009 \\
\text { Von Korff, } 2005\end{array}$ & Moderate & Consistent & Precise & Undetected & Moderate & $\begin{array}{l}\text { Pooled difference }-0.31,95 \% \mathrm{Cl} \\
-0.51 \text { to }-0.11, \mathrm{I}^{2}=0 \% \text {, on a } 0 \text { to } 10 \\
\text { scale }\end{array}$ \\
\hline & $\begin{array}{l}\text { Pain } \\
\text { Short term }\end{array}$ & $\begin{array}{l}2(\mathrm{~N}=721) \\
\text { Mas, } 2019 \\
\text { von Korff, } 2005\end{array}$ & Moderate & Consistent & Imprecise & Undetected & Low & $\begin{array}{l}\text { Pooled difference }-0.59,95 \% \mathrm{Cl} \\
-1.17 \text { to }-0.07, \mathrm{I}^{2}=0 \% \text {, on a } 0 \text { to } 10 \\
\text { scale }\end{array}$ \\
\hline & $\begin{array}{l}\text { Pain } \\
\text { Intermediate term }\end{array}$ & $\begin{array}{l}1(\mathrm{~N}=197) \\
\text { von Korff, } 2005\end{array}$ & Moderate & Unknown & Imprecise & Undetected & Low & $\begin{array}{l}\text { Difference }-0.70,95 \% \mathrm{Cl}-1.13 \text { to } \\
-0.09 \text {, on a } 0 \text { to } 10 \text { scale }\end{array}$ \\
\hline & $\begin{array}{l}\text { Pain } \\
\text { Long term }\end{array}$ & $\begin{array}{l}2(\mathrm{~N}=688) \\
\text { Mas, } 2019 \\
\text { von Korff, } 2005\end{array}$ & Moderate & Consistent & Imprecise & Undetected & Low & $\begin{array}{l}\text { Pooled difference }-0.28,95 \% \mathrm{Cl} \\
-0.80 \text { to } 0.23, \mathrm{I}^{2}=0 \% \text {, on a } 0 \text { to } 10 \text { scale }\end{array}$ \\
\hline & $\begin{array}{l}\text { Function } \\
\text { Postintervention }^{a}\end{array}$ & $\begin{array}{l}\text { Continuous } \\
4(\mathrm{~N}=1142) \\
\text { Allen } 2016 \\
\text { Allen, 2016 } \\
\text { Dobscha, } 2009 \\
\text { von Korff, } 2005 \\
\text { RMD success } \\
2(\mathrm{~N}=608) \\
\text { Dobscha, } 2009 \\
\text { Von Korff, } 2005 \\
\text { wOMAC success } \\
2 \text { (N=399) } \\
\text { Allen, 2016 } \\
\text { Allen, 2017 }\end{array}$ & Moderate & $\begin{array}{l}\text { Consistent } \\
\text { (based on } \\
\text { pooled } \\
\text { continuous) }\end{array}$ & Precise & Undetected & $\begin{array}{l}\text { Moderate } \\
\text { (based on } \\
\text { pooled } \\
\text { continuous } \\
\text { data) }\end{array}$ & $\begin{array}{l}\text { Continuous (across conditions) } \\
\text { Pooled SMD, }-0.20,95 \% \mathrm{Cl}-0.34 \text { to } \\
-0.06, \mathrm{I}^{2}=0 \% \\
\text { RMD success ( } \geq 30 \% \text { improvement, } 0 \\
\text { to } 23 \text { or } 24 \text { scale) } \\
2 \text { trials (LBP, MSK pain): } 23 \% \text { vs. } 13 \% \text {, } \\
\text { pooled RR } 1.73,95 \% \mathrm{Cl} 1.14 \text { to } 2.80, \\
\mathrm{I}^{2}=0 \\
\text { womAC function success ( } \geq 18 \% \\
\text { improvement ( } 0 \text { to } 68 \text { scale) } \\
2 \text { trials (OA): } 18 \% \text { vs. } 21 \%, \\
\text { pooled RR } 1.05,95 \% \mathrm{Cl} 0.69 \text { to } 1.65, \\
I^{2}=0 \%\end{array}$ \\
\hline
\end{tabular}




\begin{tabular}{|c|c|c|c|c|c|c|c|c|}
\hline Comparison & Outcome & $\begin{array}{c}\text { Number of RCTs } \\
\text { (Patients) } \\
\text { Author Year }\end{array}$ & $\begin{array}{c}\text { Study } \\
\text { Limitations }\end{array}$ & Consistency & Precision & $\begin{array}{l}\text { Reporting } \\
\text { Bias }\end{array}$ & $\begin{array}{l}\text { Strength } \\
\text { of } \\
\text { Evidence }\end{array}$ & $\begin{array}{l}\text { Findings, Direction, and Magnitude } \\
\text { of Effect }\end{array}$ \\
\hline & $\begin{array}{l}\text { Function } \\
\text { Short term }\end{array}$ & $\begin{array}{l}\text { RMDQ success } \\
1(\mathrm{~N}=207) \\
\text { Von, Korff } 2005 \\
\text { Continuous } \\
2(\mathrm{~N}=721) \\
\\
\text { Mas, } 2019 \\
\text { Von, Korff } 2005\end{array}$ & Moderate & Consistent & Precise & Undetected & Moderate & $\begin{array}{l}\text { RMD success ( } \geq 30 \% \text { improvement, } 0 \\
\text { to } 23 \text { or } 24 \text { scale) } \\
42 \% \text { vs. } 23 \% \\
\text { RR } 1.81,95 \% \mathrm{Cl} 1.20 \text { to } 2.72 \\
\text { Continuous } \\
\text { SMD }-0.23,95 \% \mathrm{Cl}-0.40 \text { to }-0.02 \text {, } \\
I^{2}=0 \%\end{array}$ \\
\hline & $\begin{array}{l}\text { Function } \\
\text { Intermediate term }\end{array}$ & $\begin{array}{l}\text { RMDQ success } \\
1(\mathrm{~N}=207) \\
\text { Von, Korff } 2005 \\
\text { Continuous } \\
1(\mathrm{~N}=220) \\
\text { Von, Korff } 2005\end{array}$ & Moderate & Unknown & Precise & Undetected & Low & $\begin{array}{l}\text { RMDQ success ( } \geq 30 \% \text { improvement, } \\
\text { 0 to } 23 \text { or } 24 \text { scale) } \\
45 \% \text { vs } 23 \% \\
\text { RR } 1.97,95 \% \mathrm{Cl} 1.30 \text { to } 2.98 \\
\text { Continuous } \\
\text { SMD }-0.10,95 \% \mathrm{Cl}-0.38 \text { to } 0.17\end{array}$ \\
\hline & $\begin{array}{l}\text { Function } \\
\text { Long term }\end{array}$ & $\begin{array}{l}\text { RMDQ success } \\
1(\mathrm{~N}=207) \\
\text { Von, Korff } 2005 \\
\text { Continuous } \\
2(\mathrm{~N}=688) \\
\\
\text { Mas, 2019 } \\
\text { von Korff, } 2005 \\
\end{array}$ & Moderate & Unknown & Imprecise & Undetected & Low & $\begin{array}{l}\text { RMDQ success ( } \geq \mathbf{3 0} \% \text { improvement, } \\
\mathbf{0} \text { to } 23 \text { or } \mathbf{2 4} \text { scale) } \\
49 \% \text { vs. } 37 \% \\
\text { RR } 1.35,95 \% \mathrm{Cl} 0.98 \text { to } 1.85 \\
\text { Continuous } \\
\text { SMD }-0.19,95 \% \mathrm{Cl}-0.36 \text { to } 0.01 \text {, } \\
I^{2}=0 \%\end{array}$ \\
\hline & $\begin{array}{l}\text { Opioid Use } \\
\text { Postintervention }^{b}\end{array}$ & $\begin{array}{l}1(\mathrm{~N}=397) \\
\text { Dobscha, } 2009\end{array}$ & Moderate & Unknown & Precise & Undetected & Low & $\begin{array}{l}\text { Adjusted estimates } \mathrm{c} \text { : } \\
\text { Opioid prescription during intervention } \\
(65 \% \text { vs. } 61 \%, p=0.56) \\
\\
\text { Receipt of long-acting opioids when } \\
\text { prescribed ( } 31 \% \text { vs. } 18 \%, p=0.03)\end{array}$ \\
\hline & $\begin{array}{l}\text { Opioid Use } \\
\text { Intermediate term }\end{array}$ & $\begin{array}{l}1(\mathrm{~N}=41) \\
\text { Angeles, } 2013\end{array}$ & High & Unknown & Imprecise & Undetected & Insufficient & $\begin{array}{l}\text { Early opioid refill } \\
7.7 \%(1 / 19) \text { vs. } 25 \%(6 / 22), p=0.08 ; R R \\
0.19,95 \% \mathrm{Cl} 0.03 \text { to } 1.46 \\
\text { Increase in opioid dose } \\
11.5 \%(2 / 19) \text { vs. } 9.4 \%(2 / 22), p=0.56 ; \\
\text { RR } 1.16,95 \% \mathrm{Cl} 0.18 \text { to } 7.45\end{array}$ \\
\hline
\end{tabular}




\begin{tabular}{|c|c|c|c|c|c|c|c|c|}
\hline Comparison & Outcome & $\begin{array}{l}\text { Number of RCTs } \\
\text { (Patients) } \\
\text { Author Year }\end{array}$ & $\begin{array}{c}\text { Study } \\
\text { Limitations }\end{array}$ & Consistency & Precision & $\begin{array}{l}\text { Reporting } \\
\text { Bias }\end{array}$ & $\begin{array}{l}\text { Strength } \\
\text { of } \\
\text { Evidence }\end{array}$ & $\begin{array}{l}\text { Findings, Direction, and Magnitude } \\
\text { of Effect }\end{array}$ \\
\hline & Harms & $\begin{array}{l}2(\mathrm{~N}=837) \\
\\
\text { Allen } 2016 \\
\text { Allen } 2017\end{array}$ & Moderate & Unknown & Imprecise & Undetected & Insufficient & $\begin{array}{l}\text { No intervention-specific adverse events } \\
\text { were seen in two trials. Harms reported } \\
\text { in a third trial were not attributed to the } \\
\text { intervention. }\end{array}$ \\
\hline \multirow[t]{4}{*}{$\begin{array}{l}\text { IPMPs vs. } \\
\text { physical activity }\end{array}$} & $\begin{array}{l}\text { Function } \\
\text { Postintervention }^{d}\end{array}$ & $\begin{array}{l}1(\mathrm{~N}=152) \\
\text { McBeth, } 2012\end{array}$ & Moderate & Unknown & Imprecise & Undetected & Low & $\begin{array}{l}\text { Chronic Pain Grade Category 0, I, II } \\
\text { instead of III/IV } \\
92 \% \text { vs. } 88 \%, \text { RR } 1.04,95 \% \text { Cl } 0.94 \text { to } \\
1.16\end{array}$ \\
\hline & $\begin{array}{l}\text { Function } \\
\text { Short term }\end{array}$ & $\begin{array}{l}1(\mathrm{~N}=145) \\
\text { McBeth, } 2012\end{array}$ & Moderate & Unknown & Imprecise & Undetected & Low & $\begin{array}{l}\text { Chronic Pain Grade Category 0, I, II } \\
\text { instead of III/IV } \\
86 \% \text { vs. } 92 \%, \text { RR } 0.94,95 \% \text { Cl } 0.83 \text { to } \\
1.05\end{array}$ \\
\hline & $\begin{array}{l}\text { Function } \\
\text { Long term }\end{array}$ & $\begin{array}{l}1(\mathrm{~N}=145) \\
\text { Beasley, } 2015\end{array}$ & Moderate & Unknown & Imprecise & Undetected & Low & $\begin{array}{l}\text { Chronic Pain Grade Category 0, I, II } \\
\text { instead of III/IV } \\
81 \% \text { vs } 69 \%, \text { RR } 1.17,95 \% \text { Cl } 0.97 \text { to } \\
1.42\end{array}$ \\
\hline & Harms & $\begin{array}{l}1(\mathrm{~N}=221) \\
\text { McBeth, } 2012 \\
\text { Beasley, } 2015\end{array}$ & Moderate & Unknown & Imprecise & Undetected & Insufficient & $\begin{array}{l}\text { No intervention-related harms were } \\
\text { seen. One patient in the exercise group } \\
\text { died of cancer. }\end{array}$ \\
\hline \multirow[t]{4}{*}{$\begin{array}{l}\text { IPMPs vs. } \\
\text { telephone CBT }\end{array}$} & $\begin{array}{l}\text { Function } \\
\text { Postintervention }^{d}\end{array}$ & $\begin{array}{l}1(\mathrm{~N}=134) \\
\text { McBeth, } 2012\end{array}$ & Moderate & Unknown & Precise & Undetected & Low & $\begin{array}{l}\text { Chronic Pain Grade Category } 0, \text { I, II } \\
\text { instead of III/IV } \\
92 \% \text { vs. } 81 \% \text {, RR } 1.1495 \% \text { Cl } 1.0 \text { to } \\
1.31\end{array}$ \\
\hline & $\begin{array}{l}\text { Function } \\
\text { Short term }\end{array}$ & $\begin{array}{l}1(\mathrm{~N}=129) \\
\text { McBeth, } 2012\end{array}$ & Moderate & Unknown & Imprecise & Undetected & Low & $\begin{array}{l}\text { Chronic Pain Grade Category 0, I, II } \\
\text { instead of III/IV } \\
86 \% \text { vs. } 79 \%, \text { RR } 1.10,95 \% \text { Cl } 0.92 \text { to } \\
1.27\end{array}$ \\
\hline & $\begin{array}{l}\text { Function } \\
\text { Long term }\end{array}$ & $\begin{array}{l}1(\mathrm{~N}=140) \\
\text { Beasley, } 2015\end{array}$ & Moderate & Unknown & Imprecise & Undetected & Low & $\begin{array}{l}\text { Chronic Pain Grade Category 0, I, II } \\
\text { instead of III/IVe } \\
81 \% \text { vs. } 82 \%, \text { RR } 1.0,95 \% \text { Cl } 0.85 \text { to } \\
1.16\end{array}$ \\
\hline & Harms & $\begin{array}{l}1(\mathrm{~N}=224) \\
\text { McBeth, } 2012 \\
\text { Beasley, } 2015\end{array}$ & Moderate & Unknown & Imprecise & Undetected & Insufficient & $\begin{array}{l}\text { No intervention-related harms were } \\
\text { seen. One patient in the TCBT group } \\
\text { died of cancer }\end{array}$ \\
\hline
\end{tabular}

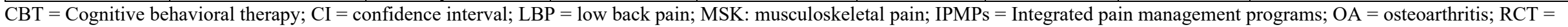

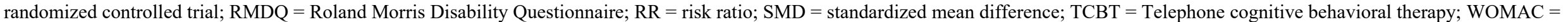
Western Ontario and McMaster University Osteoarthritis Index 
${ }^{a}$ Intervention durations were 2 months (von Korff 2005) and 12 months (Dobscha 2009, Allen 2016, Allen 2017)

${ }^{\mathrm{b}}$ Intervention duration was 12 months (Dobscha 2009)

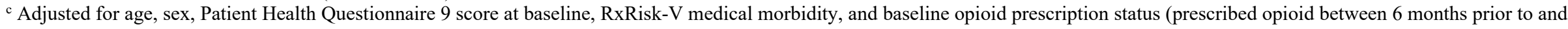

including enrollment date)

${ }^{\mathrm{d}}$ Intervention duration was 6.5 months (McBeth 2012/Beasley 2015)

${ }^{\mathrm{e}}$ Grades of 0 (no pain), I (low disability/low intensity pain), II (low disability/high intensity pain), III (high disability, low intensity pain) IV (high disability, high intensity pain).

\section{Table G-2. CPMPs addressing Key Question 1 strength of evidence}

\begin{tabular}{|c|c|c|c|c|c|c|c|c|}
\hline Comparison & Outcome & $\begin{array}{l}\text { Number of } \\
\text { RCTs (Patients) } \\
\text { Author Year }\end{array}$ & $\begin{array}{c}\text { Study } \\
\text { Limitations }\end{array}$ & Consistency & Precision & $\begin{array}{l}\text { Reporting } \\
\text { Bias }\end{array}$ & $\begin{array}{l}\text { Strength } \\
\text { of } \\
\text { Evidence }\end{array}$ & $\begin{array}{l}\text { Findings, Direction, and } \\
\text { Magnitude of Effect }\end{array}$ \\
\hline \multirow[t]{4}{*}{$\begin{array}{l}\text { CPMPs vs. usual } \\
\text { care or waitlist }\end{array}$} & $\begin{array}{l}\text { Pain } \\
\text { Postintervention }^{\text {a }}\end{array}$ & $\begin{array}{l}11(\mathrm{~N}=764) \\
\\
\text { Abbasi, } 2012 \\
\text { Basler, 1997 } \\
\text { Browne, 2013 } \\
\text { Johansson, } 1998 \\
\text { Lemstra, } 2005 \\
\text { Peters, } 1990 \\
\text { Smeets, } 2006 a \\
\text { Smith, 2019 } \\
\text { Turner, } 1990 \\
\text { van Eijk-Hustings, } \\
2013 \\
\text { Weiner, } 2020\end{array}$ & Moderate & Consistent & Precise & Undetected & Moderate & $\begin{array}{l}\text { Pooled difference }-0.53,95 \% \mathrm{Cl} \\
-0.80 \text { to }-0.25, \mathrm{I}^{2}=0 \% \text {, on a } 0 \text { to } 10 \\
\text { scale }\end{array}$ \\
\hline & $\begin{array}{l}\text { Pain } \\
\text { Short term }\end{array}$ & $\begin{array}{l}6 \text { (N=943) } \\
\text { Amris, } 2014 \\
\text { Bendix, } 1996 \\
\text { Härkäpää, } 1989 \\
\text { Johansson, } 1998 \\
\text { Smith, 2019 } \\
\text { Williams, } 1996\end{array}$ & Moderate & Consistent & Imprecise & Undetected & Low & $\begin{array}{l}\text { Pooled difference }-0.39,95 \% \mathrm{Cl} \\
-0.83 \text { to } 0.04, \mathrm{I}^{2}=36.6 \% \text {, on a } 0 \text { to } 10 \\
\text { scale }\end{array}$ \\
\hline & $\begin{array}{l}\text { Pain } \\
\text { Intermediate term }\end{array}$ & $\begin{array}{l}4(\mathrm{~N}=690) \\
\text { de Buck, } 2005 \\
\text { Härkäpää, } 1990 \\
\text { Saral, } 2016 \\
\text { Whitfill, } 2010\end{array}$ & Moderate & Inconsistent & Imprecise & Undetected & Low & $\begin{array}{l}\text { Pooled difference }-0.85,95 \% \mathrm{Cl} \\
-2.01 \text { to } 0.21, \mathrm{I}^{2}=83.5 \% \text {, on a } 0 \text { to } 10 \\
\text { scale }\end{array}$ \\
\hline & $\begin{array}{l}\text { Pain } \\
\text { Long term }\end{array}$ & $\begin{array}{l}6 \text { (N=906) } \\
\text { Abbasi, 2012 } \\
\text { Bendix, 1998b } \\
\text { de Buck, } 2005 \\
\text { Härkäpää, } 1990 \\
\text { Linton, 2005 } \\
\text { van Eijk-Hustings, } \\
2013\end{array}$ & Moderate & Consistent & Imprecise & Undetected & Low & $\begin{array}{l}\text { Pooled difference }-0.13,95 \% \mathrm{Cl}-0.71 \\
\text { to } 0.22, \mathrm{I}^{2}=19.5 \% \text {, on a } 0 \text { to } 10 \text { scale }\end{array}$ \\
\hline
\end{tabular}




\begin{tabular}{|c|c|c|c|c|c|c|c|c|}
\hline Comparison & Outcome & $\begin{array}{c}\text { Number of } \\
\text { RCTs (Patients) } \\
\text { Author Year }\end{array}$ & $\begin{array}{c}\text { Study } \\
\text { Limitations }\end{array}$ & Consistency & Precision & $\begin{array}{c}\text { Reporting } \\
\text { Bias }\end{array}$ & $\begin{array}{l}\text { Strength } \\
\text { of } \\
\text { Evidence }\end{array}$ & $\begin{array}{l}\text { Findings, Direction, and } \\
\text { Magnitude of Effect }\end{array}$ \\
\hline & $\begin{array}{l}\text { Function } \\
\text { Postintervention }^{\mathrm{b}}\end{array}$ & $\begin{array}{l}13(\mathrm{~N}=981) \\
\text { Abbasi, 2012 } \\
\text { Ahlmen, 1988 } \\
\text { Basler, 1997 } \\
\text { Browne, 2013 } \\
\text { Lemstra, 2005 } \\
\text { Peters, 1990 } \\
\text { Scholten, 1999 } \\
\text { Smeets, 2006a } \\
\text { Smith, 2019 } \\
\text { Turner, 1990 } \\
\text { van Eijk-Hustings, } \\
2013 \\
\text { van Koulil } 2010 \\
\text { Weiner, 2020 }\end{array}$ & Moderate & Inconsistent & Precise & Undetected & Low & $\begin{array}{l}\text { Pooled SMD }-0.52,95 \% \mathrm{Cl}-0.88 \text { to } \\
-0.16, \mathrm{I}^{2}=83.0 \%\end{array}$ \\
\hline & $\begin{array}{l}\text { Function } \\
\text { Short term }\end{array}$ & $\begin{array}{l}7(\mathrm{~N}=1,097) \\
\text { Amris, } 2014 \\
\text { Bendix, 1996 } \\
\text { Härkäpää,1989 } \\
\text { Scholten, 1999 } \\
\text { Smith, 2019 } \\
\text { van Koulil, } 2010 \\
\text { Williams, 1996 } \\
\end{array}$ & Moderate & Inconsistent & Precise & Undetected & Low & $\begin{array}{l}\text { Pooled SMD }-0.62,95 \% \mathrm{Cl}-1.02 \text { to } \\
-0.24, I^{2}=83.7 \%\end{array}$ \\
\hline & $\begin{array}{l}\text { Function } \\
\text { Intermediate term }\end{array}$ & $\begin{array}{l}4(\mathrm{~N}=656) \\
\\
\text { de Buck, } 2005 \\
\text { Härkäpää, } 1990 \\
\text { Saral, 2016 } \\
\text { Scholten, } 1999 \\
\end{array}$ & Moderate & Inonsistent & Imprecise & Undetected & Low & $\begin{array}{l}\text { Pooled SMD }-0.33,95 \% \mathrm{Cl}-0.81 \text { to } \\
0.05, \mathrm{I}^{2}=66.9 \%\end{array}$ \\
\hline & $\begin{array}{l}\text { Function } \\
\text { Long term }\end{array}$ & $\begin{array}{l}6 \text { (N=906) } \\
\text { Abbasi, 2012 } \\
\text { Bendix, 1998b } \\
\text { de Buck, 2005 } \\
\text { Härkäpää, 1990 } \\
\text { Linton, 2005 } \\
\text { van Eijk-Hustings, } \\
2013\end{array}$ & Moderate & Consistent & Precise & Undetected & Low & $\begin{array}{l}\text { Pooled SMD }-0.27(95 \% \mathrm{Cl}-0.47 \text { to } \\
-0.00), I^{2}=42.1 \%\end{array}$ \\
\hline & $\begin{array}{l}\text { Opioid Use } \\
\text { Postintervention }\end{array}$ & $\begin{array}{l}1(\mathrm{~N}=80) \\
\text { Smith, } 2019\end{array}$ & Moderate & Unknown & Imprecise & Undetected & Insufficient & $\begin{array}{l}\text { Insufficient data on opioid use from } 1 \\
\text { trial. }\end{array}$ \\
\hline & $\begin{array}{l}\text { Opioid Use } \\
\text { Long term }\end{array}$ & $\begin{array}{l}1(\mathrm{~N}=121) \\
\text { Williams, } 1996\end{array}$ & High & Unknown & Imprecise & Undetected & Insufficient & $\begin{array}{l}\text { Insufficient evidence from } 1 \text { poor- } \\
\text { quality trial }\end{array}$ \\
\hline
\end{tabular}




\begin{tabular}{|c|c|c|c|c|c|c|c|c|}
\hline Comparison & Outcome & $\begin{array}{c}\text { Number of } \\
\text { RCTs (Patients) } \\
\text { Author Year }\end{array}$ & $\begin{array}{c}\text { Study } \\
\text { Limitations }\end{array}$ & Consistency & Precision & $\begin{array}{l}\text { Reporting } \\
\text { Bias }\end{array}$ & $\begin{array}{l}\text { Strength } \\
\text { of } \\
\text { Evidence }\end{array}$ & $\begin{array}{l}\text { Findings, Direction, and } \\
\text { Magnitude of Effect }\end{array}$ \\
\hline & Harms & $\begin{array}{l}2(N=178) \\
\text { Saral, 2016 } \\
\text { Smeets, 2006a, } \\
2008\end{array}$ & Moderate & Unknown & Imprecise & Undetected & Insufficient & $\begin{array}{l}\text { Two trials reported adverse events } \\
\text { due to the interventions (increased } \\
\text { pain). }\end{array}$ \\
\hline \multirow[t]{4}{*}{$\begin{array}{l}\text { CPMPs vs. } \\
\text { physical activity }\end{array}$} & $\begin{array}{l}\text { Pain } \\
\text { Postintervention }^{\mathrm{d}}\end{array}$ & $\begin{array}{l}8(\mathrm{~N}=1,312) \\
\text { Kappa, } 2006 \\
\text { Roche, 2007 } \\
\text { Schweikert, 2006 } \\
\text { Smeets, 2006a } \\
\text { Turner, 1990 } \\
\text { van Eijk-Hustings, } \\
2013 \\
\text { Meyer, } 2005 \\
\text { Mangels, 2009 } \\
\end{array}$ & Moderate & Consistent & Precise & Undetected & Moderate & $\begin{array}{l}\text { Pooled difference }-0.05,95 \% \mathrm{Cl} \\
-0.32 \text { to } 0.19, I^{2}=0 \%\end{array}$ \\
\hline & $\begin{array}{l}\text { Pain } \\
\text { Short term }\end{array}$ & $\begin{array}{l}1(\mathrm{~N}=106) \\
\text { Bendix, } 1995\end{array}$ & Moderate & Unknown & Imprecise & Undetected & Low & $\begin{array}{l}\text { Difference }-0.35,95 \% \mathrm{Cl}-1.49 \text { to } \\
0.79\end{array}$ \\
\hline & $\begin{array}{l}\text { Pain } \\
\text { Intermediate term }\end{array}$ & $\begin{array}{l}4(\mathrm{~N}=341) \\
\\
\text { Jousset, } 2004 \\
\text { Kaapa, } 2006 \\
\text { Smeets, } 2008 \\
\text { Turner, } 1990\end{array}$ & Moderate & Consistent & Imprecise & Undetected & Low & $\begin{array}{l}\text { Pooled difference }-0.15,95 \% \mathrm{Cl} \\
-0.73 \text { to } 0.38, I^{2}=0 \%\end{array}$ \\
\hline & $\begin{array}{l}\text { Pain } \\
\text { Long term }\end{array}$ & $\begin{array}{l}9(\mathrm{~N}=2,492) \\
\text { Bendix, 1998b } \\
\text { Bendix, 2000 } \\
\text { Kaapa, 2006 } \\
\text { Roche- } \\
\text { LeBoucher, } 2011 \\
\text { Ronzi, 2017 } \\
\text { Smeets, } 2008 \\
\text { Turner, } 1990 \\
\text { van Eijk- } \\
\text { Hustings, } 2013 \\
\text { Mangels, } 2009\end{array}$ & Moderate & Consistent & Precise & Undetected & Moderate & $\begin{array}{l}\text { Pooled difference } 0.05,95 \% \mathrm{Cl}-0.30 \\
\text { to } 0.42, I^{2}=0 \%\end{array}$ \\
\hline
\end{tabular}




\begin{tabular}{|c|c|c|c|c|c|c|c|c|}
\hline Comparison & Outcome & $\begin{array}{l}\text { Number of } \\
\text { RCTs (Patients) } \\
\text { Author Year }\end{array}$ & $\begin{array}{c}\text { Study } \\
\text { Limitations }\end{array}$ & Consistency & Precision & $\begin{array}{l}\text { Reporting } \\
\text { Bias }\end{array}$ & $\begin{array}{c}\text { Strength } \\
\text { of } \\
\text { Evidence }\end{array}$ & $\begin{array}{l}\text { Findings, Direction, and } \\
\text { Magnitude of Effect }\end{array}$ \\
\hline & $\begin{array}{l}\text { Function } \\
\text { Postintervention }\end{array}$ & $\begin{array}{l}9(\mathrm{~N}=1,379) \\
\\
\text { Henchoz, } 2010 \\
\text { Kaapa, } 2006 \\
\text { Roche, } 2007 \\
\text { Schweikert, } 2006 \\
\text { Smeets, 2006a } \\
\text { Turner, } 1990 \\
\text { van Eijk-Hustings, } \\
2013 \\
\text { Meyer, } 2005 \\
\text { Mangels, } 2009\end{array}$ & Moderate & Consistent & Precise & Undetected & Moderate & $\begin{array}{l}\text { Pooled SMD }-0.05,95 \% \mathrm{Cl}-0.16 \text { to } \\
0.05, \mathrm{I}^{2}=0 \%\end{array}$ \\
\hline & $\begin{array}{l}\text { Function } \\
\text { Short term }\end{array}$ & $\begin{array}{l}3(\mathrm{~N}=459) \\
\text { Alaranta, } 1994 \\
\text { Bendix, } 1995 \\
\text { Henchoz, } 2010\end{array}$ & Moderate & Consistent & Precise & Undetected & Moderate & $\begin{array}{l}\text { Pooled SMD }-0.37,95 \% \mathrm{Cl}-0.61 \text { to } \\
-0.16, \mathrm{I}^{2}=0 \%\end{array}$ \\
\hline & $\begin{array}{l}\text { Function } \\
\text { Intermediate term }\end{array}$ & $\begin{array}{l}6(\mathrm{~N}=695) \\
\text { Alaranta, } 1994 \\
\text { Henchoz, } 2010 \\
\text { Jousset, } 2004 \\
\text { Kaapa, } 2006 \\
\text { Smeets, } 2008 \\
\text { Turner, } 1990 \\
\end{array}$ & Moderate & Consistent & Precise & Undetected & Moderate & $\begin{array}{l}\text { Pooled SMD }-0.11,95 \% \mathrm{Cl}-0.36 \text { to } \\
0.13, \mathrm{I}^{2}=38.3 \%\end{array}$ \\
\hline & $\begin{array}{l}\text { Function } \\
\text { Long term }\end{array}$ & $\begin{array}{l}10(\mathrm{~N}=1,214) \\
\\
\text { Bendix, 1998b } \\
\text { Bendix, 2000 } \\
\text { Henchoz, 2010 } \\
\text { Kaapa, 2006 } \\
\text { Roche- } \\
\text { LeBoucher, } 2011 \\
\text { Ronzi, 2017 } \\
\text { Smeets, } 2008 \\
\text { Turner, } 1990 \\
\text { van Eijk- } \\
\text { Hustings, } 2013 \\
\text { Mangels, } 2009 \\
\end{array}$ & Moderate & Consistent & Precise & Undetected & Moderate & $\begin{array}{l}\text { Pooled SMD }-0.12,95 \% \mathrm{Cl}-0.31 \text { to } \\
0.06, \mathrm{I}^{2}=43.3 \%\end{array}$ \\
\hline & Harms & $\begin{array}{l}1(\mathrm{~N}=116) \\
\text { Ronzi, } 2017\end{array}$ & High & Unknown & Imprecise & Undetected & Insufficient & $\begin{array}{l}\text { One trial reported no adverse events } \\
\text { related to the interventions. }\end{array}$ \\
\hline
\end{tabular}




\begin{tabular}{|c|c|c|c|c|c|c|c|c|}
\hline Comparison & Outcome & $\begin{array}{c}\text { Number of } \\
\text { RCTs (Patients) } \\
\text { Author Year }\end{array}$ & $\begin{array}{c}\text { Study } \\
\text { Limitations }\end{array}$ & Consistency & Precision & $\begin{array}{c}\text { Reporting } \\
\text { Bias }\end{array}$ & $\begin{array}{l}\text { Strength } \\
\text { of } \\
\text { Evidence }\end{array}$ & $\begin{array}{l}\text { Findings, Direction, and } \\
\text { Magnitude of Effect }\end{array}$ \\
\hline \multirow[t]{4}{*}{$\begin{array}{l}\text { CPMPs vs. } \\
\text { pharmacologic } \\
\text { therapy }\end{array}$} & $\begin{array}{l}\text { Pain } \\
\text { Postintervention }^{\mathrm{f}}\end{array}$ & $\begin{array}{l}\text { Continuous } \\
2(\mathrm{~N}=204) \\
\text { Onac } 2012 \\
\text { Castel } 2013 \\
\text { Success } \\
1(\mathrm{~N}=155) \\
\text { Castel } 2013\end{array}$ & Moderate & Consistent & Imprecise & Undetected & Low & $\begin{array}{l}\text { Continuous } \\
\text { VAS or NRS }(0-10 \text { scale) } \\
\text { Pooled difference }-1.28,95 \% \mathrm{Cl} \\
-2.14 \text { to }-0.63, \mathrm{I}^{2}=0 \% \\
\text { Success: } \geq 30 \% \text { improvement on } \\
\text { the NRS } \\
22.2 \% \text { vs. } 6.7 \%, \text { RR } 3.3(95 \% \mathrm{Cl} 1.3 \\
\text { to } 8.4)\end{array}$ \\
\hline & $\begin{array}{l}\text { Pain } \\
\text { Short term }\end{array}$ & $\begin{array}{l}1(\mathrm{~N}=155) \\
\text { Castel } 2013\end{array}$ & Moderate & Unknown & Imprecise & Undetected & Low & $\begin{array}{l}\text { Continuous } \\
\text { VAS or NRS }(0-10 \text { scale) } \\
\text { Difference }-0.40,95 \% \mathrm{Cl}-0.98 \text { to } \\
0.18 \\
\\
\text { Success: } \geq 30 \% \text { improvement on } \\
\text { the NRS } \\
13.6 \% \text { vs. } 10.8 \%, \text { RR } 1.3(95 \% \mathrm{Cl} \\
0.53 \text { to } 3.0)\end{array}$ \\
\hline & $\begin{array}{l}\text { Pain } \\
\text { Intermediate term }\end{array}$ & $\begin{array}{l}\text { Continuous } \\
2(N=265) \\
\text { Martin } 2014 \mathrm{c} \\
\text { Castel } 2013 \\
\text { Success } \\
1(\mathrm{~N}=155) \\
\text { Castel } 2013\end{array}$ & Moderate & Consistent & Imprecise & Undetected & Low & $\begin{array}{l}\text { Continuous } \\
\text { VAS or NRS }(0-10 \text { scale) } \\
\text { Pooled difference }-0.85,95 \% \mathrm{Cl}- \\
1.54 \text { to }-0.15, \mathrm{I}^{2}=0 \% \\
\\
\text { Success: } \geq 30 \% \text { improvement on } \\
\text { the NRS } \\
16.0 \% \text { vs. } 5.4 \% \text {, RR } 3.0(95 \% \mathrm{Cl} 1.0 \\
\text { to } 8.7)\end{array}$ \\
\hline & $\begin{array}{l}\text { Pain } \\
\text { Long term }\end{array}$ & $\begin{array}{l}1(\mathrm{~N}=155) \\
\text { Castel 2013 }\end{array}$ & Moderate & Unknown & Imprecise & Undetected & Low & $\begin{array}{l}\text { Continuous } \\
\text { VAS or NRS }(0-10 \text { scale) } \\
\text { Difference }-0.40,95 \% \mathrm{Cl}-0.94 \text { to } \\
0.14 \\
\text { Success: } \geq 30 \% \text { improvement on } \\
\text { the NRS } \\
8.6 \% \text { vs. } 0 \%, \text { RR not calculable }\end{array}$ \\
\hline
\end{tabular}




\begin{tabular}{|c|c|c|c|c|c|c|c|c|}
\hline Comparison & Outcome & $\begin{array}{c}\text { Number of } \\
\text { RCTs (Patients) } \\
\text { Author Year }\end{array}$ & $\begin{array}{c}\text { Study } \\
\text { Limitations }\end{array}$ & Consistency & Precision & $\begin{array}{l}\text { Reporting } \\
\text { Bias }\end{array}$ & $\begin{array}{c}\text { Strength } \\
\text { of } \\
\text { Evidence }\end{array}$ & $\begin{array}{l}\text { Findings, Direction, and } \\
\text { Magnitude of Effect }\end{array}$ \\
\hline & $\begin{array}{l}\text { Function } \\
\text { Postintervention }^{\mathrm{f}}\end{array}$ & $\begin{array}{l}\text { Continuous } \\
2(N=204) \\
\text { Onac } 2012 \\
\text { Castel } 2013 \\
\text { Success } \\
1(N=155) \\
\text { Castel } 2013\end{array}$ & Moderate & Unknown & Imprecise & Undetected & $\begin{array}{c}\text { Low } \\
\text { (based on } \\
\text { better quality } \\
\text { trial) }\end{array}$ & $\begin{array}{l}\text { Continuous } \\
\text { Pooled analysis ( } 2 \text { trials, } \mathrm{N}=204, \\
\mathrm{SMD}-0.57,95 \% \mathrm{Cl}-1.22 \text { to } 0.62 \text {, } \\
\mathrm{I}^{2}=74.5 \% \\
\\
\text { Individual studies } \\
\text { Fair-quality trial ( } \mathrm{N}=155) \text { : } \mathrm{FIQ}(0- \\
100) \text {, difference }-18.2,95 \% \mathrm{Cl}-24.0 \\
\text { to }-12.4) \\
\text { Poor-quality trial: } \mathrm{RMDQ}(0-24 \text { scale), } \\
\text { difference }-0.24 \text { ( } 95 \% \mathrm{Cl}-4.2 \text { to } 3.8) \\
\\
\text { Success } \geq 14 \% \text { improvement } \mathrm{FIQ} \\
\text { (0-100 scale) } \\
64.2 \% \text { vs. } 24.3 \%, \mathrm{RR} 2.6(95 \% \mathrm{Cl} \\
1.7 \text { to } 4.1\end{array}$ \\
\hline & $\begin{array}{l}\text { Function } \\
\text { Short term }\end{array}$ & $\begin{array}{l}\text { Continuous } \\
2(\mathrm{~N}=342) \\
\text { Tavafian } 2011 \\
\text { Castel } 2013 \\
\text { Success } \\
1(\mathrm{~N}=155) \\
\text { Castel } 2013\end{array}$ & Moderate & Consistent & Imprecise & Undetected & Low & $\begin{array}{l}\text { Continuous } \\
\text { Pooled SMD }-0.37,95 \% \mathrm{Cl}-0.67 \text { to } \\
-0.08, \mathrm{I}^{2}=0 \% \\
\\
\text { Success: } \geq 14 \% \text { improvement FIQ } \\
\text { (0-100 scale) } \\
48.1 \% \text { vs. } 23.0 \%, \text { RR } 2.1(95 \% \mathrm{Cl} \\
1.3 \text { to } 3.4)\end{array}$ \\
\hline & $\begin{array}{l}\text { Function } \\
\text { Intermediate term }\end{array}$ & $\begin{array}{l}\text { Continuous } \\
3(\mathrm{~N}=453) \\
\text { Tavafian } 2011 \\
\text { Castel } 2013 \\
\text { Martin 2014c } \\
\\
\text { Success } \\
1(\mathrm{~N}=155) \\
\text { Castel 2013 }\end{array}$ & Moderate & Consistent & Precise & Undetected & Moderate & $\begin{array}{l}\text { Continuous } \\
\text { Pooled SMD }-0.44,95 \% \mathrm{Cl}-0.67 \text { to } \\
-0.22, l^{2}=0 \% \\
\text { Success: } \geq 14 \% \text { improvement FIQ (0- } \\
100 \text { scale) } \\
42.0 \% \text { vs. } 18.9 \%, \text { RR } 2.2,95 \% \mathrm{Cl} 1.3 \\
\text { to } 3.8\end{array}$ \\
\hline & $\begin{array}{l}\text { Function } \\
\text { Long term }\end{array}$ & $\begin{array}{l}\text { Continuous } \\
2(N=301) \\
\text { Castel 2013 } \\
\text { Tavafian } 2017 b \\
\text { Success } \\
1(N=155) \\
\text { Castel } 2013\end{array}$ & Moderate & Consistent & Imprecise & Undetected & Low & $\begin{array}{l}\text { Continuous } \\
\text { Pooled SMD }-0.46,95 \% \mathrm{Cl}-0.76 \text { to } \\
\left.-0.16, \mathrm{I}^{2}=0 \%\right) \\
\text { Success: } \geq 14 \% \text { improvement FIQ } \\
\text { (0-100 scale) } \\
27.2 \% \text { vs. } 4.0 \% \text {, RR } 6.7,95 \% \mathrm{Cl} 2.1 \\
\text { to } 21.5\end{array}$ \\
\hline & Harms & None & ---- & ---- & ---- & ---- & ----- & No evidence \\
\hline
\end{tabular}




\begin{tabular}{|c|c|c|c|c|c|c|c|c|}
\hline Comparison & Outcome & $\begin{array}{c}\text { Number of } \\
\text { RCTs (Patients) } \\
\text { Author Year }\end{array}$ & $\begin{array}{c}\text { Study } \\
\text { Limitations }\end{array}$ & Consistency & Precision & $\begin{array}{l}\text { Reporting } \\
\text { Bias }\end{array}$ & $\begin{array}{l}\text { Strength } \\
\text { of } \\
\text { Evidence }\end{array}$ & $\begin{array}{l}\text { Findings, Direction, and } \\
\text { Magnitude of Effect }\end{array}$ \\
\hline \multirow[t]{8}{*}{$\begin{array}{l}\text { CPMPs vs. } \\
\text { pharmacologic } \\
\text { therapy plus } \\
\text { physical activity }\end{array}$} & $\begin{array}{l}\text { Pain } \\
\text { Postintervention }\end{array}$ & $\begin{array}{l}2(\mathrm{~N}=118) \\
\text { Thieme } 2003 \\
\text { Onac } 2012\end{array}$ & Moderate & Unknown & Imprecise & Undetected & \begin{tabular}{|c|} 
Low \\
(based on \\
better quality \\
trial)
\end{tabular} & $\begin{array}{l}1 \text { trial: VAS or NRS (0-10 scale) } \\
\text { Difference } 0.93,95 \% \mathrm{Cl}-0.19 \text { to } 2.1 \\
1 \text { trial: MPI-Pain intensity (0-6 scale) } \\
\text { Difference }-1.7,95 \% \mathrm{Cl}-2.2 \text { to }-1.1 \\
\text { (antidepressants) }\end{array}$ \\
\hline & $\begin{array}{l}\text { Pain } \\
\text { Intermediate term }\end{array}$ & $\begin{array}{l}1(\mathrm{~N}=61) \\
\text { Thieme } 2003\end{array}$ & Moderate & Unknown & Imprecise & Undetected & Low & $\begin{array}{l}\text { MPI-Pain intensity (0-6 scale) } \\
\text { Difference }-1.2,95 \% \mathrm{Cl}-1.8 \text { to - } \\
0.59\end{array}$ \\
\hline & $\begin{array}{l}\text { Pain } \\
\text { Long term }\end{array}$ & $\begin{array}{l}1(\mathrm{~N}=61) \\
\text { Thieme } 2003\end{array}$ & Moderate & Unknown & Imprecise & Undetected & Low & $\begin{array}{l}\text { MPI-Pain intensity (0-6 scale) } \\
\text { Difference }-2.1,95 \% \mathrm{Cl}-2.7 \text { to }-1.5\end{array}$ \\
\hline & $\begin{array}{l}\text { Function } \\
\text { Postinterventiong }\end{array}$ & $\begin{array}{l}2(\mathrm{~N}=136) \\
\text { Thieme 2003 } \\
\text { Onac } 2012\end{array}$ & Moderate & Unknown & Imprecise & Undetected & \begin{tabular}{|c|} 
Low \\
(based on \\
better quality \\
trial)
\end{tabular} & $\begin{array}{l}1 \text { trial: RMDQ }(0-24 \text { scale }) \\
\text { Difference } 1.5,95 \% \mathrm{Cl}-2.0 \text { to } 5.0 \\
1 \text { trial: } \mathrm{MPI}-\text { Total activity scale }(0-6) \\
\text { Difference }-0.25,95 \% \mathrm{Cl}-0.66 \text { to } \\
0.16\end{array}$ \\
\hline & $\begin{array}{l}\text { Function } \\
\text { Intermediate term }\end{array}$ & $\begin{array}{l}1(\mathrm{~N}=61) \\
\text { Thieme } 2003\end{array}$ & Moderate & Unknown & Imprecise & Undetected & Low & $\begin{array}{l}\text { MPI-Total activity scale (0-6 scale) } \\
\text { Difference }-0.22,95 \% \mathrm{Cl}-0.68 \text { to } \\
0.24\end{array}$ \\
\hline & $\begin{array}{l}\text { Function } \\
\text { Long term }\end{array}$ & $\begin{array}{l}1(\mathrm{~N}=61) \\
\text { Thieme } 2003\end{array}$ & Moderate & Unknown & Imprecise & Undetected & Low & $\begin{array}{l}\text { MPI-Total activity scale ( } 0-6 \text { scale) } \\
\text { Difference }-0.27,95 \% \mathrm{Cl}-0.69 \text { to } \\
0.15\end{array}$ \\
\hline & $\begin{array}{l}\text { Opioid Use } \\
\text { Long term }\end{array}$ & $\begin{array}{l}1(\mathrm{~N}=61) \\
\text { Thieme } 2003\end{array}$ & Moderate & Unknown & Imprecise & Undetected & Insufficient & $\begin{array}{l}\text { Authors report reduced use of opioids } \\
\text { following CPMP }(p, 0.001) \text { but do not } \\
\text { provide relevant data }\end{array}$ \\
\hline & Harms & None & ----- & ----- & ----- & ----- & ----- & No evidence \\
\hline \multirow[t]{2}{*}{$\begin{array}{l}\text { CPMPs vs. } \\
\text { psychological } \\
\text { therapy }\end{array}$} & $\begin{array}{l}\text { Pain } \\
\text { Postintervention }^{\mathrm{h}}\end{array}$ & $\begin{array}{l}3(\mathrm{~N}=259) \\
\text { Turner, } 1990 \\
\text { Turner-Stokes, } \\
2003 \\
\text { Smeets, } 2008\end{array}$ & Moderate & Consistent & Imprecise & Undetected & Low & $\begin{array}{l}\text { Pooled SMD } 0.03,95 \% \mathrm{Cl}-0.30 \text { to } \\
0.31, \mathrm{I}^{2}=0 \%\end{array}$ \\
\hline & $\begin{array}{l}\text { Pain } \\
\text { Intermediate term }\end{array}$ & $\begin{array}{l}3(\mathrm{~N}=228) \\
\text { Turner, } 1990 \\
\text { Turner-Stokes, } \\
2003 \\
\text { Smeets, } 2008\end{array}$ & Moderate & Consistent & Imprecise & Undetected & Low & $\begin{array}{l}\text { Pooled SMD }-0.09,95 \% \mathrm{Cl}-0.50 \text { to } \\
0.21, \mathrm{I}^{2}=0 \%\end{array}$ \\
\hline
\end{tabular}




\begin{tabular}{|c|c|c|c|c|c|c|c|c|}
\hline Comparison & Outcome & $\begin{array}{c}\text { Number of } \\
\text { RCTs (Patients) } \\
\text { Author Year }\end{array}$ & $\begin{array}{c}\text { Study } \\
\text { Limitations }\end{array}$ & Consistency & Precision & $\begin{array}{l}\text { Reporting } \\
\text { Bias }\end{array}$ & $\begin{array}{l}\text { Strength } \\
\text { of } \\
\text { Evidence }\end{array}$ & $\begin{array}{l}\text { Findings, Direction, and } \\
\text { Magnitude of Effect }\end{array}$ \\
\hline & $\begin{array}{l}\text { Pain } \\
\text { Long term }\end{array}$ & $\begin{array}{l}3(\mathrm{~N}=256) \\
\text { Turner, } 1990 \\
\text { Linton, } 2005 \\
\text { Smeets, } 2008\end{array}$ & Moderate & Consistent & Imprecise & Undetected & Low & $\begin{array}{l}\text { Pooled SMD } 0.05,95 \% \mathrm{Cl}-0.35 \text { to } \\
0.48, \mathrm{I}^{2}=26.1 \%\end{array}$ \\
\hline & $\begin{array}{l}\text { Function } \\
\text { Postintervention }^{\mathrm{h}}\end{array}$ & $\begin{array}{l}3(\mathrm{~N}=262) \\
\text { Turner, 1990 } \\
\text { Turner-Stokes, } \\
2003 \\
\text { Smeets, } 2008\end{array}$ & Moderate & Consistent & Imprecise & Undetected & Low & $\begin{array}{l}\text { Pooled SMD } 0.10,95 \% \mathrm{Cl}-0.23 \text { to } \\
0.36, \mathrm{I}^{2}=0.0 \%\end{array}$ \\
\hline & $\begin{array}{l}\text { Function } \\
\text { Intermediate term }\end{array}$ & $\begin{array}{l}3(\mathrm{~N}=231) \\
\text { Turner, } 1990 \\
\text { Turner-Stokes, } \\
2003 \\
\text { Smeets, } 2008\end{array}$ & Moderate & Consistent & Imprecise & Undetected & Low & $\begin{array}{l}\text { Pooled SMD } 0.11,95 \% \mathrm{Cl}-0.32 \text { to } \\
0.41, \mathrm{I}^{2}=0 \%\end{array}$ \\
\hline & $\begin{array}{l}\text { Function } \\
\text { Long term }\end{array}$ & $\begin{array}{l}3(\mathrm{~N}=259) \\
\text { Turner, } 1990 \\
\text { Linton, } 2005 \\
\text { Smeets, } 2008\end{array}$ & Moderate & Consistent & Imprecise & Undetected & Low & $\begin{array}{l}\text { Pooled SMD } 0.16,95 \% \mathrm{Cl}-0.18 \text { to } \\
0.45, \mathrm{I}^{2}=0 \%\end{array}$ \\
\hline & Harms & $\begin{array}{l}1(\mathrm{~N}=110) \\
\text { Smeets, } 2006 \mathrm{a}\end{array}$ & Moderate & Unknown & Imprecise & Undetected & Insufficient & $\begin{array}{l}\text { Increased pain the low back or } \\
\text { radiating leg pain leading to withdrawal } \\
\text { from the program: } 5.5 \%(3 / 55) \text { vs. } 0 \% \\
(0 / 55)\end{array}$ \\
\hline
\end{tabular}

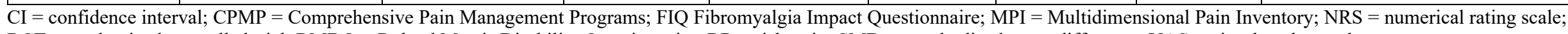
$\mathrm{RCT}=$ randomized controlled trial; RMDQ = Roland Morris Disability Questionnaire; RR = risk ratio; SMD = standardized mean difference; VAS = visual analog scale.

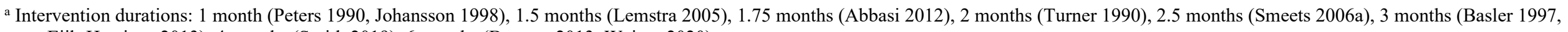
van Eijk-Hustings 2013), 4 months (Smith 2019), 6 months (Browne 2013, Weiner 2020)

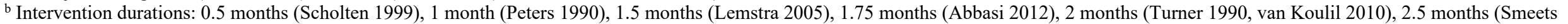

2006a), 3 months (Basler 1997, van Eijk-Hustings 2013), 4 months (Smith 2019), 6 months (Browne 2013, Weiner 2020), 12 months (Ahlmen 1988)

${ }^{\mathrm{c}}$ Intervention duration: 4 months (Smith 2019)

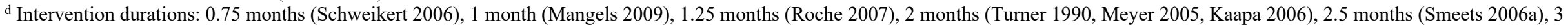
months (van Eijk-Hustings 2013)

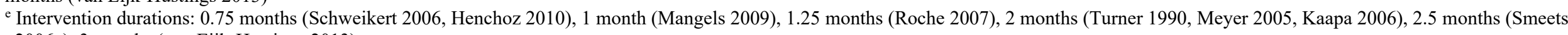
2006a), 3 months (van Eijk-Hustings 2013)

${ }^{\mathrm{f}}$ Intervention durations: 0.5 months (Onac 2012) and 3 months (Castel 2013)

g Intervention durations: 0.5 months (Onac 2012) and 1.25 months (Thieme 2003)

${ }^{\mathrm{h}}$ Intervention durations: 2 months (Turner 1990, Turner-Stokes 2003), 2.5 months (Smeets, 2008) 


\section{Appendix H. Excluded Studies List}

Table H-1. Key to exclusion codes

\begin{tabular}{|c|l|}
\hline Exclusion Code & Exclusion Reason \\
\hline 2 & Case-series, may be applicable \\
\hline 3 & Ineligible population \\
\hline 4 & Ineligible intervention \\
\hline 5 & Ineligible comparator \\
\hline 6 & Ineligible outcomes \\
\hline 7 & Ineligible setting \\
\hline 8 & Ineligible study design \\
\hline 10 & Not a study (trial protocol, letter, editorial, nonsystematic review article) \\
\hline 11 & Systematic review, not directly used, but studies checked for inclusion \\
\hline 12 & Not English language but possibly relevant \\
\hline & Not English language and not relevant \\
\hline
\end{tabular}

\section{Excluded from systematic literature search and hand searching/bibliography review:}

1.

Aasdahl L, Pape K, Vasseljen O, et al.

Effects of Inpatient Multicomponent

Occupational Rehabilitation versus Less

Comprehensive Outpatient Rehabilitation on

Somatic and Mental Health: Secondary

Outcomes of a Randomized Clinical Trial.

Journal of Occupational Rehabilitation.

2017 Sep;27(3):456-66. doi:

https://dx.doi.org/10.1007/s10926-016-

9679-5. PMID: 27815771. Exclusion: 3

2. Akerblom S, Perrin S, Fischer MR, et al.

The mediating role of acceptance in multidisciplinary cognitive-behavioral therapy for chronic pain. The Journal of Pain. 2015 Jul;16(7):606-15. doi: http://dx.doi.org/10.1016/j.jpain.2015.03.00 7. PMID: 2015-22349-001. Exclusion: 2

3. Amris K, Luta G, Christensen R, et al. Predictors of improvement in observed functional ability in patients with fibromyalgia as an outcome of rehabilitation. Journal of Rehabilitation Medicine. 2016 Jan;48(1):65-71. doi: https://dx.doi.org/10.2340/16501977-2036. PMID: 26660148. Exclusion: 8
4. Andersen A, Larsson K, Lytsy P, et al. Strengthened General Self-Efficacy with Multidisciplinary Vocational Rehabilitation in Women on Long-Term Sick Leave: A Randomised Controlled Trial. Journal of Occupational Rehabilitation. 2018 12;28(4):691-700. doi: https://dx.doi.org/10.1007/s10926-0179752-8. PMID: 29318421. Exclusion: 3

5. Anderson FJ, Winkler AE. Benefits of Long-Term Fibromyalgia Syndrome Treatment with a Multidisciplinary Program. Journal of Musculoskeletal Pain. 2006;14(4):11-25. doi: http://dx.doi.org/10.1300/J094v14n04 03. PMID: 2007-00079-002. Exclusion: 8

6. Anderson FJ, Winkler AE. An integrated model of group psychotherapy for patients with fibromyalgia. International Journal of Group Psychotherapy. 2007 Oct;57(4):45174. doi: http://dx.doi.org/10.1521/ijgp.2007.57.4.451 . PMID: 2007-14773-002. Exclusion: 4

7. Andersson S, Sundberg T, Johansson E, et al. Patients' experiences and perceptions of integrative care for back and neck pain. Alternative Therapies in Health \& Medicine. 2012 May-Jun;18(3):25-32. PMID: 22875559. Exclusion: 8 
8. Anema JR, Steenstra IA, Bongers PM, et al. Multidisciplinary rehabilitation for subacute low back pain: graded activity or workplace intervention or both? A randomized controlled trial. Spine (Phila Pa 1976). 2007

Feb 1;32(3):291-8; discussion 9-300. doi:

10.1097/01.brs.0000253604.90039.ad.

PMID: 17268258. Exclusion: 4

9. Angst F, Brioschi R, Main CJ, et al. Interdisciplinary rehabilitation in fibromyalgia and chronic back pain: a prospective outcome study. J Pain. 2006

Nov;7(11):807-15. doi:

10.1016/j.jpain.2006.03.009. PMID:

17074622. Exclusion: 2

10. Angst F, Verra ML, Lehmann S, et al. Effects of inpatient rehabilitation in hip and knee osteoarthritis: a naturalistic prospective cohort study with intraindividual control of effects. Archives of Physical Medicine \& Rehabilitation. 2013 Nov;94(11):2139-45. doi:

https://dx.doi.org/10.1016/j.apmr.2013.03.0 26. PMID: 23587838. Exclusion: 2

11. Angst F, Verra ML, Lehmann S, et al. Clinical effectiveness of an interdisciplinary pain management programme compared with standard inpatient rehabilitation in chronic pain: a naturalistic, prospective controlled cohort study. J Rehabil Med. 2009 Jun;41(7):569-75. doi: 10.2340/16501977-0381. PMID: 19543669. Exclusion: 8

12. Aragones E, Lopez-Cortacans G, Caballero A, et al. Evaluation of a multicomponent programme for the management of musculoskeletal pain and depression in primary care: a cluster-randomised clinical trial (the DROP study). BMC psychiatry. 2016;16(1) PMID: CN-01158730.

Exclusion: 9

13. Aragones E, Rambla C, Lopez-Cortacans G, et al. Effectiveness of a collaborative care intervention for managing major depression and chronic musculoskeletal pain in primary care: A cluster-randomised controlled trial. Journal of Affective Disorders. 201906 01;252:221-9. doi: https://dx.doi.org/10.1016/j.jad.2019.04.004. PMID: 30986737. Exclusion: 4
14. Arnstein P, Herr KA, Butcher HK. Evidence-Based Practice Guideline: Persistent Pain Management in Older Adults. Journal of Gerontological Nursing. 2017 Jul 01;43(7):20-31. doi: https://dx.doi.org/10.3928/0098913420170419-01. PMID: 28651032. Exclusion: 10

15. Asenlof P, Denison E, Lindberg P. Individually tailored treatment targeting activity, motor behavior, and cognition reduces pain-related disability: a randomized controlled trial in patients with musculoskeletal pain. Journal of Pain. 2005 Sep;6(9):588-603. PMID: 16139778. Exclusion: 4

16. Bailey A, Starr L, Alderson M, et al. A comparative evaluation of a fibromyalgia rehabilitation program. Arthritis Care \& Research. 1999 Oct;12(5):336-40. PMID: 11081003. Exclusion: 2

17. Bair MJ, Ang D, Wu J, et al. Evaluation of Stepped Care for Chronic Pain (ESCAPE) in veterans of the iraq and afghanistan conflicts a randomized clinical trial. JAMA internal medicine. 2015;175(5):682-9. PMID: CN01075216. Exclusion: 4

18. Baranoff J, Hanrahan S, Kapur D, et al. Acceptance as a process variable in relation to catastrophizing in multidisciplinary pain treatment. European Journal of Pain. 2013 Jan;17(1):101-10. doi: http://dx.doi.org/10.1002/j.15322149.2012.00165.x. PMID: 2012-33745013. Exclusion: 2

19. Bearne LM, Byrne AM, Segrave H, et al. Multidisciplinary team care for people with rheumatoid arthritis: a systematic review and meta-analysis. Rheumatology International. 2016 Mar;36(3):311-24. doi: https://dx.doi.org/10.1007/s00296-0153380-4. PMID: 26563338. Exclusion: 10 
20. Beltran-Alacreu H, Lopez-de-UraldeVillanueva I, Fernandez-Carnero J, et al. Manual Therapy, Therapeutic Patient Education, and Therapeutic Exercise, an Effective Multimodal Treatment of Nonspecific Chronic Neck Pain: A Randomized Controlled Trial. American Journal of Physical Medicine \& Rehabilitation. 2015 Oct;94(10 Suppl 1):887-97. doi:

https://dx.doi.org/10.1097/PHM.000000000 0000293. PMID: 25888653. Exclusion: 4

21. Bergstrom C, Jensen I, Hagberg J, et al. Effectiveness of different interventions using a psychosocial subgroup assignment in chronic neck and back pain patients: a 10year follow-up. Disability \& Rehabilitation. 2012;34(2):110-8. doi:

https://dx.doi.org/10.3109/09638288.2011.6 07218. PMID: 21988525. Exclusion: 6

22. Bontoux L, Dubus V, Roquelaure Y, et al. Return to work of 87 severely impaired low back pain patients two years after a program of intensive functional rehabilitation. Ann Phys Rehabil Med. 2009 Feb;52(1):17-29. doi: 10.1016/j.rehab.2008.12.005. PMID: 19419656. Exclusion: 2

23. Bontoux L, Roquelaure $\mathrm{Y}$, Billabert C, et al. [Prospective study of the outcome at one year of patients with chronic low back pain in a program of intensive functional restoration and ergonomic intervention. Factors predicting their return to work]. Ann Readapt Med Phys. 2004 Oct;47(8):563-72. doi: 10.1016/j.annrmp.2004.03.006. PMID: 15465161. Exclusion: 2

24. Borys C, Lutz J, Strauss B, et al. Effectiveness of a multimodal therapy for patients with chronic low back pain regarding pre-admission healthcare utilization. Plos one. 2015;10(11) PMID: CN-01138991. Exclusion: 8

25. Boschen KA, Robinson E, Campbell KA, et al. Results from 10 years of a CBT pain selfmanagement outpatient program for complex chronic conditions. Pain research \& management. 2016(pagination) PMID: CN01327924. Exclusion: 2
26. Bourgault P, Lacasse A, Marchand S, et al. Multicomponent interdisciplinary group intervention for self-management of fibromyalgia: a mixed-methods randomized controlled trial. PLoS One.

2015;10(5):e0126324. doi:

10.1371/journal.pone.0126324. PMID: 25978402. Exclusion: 4

27. Bourke JH, Johnson AL, Sharpe M, et al. Pain in chronic fatigue syndrome: response to rehabilitative treatments in the PACE trial. Psychological Medicine. 2014 May;44(7):1545-52. doi: https://dx.doi.org/10.1017/S0033291713002 201. PMID: 23967878. Exclusion: 4

28. Brendbekken R, Harris A, Ursin H, et al. Multidisciplinary Intervention in Patients with Musculoskeletal Pain: a Randomized Clinical Trial. International Journal of Behavioral Medicine. 2016;23(1):1-11. doi: 10.1007/s12529-015-9486-y. PMID: 112860024. Language: English. Entry Date: 20160212. Revision Date: 20170131. Publication Type: Article. Exclusion: 4

29. Brockow T, Wagner A, Franke A, et al. A randomized controlled trial on the effectiveness of mild water-filtered near infrared whole-body hyperthermia as an adjunct to a standard multimodal rehabilitation in the treatment of fibromyalgia. The Clinical Journal of Pain. 2007 Jan;23(1):67-75. doi: http://dx.doi.org/10.1097/AJP.0b013e31802 b4f80. PMID: 2006-23493-010. Exclusion: 4

30. Bron C, de Gast A, Dommerholt J, et al. Treatment of myofascial trigger points in patients with chronic shoulder pain: a randomized, controlled trial. BMC Medicine. 2011 Jan 24;9:8. doi: https://dx.doi.org/10.1186/1741-7015-9-8. PMID: 21261971. Exclusion: 4

31. Bronfort G, Evans R, Anderson AV, et al. Spinal manipulation, medication, or home exercise with advice for acute and subacute neck pain: a randomized trial. Annals of Internal Medicine. 2012 Jan 03;156(1 Pt 1):1-10. doi: https://dx.doi.org/10.7326/0003-4819-1561-201201030-00002. PMID: 22213489.

Exclusion: 4 
32. Bronfort G, Hondras MA, Schulz CA, et al. Spinal manipulation and home exercise with advice for subacute and chronic back-related leg pain: a trial with adaptive allocation. Annals of Internal Medicine. 2014 Sep 16;161(6):381-91. doi: https://dx.doi.org/10.7326/M14-0006. PMID: 25222385. Exclusion: 4

33. Brown CA, Jones AK. Psychobiological correlates of improved mental health in patients with musculoskeletal pain after a mindfulness-based pain management program. Clinical Journal of Pain. 2013 Mar;29(3):233-44. doi: https://dx.doi.org/10.1097/AJP.0b013e3182 4c5d9f. PMID: 22874090. Exclusion: 4

34. Brown M, Dean S, Hay-Smith E, et al. Musculoskeletal pain and treatment choice: An exploration of illness perceptions and choices of conventional or complementary therapies. Disability and Rehabilitation: An International, Multidisciplinary Journal. 2010;32(20):1645-57. doi: http://dx.doi.org/10.3109/096382810036498 96. PMID: 2010-17687-003. Exclusion: 8

35. Brown R, Peikes D, Chen A, et al. 15-site randomized trial of coordinated care in Medicare FFS. Health care financing review. 2008;30(1):5-25. PMID: CN00665022. Exclusion: 3

36. Brox JI, Reikerås O, Nygaard Ø, et al. Lumbar instrumented fusion compared with cognitive intervention and exercises in patients with chronic back pain after previous surgery for disc herniation: a prospective randomized controlled study. Pain. 2006 May;122(1-2):145-55. doi: 10.1016/j.pain.2006.01.027. PMID: 16545523. Exclusion: 5

37. Brox JI, Sørensen R, Friis A, et al. Randomized clinical trial of lumbar instrumented fusion and cognitive intervention and exercises in patients with chronic low back pain and disc degeneration. Spine (Phila Pa 1976). 2003

Sep 1;28(17):1913-21. doi: 10.1097/01.Brs.0000083234.62751.7a. PMID: 12973134. Exclusion: 5
38. Bruce B, Lorig K, Laurent D, et al. The impact of a moderated e-mail discussion group on use of complementary and alternative therapies in subjects with recurrent back pain. Patient Education \& Counseling. 2005 Sep;58(3):305-11. PMID: 16122642. Exclusion: 4

39. Brunahl CA, Klotz SGR, Dybowski C, et al. Combined Cognitive-Behavioural and Physiotherapeutic Therapy for Patients with Chronic Pelvic Pain Syndrome (COMBICPPS): study protocol for a controlled feasibility trial. Trials. 2018;19(1) PMID: CN-01449690. Exclusion: 9

40. Busch H, Bjork Bramberg E, Hagberg J, et al. The effects of multimodal rehabilitation on pain-related sickness absence-An observational study. Disability and Rehabilitation: An International, Multidisciplinary Journal. 2018 Jul;40(14):1646-53. doi: http://dx.doi.org/10.1080/09638288.2017.13 05456. PMID: 2018-13188-005. Exclusion: 6

41. Busch H, Bodin L, Bergstrom G, et al. Patterns of sickness absence a decade after pain-related multidisciplinary rehabilitation. Pain. 2011 PMID: CN-01770749 NEW. Exclusion: 6

42. Buttagat V, Techakhot $\mathrm{P}$, Wiriya $\mathrm{W}$, et al. Effectiveness of traditional Thai selfmassage combined with stretching exercises for the treatment of patients with chronic non-specific low back pain: A singleblinded randomized controlled trial. Journal of Bodywork \& Movement Therapies. 2020;24(1):19-24. doi: 10.1016/j.jbmt.2019.03.017. PMID: 141379770. Language: English. Entry Date: 20200129. Revision Date: 20200131. Publication Type: Article. Exclusion: 4

43. Cabak A, Rudnicka A, Kulej L, et al. Biopsychosocial Rehabilitation Programme for Patients with Chronic Back Pain. Pilot Study. Ortopedia Traumatologia Rehabilitacja. 2017 Apr 12;19(2):165-74. PMID: 28508767. Exclusion: 4 
44. Callahan CM. Controversies regarding comprehensive chronic care: Coordinated care: The drug-free wonder drug. Journal of the American Geriatrics Society. 2015 Sep;63(9):1938-40. doi: http://dx.doi.org/10.1111/jgs.13599. PMID: 2015-43892-029. Exclusion: 8

45. Callahan LF, Schoster B, Hootman J, et al. Modifications to the Active Living Every Day (ALED) course for adults with arthritis. Preventing Chronic Disease. 2007 Jul;4(3):A58. PMID: 17572962. Exclusion: 8

46. Camargo PR, Alburquerque-SendÍN F, Avila MA, et al. Effects of Stretching and Strengthening Exercises, With and Without Manual Therapy, on Scapular Kinematics, Function, and Pain in Individuals With Shoulder Impingement: A Randomized Controlled Trial. Journal of Orthopaedic \& Sports Physical Therapy. 2015;45(12):98497. doi: 10.2519/jospt.2015.5939. PMID: 111468870. Language: English. Entry Date: 20170401. Revision Date: 20190620. Publication Type: Article. Exclusion: 4

47. Campello M, Ziemke G, Hiebert R, et al. Implementation of a multidisciplinary program for active duty personnel seeking care for low back pain in a U.S. Navy Medical Center: a feasibility study. Military Medicine. 2012 Sep;177(9):1075-80. PMID: 23025138. Exclusion: 3

48. Cantero-Braojos MA, Cabrera-Leon A, Lopez-Gonzalez MA, et al. Group intervention from a sensorimotor approach to reduce the intensity of chronic pain. Atencion primaria / sociedad espanola de medicina de familia y comunitaria. 2018 PMID: CN-01571751. Exclusion: 11

49. Cao Y, Zhan H, Pang J, et al. Individually integrated traditional Chinese medicine approach in the management of knee osteoarthritis: study protocol for a randomized controlled trial. Trials [Electronic Resource]. 2011 Jun 22;12:160. doi: https://dx.doi.org/10.1186/1745-621512-160. PMID: 21696615. Exclusion: 4
50. Carbonell-Baeza A, Aparicio VA, Chillón P, et al. Effectiveness of multidisciplinary therapy on symptomatology and quality of life in women with fibromyalgia. Clin Exp Rheumatol. 2011 Nov-Dec;29(6 Suppl 69):S97-103. PMID: 22243556. Exclusion: 4

51. Carbonell-Baeza A, Aparicio VA, Ortega FB, et al. Does a 3-month multidisciplinary intervention improve pain, body composition and physical fitness in women with fibromyalgia? Br J Sports Med. 2011 Dec;45(15):1189-95. doi: 10.1136/bjsm.2009.070896. PMID: 20542976. Exclusion: 6

52. Carbonell-Baeza A, Ruiz JR, Aparicio VA, et al. Multidisciplinary and biodanza intervention for the management of fibromyalgia. Acta Reumatol Port. 2012 JulSep;37(3):240-50. PMID: 23348113. Exclusion: 4

53. Carnes D, Taylor SJC, Homer K, et al. Effectiveness and cost-effectiveness of a novel, group Self-management course for adults with Chronic musculoskeletal pain: study protocol for a multicentre, randomised controlled trial (COPERS). BMJ open. 2013;3(1) PMID: CN-00912086. Exclusion: 9

54. Carty JN, Ziadni MS, Holmes HJ, et al. Effects of a Life Stress Emotional Awareness and Expression Interview for Women with Chronic Urogenital Pain: a Randomized Controlled Trial. Pain medicine (Malden, Mass.). 2019;20(7):1321-9. PMID: CN-02117135 NEW. Exclusion: 4

55. Casanueva-Fernandez B, Llorca J, Rubio JB, et al. Efficacy of a multidisciplinary treatment program in patients with severe fibromyalgia. Rheumatology International. 2012 Aug;32(8):2497-502. doi: https://dx.doi.org/10.1007/s00296-0112045-1. PMID: 21785956. Exclusion: 4

56. Cederbom S, Leveille SG, Bergland A. Effects of a behavioral medicine intervention on pain, health, and behavior among community-dwelling older adults: a randomized controlled trial. Clinical Interventions In Aging. 2019;14:1207-20. doi:

https://dx.doi.org/10.2147/CIA.S208102. PMID: 31308644. Exclusion: 4 
57. Cedraschi C, Desmeules J, Rapiti E, et al. Fibromyalgia: a randomised, controlled trial of a treatment programme based on self management. Annals of the Rheumatic Diseases. 2004 Mar;63(3):290-6. PMID: 14962965. Exclusion: 4

58. Champagne R, Ronzi Y, Roche-Leboucher $\mathrm{G}$, et al. Effectiveness of an outpatient rehabilitation program with multidisciplinary approach on return to work for patients with non-specific chronic lombal pain. Annals of physical and rehabilitation medicine. 2018 PMID: CN01920291 NEW. Exclusion: 9

59. Chelimsky TC, Fischer RL, Levin JB, et al. The primary practice physician program for chronic pain (C) 4PCP): outcomes of a primary physician-pain specialist collaboration for community-based training and support. Clinical Journal of Pain. 2013 Dec;29(12):1036-43. doi: https://dx.doi.org/10.1097/AJP.0b013e3182 851584. PMID: 23459398. Exclusion: 2

60. Chen LX, Mao JJ, Fernandes S, et al. Integrating acupuncture with exercise-based physical therapy for knee osteoarthritis: a randomized controlled trial. JCR: Journal of Clinical Rheumatology. 2013

Sep;19(6):308-16. doi: https://dx.doi.org/10.1097/RHU.0b013e3182 a21848. PMID: 23965480. Exclusion: 4

61. Chen YL, Francis AJ. Relaxation and imagery for chronic, nonmalignant pain: effects on pain symptoms, quality of life, and mental health. Pain Management Nursing. 2010 Sep;11(3):159-68. doi: https://dx.doi.org/10.1016/j.pmn.2009.05.00 5. PMID: 20728065. Exclusion: 4

62. Childs JD, Wu SS, Teyhen DS, et al. Prevention of low back pain in the military cluster randomized trial: effects of brief psychosocial education on total and low back pain-related health care costs. Spine Journal: Official Journal of the North American Spine Society. 2014 Apr;14(4):571-83. doi: https://dx.doi.org/10.1016/j.spinee.2013.03. 019. PMID: 23608562. Exclusion: 4
63. Chobe S, Bhargav H, Raghuram N, et al. Effect of integrated Yoga and Physical therapy on audiovisual reaction time, anxiety and depression in patients with chronic multiple sclerosis: a pilot study. Journal of Complementary \& Integrative Medicine. 2016 Sep 01;13(3):301-9. doi: https://dx.doi.org/10.1515/jcim-2015-0105. PMID: 27337744. Exclusion: 4

64. Christiansen S, Oettingen G, Dahme B, et al. A short goal-pursuit intervention to improve physical capacity: a randomized clinical trial in chronic back pain patients. Pain. 2010 Jun;149(3):444-52. doi: https://dx.doi.org/10.1016/j.pain.2009.12.01 5. PMID: 20199846. Exclusion: 4

65. Coelho Cde F, Leal-Junior EC, BiasottoGonzalez DA, et al. Effectiveness of phototherapy incorporated into an exercise program for osteoarthritis of the knee: study protocol for a randomized controlled trial. Trials [Electronic Resource]. 2014 Jun 11;15:221. doi: https://dx.doi.org/10.1186/1745-6215-15221. PMID: 24919587. Exclusion: 4

66. Coffey CP, Ulbrich TR, Baughman K, et al. The effect of an interprofessional pain service on nonmalignant pain control. American Journal of Health-System Pharmacy. 2019 May 17;76(Supplement_2):S49-S54. doi: https://dx.doi.org/10.1093/ajhp/zxy084. PMID: 30854542. Exclusion: 4

67. Collado-Mateo D, Dominguez-Munoz FJ, Adsuar JC, et al. Exergames for women with fibromyalgia: a randomised controlled trial to evaluate the effects on mobility skills, balance and fear of falling. Peerj. 2017(pagination) PMID: CN-01370679 NEW. Exclusion: 4

68. Coole C, Drummond A, Watson PJ. Individual work support for employed patients with low back pain: a randomized controlled pilot trial. Clin Rehabil. 2013 Jan;27(1):40-50. doi: 10.1177/0269215512446839. PMID: 22701039. Exclusion: 4 
69. Critchley DJ. For sick-listed people with chronic low back pain, an integrated care programme costs society less and returns participants to work faster than usual management. Evidence based medicine. 2011;16(4):105-6. PMID: CN-02128525 NEW. Exclusion: 9

70. Cuesta-Vargas AI, Garcia-Romero JC, Arroyo-Morales M, et al. Exercise, manual therapy, and education with or without highintensity deep-water running for nonspecific chronic low back pain: a pragmatic randomized controlled trial. American Journal of Physical Medicine \& Rehabilitation. 2011 Jul;90(7):526-34; quiz 35-8. doi:

https://dx.doi.org/10.1097/PHM.0b013e318 21a71d0. PMID: 21765272. Exclusion: 4

71. Cuesta-Vargas AI, Gonzalez-Sanchez M. Changes in disability, physical/mental health states and quality of life during an 8-week multimodal physiotherapy programme in patients with chronic non-specific neck pain: a prospective cohort study. PLoS ONE [Electronic Resource]. 2015;10(2):e0118395. doi: https://dx.doi.org/10.1371/journal.pone.0118 395. PMID: 25710539. Exclusion: 4

72. Cuesta-Vargas AI, White M, GonzalezSanchez M, et al. The optimal frequency of aquatic physiotherapy for individuals with chronic musculoskeletal pain: a randomised controlled trial. Disability \& Rehabilitation. 2015;37(4):311-8. doi: https://dx.doi.org/10.3109/09638288.2014.9 18191. PMID: 24819432. Exclusion: 4

73. Cuperus N, Hoogeboom TJ, Kersten CC, et al. Randomized trial of the effectiveness of a non-pharmacological multidisciplinary faceto-face treatment program on daily function compared to a telephone-based treatment program in patients with generalized osteoarthritis. Osteoarthritis \& Cartilage. 2015 Aug;23(8):1267-75. doi: https://dx.doi.org/10.1016/j.joca.2015.04.00 7. PMID: 25887365. Exclusion: 4
74. da Silva FS, de Melo FE, do Amaral MM, et al. Efficacy of simple integrated group rehabilitation program for patients with knee osteoarthritis: Single-blind randomized controlled trial. Journal of Rehabilitation Research \& Development. 2015;52(3):30922. doi:

https://dx.doi.org/10.1682/JRRD.2014.08.01 99. PMID: 26237073. Exclusion: 4

75. Davies HT, Crombie IK, Brown JH, et al. Diminishing returns or appropriate treatment strategy?--an analysis of short-term outcomes after pain clinic treatment. Pain. 1997 Apr;70(2-3):203-8. PMID: 9150294. Exclusion: 4

76. Davin S, Lapin B, Mijatovic D, et al. Comparative Effectiveness of an Interdisciplinary Pain Program for Chronic Low Back Pain, Compared to Physical Therapy Alone. Spine. 2019 Dec 15;44(24):1715-22. doi: https://dx.doi.org/10.1097/BRS.0000000000 003161. PMID: 31794508. Exclusion: 8

77. Davis LL, Kroenke K, Monahan P, et al. The SPADE Symptom Cluster in Primary Care Patients With Chronic Pain. Clinical Journal of Pain. 2016 May;32(5):388-93. doi:

https://dx.doi.org/10.1097/AJP.0000000000 000286. PMID: 26295379. Exclusion: 6

78. de Heer EW, Dekker J, Beekman AT, et al. Comparative effect of collaborative care, pain medication, and duloxetine in the treatment of major depressive disorder and comorbid (sub)chronic pain: Results of an exploratory randomized, placebo-controlled, multicenter trial (CC:PAINDIP). Frontiers in Psychiatry Vol 9 2018, ArtID 118. 2018 Apr;9doi:

http://dx.doi.org/10.3389/fpsyt.2018.00118. PMID: 2018-16083-001. Exclusion: 4

79. de Heer EW, Dekker J, van Eck van der Sluijs JF, et al. Effectiveness and costeffectiveness of transmural collaborative care with consultation letter (TCCCL) and duloxetine for major depressive disorder (MDD) and (sub)chronic pain in collaboration with primary care: design of a randomized placebo-controlled multi-Centre trial: TCC:PAINDIP. BMC Psychiatry. 2013 May 24;13:147. doi: https://dx.doi.org/10.1186/1471-244X-13147. PMID: 23705849. Exclusion: 9 
80. De Oliveira Silva D, Pazzinatto MF, Crossley KM, et al. Novel Stepped Care Approach to Provide Education and Exercise Therapy for Patellofemoral Pain: feasibility Study. Journal of medical Internet research. 2020;22(7):e18584-. PMID: CN02139831 NEW. Exclusion: 4

81. de Paula Gomes CAF, Leal-Junior ECP, Dibai-Filho AV, et al. Incorporation of photobiomodulation therapy into a therapeutic exercise program for knee osteoarthritis: A placebo-controlled, randomized, clinical trial. Lasers in Surgery \& Medicine. 2018 10;50(8):819-28. doi: https://dx.doi.org/10.1002/1sm.22939. PMID: 29733117. Exclusion: 4

82. de Rooij A, van der Leeden M, de Boer MR, et al. Fatigue in patients with chronic widespread pain participating in multidisciplinary rehabilitation treatment: A prospective cohort study. Disability and Rehabilitation: An International, Multidisciplinary Journal. 2015 Mar;37(6):490-8. doi: http://dx.doi.org/10.3109/09638288.2014.92 3530. PMID: 2015-10734-002. Exclusion: 2

83. Dear BF, Gandy M, Karin E, et al. The Pain Course: 12- and 24-Month Outcomes From a Randomized Controlled Trial of an Internet-Delivered Pain Management Program Provided With Different Levels of Clinician Support. Journal of pain. 2018;19(12):1491-503. PMID: CN01710336. Exclusion: 4

84. Deckert S, Kaiser U, Kopkow C, et al. A systematic review of the outcomes reported in multimodal pain therapy for chronic pain. European Journal of Pain. 2016 Jan;20(1):51-63. doi: http://dx.doi.org/10.1002/ejp.721. PMID: 2015-58820-005. Exclusion: 10

85. Del Pozo-Cruz B, Adsuar JC, Parraca J, et al. A web-based intervention to improve and prevent low back pain among office workers: a randomized controlled trial. Journal of Orthopaedic \& Sports Physical Therapy. 2012 Oct;42(10):831-41. doi: https://dx.doi.org/10.2519/jospt.2012.3980. PMID: 22951407. Exclusion: 4
86. Demirjian EL. Treatment of chronic pain in a multidisciplinary program: A longitudinal study. Dissertation Abstracts International: Section B: The Sciences and Engineering. 2004;64(8-B):4031. PMID: 2004-99004068. Exclusion: 8

87. Denneson LM, Corson K, Dobscha SK. Complementary and alternative medicine use among veterans with chronic noncancer pain. Journal of Rehabilitation Research \& Development. 2011;48(9):1119-28. PMID: 22234716. Exclusion: 5

88. Deveza LA, Hunter DJ, Wajon A, et al. Efficacy of combined conservative therapies on clinical outcomes in patients with thumb base osteoarthritis: protocol for a randomised, controlled trial (COMBO). BMJ Open. 201701 12;7(1):e014498. doi: https://dx.doi.org/10.1136/bmjopen-2016014498. PMID: 28082368. Exclusion: 4

89. Dobson F, Hinman RS, French S, et al. Internet-mediated physiotherapy and pain coping skills training for people with persistent knee pain (IMPACT - Knee pain): a randomised controlled trial protocol. BMC musculoskeletal disorders. 2014;15(1) PMID: CN-01022892. Exclusion: 4

90. Douglas W, Graham C, Anderson D, et al. Managing chronic pain through cognitive change and multidisciplinary treatment program. Australian Psychologist. 2004 Nov;39(3):201-7. doi: http://dx.doi.org/10.1080/000500604123312 95045. PMID: 2004-21752-003. Exclusion: 8

91. Drager D, Budnick A, Kuhnert R, et al. Pain management intervention targeting nursing staff and general practitioners: Pain intensity, consequences and clinical relevance for nursing home residents. Geriatrics \& gerontology international. 2017 Oct;17(10):1534-43. doi: https://dx.doi.org/10.1111/ggi.12924. PMID: 27935182. Exclusion: 3 
92. Dufour N, Thamsborg G, Oefeldt A, et al. Treatment of chronic low back pain: a randomized, clinical trial comparing groupbased multidisciplinary biopsychosocial rehabilitation and intensive individual therapist-assisted back muscle strengthening exercises. Spine. 2010 Mar 01;35(5):469-76. doi:

https://dx.doi.org/10.1097/BRS.0b013e3181 b8db2e. PMID: 20147878. Exclusion: 4

93. Dunstan DA, Covic T. Can a rural community-based work-related activity program make a difference for chronic paindisabled injured workers? Aust J Rural Health. 2007 Jun;15(3):166-71. doi: 10.1111/j.1440-1584.2007.00879.x. PMID: 17542788. Exclusion: 4

94. Dysvik E, Kvaloy JT, Furnes B. Evaluating physical functioning as part of a Cognitive Behavioural Therapy approach in treatment of people suffering from chronic pain. Journal of Clinical Nursing. 2013 Mar;22(56):806-16. doi:

https://dx.doi.org/10.1111/j.13652702.2011.04040.x. PMID: 22642679.

Exclusion: 8

95. Dysvik E, Kvaloy JT, Natvig GK. The effectiveness of an improved multidisciplinary pain management programme: a 6- and 12-month follow-up study. Journal of Advanced Nursing. 2012 May;68(5):1061-72. doi:

https://dx.doi.org/10.1111/j.13652648.2011.05810.x. PMID: 22050304. Exclusion: 8

96. Dysvik E, Kvaloy JT, Stokkeland R, et al. The effectiveness of a multidisciplinary pain management programme managing chronic pain on pain perceptions, health-related quality of life and stages of change--A nonrandomized controlled study. International Journal of Nursing Studies. 2010 Jul;47(7):826-35. doi: https://dx.doi.org/10.1016/j.ijnurstu.2009.12 .001. PMID: 20036362. Exclusion: 8

97. Eaton LH, Gordon DB, Wyant S, et al. Development and implementation of a telehealth-enhanced intervention for pain and symptom management. Contemporary Clinical Trials. 2014 Jul;38(2):213-20. doi: https://dx.doi.org/10.1016/j.cct.2014.05.005. PMID: 24846620. Exclusion: 9
98. Eisenberg DM, Buring JE, Hrbek AL, et al. A model of integrative care for low-back pain. Journal of Alternative \& Complementary Medicine. 2012 Apr;18(4):354-62. doi: https://dx.doi.org/10.1089/acm.2011.0408. PMID: 22455544. Exclusion: 4

99. Ekberg K, Björkqvist B, Malm P, et al. Controlled two year follow up of rehabilitation for disorders in the neck and shoulders. Occup Environ Med. 1994 Dec;51(12):833-8. doi: 10.1136/oem.51.12.833. PMID: 7849868. Exclusion: 4

100. Esselens G, Westhovens R, Verschueren P. Effectiveness of an integrated outpatient care programme compared with present-day standard care in early rheumatoid arthritis. Musculoskeletal Care. 2009 Mar;7(1):1-16. doi: https://dx.doi.org/10.1002/msc.136. PMID: 18618520. Exclusion: 8

101. Fairbank J, Frost H, Wilson-MacDonald J, et al. Randomised controlled trial to compare surgical stabilisation of the lumbar spine with an intensive rehabilitation programme for patients with chronic low back pain: the MRC spine stabilisation trial. Bmj. 2005 May 28;330(7502):1233. doi: 10.1136/bmj.38441.620417.8F. PMID: 15911537. Exclusion: 5

102. Freiwald J, Hoppe MW, Beermann W, et al. Effects of supplemental heat therapy in multimodal treated chronic low back pain patients on strength and flexibility. Clinical biomechanics. 2018;57:107-13. PMID: CN01618661. Exclusion: 4

103. Frettloh J, Kroner-Herwig B. Individual versus group training in the treatment of chronic pain: Which is more efficacious? Zeitschrift fur Klinische Psychologie. 1999;28(4):256-66. doi: http://dx.doi.org/10.1026//00845345.28.4.256. PMID: 1999-01912-004. Exclusion: 11

104. Friedrich M, Gittler G, Halberstadt Y, et al. Combined exercise and motivation program: effect on the compliance and level of disability of patients with chronic low back pain: a randomized controlled trial. Arch Phys Med Rehabil. 1998 May;79(5):475-87. doi: 10.1016/s0003-9993(98)90059-4. PMID: 9596385. Exclusion: 4 
105. Furlan AD, Irvin E, Munhall C, et al. Rehabilitation service models for people with physical and/or mental disability living in low- and middle-income countries: A systematic review. Journal of Rehabilitation Medicine. 2018 Jun 15;50(6):487-98. doi: https://dx.doi.org/10.2340/16501977-2325. PMID: 29616278. Exclusion: 10

106. Gagnon S, Lensel-Corbeil G, Duquesnoy B. Multicenter multidisciplinary training program for chronic low back pain: French experience of the Renodos back pain network (Réseau Nord-Pas-de-Calais du DOS). Ann Phys Rehabil Med. 2009 Feb;52(1):3-16. doi: 10.1016/j.rehab.2008.10.001. PMID: 19419655. Exclusion: 2

107. Garschagen A, Steegers MA, van Bergen $\mathrm{AH}$, et al. Is there a need for including spiritual care in interdisciplinary rehabilitation of chronic pain patients? Investigating an innovative strategy. Pain Practice. 2015 Sep;15(7):671-87. doi: http://dx.doi.org/10.1111/papr.12234. PMID: 2015-46970-010. Exclusion: 10

108. Giraudet-Le Quintrec JS, MayouxBenhamou A, Ravaud P, et al. Effect of a collective educational program for patients with rheumatoid arthritis: a prospective 12month randomized controlled trial. J Rheumatol. 2007 Aug;34(8):1684-91. PMID: 17610321. Exclusion: 4

109. Giusti EM, Castelnuovo G, Molinari E. Differences in multidisciplinary and interdisciplinary treatment programs for fibromyalgia: A mapping review. Pain Research \& Management Vol 2017 2017, ArtID 7261468. 2017 May;2017 PMID: 2017-30713-001. Exclusion: 10

110. Godfrey E, Galea Holmes M, Wileman V, et al. Physiotherapy informed by Acceptance and Commitment Therapy (PACT): protocol for a randomised controlled trial of PACT versus usual physiotherapy care for adults with chronic low back pain. BMJ Open. 201606 07;6(6):e011548. doi: https://dx.doi.org/10.1136/bmjopen-2016011548. PMID: 27267109. Exclusion: 9
111. Goertz CM, Salsbury SA, Long CR, et al. Patient-centered professional practice models for managing low back pain in older adults: a pilot randomized controlled trial. BMC Geriatrics. 201710 13;17(1):235. doi: https://dx.doi.org/10.1186/s12877-0170624-z. PMID: 29029606. Exclusion: 4

112. Guck TP, Skultety FM, Meilman PW, et al. Multidisciplinary pain center follow-up study: evaluation with a no-treatment control group. Pain. 1985 Mar;21(3):295306. doi: 10.1016/0304-3959(85)90093-4. PMID: 3991232. Exclusion: 8

113. Guilkey RE, Draucker CB, Wu J, et al. Acceptability of a telecare intervention for persistent musculoskeletal pain. Journal of Telemedicine \& Telecare. 2018

Jan;24(1):44-50. doi: https://dx.doi.org/10.1177/1357633X166708 15. PMID: 27681227. Exclusion: 4

114. Gustafsson M, Ekholm J, Broman L. Effects of a multiprofessional rehabilitation programme for patients with fibromyalgia syndrome. J Rehabil Med. 2002

May;34(3):119-27. doi: 10.1080/165019702753714147. PMID: 12395939. Exclusion: 4

115. Gustavsson C, Denison E, von Koch L. Selfmanagement of persistent neck pain: a randomized controlled trial of a multicomponent group intervention in primary health care. Eur J Pain. 2010

Jul;14(6):630.e1-.e11. doi: 10.1016/j.ejpain.2009.10.004. PMID: 19939717. Exclusion: 4

116. Gustavsson C, Denison E, von Koch L. Selfmanagement of persistent neck pain: twoyear follow-up of a randomized controlled trial of a multicomponent group intervention in primary health care. Spine (Phila Pa 1976). 2011 Dec 1;36(25):2105-15. doi: 10.1097/BRS.0b013e3182028b04. PMID: 21358487. Exclusion: 4

117. Gustavsson C, von Koch L. A 9-year follow-up of a self-management group intervention for persistent neck pain in primary health care: a randomized controlled trial. J Pain Res. 2017;10:53-64. doi: 10.2147/jpr.S125074. PMID: 28115865. Exclusion: 4 
118. Guzman J, Esmail R, Karjalainen K, et al. Multidisciplinary rehabilitation for chronic low back pain: Systematic review. BMJ: British Medical Journal. 2001 Jun;322(7301):1511-6. doi: http://dx.doi.org/10.1136/bmj.322.7301.151 1. PMID: 2001-07462-001. Exclusion: 10

119. Guzman J, Esmail R, Karjalainen K, et al. Multidisciplinary bio-psycho-social rehabilitation for chronic low back pain. Cochrane database of systematic reviews. 2002;1 PMID: CN-01759922 NEW.

Exclusion: 10

120. Haldorsen E, Kronholm K, Skouen J, et al. Predictors for outcome of a multi-modal cognitive behavioural treatment program for low back pain patients--a 12-month followup study. European Journal of Pain. 1998;2(4):293-307. doi: http://dx.doi.org/10.1016/S10903801\%2898\%2990028-3. PMID: 200600360-003. Exclusion: 8

121. Haldorsen EM, Grasdal AL, Skouen JS, et al. Is there a right treatment for a particular patient group? Comparison of ordinary treatment, light multidisciplinary treatment, and extensive multidisciplinary treatment for long-term sick-listed employees with musculoskeletal pain. Pain. 2002 Jan;95(12):49-63. PMID: 11790467. Exclusion: 6

122. Haldorsen EM, Kronholm K, Skouen JS, et al. Multimodal cognitive behavioral treatment of patients sicklisted for musculoskeletal pain: a randomized controlled study. Scandinavian Journal of Rheumatology. 1998;27(1):16-25. PMID: 9506873. Exclusion: 4

123. Hammond A, Freeman K. Community patient education and exercise for people with fibromyalgia: a parallel group randomized controlled trial. Clin Rehabil. 2006 Oct;20(10):835-46. doi: 10.1177/0269215506072173. PMID: 17008336. Exclusion: 4

124. Hamnes B, Mowinckel P, Kjeken I, et al. Effects of a one week multidisciplinary inpatient self-management programme for patients with fibromyalgia: a randomised controlled trial. BMC Musculoskeletal Disorders. 2012 Sep 26;13:189. doi: https://dx.doi.org/10.1186/1471-2474-13189. PMID: 23013162. Exclusion: 4
125. Hay EM, Foster NE, Thomas E, et al. Effectiveness of community physiotherapy and enhanced pharmacy review for knee pain in people aged over 55 presenting to primary care: pragmatic randomised trial. BMJ. 2006 Nov 11;333(7576):995. PMID: 17056608. Exclusion: 4

126. Hazard RG, Fenwick JW, Kalisch SM, et al. Functional restoration with behavioral support. A one-year prospective study of patients with chronic low-back pain. Spine (Phila Pa 1976). 1989 Feb;14(2):157-61. PMID: 2522243. Exclusion: 8

127. Heidari J, Belz J, Hasenbring MI, et al. Evaluation of the short-term effects of recovery tools in the rehabilitation of chronic back pain: a feasibility study. European journal of physiotherapy. 2018 PMID: CN-01568795. Exclusion: 4

128. Hellum C, Johnsen LG, Storheim K, et al. Surgery with disc prosthesis versus rehabilitation in patients with low back pain and degenerative disc: two year follow-up of randomised study. BMJ. 2011 May 19;342:d2786. doi: 10.1136/bmj.d2786. PMID: 21596740. Exclusion: 5

129. Henschke N, Ostelo RW, van Tulder MW, et al. Behavioural treatment for chronic lowback pain. Cochrane Database Syst Rev. 2010 Jul 7(7):CD002014. doi: 10.1002/14651858.CD002014.pub3. PMID: 20614428. Exclusion: 10

130. Hill J, Thorpe R, Bird H. Outcomes for patients with RA: a rheumatology nurse practitioner clinic compared to standard outpatient care. Musculoskeletal Care. 2003 Mar;1(1):5-20. doi: 10.1002/msc.35. PMID: 20217660. Exclusion: 4

131. Hoogeboom TJ, Kwakkenbos L, Rietveld L, et al. Feasibility and potential effectiveness of a non-pharmacological multidisciplinary care programme for persons with generalised osteoarthritis: a randomised, multiple-baseline single-case study. BMJ open. 2012;2(4) PMID: CN-00903687. Exclusion: 4 
132. Hooten W, Knight-Brown M, Townsend $\mathrm{CO}$, et al. Clinical outcomes of multidisciplinary pain rehabilitation among African American compared with Caucasian patients with chronic pain. Pain Medicine. 2012 Nov;13(11):1499-508. doi: http://dx.doi.org/10.1111/j.15264637.2012.01489.x. PMID: 2012-31057014. Exclusion: 5

133. Hooten WM, Townsend CO, Decker PA. Gender differences among patients with fibromyalgia undergoing multidisciplinary pain rehabilitation. Pain Med. 2007 NovDec;8(8):624-32. doi: 10.1111/j.15264637.2006.00202.x. PMID: 18028040. Exclusion: 8

134. Hooten WM, Townsend CO, Sletten CD, et al. Treatment outcomes after multidisciplinary pain rehabilitation with analgesic medication withdrawal for patients with fibromyalgia. Pain Med. 2007 JanFeb;8(1):8-16. doi: 10.1111/j.15264637.2007.00253.x. PMID: 17244099. Exclusion: 8

135. Hudon C, Chouinard MC, Dubois MF, et al. Case Management in Primary Care for Frequent Users of Health Care Services: A Mixed Methods Study. Annals of Family Medicine. 2018 05;16(3):232-9. doi: https://dx.doi.org/10.1370/afm.2233. PMID: 29760027. Exclusion: 3

136. Huge V, Schloderer U, Steinberger M, et al. Impact of a functional restoration program on pain and health-related quality of life in patients with chronic low back pain. Pain Medicine. 2006 Nov-Dec;7(6):501-8. PMID: 17112363. Exclusion: 8

137. Hunt MA, Keefe FJ, Bryant C, et al. A physiotherapist-delivered, combined exercise and pain coping skills training intervention for individuals with knee osteoarthritis: a pilot study. Knee. 2013 Mar;20(2):106-12. doi: https://dx.doi.org/10.1016/j.knee.2012.07.00 8. PMID: 22921688. Exclusion: 4
138. Hurley MV, Walsh NE, Mitchell H, et al. Long-term outcomes and costs of an integrated rehabilitation program for chronic knee pain: a pragmatic, cluster randomized, controlled trial. Arthritis care \& research. 2012 Feb;64(2):238-47. doi: https://dx.doi.org/10.1002/acr.20642. PMID: 21954131. Exclusion: 4

139. Ismael Martins MR, Gritti CC, Dos Santos Junior R, et al. Randomized controlled trial of a therapeutic intervention group in patients with fibromyalgia syndrome. Revista brasileira de reumatologia. 2014;54(3):179-84. PMID: CN-00999361. Exclusion: 3

140. Jensen C, Jensen OK, Christiansen DH, et al. One-year follow-up in employees sicklisted because of low back pain: randomized clinical trial comparing multidisciplinary and brief intervention. Spine. $2011 \mathrm{Jul}$ 01;36(15):1180-9. doi: https://dx.doi.org/10.1097/BRS.0b013e3181 eba711. PMID: 21217456. Exclusion: 4

141. Jensen C, Jensen OK, Nielsen CV. Sustainability of return to work in sick-listed employees with low-back pain. Two-year follow-up in a randomized clinical trial comparing multidisciplinary and brief intervention. BMC musculoskeletal disorders. 2012;13 PMID: CN-01019730. Exclusion: 4

142. Jensen MP, Turner JA, Romano JM. Changes in beliefs, catastrophizing, and coping are associated with improvement in multidisciplinary pain treatment. Journal of Consulting \& Clinical Psychology. 2001 Aug;69(4):655-62. PMID: 11550731. Exclusion: 5

143. Johnson RE, Jones GT, Wiles NJ, et al. Active exercise, education, and cognitive behavioral therapy for persistent disabling low back pain: a randomized controlled trial. Spine (Phila Pa 1976). 2007 Jul 1;32(15):1578-85. doi: 10.1097/BRS.0b013e318074f890. PMID: 17621203. Exclusion: 4 
144. Kamper SJ, Apeldoorn A, Chiarotto A, et al. Multidisciplinary biopsychosocial rehabilitation for chronic low back pain: Cochrane systematic review and metaanalysis. BMJ: British Medical Journal Vol 350 2015, ArtID h444. 2015 Feb;350 PMID: 2015-14057-001. Exclusion: 10

145. Kamper SJ, Apeldoorn AT, Chiarotto A, et al. Multidisciplinary biopsychosocial rehabilitation for chronic low back pain. Cochrane Database of Systematic Reviews. 2014(9) PMID: 00075320-10000000000466. Exclusion: 10

146. Kankaanpää M, Taimela S, Airaksinen O, et al. The efficacy of active rehabilitation in chronic low back pain. Effect on pain intensity, self-experienced disability, and lumbar fatigability. Spine (Phila Pa 1976). 1999 May 15;24(10):1034-42. doi: 10.1097/00007632-199905150-00019. PMID: 10332798. Exclusion: 4

147. Karjalainen K, Malmivaara A, Pohjolainen $\mathrm{T}$, et al. Mini-intervention for subacute low back pain: a randomized controlled trial. Spine (Phila Pa 1976). 2003 Mar 15;28(6):533-40; discussion 40-1. doi: 10.1097/01.Brs.0000049928.52520.69. PMID: 12642757. Exclusion: 4

148. Karjalainen K, Malmivaara A, van Tulder $\mathrm{M}$, et al. Multidisciplinary biopsychosocial rehabilitation for neck and shoulder pain among working age adults. Cochrane database of systematic reviews. 2003;2 PMID: CN-01773940 NEW. Exclusion: 10

149. Keller S, Ehrhardt-Schmelzer S, Herda C, et al. Multidisciplinary rehabilitation for chronic back pain in an outpatient setting: a controlled randomized trial. European journal of pain (London, England). 1997;1(4):279-92. PMID: CN-01261620. Exclusion: 4

150. Kendall NAS, Thompson BF. A Pilot Program for Dealing with the Comorbidity of Chronic Pain and Long-Term Unemployment. Journal of Occupational Rehabilitation. 1998;8(1):5-26. Exclusion: 8
151. Kole-Snijders AM, Vlaeyen JW, Goossens ME, et al. Chronic low-back pain: what does cognitive coping skills training add to operant behavioral treatment? Results of a randomized clinical trial. J Consult Clin Psychol. 1999 Dec;67(6):931-44. doi: 10.1037//0022-006x.67.6.931. PMID: 10596514. Exclusion: 8

152. Kool J, Bachmann S, Oesch P, et al. Function-centered rehabilitation increases work days in patients with nonacute nonspecific low back pain: 1-year results from a randomized controlled trial. Arch Phys Med Rehabil. 2007 Sep;88(9):1089-94. doi: 10.1016/j.apmr.2007.05.022. PMID: 17826451. Exclusion: 6

153. Koopman FS, Edelaar M, Slikker R, et al. Effectiveness of a multidisciplinary occupational training program for chronic low back pain: a prospective cohort study. Am J Phys Med Rehabil. 2004 Feb;83(2):94-103. doi: 10.1097/01.Phm.0000107482.35803.11. PMID: 14758295. Exclusion: 2

154. Lamb SE, Hansen Z, Lall R, et al. Group cognitive behavioural treatment for lowback pain in primary care: a randomised controlled trial and cost-effectiveness analysis. Lancet. $2010 \mathrm{Mar}$ 13;375(9718):916-23. doi: 10.1016/s01406736(09)62164-4. PMID: 20189241. Exclusion: 4

155. Lamb SE, Lall R, Hansen Z, et al. A multicentred randomised controlled trial of a primary care-based cognitive behavioural programme for low back pain. The Back Skills Training (BeST) trial. Health Technol Assess. 2010 Aug;14(41):1-253, iii-iv. doi: 10.3310/hta14410. PMID: 20807469.

Exclusion: 4

156. Lamb SE, Mistry D, Lall R, et al. Group cognitive behavioural interventions for low back pain in primary care: extended followup of the Back Skills Training Trial (ISRCTN54717854). Pain. 2012 Feb;153(2):494-501. doi: 10.1016/j.pain.2011.11.016. PMID: 22226729. Exclusion: 4 
157. Lambeek LC, Anema JR, van Royen BJ, et al. Multidisciplinary outpatient care program for patients with chronic low back pain: design of a randomized controlled trial and cost-effectiveness study [ISRCTN28478651]. BMC Public Health. 2007 Sep 20;7:254. doi: 10.1186/14712458-7-254. PMID: 17883853. Exclusion: 4

158. Lambeek LC, Bosmans JE, Van Royen BJ, et al. Effect of integrated care for sick listed patients with chronic low back pain: economic evaluation alongside a randomised controlled trial. BMJ. $2010 \mathrm{Nov}$ 30;341:c6414. doi: 10.1136/bmj.c6414. PMID: 21118874. Exclusion: 4

159. Lambeek LC, van Mechelen W, Buijs PC, et al. An integrated care program to prevent work disability due to chronic low back pain: a process evaluation within a randomized controlled trial. BMC Musculoskelet Disord. 2009 Nov 30;10:147. doi: 10.1186/1471-2474-10-147. PMID: 19943976. Exclusion: 4

160. Lambeek LC, van Mechelen W, Knol DL, et al. Randomised controlled trial of integrated care to reduce disability from chronic low back pain in working and private life. BMJ. 2010 Mar 16;340:c1035. doi:

10.1136/bmj.c1035. PMID: 20234040. Exclusion: 4

161. Lang E, Liebig K, Kastner S, et al. Multidisciplinary rehabilitation versus usual care for chronic low back pain in the community: effects on quality of life. Spine J. 2003 Jul-Aug;3(4):270-6. doi: 10.1016/s1529-9430(03)00028-7. PMID: 14589185. Exclusion: 8

162. Letzel J, Angst F, Weigl MB.

Multidisciplinary biopsychosocial rehabilitation in chronic neck pain: a naturalistic prospective cohort study with intraindividual control of effects and 12month follow-up. European journal of physical \& rehabilitation medicine. 2019 Oct;55(5):665-75. doi:

https://dx.doi.org/10.23736/S19739087.18.05348-0. PMID: 30468363.

Exclusion: 2
163. Liang L, Pan Y, Wu D, et al. Effects of Multidisciplinary Team-Based Nurse-led Transitional Care on Clinical Outcomes and Quality of Life in Patients With Ankylosing Spondylitis. Asian Nursing Research. 2019;13(2):107-14. doi: 10.1016/j.anr.2019.02.004. PMID: 136729282. Language: English. Entry Date: 20190606. Revision Date: 20190606. Publication Type: Article. Exclusion: 4

164. Lin EH, Katon W, Von Korff M, et al. Effect of improving depression care on pain and functional outcomes among older adults with arthritis: a randomized controlled trial. Jama. 2003 Nov 12;290(18):2428-9. doi: 10.1001/jama.290.18.2428. PMID: 14612479. Exclusion: 4

165. Lipchik GL, Milles K, Covington EC. The effects of multidisciplinary pain management treatment on locus of control and pain beliefs in chronic non-terminal pain. Clinical Journal of Pain. 1993 Mar;9(1):49-57. PMID: 8477140. Exclusion: 8

166. Macedo LG, Latimer J, Maher CG, et al. Effect of motor control exercises versus graded activity in patients with chronic nonspecific low back pain: a randomized controlled trial. Phys Ther. 2012 Mar;92(3):363-77. doi: 10.2522/ptj.20110290. PMID: 22135712. Exclusion: 4

167. Marcus DA, Bernstein CD, Haq A, et al. Including a range of outcome targets offers a broader view of fibromyalgia treatment outcome: results from a retrospective review of multidisciplinary treatment. Musculoskeletal Care. 2014 Jun;12(2):7481. doi: 10.1002/msc.1056. PMID: 23878014. Exclusion: 8

168. Marin TJ, Van Eerd D, Irvin E, et al. Multidisciplinary biopsychosocial rehabilitation for subacute low back pain. Cochrane Database of Systematic Reviews. 2017(6) PMID: 00075320-10000000001656. Exclusion: 10 
169. Mayer TG, Gatchel RJ, Kishino N, et al. Objective assessment of spine function following industrial injury. A prospective study with comparison group and one-year follow-up. Spine (Phila Pa 1976). 1985 JulAug;10(6):482-93. doi: 10.1097/00007632198507000-00002. PMID: 2934829.

Exclusion: 8

170. Mayer TG, Gatchel RJ, Mayer H, et al. A prospective two-year study of functional restoration in industrial low back injury. An objective assessment procedure. JAMA. 1987 Oct 2;258(13):1763-7. PMID: 2957520. Exclusion: 8

171. McCracken LM, Davies M, Scott W, et al. Can a psychologically based treatment help people to live with chronic pain when they are seeking a procedure to reduce it? Pain Medicine. 2015 Mar;16(3):451-9. doi: http://dx.doi.org/10.1111/pme.12623. PMID: 2015-12616-009. Exclusion: 8

172. Mellin G, Härkäpää K, Hurri H, et al. A controlled study on the outcome of inpatient and outpatient treatment of low back pain. Part IV. Long-term effects on physical measurements. Scand J Rehabil Med. 1990;22(4):189-94. PMID: 2148222. Exclusion: 4

173. Mellin G, Hurri H, Härkäpää K, et al. A controlled study on the outcome of inpatient and outpatient treatment of low back pain. Part II. Effects on physical measurements three months after treatment. Scand J Rehabil Med. 1989;21(2):91-5. PMID: 2526365. Exclusion: 4

174. Meng K, Seekatz B, Roband H, et al. Intermediate and long-term effects of a standardized back school for inpatient orthopedic rehabilitation on illness knowledge and self-management behaviors: a randomized controlled trial. Clinical Journal of Pain. 2011 Mar-Apr;27(3):24857. doi:

https://dx.doi.org/10.1097/AJP.0b013e3181f fbfaf. PMID: 21178600. Exclusion: 4

175. Mitchell RI, Carmen GM. The functional restoration approach to the treatment of chronic pain in patients with soft tissue and back injuries. Spine (Phila Pa 1976). 1994 Mar 15;19(6):633-42. doi: 10.1097/00007632-199403001-00001. PMID: 8009327. Exclusion: 6
176. Moe RH, Grotle M, Kjeken I, et al. Effectiveness of an Integrated Multidisciplinary Osteoarthritis Outpatient Program versus Outpatient Clinic as Usual: A Randomized Controlled Trial. Journal of Rheumatology. 2016 Feb;43(2):411-8. doi: https://dx.doi.org/10.3899/jrheum.150157. PMID: 26669917. Exclusion: 4

177. Moe RH, Uhlig T, Kjeken I, et al. Multidisciplinary and multifaceted outpatient management of patients with osteoarthritis: protocol for a randomised, controlled trial. BMC Musculoskeletal Disorders. 2010 Nov 01;11:253. doi: https://dx.doi.org/10.1186/1471-2474-11253. PMID: 21040556. Exclusion: 4

178. Moll LT, Jensen OK, Schiottz-Christensen B, et al. Return to Work in Employees on Sick Leave due to Neck or Shoulder Pain: A Randomized Clinical Trial Comparing Multidisciplinary and Brief Intervention with One-Year Register-Based Follow-Up. Journal of Occupational Rehabilitation. 2018 06;28(2):346-56. doi: https://dx.doi.org/10.1007/s10926-0179727-9. PMID: 28836120. Exclusion: 4

179. Momsen A, Nielsen C, Jensen C. Somatisation and sickness absence in low back pain patients. A randomised comparison of a brief and a multidisciplinary intervention [abstract]. Journal of psychosomatic research [abstracts of the 15th annual meeting of the european association for consultation-liaison psychiatry and psychosomatics, EACLPP and 29th european conference on psychosomatic research, ecpr. 2012;2012 jun 27-30; aarhus denmark] 72(6):495. PMID: CN-00852165 UPDATE. Exclusion: 9

180. Monticone M, Ambrosini E, Rocca B, et al. Group-based task-oriented exercises aimed at managing kinesiophobia improved disability in chronic low back pain. European journal of pain (united kingdom). 2016;20(4):541-51. PMID: CN-01142007. Exclusion: 4 
181. Monticone M, Ambrosini E, Rocca B, et al. Group-based multimodal exercises integrated with cognitive-behavioural therapy improve disability, pain and quality of life of subjects with chronic neck pain: a randomized controlled trial with one-year follow-up. Clinical rehabilitation. 2017;31(6):742-52. PMID: CN-01465277 NEW. Exclusion: 4

182. Monticone M, Ambrosini E, Rocca B, et al. A multidisciplinary rehabilitation programme improves disability, kinesiophobia and walking ability in subjects with chronic low back pain: results of a randomised controlled pilot study. European Spine Journal. 2014 Oct;23(10):2105-13. doi: https://dx.doi.org/10.1007/s00586-0143478-5. PMID: 25064093. Exclusion: 4

183. Monticone M, Ferrante S, Rocca B, et al. Effect of a long-lasting multidisciplinary program on disability and fear-avoidance behaviors in patients with chronic low back pain: results of a randomized controlled trial. Clinical Journal of Pain. 2013 Nov;29(11):929-38. doi: https://dx.doi.org/10.1097/AJP.0b013e3182 7fef7e. PMID: 23328343. Exclusion: 4

184. Morasco BJ, Corson K, Turk DC, et al. Association between substance use disorder status and pain-related function following 12 months of treatment in primary care patients with musculoskeletal pain. Journal of Pain. 2011 Mar;12(3):352-9. doi: https://dx.doi.org/10.1016/j.jpain.2010.07.01 0. PMID: 20851057. Exclusion: 8

185. Morone G, Iosa M, Paolucci T, et al. Efficacy of perceptive rehabilitation in the treatment of chronic nonspecific low back pain through a new tool: a randomized clinical study. Clin Rehabil. 2012 Apr;26(4):339-50. doi: 10.1177/0269215511414443. PMID: 21965520. Exclusion: 4

186. Morone G, Paolucci T, Alcuri MR, et al. Quality of life improved by multidisciplinary back school program in patients with chronic non-specific low back pain: a single blind randomized controlled trial. European journal of physical \& rehabilitation medicine. 2011

Dec;47(4):533-41. PMID: 21508915. Exclusion: 4
187. Motoya R, Otani K, Nikaido T, et al. Shortterm effect of back school based on cognitive behavioral therapy involving multidisciplinary collaboration. Fukushima Journal of Medical Science. 2017 Aug 09;63(2):81-9. doi:

https://dx.doi.org/10.5387/fms.2016-07. PMID: 28724842. Exclusion: 8

188. Myhre K, Marchand GH, Leivseth G, et al. The effect of work-focused rehabilitation among patients with neck and back pain: a randomized controlled trial. Spine. 2014 Nov 15;39(24):1999-2006. doi: https://dx.doi.org/10.1097/BRS.0000000000 000610. PMID: 25271499. Exclusion: 6

189. Nicholas MK, Asghari A, Blyth FM, et al. Long-term outcomes from training in selfmanagement of chronic pain in an elderly population: a randomized controlled trial. Pain. 2017 01;158(1):86-95. PMID: 27682207. Exclusion: 4

190. Nicholas MK, Wilson PH, Goyen J. Operant-behavioural and cognitivebehavioural treatment for chronic low back pain. Behaviour Research \& Therapy. 1991;29(3):225-38. PMID: 1831972. Exclusion: 4

191. Nicholas MK, Wilson PH, Goyen J. Comparison of cognitive-behavioral group treatment and an alternative nonpsychological treatment for chronic low back pain. Pain. 1992 Mar;48(3):339-47. doi: 10.1016/0304-3959(92)90082-m. PMID: 1534400. Exclusion: 4

192. Nielson WR, Jensen MP. Relationship between changes in coping and treatment outcome in patients with Fibromyalgia Syndrome. Pain. 2004 Jun;109(3):233-41. PMID: 15157683. Exclusion: 8

193. Nielson WR, Weir R, Smith B, et al. Biopsychosocial approaches to the treatment of chronic pain. Clinical journal of pain. 2001;17(4 SUPPL.):S114-S27. PMID: CN01719575 NEW. Exclusion: 10

194. Nielssen O, Karin E, Staples L, et al. Opioid use before and after completion of an online pain management program. Journal of Consulting \& Clinical Psychology. 2019 Oct;87(10):904-17. doi: https://dx.doi.org/10.1037/ccp0000407. PMID: 31556667. Exclusion: 4 
195. Niemisto L, Lahtinen-Suopanki T, Rissanen $\mathrm{P}$, et al. A randomized trial of combined manipulation, stabilizing exercises, and physician consultation compared to physician consultation alone for chronic low back pain. Spine. 2003 Oct 01;28(19):218591. PMID: 14520029. Exclusion: 4

196. Niemisto L, Rissanen P, Sarna S, et al. Costeffectiveness of combined manipulation, stabilizing exercises, and physician consultation compared to physician consultation alone for chronic low back pain: a prospective randomized trial with 2year follow-up. Spine. 2005 May 15;30(10):1109-15. PMID: 15897822. Exclusion: 4

197. Nordin CA, Michaelson P, Gard G, et al. Effects of the Web Behavior Change Program for Activity and Multimodal Pain Rehabilitation: Randomized Controlled Trial. Journal of Medical Internet Research. 201610 05;18(10):e265. PMID: 27707686. Exclusion: 4

198. Norrbrink Budh C, Kowalski J, Lundeberg T. A comprehensive pain management programme comprising educational, cognitive and behavioural interventions for neuropathic pain following spinal cord injury. Journal of Rehabilitation Medicine. 2006 May;38(3):172-80. PMID: 16702084. Exclusion: 8

199. Nost TH, Steinsbekk A, Bratas O, et al. Twelve-month effect of chronic pain selfmanagement intervention delivered in an easily accessible primary healthcare service - a randomised controlled trial. BMC Health Services Research. 2018 Dec 29;18(1):1012. doi: https://dx.doi.org/10.1186/s12913-0183843-x. PMID: 30594190. Exclusion: 4

200. Nygaard AS, Rydningen MB, Stedenfeldt $\mathrm{M}$, et al. Group-based multimodal physical therapy in women with chronic pelvic pain: a randomized controlled trial. Acta obstetricia et gynecologica Scandinavica. 2020 PMID: CN-02132221 NEW. Exclusion: 4
201. O'Keeffe M, O'Sullivan P, Purtill H, et al. Cognitive functional therapy compared with a group-based exercise and education intervention for chronic low back pain: a multicentre randomised controlled trial (RCT). British Journal of Sports Medicine. 2020 Jul;54(13):782-9. doi: https://dx.doi.org/10.1136/bjsports-2019100780. PMID: 31630089. Exclusion: 4

202. O'Keeffe M, Purtill H, Kennedy N, et al. Comparative effectiveness of conservative interventions for nonspecific chronic spinal pain: Physical, behavioral/psychologically informed, or combined? A systematic review and meta-analysis. The Journal of Pain. 2016 Jul;17(7):755-74. doi: http://dx.doi.org/10.1016/j.jpain.2016.01.47 3. PMID: 2016-14223-001. Exclusion: 10

203. O'Keeffe M, Purtill H, Kennedy N, et al. Individualised cognitive functional therapy compared with a combined exercise and pain education class for patients with nonspecific chronic low back pain: study protocol for a multicentre randomised controlled trial. BMJ Open. 2015 Jun 01;5(6):e007156. doi: https://dx.doi.org/10.1136/bmjopen-2014007156. PMID: 26033941. Exclusion: 9

204. Olason M, Andrason RH, Jonsdottir IH, et al. Cognitive Behavioral Therapy for Depression and Anxiety in an Interdisciplinary Rehabilitation Program for Chronic Pain: a Randomized Controlled Trial with a 3-Year Follow-up. International Journal of Behavioral Medicine. 2018 Feb;25(1):55-66. doi: https://dx.doi.org/10.1007/s12529-0179690-z. PMID: 29094283. Exclusion: 4

205. Osteras N, Moseng T, van Bodegom-Vos L, et al. Implementing a structured model for osteoarthritis care in primary healthcare: A stepped-wedge cluster-randomised trial. PLoS Medicine / Public Library of Science. 2019 10;16(10):e1002949. doi: https://dx.doi.org/10.1371/journal.pmed.100 2949. PMID: 31613885. Exclusion: 4 
206. Ozsoy G, Ilcin N, Ozsoy I, et al. The Effects Of Myofascial Release Technique Combined With Core Stabilization Exercise In Elderly With Non-Specific Low Back Pain: A Randomized Controlled, SingleBlind Study. Clinical Interventions In Aging. 2019;14:1729-40. doi: https://dx.doi.org/10.2147/CIA.S223905. PMID: 31631992. Exclusion: 4

207. Panton LB, Figueroa A, Kingsley JD, et al. Effects of resistance training and chiropractic treatment in women with fibromyalgia. Journal of Alternative \& Complementary Medicine. 2009 Mar;15(3):321-8. doi: https://dx.doi.org/10.1089/acm.2008.0132. PMID: 19249999. Exclusion: 4

208. Paolucci T, Morone G, Iosa M, et al. Psychological features and outcomes of the Back School treatment in patients with chronic non-specific low back pain. A randomized controlled study. European journal of physical \& rehabilitation medicine. 2012 Jun;48(2):245-53. PMID: 22095057. Exclusion: 4

209. Pape E, Hagen KB, Brox JI, et al. Early multidisciplinary evaluation and advice was ineffective for whiplash-associated disorders. European Journal of Pain. 2009 Nov;13(10):1068-75. doi: http://dx.doi.org/10.1016/j.ejpain.2008.12.0 06. PMID: 2009-21475-011. Exclusion: 4

210. Park J, Hughes AK. Nonpharmacological approaches to the management of chronic pain in community-dwelling older adults: a review of empirical evidence. Journal of the American Geriatrics Society. 2012 Mar;60(3):555-68. doi: https://dx.doi.org/10.1111/j.15325415.2011.03846.x. PMID: 22288789.

Exclusion: 10

211. Parlar S, Fadiloglu C, Argon G, et al. The effects of self-pain management on the intensity of pain and pain management methods in arthritic patients. Pain Management Nursing. 2013 Sep;14(3):13342. doi:

https://dx.doi.org/10.1016/j.pmn.2010.08.00

2. PMID: 23972864. Exclusion: 5
212. Patrick LE. Multidisciplinary treatment of low back pain: long-term follow-up of a randomized, controlled trial. Dissertation/ thesis. 2000;122 PMID: CN-02109307. Exclusion: 8

213. Patwardhan A, Matika R, Gordon J, et al. Exploring the Role of Chronic Pain Clinics: Potential for Opioid Reduction. Pain Physician. 2018 11;21(6):E603-E10. PMID: 30508991. Exclusion: 2

214. Pavlek M. Paining out: An integrative pain therapy model. Clinical Social Work Journal. 2008 Dec;36(4):385-93. doi: http://dx.doi.org/10.1007/s10615-007-0136y. PMID: 2008-14743-007. Exclusion: 2

215. Pedersen P, Nielsen CV, Jensen OK, et al. Employment status five years after a randomised controlled trial comparing multidisciplinary and brief intervention in employees on sick leave due to low back pain. Scandinavian journal of public health. 2018;46(3):383-8. PMID: CN-01607473 NEW. Exclusion: 6

216. Perry KN, Nicholas MK, Middleton JW. Comparison of a pain management program with usual care in a pain management center for people with spinal cord injury-related chronic pain. Clin J Pain. 2010 MarApr;26(3):206-16. doi: 10.1097/AJP.0b013e3181bff8f3. PMID: 20173434. Exclusion: 8

217. Peters AA, van Dorst E, Jellis B, et al. A randomized clinical trial to compare two different approaches in women with chronic pelvic pain. Obstetrics \& Gynecology. 1991 May;77(5):740-4. PMID: 1826544. Exclusion: 4

218. Petrozzi MJ, Leaver A, Ferreira PH, et al. Addition of MoodGYM to physical treatments for chronic low back pain: A randomized controlled trial. Chiropractic \& manual therapies. 2019;27:54. doi: https://dx.doi.org/10.1186/s12998-0190277-4. PMID: 31673330. Exclusion: 4

219. Pimm TJ, Williams LJ, Reay M, et al. An evaluation of a digital pain management programme: clinical effectiveness and cost savings. British journal of pain. 2019 PMID: CN-01979036 NEW. Exclusion: 2 
220. Pimple CJ. Evaluation of a chronic care management program in a rural primary care clinic. Dissertation Abstracts International: Section B: The Sciences and Engineering. 2020;81(10-B):No Pagination Specified. PMID: 2020-31777-190. Exclusion: 2

221. Poirier RH, Brown CS, Baggenstos YT, et al. Impact of a pharmacist-directed pain management service on inpatient opioid use, pain control, and patient safety. American Journal of Health-System Pharmacy. 2019 Jan 01;76(1):17-25. doi: https://dx.doi.org/10.1093/ajhp/zxy003. PMID: 31381097. Exclusion: 4

222. Pouyanfard S, ParviziFard AA, Mohammadpour M, et al. Effectiveness of mindfulness-integrated cognitive behavior therapy on anxiety, depression and hope in multiple sclerosis patients: a randomized clinical trial. Trends in psychiatry and psychotherapy. 2020;42(1):55-63. PMID: CN-02122739 NEW. Exclusion: 3

223. Price CJ, McBride B, Hyerle L, et al. Mindful awareness in body-oriented therapy for female veterans with post-traumatic stress disorder taking prescription analgesics for chronic pain: a feasibility study.

Alternative Therapies in Health \& Medicine. 2007 Nov-Dec;13(6):32-40. PMID: 17985809. Exclusion: 3

224. Provinciali L, Baroni M, Illuminati L, et al. Multimodal treatment to prevent the late whiplash syndrome. Scandinavian Journal of Rehabilitation Medicine. 1996 May;28(2):105-11. PMID: 8815995. Exclusion: 4

225. Prvu Bettger JA, Stineman MG. Effectiveness of multidisciplinary rehabilitation services in postacute care: state-of-the-science. A review. Archives of Physical Medicine \& Rehabilitation. 2007 Nov;88(11):1526-34. PMID: 17964900. Exclusion: 10

226. Rantonen J, Karppinen J, Vehtari A, et al. Effectiveness of three interventions for secondary prevention of low back pain in the occupational health setting - a randomised controlled trial with a natural course control. BMC Public Health. 201805 08;18(1):598. doi:

https://dx.doi.org/10.1186/s12889-0185476-8. PMID: 29739371. Exclusion: 4
227. Rasmussen CD, Holtermann A, Mortensen OS, et al. Prevention of low back pain and its consequences among nurses' aides in elderly care: a stepped-wedge multi-faceted cluster-randomized controlled trial. BMC Public Health. 2013 Nov 21;13:1088. doi: https://dx.doi.org/10.1186/1471-2458-131088. PMID: 24261985. Exclusion: 4

228. Raspe HH, Deck R, Mattussek S. The outcome of traditional or comprehensive outpatient care for rheumatoid arthritis (RA). Results of an open, non-randomized, 2-year prospective study. Zeitschrift fur Rheumatologie. 1992;51 Suppl 1:61-6. PMID: 1471435. Exclusion: 8

229. Riberto M, Alfieri FM, de Benedetto Pacheco KM, et al. Efficacy of transcranial direct current stimulation coupled with a multidisciplinary rehabilitation program for the treatment of fibromyalgia. Open rheumatology journal. 2011;5(1):45-50. PMID: CN-00893536. Exclusion: 4

230. Ricci M, Fabbri L, Pirotti S, et al. Scrambler therapy: what's new after 15 years? The results from 219 patients treated for chronic pain. Medicine. 2019 Jan;98(2):e13895. doi: https://dx.doi.org/10.1097/MD.0000000000 013895. PMID: 30633163. Exclusion: 4

231. Riipinen M, Niemisto L, Lindgren KA, et al. Psychosocial differences as predictors for recovery from chronic low back pain following manipulation, stabilizing exercises and physician consultation or physician consultation alone. Journal of Rehabilitation Medicine. 2005 May;37(3):152-8. PMID: 16040472. Exclusion: 4

232. Ris I, Sogaard K, Gram B, et al. Does a combination of physical training, specific exercises and pain education improve health-related quality of life in patients with chronic neck pain? A randomised control trial with a 4-month follow up. Manual Therapy. 2016 Dec;26:132-40. doi: https://dx.doi.org/10.1016/j.math.2016.08.00 4. PMID: 27598552. Exclusion: 4

233. Robbins SR, Melo LRS, Urban H, et al. Is a stepped-care intervention effective in overweight and obese people with medial tibiofemoral osteoarthritis? The STrEAMline study: a randomised controlled trial. Arthritis care \& research. 2020 PMID: CN-02072889 NEW. Exclusion: 4 
234. Rodríguez-Torres J, López-López L, Cabrera-Martos I, et al. Effects of an Individualized Comprehensive Rehabilitation Program on Impaired Postural Control in Women With Chronic Pelvic Pain: A Randomized Controlled Trial. Archives of Physical Medicine \& Rehabilitation. 2020;101(8):1304-12. doi: 10.1016/j.apmr.2020.02.019. PMID: 144727206. Language: English. Entry Date: 20200730. Revision Date: 20200730. Publication Type: Article. Exclusion: 4

235. Rosemann T, Joos S, Laux G, et al. Case management of arthritis patients in primary care: a cluster-randomized controlled trial. Arthritis \& Rheumatism. 2007 Dec 15;57(8):1390-7. PMID: 18050178. Exclusion: 4

236. Ross LA, Bloodworth LS. Patient-centered health care using pharmacist-delivered medication therapy management in rural Mississippi. Journal of the American Pharmacists Association: JAPhA. 2012;52(6):802-9. doi: https://dx.doi.org/10.1331/JAPhA.2012.101 92. PMID: 23229968. Exclusion: 3

237. Şahin N, Karahan AY, Albayrak İ. Effectiveness of physical therapy and exercise on pain and functional status in patients with chronic low back pain: a randomized-controlled trial. Turkish Journal of Physical Medicine \& Rehabilitation (2587-0823). 2018;64(1):52-8. doi: 10.5606/tttrd.2018.1238. PMID: 128703315. Language: English. Entry Date: 20180410. Revision Date: 20180613. Publication Type: Article. Exclusion: 4

238. Salaffi F, Carotti M, Ciapetti A, et al. Effectiveness of a telemonitoring intensive strategy in early rheumatoid arthritis: comparison with the conventional management approach. BMC musculoskeletal disorders. 2016;17(1) PMID: CN-01154525. Exclusion: 4

239. Salaffi F, Ciapetti A, Gasparini S, et al. Web/Internet-based telemonitoring of a randomized controlled trial evaluating the time-integrated effects of a 24 -week multicomponent intervention on key health outcomes in patients with fibromyalgia. Clinical \& Experimental Rheumatology. 2015 Jan-Feb;33(1 Suppl 88):S93-101. PMID: 25786050. Exclusion: 4
240. Salisbury C, Man MS, Bower P, et al. Management of multimorbidity using a patient-centred care model: a pragmatic cluster-randomised trial of the 3D approach. Lancet. 201807 07;392(10141):41-50. doi: https://dx.doi.org/10.1016/S01406736(18)31308-4. PMID: 29961638. Exclusion: 3

241. Samwel HJ, Kraaimaat FW, Crul BJ, et al. Multidisciplinary allocation of chronic pain treatment: Effects and cognitive-behavioural predictors of outcome. British Journal of Health Psychology. 2009 Sep;14(3):405-21. doi:

http://dx.doi.org/10.1348/135910708X3377 60. PMID: 2010-00046-001. Exclusion: 4

242. Santolaya-Perrin R, Jimenez-Diaz G, GalanRamos N, et al. A randomised controlled trial on the efficacy of a multidisciplinary health care team on morbidity and mortality of elderly patients attending the Emergency Department. Study design and preliminary results. Farmacia Hospitalaria. 2016 Sep 01;40(5):371-84. doi:

https://dx.doi.org/10.7399/fh.2016.40.5.104 65. PMID: 27570988. Exclusion: 4

243. Schaller A, Dintsios CM, Icks A, et al. Promoting physical activity in low back pain patients: six months follow-up of a randomised controlled trial comparing a multicomponent intervention with a low intensity intervention. Clinical Rehabilitation. 2016 Sep;30(9):865-77. doi: https://dx.doi.org/10.1177/02692155156187 30. PMID: 27496696. Exclusion: 4

244. Schaller A, Petrowski K, Pfoertner TK, et al. Effectiveness of a theory-based multicomponent intervention (Movement Coaching) on the promotion of total and domain-specific physical activity: a randomised controlled trial in low back pain patients. BMC musculoskeletal disorders. 2017;18(1) PMID: CN-01429134.

Exclusion: 4

245. Scharf HP, Mansmann U, Streitberger K, et al. Acupuncture and knee osteoarthritis: a three-armed randomized trial. Annals of Internal Medicine. 2006 Jul 04;145(1):1220. PMID: 16818924. Exclusion: 4 
246. Schiltenwolf M, Buchner M, Heindl B, et al. Comparison of a biopsychosocial therapy (BT) with a conventional biomedical therapy (MT) of subacute low back pain in the first episode of sick leave: a randomized controlled trial. European Spine Journal. 2006 Jul;15(7):1083-92. PMID: 16311751. Exclusion: 4

247. Schreurs K, Veehof M, Passade L, et al. Cognitive behavioural treatment for chronic fatigue syndrome in a rehabilitation setting: Effectiveness and predictors of outcome. Behaviour Research and Therapy. 2011 Dec;49(12):908-13. doi: http://dx.doi.org/10.1016/j.brat.2011.09.004. PMID: 2011-25847-009. Exclusion: 3

248. Schutze R, Rees C, Preece M, et al. Low mindfulness predicts pain catastrophizing in a fear-avoidance model of chronic pain. Pain. 2010 Jan;148(1):120-7. doi: https://dx.doi.org/10.1016/j.pain.2009.10.03 0. PMID: 19944534. Exclusion: 8

249. Schutze R, Slater H, O'Sullivan P, et al. Mindfulness-Based Functional Therapy: A preliminary open trial of an integrated model of care for people with persistent low back pain. Frontiers in Psychology Vol 5 2014, ArtID 839. 2014 Aug;5 PMID: 2014-38857001. Exclusion: 4

250. Scott LJ, Kesten JM, Bache K, et al. Evaluation of a primary care-based opioid and pain review service: a mixed-methods evaluation in two GP practices in England. British journal of general practice. 2019 PMID: CN-02052022 NEW. Exclusion: 4

251. Scott W, Chilcot J, Guildford B, et al. Feasibility randomized-controlled trial of online Acceptance and Commitment Therapy for patients with complex chronic pain in the United Kingdom. European journal of pain (united kingdom). 2018;22(8):1473-84. PMID: CN-01630124. Exclusion: 4

252. Sennehed CP, Stigmar K, Grahn B, et al. Evaluation of a multimodal pain rehabilitation programme in primary care based on clinical register data: a feasibility study. Primary health care research \& development. 2020;21 PMID: CN-02076527 NEW. Exclusion: 6
253. Sharpe L, Sensky T, Timberlake N, et al. Long-term efficacy of a cognitive behavioural treatment from a randomized controlled trial for patients recently diagnosed with rheumatoid arthritis. Rheumatology. 2003 Mar;42(3):435-41. PMID: 12626793. Exclusion: 4

254. Sheng X, Simpson PM. Health care information seeking and seniors: determinants of Internet use. Health Marketing Quarterly. 2015;32(1):96-112. doi:

https://dx.doi.org/10.1080/07359683.2015.1 000758. PMID: 25751321. Exclusion: 8

255. Shim YW, Chua SS, Wong HC, et al. Collaborative intervention between pharmacists and physicians on elderly patients: a randomized controlled trial. Therapeutics and clinical risk management. 1115;14:1115-25. PMID: CN-01618320. Exclusion: 4

256. Silvemark A, Kallmen H, Molander C. Improved life satisfaction and pain reduction: follow-up of a 5-week multidisciplinary long-term pain rehabilitation programme. Upsala Journal of Medical Sciences. 2014 Aug;119(3):278-86. doi:

https://dx.doi.org/10.3109/03009734.2014.9 08252. PMID: 24878367. Exclusion: 8

257. Singh J, Singh P, Sohal MS. Effect of Exercise Rehabilitation Programme on Clinical Health Status of Osteoarthritis Knee Patients. Indian Journal of Physiotherapy \& Occupational Therapy. 2011;5(3):191-8. PMID: 104693817. Language: English. Entry Date: 20111125. Revision Date: 20150711. Publication Type: Journal Article. Exclusion: 8

258. Skouen JS, Grasdal A, Haldorsen EM. Return to work after comparing outpatient multidisciplinary treatment programs versus treatment in general practice for patients with chronic widespread pain. European Journal of Pain. 2006 Feb;10(2):145-52. PMID: 16310718. Exclusion: 6 
259. Skouen JS, Grasdal AL, Haldorsen EM, et al. Relative cost-effectiveness of extensive and light multidisciplinary treatment programs versus treatment as usual for patients with chronic low back pain on longterm sick leave: randomized controlled study. Spine. 2002 May 01;27(9):901-9; discussion 9-10. PMID: 11979157. Exclusion: 6

260. Skouen JS, Kvale A. Different outcomes in subgroups of patients with long-term musculoskeletal pain. Norsk epidemiologi. 2006;16(2):127-35. PMID: CN-01728841 NEW. Exclusion: 6

261. Slater MA, Weickgenant AL, Greenberg MA, et al. Preventing progression to chronicity in first onset, subacute low back pain: an exploratory study. Arch Phys Med Rehabil. 2009 Apr;90(4):545-52. doi: 10.1016/j.apmr.2008.10.032. PMID: 19345767. Exclusion: 4

262. Smeenk FW, de Witte LP, Nooyen IW, et al. Effects of transmural care on coordination and continuity of care. Patient Education and Counseling. 2000 Aug;41(1):73-81. doi: http://dx.doi.org/10.1016/S07383991\%2800\%2900117-8. PMID: 200005175-003. Exclusion: 3

263. Smeets RJ, Vlaeyen JW, Kester AD, et al. Reduction of pain catastrophizing mediates the outcome of both physical and cognitivebehavioral treatment in chronic low back pain. J Pain. 2006 Apr;7(4):261-71. doi: 10.1016/j.jpain.2005.10.011. PMID: 16618470. Exclusion: 6

264. Smith SM, Allwright S, O'Dowd T. Effectiveness of shared care across the interface between primary and specialty care in chronic disease management. Cochrane database of systematic reviews. 2007;3 PMID: CN-01770790 NEW. Exclusion: 10

265. Soderlund A, Lindberg P. Cognitive behavioural components in physiotherapy management of chronic whiplash associated disorders (WAD)--a randomised group study. Giornale Italiano di Medicina del Lavoro Ed Ergonomia. 2007 Jan-Mar;29(1 Suppl A):A5-11. PMID: 17650736. Exclusion: 4
266. Sommers LS, Marton KI, Barbaccia JC, et al. Physician, nurse, and social worker collaboration in primary care for chronically ill seniors. Archives of Internal Medicine. 2000 Jun 26;160(12):1825-33. PMID: 10871977. Exclusion: 3

267. Spinhoven P, Ter Kuile M, Kole-Snijders $\mathrm{AM}$, et al. Catastrophizing and internal pain control as mediators of outcome in the multidisciplinary treatment of chronic low back pain. European Journal of Pain. 2004 Jun;8(3):211-9. PMID: 15109971.

Exclusion: 4

268. Staats P. Trial Design and Endpoint Evaluation in Clinical Studies Addressing Chronic Back Pain. Spine. 2017(pagination) PMID: CN-01365422. Exclusion: 10

269. Stapelfeldt CM, Christiansen DH, Jensen OK, et al. Subgroup analyses on return to work in sick-listed employees with low back pain in a randomised trial comparing brief and multidisciplinary intervention. BMC musculoskeletal disorders. 2011;12 PMID: CN-01016316. Exclusion: 6

270. Steinmetz A, Psczolla M, Seidel W, et al. Effect of subgroup-specific multimodal therapy on chronic spinal back pain and function-a prospective inpatient multicentre clinical trial in Germany. Medicine. 2019 Jan;98(1):e13825. doi: https://dx.doi.org/10.1097/MD.0000000000 013825. PMID: 30608395. Exclusion: 8

271. Stoffer-Marx MA, Klinger M, Luschin S, et al. Functional consultation and exercises improve grip strength in osteoarthritis of the hand - a randomised controlled trial. Arthritis Research \& Therapy. 2018 Nov 09;20(1):253. doi: https://dx.doi.org/10.1186/s13075-0181747-0. PMID: 30413191. Exclusion: 4

272. Storro S, Moen J, Svebak S. Effects on sickleave of a multidisciplinary rehabilitation programme for chronic low back, neck or shoulder pain: comparison with usual treatment. Journal of Rehabilitation Medicine. 2004 Jan;36(1):12-6. PMID: 15074433. Exclusion: 6 
273. Strand LI, Ljunggren AE, Haldorsen EM, et al. The impact of physical function and pain on work status at 1-year follow-up in patients with back pain. Spine (Phila Pa 1976). 2001 Apr 1;26(7):800-8. doi: 10.1097/00007632-200104010-00022. PMID: 11295903. Exclusion: 4

274. Strong J. Incorporating cognitive-behavioral therapy with occupational therapy: A comparative study with patients with low back pain. Journal of Occupational Rehabilitation. 1998 Mar;8(1):61-71. doi: http://dx.doi.org/10.1023/A:1023008632131 . PMID: 1998-04347-004. Exclusion: 4

275. Stukstette MJ, Dekker J, den Broeder AA, et al. No evidence for the effectiveness of a multidisciplinary group based treatment program in patients with osteoarthritis of hands on the short term; results of a randomized controlled trial. Osteoarthritis \& Cartilage. 2013 Jul;21(7):901-10. doi: https://dx.doi.org/10.1016/j.joca.2013.03.01 6. PMID: 23583457. Exclusion: 4

276. Sundberg T, Petzold M, Wandell P, et al. Exploring integrative medicine for back and neck pain - a pragmatic randomised clinical pilot trial. BMC Complementary \& Alternative Medicine. 2009 Sep 07;9:33. doi: https://dx.doi.org/10.1186/1472-6882-933. PMID: 19735542. Exclusion: 4

277. Suni JH, Kolu P, Tokola K, et al. Effectiveness and cost-effectiveness of neuromuscular exercise and back care counseling in female healthcare workers with recurrent non-specific low back pain: a blinded four-arm randomized controlled trial. BMC Public Health. 2018 Dec 17;18(1):1376. doi: https://dx.doi.org/10.1186/s12889-0186293-9. PMID: 30558592. Exclusion: 4

278. Taimela S, Takala EP, Asklof T, et al. Active treatment of chronic neck pain: a prospective randomized intervention. Spine. 2000 Apr 15;25(8):1021-7. PMID: 10767816. Exclusion: 4
279. Taylor AM, Bingham J, Schussel K, et al. Integrating Innovative Telehealth Solutions into an Interprofessional Team-Delivered Chronic Care Management Pilot Program. Journal of Managed Care \& Specialty Pharmacy. 2018 Aug;24(8):813-8. doi: https://dx.doi.org/10.18553/jmcp.2018.24.8. 813. PMID: 30058982. Exclusion: 4

280. Taylor RR. Rehabilitation Programs for Individuals with Chronic Fatigue Syndrome: A Review. Journal of Chronic Fatigue Syndrome. 2006;13(1):41-55. doi: http://dx.doi.org/10.1300/J092v13n01 05. PMID: 2006-22188-004. Exclusion: 10

281. Taylor SS, Oddone EZ, Coffman CJ, et al. Cognitive Mediators of Change in Physical Functioning in Response to a Multifaceted Intervention for Managing Osteoarthritis. International Journal of Behavioral Medicine. 2018 Apr;25(2):162-70. doi: https://dx.doi.org/10.1007/s12529-0179689-5. PMID: 29453622. Exclusion: 6

282. Teitelbaum JE, Bird B, Greenfield RM, et al. Effective treatment of chronic fatigue syndrome and fibromyalgia - A randomized, double-blind, placebo-controlled, intent-totreat study. Journal of chronic fatigue syndrome. 2001;8(2):3-28. PMID: CN00369061. Exclusion: 4

283. Tejedor CC, Requena GC, Palacios PL, et al. A multidisciplinary approach for patients with non-specific chronic low back pain: Study protocol and preliminary findings. International Journal of Psychology \& Psychological Therapy. 2015;15(3):433-42. PMID: 2015-51644-009. Exclusion: 8

284. Thompson DP, Oldham JA, Woby SR. Does adding cognitive-behavioural physiotherapy to exercise improve outcome in patients with chronic neck pain? A randomised controlled trial. Physiotherapy. 2016 Jun;102(2):170-7. doi:

https://dx.doi.org/10.1016/j.physio.2015.04. 008. PMID: 26383695. Exclusion: 4

285. Thorn J, Man MS, Chaplin K, et al. Costeffectiveness of a patient-centred approach to managing multimorbidity in primary care: a pragmatic cluster randomised controlled trial. BMJ open. 2020;10(1) PMID: CN02086727 NEW. Exclusion: 3 
286. Tijhuis GJ, Zwinderman AH, Hazes JM, et al. Two-year follow-up of a randomized controlled trial of a clinical nurse specialist intervention, inpatient, and day patient team care in rheumatoid arthritis. Journal of Advanced Nursing. 2003 Jan;41(1):34-43. PMID: 12519286. Exclusion: 4

287. Tijhuis GJ, Zwinderman AH, Hazes JM, et al. A randomized comparison of care provided by a clinical nurse specialist, an inpatient team, and a day patient team in rheumatoid arthritis. Arthritis \& Rheumatism. 2002 Oct 15;47(5):525-31. PMID: 12382302. Exclusion: 4

288. Toelle TR, Utpadel-Fischler DA, Haas KK, et al. App-based multidisciplinary back pain treatment versus combined physiotherapy plus online education: a randomized controlled trial. Npj digital medicine. 2019;2(1) PMID: CN-01942671. Exclusion: 4

289. Tokem Y, Argon G, Keser G. Case management in care of Turkish rheumatoid arthritis patients. Rehabilitation Nursing Journal. 2011 Sep-Oct;36(5):205-13. PMID: 21882799. Exclusion: 4

290. Toprak Celenay S, Anaforoglu Kulunkoglu B, Yasa ME, et al. A comparison of the effects of exercises plus connective tissue massage to exercises alone in women with fibromyalgia syndrome: a randomized controlled trial. Rheumatology International. 2017 Nov;37(11):1799-806. doi: https://dx.doi.org/10.1007/s00296-0173805-3. PMID: 28840379. Exclusion: 4

291. Toprak Celenay S, Mete O, Akan S, et al. Comparison of the effects of stabilization exercise plus kinesio taping and stabilization exercise alone on pain and well-being in fibromyalgia. Complementary Therapies in Clinical Practice. 2020;38:N.PAG-N.PAG. doi: 10.1016/j.ctcp.2019.101076. PMID: 141784937. Language: English. Entry Date: 20200225. Revision Date: 20200227. Publication Type: Article. Exclusion: 4

292. Tracy B, Sean Morrison R. Pain management in older adults. Clinical Therapeutics. 2013 Nov;35(11):1659-68. doi: https://dx.doi.org/10.1016/j.clinthera.2013.0 9.026. PMID: 24148553. Exclusion: 10
293. Traeger AC, Lee H, Hubscher M, et al. Effect of Intensive Patient Education vs Placebo Patient Education on Outcomes in Patients With Acute Low Back Pain: A Randomized Clinical Trial. JAMA Neurology. 201902 01;76(2):161-9. doi: https://dx.doi.org/10.1001/jamaneurol.2018. 3376. PMID: 30398542. Exclusion: 4

294. Tricco AC, Antony J, Ivers NM, et al. Effectiveness of quality improvement strategies for coordination of care to reduce use of health care services: A systematic review and meta-analysis. Canadian Medical Association Journal. 2014 Oct;186(15):E568-E78. doi: http://dx.doi.org/10.1503/cmaj.140289. PMID: 2015-18141-002. Exclusion: 10

295. Trivedi D, Goodman C, Gage H, et al. The effectiveness of inter-professional working for older people living in the community: a systematic review. Health \& Social Care in the Community. 2013 Mar;21(2):113-28. doi: https://dx.doi.org/10.1111/j.13652524.2012.01067.x. PMID: 22891915. Exclusion: 10

296. Truchon M, Fillion L. Biopsychosocial determinants of chronic disability and lowback pain: a review. Journal of occupational rehabilitation. 2000;10(2):117-42. PMID: CN-01737224 NEW. Exclusion: 10

297. Tsang SMH, So BCL, Lau RWL, et al. Comparing the effectiveness of integrating ergonomics and motor control to conventional treatment for pain and functional recovery of work-related neckshoulder pain: A randomized trial. European Journal of Pain. 2019 07;23(6):1141-52. doi: https://dx.doi.org/10.1002/ejp.1381. PMID: 30793422. Exclusion: 4

298. Tse M, Li Y, Tang SK, et al. An exploration of the effectiveness of a peer-led pain management program (PAP) for nursing home residents with chronic pain and an evaluation of their experiences: a pilot randomized controlled trial. International journal of environmental research and public health. 2020;17(11):1-11. PMID: CN02143966 NEW. Exclusion: 4 
299. Tse MM, Ho SS. Pain management for older persons living in nursing homes: a pilot study. Pain Management Nursing. 2013 Jun;14(2):e10-21. doi: https://dx.doi.org/10.1016/j.pmn.2011.01.00 4. PMID: 23688367. Exclusion: 4

300. Tse MM, Tang SK, Wan VT, et al. The effectiveness of physical exercise training in pain, mobility, and psychological well-being of older persons living in nursing homes. Pain management nursing. 2014;15(4):77888. PMID: CN-01367707. Exclusion: 4

301. Tse MM, Vong SK, Ho SS. The effectiveness of an integrated pain management program for older persons and staff in nursing homes. Arch Gerontol Geriatr. 2012 Mar-Apr;54(2):e203-12. doi: 10.1016/j.archger.2011.04.015. PMID: 21592596. Exclusion: 4

302. Tse MM, Yeung SS, Lee PH, et al. Effects of a Peer-Led Pain Management Program for Nursing Home Residents with Chronic Pain: A Pilot Study. Pain Medicine. 2016 09;17(9):1648-57. doi: https://dx.doi.org/10.1093/pm/pnv121. PMID: 26893112. Exclusion: 4

303. Tummers M, Knoop H, Bleijenberg G. Effectiveness of stepped care for chronic fatigue syndrome: a randomized noninferiority trial. Journal of Consulting \& Clinical Psychology. 2010 Oct;78(5):72431. doi: https://dx.doi.org/10.1037/a0020052. PMID: 20873907. Exclusion: 4

304. Turner JA, Jensen MP, Romano JM. Do beliefs, coping, and catastrophizing independently predict functioning in patients with chronic pain? Pain. 2000 Mar;85(12):115-25. PMID: 10692610. Exclusion: 8

305. Vaegter HB, Stoten M, Silseth SL, et al. Cause-specific mortality of patients with severe chronic pain referred to a multidisciplinary pain clinic: a cohort register-linkage study. Scandinavian Journal of Pain. 201901 28;19(1):93-9. doi: https://dx.doi.org/10.1515/sjpain-2018-0094. PMID: 30205653. Exclusion: 6
306. van Baar ME, Dekker J, Oostendorp RA, et al. Effectiveness of exercise in patients with osteoarthritis of hip or knee: nine months' follow up. Annals of the Rheumatic Diseases. 2001 Dec;60(12):1123-30. PMID: 11709454. Exclusion: 4

307. van den Driest JJ, Schiphof D, Luijsterburg PAJ, et al. Effectiveness and costeffectiveness of duloxetine added to usual care for patients with chronic pain due to hip or knee osteoarthritis: protocol of a pragmatic open-label cluster randomised trial (the DUO trial). BMJ Open. 2017 Sep 11;7(9):e018661. doi: https://dx.doi.org/10.1136/bmjopen-2017018661. PMID: 28893757. Exclusion: 4

308. van den Hout JH, Vlaeyen JW, Heuts PH, et al. Secondary prevention of work-related disability in nonspecific low back pain: does problem-solving therapy help? A randomized clinical trial. Clinical Journal of Pain. 2003 Mar-Apr;19(2):87-96. PMID: 12616178. Exclusion: 4

309. van der Hulst M, Vollenbroek-Hutten MM, Groothuis-Oudshoorn KG, et al.

Multidisciplinary rehabilitation treatment of patients with chronic low back pain: a prognostic model for its outcome. Clinical Journal of Pain. 2008 Jun;24(5):421-30. doi: https://dx.doi.org/10.1097/AJP.0b013e3181 6719f5. PMID: 18496307. Exclusion: 6

310. van der Maas LC, Koke A, Bosscher RJ, et al. Body Awareness as an Important Target in Multidisciplinary Chronic Pain Treatment: Mediation and Subgroup Analyses. Clinical Journal of Pain. 2016 09;32(9):763-72. doi:

https://dx.doi.org/10.1097/AJP.0000000000 000320. PMID: 26550959. Exclusion: 6

311. van der Roer N, van Tulder M, Barendse J, et al. Intensive group training protocol versus guideline physiotherapy for patients with chronic low back pain: a randomised controlled trial. Eur Spine J. 2008 Sep;17(9):1193-200. doi: 10.1007/s00586008-0718-6. PMID: 18663487. Exclusion: 4 
312. van der Roer N, van Tulder M, van Mechelen W, et al. Economic evaluation of an intensive group training protocol compared with usual care physiotherapy in patients with chronic low back pain. Spine (Phila Pa 1976). 2008 Feb 15;33(4):445-51. doi: 10.1097/BRS.0b013e318163fa59. PMID: 18277878. Exclusion: 4

313. van der Sluis CK, Datema L, Saan I, et al. Effects of a nurse practitioner on a multidisciplinary consultation team. Journal of Advanced Nursing. 2009 Mar;65(3):62533. doi: https://dx.doi.org/10.1111/j.13652648.2008.04916.x. PMID: 19222660.

Exclusion: 4

314. van Eck van der Sluijs JF, Castelijns H, Eijsbroek V, et al. Illness burden and physical outcomes associated with collaborative care in patients with comorbid depressive disorder in chronic medical conditions: A systematic review and metaanalysis. General Hospital Psychiatry. 2018 Jan-Feb;50:1-14. doi: http://dx.doi.org/10.1016/j.genhosppsych.20 17.08.003. PMID: 2018-04112-002.

Exclusion: 10

315. van Eeghen CO, Littenberg B, Kessler R. Chronic care coordination by integrating care through a team-based, populationdriven approach: A case study. Translational Behavioral Medicine. 2018 Jun;8(3):468-80. doi: http://dx.doi.org/10.1093/tbm/ibx073. PMID: 2018-29830-018. Exclusion: 3

316. van Erp RM, Huijnen IP, Jakobs ML, et al. Effectiveness of primary care interventions using a biopsychosocial approach in chronic low back pain: A systematic review. Pain Practice. 2019 Feb;19(2):224-41. doi: http://dx.doi.org/10.1111/papr.12735. PMID: 2019-06605-011. Exclusion: 10

317. van Erp RMA, Huijnen IPJ, Ambergen AW, et al. Biopsychosocial primary care versus physiotherapy as usual in chronic low back pain: results of a pilot-randomised controlled trial. European journal of physiotherapy. 2019 PMID: CN-01959019 NEW. Exclusion: 4
318. van Gils RF, Boot CR, Knol DL, et al. The effectiveness of integrated care for patients with hand eczema: results of a randomized, controlled trial. Contact Dermatitis. 2012 Apr;66(4):197-204. doi:

https://dx.doi.org/10.1111/j.16000536.2011.02024.x. PMID: 22404194.

Exclusion: 4

319. van Gils RF, Bosmans JE, Boot CR, et al. Economic evaluation of an integrated care programme for patients with hand dermatitis. Contact Dermatitis. 2013 Sep;69(3):144-52. doi: https://dx.doi.org/10.1111/cod.12095. PMID: 23808896. Exclusion: 6

320. van Gils RF, van der Valk PG, Bruynzeel D, et al. Integrated, multidisciplinary care for hand eczema: design of a randomized controlled trial and cost-effectiveness study. BMC Public Health. 2009 Dec 01;9:438. doi: https://dx.doi.org/10.1186/1471-2458-9438. PMID: 19951404. Exclusion: 4

321. Van Middelkoop M, Rubinstein SM, Kuijpers T, et al. A systematic review on the effectiveness of physical and rehabilitation interventions for chronic non-specific low back pain. European spine journal. 2011;20(1):19-39. PMID: CN-01732773 NEW. Exclusion: 10

322. van Vilsteren M, Boot CR, Twisk JW, et al. One Year Effects of a Workplace Integrated Care Intervention for Workers with Rheumatoid Arthritis: results of a Randomized Controlled Trial. Journal of occupational rehabilitation. 2017;27(1):12836. PMID: CN-01464204 NEW. Exclusion: 4

323. van Vilsteren M, Boot CR, Twisk JW, et al. Effectiveness of an integrated care intervention on supervisor support and work functioning of workers with rheumatoid arthritis. Disability and rehabilitation. 2017;39(4):354-62. PMID: CN-01460793 NEW. Exclusion: 6

324. Van Zundert J. Clinical research in interventional pain management techniques: The clinician's point of view. Pain Practice. 2007 Sep;7(3):221-9. doi: http://dx.doi.org/10.1111/j.15332500.2007.00139.x. PMID: 2007-12641001. Exclusion: 10 
325. Vanderboom CE, Holland DE, Lohse CM, et al. Enhancing patient-centered care: Pilot study results of a community care team intervention. Western Journal of Nursing Research. 2014 Jan;36(1):47-65. doi: http://dx.doi.org/10.1177/019394591349084 1. PMID: 2013-43209-004. Exclusion: 3

326. Vanhaudenhuyse A, Gillet A, Malaise N, et al. Efficacy and cost-effectiveness: a study of different treatment approaches in a tertiary pain centre. European journal of pain (united kingdom). 2015;19(10):143746. PMID: CN-01131955. Exclusion: 8

327. Vas J, Ortega C, Olmo V, et al. Single-point acupuncture and physiotherapy for the treatment of painful shoulder: a multicentre randomized controlled trial. Rheumatology. 2008 Jun;47(6):887-93. doi: https://dx.doi.org/10.1093/rheumatology/ken 040. PMID: 18403402. Exclusion: 4

328. Vera M, Perez-Pedrogo C, Huertas SE, et al. Collaborative care for depressed patients with chronic medical conditions: a randomized trial in Puerto Rico. Psychiatric Services. 2010 Feb;61(2):144-50. doi: https://dx.doi.org/10.1176/ps.2010.61.2.144. PMID: 20123819. Exclusion: 3

329. Verhagen AP, Bierma-Zeinstra SM, Feleus A, et al. Ergonomic and physiotherapeutic interventions for treating upper extremity work related disorders in adults. Cochrane database of systematic reviews. 2004;1 PMID: CN-01766650 NEW. Exclusion: 10

330. Vermunt N, Harmsen M, Westert GP, et al. Collaborative goal setting with elderly patients with chronic disease or multimorbidity: a systematic review. BMC Geriatrics. 201707 31;17(1):167. doi: https://dx.doi.org/10.1186/s12877-0170534-0. PMID: 28760149. Exclusion: 10

331. Verwoerd AJH, Luijsterburg PAJ, Koes BW, et al. Does Kinesiophobia Modify the Effects of Physical Therapy on Outcomes in Patients With Sciatica in Primary Care? Subgroup Analysis From a Randomized Controlled Trial. Physical Therapy. 2015;95(9):1217-23. doi: 10.2522/ptj.20140458. PMID: 109244721. Language: English. Entry Date: 20151013. Revision Date: 20151013. Publication Type: Article. Exclusion: 3
332. Vibe Fersum K, O'Sullivan P, Skouen JS, et al. Efficacy of classification-based cognitive functional therapy in patients with nonspecific chronic low back pain: a randomized controlled trial. Eur J Pain. 2013 Jul;17(6):916-28. doi: 10.1002/j.15322149.2012.00252.x. PMID: 23208945. Exclusion: 4

333. Villafane JH, Cleland JA, Fernandez-deLas-Penas C. The effectiveness of a manual therapy and exercise protocol in patients with thumb carpometacarpal osteoarthritis: a randomized controlled trial. Journal of Orthopaedic \& Sports Physical Therapy. 2013 Apr;43(4):204-13. doi: https://dx.doi.org/10.2519/jospt.2013.4524. PMID: 23485660. Exclusion: 4

334. Viner R, Gregorowski A, Wine C, et al. Outpatient rehabilitative treatment of chronic fatigue syndrome (CFS/ME). Archives of disease in childhood. 2004;89(7):615-9. PMID: CN-00893173. Exclusion: 3

335. Visser LH, Woudenberg NP, de Bont J, et al. Treatment of the sacroiliac joint in patients with leg pain: a randomizedcontrolled trial. European Spine Journal. 2013 Oct;22(10):2310-7. doi: https://dx.doi.org/10.1007/s00586-0132833-2. PMID: 23720124. Exclusion: 4

336. Vliet Vlieland TP, Breedveld FC, Hazes JM. The two-year follow-up of a randomized comparison of in-patient multidisciplinary team care and routine out-patient care for active rheumatoid arthritis. British Journal of Rheumatology. 1997 Jan;36(1):82-5. PMID: 9117182. Exclusion: 4

337. Vliet Vlieland TP, Zwinderman AH, Breedveld FC, et al. Measurement of morning stiffness in rheumatoid arthritis clinical trials. Journal of Clinical Epidemiology. 1997 Jul;50(7):757-63. PMID: 9253385. Exclusion: 6

338. Vliet Vlieland TP, Zwinderman AH, Vandenbroucke JP, et al. A randomized clinical trial of in-patient multidisciplinary treatment versus routine out-patient care in active rheumatoid arthritis. British Journal of Rheumatology. 1996 May;35(5):475-82. PMID: 8646440. Exclusion: 4 
339. Vollenbroek-Hutten MM, Hermens HJ, Wever D, et al. Differences in outcome of a multidisciplinary treatment between subgroups of chronic low back pain patients defined using two multiaxial assessment instruments: the multidimensional pain inventory and lumbar dynamometry. Clinical Rehabilitation. 2004

Aug;18(5):566-79. PMID: 15293491. Exclusion: 4

340. von Bulow C, Amris K, Bandak E, et al. Improving activities of daily living ability in women with fibromyalgia: An exploratory, quasi-randomized, phase-two study, IMPROvE trial. Journal of Rehabilitation Medicine. 2017 Mar 06;49(3):241-50. doi: https://dx.doi.org/10.2340/16501977-2198. PMID: 28240340. Exclusion: 4

341. Vos-Vromans DC, Huijnen IP, Rijnders LJ, et al. Treatment expectations influence the outcome of multidisciplinary rehabilitation treatment in patients with CFS. Journal of Psychosomatic Research. 2016 Apr;83:40-5. doi:

https://dx.doi.org/10.1016/j.jpsychores.2016. 02.004. PMID: 27020075. Exclusion: 6

342. Vos-Vromans DC, Smeets RJ, Huijnen IP, et al. Multidisciplinary rehabilitation treatment versus cognitive behavioural therapy for patients with chronic fatigue syndrome: a randomized controlled trial. Journal of Internal Medicine. 2016 Mar;279(3):268-82. doi: https://dx.doi.org/10.1111/joim.12402. PMID: 26306716. Exclusion: 3

343. Vugts MA, Joosen MC, Mert A, et al. Effectiveness of serious gaming during the multidisciplinary rehabilitation of patients with complex chronic pain or fatigue: Natural quasi-experiment. Journal of Medical Internet Research Vol 20(8), 2018, ArtID e250. 2018 Aug;20(8)doi: http://dx.doi.org/10.2196/jmir.9739. PMID: 2018-57227-001. Exclusion: 5

344. Wagle KC, Skopelja EN, Campbell NL. Caregiver-Based Interventions to Optimize Medication Safety in Vulnerable Elderly Adults: A Systematic Evidence-Based Review. Journal of the American Geriatrics Society. 2018 11;66(11):2128-35. doi: https://dx.doi.org/10.1111/jgs.15556. PMID: 30136714. Exclusion: 10
345. Walker JM. A 6 week psychoeducational programme reduced pain and improved quality of life in adults with chronic idiopathic pain. Evidence based nursing. 1998 PMID: CN-02107371 NEW. Exclusion: 8

346. Walti P, Kool J, Luomajoki H. Short-term effect on pain and function of neurophysiological education and sensorimotor retraining compared to usual physiotherapy in patients with chronic or recurrent non-specific low back pain, a pilot randomized controlled trial. BMC Musculoskeletal Disorders. 2015 Apr 10;16:83. doi: https://dx.doi.org/10.1186/s12891-0150533-2. PMID: 25887550. Exclusion: 4

347. Wand BM, Bird C, McAuley JH, et al. Early intervention for the management of acute low back pain: a single-blind randomized controlled trial of biopsychosocial education, manual therapy, and exercise. Spine. 2004 Nov 01;29(21):2350-6. PMID: 15507794. Exclusion: 4

348. Wearden AJ, Morriss RK, Mullis R, et al. Randomised, double-blind, placebocontrolled treatment trial of fluoxetine and graded exercise for chronic fatigue syndrome. British Journal of Psychiatry. 1998 Jun;172:485-90. PMID: 9828987. Exclusion: 4

349. Wertli MM, Kessels AG, Perez RS, et al. Rational pain management in complex regional pain syndrome 1 (CRPS 1)--a network meta-analysis. Pain Medicine. 2014 Sep;15(9):1575-89. doi: https://dx.doi.org/10.1111/pme.12466. PMID: 25234478. Exclusion: 10

350. Westman A, Linton SJ, Ohrvik J, et al. Controlled 3-year follow-up of a multidisciplinary pain rehabilitation program in primary health care. Disability and Rehabilitation: An International, Multidisciplinary Journal. 2010;32(4):30716. doi: http://dx.doi.org/10.3109/096382809030959 24. PMID: 2010-00475-002. Exclusion: 8 
351. Wilkey A, Gregory M, Byfield D, et al. A comparison between chiropractic management and pain clinic management for chronic low-back pain in a national health service outpatient clinic. Journal of Alternative \& Complementary Medicine. 2008 Jun;14(5):465-73. doi: https://dx.doi.org/10.1089/acm.2007.0796. PMID: 18564952. Exclusion: 4

352. Williams AC, Nicholas MK, Richardson $\mathrm{PH}$, et al. Generalizing from a controlled trial: The effects of patient preference versus randomization on the outcome of inpatient versus outpatient chronic pain management. Pain. 1999 Oct;83(1):57-65. doi: http://dx.doi.org/10.1016/S03043959\%2899\%2900074-3. PMID: 199901393-007. Exclusion: 8

353. Williams DA, Cary MA, Groner KH, et al. Improving physical functional status in patients with fibromyalgia: a brief cognitive behavioral intervention. Journal of Rheumatology. 2002 Jun;29(6):1280-6. PMID: 12064847. Exclusion: 4

354. Williams DA, Kuper D, Segar M, et al. Internet-enhanced management of fibromyalgia: a randomized controlled trial. Pain. 2010 Dec;151(3):694-702. doi: https://dx.doi.org/10.1016/j.pain.2010.08.03 4. PMID: 20855168. Exclusion: 4

355. Wilson M, Roll JM, Corbett C, et al. Empowering Patients with Persistent Pain Using an Internet-based Self-Management Program. Pain management nursing. 2015;16(4):503-14. PMID: CN-01366690. Exclusion: 4

356. Wippert PM, Driesslein D, Beck H, et al. The feasibility and effectiveness of a new practical multidisciplinary treatment for low-back pain: a randomized controlled trial. Journal of clinical medicine. 2020;9(1) PMID: CN-02074928. Exclusion: 4

357. Witt CM, Michalsen A, Roll S, et al. Comparative effectiveness of a complex Ayurvedic treatment and conventional standard care in osteoarthritis of the knee-study protocol for a randomized controlled trial. Trials [Electronic Resource]. 2013 May 23;14:149. doi: https://dx.doi.org/10.1186/1745-6215-14149. PMID: 23701973. Exclusion: 4
358. Wong SY, Chan FW, Wong RL, et al. Comparing the effectiveness of mindfulnessbased stress reduction and multidisciplinary intervention programs for chronic pain: a randomized comparative trial. Clinical Journal of Pain. 2011 Oct;27(8):724-34. doi: https://dx.doi.org/10.1097/AJP.0b013e3182 183c6e. PMID: 21753729. Exclusion: 4

359. Wong WS, Chen PP, Yap J, et al. Chronic pain and psychiatric morbidity: A comparison between patients attending specialist orthopedics clinic and multidisciplinary pain clinic. Pain Medicine. 2011 Feb;12(2):246-59. doi: http://dx.doi.org/10.1111/j.15264637.2010.01044.x. PMID: 2011-03032009. Exclusion: 8

360. Wood S, Gehlfuss S, Greisl B, et al. Exploring the Multimodal Theory of Pain Management: Results of a Randomized Controlled Trial. Journal of PeriAnesthesia Nursing. 2015;30(4):e37-e. doi: 10.1016/j.jopan.2015.05.099. PMID: 108506091. Language: English. Entry Date: 20151012. Revision Date: 20151012. Publication Type: Article. Exclusion: 9

361. Worm-Smeitink M, Janse A, van Dam A, et al. Internet-Based Cognitive Behavioral Therapy in Stepped Care for Chronic Fatigue Syndrome: Randomized Noninferiority Trial. J Med Internet Res. 2019 Mar 14;21(3):e11276. doi: 10.2196/11276. PMID: 30869642. Exclusion: 4

362. Wright K, Hazelett S, Jarjoura D, et al. The AD-LIFE trial: working to integrate medical and psychosocial care management models. Home healthcare nurse. 2007;25(5):308-14. PMID: CN-01726008 NEW. Exclusion: 3

363. Yang EJ, Park WB, Shin HI, et al. The effect of back school integrated with core strengthening in patients with chronic lowback pain. American Journal of Physical Medicine \& Rehabilitation. 2010 Sep;89(9):744-54. doi: https://dx.doi.org/10.1097/PHM.0b013e318 1e72239. PMID: 20581648. Exclusion: 4 
364. Yang S-Y, McCracken LM, Moss-Morris R. Psychological treatment needs for chronic pain in Singapore and the relevance of the psychological flexibility model. Pain Medicine. 2017 Sep;18(9):1679-94. PMID: 2018-46562-009. Exclusion: 8

365. Yau CY. The Effects of Nurse-Initiated Early Pain Management Program (NIEPMP) for Acute Back Pain in Emergency Medicine Ward: A Randomized Control Trial: Chinese University of Hong Kong (Hong Kong); 2013. Exclusion: 9

366. Yoon J, Chang E, Rubenstein LV, et al. Impact of Primary Care Intensive Management on High-Risk Veterans' Costs and Utilization A Randomized Quality Improvement Trial. Annals of internal medicine. 2018;168(12):846-54. PMID: CN-01611132. Exclusion: 3

367. Zhang M, Wang H, Pan X, et al. Efficacy of whole-course pain intervention on healthrelated quality of life for patients after esophagectomy. Journal of B.U.On. 2016 Nov-Dec;21(6):1546-51. PMID: 28039721. Exclusion: 3

368. Zhang NJ, Wan TT, Rossiter LF, et al. Evaluation of chronic disease management on outcomes and cost of care for Medicaid beneficiaries. Health Policy. 2008 May;86(2-3):345-54. doi: https://dx.doi.org/10.1016/j.healthpol.2007.1 1.011. PMID: 18207282. Exclusion: 3

369. Zulman DM, Pal Chee C, Ezeji-Okoye SC, et al. Effect of an Intensive Outpatient Program to Augment Primary Care for High-Need Veterans Affairs Patients: A Randomized Clinical Trial. JAMA Internal Medicine. 201702 01;177(2):166-75. doi: https://dx.doi.org/10.1001/jamainternmed.20 16.8021. PMID: 28027338. Exclusion: 3

370. Zyczkowska J, Szczerbinska K, Jantzi MR, et al. Pain among the oldest old in community and institutional settings. Pain. 2007 May;129(1-2):167-76. PMID:

17250966. Exclusion: 4 


\section{Excluded from peer/public review:}

1. Ahles TA, Wasson JH, Seville JL, et al. A controlled trial of methods for managing pain in primary care patients with or without co-occurring psychosocial problems. Ann Fam Med. 2006 Jul-Aug;4(4):341-50. doi: 10.1370/afm.527. PMID:

16868238.Exclusion: 4

2. American Massage Therapy Association. Massage Therapy in Integrative Care \& Pain Management. American Massage Therapy Association; 2018. https:/www.amtamassage.org/globalassets/d ocuments/publications-andresearch $/ \mathrm{mt}$ in integrative care and pain management.pdf.Exclusion: 9

3. Bosy D, Etlin D, Corey D, et al. An interdisciplinary pain rehabilitation programme: description and evaluation of outcomes. Physiother Can. 2010

Fall;62(4):316-26. doi: 10.3138/physio.62.4.316. PMID: 21886371.Exclusion: 8

4. Burns J, Mullen TA. The role of traditional Chinese medicine in the management of chronic pain: a biopsychosocial approach. J Patient Cent Res Rev. 2015;2:192-6. doi: 10.17294/2330-0698.1206.Exclusion: 9

5. Burns JR, Kram JJ, Xiong V, et al. Utilization of acupuncture services in the emergency department setting: a quality improvement study. J Patient Cent Res Rev. 2019;6:172-8. doi: 10.17294/2330-

0698.1688.Exclusion: 8

6. Chao MT, Hurstak E, Leonoudakis-Watts K, et al. Patient-Reported Outcomes of an Integrative Pain Management Program Implemented in a Primary Care Safety Net Clinic: a Quasi-experimental Study. J Gen Intern Med. 2019 Jul;34(7):1105-7. doi: 10.1007/s11606-019-04868-0. PMID: 30783878.Exclusion: 8

7. Chu H, Seo J, Kim C, et al.

Electroacupuncture for migraine protocol for a systematic review of controlled trials.

Medicine (Baltimore). 2018

Apr;97(17):e9999. doi:

10.1097/MD.0000000000009999. PMID:

29703068.Exclusion: 4
8. Darnall BD, Krishnamurthy P, Tsuei J, et al. Self-Administered Skills-Based Virtual Reality Intervention for Chronic Pain: Randomized Controlled Pilot Study. JMIR Form Res. 2020 Jul 7;4(7):e17293. doi: 10.2196/17293. PMID: 32374272.Exclusion: 4

9. Dusek JA, Finch M, Plotnikoff G, et al. The impact of integrative medicine on pain management in a tertiary care hospital. J Patient Saf. 2010 Mar;6(1):48-51. doi: 10.1097/PTS.0b013e3181d10ad5. PMID: 22130304.Exclusion: 8

10. Dusek JA, Rivard RL, Griffin KH, et al. Significant Pain Reduction in Hospitalized Patients Receiving Integrative Medicine Interventions by Clinical Population and Accounting for Pain Medication. J Altern Complement Med. 2021 Mar;27(S1):S28S36. doi: 10.1089/acm.2021.0051. PMID: 33788611.Exclusion: 8

11. Dyer NL, Surdam J, Dusek JA. A Systematic Review of Practiced-Based Research of Complementary and Integrative Health Therapies as Provided for Pain Management in Clinical Settings: Recommendations for the Future and A Call to Action. Pain Med. 2021 May 1doi: 10.1093/pm/pnab151. PMID: 34009391.Exclusion: 8

12. Elwy AR, Taylor SL. Progress of Veterans Health Administration Complementary and Integrative Health Research Along the Quality Enhancement Research Initiative Implementation Roadmap. Med Care. 2020 Sep;58 Suppl 2 9S:S75-S7. doi: 10.1097/MLR.0000000000001382. PMID: 32826774.Exclusion: 9

13. Elwy AR, Taylor SL, Zhao S, et al. Participating in Complementary and Integrative Health Approaches Is Associated With Veterans' Patient-reported Outcomes Over Time. Med Care. 2020 Sep;58 Suppl 2 9S:S125-S32. doi: 10.1097/MLR.0000000000001357. PMID: 32826782.Exclusion: 8 
14. Esparham A, Herbert A, Pierzchalski E, et al. Pediatric Headache Clinic Model: Implementation of Integrative Therapies in Practice. Children (Basel). 2018 Jun 12;5(6)doi: 10.3390/children5060074. PMID: 29895725.Exclusion: 3

15. Faber T, Ravaud P, Riveros C, et al. Metaanalyses including non-randomized studies of therapeutic interventions: a methodological review. BMC Med Res Methodol. 2016 Mar 22;16:35. doi: 10.1186/s12874-016-0136-0. PMID: 27004721.Exclusion: 9

16. Fan AY, Miller DW, Bolash B, et al. Acupuncture's Role in Solving the Opioid Epidemic: Evidence, Cost-Effectiveness, and Care Availability for Acupuncture as a Primary, Non-Pharmacologic Method for Pain Relief and Management-White Paper 2017. J Integr Med. 2017 Nov;15(6):411-25. doi: 10.1016/S2095-4964(17)60378-9. PMID: 29103410.Exclusion: 8

17. Fink J, Burns J, Perez Moreno AC, et al. A Quality Brief of an Oncological Multisite Massage and Acupuncture Therapy Program to Improve Cancer-Related Outcomes. J Altern Complement Med. 2020

Sep;26(9):820-4. doi:

10.1089/acm.2019.0371. PMID: 32924553.Exclusion: 3

18. Flynn D, Eaton LH, Langford DJ, et al. A SMART design to determine the optimal treatment of chronic pain among military personnel. Contemp Clin Trials. 2018 Oct;73:68-74. doi: 10.1016/j.cct.2018.08.008. PMID: 30145267.Exclusion: 8

19. Flynn DM, Cook K, Kallen M, et al. Use of the pain assessment screening tool and outcomes registry in an army interdisciplinary pain management center, lessons learned and future implications of a 10 -month beta test. Military medicine. 2017;182(suppl_1):167-74.Exclusion: 8

20. Flynn DM, Eaton LH, McQuinn H, et al. TelePain: Primary Care Chronic Pain Management through Weekly Didactic and Case-based Telementoring. Contemp Clin Trials Commun. 2017 Dec;8:162-6. doi: 10.1016/j.conctc.2017.10.004. PMID: 29497708.Exclusion: 8
21. Garcia LM, Birckhead BJ, Krishnamurthy P, et al. An 8-Week Self-Administered AtHome Behavioral Skills-Based Virtual Reality Program for Chronic Low Back Pain: Double-Blind, Randomized, PlaceboControlled Trial Conducted During COVID19. J Med Internet Res. 2021 Feb 22;23(2):e26292. doi: 10.2196/26292. PMID: 33484240.Exclusion: 4

22. Gardiner P, Lestoquoy AS, Gergen-Barnett $\mathrm{K}$, et al. Design of the integrative medical group visits randomized control trial for underserved patients with chronic pain and depression. Contemp Clin Trials. 2017 Mar;54:25-35. doi: 10.1016/j.cct.2016.12.013. PMID: 27979754.Exclusion: 9

23. Gardiner P, Luo M, D'Amico S, et al. Effectiveness of integrative medicine group visits in chronic pain and depressive symptoms: A randomized controlled trial. PLoS One. 2019;14(12):e0225540. doi: 10.1371/journal.pone.0225540. PMID: 31851666.Exclusion: 8

24. Gentry KR, McGinn KL, Kundu A, et al. Acupuncture therapy for infants: a preliminary report on reasons for consultation, feasibility, and tolerability. Paediatr Anaesth. 2012 Jul;22(7):690-5. doi: 10.1111/j.1460-9592.2011.03743.x. PMID: 22141917.Exclusion: 3

25. Georgoudis G, Felah B, Nikolaidis PT, et al. The effect of physiotherapy and acupuncture on psychocognitive, somatic, quality of life, and disability characteristics in TTH patients. J Pain Res. 2018;11:2527-35. doi: 10.2147/JPR.S178110. PMID: 30425565.Exclusion: 4

26. Gilliam WP, Craner JR, Cunningham JL, et al. Longitudinal Treatment Outcomes for an Interdisciplinary Pain Rehabilitation Program: Comparisons of Subjective and Objective Outcomes on the Basis of Opioid Use Status. J Pain. 2018 Jun;19(6):678-89. doi: 10.1016/j.jpain.2018.02.010. PMID: 29496637.Exclusion: 8 
27. Gold JI, Mahrer NE. Is Virtual Reality Ready for Prime Time in the Medical Space? A Randomized Control Trial of Pediatric Virtual Reality for Acute Procedural Pain Management. J Pediatr Psychol. 2018 Apr 1;43(3):266-75. doi: 10.1093/jpepsy/jsx129. PMID: 29053848.Exclusion: 3

28. Hansen KA, McKernan LC, Carter SD, et al. A Replicable and Sustainable Whole Person Care Model for Chronic Pain. J Altern Complement Med. 2019 Mar;25(S1):S86S94. doi: 10.1089/acm.2018.0420. PMID: 30870025.Exclusion: 9

29. Huffman KL, Rush TE, Fan Y, et al. Sustained improvements in pain, mood, function and opioid use post interdisciplinary pain rehabilitation in patients weaned from high and low dose chronic opioid therapy. Pain. 2017 Jul;158(7):1380-94. doi: 10.1097/j.pain.0000000000000907. PMID: 28328578.Exclusion: 4

30. Hurstak E, Chao MT, Leonoudakis-Watts K, et al. Design, Implementation, and Evaluation of an Integrative Pain Management Program in a Primary Care Safety-Net Clinic. J Altern Complement Med. 2019 Mar;25(S1):S78-S85. doi: 10.1089/acm.2018.0398. PMID: 30870021.Exclusion: 8

31. Jiang Y, Bai P, Chen H, et al. The Effect of Acupuncture on the Quality of Life in Patients With Migraine: A Systematic Review and Meta-Analysis. Front Pharmacol. 2018;9:1190. doi: 10.3389/fphar.2018.01190. PMID: 30416444.Exclusion: 4

32. Johnson Rolfes J, Christensen K, Gershan LA. Acceptance of Traditional Chinese Medicine in the Neonatal Intensive Care Unit: A Launching Point. Glob Adv Health Med. 2020;9:2164956120924644. doi: 10.1177/2164956120924644. PMID: 32426181.Exclusion: 3

33. Kim H, Mawla I, Lee J, et al. Reduced tactile acuity in chronic low back pain is linked with structural neuroplasticity in primary somatosensory cortex and is modulated by acupuncture therapy. Neuroimage. 2020 Aug 15;217:116899. doi: 10.1016/j.neuroimage.2020.116899. PMID: 32380138.Exclusion: 4
34. Li H, Flynn DM, Highland KB, et al. Relationship between Post-Traumatic Stress Disorder Symptoms and Chronic Pain Related Symptom Domains among Military Active Duty Service Members. Pain Med. 2021 Mar 4doi: 10.1093/pm/pnab087. PMID: 33690851.Exclusion: 8

35. Martin DP, Sletten CD, Williams BA, et al. Improvement in fibromyalgia symptoms with acupuncture: results of a randomized controlled trial. Mayo Clin Proc. 2006 Jun;81(6):749-57. doi: 10.4065/81.6.749. PMID: 16770975.Exclusion: 4

36. McDonald J, Janz S. The acupuncture evidence project. A comparative literature review: Australian acupuncture and Chinese Medicine Association Ltd. 2017.Exclusion: 4

37. Miaskowski C, Blyth F, Nicosia F, et al. A Biopsychosocial Model of Chronic Pain for Older Adults. Pain Med. 2020 Sep 1;21(9):1793-805. doi: 10.1093/pm/pnz329. PMID: 31846035.Exclusion: 9

38. Mosadeghi S, Reid MW, Martinez B, et al. Feasibility of an Immersive Virtual Reality Intervention for Hospitalized Patients: An Observational Cohort Study. JMIR Ment Health. 2016 Jun 27;3(2):e28. doi: 10.2196/mental.5801. PMID: 27349654.Exclusion: 3

39. Murad MH, Asi N, Alsawas M, et al. New evidence pyramid. Evid Based Med. 2016 Aug;21(4):125-7. doi: 10.1136/ebmed-2016110401. PMID: 27339128.Exclusion: 9

40. Murphy JL, Clark ME, Banou E. Opioid cessation and multidimensional outcomes after interdisciplinary chronic pain treatment. Clin J Pain. 2013 Feb;29(2):10917. doi: 10.1097/AJP.0b013e3182579935. PMID: 22751033.Exclusion: 8

41. Murphy JL, Palyo SA, Schmidt ZS, et al. The Resurrection of Interdisciplinary Pain Rehabilitation: Outcomes Across a Veterans Affairs Collaborative. Pain Med. $2021 \mathrm{Feb}$ 23;22(2):430-43. doi: 10.1093/pm/pnaa417. PMID: 33496787.Exclusion: 8 
42. Musil F, Pokladnikova J, Pavelek Z, et al. Acupuncture in migraine prophylaxis in Czech patients: an open-label randomized controlled trial. Neuropsychiatr Dis Treat. 2018;14:1221-8. doi:

10.2147/NDT.S155119. PMID:

29785113.Exclusion: 4

43. Naderinabi B, Saberi A, Hashemi M, et al. Acupuncture and botulinum toxin A injection in the treatment of chronic migraine: A randomized controlled study. Caspian J Intern Med. 2017

Summer;8(3):196-204. doi: 10.22088/cjim.8.3.196. PMID: 28932372.Exclusion: 4

44. Pokladnikova J, Maresova P, Dolejs J, et al. Economic analysis of acupuncture for migraine prophylaxis. Neuropsychiatr Dis Treat. 2018;14:3053-61. doi: 10.2147/NDT.S174870. PMID: 30519026.Exclusion: 4

45. Reinstein AS, Erickson LO, Griffin KH, et al. Acceptability, Adaptation, and Clinical Outcomes of Acupuncture Provided in the Emergency Department: A Retrospective Pilot Study. Pain Med. 2017 Jan 30;18(1):169-78. doi: 10.1093/pm/pnv114. PMID: 26917627.Exclusion: 8

46. Ringqvist A, Dragioti E, Bjork M, et al. Moderate and Stable Pain Reductions as a Result of Interdisciplinary Pain Rehabilitation-A Cohort Study from the Swedish Quality Registry for Pain Rehabilitation (SQRP). J Clin Med. 2019 Jun 24;8(6)doi: 10.3390/jcm8060905. PMID: 31238588.Exclusion: 8

47. Sikka N, Shu L, Ritchie B, et al. Virtual Reality-Assisted Pain, Anxiety, and Anger Management in the Emergency Department. Telemed J E Health. 2019 Dec;25(12):120715. doi: 10.1089/tmj.2018.0273. PMID: 30785860.Exclusion: 4

48. Silver Highfield E, Longacre M, Chuang YH, et al. Does Acupuncture Treatment Affect Utilization of Other Hospital Services at an Urban Safety-Net Hospital? J Altern Complement Med. 2016 Apr;22(4):323-7. doi: 10.1089/acm.2015.0151. PMID: 26982686.Exclusion: 4
49. Singh G, Willen SN, Boswell MV, et al. The value of interdisciplinary pain management in complex regional pain syndrome type I: a prospective outcome study. Pain Physician. 2004 Apr;7(2):203-9. PMID: 16868593.Exclusion: 8

50. Sletten CD, Kurklinsky S, Chinburapa V, et al. Economic analysis of a comprehensive pain rehabilitation program: a collaboration between Florida Blue and Mayo Clinic Florida. Pain Med. 2015 May;16(5):898904. doi: 10.1111/pme.12679. PMID: 25645237.Exclusion: 8

51. Spiegel B, Fuller G, Lopez M, et al. Virtual reality for management of pain in hospitalized patients: A randomized comparative effectiveness trial. PLoS One. 2019;14(8):e0219115. doi: 10.1371/journal.pone.0219115. PMID: 31412029. Exclusion: 4

52. Stewart MC U, Justin J, Flynn D, Steffen $\mathrm{AD}$, et al. Evaluating the Relationship Between Initial Injury, Referral to A Pain Clinic, and Medical Retirement from the Army: A Retrospective Analysis. Military Medicine. 2021;186(Supplement_1):5025.Exclusion: 4

53. Tastan K, Ozer Disci O, Set T. A Comparison of the Efficacy of Acupuncture and Hypnotherapy in Patients With Migraine. Int J Clin Exp Hypn. 2018 OctDec;66(4):371-85. doi: 10.1080/00207144.2018.1494444. PMID: 30152732.Exclusion: 4

54. Taylor-Swanson L, Budhathoki A, White S, et al. Enhancing Interoceptive Awareness:

Mindfulness and Acupuncture as a Combined Intervention for Chronic Pain. Advances in Integrative Medicine. 2019;6:S45.Exclusion: 4

55. Tick H, Nielsen A, Pelletier KR, et al. Evidence-based nonpharmacologic strategies for comprehensive pain care: the consortium pain task force white paper. Explore. 2018;14(3):177-211.Exclusion: 9

56. Venuturupalli RS, Chu T, Vicari M, et al. Virtual Reality-Based Biofeedback and Guided Meditation in Rheumatology: A Pilot Study. ACR Open Rheumatol. 2019 Dec;1(10):667-75. doi: 10.1002/acr2.11092. PMID: 31872189.Exclusion: 4 
57. Vickers AJ, Vertosick EA, Lewith G, et al. Acupuncture for Chronic Pain: Update of an Individual Patient Data Meta-Analysis. J

Pain. 2018 May;19(5):455-74. doi:

10.1016/j.jpain.2017.11.005. PMID:

29198932.Exclusion: 4

58. Wayne PM, Buring JE, Eisenberg DM, et al. Cost-Effectiveness of a Team-Based Integrative Medicine Approach to the Treatment of Back Pain. J Altern Complement Med. 2019 Mar;25(S1):S138s46. doi: 10.1089/acm.2018.0503. PMID:

30870015.Exclusion: 4
59. Wenham A, Atkin K, Woodman J, et al. Self-efficacy and embodiment associated with Alexander Technique lessons or with acupuncture sessions: A longitudinal qualitative sub-study within the ATLAS trial. Complement Ther Clin Pract. 2018 May;31:308-14. doi: 10.1016/j.ctcp.2018.03.009. PMID: 29705473.Exclusion: 4

60. Yin C, Buchheit TE, Park JJ. Acupuncture for chronic pain: an update and critical overview. Curr Opin Anaesthesiol. 2017 Oct;30(5):583-92. doi: 10.1097/ACO.0000000000000501. PMID: 28719458.Exclusion: 4

61. Znidarsic J, Kirksey KN, Dombrowski SM, et al. "Living Well with Chronic Pain": Integrative Pain Management via Shared Medical Appointments. Pain Med. 2021 Feb 4;22(1):181-90. doi: 10.1093/pm/pnaa418. PMID: 33543263.Exclusion: 8 


\section{Appendix I. Forest Plots}

\section{Key Question 1}

Figure I-1. IPMP versus UC: SF-36 or SF-12 PCS at postintervention, short term and long term

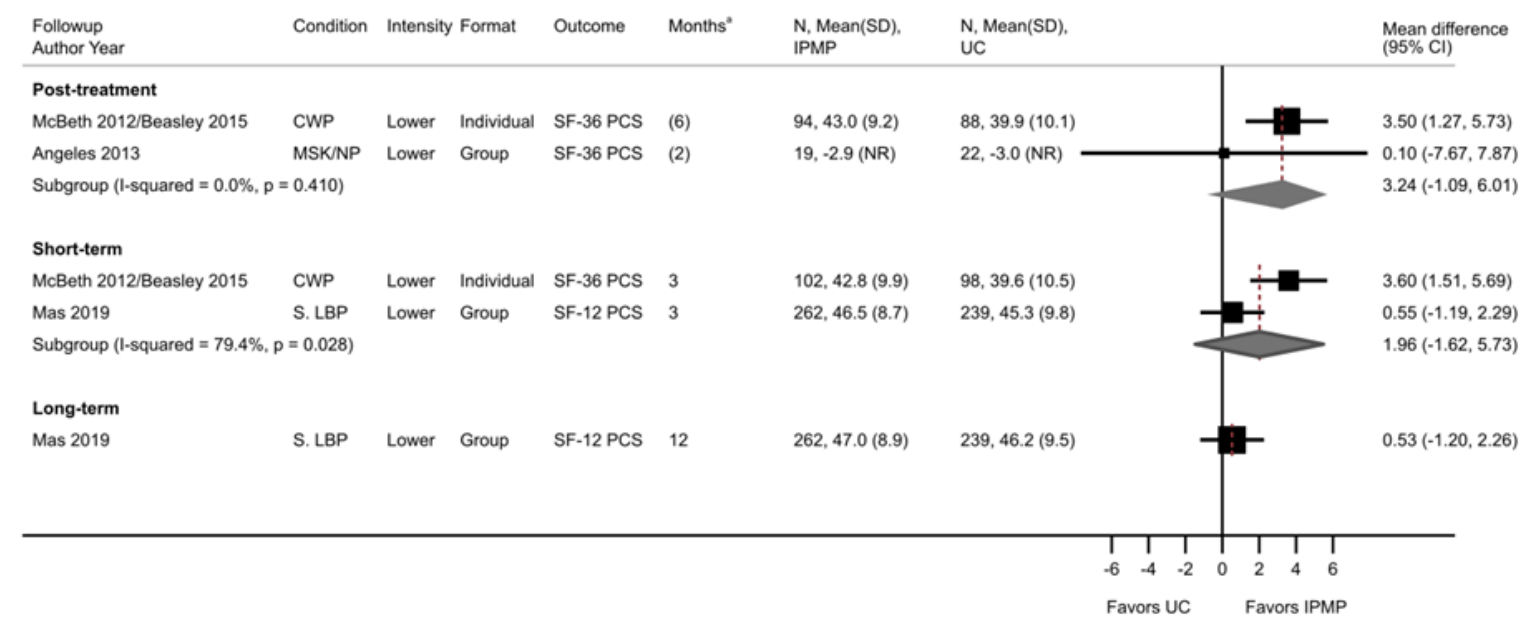

$\mathrm{CI}=$ confidence interval; $\mathrm{CWP}=$ chronic wide-spread pain; $\mathrm{IPMP}=$ integrated pain management program; $\mathrm{MSK} / \mathrm{NP}=$ musculoskeletal pain/neck pain; OA = osteoarthritis; $\mathrm{SD}=$ standard deviation; SF-36 PCS = Short Form-36 Physical Component Score; S. LBP = subacute low back pain; $\mathrm{UC}=$ usual care.

${ }^{a}$ Number of months in parentheses for the post-treatment timeframe indicate the duration of the intervention; followup for the remaining timeframes is in months following the end of the intervention.

Figure I-2. IPMP versus UC: SF-36 or SF-12 MCS at postintervention, short term and long term

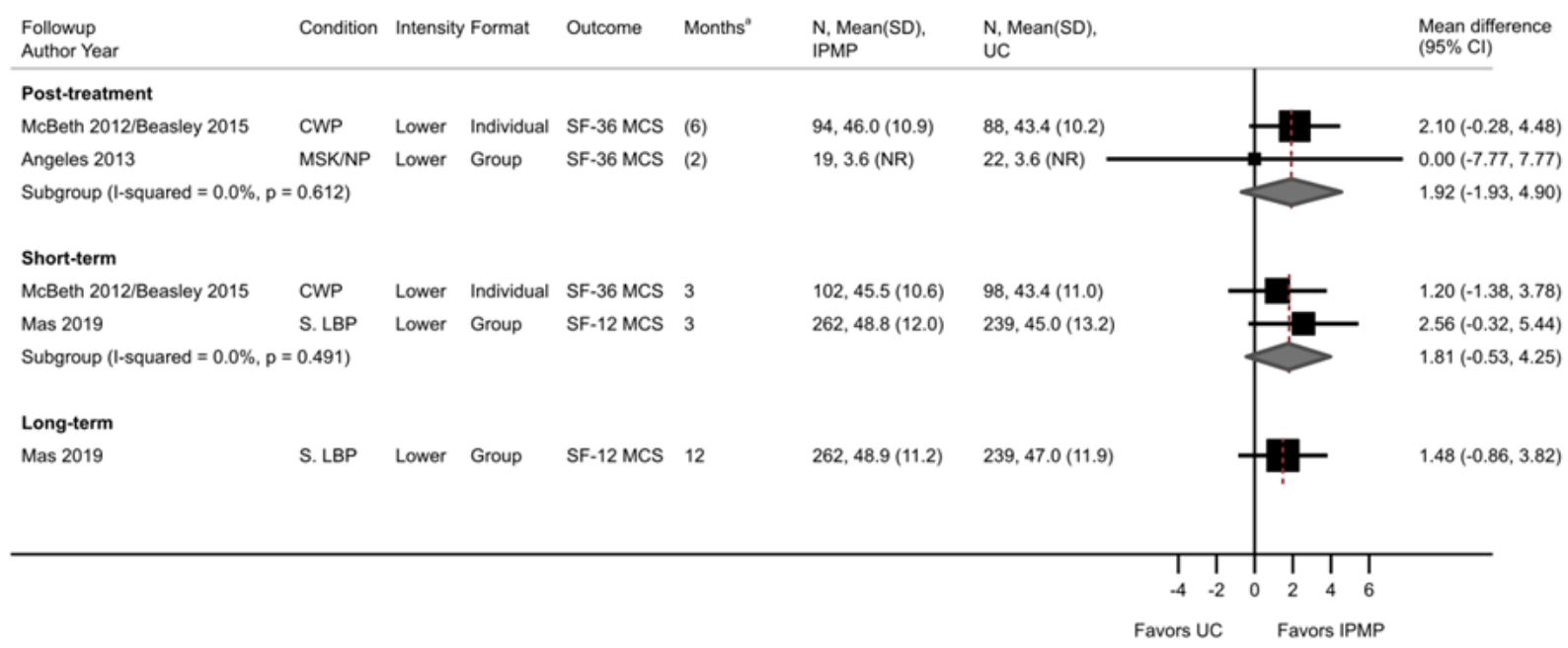

$\mathrm{CI}=$ confidence interval; $\mathrm{CWP}=$ chronic wide-spread pain; $\mathrm{IPMP}=$ integrated pain management program; $\mathrm{MSK} / \mathrm{NP}=$ musculoskeletal pain/neck pain; OA = osteoarthritis; SD = standard deviation; SF-36 MCS = Short Form-36 Mental Component Score; $\mathrm{S}$. LBP = subacute low back pain; $\mathrm{UC}=$ usual care.

${ }^{\text {a }}$ Number of months in parentheses for the post-treatment timeframe indicate the duration of the intervention; followup for the remaining timeframes is in months following the end of the intervention. 
Figure I-3. IPMP versus UC: Depression at postintervention

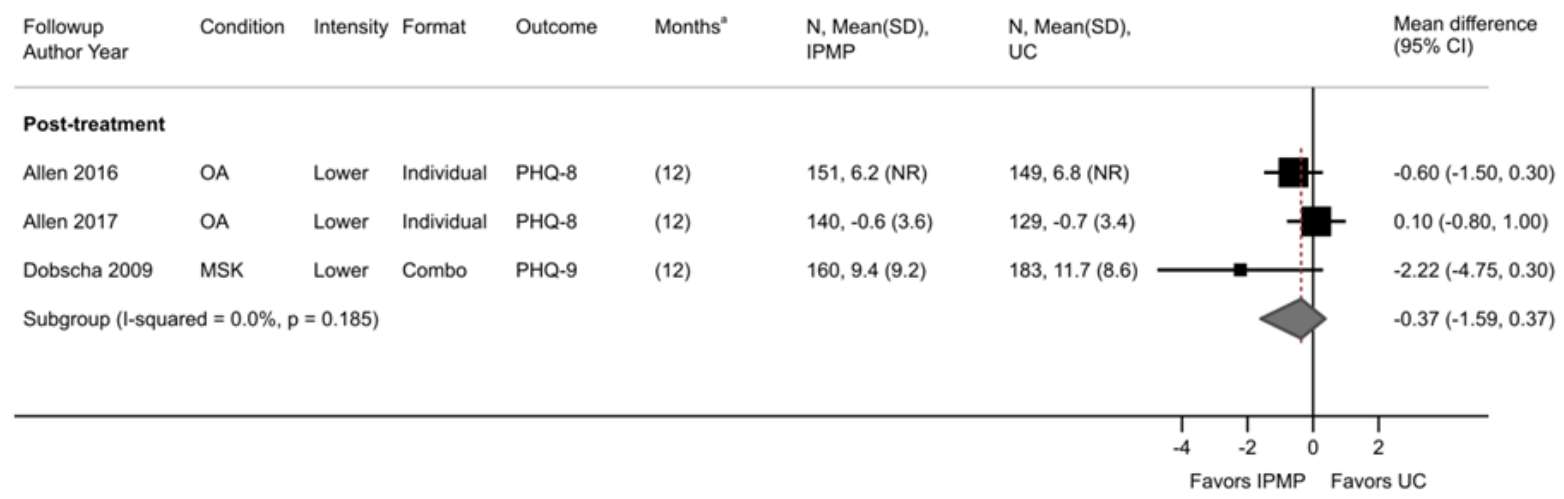

$\mathrm{CI}=$ confidence interval; Combo $=$ combination group and individual sessions; IPMP = integrated pain management program; MSK = musculoskeletal pain; $\mathrm{OA}=$ osteoarthritis; $\mathrm{PHQ}=$ Patient Health Questionnaire-8 or $-9 ; \mathrm{SD}=$ standard deviation; $\mathrm{UC}=$ usual care.

${ }^{\text {a }}$ Number of months in parentheses for the post-treatment timeframe indicate the duration of the intervention

Figure I-4. CPMP versus UC: Sensitivity analysis for pain excluding poor quality trials

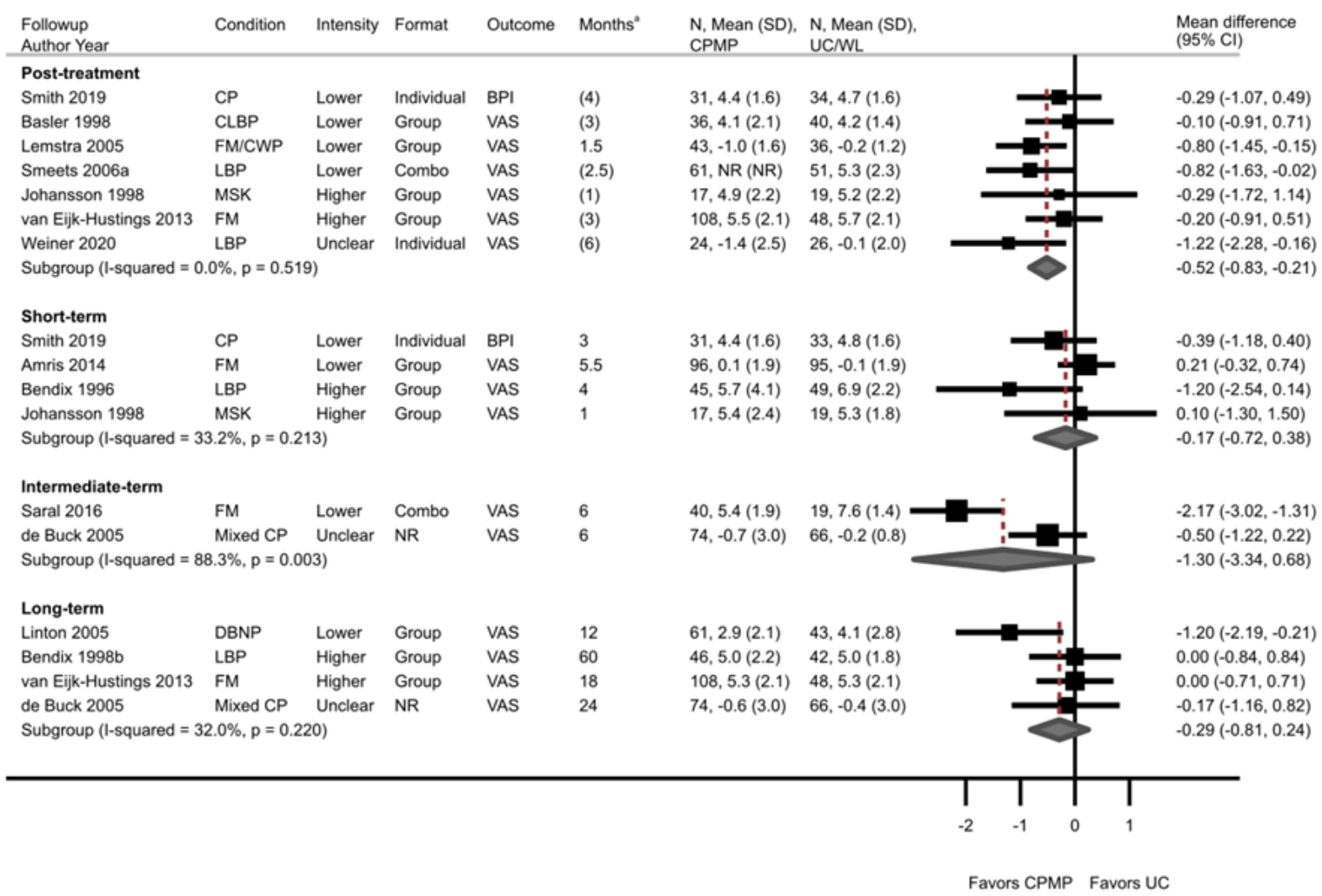

$\mathrm{BPI}=$ Brief Pain Inventory; $\mathrm{CI}=$ confidence interval; $\mathrm{CLBP}=$ chronic low back pain; Combo = combination group and individual sessions; $\mathrm{CP}=$ chronic pain; $\mathrm{CPMP}=$ comprehensive pain management program; $\mathrm{CWP}=$ chronic widespread pain; $\mathrm{DBNP}=$ back/neck pain; $\mathrm{FM}=$ fibromyalgia; $\mathrm{LBP}=$ low back pain; $\mathrm{MSK}=$ musculoskeletal pain; $\mathrm{NR}=$ not reported; $\mathrm{SD}=$ standard deviation; $\mathrm{UC}=$ usual care; $\mathrm{VAS}=$ visual analog scale; $\mathrm{WL}=$ waitlist.

${ }^{a}$ Number of months in parentheses for the post-treatment timeframe indicate the duration of the intervention; followup for the remaining timeframes is in months following the end of the intervention. 
Figure I-5. CPMP versus UC: Sensitivity analysis for pain excluding trial in patients with acute $(<4$ weeks) trauma

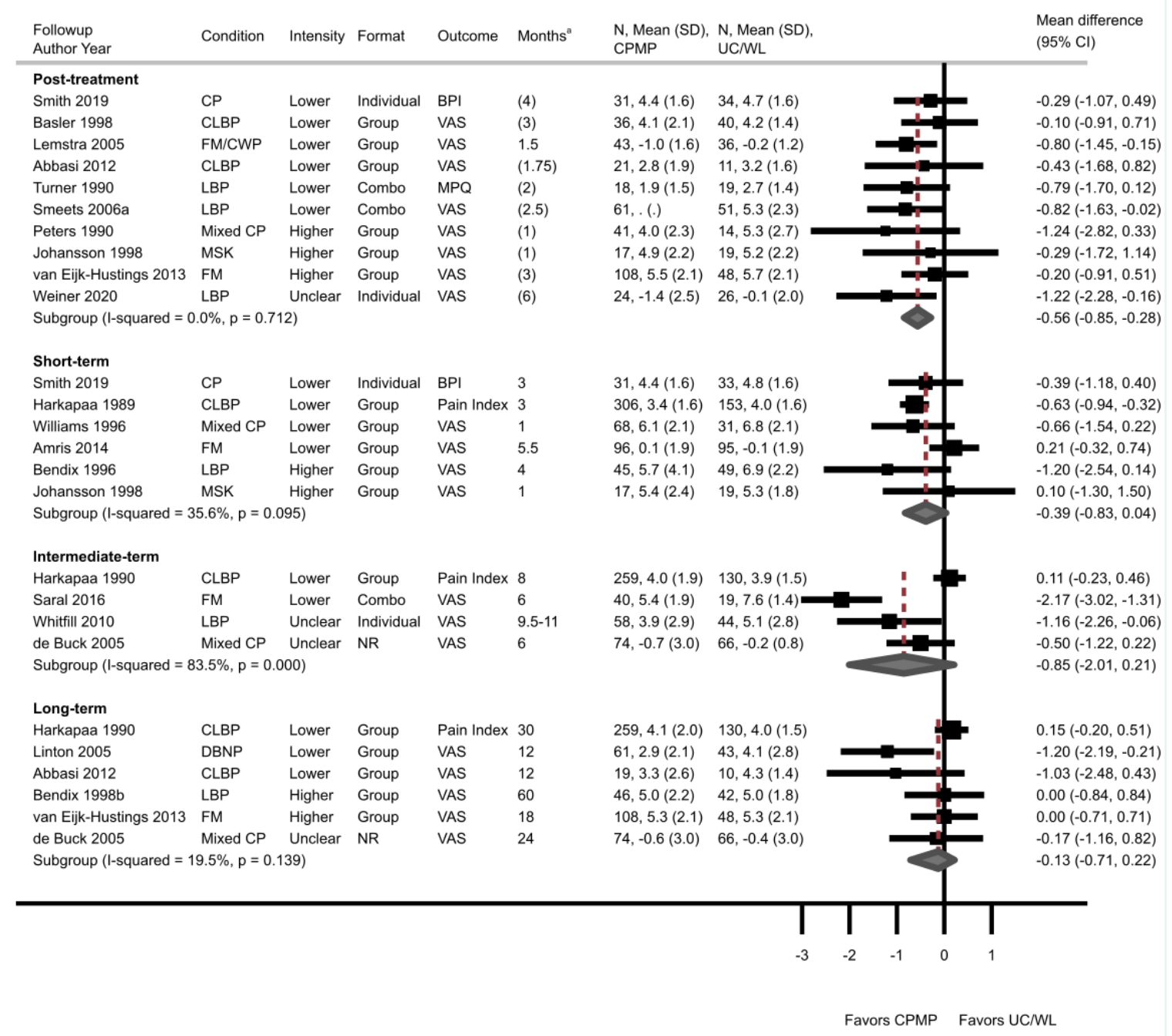

$\mathrm{BPI}=$ Brief Pain Inventory; $\mathrm{CI}=$ confidence interval $\mathrm{CLBP}=$ chronic low back pain; Combo = combination group and individual sessions; $\mathrm{CP}=$ chronic pain; $\mathrm{CPMP}=$ comprehensive pain management program; $\mathrm{CWP}=$ chronic widespread pain; $\mathrm{DBNP}=$ back/neck pain; FM = fibromyalgia; $\mathrm{LBP}=$ low back pain; $\mathrm{MPQ}=$ The McGill Pain Questionnaire; $\mathrm{MSK}=$ musculoskeletal pain; $\mathrm{NR}=$ not reported; $\mathrm{SD}=$ standard deviation; $\mathrm{UC}=$ usual care; $\mathrm{VAS}=$ visual analog scale; $\mathrm{WL}=$ waitlist.

${ }^{\text {a }}$ Number of months in parentheses for the post-treatment timeframe indicate the duration of the intervention; followup for the remaining timeframes is in months following the end of the intervention. 
Figure I-6. CPMP versus UC: Sensitivity analysis for pain using the most common duration for long-term followup

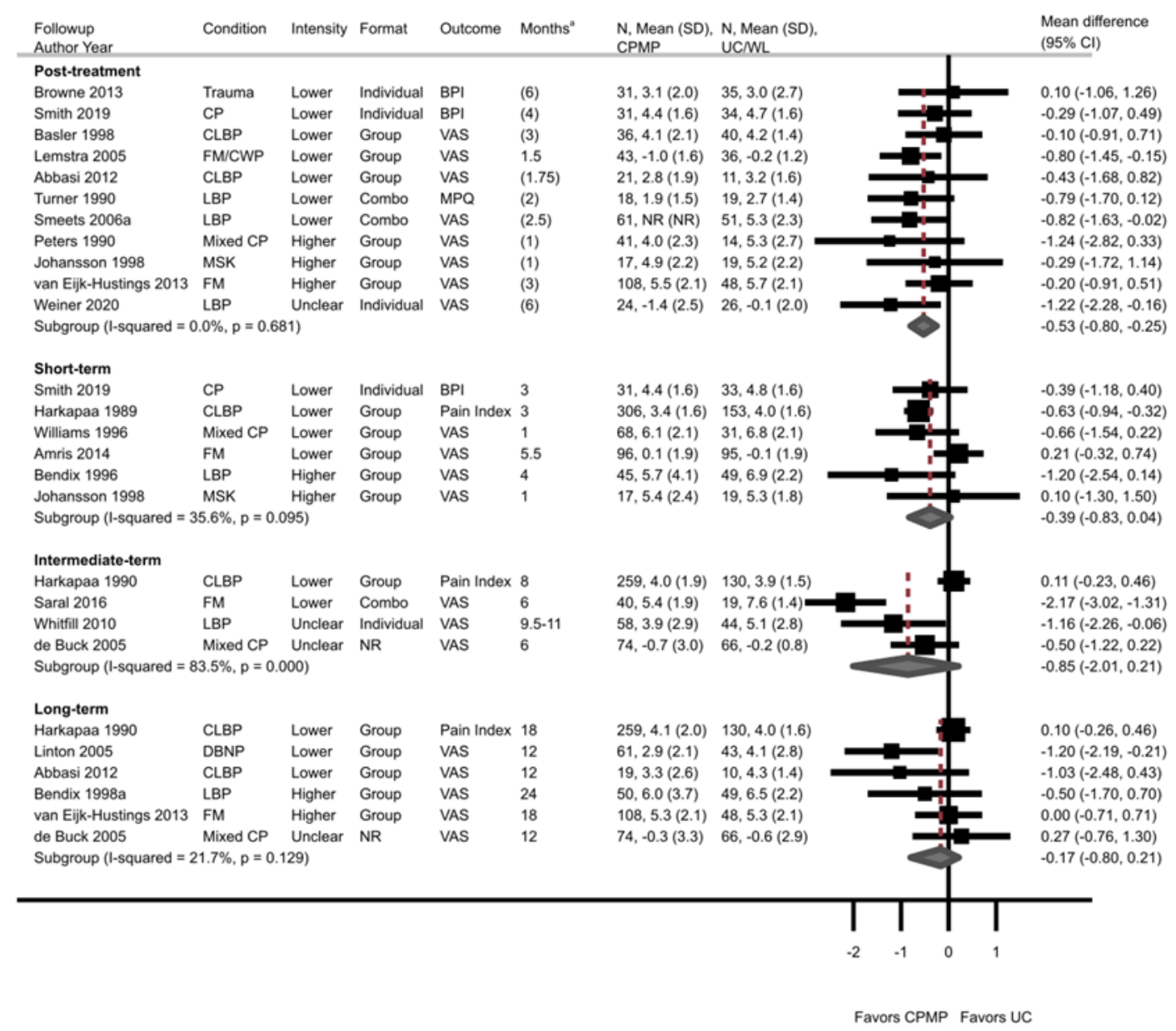

$\mathrm{BPI}=$ Brief Pain Inventory; $\mathrm{CI}=$ confidence interval; $\mathrm{CLBP}=$ chronic low back pain; Combo = combination group and individual sessions; $\mathrm{CP}=$ chronic pain; $\mathrm{CPMP}=$ comprehensive pain management program; $\mathrm{CWP}=$ chronic widespread pain; $\mathrm{DBNP}=$ back/neck pain; FM = fibromyalgia; $\mathrm{LBP}=$ low back pain; MPQ = The McGill Pain Questionnaire; MSK = musculoskeletal pain; $\mathrm{NR}=$ not reported; $\mathrm{SD}=$ standard deviation; $\mathrm{UC}=$ usual care; $\mathrm{VAS}=$ visual analog scale; $\mathrm{WL}=$ waitlist.

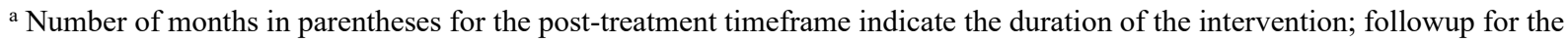
remaining timeframes is in months following the end of the intervention. 
Figure I-7. CPMP versus UC: Sensitivity analysis for pain excluding the MPQ

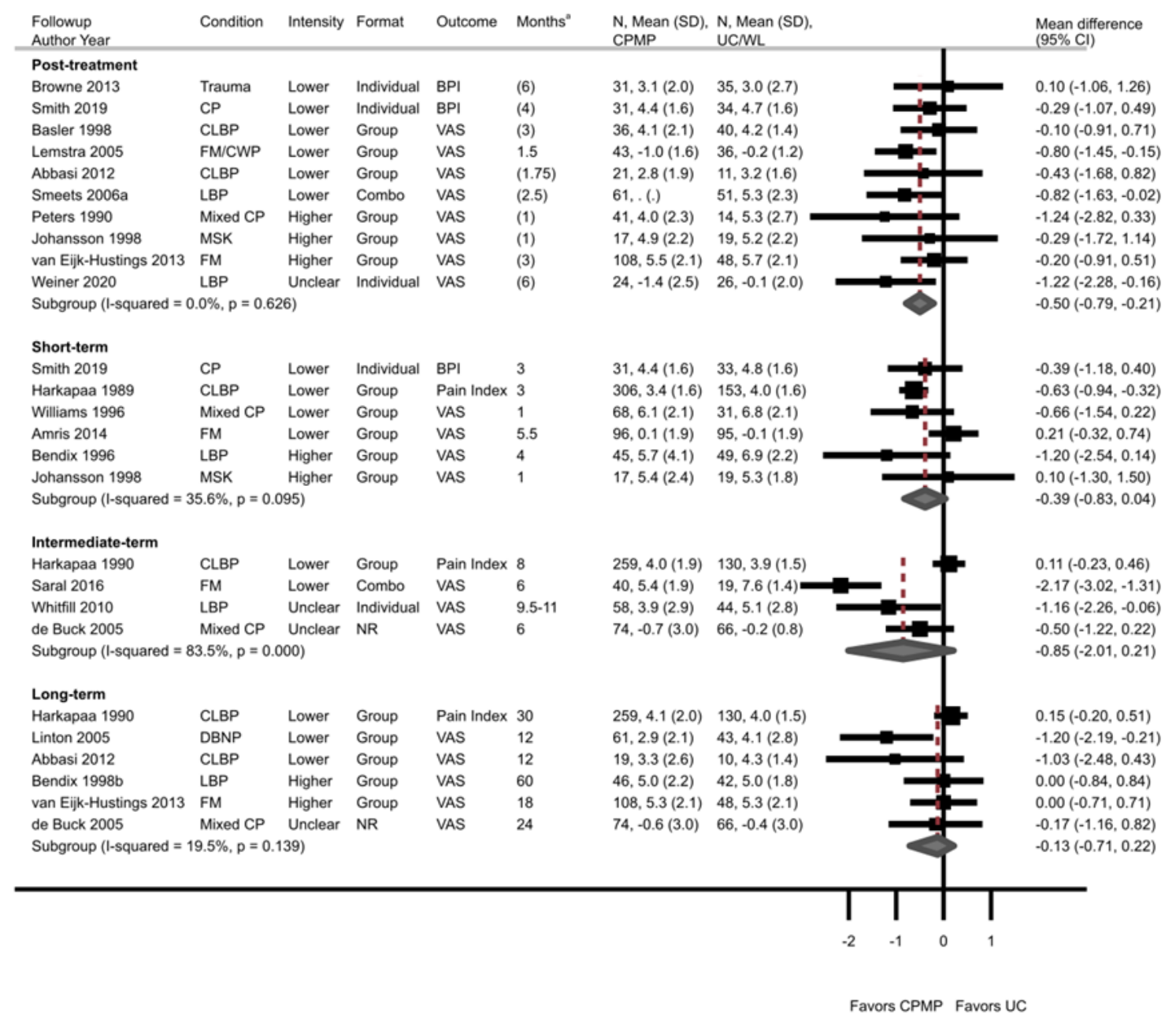

BPI = Brief Pain Inventory $\mathrm{CI}=$ confidence interval; $\mathrm{CLBP}=$ chronic low back pain; Combo $=$ combination group and individual sessions; $\mathrm{CP}=$ chronic pain; $\mathrm{CPMP}=$ comprehensive pain management program; $\mathrm{CWP}=$ chronic widespread pain; $\mathrm{DBNP}=$ back/neck pain; FM = fibromyalgia; $\mathrm{LBP}=$ low back pain; $\mathrm{MPQ}=$ The McGill Pain Questionnaire; $\mathrm{MSK}=$ musculoskeletal pain; $\mathrm{NR}=$ not reported; $\mathrm{SD}=$ standard deviation; $\mathrm{UC}=$ usual care; $\mathrm{VAS}=$ visual analog $\mathrm{scale} ; \mathrm{WL}=$ waitlist.

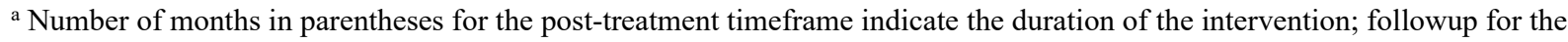
remaining timeframes is in months following the end of the intervention. 
Figure I-8. CPMP versus UC: Sensitivity analysis for function excluding an outlier trial

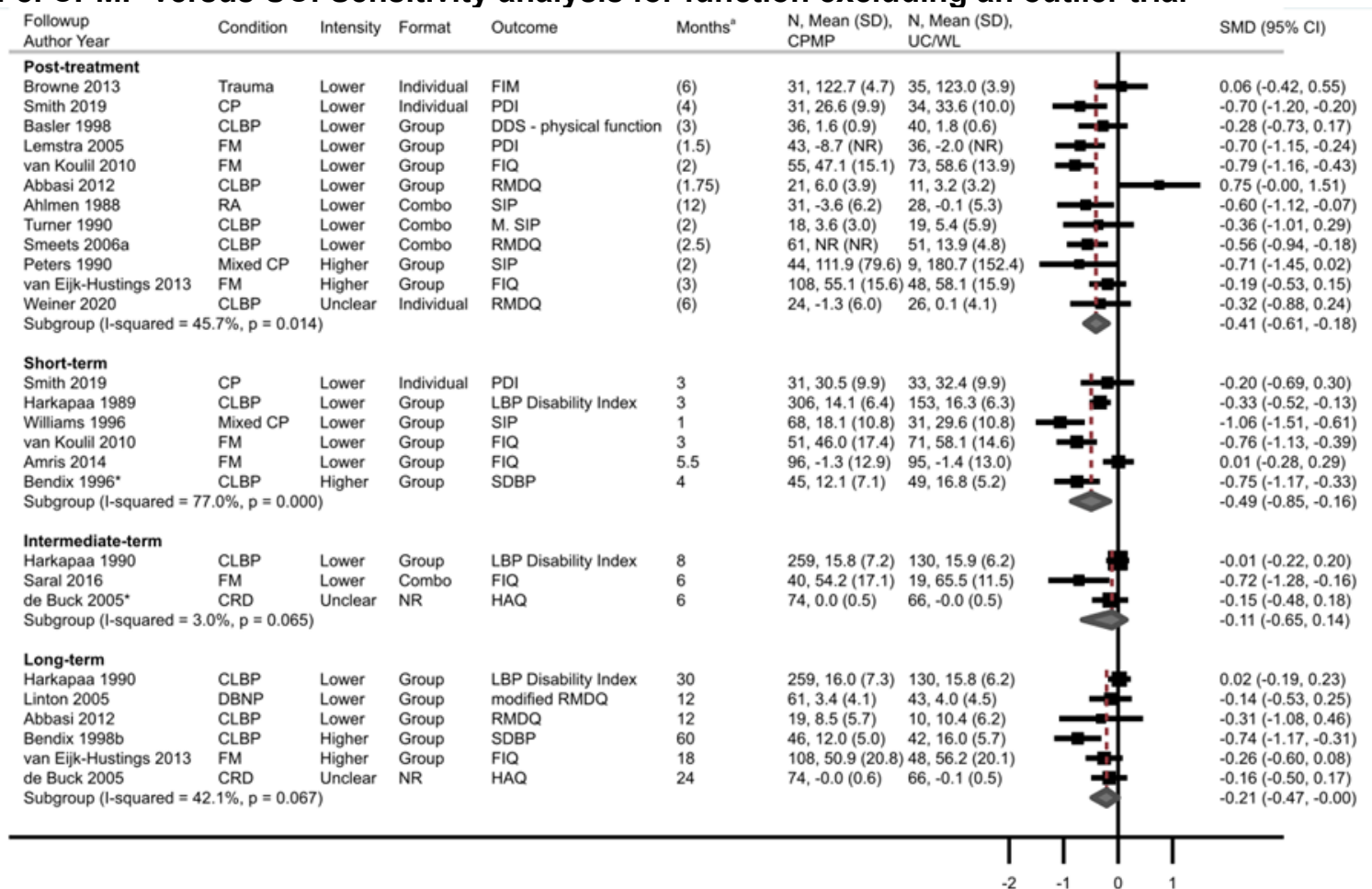

Favors CPMP Favors UC

$\mathrm{CI}=$ confidence interval; $\mathrm{CLBP}=$ chronic low back pain; Combo $=$ combination group and individual sessions; $\mathrm{CP}=$ chronic pain; $\mathrm{CPMP}=$ comprehensive pain management program; $\mathrm{CRD}=$ chronic rheumatoid arthritis; $\mathrm{DBNP}=$ back/neck pain; $\mathrm{DDS}=$ Dusseldorf Disability Scale; FIM = Functional independence measure; FIQ = Fibromyalgia Impact Questionnaire; FM = fibromyalgia; HAQ = Health Assessment Questionnaire LPB = Low back pain; M. SIP = Modified Sickness Impact Profile; NR $=$ not reported; SIP $=$ Sickness Impact Profile; PDI = Pain Disability Index; RA = Rheumatoid arthritis; RMDQ = Roland and Morris Disability Questionnaire; SD = standard deviation; SDBP = Self-reported disability of back pain scale; SIP = Sickness Impact Profile; $\mathrm{SMD}=$ standardized mean difference; $\mathrm{UC}=$ usual care; $\mathrm{WL}=$ waitlist.

${ }^{\text {a }}$ Number of months in parentheses for the post-treatment timeframe indicate the duration of the intervention; followup for the remaining timeframes is in months following the end of the intervention. 
Figure I-9. CPMP versus UC: Sensitivity analysis for function excluding poor-quality trials

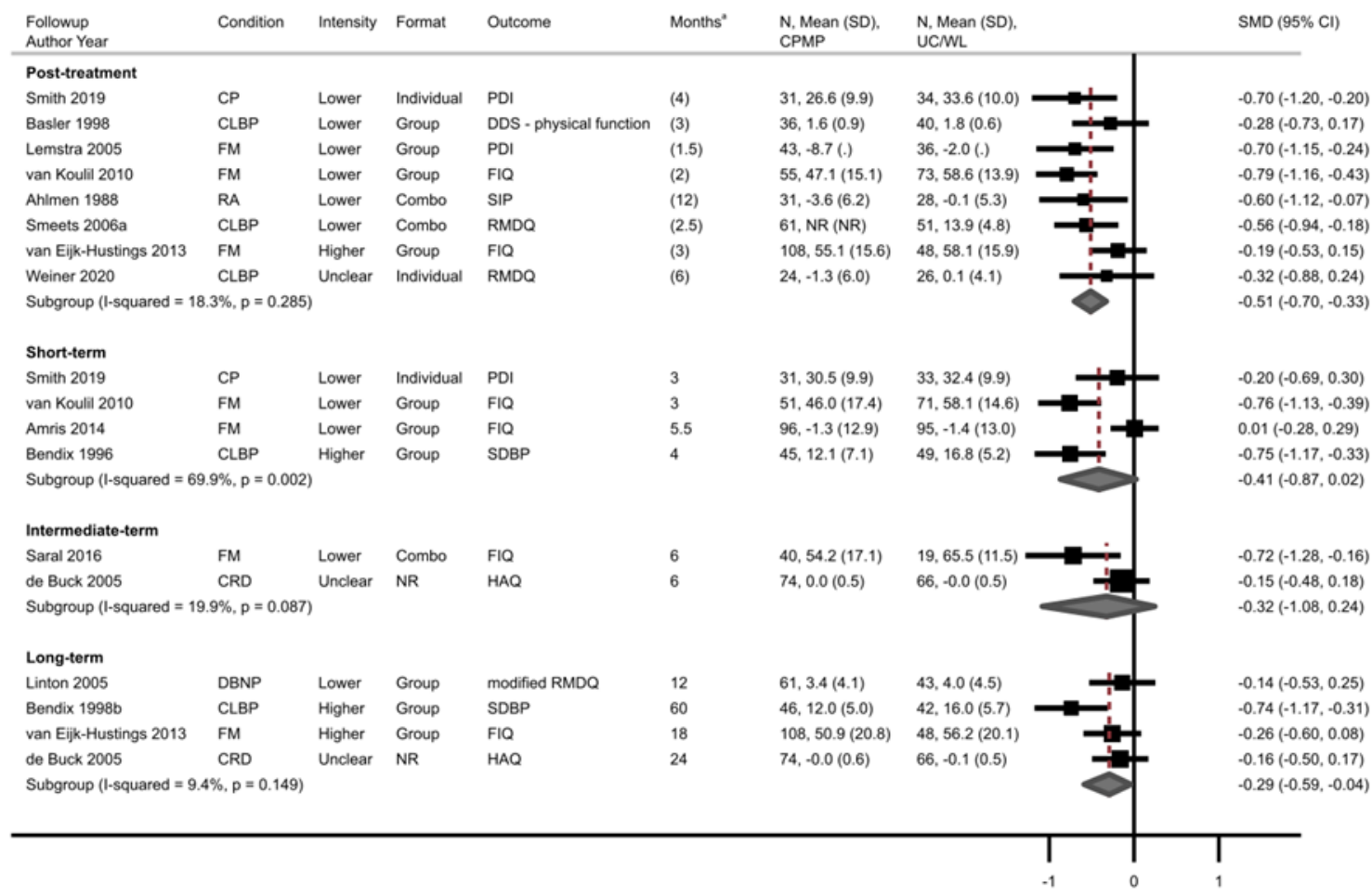

Favors CPMP Favors UC

$\mathrm{CI}=$ confidence interval; $\mathrm{CLBP}=$ chronic low back pain; Combo $=$ combination group and individual sessions; $\mathrm{CP}=$ chronic pain; $\mathrm{CPMP}=$ comprehensive pain management program; $\mathrm{CRD}=$ chronic rheumatoid arthritis; $\mathrm{DBNP}=$ back/neck pain; DDS= Dusseldorf Disability Scale; FIM = Functional Independence Measure; FIQ = Fibromyalgia Impact Questionnaire; FM = fibromyalgia; HAQ = Health Assessment Questionnaire LPB = low back pain; NR = not reported; SIP = Sickness Impact Profile; PDI = Pain Disability Index; RA = rheumatoid arthritis; RMDQ = Roland and Morris Disability Questionnaire; $\mathrm{SD}=\mathrm{standard}$ deviation; SDBP = Self-reported disability of back pain scale; SIP = Sickness Impact Profile; SMD = standardized mean difference; $\mathrm{UC}=$ usual care; $\mathrm{WL}=$ waitlist.

${ }^{\text {a }}$ Number of months in parentheses for the post-treatment timeframe indicate the duration of the intervention; followup for the remaining timeframes is in months following the end of the intervention. 
Figure I-10. CPMP versus UC: Sensitivity analysis for function excluding trial in patients with acute (<4 weeks) trauma

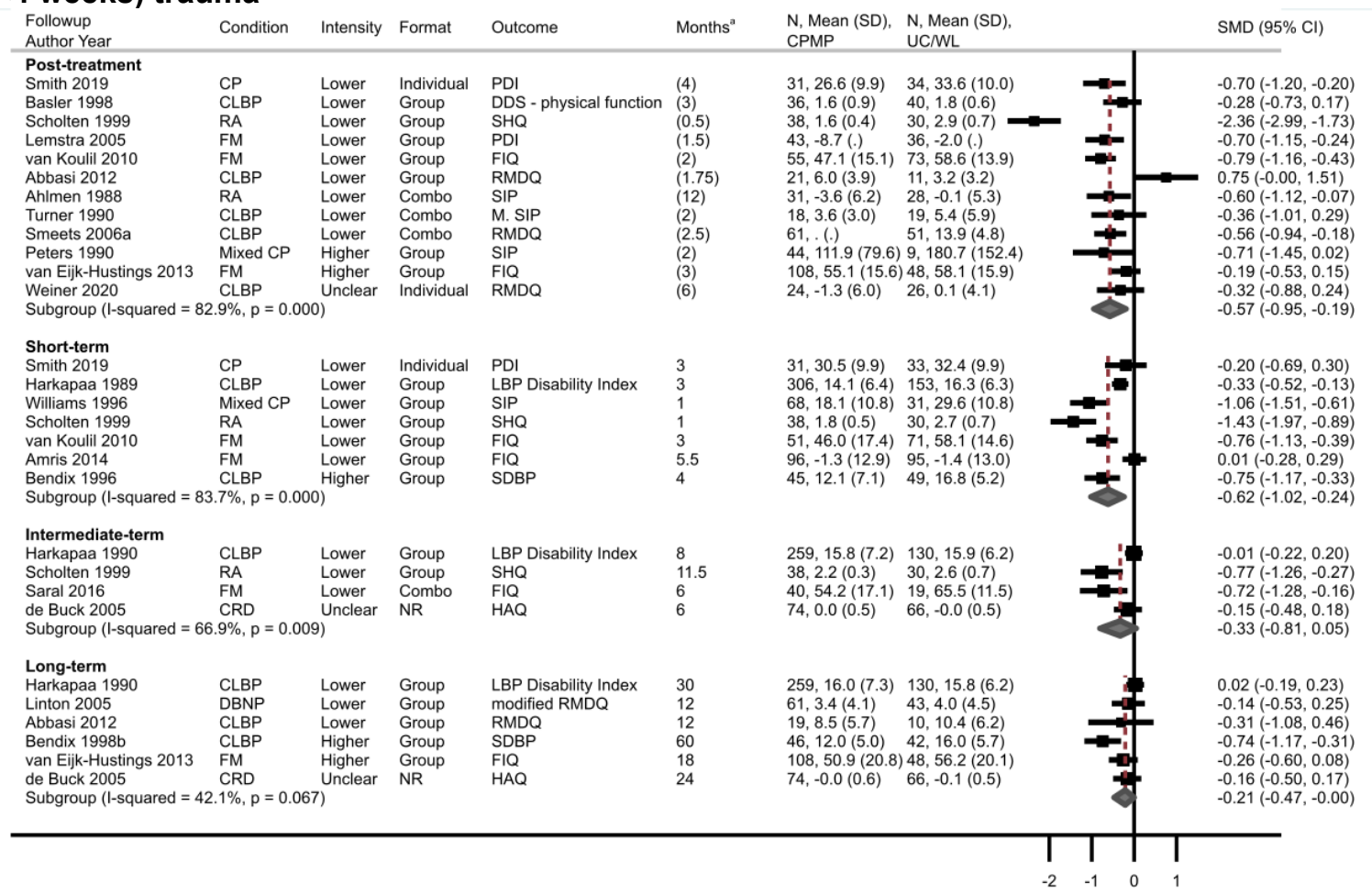

Favors CPMP Favors UC

$\mathrm{CI}=$ confidence interval; $\mathrm{CLBP}=$ chronic low back pain; Combo $=$ combination group and individual sessions; $\mathrm{CP}=\mathrm{chronic}$ pain; $\mathrm{CPMP}=$ comprehensive pain management program; $\mathrm{CRD}=$ chronic rheumatoid arthritis; $\mathrm{DBNP}=$ back/neck pain; $\mathrm{DDS}=$ Dusseldorf Disability Scale; FIM = Functional Independence Measure; FIQ = Fibromyalgia Impact Questionnaire; FM = fibromyalgia; HAQ = Health Assessment Questionnaire LPB = low back pain; NR = not reported; SIP = Sickness Impact Profile; PDI = Pain Disability Index; RA = rheumatoid arthritis; RMDQ = Roland and Morris Disability Questionnaire; SD = standard deviation; SDBP = Self-reported disability of back pain scale; SHQ = Stanford Health Questionnaire; SIP = Sickness Impact Profile; $\mathrm{SMD}=$ standardized mean difference; $\mathrm{UC}=$ usual care; $\mathrm{WL}=$ waitlist.

${ }^{a}$ Number of months in parentheses for the post-treatment timeframe indicate the duration of the intervention; followup for the remaining timeframes is in months following the end of the intervention. 
Figure I-11. CPMP versus UC: Sensitivity analysis for function using the most common duration for long-term followup

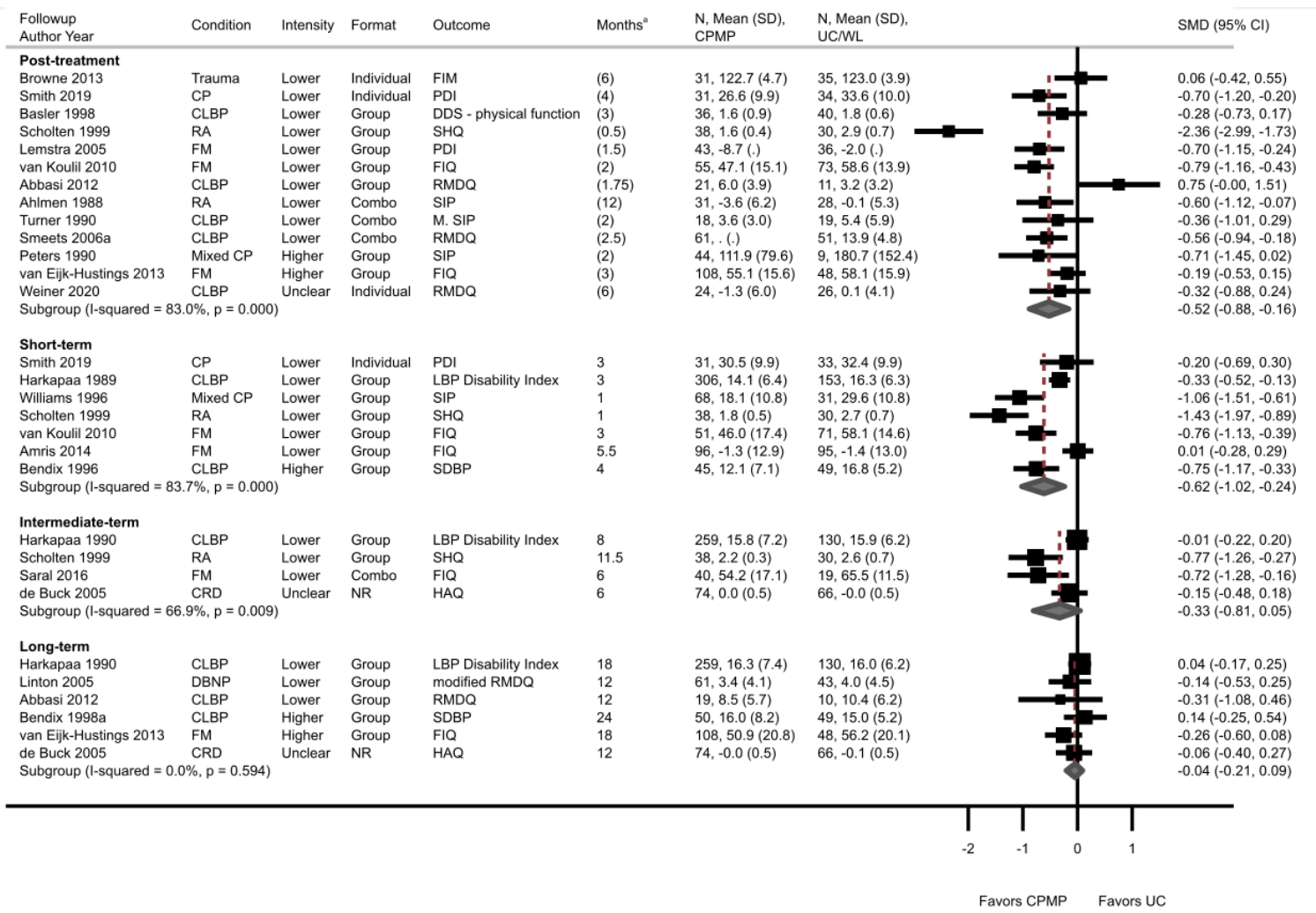

$\mathrm{CI}=$ confidence interval $\mathrm{CLBP}=$ chronic low back pain; Combo $=$ combination group and individual sessions; $\mathrm{CP}=$ chronic pain; $\mathrm{CPMP}=$ comprehensive pain management program; $\mathrm{CRD}=$ chronic rheumatoid arthritis; $\mathrm{DBNP}=$ back/neck pain; $\mathrm{DDS}=$ Dusseldorf Disability Scale; FIM = Functional independence measure; FIQ = Fibromyalgia Impact Questionnaire; FM = fibromyalgia; HAQ = Health Assessment Questionnaire LPB = Low back pain; M. SIP = Modified Sickness Impact Profile; NR $=$ not reported; SIP $=$ Sickness Impact Profile; PDI $=$ Pain Disability Index; RA $=$ Rheumatoid arthritis; RMDQ $=$ Roland and Morris Disability Questionnaire; SD = standard deviation; SDBP = Self-reported disability of back pain scale; SHQ = Stanford Health Questionnaire; SIP = Sickness Impact Profile; SMD = standardized mean difference; UC $=$ usual care; WL = waitlist.

${ }^{a}$ Number of months in parentheses for the post-treatment timeframe indicate the duration of the intervention; followup for the remaining timeframes is in months following the end of the intervention. 
Figure I-12. CPMP versus UC: Pain interference

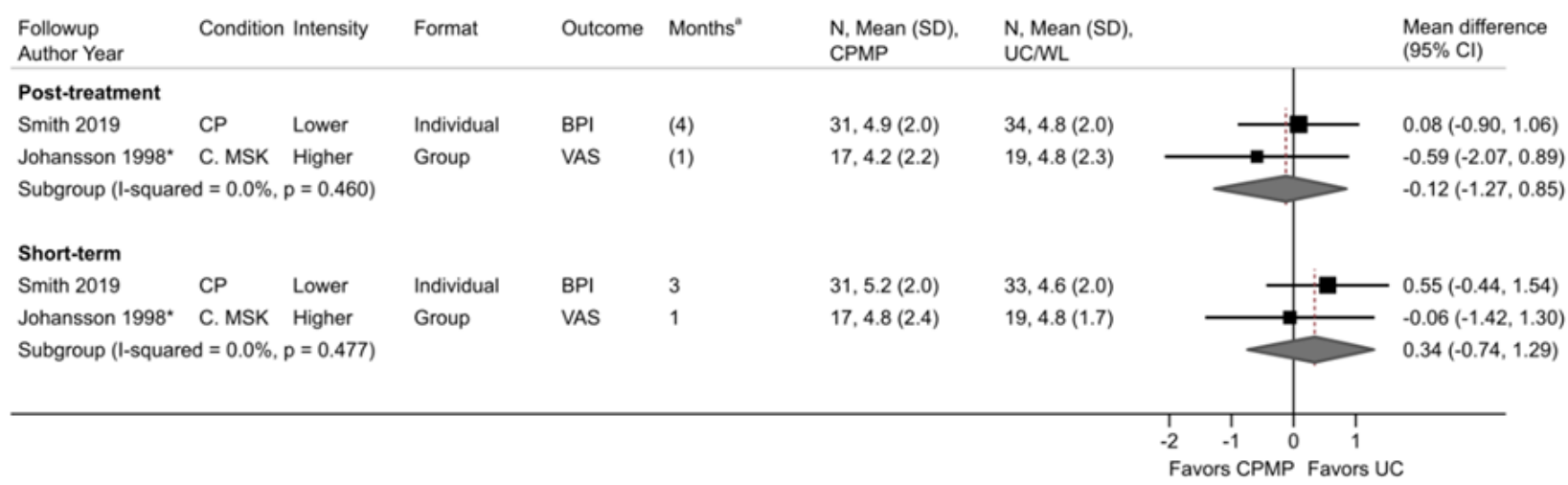

BPI = Brief Pain Inventory; $\mathrm{CI}=$ confidence interval; $\mathrm{C} . \mathrm{MSK}=$ Chronic musculoskeletal pain; Combo $=$ combination group and individual sessions; $\mathrm{CP}=$ Chronic pain; $\mathrm{CPMP}=$ Comprehensive pain management program; $\mathrm{SD}=$ standard deviation; $\mathrm{UC}=$ usual care; $\mathrm{VAS}=$ visual analog scale; $\mathrm{WL}=$ waitlist

a Number of months in parentheses for the post-treatment timeframe indicate the duration of the intervention; followup for the remaining timeframes is in months following the end of the intervention.

\section{Figure I-13. CPMP versus UC: SF-36 PCS}

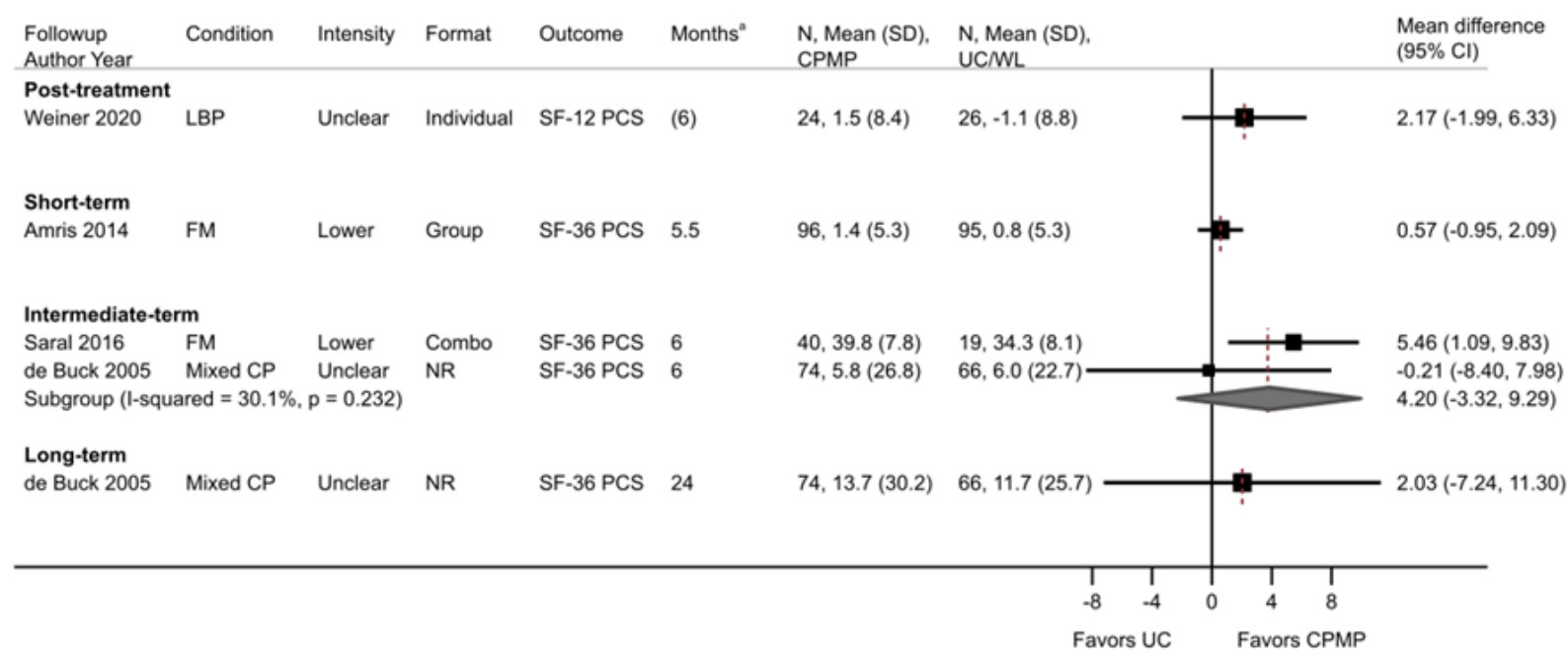

$\mathrm{CI}=$ confidence interval; $\mathrm{Combo}=$ combination group and individual sessions; $\mathrm{CP}=$ Chronic pain; $\mathrm{CPMP}=\mathrm{Comprehensive} \mathrm{pain}$ management program; FM = fibromyalgia; LBP = low back pain; NR = not reported; $\mathrm{SD}=$ standard deviation; SF-36 or -12 PCS $=$ Short-Form 36 or 12 Physical Component Score; $\mathrm{UC}=$ usual care; $\mathrm{WL}=$ waitlist

${ }^{a}$ Number of months in parentheses for the post-treatment timeframe indicate the duration of the intervention; followup for the remaining timeframes is in months following the end of the intervention. 
Figure I-14. CPMP versus UC: SF-36 MCS

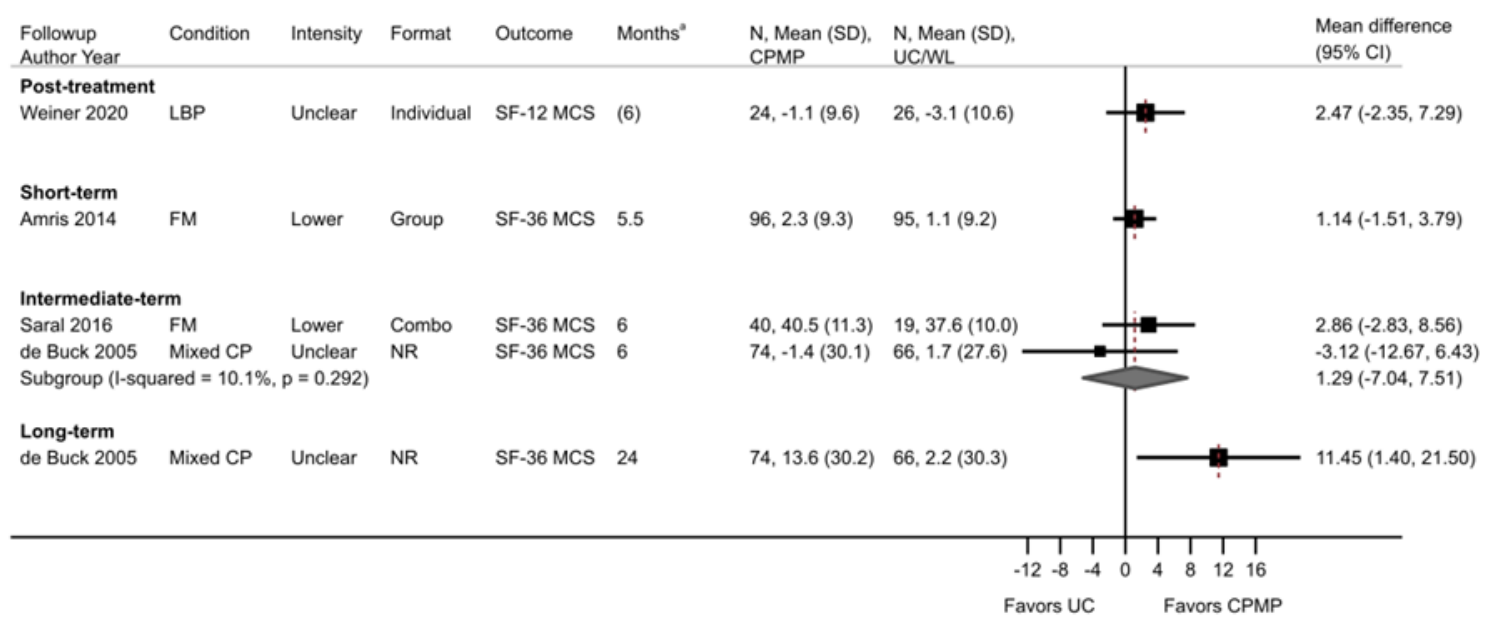

$\mathrm{CI}=$ confidence interval; $\mathrm{Combo}=$ combination group and individual sessions; $\mathrm{CP}=\mathrm{Chronic}$ pain; $\mathrm{CPMP}=\mathrm{Comprehensive} \mathrm{pain}$ management program; FM = fibromyalgia; $\mathrm{LBP}=$ low back pain; $\mathrm{NR}=$ not reported; $\mathrm{SD}=$ standard deviation; $\mathrm{SF}-36$ or -12 MCS $=$ Short-Form 36 or 12 Mental Component Score; UC = usual care; WL = waitlist

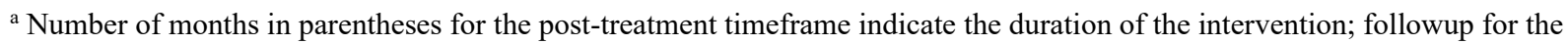
remaining timeframes is in months following the end of the intervention. 
Figure I-15. CPMP versus UC: Depression

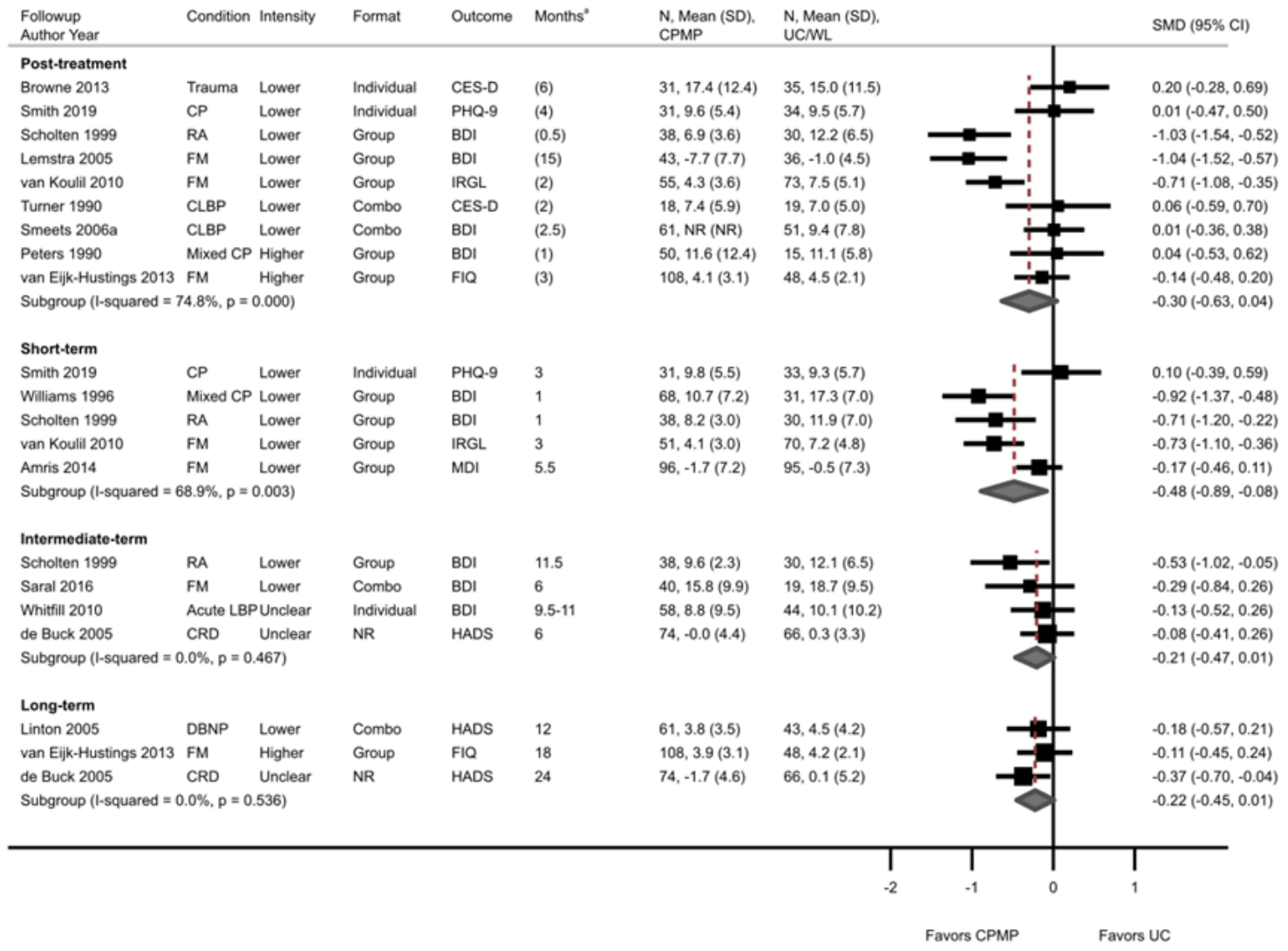

$\mathrm{BDI}=$ Beck Depression Inventory; CBNP = chronic back/neck pain; CES-D = Center for Epidemiological Studies Depression Scale; $\mathrm{CI}=$ confidence interval; $\mathrm{CLBP}=$ Chronic low back pain; Combo $=$ combination group and individual sessions; $\mathrm{CP}=$ Chronic pain; $\mathrm{CPMP}=$ Comprehensive pain management program; $\mathrm{CRD}=$ Chronic rheumatoid arthritis; FIQ = Fibromyalgia Impact Questionnaire; FM = fibromyalgia; HADS = Hospital Anxiety and Depression Scale; IRGL = Impact of Rheumatic Diseases on General Health and Lifestyle Instrument; LBP = Low back pain; MDI = Major Depression Inventory; NR = not reported; PHQ-9 = Patient Health Questionnaire-9; RA = Rheumatoid arthritis; SD = standard deviation; SMD = standard mean difference; $\mathrm{UC}=$ usual care; $\mathrm{WL}=$ waitlist

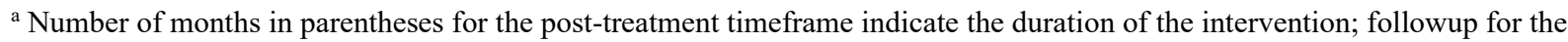
remaining timeframes is in months following the end of the intervention. 
Figure I-16. CPMP versus UC: Sensitivity analysis for depression excluding poor-quality trials

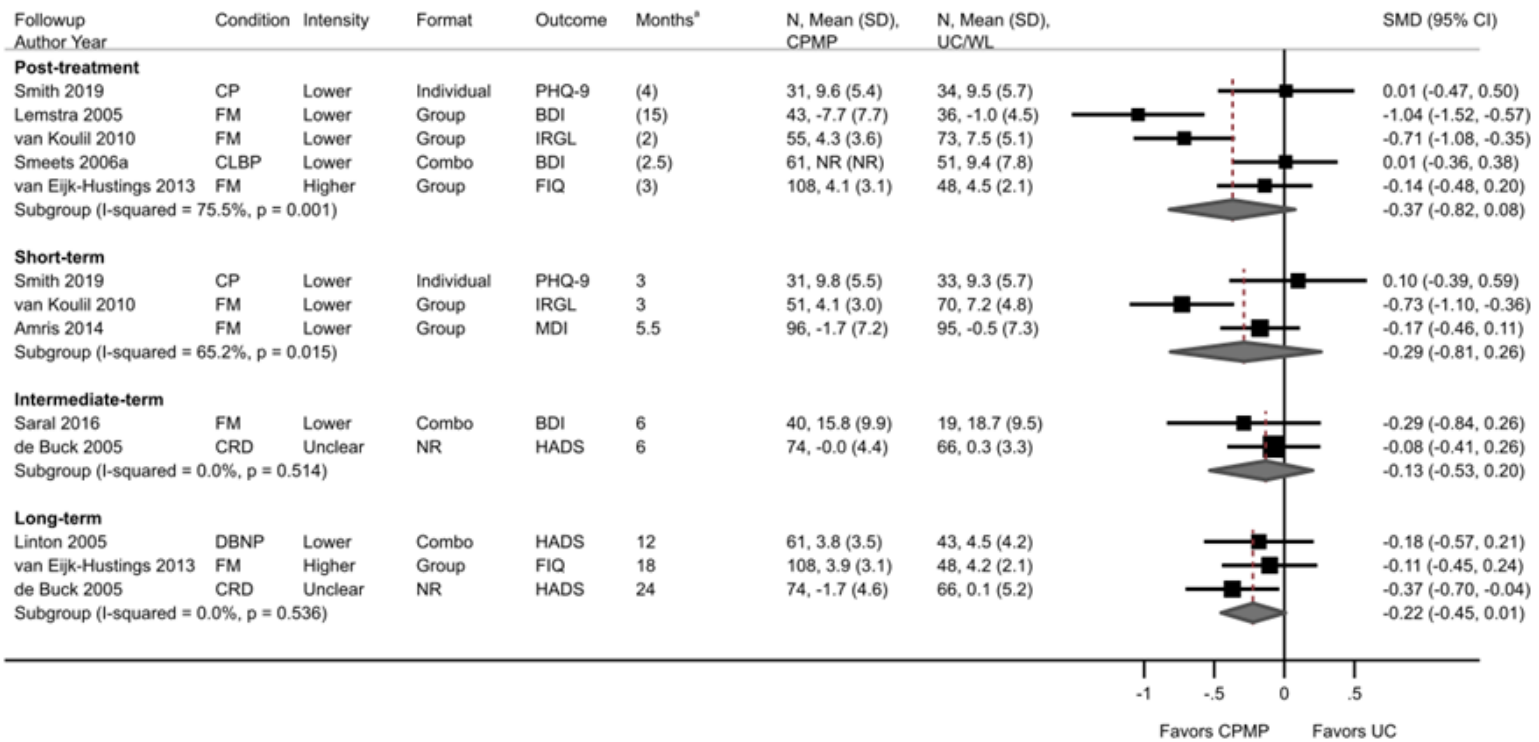

$\mathrm{BDI}=$ Beck Depression Inventory $\mathrm{CI}=$ confidence interval; $\mathrm{CLBP}=$ Chronic low back pain; Combo $=$ combination group and individual sessions; $\mathrm{CP}=$ Chronic pain; $\mathrm{CPMP}=$ Comprehensive pain management program; $\mathrm{CRD}=\mathrm{Chronic}$ rheumatoid arthritis; DBNP = back/neck pain; FIQ = Fibromyalgia Impact Questionnaire; FM = fibromyalgia; HADS = Hospital Anxiety and Depression Scale; IRGL = Impact of Rheumatic Diseases on General Health and Lifestyle Instrument; MDI = Major Depression Inventory; NR = not reported; PHQ-9 = Patient Health Questionnaire-9; RA = Rheumatoid arthritis; SD = standard deviation; $\mathrm{SMD}=$ standardized mean difference; $\mathrm{UC}=$ usual care; $\mathrm{WL}=$ waitlist.

${ }^{a}$ Number of months in parentheses for the post-treatment timeframe indicate the duration of the intervention; followup for the remaining timeframes is in months following the end of the intervention. 


\section{Figure I-17. CPMP versus UC: Sensitivity analysis for depression using the most common}

duration for long-term followup

\begin{tabular}{|c|c|c|c|c|c|c|c|c|}
\hline $\begin{array}{l}\text { Followup } \\
\text { Author Year }\end{array}$ & Condition & Intensity & Format & Outcome & Months" & $\begin{array}{l}\text { N, Mean (SD), } \\
\text { CPMP }\end{array}$ & $\begin{array}{l}\text { N, Mean (SD), } \\
\text { UC/WL }\end{array}$ & SMD $(95 \% \mathrm{Cl})$ \\
\hline \multicolumn{9}{|l|}{ Post-treatment } \\
\hline Browne 2013 & Trauma & Lower & Individual & CES-D & (6) & $31,17.4(12.4)$ & $35,15.0(11.5)$ & $0.20(-0.28,0.69)$ \\
\hline Smith 2019 & $\mathrm{CP}$ & Lower & Individual & PHQ-9 & (4) & $31,9.6(5.4)$ & $34,9.5(5.7)$ & $0.01(-0.47,0.50)$ \\
\hline Scholten 1999 & RA & Lower & Group & BDI & (0.5) & $38,6.9(3.6)$ & $30,12.2(6.5)$ & $-1.03(-1.54,-0.52)$ \\
\hline Lemstra 2005 & FM & Lower & Group & BDI & (15) & $43,-7.7(7.7)$ & $36,-1.0(4.5)$ & $-1.04(-1.52,-0.57)$ \\
\hline van Koulil 2010 & FM & Lower & Group & IRGL & (2) & $55,4.3(3.6)$ & $73,7.5(5.1)$ & $-0.71(-1.08,-0.35)$ \\
\hline Turner 1990 & CLBP & Lower & Combo & CES-D & (2) & $18,7.4(5.9)$ & $19,7.0(5.0)$ & $0.06(-0.59,0.70)$ \\
\hline Smeets 2006a & CLBP & Lower & Combo & $\mathrm{BDI}$ & (2.5) & 61, NR (NR) & $51.9 .4(7.8)$ & $0.01(-0.36,0.38)$ \\
\hline Peters 1990 & Mixed CP & Higher & Group & BDI & (1) & $50,11.6(12.4)$ & $15,11.1(5.8)$ & $0.04(-0.53,0.62)$ \\
\hline van Eijk-Hustings 2013 & FM & Higher & Group & FIQ & (3) & $108,4.1(3.1)$ & $48,4.5(2.1)$ & $-0.14(-0.48,0.20)$ \\
\hline \multicolumn{8}{|c|}{ Subgroup (l-squared $=74.8 \%, p=0.000$ ) } & $-0.30(-0.63,0.04)$ \\
\hline \multicolumn{9}{|l|}{ Short-term } \\
\hline Smith 2019 & $\mathrm{CP}$ & Lower & Individual & PHQ-9 & 3 & $31,9.8(5.5)$ & $33,9.3(5.7)$ & $0.10(-0.39,0.59)$ \\
\hline Williams 1996 & Mixed CP & Lower & Group & BDI & 1 & $68,10.7(7.2)$ & $31,17.3(7.0)$ & $-0.92(-1.37,-0.48)$ \\
\hline Scholten 1999 & RA & Lower & Group & BDI & 1 & $38,8.2(3.0)$ & $30,11.9(7.0)$ & $-0.71(-1.20,-0.22)$ \\
\hline van Koulil 2010 & FM & Lower & Group & IRGL & 3 & $51,4.1(3.0)$ & $70,7.2(4.8)$ & $-0.73(-1.10,-0.36)$ \\
\hline Amris 2014 & FM & Lower & Group & MDI & 5.5 & $96,-1.7(7.2)$ & $95,-0.5(7.3)$ & $-0.17(-0.46,0.11)$ \\
\hline \multicolumn{8}{|c|}{ Subgroup $(1-$ squared $=68.9 \%, p=0.003$ ) } & $-0.48(-0.89,-0.08)$ \\
\hline \multicolumn{9}{|l|}{ Intermediate-term } \\
\hline Scholten 1999 & RA & Lower & Group & BDI & 11.5 & $38,9.6(2.3)$ & $30,12.1(6.5)$ & $-0.53(-1.02,-0.05)$ \\
\hline Saral 2016 & FM & Lower & Combo & BDI & 6 & $40,15.8(9.9)$ & $19,18.7(9.5)$ & $-0.29(-0.84,0.26)$ \\
\hline Whitfill 2010 & Acute LBP & PUnclear & Individual & BDI & $9.5-11$ & $58,8.8(9.5)$ & $44,10.1(10.2)$ & $-0.13(-0.52,0.26)$ \\
\hline de Buck 2005 & CRD & Unclear & NR & HADS & 6 & $74,-0.0(4.4)$ & $66,0.3(3.3)$ & $-0.08(-0.41,0.26)$ \\
\hline \multicolumn{8}{|c|}{ Subgroup (l-squared $=0.0 \%, p=0.467$ ) } & $-0.21(-0.47,0.01)$ \\
\hline \multicolumn{9}{|l|}{ Long-term } \\
\hline Linton 2005 & DBNP & Lower & Combo & HADS & 12 & $61,3.8(3.5)$ & $43,4.5(4.2)$ & $-0.18(-0.57,0.21)$ \\
\hline van Eijk-Hustings 2013 & FM & Higher & Group & FIQ & 18 & $108,3.9(3.1)$ & $48,4.2(2.1)$ & $-0.11(-0.45,0.24)$ \\
\hline de Buck 2005 & CRD & Unclear & NR & HADS & 12 & $74,-0.5(4.5)$ & $66,0.0(3.7)$ & $-0.12(-0.45,0.22)$ \\
\hline \multirow{3}{*}{\multicolumn{8}{|c|}{ Subgroup (l-squared $=0.0 \%, p=0.954)$}} & $-0.13(-0.34,0.08)$ \\
\hline & & & & & & & & \\
\hline & & & & & & & & \\
\hline
\end{tabular}

$\mathrm{BDI}=$ Beck Depression Inventory; CES-D = Center for Epidemiological Studies Depression Scale; CI = confidence interval; $\mathrm{CLBP}=$ Chronic low back pain; Combo $=$ combination group and individual sessions; $\mathrm{CP}=\mathrm{Chronic}$ pain; $\mathrm{CPMP}=$

Comprehensive pain management program; $\mathrm{CRD}=$ Chronic rheumatoid arthritis; DBNP = back/neck pain; FIQ = Fibromyalgia Impact Questionnaire; FM = fibromyalgia; HADS = Hospital Anxiety and Depression Scale; IRGL = Impact of Rheumatic Diseases on General Health and Lifestyle Instrument; LBP = Low back pain; MDI = Major Depression Inventory; NR = not reported; PHQ-9 = Patient Health Questionnaire-9; RA = Rheumatoid arthritis; SD = standard deviation; SMD = standard mean difference; $\mathrm{UC}=$ usual care; $\mathrm{WL}=$ waitlist

${ }^{a}$ Number of months in parentheses for the post-treatment timeframe indicate the duration of the intervention; followup for the remaining timeframes is in months following the end of the intervention. 
Figure I-18. CPMP versus UC: Anxiety

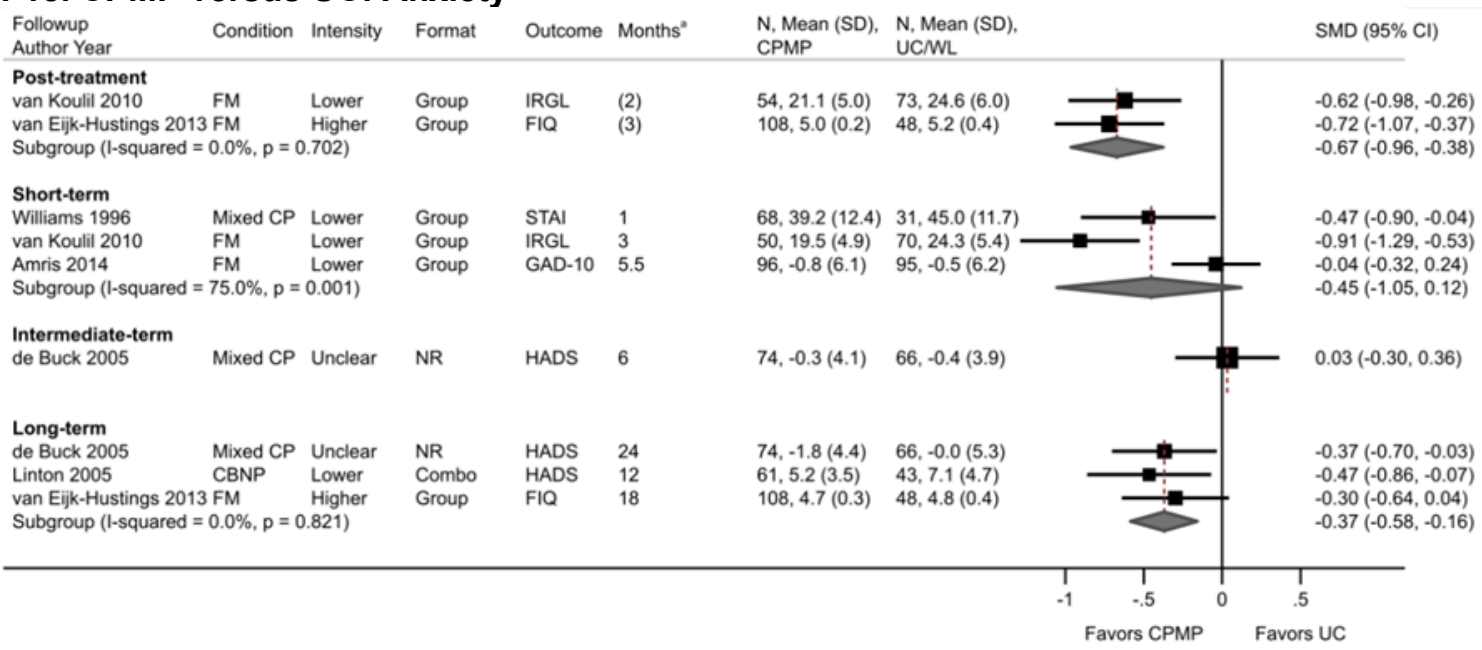

$\mathrm{CBNP}=$ chronic back/neck pain $\mathrm{CI}=$ confidence interval $; \mathrm{Combo}=$ combination group and individual sessions $\mathrm{CP}=\mathrm{Chronic}$ pain; $\mathrm{CPMP}=$ Comprehensive pain management program; $\mathrm{CRD}=$ Chronic rheumatoid arthritis; FIQ = Fibromyalgia Impact Questionnaire; FM = fibromyalgia; GAD-10 = Generalized Anxiety Disorder inventory; HADS = Hospital Anxiety and Depression Scale Anxiety; IRGL = Impact of Rheumatic Diseases on General Health and Lifestyle Instrument; NR = not reported; SD = standard deviation; SMD = standard mean deviation; STAI = Spielberger State-Trait Anxiety Inventory; UC = usual care; $\mathrm{WL}=$ waitlist

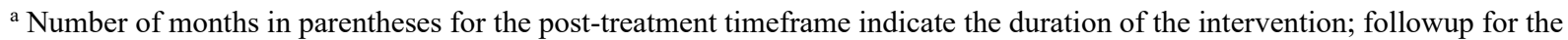
remaining timeframes is in months following the end of the intervention

Figure I-19. CPMP versus UC: Sensitivity analysis for anxiety excluding the poor-quality trial

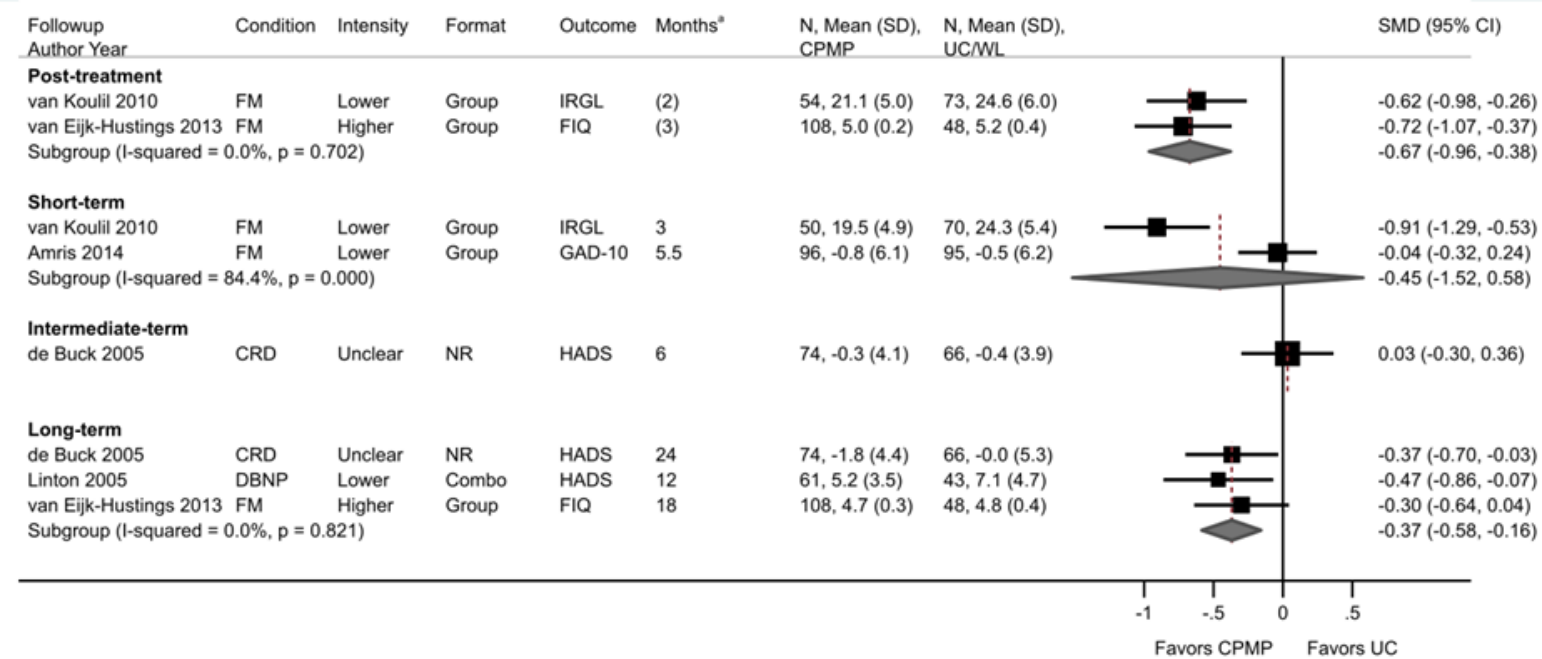

$\mathrm{CI}=$ confidence interval; $\mathrm{CP}=$ Chronic pain $\mathrm{CPMP}=$ Comprehensive pain management program; $\mathrm{CRD}=\mathrm{Chronic}$ rheumatoid arthritis; DBNP = back/neck pain; FIQ = Fibromyalgia Impact Questionnaire; FM = fibromyalgia; GAD-10 = Generalized Anxiety Disorder inventory; HADS = Hospital Anxiety and Depression Scale Anxiety; IRGL = Impact of Rheumatic Diseases on General Health and Lifestyle Instrument; NR = not reported; SD = standard deviation; SMD = standard mean deviation; UC = usual care; $\mathrm{WL}=$ waitlist

${ }^{\text {a }}$ Number of months in parentheses for the post-treatment timeframe indicate the duration of the intervention; followup for the remaining timeframes is in months following the end of the intervention 
Figure I-20. CPMP versus UC: Sensitivity analysis for anxiety using the most common duration for long-term followup

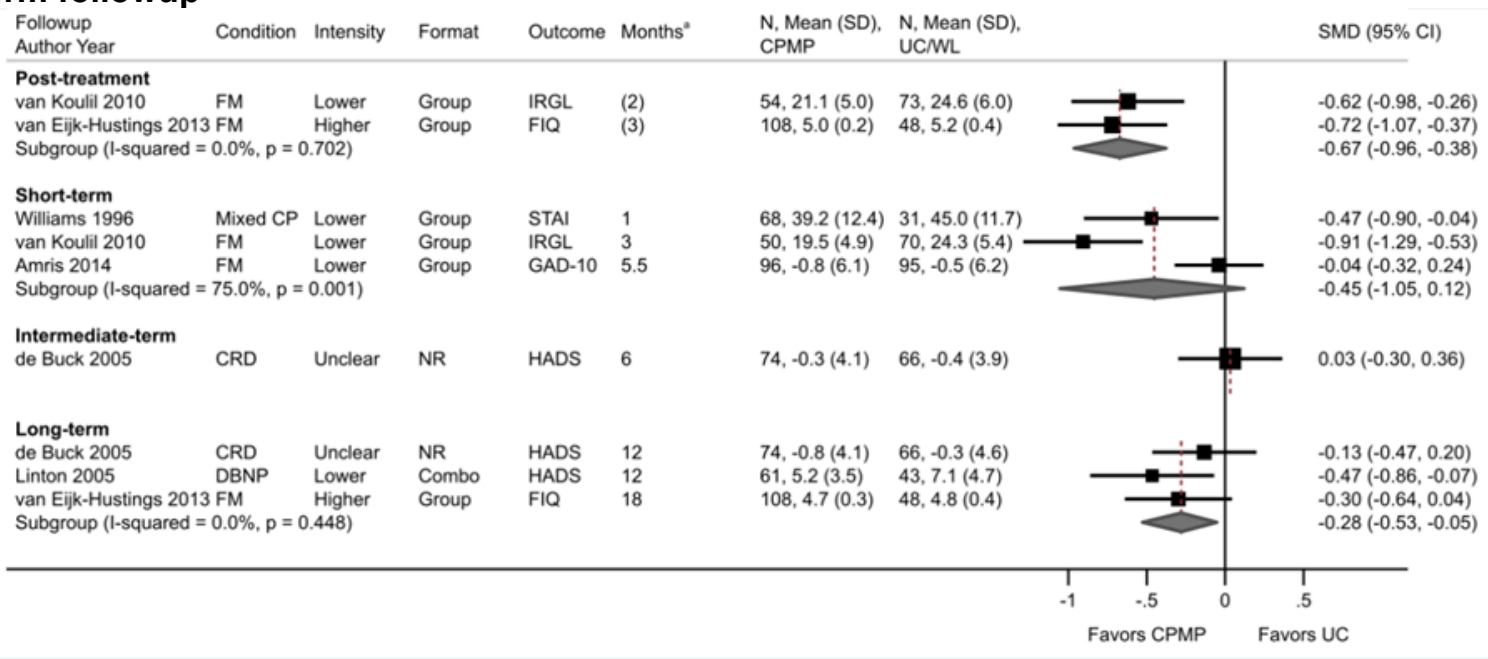

$\mathrm{CI}=$ confidence interval $\mathrm{CP}=$ Chronic pain $; \mathrm{CPMP}=$ Comprehensive pain management program $\mathrm{CRD}=$ Chronic rheumatoid arthritis; DBNP = back/neck pain; FIQ = Fibromyalgia Impact Questionnaire; FM = fibromyalgia; GAD-10 = Generalized Anxiety Disorder inventory; HADS = Hospital Anxiety and Depression Scale Anxiety; IRGL = Impact of Rheumatic Diseases on General Health and Lifestyle Instrument; NR = not reported; SD = standard deviation; SMD = standard mean deviation; UC = usual care; $\mathrm{WL}=$ waitlist

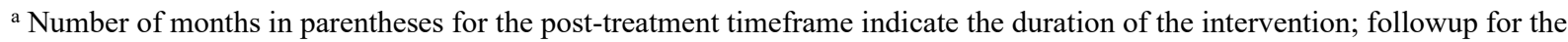
remaining timeframes is in months following the end of the intervention 
Figure I-21. CPMP versus physical activity: Sensitivity analysis for back pain excluding poorquality trials

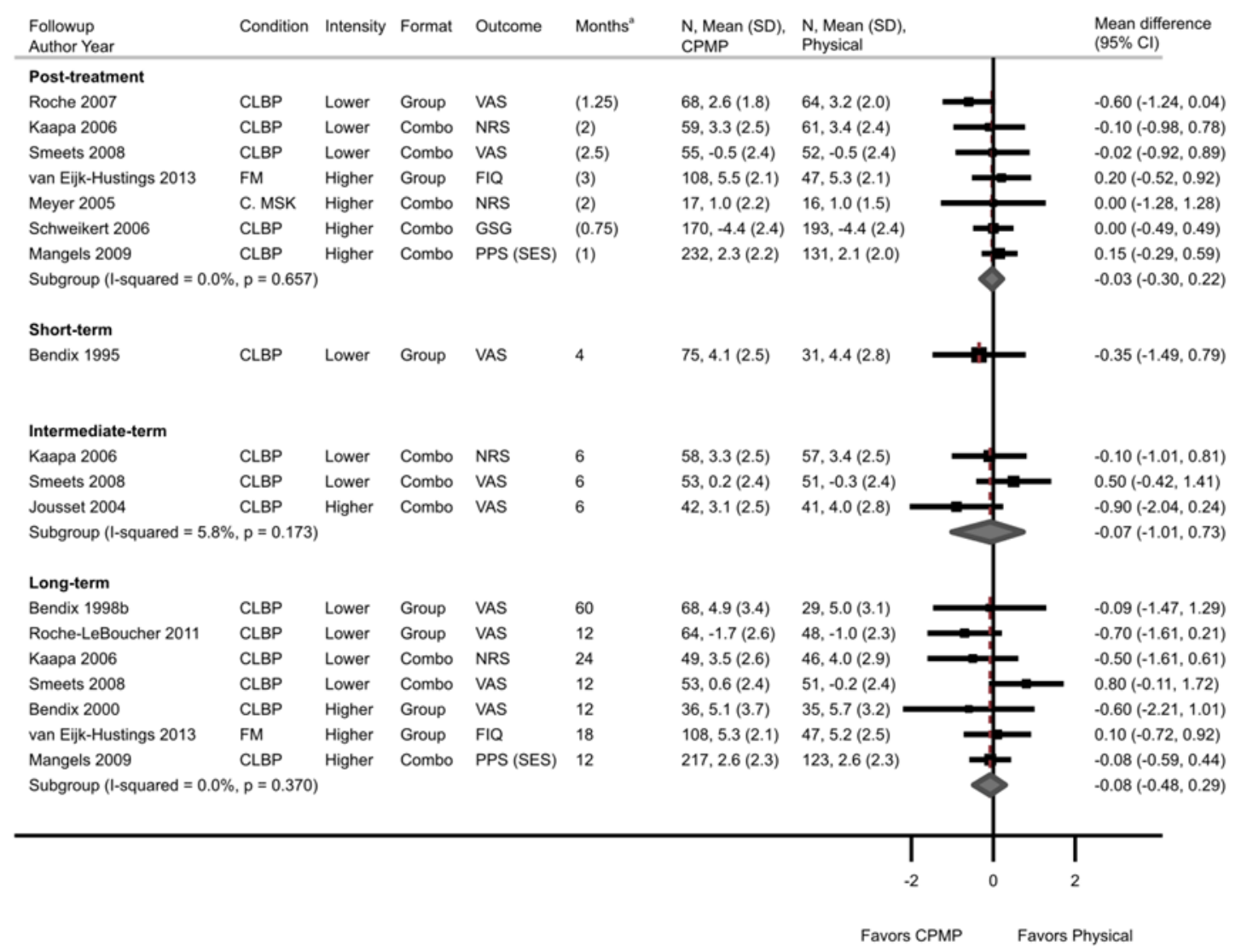

$\mathrm{CI}=$ confidence interval; $\mathrm{CLBP}=$ chronic low back pain; $\mathrm{C}$. MSK = chronic musculoskeletal pain; Combo = combination group and individual sessions; CPMP = comprehensive pain management program; FIQ = Fibromyalgia Impact Questinnaire; FM = fibromyalgia; GSG = German School Grades; NR = not reported; NRS = numerical rating scale; PPS = Pain Perception Scale; $\mathrm{SD}=$ standard deviation; $\mathrm{VAS}=$ visual analog scale.

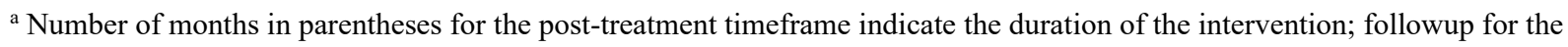
remaining timeframes is in months following the end of the intervention. 
Figure I-22. CPMP versus physical activity: Sensitivity analysis for back pain using the most common duration for long-term followup

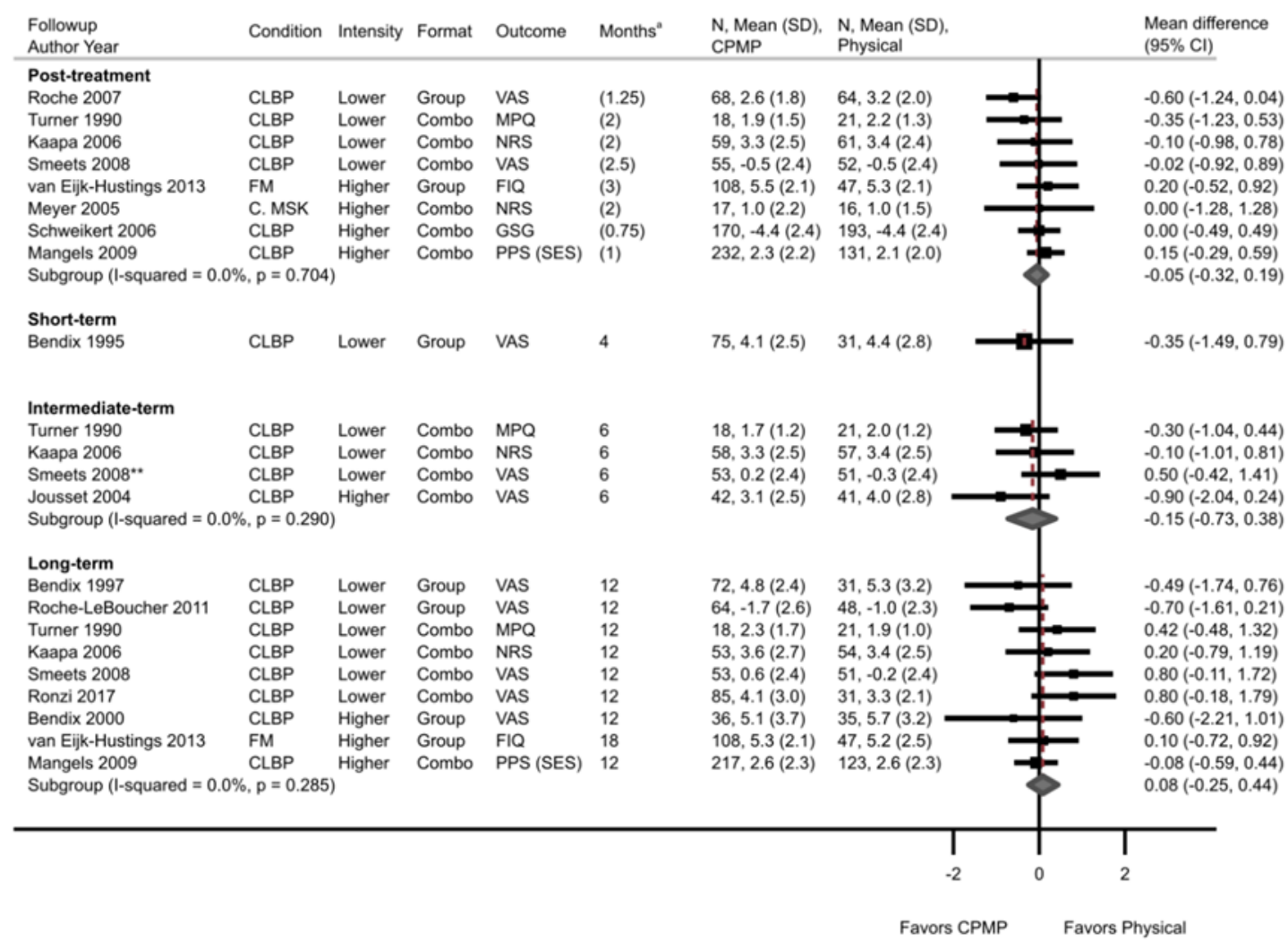

$\mathrm{CI}=$ confidence interval; $\mathrm{CLBP}=$ chronic low back pain; $\mathrm{C}$. MSK = chronic musculoskeletal pain; Combo = combination group and individual sessions; $\mathrm{CPMP}=$ comprehensive pain management program; FIQ = Fibromyalgia Impact Questinnaire; FM = fibromyalgia; GSG = German School Grades; MPQ = McGill Pain Questionnaire; NR = not reported; NRS = numerical rating scale; PPS = Pain Perception Scale; SD = standard deviation; VAS = visual analog scale.

${ }^{a}$ Number of months in parentheses for the post-treatment timeframe indicate the duration of the intervention; followup for the remaining timeframes is in months following the end of the intervention. 
Figure I-23. CPMP versus physical activity: Leg pain

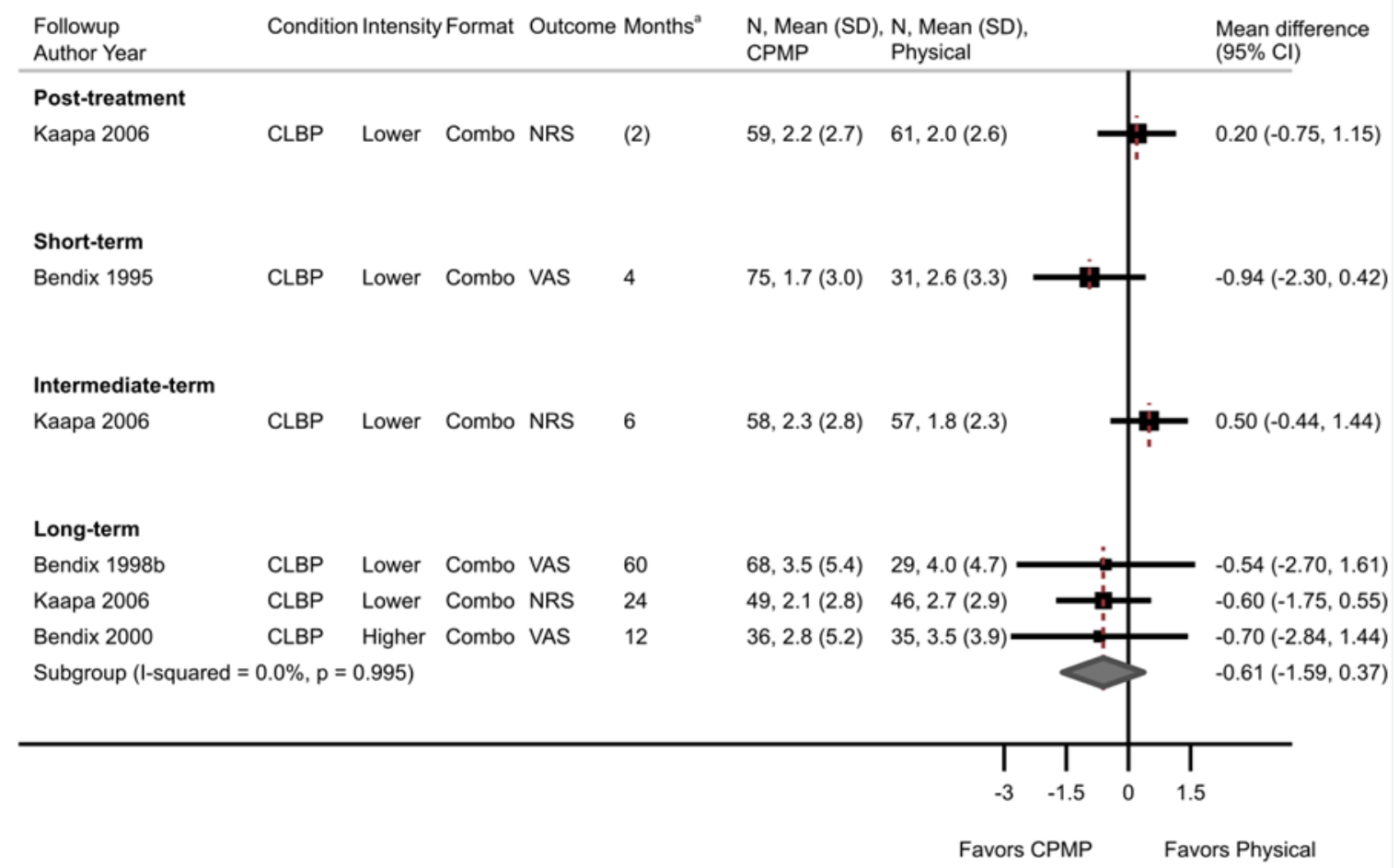

$\mathrm{CI}=$ confidence interval; $\mathrm{CLBP}=$ chronic low back pain; $\mathrm{C}$. Combo $=$ combination group and individual sessions; $\mathrm{CPMP}=$ comprehensive pain management program; $\mathrm{NRS}=$ numerical rating scale; $\mathrm{SD}=$ standard deviation; VAS = visual analog scale.

${ }^{\text {a }}$ Number of months in parentheses for the post-treatment timeframe indicate the duration of the intervention; followup for the remaining timeframes is in months following the end of the intervention. 
Figure I-24. CPMP versus physical activity: Sensitivity analysis for function excluding poor-quality trials

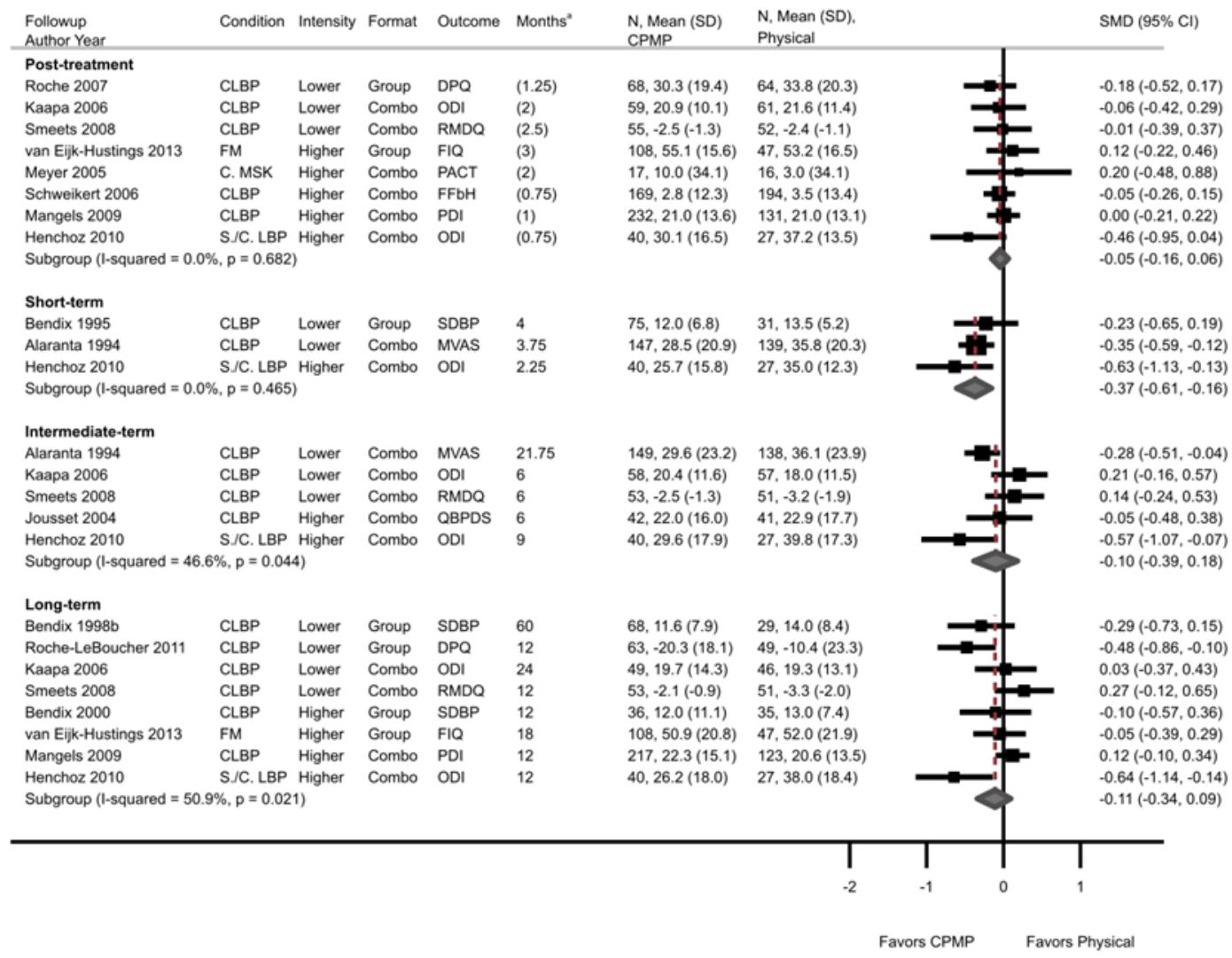

$\mathrm{CI}=$ confidence interval; $\mathrm{CLBP}=$ chronic low back pain; $\mathrm{C} . \mathrm{MSK}=$ chronic musculoskeletal disease; Combo = combination group and individual sessions; $\mathrm{CPMP}=$ comprehensive pain management program; $\mathrm{DPQ}=$ Dallas Pain Questionnaire; $\mathrm{FFbH}=$ Hannover functional questionnaire; FIQ = Fibromyalgia Impact Questionnaire; FM = fibromyalgia; S./C. LBP = subacute and chronic low back pain; MVAS = Million visual analog scale; ODI = Oswestry Disability Index; PACT $=$ Performance Assessment of Capacity Testing; PDI = Pain Disability Index; QBPDS = Quebec Back Pain Disability Scale; RMDQ = Roland Morris Disability Index; S/C. LBP = subacute and chronic low back pain; SD = standard deviation; SDBP = Self-reported disability of back pain scale; $\mathrm{SMD}=$ standardized mean difference.

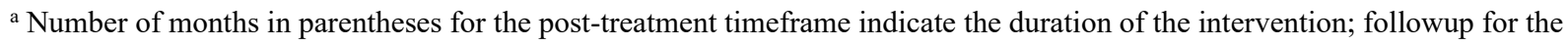
remaining timeframes is in months following the end of the intervention. 
Figure 1-25. CPMP versus physical activity: Sensitivity analysis for function using the most common duration for long-term followup

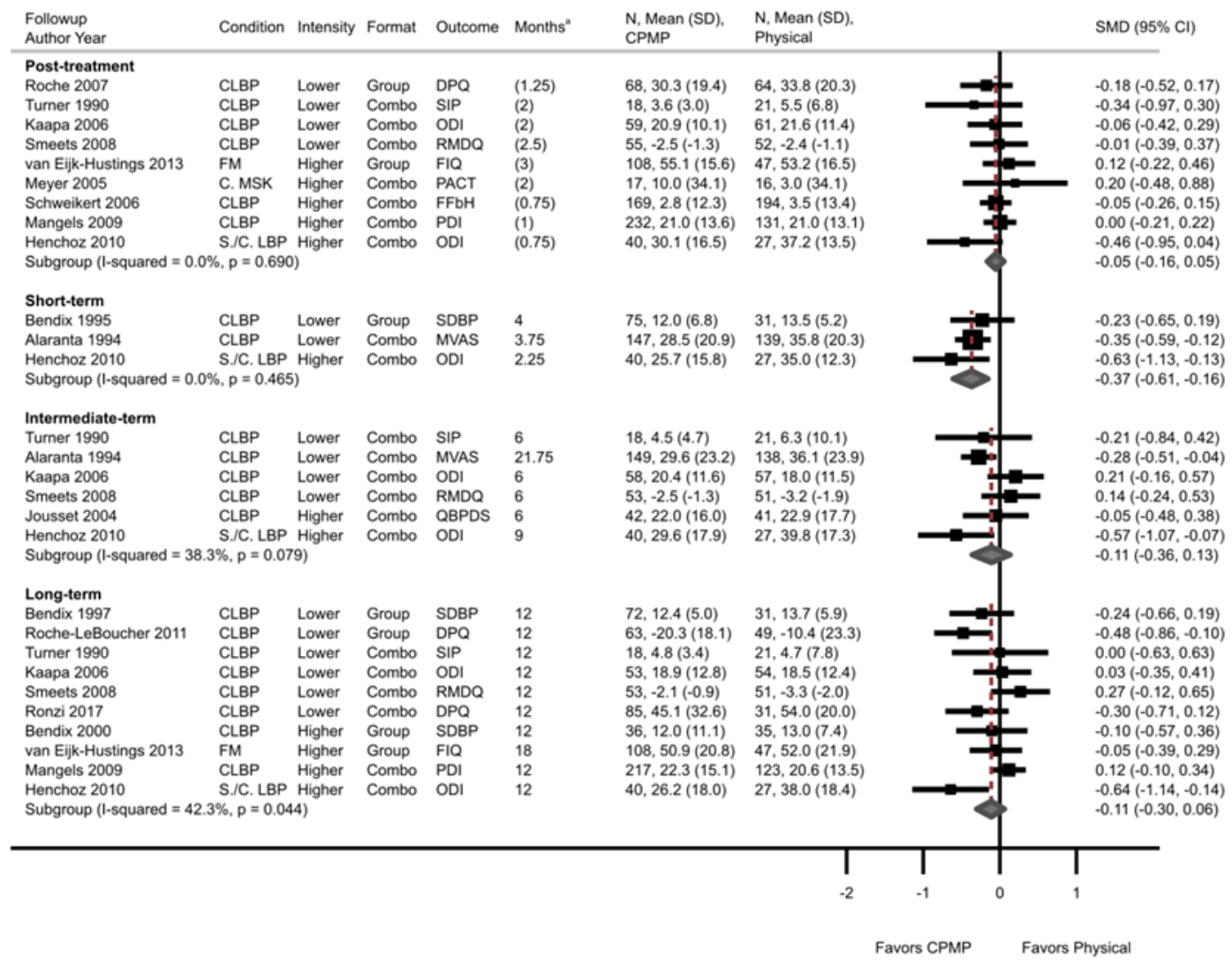

$\mathrm{CI}=$ confidence interval; $\mathrm{CLBP}=$ chronic low back pain; $\mathrm{C}$. MSK = chronic musculoskeletal disease; Combo = combination group and individual sessions; $\mathrm{CPMP}=$ comprehensive pain management program; $\mathrm{DPQ}=$ Dallas Pain Questionnaire; $\mathrm{FFbH}=$ Hannover functional questionnaire; FIQ = Fibromyalgia Impact Questionnaire; FM = fibromyalgia; S./C. LBP = subacute and chronic low back pain; MVAS = Million visual analog scale; ODI = Oswestry Disability Index; PACT = Performance Assessment of Capacity Testing; PDI = Pain Disability Index; QBPDS = Quebec Back Pain Disability Scale; RMDQ = Roland Morris Disability Index; S/C. LBP = subacute and chronic low back pain; SD = standard deviation; SDBP = Self-reported disability of back pain scale; SIP = Sickness Impact Profile; SMD = standardized mean difference.

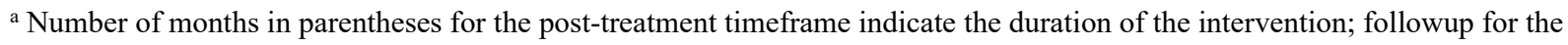
remaining timeframes is in months following the end of the intervention. 
Figure I-26. CPMP versus physical activity: SF-36 or SF-12 PCS

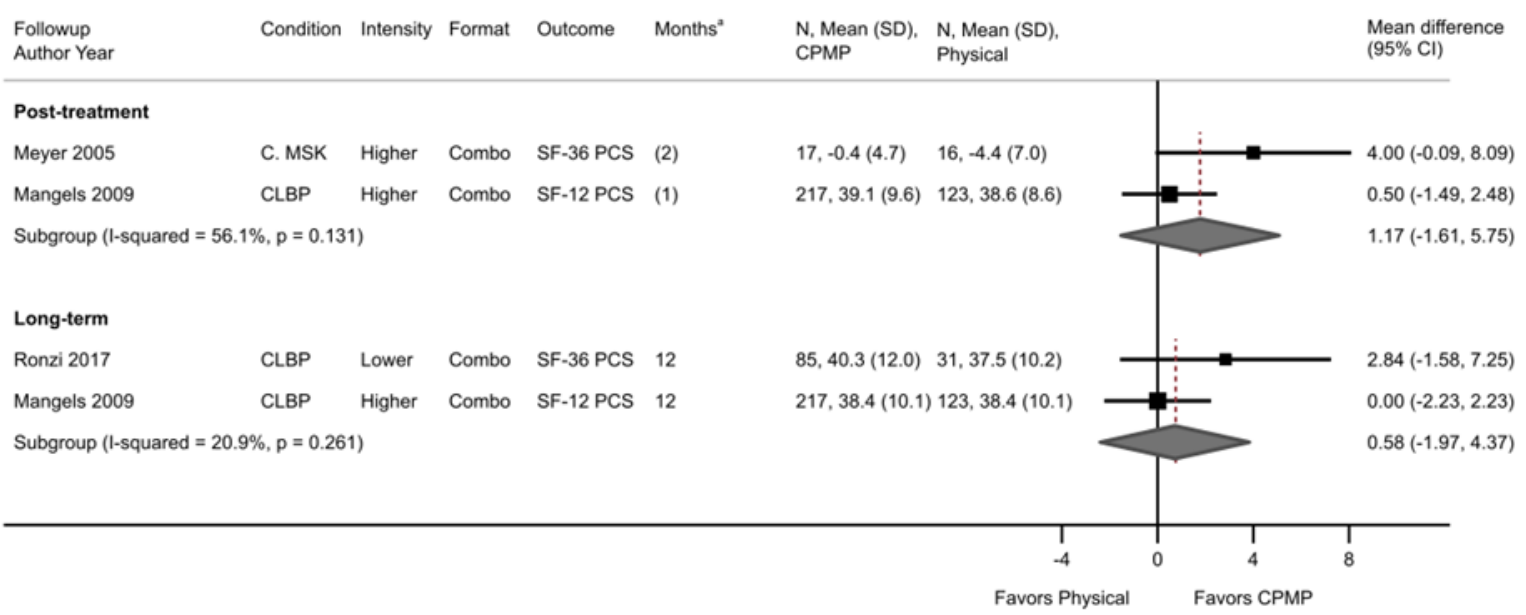

$\mathrm{CI}=$ confidence interval; $\mathrm{CLBP}=$ chronic low back pain; $\mathrm{C} . \mathrm{MSK}=$ chronic musculoskeletal disease; Combo $=$ combination group and individual sessions; $\mathrm{CPMP}=$ comprehensive pain management program; $\mathrm{SD}=$ standard deviation; $\mathrm{SF}-36 \mathrm{PCS}=$ ShortForm 36 Physical Component Score.

${ }^{a}$ Number of months in parentheses for the post-treatment timeframe indicate the duration of the intervention; followup for the remaining timeframes is in months following the end of the intervention.

Figure I-27. CPMP versus physical activity: Sensitivity analysis for the SF-36 or SF-12 PCS excluding the poor-quality trial

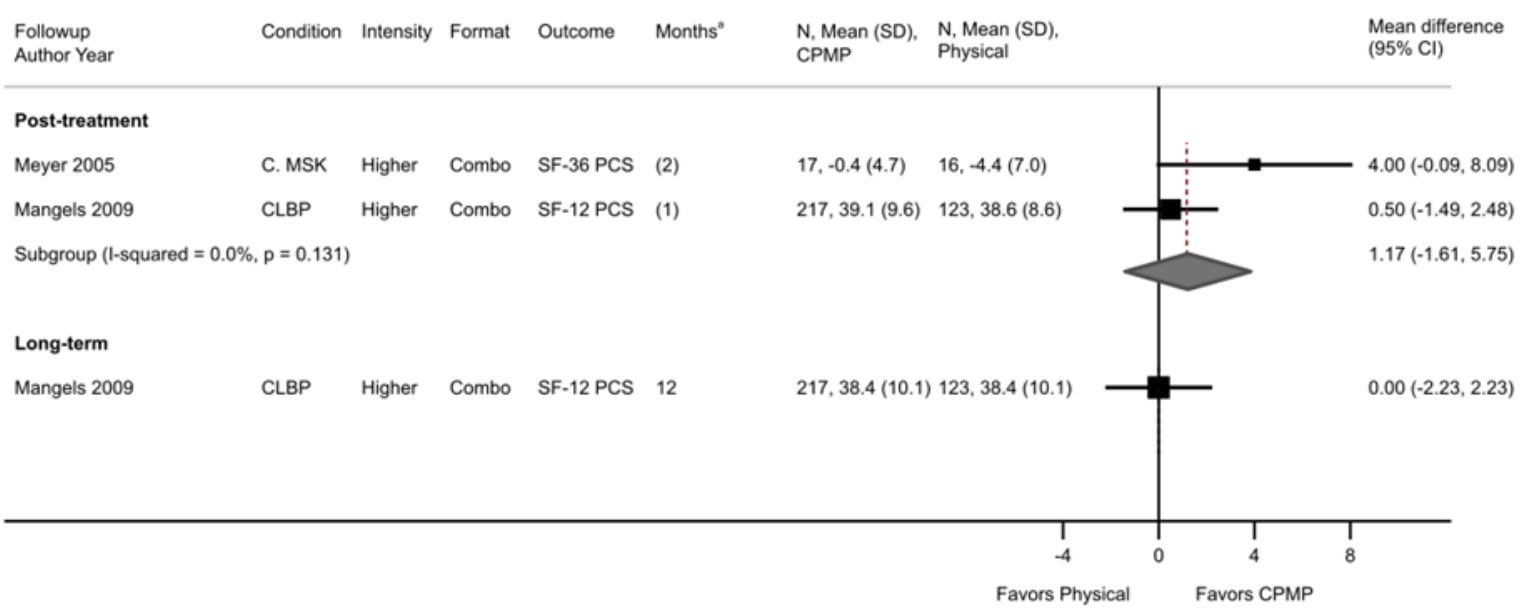

$\mathrm{CI}=$ confidence interval; $\mathrm{CLBP}=$ chronic low back pain; $\mathrm{C} . \mathrm{MSK}=$ chronic musculoskeletal disease; Combo $=$ combination group and individual sessions; CPMP = comprehensive pain management program; SD = standard deviation; SF-36 PCS = ShortForm 36 Physical Component Score.

${ }^{a}$ Number of months in parentheses for the post-treatment timeframe indicate the duration of the intervention; followup for the remaining timeframes is in months following the end of the intervention. 
Figure I-28. CPMP versus physical activity: SF-36 or SF-12 MCS

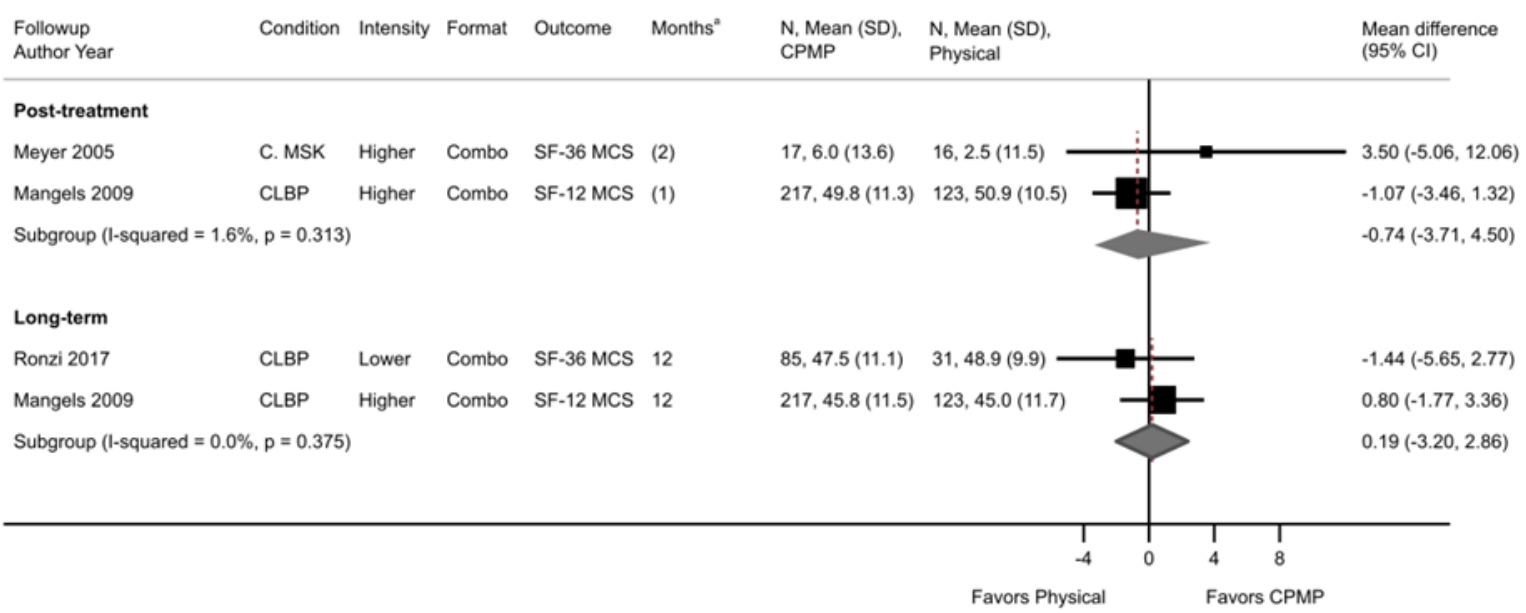

$\mathrm{CI}=$ confidence interval; $\mathrm{CLBP}=$ chronic low back pain; $\mathrm{C} . \mathrm{MSK}=$ chronic musculoskeletal disease; Combo = combination group and individual sessions; $\mathrm{CPMP}=$ comprehensive pain management program; $\mathrm{SD}=$ standard deviation; $\mathrm{SF}-36 \mathrm{MCS}=$ Short-Form 36 Mental Component Score.

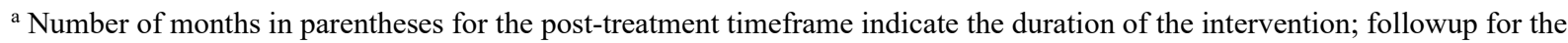
remaining timeframes is in months following the end of the intervention.

Figure I-29. CPMP versus physical activity: Sensitivity analysis for the SF-36 or SF-12 MCS excluding the poor-quality trial

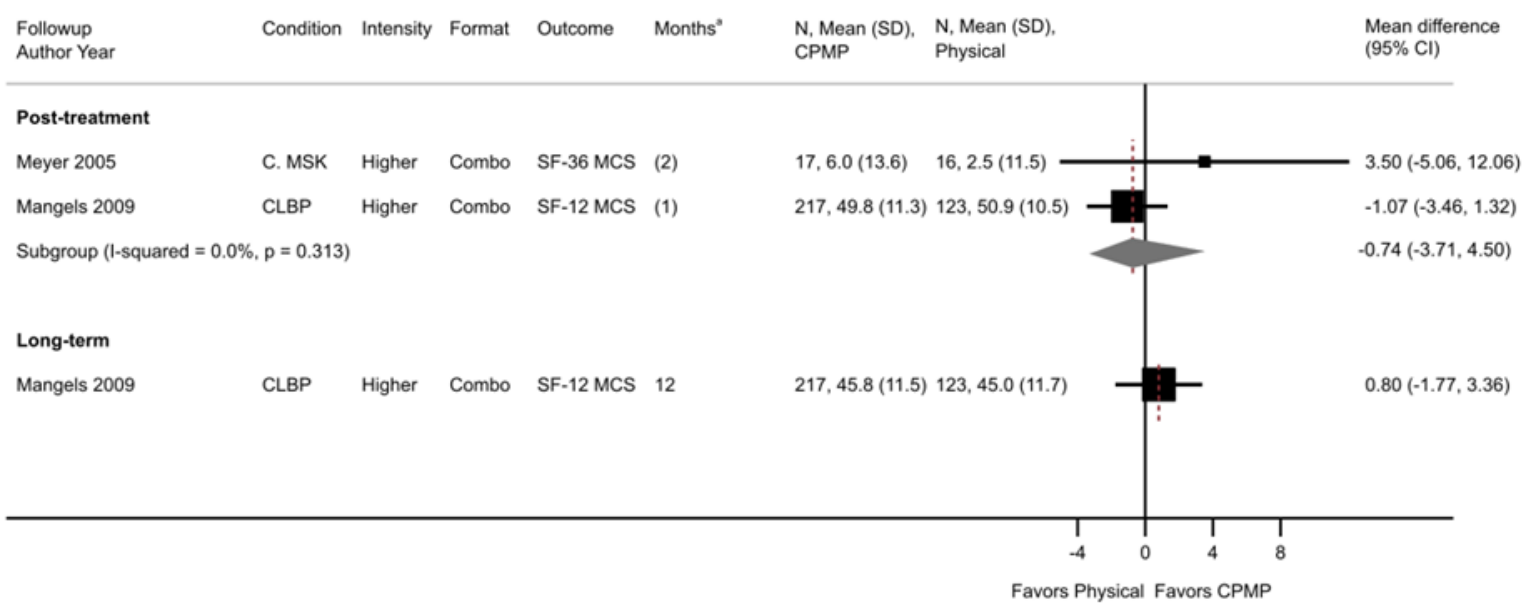

$\mathrm{CI}=$ confidence interval; $\mathrm{CLBP}=$ chronic low back pain; $\mathrm{C}$. MSK = chronic musculoskeletal disease; Combo = combination group and individual sessions; $\mathrm{CPMP}=$ comprehensive pain management program; $\mathrm{SD}=$ standard deviation; $\mathrm{SF}-36 \mathrm{MCS}=$ Short-Form 36 Mental Component Score.

${ }^{\text {a }}$ Number of months in parentheses for the post-treatment timeframe indicate the duration of the intervention; followup for the remaining timeframes is in months following the end of the intervention. 
Figure I-30. CPMP versus physical activity: Depression

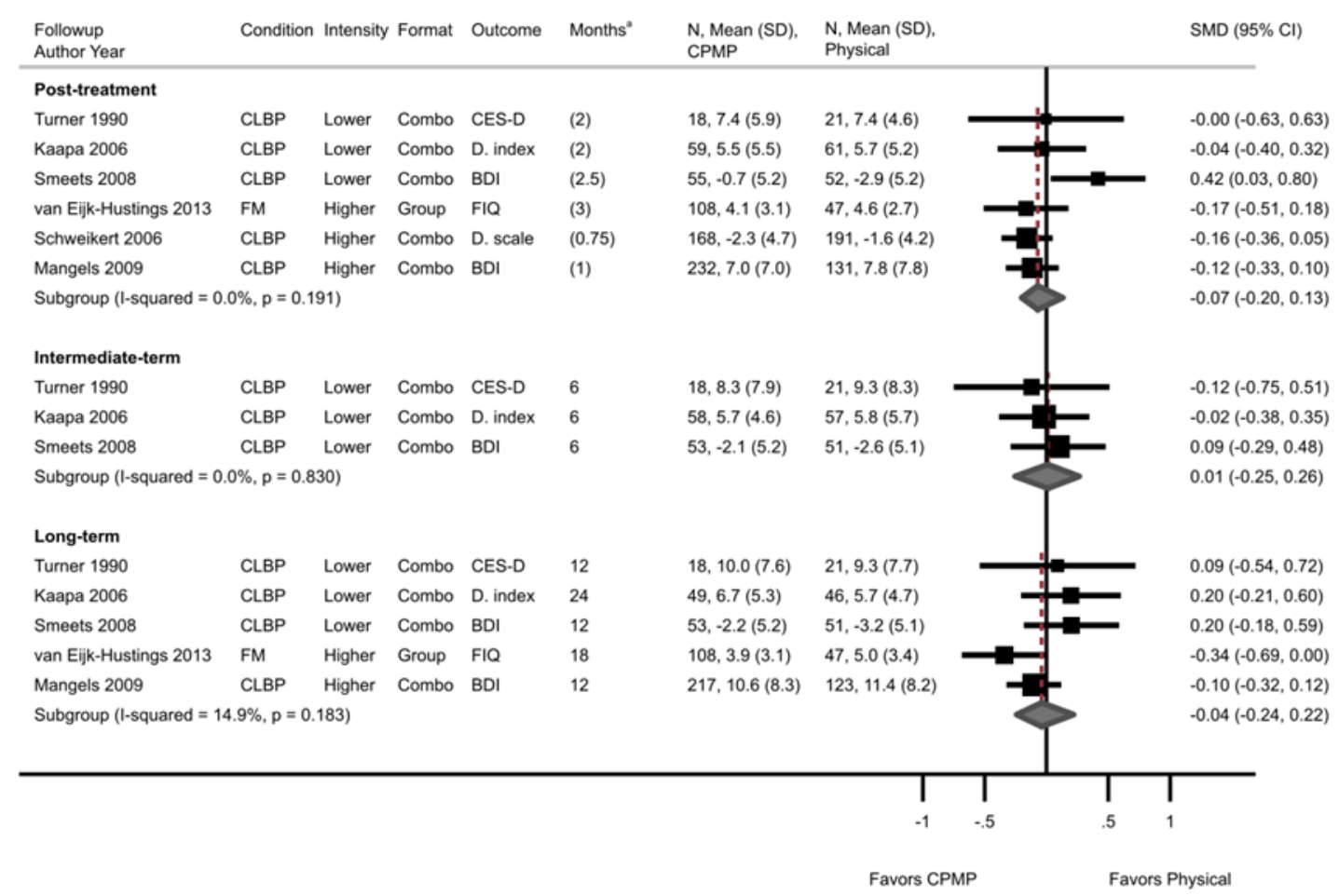

BDI = Beck Depression Inventory; CES-D = Center for Epidemiological Studies Depression; $\mathrm{CI}=$ confidence interval; $\mathrm{CLBP}=$ chronic low back pain; Combo = combination group and individual sessions; CPMP = comprehensive pain management program; D. index = Symptoms of Depression; FIQ = Fibromyalgia Impact Questionnaire; FM = fibromyalgia; SD = standard deviation; $\mathrm{SMD}=$ standardized mean difference.

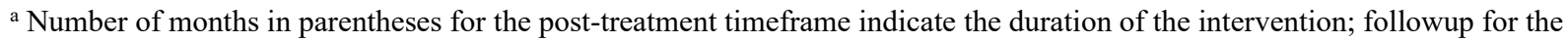
remaining timeframes is in months following the end of the intervention. 
Figure I-31. CPMP versus physical activity: Sensitivity analysis for depression excluding the poorquality trial

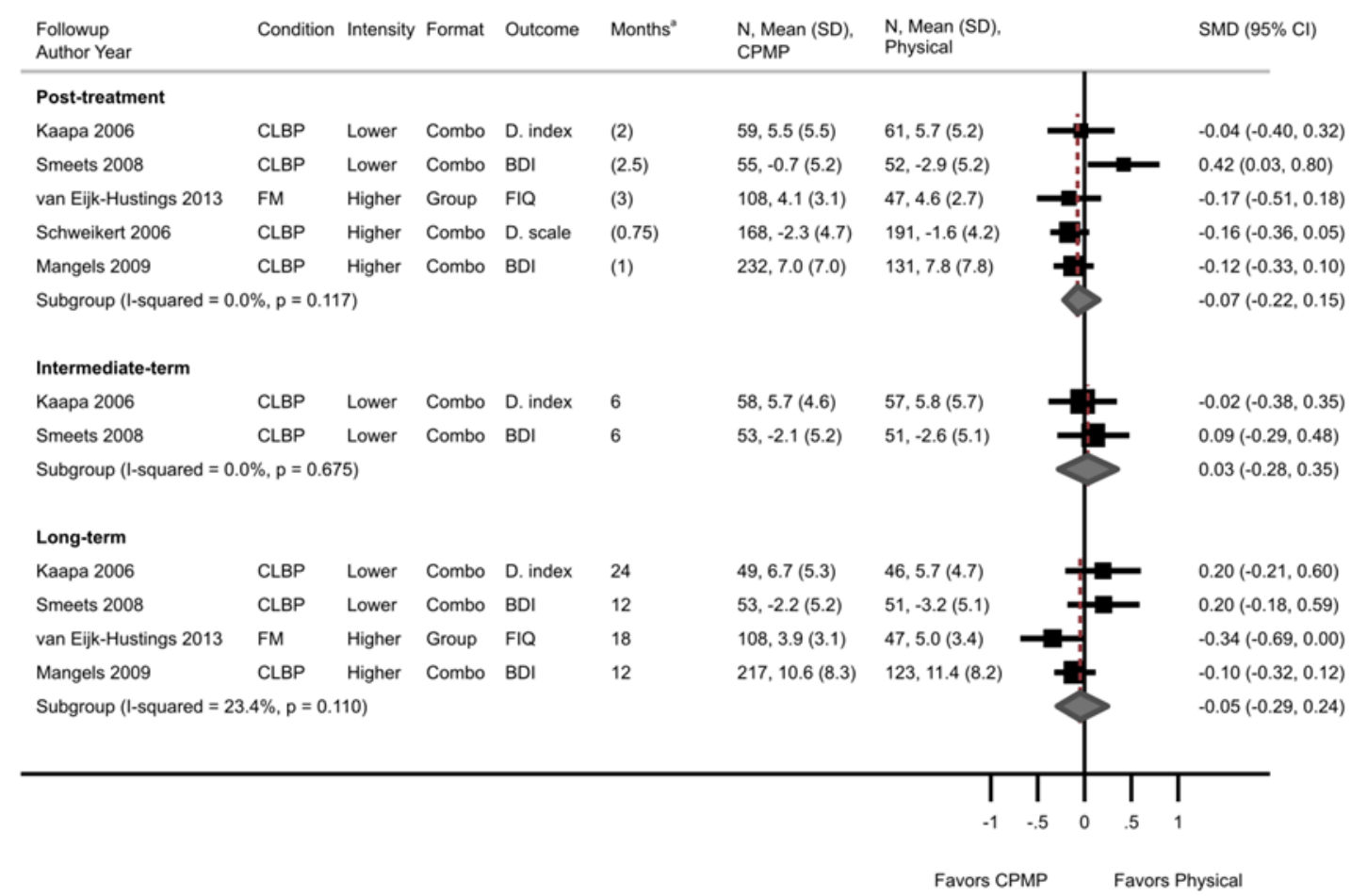

$\mathrm{BDI}=$ Beck Depression Inventory; $\mathrm{CI}=$ confidence interval; $\mathrm{CLBP}=$ chronic low back pain; Combo = combination group and individual sessions; $\mathrm{CPMP}=$ comprehensive pain management program; D. index $=$ Symptoms of Depression; FIQ = Fibromyalgia Impact Questionnaire; FM = fibromyalgia; SD = standard deviation; SMD = standardized mean difference.

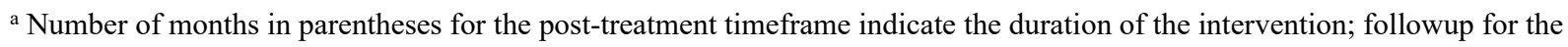
remaining timeframes is in months following the end of the intervention. 
Figure 1-32. CPMP versus physical activity: Sensitivity analysis for depression using the most common duration for long-term followup

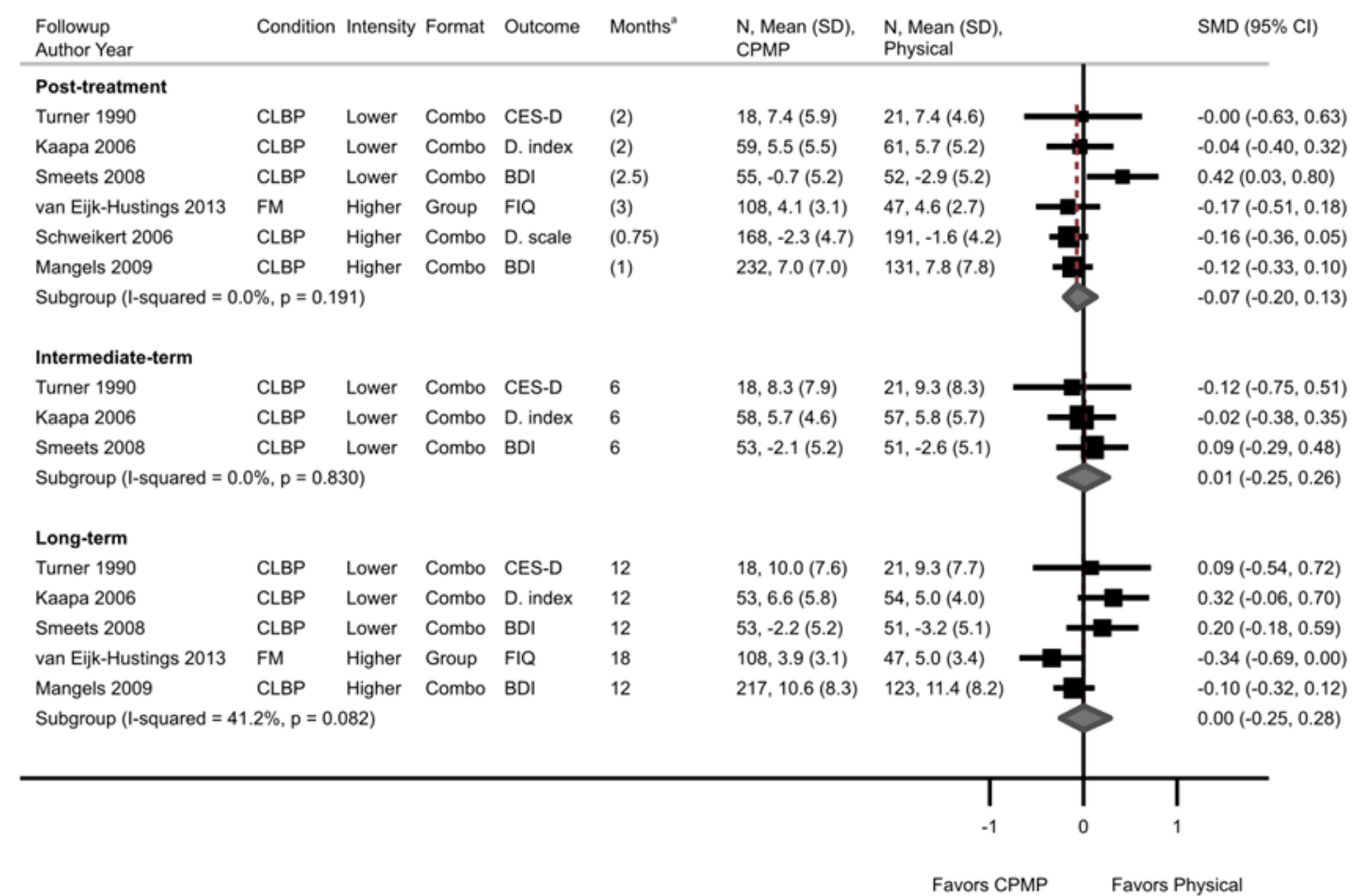

BDI = Beck Depression Inventory; CES-D = Center for Epidemiological Studies Depression; CI = confidence interval; CLBP = chronic low back pain; Combo = combination group and individual sessions; $\mathrm{CPMP}=$ comprehensive pain management program; D. index = Symptoms of Depression; FIQ = Fibromyalgia Impact Questionnaire; FM = fibromyalgia; SD = standard deviation; $\mathrm{SMD}=$ standardized mean difference.

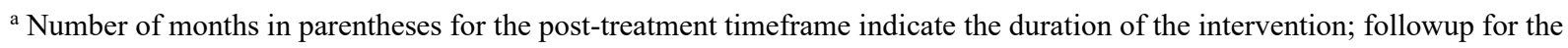
remaining timeframes is in months following the end of the intervention.

Figure I-33. CPMP versus physical activity: Anxiety

\begin{tabular}{|c|c|c|c|c|c|c|c|c|}
\hline $\begin{array}{l}\text { Followup } \\
\text { Author Year }\end{array}$ & Condition & Intensity & Format & Outcome & Months $s^{a}$ & $\begin{array}{l}\mathrm{N}, \text { Mean (SD), } \\
\text { CPMP }\end{array}$ & $\begin{array}{l}\text { N, Mean (SD), } \\
\text { Physical }\end{array}$ & SMD $(95 \% \mathrm{Cl})$ \\
\hline \multicolumn{9}{|l|}{ Post-treatment } \\
\hline van Eijk-Hustings 2013 & $\mathrm{FM}$ & Higher & Group & FIQ & (3) & $108,5.0(2.1)$ & $47,4.6(2.7)$ & $0.17(-0.17,0.52)$ \\
\hline Schweikert 2006 & CLBP & Higher & Combo & STAI & $(0.75)$ & $165,-2.7(6.9)$ & $192,-2.3(6.3)$ & $-0.06(-0.27,0.15)$ \\
\hline \multicolumn{9}{|c|}{ Subgroup $(1-$ squared $=0.0 \%, p=0.253$ ) } \\
\hline \multicolumn{9}{|l|}{ Long-term } \\
\hline van Eijk-Hustings 2013 & $\mathrm{FM}$ & Higher & Group & FIQ & 18 & $108,4.7(3.1)$ & $47,5.0(3.4)$ & $-0.09(-0.44,0.25)$ \\
\hline & & & & & & & $\begin{array}{l}1 \\
-.5\end{array}$ & 5 \\
\hline & & & & & & & Favors CPMP & Physical \\
\hline
\end{tabular}

$\mathrm{CI}=$ confidence interval; $\mathrm{CLBP}=$ chronic low back pain; $\mathrm{Combo}=$ combination group and individual sessions; $\mathrm{CPMP}=$ comprehensive pain management program; FIQ = Fibromyalgia Impact Questionnaire; FM = fibromyalgia; SD = standard deviation; SMD = standardized mean difference; STAI = State-Trait Anxiety Inventory.

${ }^{\text {a }}$ Number of months in parentheses for the post-treatment timeframe indicate the duration of the intervention; followup for the remaining timeframes is in months following the end of the intervention. 
Figure I-34. CPMP versus pharmacologic therapy alone: Sensitivity analysis for function excluding the poor-quality trial

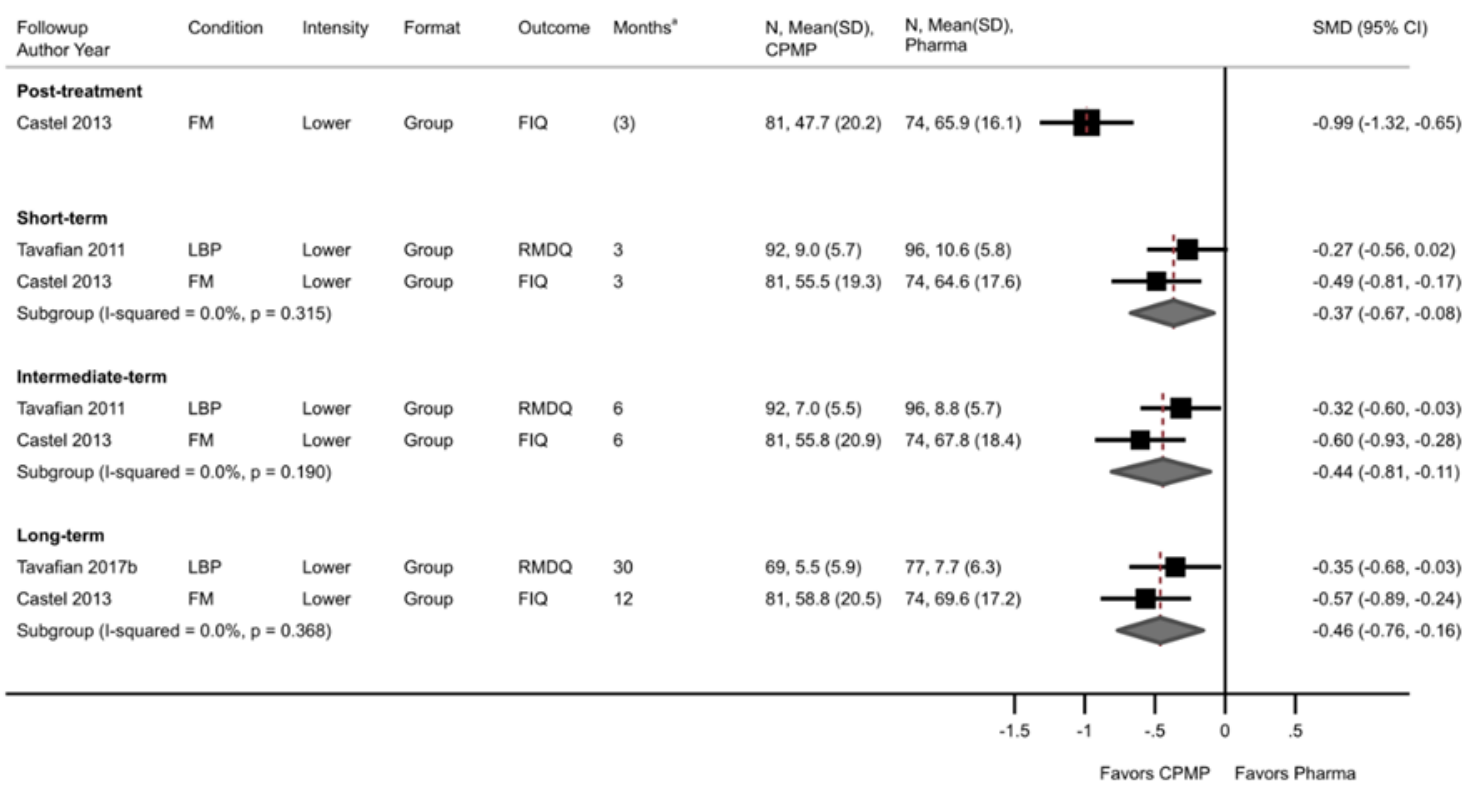

$\mathrm{CI}=$ confidence interval; $\mathrm{CPMP}=$ comprehensive pain management program; FIQ = Fibromyalgia Impact Questionnaire; FM = fibromyalgia; LBP = low back pain; RMDQ = Roland Morris Disability Index; SD = standard deviation; SMD = standardized mean difference.

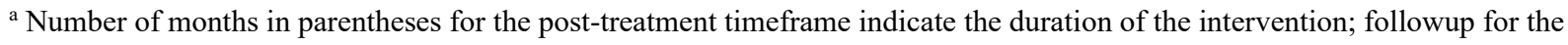
remaining timeframes is in months following the end of the intervention. 
Figure I-35. CPMP versus pharmacologic therapy alone: Sensitivity analysis for function using the most common duration for long-term followup

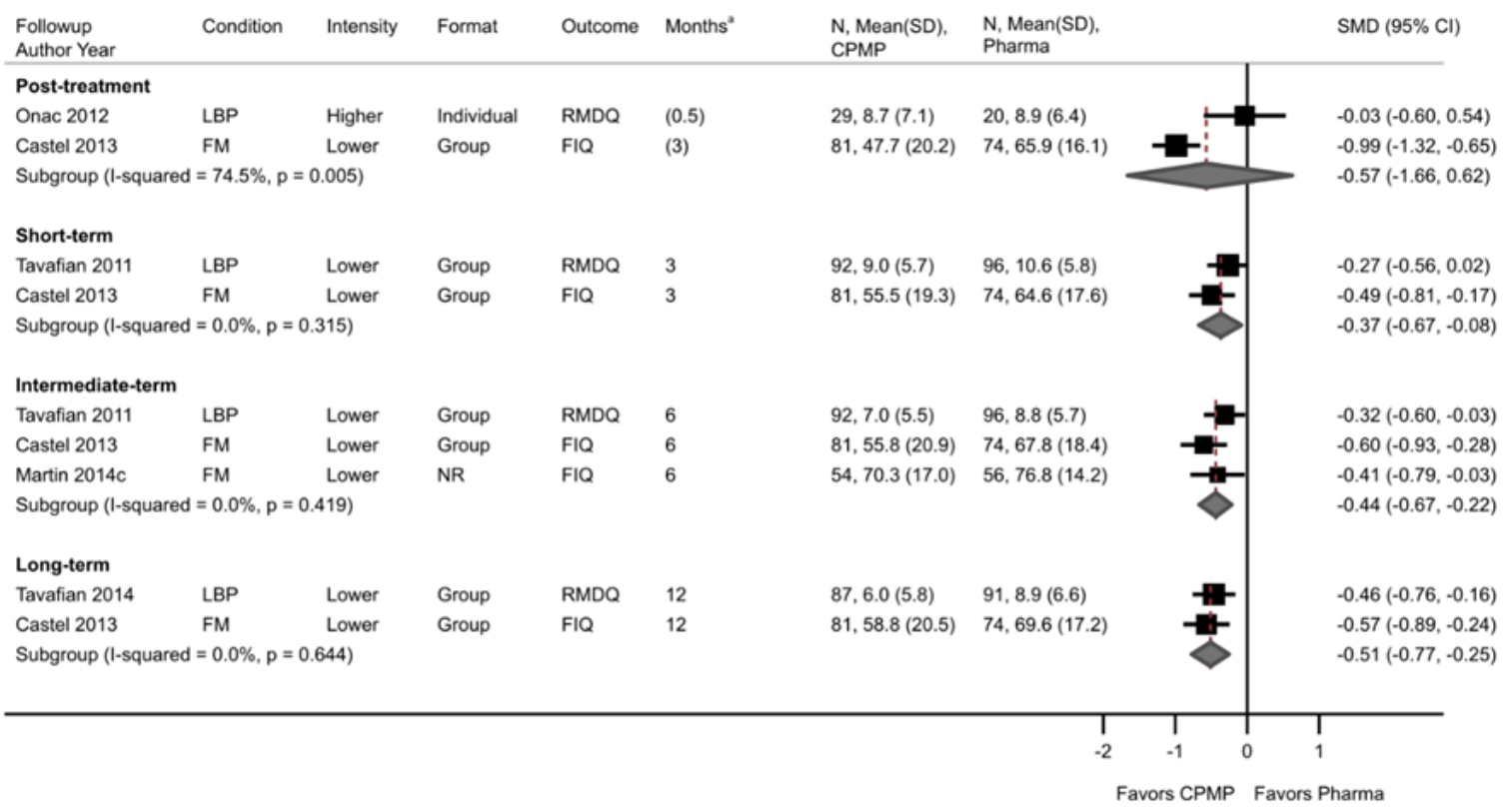

$\mathrm{CI}=$ confidence interval; $\mathrm{CPMP}=$ comprehensive pain management program; FIQ = Fibromyalgia Impact Questionnaire; FM = fibromyalgia; LBP = low back pain; RMDQ = Roland Morris Disability Index; SD = standard deviation; SMD = standardized mean difference.

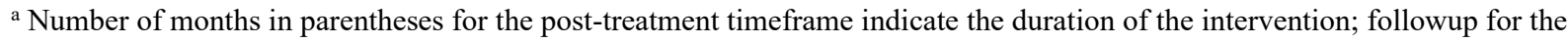
remaining timeframes is in months following the end of the intervention.

\section{Figure I-36. CPMP versus psychological therapy alone: Sensitivity analysis for pain excluding poor-quality trials}

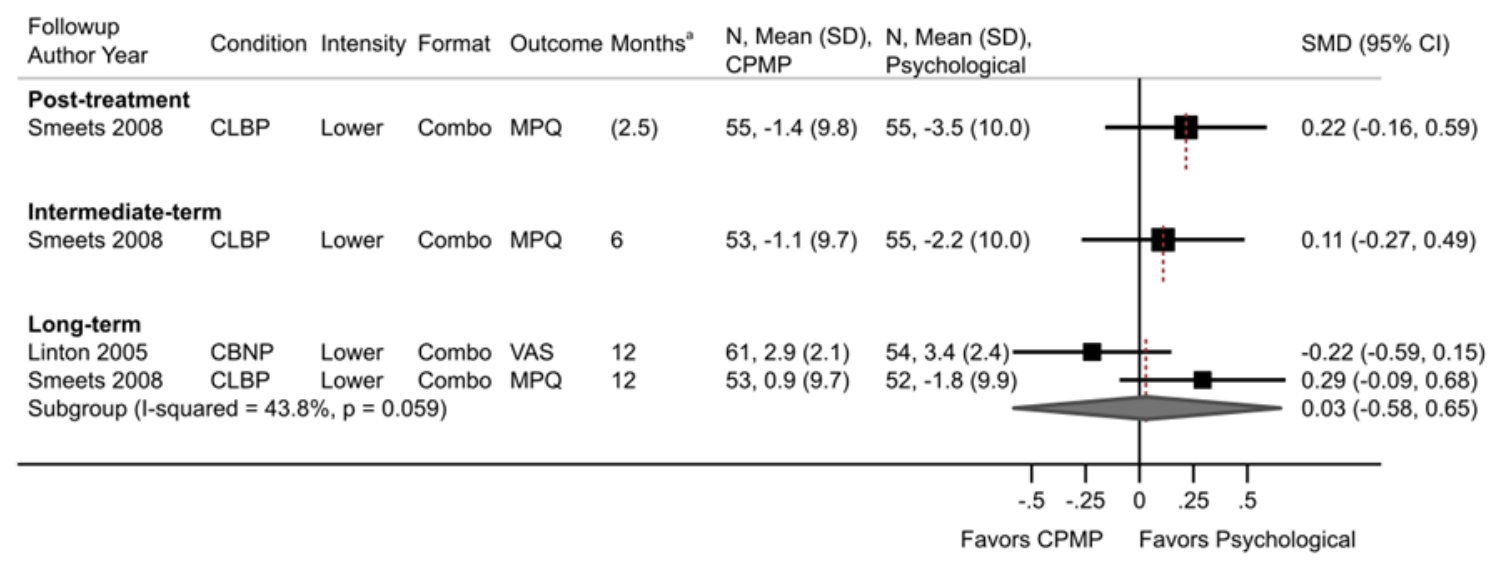

$\mathrm{CBNP}=$ chronic back/neck pain; $\mathrm{CI}=$ confidence interval; $\mathrm{CLBP}=$ chronic low back pain; $\mathrm{CPMP}=$ comprehensive pain management program; MPQ = McGill Pain Questionnaire; $\mathrm{SD}=$ standard deviation; $\mathrm{SMD}=$ standardized mean difference; VAS $=$ visual analog scale.

${ }^{\text {a }}$ Number of months in parentheses for the post-treatment timeframe indicate the duration of the intervention; followup for the remaining timeframes is in months following the end of the intervention. 
Figure I-37. CPMP versus psychological therapy alone: Sensitivity analysis for function excluding poor-quality trials

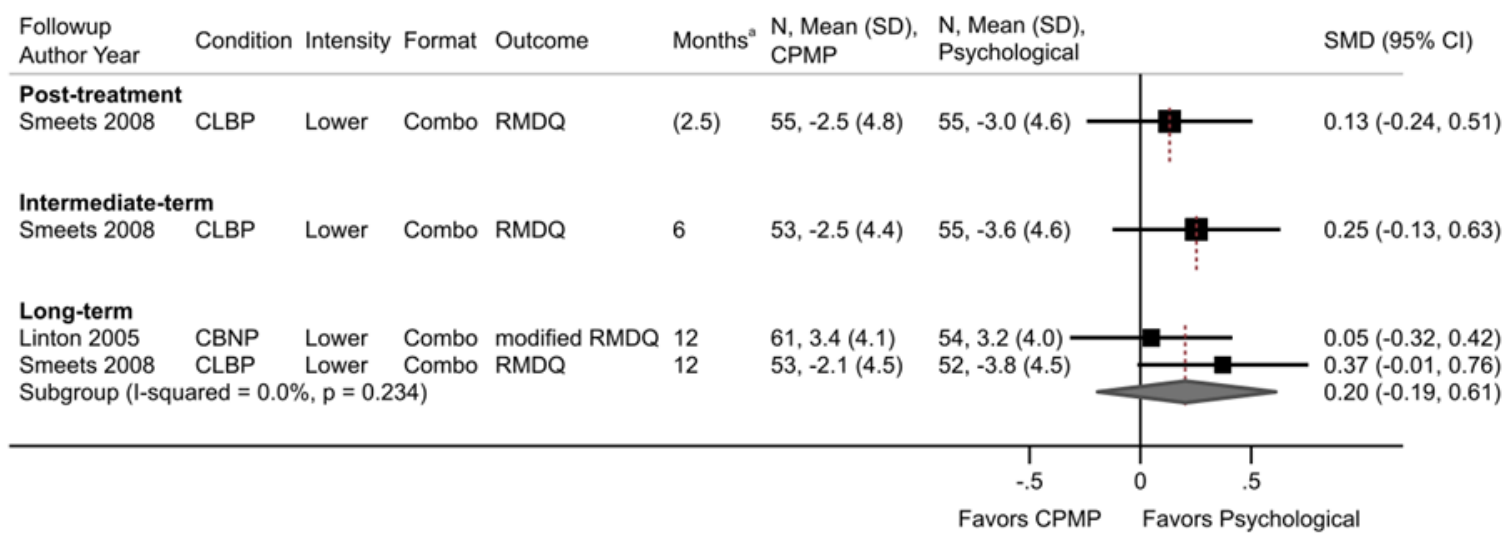

$\mathrm{CBNP}=$ chronic back/neck pain; $\mathrm{CI}=$ confidence interval; $\mathrm{CLBP}=$ chronic low back pain; $\mathrm{CPMP}=$ comprehensive pain management program; RMDQ = Roland Morris Disability Questinnaire; SD = standard deviation; $\mathrm{SMD}=$ standardized mean difference.

${ }^{a}$ Number of months in parentheses for the post-treatment timeframe indicate the duration of the intervention; followup for the remaining timeframes is in months following the end of the intervention.

Figure I-38. CPMP versus psychological therapy alone: Depression

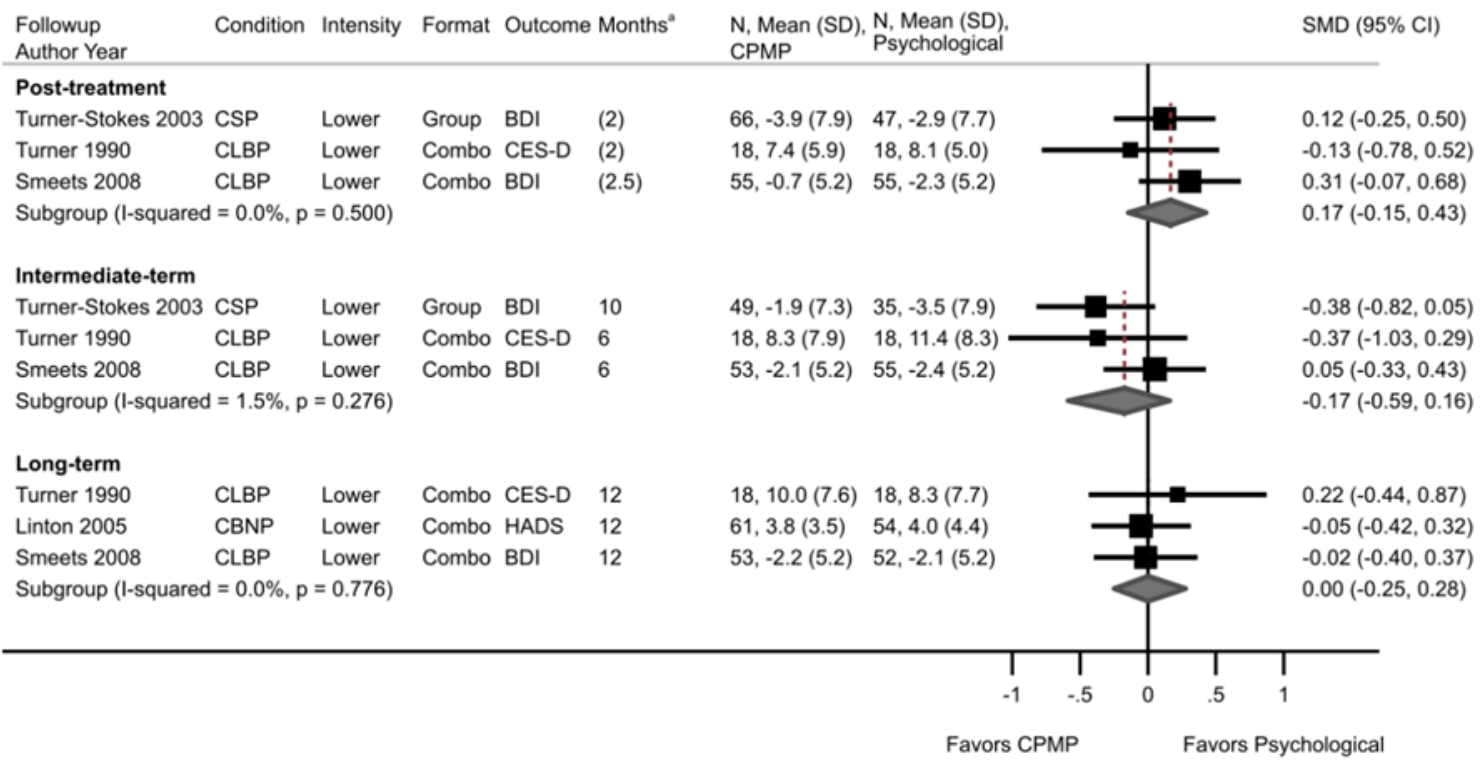

BDI = Beck Depression Inventory; CES-D = Center for Epidemiological Studies Depression; CI = confidence interval; CLBP = chronic low back pain; Combo = combination group and individual sessions; $\mathrm{CPMP}=$ comprehensive pain management program; CSP = chronic spinal pain; HADS = Hospital Anxiety and Depression Scale; $\mathrm{SD}=$ standard deviation; $\mathrm{SMD}=$ standardized mean difference.

${ }^{\text {a }}$ Number of months in parentheses for the post-treatment timeframe indicate the duration of the intervention; followup for the remaining timeframes is in months following the end of the intervention. 
Figure 1-39. CPMP versus psychological therapy alone: Sensitivity analysis for depression excluding poor-quality trials

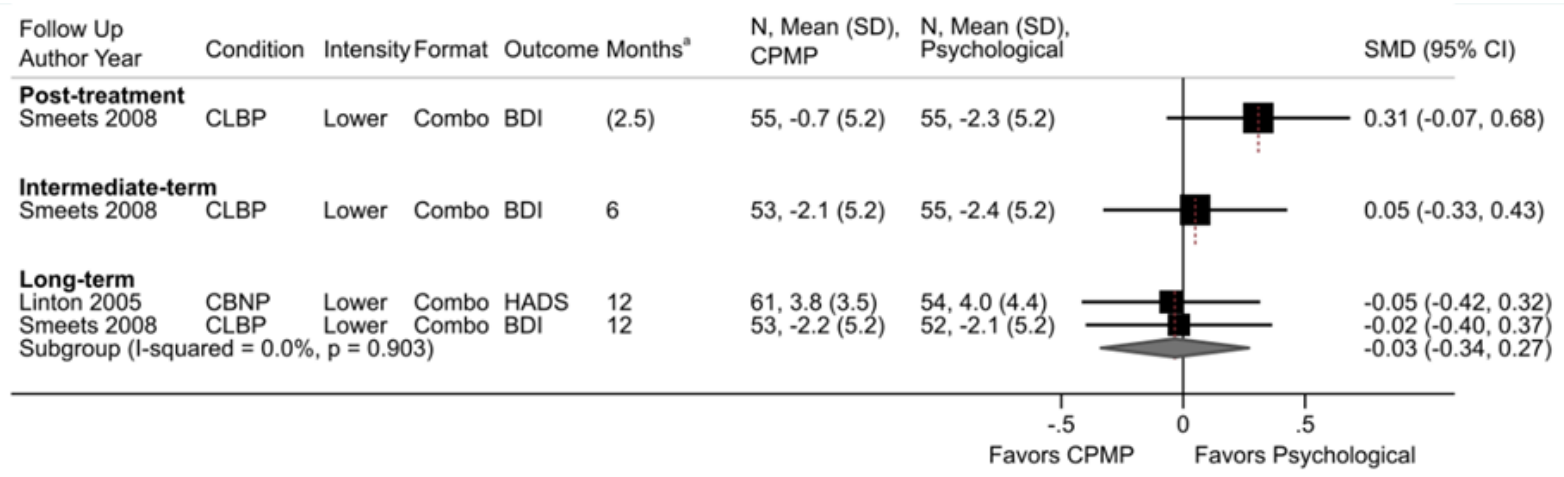

BDI = Beck Depression Inventory CI = confidence interval; CLBP = chronic low back pain; Combo = combination group and individual sessions; $\mathrm{CPMP}=$ comprehensive pain management program; CSP = chronic spinal pain; HADS = Hospital Anxiety and Depression Scale; $\mathrm{SD}=$ standard deviation; $\mathrm{SMD}=$ standardized mean difference.

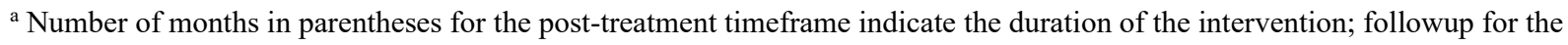
remaining timeframes is in months following the end of the intervention.

\section{Key Question 2}

Figure I-40. CPMP with greater versus fewer total hours: Pain at intermediate term

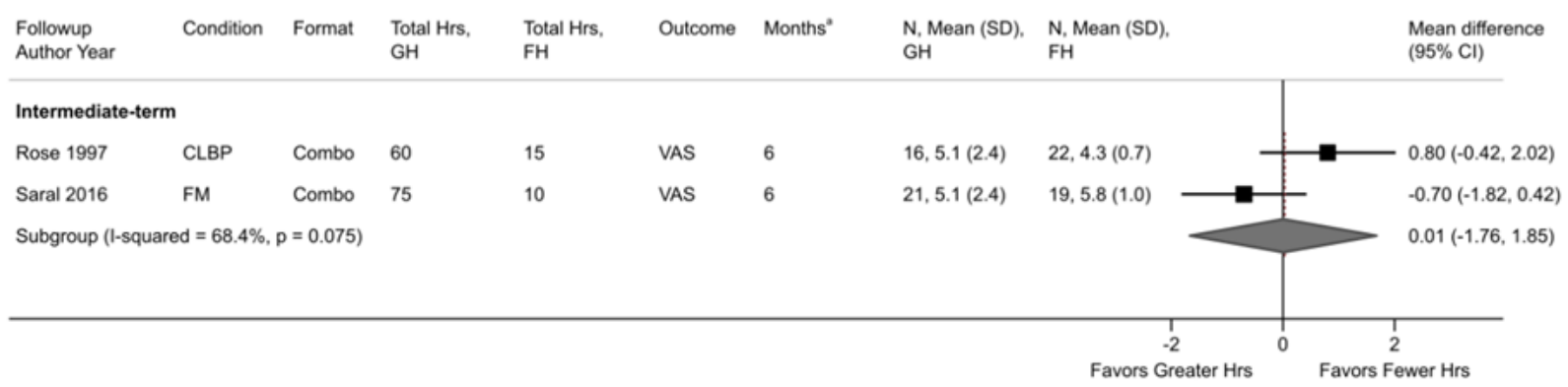

$\mathrm{CI}=$ confidence interval; $\mathrm{CLBP}=$ chronic low back pain; Combo $=$ combination group and individual sessions; $\mathrm{CPMP}=$ comprehensive pain management program; $\mathrm{FH}=$ fewer hours; $\mathrm{FM}=$ fibromyalgia; $\mathrm{GH}=$ greater hours; Hrs = hours; $\mathrm{SD}=$ standard deviation; VAS = visual analog scale

${ }^{\text {a }}$ Followup is in months following the end of the intervention. 
Figure I-41. CPMP with greater versus fewer total hours: Sensitivity analysis for pain at intermediate term using data for the group with 30 hours data for Rose 1997

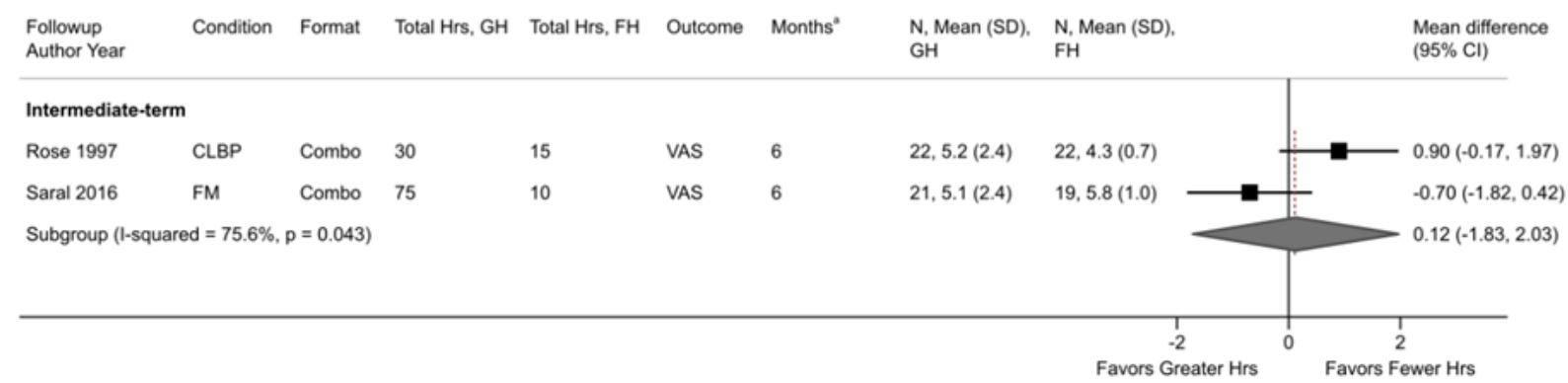

$\mathrm{CI}=$ confidence interval; $\mathrm{CLBP}=$ chronic low back pain; Combo $=$ combination group and individual sessions; $\mathrm{CPMP}=$ comprehensive pain management program; $\mathrm{FH}=$ fewer hours; $\mathrm{FM}=$ fibromyalgia; $\mathrm{GH}=$ greater hours; Hrs = hours; $\mathrm{SD}=$ standard deviation; VAS $=$ visual analog scale.

${ }^{\text {a }}$ Followup is in months following the end of the intervention.

Figure I-42. CPMP with greater versus fewer total hours: Function at intermediate term

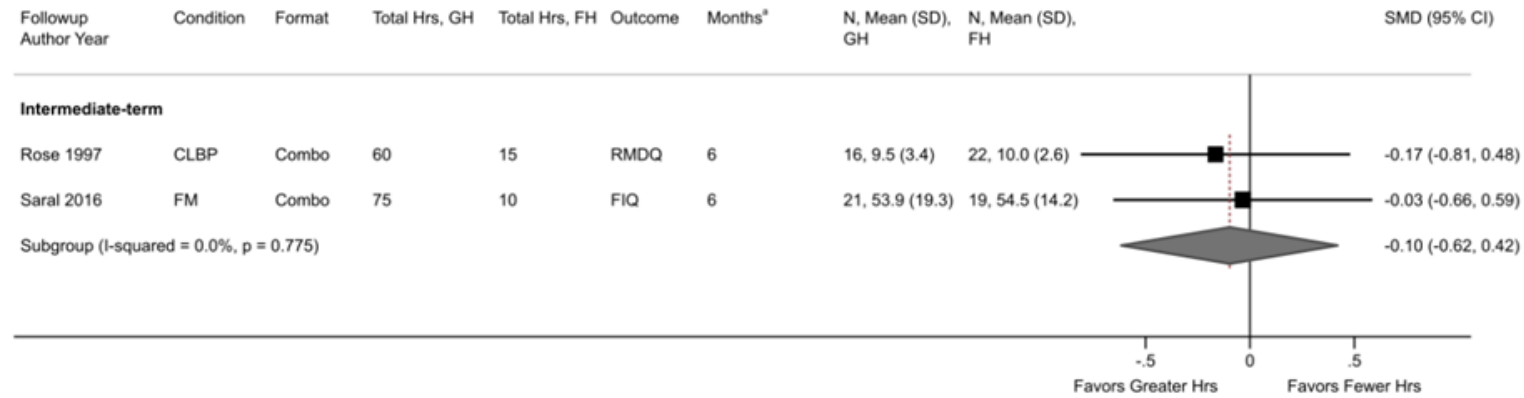

$\mathrm{CI}=$ confidence interval; $\mathrm{CLBP}=$ chronic low back pain; Combo $=$ combination group and individual sessions; $\mathrm{CPMP}=$ comprehensive pain management program; FH = fewer hours; FIQ = Fibromyalgia Impact Questionnaire; FM = fibromyalgia; $\mathrm{GH}=$ greater hours; Hrs = hours; RMDQ = Roland Morris Disability Index; SD = standard deviation.

${ }^{\text {a }}$ Followup is in months following the end of the intervention.

Figure 1-43. CPMP with greater versus fewer total hours: Sensitivity analysis for function at intermediate term using data for the group with 30 hours data for Rose 1997

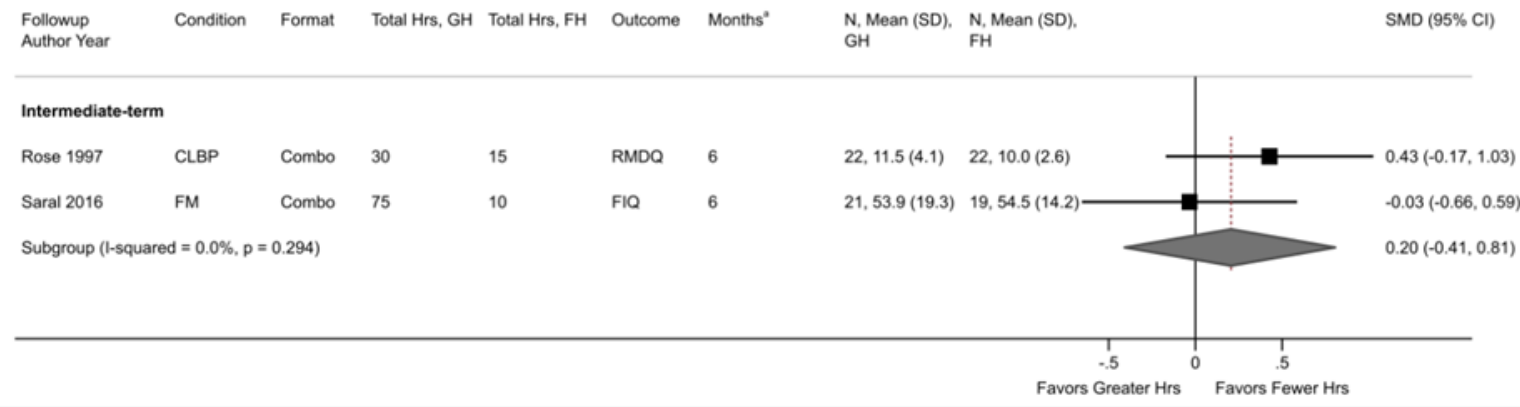

$\mathrm{CI}=$ confidence interval CLBP $=$ chronic low back pain Combo $=$ combination group and individual sessions; $\mathrm{CPMP}=$ comprehensive pain management program; FH = fewer hours; FIQ = Fibromyalgia Impact Questionnaire; FM = fibromyalgia; $\mathrm{GH}=$ greater hours; Hrs = hours; RMDQ = Roland Morris Disability Index; SD = standard deviation.

${ }^{a}$ Followup is in months following the end of the intervention. 
Figure I-44. CPMP with greater versus fewer total hours: Depression at intermediate term

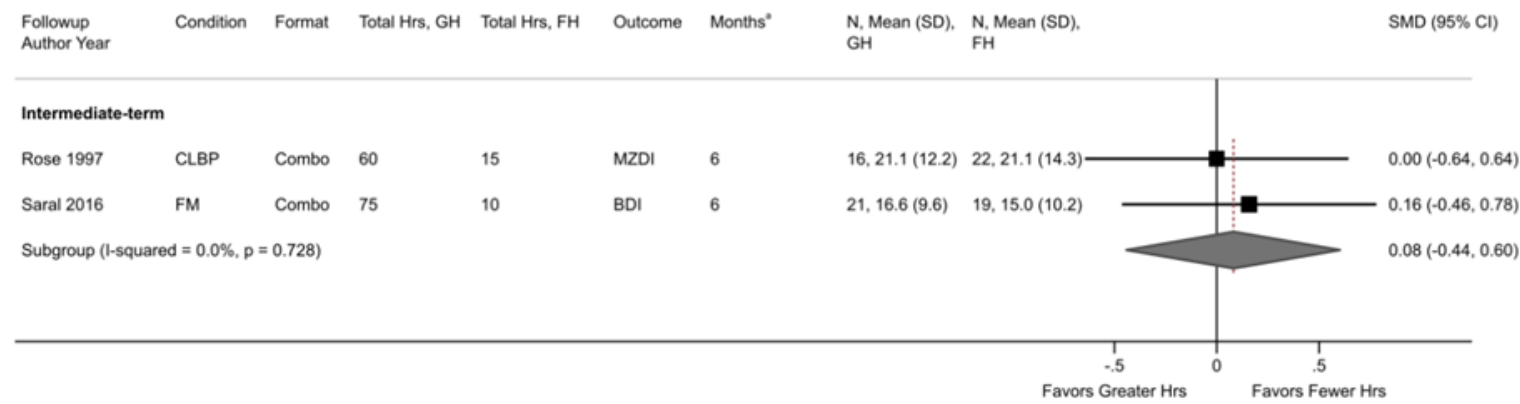

$\mathrm{BDI}=$ Beck Depression Inventory $\mathrm{CI}=$ confidence interval; $\mathrm{CLBP}=$ chronic low back pain; Combo = combination group and individual sessions; $\mathrm{CPMP}=$ comprehensive pain management program; $\mathrm{FH}=$ fewer hours; $\mathrm{FM}=$ fibromyalgia; $\mathrm{GH}=$ greater hours; Hrs = hours; MZDI = modified Zung Depression Inventory; SD = standard deviation.

${ }^{\text {a }}$ Followup is in months following the end of the intervention.

Figure I-45. CPMP with greater versus fewer total hours: Sensitivity analysis for depression at intermediate term using data for the group with 30 hours data for Rose 1997

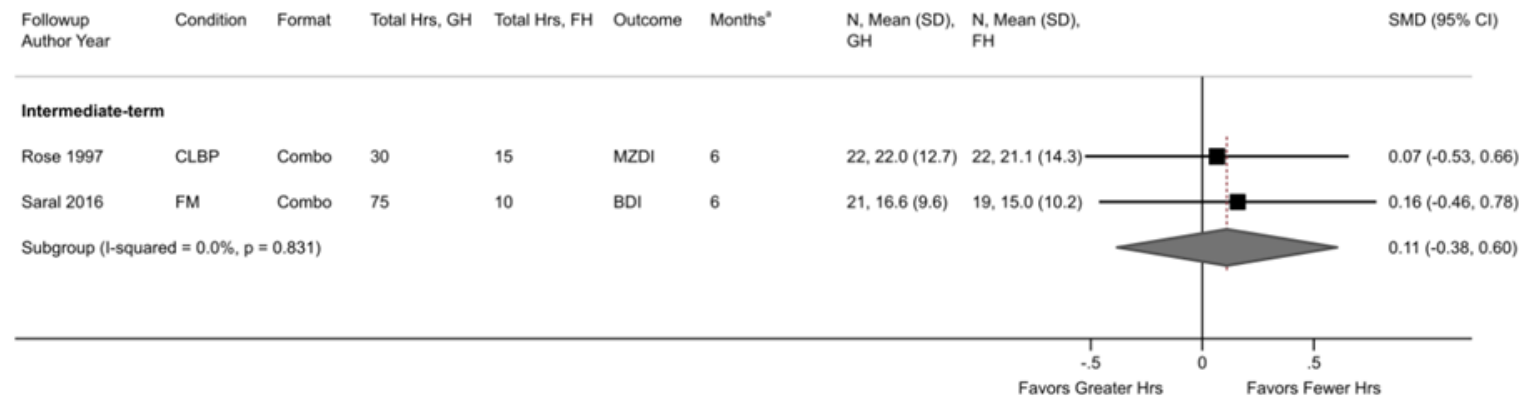

$\mathrm{BDI}=$ Beck Depression Inventory $\mathrm{CI}=$ confidence interval; $\mathrm{CLBP}=$ chronic low back pain; Combo = combination group and individual sessions; $\mathrm{CPMP}=$ comprehensive pain management program; $\mathrm{FH}=$ fewer hours; $\mathrm{FM}=$ fibromyalgia; $\mathrm{GH}=$ greater hours; Hrs = hours; MZDI = modified Zung Depression Inventory; SD = standard deviation.

${ }^{\text {a }}$ Followup is in months following the end of the intervention. 
Figure I-46. CPMP conducted in an inpatient versus outpatient setting: Pain at short and long term

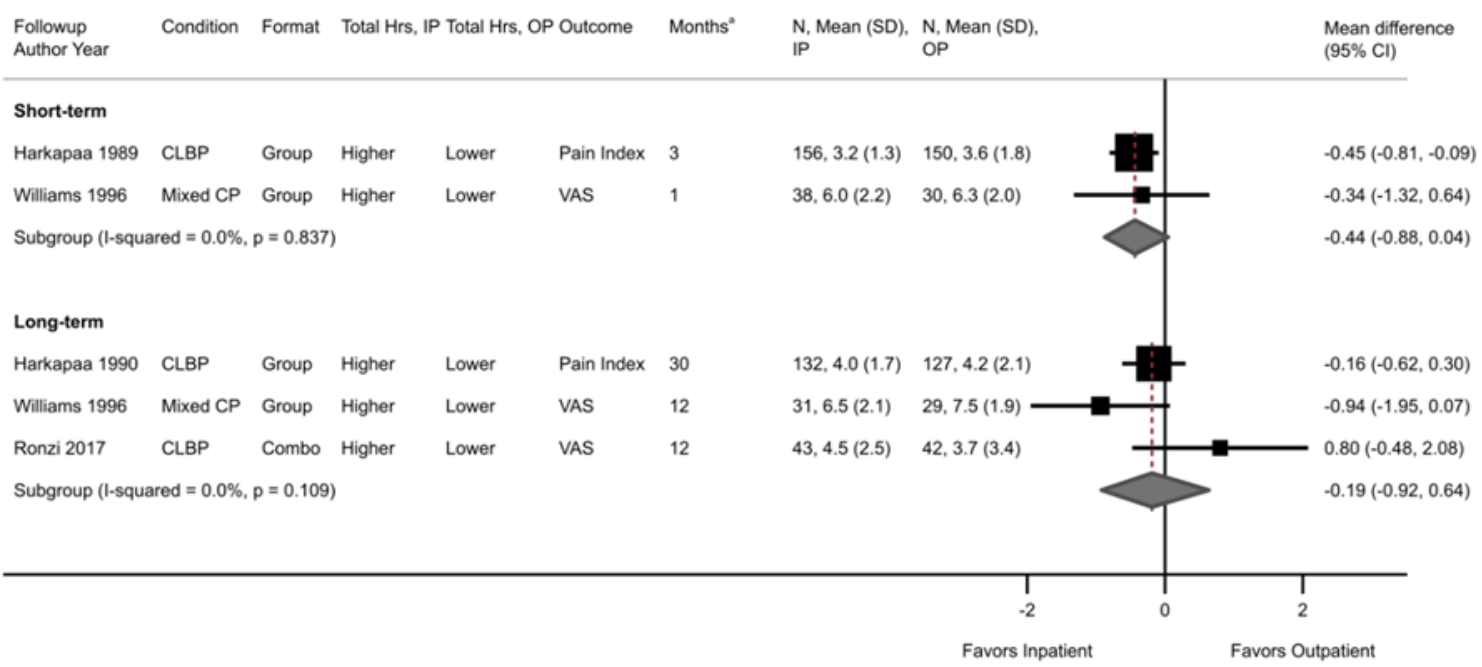

$\mathrm{CI}=$ confidence interval; $\mathrm{CLBP}=$ chronic low back pain $; \mathrm{Combo}=$ combination group and individual sessions; $\mathrm{CP}=$ chronic pain; $\mathrm{CPMP}=$ comprehensive pain management program; $\mathrm{IP}$ = inpatient; $\mathrm{OP}=$ outpatient; $\mathrm{SD}$ = standard deviation; $\mathrm{VAS}$ = visual analog scale.

Figure I-47. CPMP conducted in an inpatient versus outpatient setting: Sensitivity analysis for pain using the most common duration for long-term followup

\begin{tabular}{|c|c|c|c|c|c|c|c|c|c|}
\hline $\begin{array}{l}\text { Followup } \\
\text { Author Year }\end{array}$ & Condition & Format & $\begin{array}{l}\text { Total Hrs, } \\
\text { IP }\end{array}$ & $\begin{array}{l}\text { Total Hrs, } \\
\text { OP }\end{array}$ & Outcome & Months" & N, Mean (SD), & N. Mean (SD), OP & $\begin{array}{l}\text { Mean difference } \\
(95 \% \mathrm{Cl})\end{array}$ \\
\hline \multicolumn{10}{|l|}{ Short-term } \\
\hline Harkapaa 1989 & CLBP & Group & Higher & Lower & Pain Index & 3 & $156,3.2(1.3)$ & $150,3.6(1.8)$ & $-0.45(-0.81,-0.09)$ \\
\hline Williams 1996 & Mixed CP & Group & Higher & Lower & VAS & 1 & $38,6.0(2.2)$ & $30,6.3(2.0)$ & $-0.34(-1.32,0.64)$ \\
\hline \multicolumn{9}{|c|}{ Subgroup (1-squared $=0.0 \%, p=0.837$ ) } & $-0.44(-0.88,0.04)$ \\
\hline \multicolumn{10}{|l|}{ Long-term } \\
\hline Harkapaa 1990 & CLBP & Group & Higher & Lower & Pain Index & 18 & $132,3.9(1.6)$ & $127,4.3(2.2)$ & $-0.44(-0.90,0.03)$ \\
\hline Williams 1996 & Mixed CP & Group & Higher & Lower & VAS & 12 & $31,6.5(2.1)$ & $29,7.5(1.9)$ & $-0.94(-1.95,0.07)$ \\
\hline Ronzi 2017 & CLBP & Combo & Higher & Lower & VAS & 12 & $43,4.5(2.5)$ & $42,3.7(3.4)$ & $0.80(-0.48,2.08)$ \\
\hline \multicolumn{9}{|c|}{ Subgroup (l-squared $=0.0 \%, p=0.105$ ) } & $-0.39(-1.04,0.53)$ \\
\hline & & & & & & & & $T$ & \\
\hline & & & & & & & & Favors Inpatient & itpatient \\
\hline
\end{tabular}

$\mathrm{CI}=$ confidence interval; $\mathrm{CLBP}=$ chronic low back pain; Combo $=$ combination group and individual sessions; $\mathrm{CP}=$ chronic pain; $\mathrm{CPMP}=$ comprehensive pain management program; $\mathrm{IP}=$ inpatient; $\mathrm{OP}=$ outpatient; $\mathrm{SD}=$ standard deviation; $\mathrm{VAS}=$ visual analog scale. 
Figure I-48. CPMP conducted in an inpatient versus outpatient setting: Sensitivity analysis for pain at long term excluding the poor quality, outlier trial

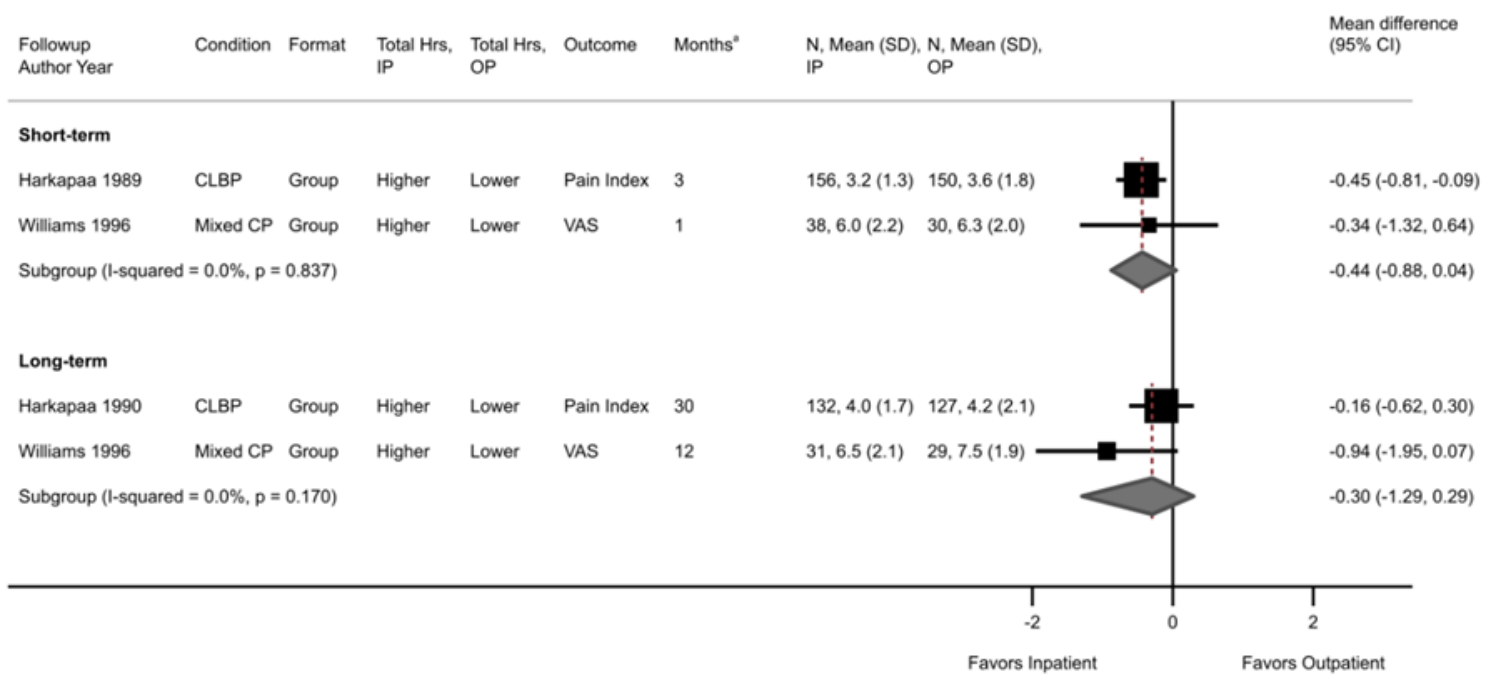

$\mathrm{CI}=$ confidence interval $\mathrm{CLBP}=$ chronic low back pain; Combo $=$ combination group and individual sessions; $\mathrm{CP}=$ chronic pain; $\mathrm{CPMP}=$ comprehensive pain management program; $\mathrm{IP}=$ inpatient; $\mathrm{OP}=$ outpatient; $\mathrm{SD}=$ standard deviation; $\mathrm{VAS}=$ visual analog scale.

Figure I-49. CPMP conducted in an inpatient versus outpatient setting: Function at short and long term

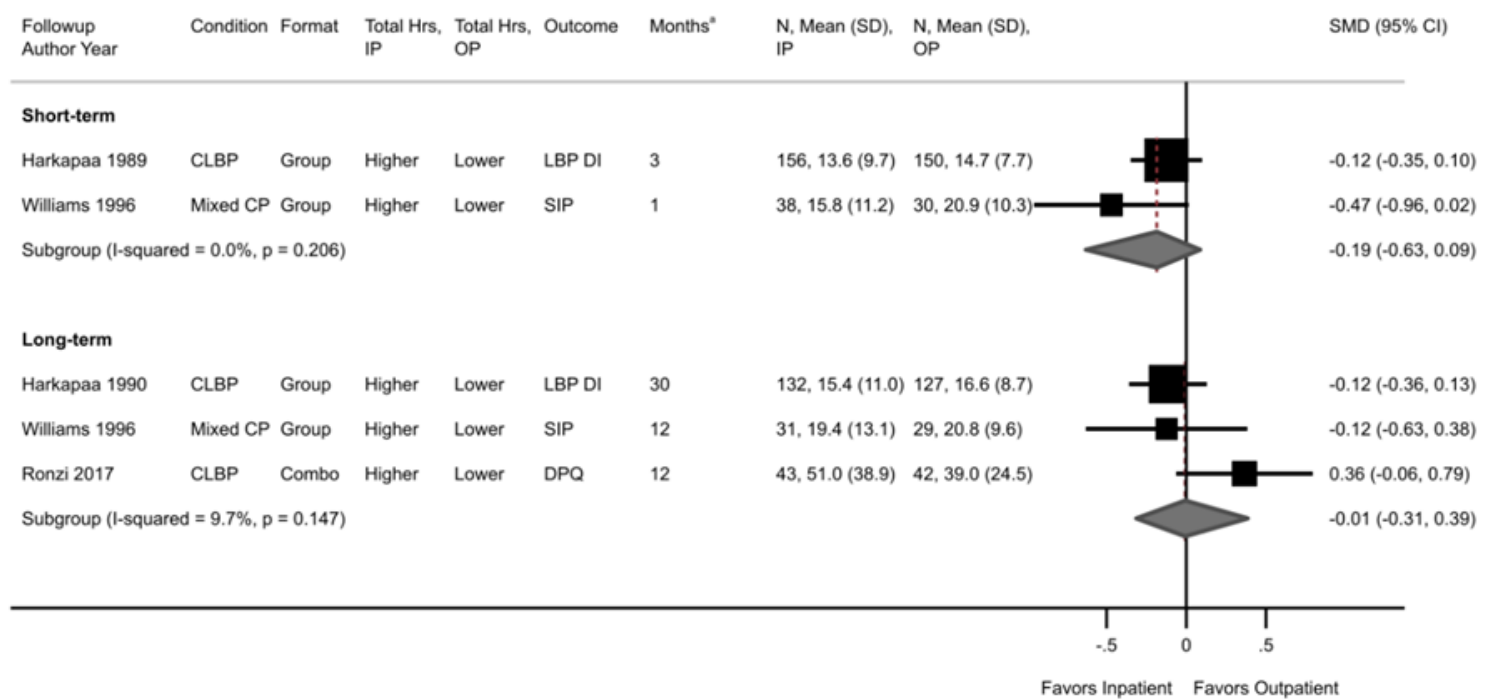

$\mathrm{CI}=$ confidence interval $\mathrm{CLBP}=$ chronic low back pain; Combo $=$ combination group and individual sessions; $\mathrm{CP}=$ chronic pain; $\mathrm{CPMP}=$ comprehensive pain management program; DPQ = Dallas Pain Questionnaire; IP = inpatient; LBP DI = Low Back Pain Disability Index; OP = outpatient; SD = standard deviation; SIP = Sickness Impact Profile; SMD = standardized mean difference. 
Figure I-50. CPMP conducted in an inpatient versus outpatient setting: Sensitivity analysis for function using the most common duration for long-term followup

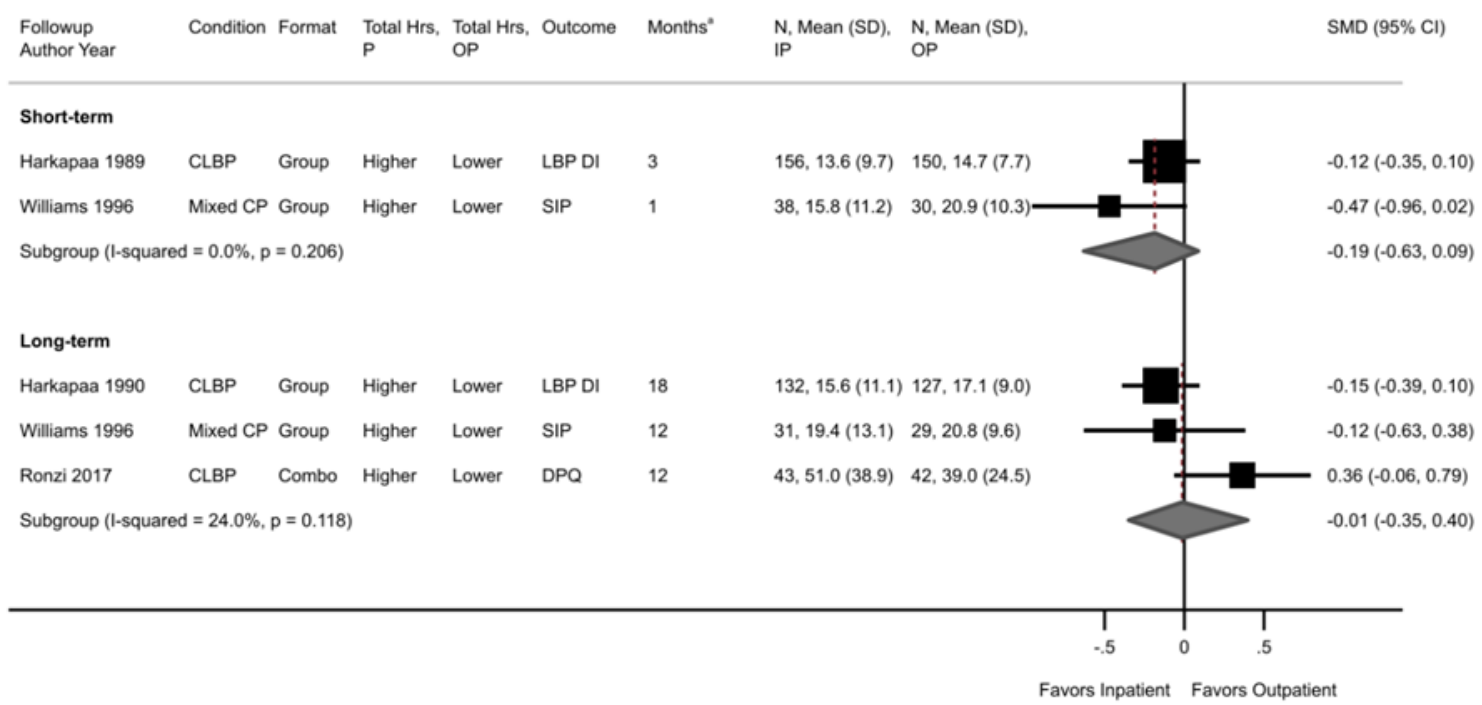

$\mathrm{CI}=$ confidence interval; $\mathrm{CLBP}=$ chronic low back pain $; \mathrm{Combo}=$ combination group and individual sessions; $\mathrm{CP}=$ chronic pain; $\mathrm{CPMP}=$ comprehensive pain management program; DPQ = Dallas Pain Questionnaire; IP = inpatient; LBP DI = Low Back Pain Disability Index; OP = outpatient; SD = standard deviation; SIP = Sickness Impact Profile; SMD = standardized mean difference.

Figure I-51. CPMP conducted in an inpatient versus outpatient setting: Sensitivity analysis for function at long term excluding the poor quality, outlier trial

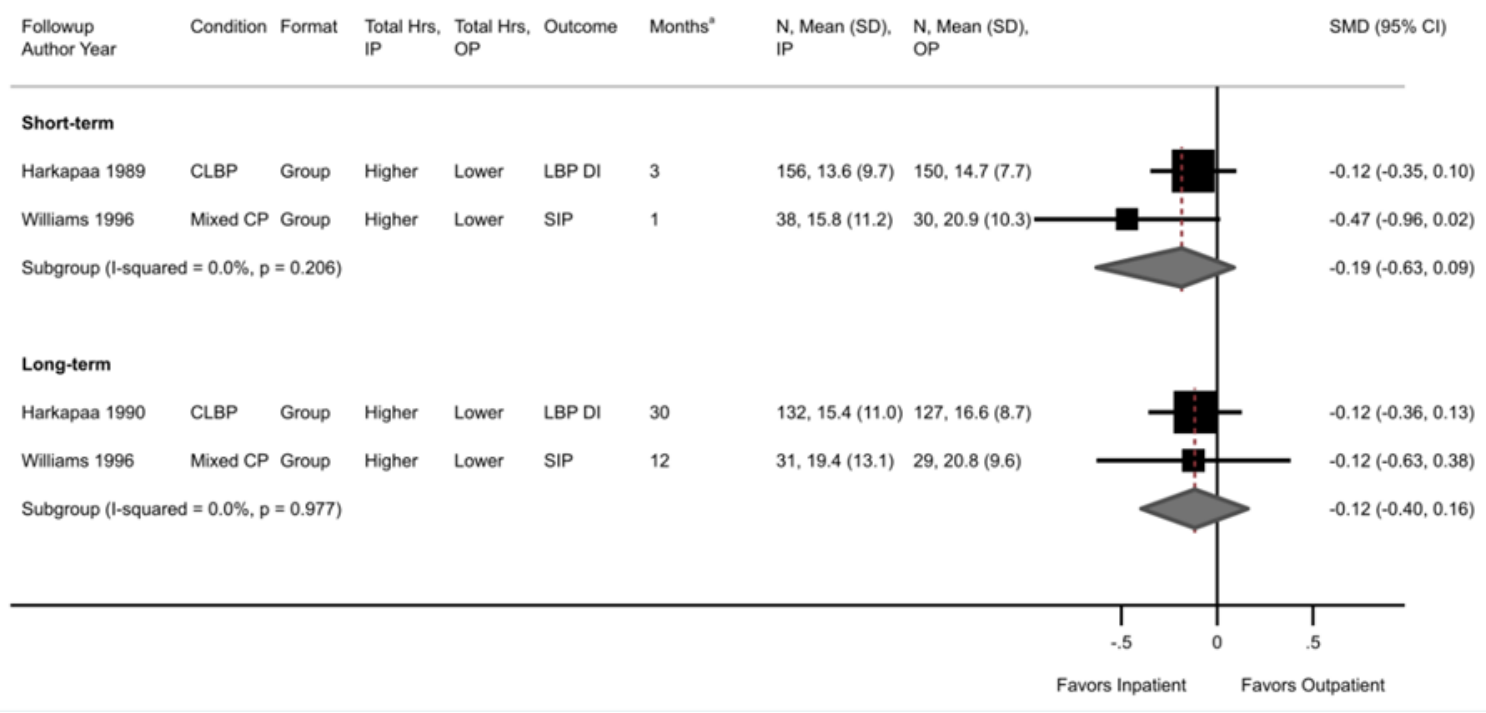

$\mathrm{CI}=$ confidence interval; $\mathrm{CLBP}=$ chronic low back pain; Combo $=$ combination group and individual sessions; $\mathrm{CP}=$ chronic pain; CPMP = comprehensive pain management program; IP = inpatient; LBP DI = Low Back Pain Disability Index; OP = outpatient; SD = standard deviation; SIP = Sickness Impact Profile; SMD = standardized mean difference. 
Figure I-52. CPMP with versus without additional psychological components: Pain at postintervention

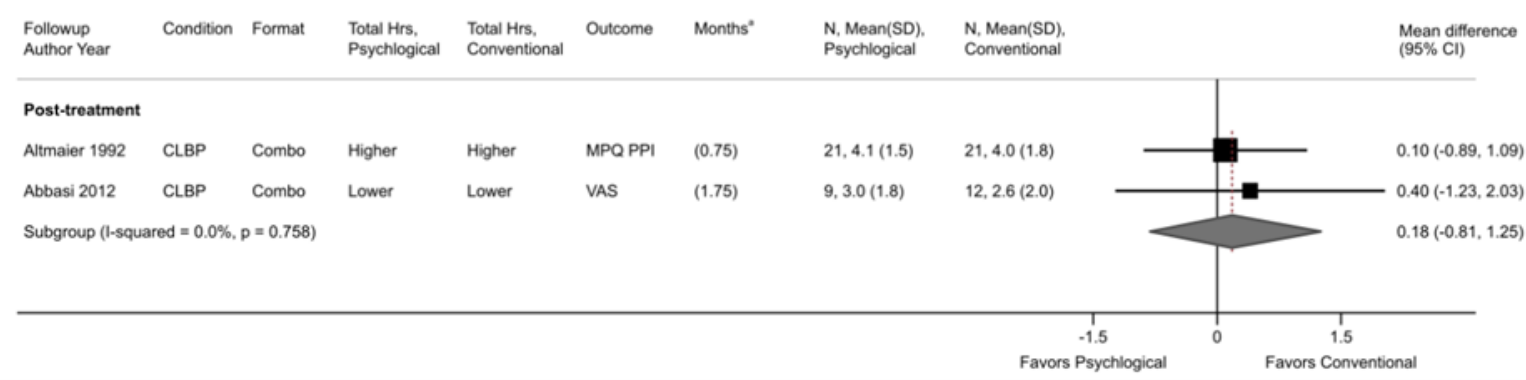

$\mathrm{CI}=$ confidence interval; $\mathrm{CLBP}=$ chronic low back pain $; \mathrm{Combo}=$ combination group and individual sessions; $\mathrm{CP}=$ chronic pain; $\mathrm{CPMP}=$ comprehensive pain management program; $\mathrm{IP}=$ inpatient; $\mathrm{OP}=$ outpatient; $\mathrm{SD}$ = standard deviation; $\mathrm{VAS}=$ visual analog scale.

Figure I-53. CPMP with versus without additional psychological components: Function at postintervention

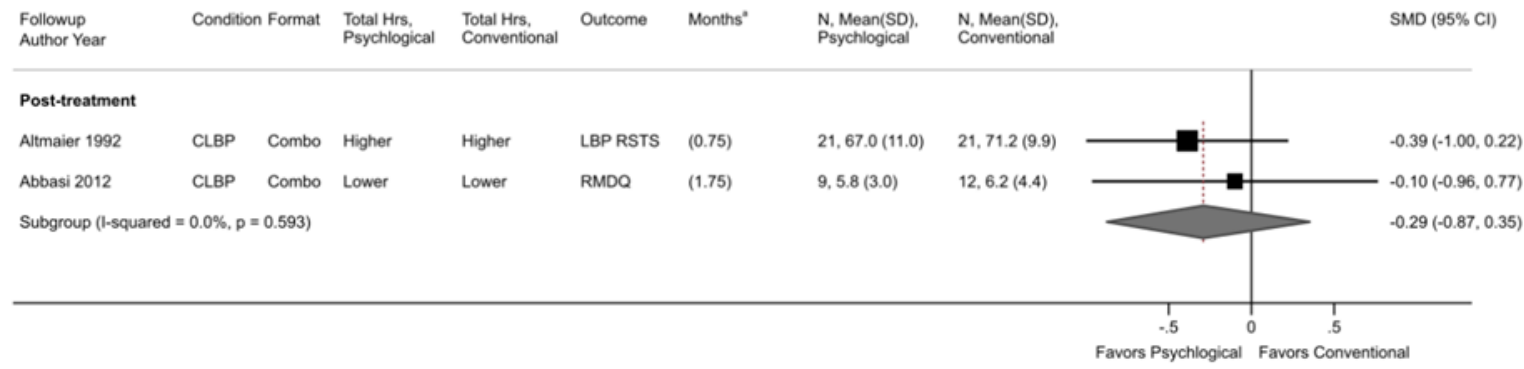

$\mathrm{CI}=$ confidence interval; $\mathrm{CLBP}=$ chronic low back pain; Combo $=$ combination group and individual sessions; $\mathrm{CP}=\mathrm{chronic}$ pain; $\mathrm{CPMP}=$ comprehensive pain management program; IP = inpatient; $\mathrm{OP}=$ outpatient; $\mathrm{SD}=$ standard deviation; VAS = visual analog scale. 


\section{Appendix J. Definitions of Magnitudes of Effect}

Table J-1. Definitions for magnitude of effects, based on mean between-group differences

\begin{tabular}{|c|c|c|c|}
\hline Outcome & Slight/Small & Moderate & Large/Substantial \\
\hline \multirow[t]{2}{*}{ Pain } & $\begin{array}{l}5-10 \text { points on a } 0 \text {-to } 100- \\
\text { point VAS or the equivalent }\end{array}$ & $\begin{array}{l}>10-20 \text { points on a } 0 \text {-to } 100- \\
\text { point VAS or the equivalent }\end{array}$ & $\begin{array}{l}>20 \text { points on a } 0 \text {-to } 100- \\
\text { point VAS or the equivalent }\end{array}$ \\
\hline & $\begin{array}{l}0.5-1.0 \text { points on a } 0 \text {-to } 10- \\
\text { point numerical } \\
\text { rating scale or the equivalent }\end{array}$ & $\begin{array}{l}>1-2 \text { points on a } 0 \text {-to } 10- \\
\text { point numerical } \\
\text { rating scale or the equivalent }\end{array}$ & $\begin{array}{l}>2 \text { points on a } 0 \text {-to } 10 \text {-point } \\
\text { numerical } \\
\text { rating scale or the equivalent }\end{array}$ \\
\hline \multirow[t]{9}{*}{ Function } & 5-10 points on the ODI & $>10-20$ points on the ODI & $>20$ points on the ODI \\
\hline & $1-2$ points on the $R D Q$ & $>2-5$ points on the $R D Q$ & $>5$ points on the $\mathrm{RDQ}$ \\
\hline & $\begin{array}{l}\text { 1-2 points on Lequesne } \\
\text { Index }\end{array}$ & $\begin{array}{l}>2-5 \text { points on the Lequesne } \\
\text { Index }\end{array}$ & $\begin{array}{l}5 \text { points on the Lequesne } \\
\text { Index }\end{array}$ \\
\hline & $5-10$ points on the WOMAC & $\begin{array}{l}>10-20 \text { points on the } \\
\text { WOMAC }\end{array}$ & $>20$ points on the WOMAC \\
\hline & $5-10$ points on the KOOS & $>10-20$ points on the KOOS & $>20$ points on the KOOS \\
\hline & 5-10 points on the NPQ & $>10-20$ points on the NPQ & $>20$ points on the NPQ \\
\hline & $\begin{array}{l}\text { 5-10 points on the FIQ Total } \\
\text { Score }\end{array}$ & $\begin{array}{l}>10-20 \text { points on the FIQ } \\
\text { Total Score }\end{array}$ & $\begin{array}{l}>20 \text { points on the FIQ Total } \\
\text { Score }\end{array}$ \\
\hline & $7.5-10$ points on the NDI & $>10-20$ on the NDI & $>20$ points on the NDI \\
\hline & $1.3-2.2$ on the PSFS & $2.3-2.6$ on the PSFS & $>2.6$ on the PSFS \\
\hline $\begin{array}{l}\text { Pain or } \\
\text { Function }\end{array}$ & $0.2-0.5$ SMD & $>0.5-0.8$ SMD & $>0.8 \mathrm{SMD}$ \\
\hline
\end{tabular}

ODI = Oswestry Disability Index; RDQ = Roland Morris Disability Questionnaire; SMD = standardized mean difference; VAS = visual analogue scale. WOMAC $=$ Western Ontario and Mc Maters Universities Osteoarthritis index; KOOS=Knee Injury and Osteoarthritis Outcome Score; NDI = neck disability index; NPQ, Northwick Park Questionnaire; PSFS, Patient-Specific Functional Scale; FIQ = Fibromyalgia Impact Questionnaire 


\section{Appendix K. Appendix References}

1. Guise JM, Butler ME, Chang C, et al. AHRQ series on complex intervention systematic reviews-paper 6: PRISMA-CI extension statement and checklist. J Clin Epidemiol. 2017 Oct;90:43-50. doi: 10.1016/j.jclinepi.2017.06.016. PMID: 28720516 .

2. Guzman J, Esmail R, Karjalainen KA, et al. Multidisciplinary bio-psycho-social rehabilitation for chronic low-back pain. Cochrane database of systematic reviews. 2002(1).

3. Lewis GN, Bean D, Mowat R. How Have Chronic Pain Management Programs Progressed? A Mapping Review. Pain Practice. 2019;19(7):767-84.

4. Merksey H, Bogduk N. Classification of chronic pain. Descriptions of Chronic Pain Syndromes and Definitions of Pain Term. 1994:59-71.

5. Kosek E, Cohen M, Baron R, et al. Do we need a third mechanistic descriptor for chronic pain states? Pain. 2016 Jul;157(7):1382-6. doi: 10.1097/j.pain.0000000000000507. PMID: 26835783 .

6. Methods Guide for Effectiveness and Comparative Effectiveness Reviews. AHRQ Publication No. 10(14)-EHC063EF. Rockville, MD: Agency for Healthcare Research and Quality. January 2014. Chapters available at: www.effectivehealthcare.ahrq.gov.

7. Chou R, Korthuis PT, Weimer M, et al. Medication-Assisted Treatment Models of Care for Opioid Use Disorder in Primary Care Settings. Technical Brief No. 28. (Prepared by the Pacific Northwest Evidence-based Practice Center under Contract No. 290-2015-00009-I.) AHRQ

Publication No. 16(17)-EHC039-EF. Rockville, MD: Agency for Healthcare Research and Quality; December 2016. https://effectivehealthcare.ahrq.gov/produc ts/opioid-use-disorder/technical-brief. PMID: 28045474.
8. Hoffmann TC, Glasziou PP, Boutron I, et al. Better reporting of interventions: template for intervention description and replication (TIDieR) checklist and guide. Bmj. 2014 Mar 7;348:g1687. doi: 10.1136/bmj.g1687. PMID: 24609605.

9. Viswanathan M, Patnode CD, Berkman ND, et al. Assessing the risk of bias in systematic reviews of health care interventions. Methods guide for effectiveness and comparative effectiveness reviews [Internet]. Agency for Healthcare Research and Quality (US); 2017

10. Higgins JP, Green S. Cochrane handbook for systematic reviews of interventions: John Wiley \& Sons; 2011.

11. Hardy RJ, Thompson SG. A likelihood approach to meta-analysis with random effects. Stat Med. 1996 Mar 30;15(6):61929. doi: 10.1002/(SICI)10970258(19960330)15:6<619::AIDSIM188>3.0.CO;2-A. PMID: 8731004.

12. Higgins JP, Thompson SG, Deeks JJ, et al. Measuring inconsistency in meta-analyses. BMJ. 2003 Sep 6;327(7414):557-60. doi: 10.1136/bmj.327.7414.557. PMID: 12958120 .

13. Pigott T, Noyes J, Umscheid CA, et al. AHRQ series on complex intervention systematic reviews-paper 5: advanced analytic methods. J Clin Epidemiol. 2017 Oct;90:37-42. doi: 10.1016/j.jclinepi.2017.06.015. PMID: 28720512 .

14. Skelly AC, Chou R, Dettori JR, et al. Noninvasive Nonpharmacological Treatment for Chronic Pain: A Systematic Review Update. Comparative Effectiveness Review No. 227. (Prepared by the Pacific Northwest Evidence-based Practice Center under Contract No. 2902015-00009-I.) AHRQ Publication No. 20EHC009. Rockville, MD: Agency for Healthcare Research and Quality; April 2020.

https://effectivehealthcare.ahrq.gov/produc ts/noninvasive-nonpharm-painupdate/research. PMID: 32338846. 
15. Skelly AC, Chou R, Dettori JR, et al. Noninvasive Nonpharmacological Treatment for Chronic Pain: A Systematic Review. Comparative Effectiveness Review No. 209. (Prepared by the Pacific Northwest Evidence-based Practice Center under Contract No. 290-2015-00009-I.) AHRQ Publication No 18-EHC013-EF. Rockville, MD: Agency for Healthcare Research and Quality; June 2018. https://effectivehealthcare.ahrq.gov/topics/ nonpharma-treatment-pain/research-2018. PMID: 30179389.

16. Chou R, Deyo R, Friedly J, et al. Noninvasive Treatment for Low Back Pain. Comparative Effectiveness Review No. 169. (Prepared by the Pacific Northwest Evidence-based Practice Center under Contract No. HHSA 290-201200014-I.) AHRQ Publication No. 16EHC004-EF. Rockville, MD: Agency for Healthcare Research and Quality; February 2016.

https://effectivehealthcare.ahrq.gov/produc ts/back-pain-treatment/research. PMID: 26985522.

17. Chou R, Hartung D, Turner J, et al. Opioid Treatments for Chronic Pain. Comparative Effectiveness Review No. 229. (Prepared by the Pacific Northwest Evidence-based Practice Center under Contract No. 2902015-00009-I.) AHRQ Publication No. 20EHC011. Rockville, MD: Agency for Healthcare Research and Quality; 2020. doi: 10.23970/AHRQEPCCER229. PMID: 32338848 .

18. Chou R, Wagner J, Ahmed AY, et al. Treatments for Acute Pain: A Systematic Review. Comparative Effectiveness Review No. 240. (Prepared by the Pacific Northwest Evidence-based Practice Center under Contract No. 290-2015-00009-I.) AHRQ Publication No. 20(21)-EHC006. Rockville, MD: Agency for Healthcare Research and Quality; December 2020. doi: 10.23970/AHRQEPCCER240. PMID: 33411426 .

19. U.S. Preventive Services Task Force. U.S. Preventive Services Task Force Procedure Manual. 2015. https://www.uspreventiveservicestaskforce .org/uspstf/procedure-manual. Accessed August 252020.
20. Allen KD, Oddone EZ, Coffman CJ, et al. Patient, Provider, and Combined Interventions for Managing Osteoarthritis in Primary Care: A Cluster Randomized Trial. Annals of Internal Medicine. 2017 Mar 21;166(6):401-11. doi: https://dx.doi.org/10.7326/M16-1245. PMID: 28114648.

21. Allen KD, Yancy WS, Jr., Bosworth HB, et al. A Combined Patient and Provider Intervention for Management of Osteoarthritis in Veterans: A Randomized Clinical Trial. Annals of Internal Medicine. 2016 Jan 19;164(2):73-83. doi: https://dx.doi.org/10.7326/M15-0378. PMID: 26720751.

22. Angeles RN, Guenter D, McCarthy L, et al. Group interprofessional chronic pain management in the primary care setting: a pilot study of feasibility and effectiveness in a family health team in Ontario. Pain Research \& Management. 2013 SepOct;18(5):237-42. PMID: 23875181.

23. Beasley M, Prescott GJ, Scotland G, et al. Patient-reported improvements in health are maintained 2 years after completing a short course of cognitive behaviour therapy, exercise or both treatments for chronic widespread pain: long-term results from the MUSICIAN randomised controlled trial. RMD Open. 2015;1(1):e000026. doi: 10.1136/rmdopen2014-000026. PMID: 26509056.

24. Dickinson KC, Sharma R, Duckart JP, et al. VA healthcare costs of a collaborative intervention for chronic pain in primary care. Medical Care. 2010 Jan;48(1):38-44. doi:

https://dx.doi.org/10.1097/MLR.0b013e31 81bd49e2. PMID: 19952802.

25. Dobscha SK, Corson K, Leibowitz RQ, et al. Rationale, design, and baseline findings from a randomized trial of collaborative care for chronic musculoskeletal pain in primary care. Pain Medicine. 2008 Nov;9(8):1050-64. doi: https://dx.doi.org/10.1111/j.15264637.2008.00457.x. PMID: 18565008. 
26. Dobscha SK, Corson K, Perrin NA, et al. Collaborative care for chronic pain in primary care: a cluster randomized trial. JAMA. 2009 Mar 25;301(12):1242-52.

doi:

https://dx.doi.org/10.1001/jama.2009.377. PMID: 19318652.

27. Mas RR, Lopez-Jimenez T, Pujol-Ribera E, et al. Effectiveness of a multidisciplinary BIOPSYCHOSOCIAL intervention for non-specific SUBACUTE low back pain in a working population: a cluster randomized clinical trial. BMC Health Services Research. 2019 Dec 12;19(1):962. doi: https://dx.doi.org/10.1186/s12913-0194810-x. PMID: 31831074.

28. McBeth J, Prescott G, Scotland G, et al. Cognitive behavior therapy, exercise, or both for treating chronic widespread pain. Archives of Internal Medicine. 2012 Jan 09;172(1):48-57. doi:

https://dx.doi.org/10.1001/archinternmed.2 011.555. PMID: 22082706.

29. Von Korff M, Balderson BH, Saunders K, et al. A trial of an activating intervention for chronic back pain in primary care and physical therapy settings. Pain. 2005

Feb;113(3):323-30. doi:

10.1016/j.pain.2004.11.007. PMID: 15661440 .

30. Calner T, Nordin C, Eriksson MK, et al. Effects of a self-guided, web-based activity programme for patients with persistent musculoskeletal pain in primary healthcare: a randomized controlled trial. European journal of pain. 2017(pagination) PMID: CN-01374487 NEW.

31. Ahlmen M, Sullivan M, Bjelle A. Team versus non-team outpatient care in rheumatoid arthritis. A comprehensive outcome evaluation including an overall health measure. Arthritis Rheum. 1988 Apr;31(4):471-9. doi: 10.1002/art.1780310403. PMID: 3358810.

32. Amris K, Wæhrens EE, Christensen R, et al. Interdisciplinary rehabilitation of patients with chronic widespread pain: primary endpoint of the randomized, nonblinded, parallel-group IMPROvE trial. Pain. 2014 Jul;155(7):1356-64. doi: 10.1016/j.pain.2014.04.012. PMID: 24727345 .
33. Bendix AE, Bendix T, Haestrup C, et al. A prospective, randomized 5-year follow-up study of functional restoration in chronic low back pain patients. Eur Spine J. 1998b;7(2):111-9. doi: 10.1007/s005860050040. PMID: 9629934.

34. Bendix AF, Bendix T, Labriola M, et al. Functional restoration for chronic low back pain. Two-year follow-up of two randomized clinical trials. Spine. 1998a Mar 15;23(6):717-25. PMID: 9549794.

35. Bendix AF, Bendix T, Vaegter K, et al. Multidisciplinary intensive treatment for chronic low back pain: a randomized, prospective study. Cleveland Clinic Journal of Medicine. 1996 JanFeb;63(1):62-9. PMID: 8590519.

36. Browne AL, Appleton S, Fong K, et al. A pilot randomized controlled trial of an early multidisciplinary model to prevent disability following traumatic injury. Disability \& Rehabilitation. 2013 Jul;35(14):1149-63. doi: https://dx.doi.org/10.3109/09638288.2012. 721047. PMID: 23083416.

37. de Buck PD, le Cessie S, van den Hout WB, et al. Randomized Comparison of a Multidisciplinary Job-Retention Vocational Rehabilitation Program With Usual Outpatient Care in Patients With Chronic Arthritis at Risk For Job Loss. Arthritis \& Rheumatism: Arthritis Care \& Research. 2005 Oct;53(5):682-90. doi: http://dx.doi.org/10.1002/art.21452. PMID: 2005-13265-006.

38. Jensen IB, Bergström G, Ljungquist $\mathrm{T}$, et al. A randomized controlled component analysis of a behavioral medicine rehabilitation program for chronic spinal pain: are the effects dependent on gender? Pain. 2001 Mar;91(1-2):65-78. doi: 10.1016/s0304-3959(00)00420-6. PMID: 11240079.

39. Johansson C, Dahl J, Jannert M, et al. Effects of a cognitive-behavioral painmanagement program. Behaviour Research \& Therapy. 1998 Oct;36(10):915-30. PMID: 9714943. 
40. Lemstra M, Olszynski W. The Effectiveness of Multidisciplinary Rehabilitation in the Treatment of Fibromyalgia: A Randomized Controlled Trial. The Clinical Journal of Pain. 2005

Mar-Apr;21(2):166-74. doi: http://dx.doi.org/10.1097/00002508200503000-00008. PMID: 2005-02073008 .

41. Peters J, Large RG, Elkind G. Follow-up results from a randomised controlled trial evaluating in- and outpatient pain management programmes. Pain. 1992 Jul;50(1):41-50. PMID: 1381070.

42. Peters JL, Large RG. A randomised control trial evaluating in- and outpatient pain management programmes. Pain. 1990 Jun;41(3):283-93. PMID: 1697057.

43. Saral I, Sindel D, Esmaeilzadeh S, et al. The effects of long- and short-term interdisciplinary treatment approaches in women with fibromyalgia: a randomized controlled trial. Rheumatology International. 2016 Oct;36(10):1379-89. doi: https://dx.doi.org/10.1007/s00296016-3473-8. PMID: 27055444.

44. Scholten C, Brodowicz T, Graninger W, et al. Persistent functional and social benefit 5 years after a multidisciplinary arthritis training program. Archives of Physical Medicine \& Rehabilitation. 1999 Oct;80(10):1282-7. PMID: 10527088.

45. Smeets RJ, Vlaeyen JW, Hidding A, et al. Chronic low back pain: physical training, graded activity with problem solving training, or both? The one-year posttreatment results of a randomized controlled trial. Pain. 2008 Feb;134(3):263-76. PMID: 17498879.

46. Smeets RJ, Vlaeyen JW, Hidding A, et al. Active rehabilitation for chronic low back pain: cognitive-behavioral, physical, or both? First direct post-treatment results from a randomized controlled trial [ISRCTN22714229]. BMC

Musculoskeletal Disorders. 2006a Jan 20;7:5. PMID: 16426449.
47. Smith J, Faux SG, Gardner T, et al. Reboot Online: a Randomized Controlled Trial Comparing an Online Multidisciplinary Pain Management Program with Usual Care for Chronic Pain. Pain medicine. 2019 PMID: CN-01987380 NEW.

48. Turner JA, Clancy S, McQuade KJ, et al. Effectiveness of behavioral therapy for chronic low back pain: a component analysis. Journal of Consulting \& Clinical Psychology. 1990 Oct;58(5):573-9. PMID: 2147702.

49. van Eijk-Hustings Y, Kroese M, Creemers A, et al. Resource utilisation and direct costs in patients with recently diagnosed fibromyalgia who are offered one of three different interventions in a randomised pragmatic trial. Clinical Rheumatology. 2016 May;35(5):1307-15. doi: https://dx.doi.org/10.1007/s10067-0153067-y. PMID: 26409883.

50. van Eijk-Hustings Y, Kroese M, Tan F, et al. Challenges in demonstrating the effectiveness of multidisciplinary treatment on quality of life, participation and health care utilisation in patients with fibromyalgia: a randomised controlled trial. Clinical Rheumatology. 2013

Feb;32(2):199-209. doi: https://dx.doi.org/10.1007/s10067-0122100-7. PMID: 23053692.

51. van Koulil S, van Lankveld W, Kraaimaat FW, et al. Tailored cognitive-behavioural therapy and exercise training improves the physical fitness of patients with fibromyalgia. Annals of the Rheumatic Diseases. 2011 Dec;70(12):2131-3. doi: https://dx.doi.org/10.1136/ard.2010.14857 7. PMID: 21926189.

52. van Koulil S, van Lankveld W, Kraaimaat FW, et al. Tailored cognitive-behavioral therapy and exercise training for high-risk patients with fibromyalgia. Arthritis Care Res (Hoboken). 2010 Oct;62(10):1377-85. doi: 10.1002/acr.20268. PMID: 20521308.

53. Weiner DK, Gentili A, Rossi M, et al. Aging Back Clinics-a Geriatric Syndrome Approach to Treating Chronic Low Back Pain in Older Adults: Results of a Preliminary Randomized Controlled Trial. Pain Med. 2020 Feb 1;21(2):274-90. doi: 10.1093/pm/pnz179. PMID: 31503275. 
54. Whitfill T, Haggard R, Bierner SM, et al. Early intervention options for acute low back pain patients: a randomized clinical trial with one-year follow-up outcomes. Journal of Occupational Rehabilitation. 2010 Jun;20(2):256-63. doi: https://dx.doi.org/10.1007/s10926-0109238-4. PMID: 20369277.

55. Williams AC, Richardson PH, Nicholas MK, et al. Inpatient vs. outpatient pain management: results of a randomised controlled trial. Pain. 1996 Jul;66(1):1322. PMID: 8857627.

56. Abbasi M, Dehghani M, Keefe FJ, et al. Spouse-assisted training in pain coping skills and the outcome of multidisciplinary pain management for chronic low back pain treatment: a 1-year randomized controlled trial. Eur J Pain. 2012 Aug;16(7):1033-43. doi: 10.1002/j.15322149.2011.00097.x. PMID: 22337646.

57. Basler HD, Jäkle C, Kröner-Herwig B. Incorporation of cognitive-behavioral treatment into the medical care of chronic low back patients: a controlled randomized study in German pain treatment centers. Patient Educ Couns. 1997 Jun;31(2):11324. doi: 10.1016/s0738-3991(97)00996-8. PMID: 9216352.

58. Härkäpää K, Järvikoski A, Mellin G, et al. A controlled study on the outcome of inpatient and outpatient treatment of low back pain. Part I. Pain, disability, compliance, and reported treatment benefits three months after treatment. Scand J Rehabil Med. 1989;21(2):81-9. PMID: 2526364.

59. Härkäpää K, Mellin G, Järvikoski A, et al. A controlled study on the outcome of inpatient and outpatient treatment of low back pain. Part III. Long-term follow-up of pain, disability, and compliance. Scand J Rehabil Med. 1990;22(4):181-8. PMID: 2148221.

60. Linton SJ, Boersma K, Jansson M, et al. The effects of cognitive-behavioral and physical therapy preventive interventions on pain-related sick leave: a randomized controlled trial. Clinical Journal of Pain. 2005 Mar-Apr;21(2):109-19. PMID: 15722803 .
61. Alaranta H, Rytökoski U, Rissanen A, et al. Intensive physical and psychosocial training program for patients with chronic low back pain. A controlled clinical trial. Spine (Phila Pa 1976). 1994 Jun 15;19(12):1339-49. doi: 10.1097/00007632-199406000-00007. PMID: 8066514.

62. Bendix AF, Bendix T, Lund C, et al. Comparison of three intensive programs for chronic low back pain patients: a prospective, randomized, observer-blinded study with one-year follow-up. Scand J Rehabil Med. 1997 Jun;29(2):81-9. PMID: 9198257.

63. Bendix AF, Bendix T, Ostenfeld S, et al. Active treatment programs for patients with chronic low back pain: a prospective, randomized, observer-blinded study. European Spine Journal. 1995;4(3):148-52. PMID: 7552649.

64. Bendix T, Bendix A, Labriola M, et al. Functional restoration versus outpatient physical training in chronic low back pain: a randomized comparative study. Spine. 2000 Oct 01;25(19):2494-500. PMID: 11013502.

65. Henchoz Y, de Goumoens P, So AK, et al. Functional multidisciplinary rehabilitation versus outpatient physiotherapy for non specific low back pain: randomized controlled trial. Swiss Medical Weekly. 2010;140:w13133. doi: https://dx.doi.org/10.4414/smw.2010.1313 3. PMID: 21181567.

66. Jousset N, Fanello S, Bontoux L, et al. Effects of functional restoration versus 3 hours per week physical therapy: a randomized controlled study. Spine (Phila Pa 1976). 2004 Mar 1;29(5):487-93; discussion 94. doi: 10.1097/01.brs.0000102320.35490.43. PMID: 15129059.

67. Kaapa EH, Frantsi K, Sarna S, et al. Multidisciplinary group rehabilitation versus individual physiotherapy for chronic nonspecific low back pain: a randomized trial. Spine. $2006 \mathrm{Feb}$ 15;31(4):371-6. PMID: 16481945. 
68. Roche G, Ponthieux A, Parot-Shinkel E, et al. Comparison of a functional restoration program with active individual physical therapy for patients with chronic low back pain: a randomized controlled trial. Arch Phys Med Rehabil. 2007 Oct;88(10):122935. doi: 10.1016/j.apmr.2007.07.014. PMID: 17908562.

69. Roche-Leboucher G, Petit-Lemanac'h A, Bontoux L, et al. Multidisciplinary intensive functional restoration versus outpatient active physiotherapy in chronic low back pain: a randomized controlled trial. Spine (Phila Pa 1976). 2011 Dec 15;36(26):2235-42. doi: 10.1097/BRS.0b013e3182191e13. PMID: 21415807.

70. Ronzi Y, Roche-Leboucher G, Begue C, et al. Efficiency of three treatment strategies on occupational and quality of life impairments for chronic low back pain patients: is the multidisciplinary approach the key feature to success? Clinical Rehabilitation. 2017 Oct;31(10):1364-73. doi:

https://dx.doi.org/10.1177/0269215517691 086. PMID: 28592147.

71. Schweikert B, Jacobi E, Seitz R, et al. Effectiveness and cost-effectiveness of adding a cognitive behavioral treatment to the rehabilitation of chronic low back pain. Journal of Rheumatology. 2006 Dec;33(12):2519-26. PMID: 17143986.

72. Mangels M, Schwarz S, Worringen U, et al. Evaluation of a behavioral-medical inpatient rehabilitation treatment including booster sessions: a randomized controlled study. Clinical Journal of Pain. 2009 Jun;25(5):356-64. doi: https://dx.doi.org/10.1097/AJP.0b013e318 1925791. PMID: 19454868.

73. Meyer K, Fransen J, Huwiler H, et al. Feasibility and results of a randomised pilot-study of a work rehabilitation programme. Journal of Back and Musculoskeletal Rehabilitation. 2005;18(3-4):67-78.
74. Turner-Stokes L, Erkeller-Yuksel F, Miles A, et al. Outpatient cognitive behavioral pain management programs: a randomized comparison of a group-based multidisciplinary versus an individual therapy model. Archives of Physical Medicine \& Rehabilitation. 2003 Jun;84(6):781-8. PMID: 12808527.

75. Castel A, Castro S, Fontova R, et al. Body mass index and response to a multidisciplinary treatment of fibromyalgia. Rheumatology International. 2015 Feb;35(2):303-14. doi: https://dx.doi.org/10.1007/s00296-0143096-x. PMID: 25080875.

76. Castel A, Fontova R, Montull S, et al. Efficacy of a multidisciplinary fibromyalgia treatment adapted for women with low educational levels: a randomized controlled trial. Arthritis Care Res (Hoboken). 2013 Mar;65(3):421-31. doi: 10.1002/acr.21818. PMID: 22899402.

77. Martin J, Torre F, Aguirre U, et al. Evaluation of the interdisciplinary PSYMEPHY treatment on patients with fibromyalgia: a randomized control trial. Pain Medicine. 2014c Apr;15(4):682-91. doi: https://dx.doi.org/10.1111/pme.12375. PMID: 24576148.

78. Martin J, Torre F, Padierna A, et al. Impact of interdisciplinary treatment on physical and psychosocial parameters in patients with fibromyalgia: results of a randomised trial. International journal of clinical practice. 2014a;68(5):618-27. PMID: CN00988924.

79. Martin J, Torre F, Padierna A, et al. Interdisciplinary treatment of patients with fibromyalgia: improvement of their healthrelated quality of life. Pain Practice. 2014b Nov;14(8):721-31. doi:

https://dx.doi.org/10.1111/papr.12134. PMID: 24279638.

80. Onac I, Moldovan A, Igna R, et al. Mechanism of change following multidisciplinary treatment of low back pain secondary to lumbar disc prolapse. Journal of Evidence-Based Psychotherapies. 2017 Sep;17(2):159-73. doi:

http://dx.doi.org/10.24193/jebp.2017.2.10. PMID: 2017-54116-010. 
81. Onac I, Moldovan A, Onac I, et al. Medication, physiotherapy and cognitive behavior therapy for the treatment of chronic back pain: a clinical trial. Journal of Cognitive and Behavioral

Psychotherapies. 2012 Mar;12(1):23-37.

82. Salvat I, Zaldivar P, Monterde S, et al. Functional status, physical activity level, and exercise regularity in patients with fibromyalgia after Multidisciplinary treatment: retrospective analysis of a randomized controlled trial. Rheumatology International. 2017 Mar;37(3):377-87. doi: https://dx.doi.org/10.1007/s00296-0163597-x. PMID: 27844124.

83. Tavafian SS, Jamshidi AR, Mohammad K. Treatment of chronic low back pain: a randomized clinical trial comparing multidisciplinary group-based rehabilitation program and oral drug treatment with oral drug treatment alone. Clinical Journal of Pain. 2011 NovDec;27(9):811-8. doi: https://dx.doi.org/10.1097/AJP.0b013e318 21e7930. PMID: 21642845.

84. Tavafian SS, Jamshidi AR, Mohammad K. Treatment of low back pain: randomized clinical trial comparing a multidisciplinary group-based rehabilitation program with oral drug treatment up to 12 months. International Journal of Rheumatic Diseases. 2014 Feb;17(2):159-64. doi: https://dx.doi.org/10.1111/1756185X.12116. PMID: 24576271.

85. Tavafian SS, Jamshidi AR, Mohammad K. Treatment of low back pain: Second extended follow up of an original trial (NCT00600197) comparing a multidisciplinary group-based rehabilitation program with oral drug treatment alone up to 30 months. International Journal of Rheumatic Diseases. 2017b Dec;20(12):1910-6. doi: https://dx.doi.org/10.1111/1756185X.12540. PMID: 25546488.

86. Tavafian SS, Jamshidi AR, Montazeri A. A randomized study of back school in women with chronic low back pain: quality of life at three, six, and twelve months follow-up. Spine (Phila Pa 1976). 2008 Jul 1;33(15):1617-21. doi: 10.1097/BRS.0b013e31817bd31c. PMID: 18580739.
87. Tavafian SS, Jamshidi AR, Shay B. Treatment of low back pain: first extended follow up of an original trial (NCT00600197) comparing a multidisciplinary group-based rehabilitation program with oral drug treatment alone up to 24 months. International journal of rheumatic diseases. 2017a;20(12):1902-9. PMID: CN01449489 NEW.

88. Thieme K, Gromnica-Ihle E, Flor H. Operant behavioral treatment of fibromyalgia: a controlled study. Arthritis Rheum. 2003 Jun 15;49(3):314-20. doi: 10.1002/art.11124. PMID: 12794785.

89. Reneman MF, Waterschoot FPC, Burgerhof JGM, et al. Dosage of pain rehabilitation programmes for patients with chronic musculoskeletal pain: a noninferiority randomised controlled trial. Disability and rehabilitation. 2020;42(6):814-21. PMID: CN-02100807 NEW.

90. Rose MJ, Reilly JP, Pennie B, et al. Chronic low back pain rehabilitation programs: a study of the optimum duration of treatment and a comparison of group and individual therapy. Spine. 1997 Oct 01;22(19):2246-51; discussion 52-3. PMID: 9346145.

91. Altmaier EM, Lehmann TR, Russell DW, et al. The effectiveness of psychological interventions for the rehabilitation of low back pain: a randomized controlled trial evaluation. Pain. 1992 Jun;49(3):329-35. doi: 10.1016/0304-3959(92)90240-c. PMID: 1408299.

92. Van der Maas LC, Koke A, Pont M, et al. Improving the Multidisciplinary Treatment of Chronic Pain by Stimulating Body Awareness: A Cluster-randomized Trial. Clinical Journal of Pain. 2015

Jul;31(7):660-9. doi: https://dx.doi.org/10.1097/AJP.000000000 0000138. PMID: 25119509. 
93. Rothman MG, Ortendahl M, Rosenblad A, et al. Improved quality of life, working ability, and patient satisfaction after a pretreatment multimodal assessment method in patients with mixed chronic muscular pain: A randomized-controlled study. The Clinical Journal of Pain. 2013 Mar;29(3):195-204. PMID: 2013-10222002 .

94. Streibelt M, Bethge M. Effects of intensified work-related multidisciplinary rehabilitation on occupational participation: a randomized-controlled trial in patients with chronic musculoskeletal disorders. International Journal of Rehabilitation Research. 2014 Mar;37(1):61-6. doi: https://dx.doi.org/10.1097/MRR.00000000 00000031. PMID: 24056065.

95. Kool J, Bachmann S, Oesch P, et al. Function-centered rehabilitation increases work days in patients with nonacute nonspecific low back pain: 1-year results from a randomized controlled trial. Arch Phys Med Rehabil. 2007 Sep;88(9):108994. doi: 10.1016/j.apmr.2007.05.022. PMID: 17826451 .

96. Leeuw M, Goossens ME, van Breukelen GJ, et al. Exposure in vivo versus operant graded activity in chronic low back pain patients: results of a randomized controlled trial. Pain. 2008 Aug 15;138(1):192-207. doi: 10.1016/j.pain.2007.12.009. PMID: 18242858 .

97. Kool JP, Oesch PR, Bachmann S, et al. Increasing days at work using functioncentered rehabilitation in nonacute nonspecific low back pain: a randomized controlled trial. Arch Phys Med Rehabil. 2005 May;86(5):857-64. doi: 10.1016/j.apmr.2004.10.044. PMID: 15895328.

98. Interagency Pain Research Coordinating Committee. National Pain Strategy: a comprehensive population health-level strategy for pain. Washington, DC: Department of Health and Human Services. 2015.
99. Institute of Medicine of the National Academies. Relieving Pain in America: A Blueprint for Transforming Prevention, Care, Education, and Research. Washington DC: The National Academies Press; 2011. http://www.ncbi.nlm.nih.gov/pubmed/2255 3896doi: 10.17226/13172.

100. U.S. Department of Health and Human Services. Pain Management Best Practices Inter-Agency Task Force Report: Updates, Gaps, Inconsistencies, and Recommendations U. S. Department of Health and Human Services. May 9, 2019 2019.

https://www.hhs.gov/sites/default/files/pmt f-final-report-2019-05-23.pdf.

101. International Association for the Study of Pain (IASP) Task Force. Task Force on Multimodal Pain Treatment Defines Terms for Chronic Pain Care. December 14, 2017. Accessed at: https://www.iasppain.org/PublicationsNews/NewsDetail.asp $\underline{x}$ ?ItemNumber $=6981$.

102. Peterson K, Anderson J, Bourne D, et al. Effectiveness of Models Used to Deliver Multimodal Care for Chronic Musculoskeletal Pain: a Rapid Evidence Review. J Gen Intern Med. 2018

May;33(Suppl 1):71-81. doi: 10.1007/s11606-018-4328-7. PMID: 29633140.

103. Gatchel RJ, McGeary DD, McGeary CA, et al. Interdisciplinary chronic pain management: past, present, and future. Am Psychol. 2014 Feb-Mar;69(2):119-30. doi: 10.1037/a0035514. PMID: 24547798.

104. Joypaul S, Kelly F, McMillan SS, et al. Multi-disciplinary interventions for chronic pain involving education: A systematic review. PLoS One. 2019;14(10):e0223306. doi: 10.1371/journal.pone.0223306. PMID: 31577827.

105. Hansen KA, McKernan LC, Carter SD, et al. A Replicable and Sustainable Whole Person Care Model for Chronic Pain. J Altern Complement Med. 2019 Mar;25(S1):S86-S94. doi: 10.1089/acm.2018.0420. PMID: 30870025 . 
106. National Academies of Sciences, Engineering, and Medicine; Health and Medicine Division; Board on Global Health; Board on Health Sciences Policy; Global Forum on Innovation in Health Professional Education; Forum on Neuroscience and Nervous System Disorders. Clare Stroud, Sheena M. Posey Norris, Lisa Bain, editors. The Role of Nonpharmacological Approaches to Pain Management: Proceedings of a Workshop. Washington, DC: Press NA; 2019. doi: 10.17226/25406. PMID: 31145569.

107. Dr. Robert Bree Collaborative. Collaborative Care for Chronic Pain Report and Recommendations. 2018. https://www.qualityhealth.org/bree/topicareas/chronic-pain/.

108. Allen KD, Bosworth HB, Brock DS, et al. Patient and provider interventions for managing osteoarthritis in primary care: protocols for two randomized controlled trials. BMC Musculoskelet Disord. 2012 Apr 24;13:60. doi: 10.1186/1471-2474-1360. PMID: 22530979.

109. Purcell N, Zamora K, Tighe J, et al. The Integrated Pain Team: A Mixed-Methods Evaluation of the Impact of an Embedded Interdisciplinary Pain Care Intervention on Primary Care Team Satisfaction, Confidence, and Perceptions of Care Effectiveness. Pain Med. 2018 Sep 1;19(9):1748-63. doi: 10.1093/pm/pnx254. PMID: 29040715.

110. Goertz CM, Salsbury SA, Long CR, et al. Patient-centered professional practice models for managing low back pain in older adults: a pilot randomized controlled trial. BMC Geriatr. 2017 Oct 13;17(1):235. doi: 10.1186/s12877-017-0624-z. PMID: 29029606.

111. Kroenke K, Bair MJ, Damush TM, et al. Optimized antidepressant therapy and pain self-management in primary care patients with depression and musculoskeletal pain: a randomized controlled trial. JAMA. 2009 May 27;301(20):2099-110. doi: 10.1001/jama.2009.723. PMID: 19470987.
112. Bokhour BG, Haun JN, Hyde J, et al. Transforming the Veterans Affairs to a Whole Health System of Care: Time for Action and Research. Med Care. 2020 Apr;58(4):295-300. doi: 10.1097/MLR.0000000000001316. PMID: 32044866 .

113. Krejci LP, Carter K, Gaudet T. Whole health: the vision and implementation of personalized, proactive, patient-driven health care for veterans. Med Care. 2014 Dec;52(12 Suppl 5):S5-8. doi: 10.1097/MLR.0000000000000226. PMID: 25397823.

114. Smeets RJ, Severens JL, Beelen S, et al. More is not always better: costeffectiveness analysis of combined, single behavioral and single physical rehabilitation programs for chronic low back pain. Eur J Pain. 2009 Jan;13(1):7181. doi: 10.1016/j.ejpain.2008.02.008. PMID: 18434221.

115. Jensen C, Nielsen CV, Jensen OK, et al. Cost-effectiveness and cost-benefit analyses of a multidisciplinary intervention compared with a brief intervention to facilitate return to work in sick-listed patients with low back pain. Spine (Phila Pa 1976). 2013 Jun 1;38(13):1059-67. doi: 10.1097/BRS.0b013e31828ca0af. PMID: 23429675.

116. Jensen IB, Busch H, Bodin L, et al. Cost effectiveness of two rehabilitation programmes for neck and back pain patients: A seven year follow-up. Pain. 2009 Apr;142(3):202-8. doi: 10.1016/j.pain.2008.12.015. PMID: 19217717.

117. van den Hout WB, de Buck PD, Vliet Vlieland TP. Cost-utility analysis of a multidisciplinary job retention vocational rehabilitation program in patients with chronic arthritis at risk of job loss. Arthritis Rheum. 2007 Jun 15;57(5):77886. doi: 10.1002/art.22786. PMID: 17530677. 